\title{
Standortbasierte Ertragsmodellierung von Pappel- und Weidenklonen in Kurzumtriebsplantagen
}

Dissertation zur Erlangung des akademischen Grades

Doctor rerum naturalium (Dr. rer. nat.)

vorgelegt von

Dipl.-Geoökol. Daniel Amthauer Gallardo

geboren am 26.10.1981 in Los Angeles, Chile

Gutachter: Prof. Dr. habil. Heinz Röhle, Technische Universität Dresden

Prof. Dr. habil. Norbert Lamersdorf, Universität Göttingen

Prof. Dr. habil. Albrecht Bemmann, Technische Universität Dresden

Tag der Verteidigung: 13.05.2014 


\section{Erklärung des Promovenden}

Die Übereinstimmung dieses Exemplars mit dem Original der Dissertation zum Thema:

„Standortbasierte Ertragsmodellierung von Pappel- und Weidenklonen in

Kurzumtriebsplantagen"

wird hiermit bestätigt.

Daniel Amthauer Gallardo

Tharandt, Mai 2014 


\section{Abstract}

The cultivation of fast growing tree species on arable land is deemed to be one of the most promising alternatives amongst the approaches to the cultivation of renewable resources currently available. As the site factors influencing the growth of poplar and willow have not yet been sufficiently identified and quantified, it is not possible to provide reliable national yield expectations as a function of the prevailing soil and climate conditions at a particular site on the basis of the data currently available. The main objective of this study, therefore, was to develop a supraregional site-based yield model for the assessment of yield expectations for poplar and willow grown on arable land over short rotations.

In order to achieve this goal, a total of 38 research sites were established across as many regions of Germany as possible. The clones selected for the experiment were the poplar clones Max 1, Hybride 275 (H 275) and AF 2, and the willow clones Inger and Tordis. Each site was also characterised, on the basis of climate and soil data. From the site parameters recorded, variables were defined for modelling purposes. The climate variables comprised temperature $(\mathrm{T})$, precipitation $(\mathrm{N})$ and aridity index (TI) derived from total or average values from selected periods during the year and during the vegetation period. Within the variables the months were indexed as numbers (January $=1$ ), periods were separated by comma. Parameters of the German soil appraisal such as the condition grade (ZS), the soil quality index value (BZ) and the arable land quality index value (AZ) were used as soil variables. The selected texture parameters were the proportions of sand $\left(\mathrm{S}_{[\%]}\right)$, silt $\left(\mathrm{U}_{[\%]}\right)$ and clay $\left(\mathrm{T}_{[\%]}\right)$. Variables of the pore space parameters were the available field moisture capacity (nFK), the air capacity (LK) and the dry bulk density (TRD). Both the texture and the pore space variables referred to a soil depth of 0 to $60 \mathrm{~cm}$. To increase the precision of the results, site clusters were derived, differentiated by main soil type, and the variables were aggregated. The dGZ, measured in odt $\mathrm{ha}^{-1} \mathrm{a}^{-1}$, at the end of the first three-year rotation was chosen as the dependent variable.

The main results of the study are outlined in the following: Negative correlations between dGZ and temperatures in the vegetation period, especially in the months July to September, were observed on the sandy and loamy soils. Precipitation variables always had a positive effect on the growth of poplar and willow across all clones and site clusters. Taking into consideration all experimental sites, precipitation in the period from May to July was most important. The parameters of the German soil appraisal revealed a moderate correlation with growth for all sites. From the appraisal of all sites and of the individual site clusters it became apparent that $\mathrm{U}_{[\%]}$ represents the most important texture variable for poplar and willow growth. Considered for all sites simultaneously, the nFK had the greatest significance for growth across all of the parameters examined.

The site-based yield models were all univariate and often comprised aggregated variables. Under consideration of all sites, the model predictors for Max 1, Inger and Tordis were $\left(\mathrm{nFK} * \mathrm{TI}_{5.7}\right)$ and $\left(\mathrm{nFK} * \mathrm{~N}_{5.7}\right)$ for AF 2. Each model was calculated with the inverse or sigmoid approach and revealed an $\mathrm{R}^{2}$ korr between 0.45 and 0.64 with a RMSE of an average of 2.0 odt ha ${ }^{-1} \mathrm{a}^{-1}$. The division into site clusters improved the accuracy of the models considerably. In the sandy site cluster, the models exhibited an $\mathrm{R}^{2}$ korr of 0.77 to 0.97 and an RMSE of 0.95 to 1.36 odt ha $^{-1} \mathrm{a}^{-1}$. These comprised $\left(\mathrm{U}_{[\%]} * \mathrm{~N}_{6.7}\right)$ for Max 1 and AF 2, $\left(\mathrm{S}_{[\%]} / \mathrm{TI}_{6.7}\right)$ for $\mathrm{H} 275$ and $\left(\mathrm{S}_{[\%]} * \mathrm{~T}_{7.8}\right)$ for Inger and Tordis. For the silty site cluster, significant models could only be determined for the clones Max 1 and Tordis. The model predictor for Max 1 was $\left(\mathrm{BZ} * \mathrm{TI}_{4.5}\right)$ and for Tordis solely (BZ). The calculated $\mathrm{R}^{2}$ korr values were 0.84 and 0.95 with a corresponding RMSE of 0.22 and 0.62 odt $\mathrm{ha}^{-1} \mathrm{a}^{-1}$, respectively. For the loamy soils the models for Max 1 and Inger comprised the variable nFK, for AF 2 the variables $\left(n F K * N_{5.6}\right) . \mathrm{R}_{\text {korr }}^{2}$ varied between 0.86 and 0.98 with RMSE between 0.56 and 1.21 odt ha ${ }^{-1} \mathrm{a}^{-1}$. 


\section{Danksagung}

Diese Arbeit basiert auf Grundlage des Verbundvorhaben PROLOC, ein Projekt mit dem Ziel die überregionalen Ertragspotentiale von Pappel- und Weidenklonen in Kurzumtriebsplantagen zu erfassen. Insgesamt wirkten 24, an der Thematik der Kurzumtriebsplantagen interessierte, deutsche Forschungsinstitutionen der Land- und Forstwirtschaft mit. Das Verbundvorhaben wurde von der Fachagentur Nachwachsende Rohstoffe e. V. finanziert. Die Koordination des Projektes übernahm das Kompetenzzentrum HessenRohstoffe (HeRo) e.V. unter der Leitung von Herrn Dr. Martin Hofmann. Ihm sowie dem damaligen HeRo-Geschäftsführer Herrn Klaus Wagner danke ich für das mir entgegengebrachte Vertrauen zur wissenschaftlichen Bearbeitung des Verbundvorhabens PROLOC.

Diese Dissertation entstand durch die wissenschaftliche Bearbeitung des Projektes: Herrn Prof. Dr. Heinz Röhle, Inhaber des Lehrstuhls für Waldwachstum und Holzmesskunde am Institut für Waldwachstum und Forstliche Informatik der Technischen Universität Dresden danke ich herzlich für seine inspirierende und stets freundliche Art sowie für die fachlich exzellente wissenschaftliche Betreuung. Ohne Frage war er für mich die wichtigste Stütze, um die wissenschaftliche Ausarbeitung des Projektes zu erzielen. Herrn Prof. Dr. Norbert Lamersdorf und Herrn Prof. Dr. Albrecht Bemmann danke ich für ihre Bereitschaft Gutachter meiner Arbeit zu sein.

Des Weiteren danke ich meinem Kollegen Christian Siebert (Projektkoordination), der mir häufig den Rücken frei hielt, damit ich mich ganz und gar der Wissenschaft widmen konnte. Nicole Heyn und Frau Dr. Christine Wachendorf vom Fachgebiet für Bodenbiologie und Pflanzenernährung der Universität Kassel danke ich für ihre Unterstützung im Rahmen des Teilprojekts zur Bodenkunde und Standortcharakterisierung.

Für wichtige Fachgespräche sowie Gedanken- und Meinungsaustausch danke ich Herrn Dr. Jan Hari Arti Khalsa (DBFZ); Herrn Dr. Oscar Thiers (Universidad Austral, Valdivia); Frau Manuela Müller (TU-Dresden); Herrn Dr. Joachim Benz und Herrn Dr. Eicke Rommelfänger (Universität Kassel); sowie Katja Skibbe und Hendrik Horn (TU-Dresden).

Als Nicht-Muttersprachler war es eine besondere Herausforderung diese Arbeit auf Deutsch zu verfassen. Für ihre unschätzbare Hilfe in der sprachlichen Überarbeitung dieser Arbeit gilt mein Dank Herrn Dr. Jan Hari Arti Khalsa, Christian Siebert und Manuela Müller. Bei Frau Gisela Metzig (TUDresden) und Frau Susanne Aigner (Biosicht) bedanke ich mich für das hervorragende Lektorat. Herrn Dr. Oscar Thiers und Herrn Dr. David Buttler-Manning (Arbora Translation) danke ich für die Unterstützung in der Erstellung der spanischen und englischen Version der Zusammenfassung. Des Weiteren danke ich Dr. Jens Schröder (HNEE Eberswalde) für die Prüfung des methodischen Teils zur Statistik.

Ein großer Dank gilt meiner Familie und meinen Freunden, die mich jederzeit unterstützten und bestärkt haben, insbesondere meiner Frau und meinen Kindern.

An dieser Stelle möchte ich auch meinen Ansprechpartnern bei den Partnerinstitutionen im Verbundvorhaben PROLOC für die Kooperation und Hilfe danken. Ich danke: Herrn Manuel Karopka (FVA Baden Württemberg); Frau Anja Chalmin, Herrn Bernd Habeck, Herrn Frieder Seidl und Herrn Klaus Mastel (LTZ Augustenberg); Frau Bettina Stoll und Herrn Dr. Frank Burger (LWF Bayern); Herrn Dr. Ansger Quinkenstein (BTU Cottbus); Herrn Rainer Schlepphorst, Herrn Holger Hartmann und Herrn Prof. Dr. Dieter Murach (HNEE Eberswalde); Herrn Dr. Dirk Knoche (FIB Finsterwalde); Herrn Peter Kaulfuß und Herrn Dr. Volkhard Scholz (ATB Potsdam Bornim); Herrn Dr. Lothar Behle Schalk (Universität Giessen); Herrn Dr. Reinhold Stülpnagel und Herrn Prof. Dr. Michael Wachendorf (Uni- 
versität Kassel); Herrn Ludger Tapken (LK Niedersachsen); Frau Dr. Marie-Luise Rottmann-Meyer (3N Niedersachsen); Frau Dr. Barbara Boelcke und Herrn Dr. Andeas Gurgel (LFA Mecklenburg Vorpommern); Herrn Holger Huffelmann und Herrn Dr. Carsten Block (LK Nordrhein-Westfalen); Herrn Norbert Hofnagel und Frau Susan Stroff (Biomassehof Borlinghausen); Herrn Dr. Nild Redde (RWE); Herrn Volker Wenghöfer und Herrn Prof. Dr. Ralf Pude (Universität Bonn); Herrn Volker Cechini und Herrn Dr. Henning Kurth (LLFG Sachsen Anhalt); Frau Karin Ruscher, Frau Sabine Mau, Herrn Pierre Seibold und Herrn Dr. Christian Röhricht (lfULG Sachsen); Katja Skibbe und Frau Dr. Dorothea Gerold (TU Dresden); Herrn Stefan Jencsik, Frau Susanne Bein und Herrn Dr. Georg von Wühlisch (TI für Forstgenetik); Herrn Dr. Hardy Dembny (Baumschule Oberdorla); Herrn Ralf Gebhardt, Herrn Ralf Köhler und Prof. Dr. Peter Heck (IfaS); Frau Heike Rudel, Frau Andrea Biertümpfel und Dr. Armin Vetter (TLL) sowie den weiteren mir unbekannten Kollegen und Hilfskräften, welche vor Ort Bonituren, Probenahmen und weitere Projektaufgaben durchführten. 


\section{Inhaltsverzeichnis}

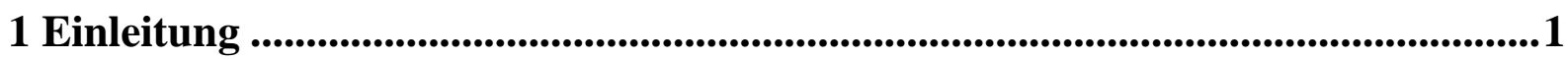

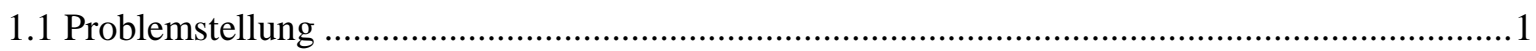

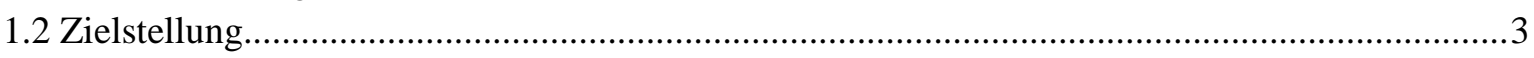

2 Hintergrund (................................................................................................................

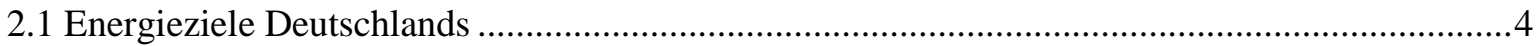

2.1.1 Flächen- und Nutzungskonkurrenz mit der Forstwirtschaft .............................................

2.1.2 Flächen- und Nutzungskonkurrenz mit der Landwirtschaft ..............................................5

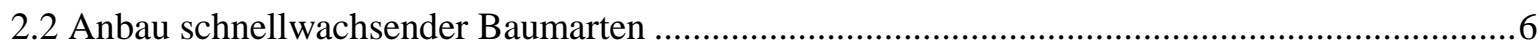

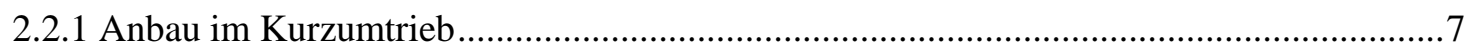

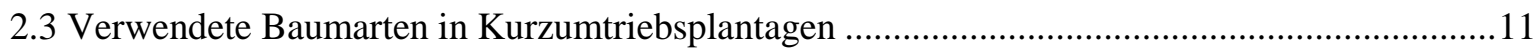

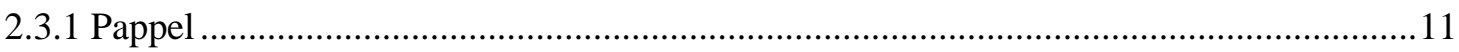

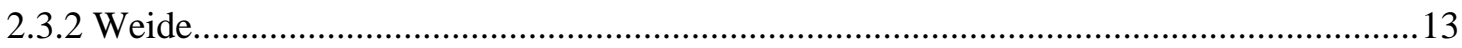

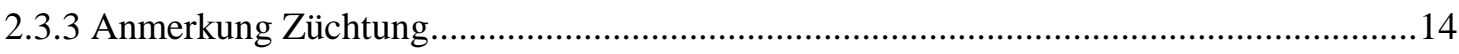

2.4 Leistung von Kurzumtriebsplantagen mit Pappel und Weide in zwei- bis dreijähriger Umtriebs-

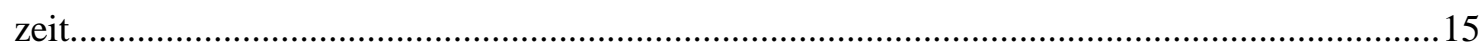

2.5 Ertragsdynamik und leistungsbeeinflussende Faktoren von Kurzumtriebsplantagen .................17

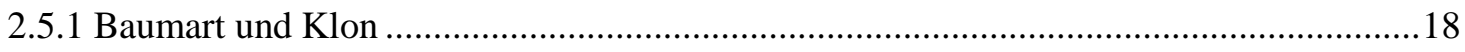

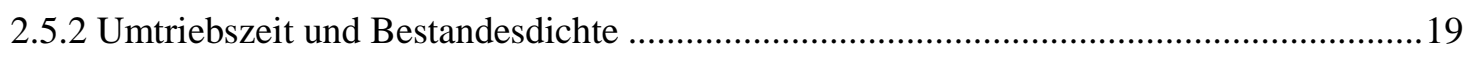

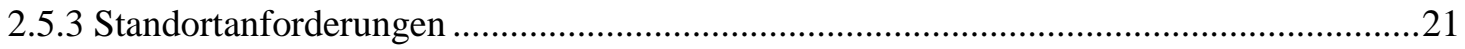

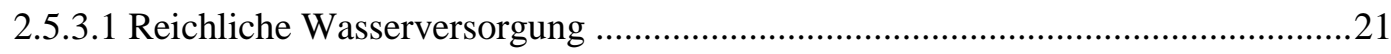

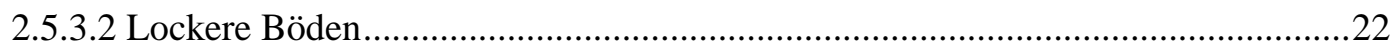

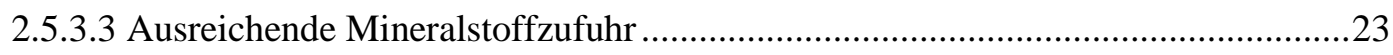

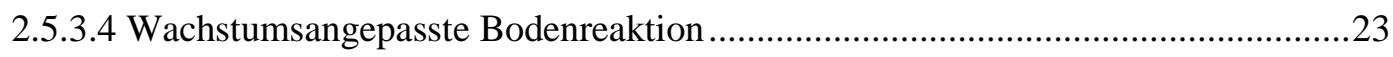

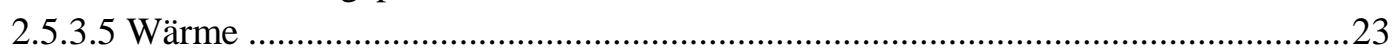

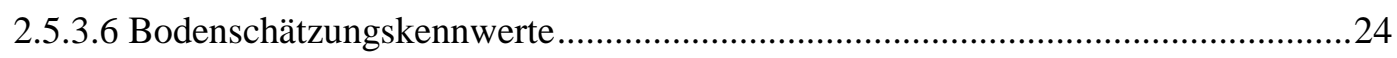

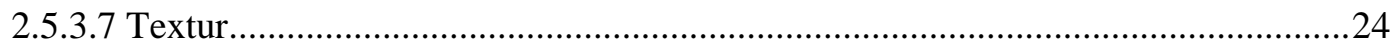

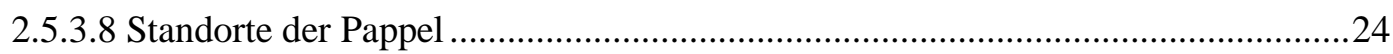

2.6 Waldwachstumsmodellierung mit Schwerpunkt in Kurzumtriebsplantagen ..............................25

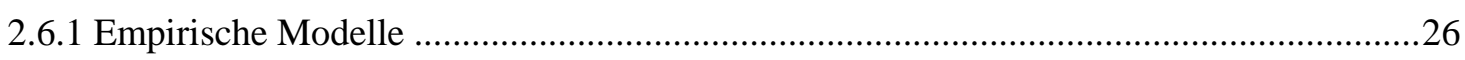

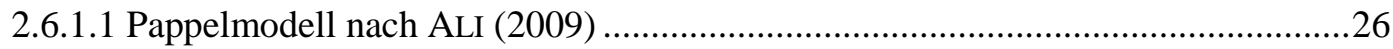

2.6.1.2 Pappel- und Weidenmodell nach AYLOTT et al. (2008) ......................................27

2.6.1.3 Leistungsbeeinflussende Standorteigenschaften für Pappel und Weide nach BERGANTE et al. (2010) ..................................................................................28

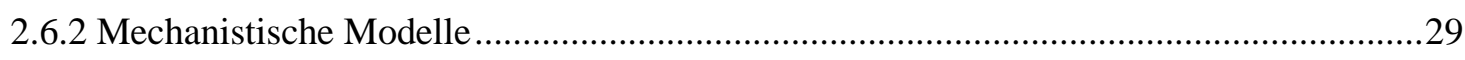

3 Material und Methoden ...................................................................................33

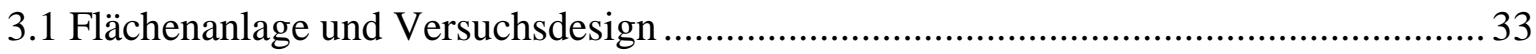




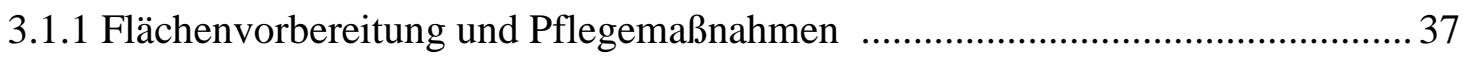

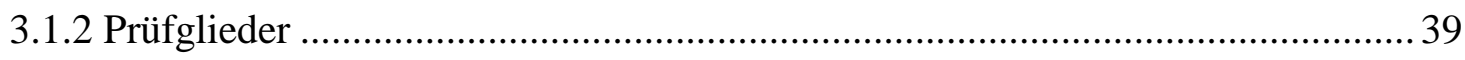

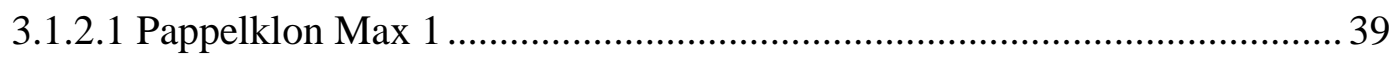

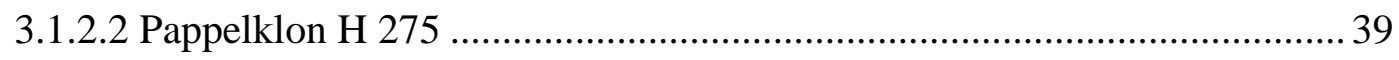

3.1.2.3 Pappelklon AF 2 ….................................................................... 40

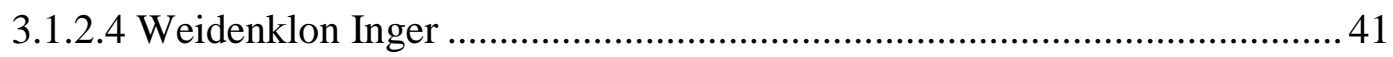

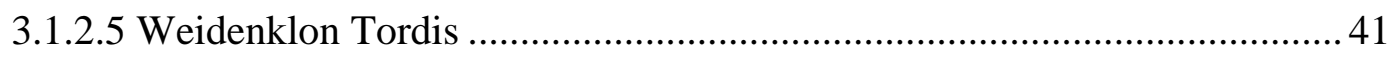

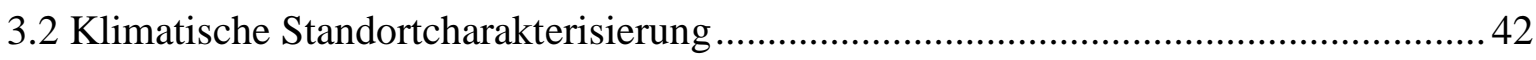

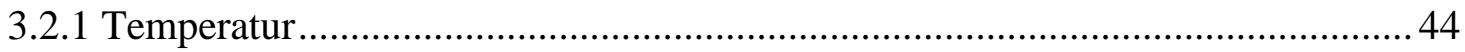

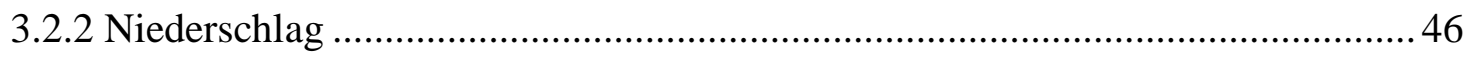

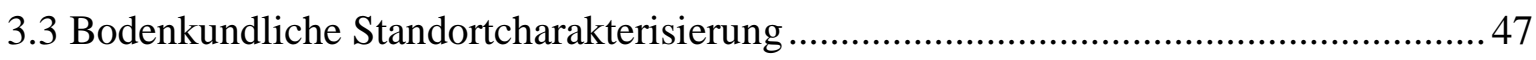

3.3.1 Profilansprache und allgemeine Standortinformationen .................................. 48

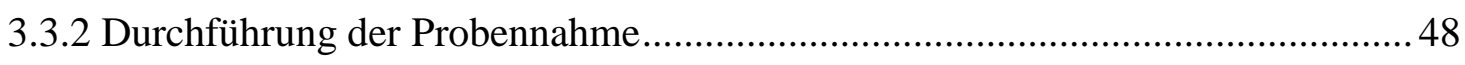

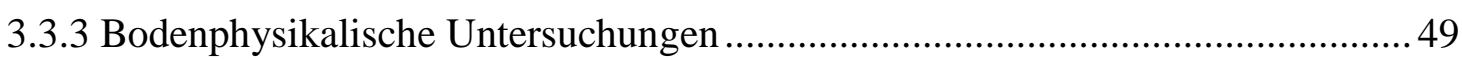

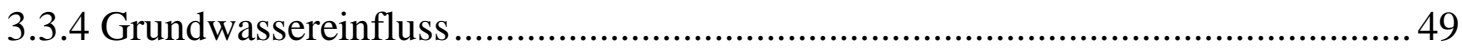

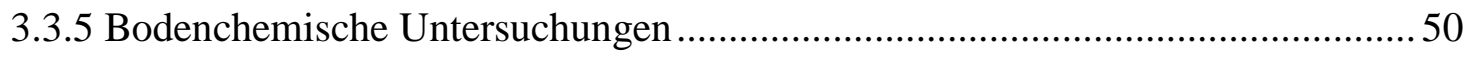

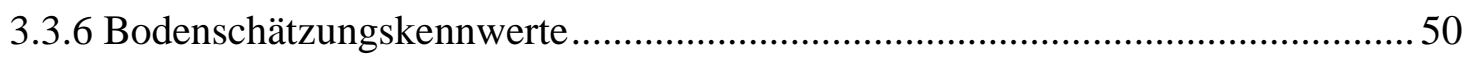

3.3.7 Zusammenfassende Betrachtung der bodenkundlichen Charakteristika ............. 51

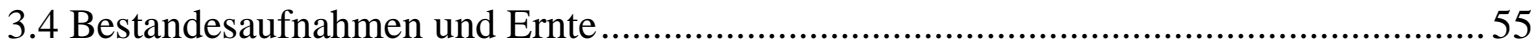

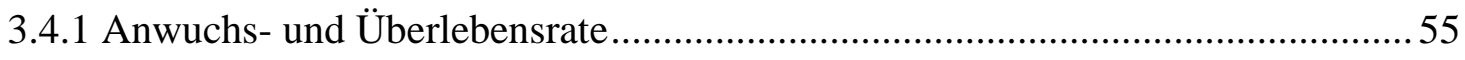

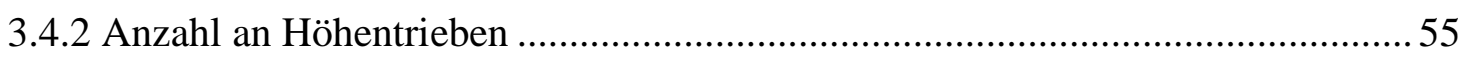

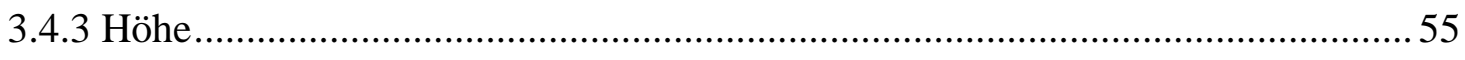

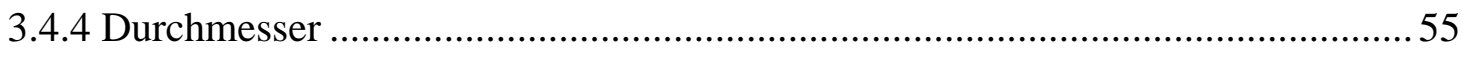

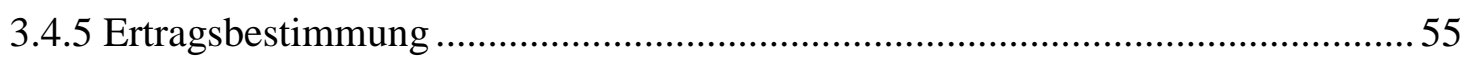

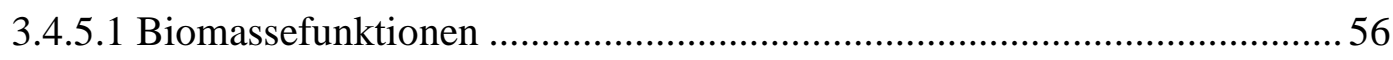

3.4.5.2 Systematische Teilbeernteung der Kernparzelle ................................... 56

3.5 Identifizierung von leistungsbeeinflussenden Standorteigenschaften und Entwicklung

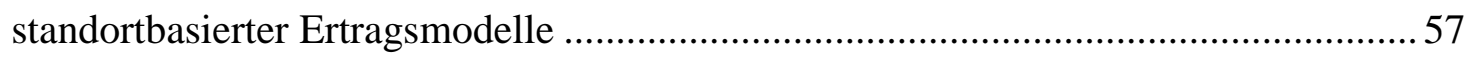

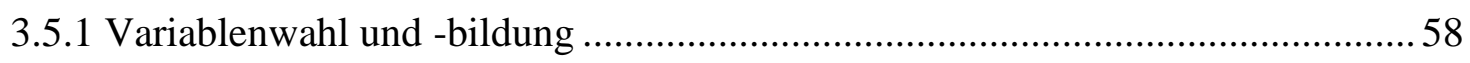

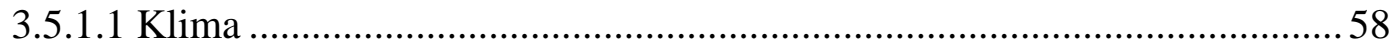

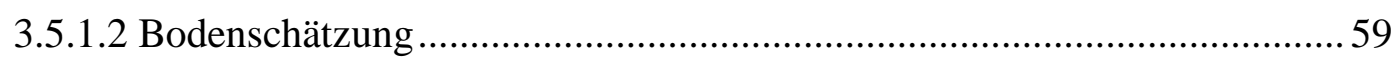

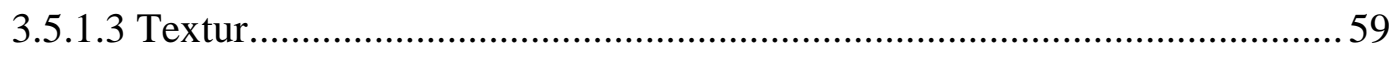

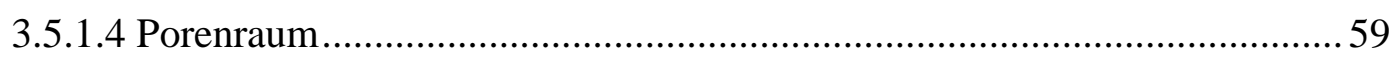

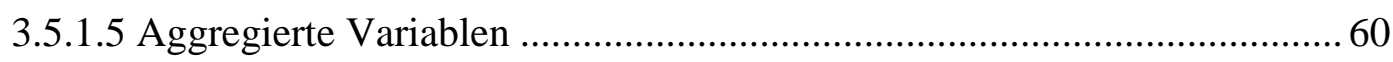

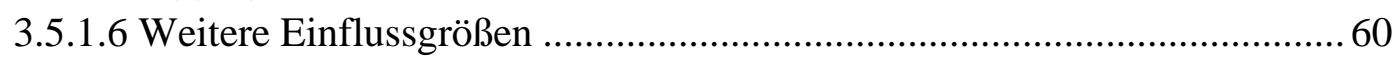

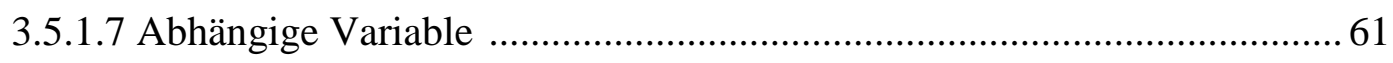

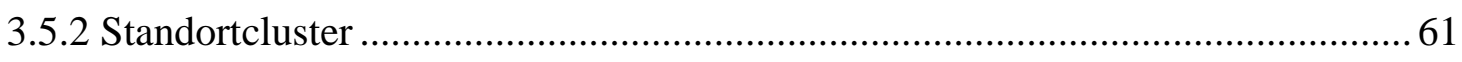

3.6 Datenbasis der standortbasierten Ertragsmodellierung.......................................... 61 


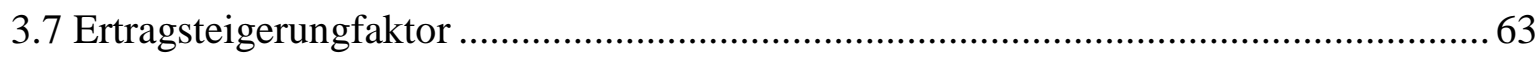

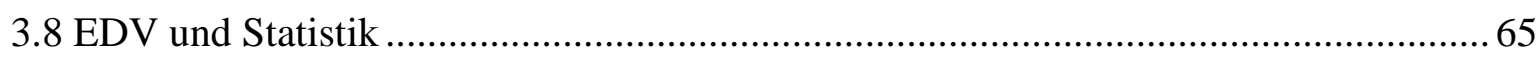

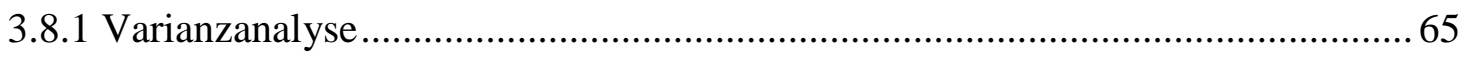

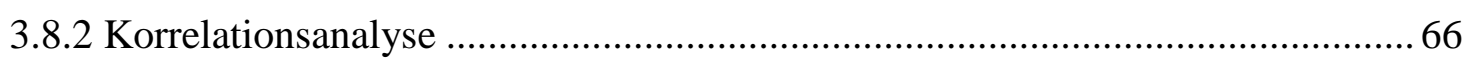

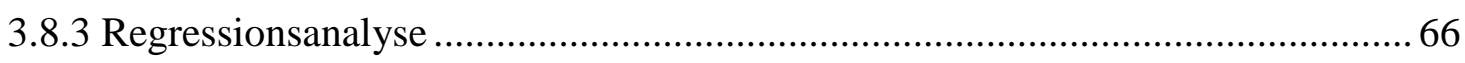

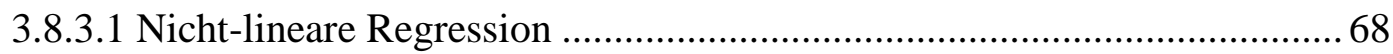

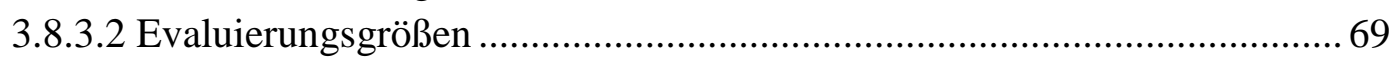

4 Ergebnisse........................................................................................................

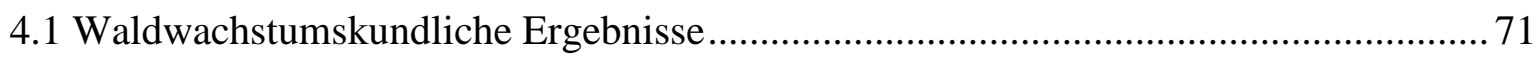

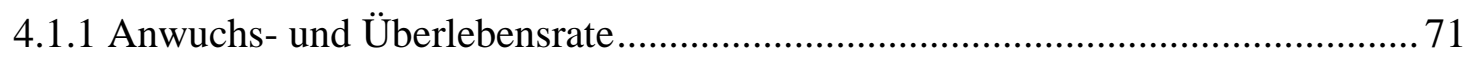

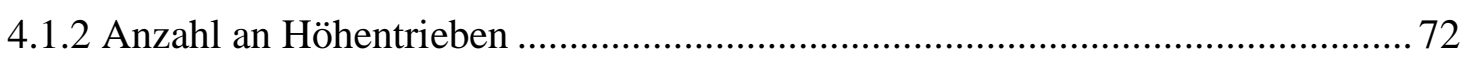

4.1.3 Übersicht Bestandesdimensionen und Wuchsleistung .................................... 73

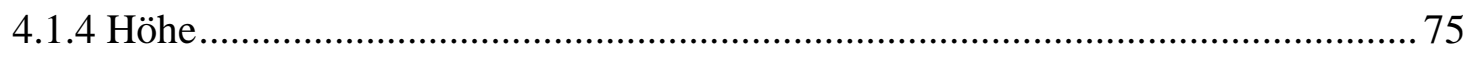

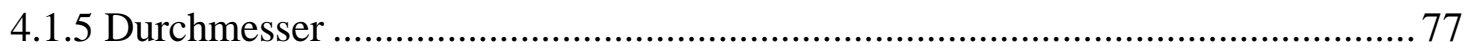

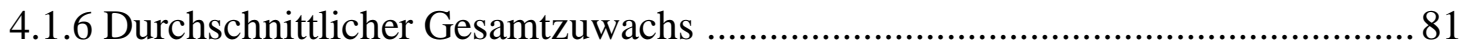

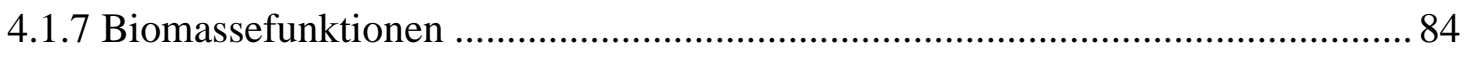

4.1.7.1 Wahl der unabhängigen Variablen ..................................................... 84

4.1.7.2 Einfluss der Gattung und des Klons ................................................. 85

4.1.7.3 Einfluss der Bestandesmittelhöhe...................................................... 85

4.1.7.4 Allgemeingültige Biomassefunktionen ............................................. 87

4.2 Leistungsbeeinflussende Standorteigenschaften.................................................. 88

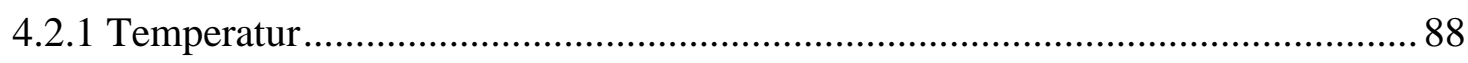

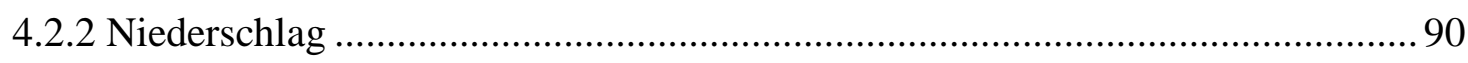

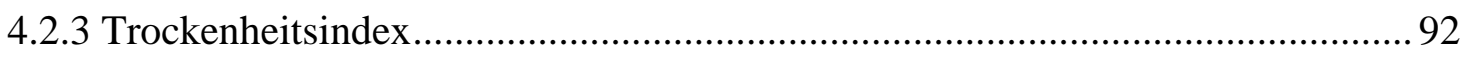

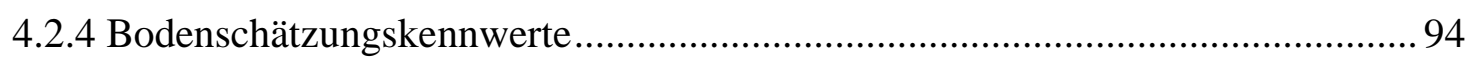

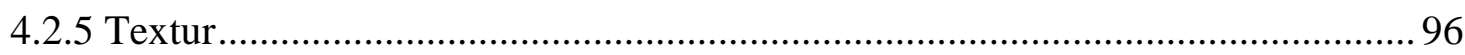

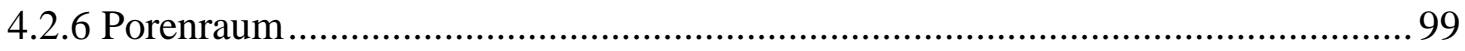

4.2.7 Korrelation zwischen unabhängigen Variablen.............................................. 102

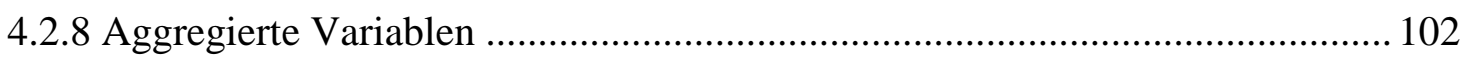

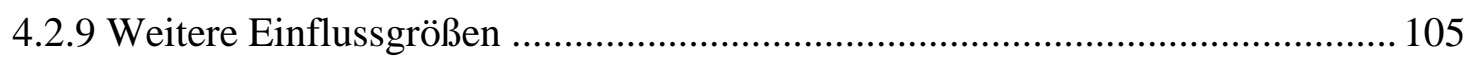

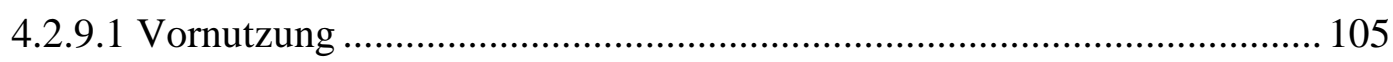

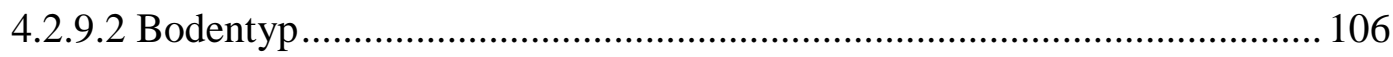

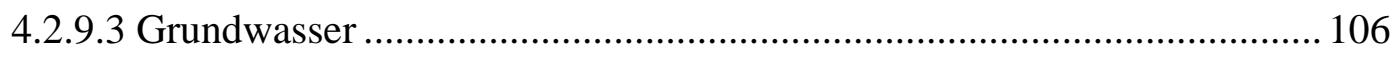

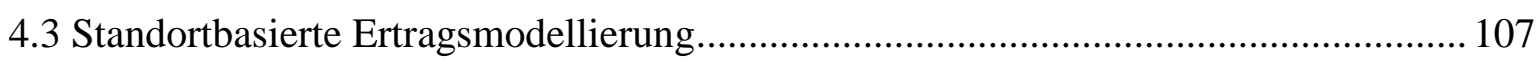

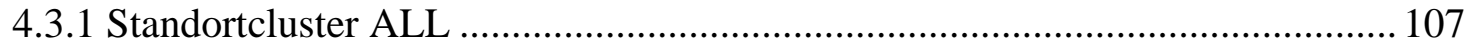

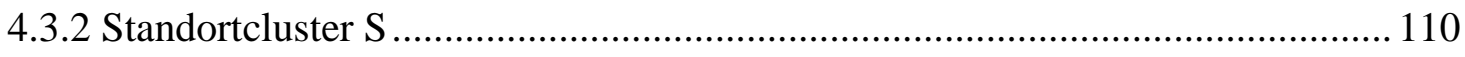

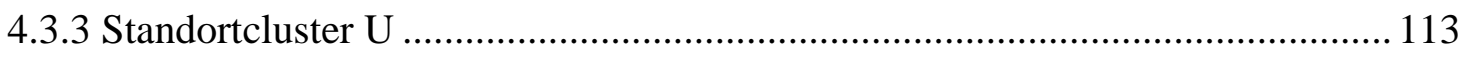




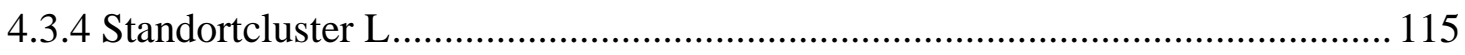

4.4 Ertragssteigerungsfaktoren und Ertragssteigerung in Folgerotationen....................... 118

5 Diskussion

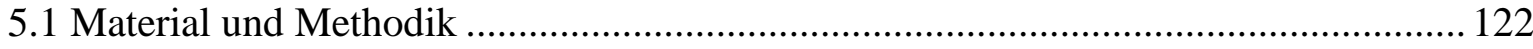

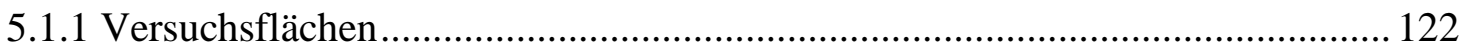

5.1.2 Modellierung und Variablenbildung............................................................ 123

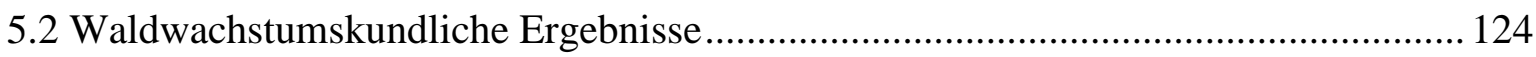

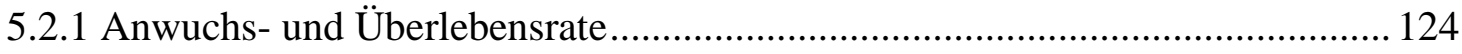

5.2.2 Anzahl an Höhentrieben ........................................................................... 125

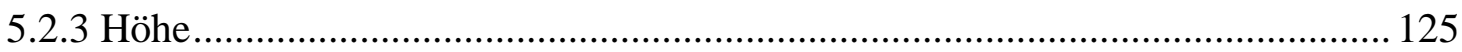

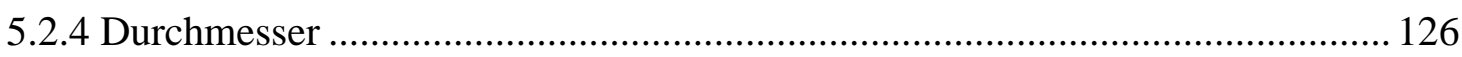

5.2.5 Durchschnittlicher Gesamtzuwachs............................................................ 126

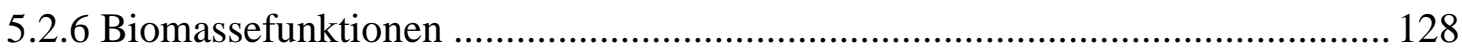

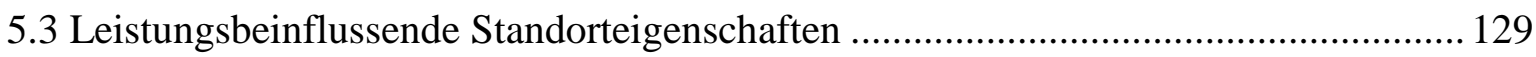

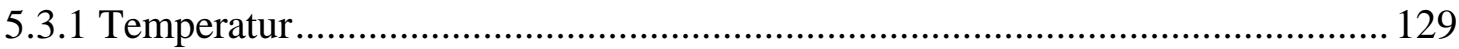

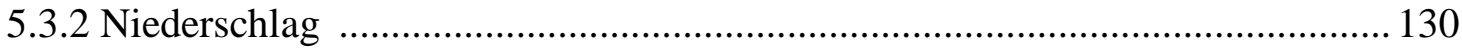

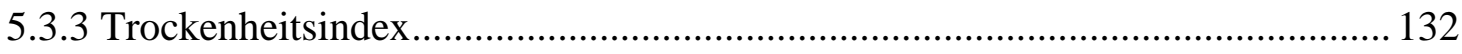

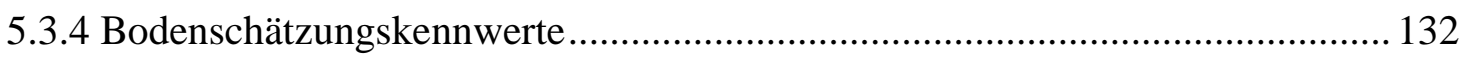

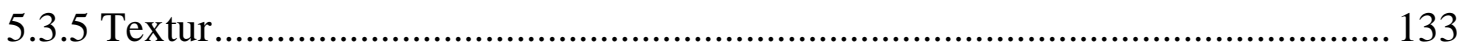

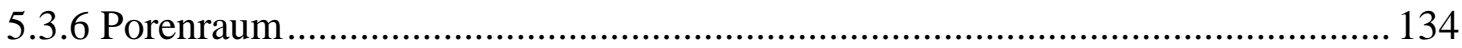

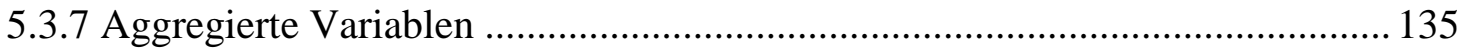

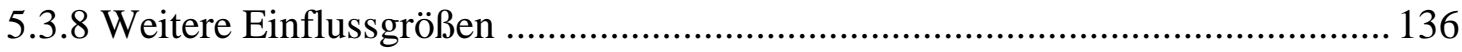

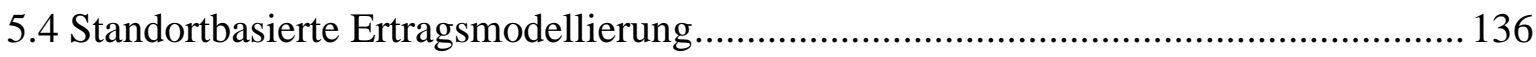

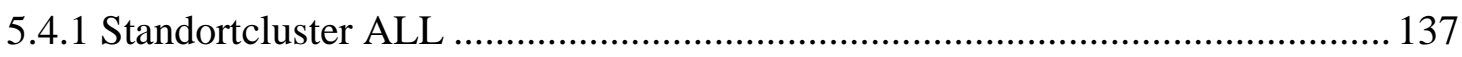

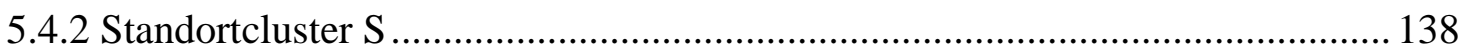

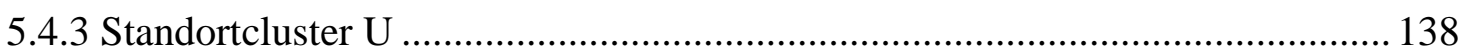

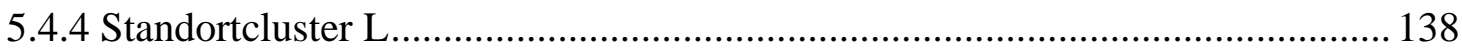

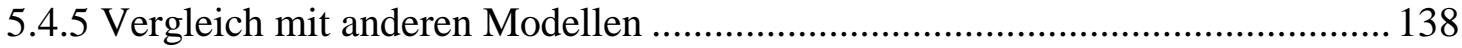

5.5 Ertragssteigerungsfaktoren und Ertragsteigerung in Folgerotationen ........................ 140

6 Schlussfolgerungen und Ausblick ...................................................................141

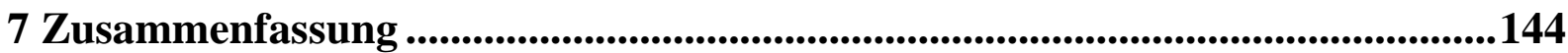

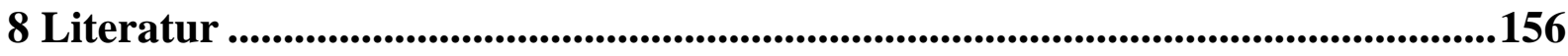

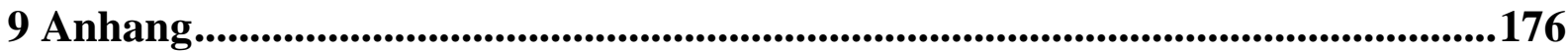




\section{Abbildungsverzeichnis}

Abb. 2-1: Pappel Kurzumtriebsplantagen a) 2-jähriger Pappelbestand Pommritz b) 4-jähriger Pappelbestand Potsdam (Stockausschlag) c) 12-jähriger Pappelbestand Methau (Bild: WoLf, H. und SCHILDBACH, M.) d) 20-jähriger Pappelbestand Deutschland

Abb. 2-2: dGZ in $\mathrm{t}_{(\text {atro) }} \mathrm{ha}^{-1} \mathrm{a}^{-1}$ von vier Langzeitbeobachtungsflächen in Thüringen und Mecklenburg-Vorpommern bei der Max-Mehrklonsorte und Max 1 in dreijähriger Rotation (Verändert nach BIERTÜMPFEL et al. 2012, BOELCKE 2006 und GURGEL 2011)...

Abb. 2-3: dGZ-Entwicklung in $\mathrm{t}_{(\text {atro) }} \mathrm{ha}^{-1} \mathrm{a}^{-1}$ der Weidenklone Tora (Versuchsfläche „Bad Salzungen“) und Zieverich (Versuchsfläche „Kalkreuth“) gegenüber Max 1 den in dreijähriger Rotation (Verändert nach BIERTüMPFEL et al. 2012 sowie RÖHRICHT 2009).

Abb. 2-4: Whiskers-Box-Plots der Leistung von Pappel und Weide in zwei bis dreijährigen Umtriebszeiten (Datengrundlage kann in Tabelle 2-5 nachgeschlagen werden).

Abb. 2-5: dGZ der ersten drei Rotationen für den Klon Max 1 (Versuchsflächen „Kalkreuth“ und „Gülzow“) sowie für MaxMehrklonsorte (Versuchsfläche „Langenwetzendorf“) bei unterschiedlichen Bestandesdichten (Verändert nach RÖHRICHT 2009, BIERTÜMPFEL et al. 2012, BOELCKE 2006 und GURGEL 2011).

Abb. 2-6: Berücksichtigte physikalisch-biologische Waldwachstumsprozesse im mechanistischen Modell FOREST GROWTH SRC (verändert nach TALLIS et al. 2013).

Abb. 3-1: Lage der Versuchsflächen.

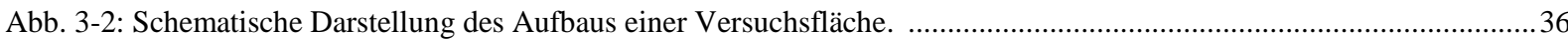

Abb. 3-3: Darstellung einer Einzelparzelle mit Kennzeichnung der Kernparzelle und des Parzellen-Randbereiches. .............36

Abb. 3-4: Versuchsflächen mit hoher Verunkrautung in der zweiten Wuchsperiode, Jahr 2009 a) Standort Nr. 2 „Lilienthal“ b) Standort Nr. 7 „Kaisheim“.

Abb. 3-5: Pappelklon Max 1.

Abb. 3-6: Pappelklon H 275.

Abb. 3-7: Pappelklon AF 2.

Abb. 3-8: Weidenklon Inger.

Abb. 3-9: Weidenklon Tordis.

Abb. 3-10: Box-Whiskers-Plots der mittleren Temperatur 2008, 2009, 2010 sowie für den Zeitraum von 1990 bis 2010 über alle Standorte für die Bezugszeitfenster a) Jahr und b) VP.

Abb. 3-11: Lage der Vergleichsstandorte mit relevanten Klimastationen (Kl-St.) zur Betrachtung des Klimas der ersten Rotation........

Abb. 3-12: Box-Whiskers-Plots der Niederschlagssummen 2008, 2009, 2010 sowie für den Zeitraum von 1990 bis 2010 über

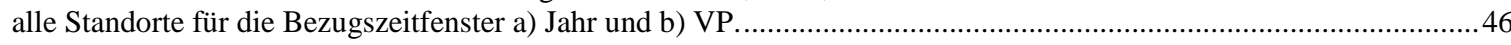

Abb. 3-13: Probenahmeschema Teilprojekt Bodenkunde und HeRo-Arbeitsgruppe. .............................................................48

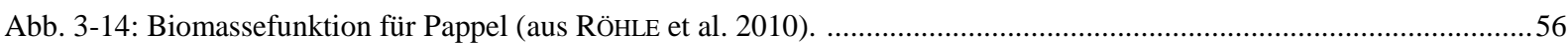

Abb. 3-15: Schematische Darstellung der Arbeitsschritte der Modellentwicklung..............................................................57

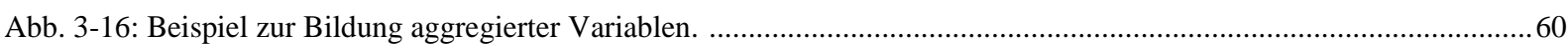

Abb. 4-1: Überlebensraten [\%] der Pappelklone Max 1, H 275 und AF 2 sowie der Weidenklone Inger und Tordis über alle Standorte a) im Anwuchsjahr sowie b) im Folgejahr nach durchgeführter Nachbesserung dargestellt mittels BoxWhiskers-Plots.

Abb. 4-2: Mittlere Anwuchsraten [\%] aller Prüfglieder in Abhängigkeit der Niederschlagssumme der Monate Mai und Juni bei der Hauptbodenart Sand...

Abb. 4-3: Mittlere Anwuchsraten [\%] aller Prüfglieder in Abhängigkeit der nFK der Bodenoberschicht $(0-30 \mathrm{~cm})$ bei den Hauptbodenarten Schluff, Lehm und Ton.

Abb. 4-4: Mittlere Anzahl an Höhentrieben der Pappelklone Max 1, H 275 und AF 2 sowie der Weidenklone Inger und Tordis über alle Standorte aus der zweiten Wuchsperiode dargestellt mittels Box-Whisker-Plots.

Abb. 4-5: Mittlere Höhen [m] der Pappelklone Max 1, H 275 und AF 2 sowie der Weidenklone Inger und Tordis für jede Wuchsperiode und Standortcluster, dargestellt mittels Box-Whisker-Plots.................................................................76

Abb. 4-6: Mittlere $\mathrm{d}_{0,1}[\mathrm{~cm}]$ der Pappelklone Max 1, H 275 und AF 2 sowie der Weidenklone Inger und Tordis für jede Wuchsperiode und Standortcluster, dargestellt mittels Box-Whisker-Plots. 
Abb 4-7: Mittlere $d_{1,3}[\mathrm{~cm}]$ der Pappelklone Max 1, H 275 und AF 2 sowie der Weidenklone Inger und Tordis für jede Wuchsperiode und Standortcluster, dargestellt mittels Box-Whisker-Plots.

Abb. 4-8: dGZ-Entwicklung der Pappelklone Max 1, H 275 und AF 2 sowie der Weidenklone Inger und Tordis in den jeweiligen Standortclustern.

Abb. 4-9: dGZ [ $\left.\mathrm{t}_{\text {(atro) }} \mathrm{ha}^{-1} \mathrm{a}^{-1}\right]$ der Pappelklone Max 1, H 275 und AF 2 sowie der Weidenklone Inger und Tordis für jede Wuchsperiode und Standortcluster, dargestellt mittels Box-Whisker-Plots.

Abb. 4-10: Gegenüberstellung der mittleren Höhe [m] mit dem $\mathrm{R}^{2}$ korr der Biomassefunktionen aller untersuchten Prüfglieder mit jeweils a) d0,1 und b) d1,3 als unabhängige Variable.

Abb. 4-11: Regressionsanalyse zwischen den Funktionskoeffizienten $\mathrm{a}_{0}$ und $\mathrm{a}_{1}$ (a) sowie zwischen der mittleren Höhe und den jeweiligen Funktionskoeffizienten (b und c) für die Klone AF 2 und Inger.

Abb. 4-12: Modellierte Biomassefunktionen nach Höhencluster sowie Hauptfunktion a) beim Pappelklon AF 2 und b) beim Weidenklon Inger. Mit H3: Funktion der Höhenstufe 3, usw..

Abb. 4-13: Allgemeingültige Biomassefunktionen für die Bestandesdichte 11.111 Stk ha ${ }^{-1}$ der Pappelklone Max 1, H 275, AF 2 sowie der Weidenklone Inger und Tordis.

Abb. 4-14: Regressionsverlauf für den dGZ in Abhängigkeit von $\mathrm{N}_{6,7}$ und im Standortcluster ALL, dargestellt am Beispiel der Klone AF 2 und Tordis.

Abb. 4-15: Regressionsverlauf für den dGZ in Abhängigkeit von $\mathrm{N}_{6,7}$ im Standortcluster $\mathrm{S}$, dargestellt am Beispiel der Klone Max 1 und $\mathrm{H} 275$.

Abb. 4-16: Regressionsverlauf für den dGZ in Abhängigkeit von $\mathrm{N}_{5,6}$ und im Standortcluster L, dargestellt am Beispiel des Klons AF 2

Abb. 4-17: Regressionsverlauf für den dGZ in Abhängigkeit von $\mathrm{TI}_{6,7}$ im Standortcluster ALL, dargestellt am Beispiel des Klon Tordis.

Abb. 4-18: Regressionsverlauf für den dGZ in Abhängigkeit von $\mathrm{TI}_{6,7}$ im Standortcluster S dargestellt am Beispiel der Klone Max 1 und Tordis.

Abb. 4-19: Regressionsverlauf für den dGZ in Abhängigkeit von $\mathrm{TI}_{5,6}$ im Standortcluster L, dargestellt am Beispiel des Klons Inger.

Abb. 4-20: Regressionsverlauf für den dGZ in Abhängigkeit von AZ im Standortcluster ALL, dargestellt am Beispiel der Klone AF 2 und Tordis.

Abb. 4-21: Regressionsverlauf für den dGZ in Abhängigkeit von BZ im Standortcluster U, dargestellt am Beispiel des Klons Tordis......

Abb. 4-22: Regressionsverlauf für den dGZ in Abhängigkeit von $\mathrm{U}_{[\%]}$ im Standortcluster ALL, dargestellt am Beispiel der Klone AF 2 und Tordis.

Abb. 4-23: Regressionsverlauf für den dGZ in Abhängigkeit von Texturvariablen im Standortcluster S. Mit a) $\mathrm{S}_{[\%]}$ dargestellt am Beispiel der Klone Max 1 und Inger und b) $\mathrm{U}_{[\%]}$ dargestellt am Beispiel der Klone AF 2 und Tordis......

Abb. 4-24: Regressionsverlauf für den dGZ in Abhängigkeit von $\mathrm{U}_{[\%]}$ im Standortcluster L, dargestellt am Beispiel der Klone AF 2 und Inger.

Abb. 4-25: Regressionsverlauf für den dGZ in Abhängigkeit von nFK im Standortcluster ALL, dargestellt am Beispiel der Klone AF 2 und Tordis.

Abb. 4-26: Regressionsverlauf für den dGZ in Abhängigkeit von LK im Standortcluster S, dargestellt am Beispiel der Klone Max 1 und AF 2.

Abb. 4-27: Regressionsverlauf für den dGZ in Abhängigkeit von nFK im Standortcluster L, dargestellt am Beispiel der Klone Max 1 und Inger.

Abb. 4-28: dGZ des Klons Max 1 bei Standorten unterschiedlicher Vornutzung in der dritten Wuchsperiode. ....................106

Abb. 4-29: dGZ des Klons Max 1 bei Standorten unterschiedlichen Bodentyps in der dritten Wuchsperiode. ..................... 106

Abb. 4-30: Standortbasierte Ertragsmodelle im Standortcluster ALL für a) Max 1 b) AF 2 c) Inger und d) Tordis.............. 108

Abb. 4-31: Dreidimensionale Darstellung der Standortbasierten Ertragsmodelle im Standortcluster ALL. Dargestellt ist der dGZ in Abhängigkeit der zwei nicht aggregierten Variablen aus denen sich der aggregierte Modellprädiktor zusammensetzt, für a) Max 1 und b) AF 2

Abb. 4-32: Standortbasierte Ertragsmodelle im Standortcluster S für a) Max 1 b) H 275 c) AF 2 d) Inger und e) Tordis. ...111

Abb. 4-33: Dreidimensionale Darstellung der Standortbasierten Ertragsmodelle im Standortcluster S. Dargestellt ist der dGZ in Abhängigkeit der zwei nicht aggregierten Variablen aus denen sich der aggregierte Modellprädiktor zusammensetzt für a) H 275 b) AF 2 und c) Tordis. 
Abb. 4-34: Vergleich beobachtete und geschätzte dGZ für die Modelle im Standortcluster S für die Modelle der Klone a) Max 1 b) H 275 c) AF 2 d) Inger e) Tordis.

Abb. 4-35: Standortbasierte Ertragsmodelle im Standortcluster U für a) Max 1 und b) Tordis.

Abb. 4-36: Dreidimensionale Darstellung des Standortbasierten Ertragsmodells im Standortcluster U. Dargestellt ist der dGZ in Abhängigkeit der zwei nicht aggregierten Variablen aus denen sich der aggregierte Modellprädiktor für den Klon Max 1 zusammensetzt.

Abb. 4-37: Vergleich beobachteter und geschätzter dGZ für die Modelle im Standortcluster U für die Modelle der Klone a) Max 1 und b) Tordis.

Abb. 4-38: Standortbasierte Ertragsmodelle im Standortcluster L für a) Max 1 b) AF 2 und c) Inger.

Abb. 4-39: Dreidimensionale Darstellung des Standortbasierten Ertragsmodells im Standortcluster L. Dargestellt ist der dGZ in Abhängigkeit der zwei nicht aggregierten Variablen, aus denen sich der aggregierte Modellprädiktor für den Klon AF 2 zusammensetzt.

Abb. 4-40: Vergleich beobachteter und geschätzter dGZ für die Modelle im Standortcluster L für die Modelle der Klone a) Max 1 b) AF 2 und c) Inger.

Abb. 4-41: dGZ Entwicklung der Klongruppen Max und BP in den Standorten „Bad Salzungen“ (Hauptbodenart S), „Dornburg“ (Hauptbodenart U) und „Langenwetzendorf“ (Hauptbodenart L).

Abb. 4-42: Standortspezifische Ertragssteigerungsfaktoren auf Basis der Referenz-dGZ der zweiten Rotation. 120

Abb. 4-43: Standortbasierte modellierte dGZ der ersten Rotation sowie mittels Faktoren ermittelte Ertragssteigerungen von fünf Folgerotationen für den Klon Max 1 in den Standortclustern a) S b) U und c) L 


\section{Tabellenverzeichnis}

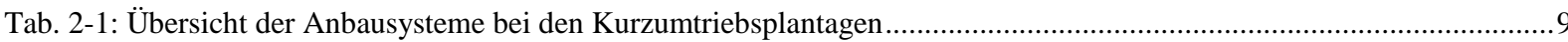

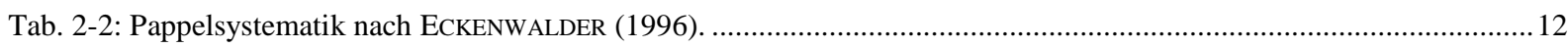

Tab. 2-3: Auswahl von Weidenarten in den jeweiligen Sektionen (nach Blanco 1993 und Newsholme 1992)....................14

Tab. 2-4: Übersicht von in Deutschland mit Pappel, Weide und Aspe erreichten dGZ in der zwei- dreijährigen Umtriebs-

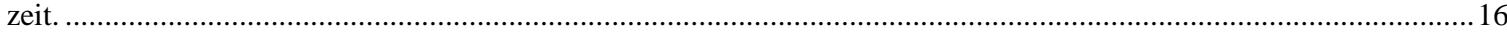

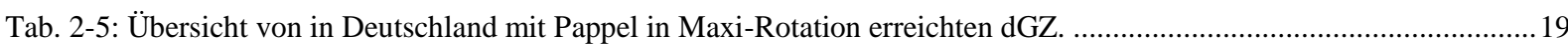

Tab. 2-6: Unabhängige Variablen für die Identifizierung leistungsbeeinflussender Standorteigenschaften nach BERGANTE

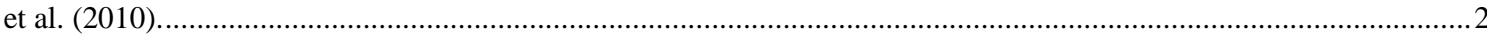

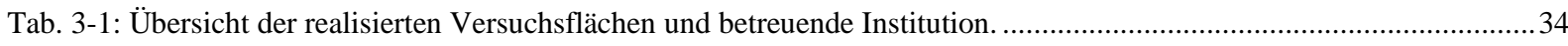

Tab. 3-2: Standortname, Bundesland, Höhe über NN, Exposition und genaue Koordinaten der Versuchsflächen....................35

Tab. 3-3: Übersicht zur Bodenvorbereitung und zu durchgeführten Pflegemaßnahmen bei der Flächenanlage. .......................37

Tab. 3-4: Nachgebesserte Prüfglieder nach Standort und Art des Pflanzmaterials (Zahlen zeigen Standortnummern, Zahl in Klammer zeigt die Gesamtanzahl der nachgebesserten Standorte) .......................................................................38

Tab. 3-5: Durchschnittliche langjährige Werte (1990 - 2010) der Niederschlagssummen, der Temperatur- und der Trockenheitsindizesmittelwerte für die Bezugszeiträume Jahr und Vegetationsperiode ...........................................................43

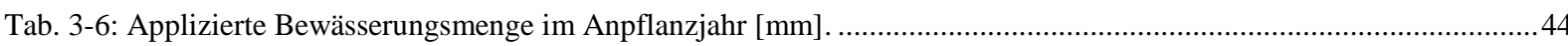

Tab. 3-7: Mittlere Monats-, Vegetationsperiode- und Jahrestemperaturen im Untersuchungszeitraum 2008 - 2010 von acht ausgewählten Standorten. Relative Vergleiche zum langjährigen Mittel wurden mittels Farbgebung gekennzeichnet...45

Tab. 3-8: Mittlere Monats-, Vegetationsperiode- und Jahresniederschlagssummen im Untersuchungszeitraum 2008 - 2010 von acht ausgewählten Standorten. Relative Vergleiche zum langjährigen Mittel wurden mittels Farbgebung gekennzeichnet.

Tab. 3-9: Methodik der bodenphysikalischen Analysen (verändert nach HEYN und WACHENDORF 2011). ..............................49

Tab. 3-10: Methodik der bodenchemischen Analysen (verändert nach HEYN und WACHENDORF 2011) .................................50

Tab. 3-11: Ehemalige Bewirtschaftung, Bodenausgangssubstrat, Bodentyp und Bodenschätzung der Versuchsflächen.........52

Tab. 3-12: Bodenphysikalische und -chemische Eigenschaften der Versuchsflächen (Die bodenphysikalischen Werte beziehen sich auf die Gesamttiefe $0-60 \mathrm{~cm}$, die bodenchemischen Werte auf die Tiefe $0-30 \mathrm{~cm}$ )

Tab. 3-13: Ausgewählte Indikatoren des Baumwachstums und deren Variablen zur Identifizierung der leistungsbeeinflussenden Standorteigenschaften und zur Modellentwicklung.

Tab. 3-14: Niederschlagsvariablen, dargestellt in unterschiedlich langen Zeitfenstern. Zahlen indizieren die Monatsnummer (Januar 1, Februar 2, usw., mit J: Jahr, VP: Vegetationsperiode).

Tab. 3-15: Temperaturvariablen, dargestellt in unterschiedlich langen Zeitfenstern. Zahlen indizieren die Monatsnummer (Januar 1, Februar 2, usw., mit J: Jahr, VP: Vegetationsperiode).

Tab. 3-16: Variablen des Trockenheitsindizes, dargestellt in unterschiedlich langen Zeitfenstern. Zahlen indizieren die Monatsnummer (Januar 1, Februar 2, usw., mit J: Jahr, VP: Vegetationsperiode). .................................................59

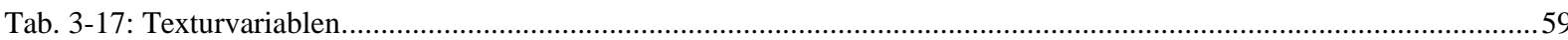

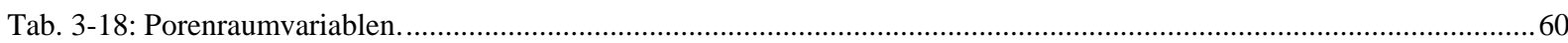

Tab. 3-19: Berücksichtigte Bodenarten bei den verschiedenen Standortclustern der deskriptiven Ergebniskapitel.................61

Tab. 3-20: Nicht berücksichtigte Standorte in der statistischen Analyse zur Ermittlung leistungsbeeinflussender Standorteigenschaften und Entwicklung Standortbasierter Ertragsmodelle.

Tab. 3-21: Berücksichtigte Bodenarten bei den verschiedenen Standortclustern zur Ermittlung leistungsbeeinflussender Standorteigenschaften und Entwicklung Standortbasierter Ertragsmodelle.

Tab. 3-22: Zusammensetzung der Standortcluster zur Ermittlung leistungsbeeinflussender Standorteigenschaften und Entwicklung Standortbasierter Ertragsmodelle.

Tab. 3-23: Wiederholungsanzahl (n) der Standortbasierten Ertragsmodelle bei den verschiedenen Klonen und Standortclustern (+: ausgewertetes Modell, -: nicht ausgewertetes Modell)

Tab. 3-24: Zusammensetzung der Klongruppen Balsampappel (BP) und Max-Klone (Max) von den Ertragssteigerungsfaktoren an den Standorten „Bad Salzungen“, „Dornburg“ und „Langenwetzendorf“. 
Tab. 4-1: Mittlere Höhe [m], $d_{1,3}[\mathrm{~cm}]$ und dGZ $\left[\mathrm{t}_{(\text {atro) }}\right.$ ha $\left.^{-1} \mathrm{a}^{-1}\right]$ der Pappelklone Max 1, H 275 und AF 2 sowie der Weidenklone Inger und Tordis in der dritten Wuchsperiodea für alle untersuchten Standorte.

Tab. 4-2: Mittlere Höhen [m] der Pappelklone Max 1, H 275 und AF 2 sowie der Weidenklone Inger und Tordis in den Wuchsperioden eins bis drei unter Berücksichtigung aller Standorte.

Tab. 4-3: Mittlere $\mathrm{d}_{0,1}[\mathrm{~cm}]$ der Pappelklone Max 1, H 275 und AF 2 sowie der Weidenklone Inger und Tordis in den Wuchsperioden eins bis drei unter Berücksichtigung aller Standorte.

Tab. 4-4: Mittlere $\mathrm{d}_{1,3}[\mathrm{~cm}]$ der Pappelklone Max 1, H 275 und AF 2 sowie der Weidenklone Inger und Tordis in den Wuchsperioden eins bis drei unter Berücksichtigung aller Standorte..

Tab. 4-5: Mittlere dGZ $\left[\mathrm{t}_{(\text {atro) }} \mathrm{ha}^{-1} \mathrm{a}^{-1}\right]$ der Pappelklone Max 1, H 275 und AF 2 sowie der Weidenklone Inger und Tordis in den Wuchsperioden eins bis drei unter Berücksichtigung aller Standorte.

Tab. 4-6: Mittlerer dGZ der Pappelklone Max 1, H 275, AF 2 und der Weidenklone Inger und Tordis im Standortcluster S, $\mathrm{U}$ und $\mathrm{L}$ für alle sowie für die in der Modellierung berücksichtigten Standorte ......................................................8 84

Tab. 4-7: Varianzanalyse der Funktionskoeffizienten $\mathrm{a}_{0}$ und $\mathrm{a}_{1}$ für die Testgrößen Klon, Gattung, Klon in der jeweiligen Gattung sowie zwischen den einzelnen Pappelklonen dargestellt durch den p-Wert.

Tab. 4-8: Statistische Kennwerte der Biomassefunktionen der Pappelklone Max 1, H 275, AF 2 sowie der Weidenklone Inger und Tordis.

Tab. 4-9: Korrelationsanalyse zwischen Temperaturvariablen und dem dGZ für die untersuchten Klone in den Standortclustern ALL, S, L und U. Dargestellt sind abnehmend die $r$-Werte der bestkorrelierten Variablen $\mathrm{V}_{1}$ bis $\mathrm{V}_{3}$ sowie der Referenzvariablen $\left(\mathrm{V}_{\mathrm{Ref}}\right)$..

Tab. 4-10: Korrelationsanalyse zwischen Niederschlagsvariablen und dem dGZ für die untersuchten Klone in den Standortclustern ALL, S, L und U. Dargestellt sind abnehmend die r-Werte der bestkorrelierten Variablen $\mathrm{V}_{1}$ bis $\mathrm{V}_{3}$ sowie der Referenzvariablen $\left(\mathrm{V}_{\mathrm{Ref}}\right)$......

Tab. 4-11: Regressionsanalyse für den dGZ in Abhängigkeit von unterschiedlichen Niederschlagsvariablen im Standortcluster ALL. Ausgewählt sind die klonspezifisch besten Anpassungen.

Tab. 4-12: Regressionsanalyse für den dGZ in Abhängigkeit von $\mathrm{N}_{6,7}$ im Standortcluster S. Ausgewählt sind die klonspezifisch besten Anpassungen.

Tab. 4-13: Regressionsanalyse für den dGZ in Abhängigkeit von unterschiedlichen Niederschlagsvariablen im Standortcluster L. Ausgewählt sind die klonspezifisch besten Anpassungen.

Tab. 4-14: Korrelationsanalyse zwischen Trockenheitsindexvariablen und dem dGZ für die untersuchten Klone in den Standortclustern ALL, S, L und U. Dargestellt sind abnehmend die r-Werte der bestkorrelierten Variablen V1 bis V3 sowie der Referenzvariablen (VRef).

Tab. 4-15: Regressionsanalyse für den dGZ in Abhängigkeit von unterschiedlichen Trockenheitsindexvariablen im Standortcluster ALL. Ausgewählt sind die klonspezifisch besten Anpassungen.

Tab. 4-16: Regressionsanalyse für den dGZ in Abhängigkeit von $\mathrm{TI}_{6,7}$ im Standortcluster S. Ausgewählt sind die klonspezifisch besten Anpassungen.

Tab. 4-17: Regressionsanalyse für den dGZ in Abhängigkeit von unterschiedlichen Trockenheitsindices im Standortcluster L. Ausgewählt sind die klonspezifisch besten Anpassungen.

Tab. 4-18: Korrelationsanalyse zwischen Bodenschätzungsvariablen und dem dGZ für die untersuchten Klone in den Standortsclustern ALL, S, L und U. Dargestellt sind die r-Werte...

Tab. 4-19: Regressionsanalyse für den dGZ in Abhängigkeit von AZ im Standortcluster ALL. Ausgewählt sind die klonspezifisch besten Anpassungen.

Tab. 4-20: Regressionsanalyse für den dGZ in Abhängigkeit von BZ im Standortcluster U. Ausgewählt sind die klonspezifisch besten Anpassungen.

Tab. 4-21: Korrelationsanalyse zwischen Texturvariablen und dem dGZ für die untersuchten Klone in den Standortclustern ALL, S, L und U. Dargestellt sind die r-Werte.

Tab. 4-22: Regressionsanalyse für den dGZ in Abhängigkeit von $U_{[\%]}$ im Standortcluster ALL. Ausgewählt sind die klonspezifisch besten Anpassungen.

Tab. 4-23: Regressionsanalyse für den dGZ in Abhängigkeit von den Texturvariablen im Standortcluster S. Ausgewählt sind

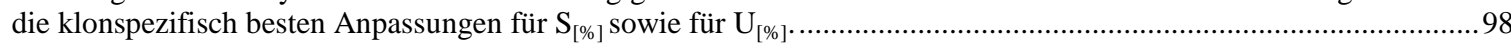

Tab. 4-24: Regressionsanalyse für den dGZ in Abhängigkeit von $\mathrm{U}_{[\%]}$ im Standortcluster L. Ausgewählt sind die klonspezifisch besten Anpassungen.

Tab. 4-25: Korrelationsanalyse zwischen Porenraumvariablen und dem dGZ für die untersuchten Klone in den Standortclustern ALL, S, U und L. Dargestellt sind die r-Werte. 
Tab. 4-26: Regressionsanalyse für den dGZ in Abhängigkeit von nFK im Standortcluster ALL. Ausgewählt sind die klon spezifisch besten Anpassungen

Tab. 4-27: Regressionsanalyse für den dGZ in Abhängigkeit von LK im Standortcluster S. Ausgewählt sind die klonspezifisch besten Anpassungen.

Tab. 4-28: Regressionsanalyse für den dGZ in Abhängigkeit von nFK im Standortcluster L. Ausgewählt sind die klonspezifisch besten Anpassungen.

Tab. 4-29:Variablenkombinationen im Standortcluster ALL

Tab. 4-30: Korrelationsanalyse der aggregierten Variablen und dem dGZ im Standortcluster ALL. Dargestellt sind die $\mathrm{r}$-Werte der aggregierten Variablen $\mathrm{V}_{\mathrm{A} 1}$ bis $\mathrm{V}_{\mathrm{A} 4}$

Tab. 4-31: Variablenkombinationen im Standortcluster S.

Tab. 4-32: Korrelationsanalyse der aggregierten Variablen und dem dGZ im Standortcluster S. Dargestellt sind die $\mathrm{r}$-Werte der aggregierten Variablen $\mathrm{V}_{\mathrm{A} 1}$ bis $\mathrm{V}_{\mathrm{A} 4}$

Tab. 4-33: Variablenkombinationen im Standortcluster U.

Tab. 4-34: Korrelationsanalyse der aggregierten Variablen und dem dGZ im Standortcluster U. Dargestellt sind die $\mathrm{r}$-Werte der aggregierten Variablen $\mathrm{V}_{\mathrm{A} 1}$ bis $\mathrm{V}_{\mathrm{A} 4}$

Tab. 4-35: Variablenkombinationen im Standortcluster L.

Tab. 4-36: Korrelationsanalyse der aggregierten Variablen und dem dGZ im Standortcluster L. Dargestellt sind die $\mathrm{r}$-Werte der aggregierten Variablen $\mathrm{V}_{\mathrm{A} 1}$ bis $\mathrm{V}_{\mathrm{A}}$

Tab. 4-37: Statistische Kennwerte der standortbasierten Ertragsmodelle im Standortcluster ALL für die Pappelklone Max 1 und AF 2 sowie für die Weidenklone Inger und Tordis

Tab. 4-38: Test auf Normalverteilung der Modellresiduen im Standortcluster ALL für die Pappelklone Max 1, AF 2 sowie für die Weidenklone Inger und Tordis. Eine Normalverteilung ist bei p-Werte $<0,05$ nicht gegeben.

Tab. 4-39: Statistische Kennwerte der Standortbasierten Ertragsmodelle im Standortcluster S für die Pappelklone Max 1, H 275, AF 2 sowie für die Weidenklone Inger und Tordis.

Tab. 4-40: Beste Modellanpassungen mit je univariate und multiple Regressionen für die Weide im Standortcluster S. ...... 112

Tab 4-41: Test auf Normalverteilung der Modellresiduen im Standortcluster S für die Pappelklone Max 1, H 275, AF 2 sowie für die Weidenklone Inger und Tordis. Eine Normalverteilung ist bei p-Werten $<0,05$ nicht gegeben.

Tab. 4-42: Statistische Kennwerte der Standortbasierten Ertragsmodelle im Standortcluster U für den Pappelklon Max 1 sowie für den Weidenklon Tordis.

Tab. 4-43: Test auf Normalverteilung der Modellresiduen im Standortcluster U für den Pappelklon Max 1 sowie für den Weidenklon Tordis. Eine Normalverteilung ist bei p-Werten $<0,05$ nicht gegeben.

Tab. 4-44: Statistische Kennwerte der Standortbasierten Ertragsmodelle im Standortcluster L für die Pappelklone Max 1, AF 2 sowie den Weidenklon Inger.

Tab. 4-45: Test auf Normalverteilung der Modellresiduen im Standortcluster L für die Pappelklone Max 1, AF 2 sowie den Weidenklon Inger. Eine Normalverteilung ist bei p-Werten $<0,05$ nicht gegeben.

Tab. 4-46: Statistische Kennwerte der Modellanpassung mittels sigmoidalem Ansatz der dGZ-Entwicklung für die Klongruppen Max und BP an den Standorten „Bad Salzungen“, „Dornburg“ und „Langenwetzendorf“.

Tab. 4-47: Standortpezifische Ertragsteigerungsfaktoren auf Basis der Referenz-dGZ der zweiten Rotation für die Klongruppe Max.

Tab. 5-1: Errechnete Interzeption nach dem Modell von HoynINGEN-HuENE (1983) für unterschiedliche Blättflächenindizes und Freilandniederschläge für Ackerkulturen.

Tab. 5-2: Vergleich der Modelle dieser Arbeit gegenüber die Ali Modelle (2009) in ihren Rahmenbedingung für die Anwendungen. 


\section{Abkürzungsverzeichnis}

\begin{tabular}{|c|c|}
\hline$\varnothing$ & Durchschnitt \\
\hline $\mathrm{a}$ & Jahr \\
\hline allo & Allometrischer Ansatz \\
\hline atro & Absolut trocken \\
\hline $\mathrm{AZ}$ & Ackerzahl \\
\hline Bergbauf. & Bergabufolgelandschaft \\
\hline BP & Balsampappel \\
\hline $\mathrm{BZ}$ & Bodenzahl \\
\hline $\mathrm{C}_{\text {org }}$ & Organischer Kohlenstoff \\
\hline $\mathrm{d}_{0,1}$ & Durchmesser, gemessen in $0,1 \mathrm{~m}$ Stammhöhe \\
\hline$d_{1,3}$ & Durchmesser, gemessen in 1,3 m Stammhöhe \\
\hline dGZ & Durchschnittlicher Gesamtzuwachs \\
\hline$\overline{\mathrm{e}}$ & Verzerrung \\
\hline EDV & Elektronische Datenverarbeitung \\
\hline ESF & Ertragssteigerungsfaktor \\
\hline $\exp 1$ & Exponentieller Ansatz 1 \\
\hline $\exp 2$ & Exponentieller Ansatz 2 \\
\hline FsB & Forschungsinstitut für schnellwachsende Baumarten \\
\hline GW & Grundwasser \\
\hline H 275 & Hybride 275 \\
\hline ha & Hektar \\
\hline HeRo & Kompetenzzentrum HessenRohstoffe (HeRo) e.V. \\
\hline $\mathrm{J}$ & Jahr \\
\hline $\mathrm{K}$ & Kalium \\
\hline Kl-St. & Klimastation \\
\hline KUP & Kurzumtriebsplantage \\
\hline $\operatorname{lin}$ & Lineare Regression \\
\hline OLS & Ordinary Last Squares \\
\hline $\mathrm{L}$ & Lehm \\
\hline LK & Luftkapazität \\
\hline M & Molar \\
\hline $\mathrm{Mx}$ & Treffgenauigkeit \\
\hline $\operatorname{Max}$ & Maxima \\
\hline $\mathrm{Mg}$ & Magnesium \\
\hline Min & Minima \\
\hline $\operatorname{mlin}$ & Multiple lineare Regression \\
\hline mnlr & Multiple nicht-lineare Regression \\
\hline $\mathrm{nFK}$ & nutzbare Feldkapazität \\
\hline $\mathrm{N}$ & Niederschlag \\
\hline $\mathrm{N}_{\min }$ & Mineralischer Stickstoff \\
\hline $\mathrm{NN}$ & Normal Null \\
\hline $\mathrm{Nt}$ & Gesamt-Stickstoff \\
\hline Org. & Organisch \\
\hline
\end{tabular}


$\mathrm{p}$

$\mathrm{P}$

$\mathrm{pH}$

r

$\mathrm{R}^{2}$

$\mathrm{R}^{2}$ korr

RMSE

S

Se

s.a.

sig

S.r.l.

SSE

SST

Sto.

$\mathrm{t}$

$\mathrm{T}$

TI

TLL

TRD

$\mathrm{U}$

VP

WP

$\mathrm{x}$

$\mathrm{y}$

ZS
Signifikanz

Phosphor

Negativer dekadischer Logarithmus der Wasserstoffionen-Aktivität

Pearsonsches Korrelationskoeffizient

Bestimmtheitsmaß

Korrigiertes Bestimmtheitsmaß

Root mean square error (Standardfehler des Mittelwertes der geschätzten)

Sand

Prognosestreuung

Standardabweichung

Sigmoidaler Ansatz

Società a responsabilità limitata

Erklärte Streuung

Gesamtstreuung

Standort

Tonne

Temperatur

Trockenheitsindex

Thüringer Landesanstalt für Landwirtschaft

Trockenrohdichte

Schluff

Vegetationsperiode

Wuchsperiode

Prädiktor

Zielgröße

Zustandstufe 
"Aber die Standortsbedingungen müssen beachtet werden, die die Pappel stellt - ihre Ansprüche an Boden und Klima. Vorweg: Klimatisch sind die meisten Pappeln, vor allem die sog. Gebrauchspappeln, bei uns allen Möglichkeiten gewachsen: sie vertragen Frost und Hitze, Regen- und Trockenzeiten, Hoch- und Tieflagen... bei richtiger Bodenwahl! Auf die Bodenansprüche kommt es an; sie erklären das Versagen der Pappel ebenso wie ihre überragenden Leistungen auf verschiedenen Standorten. “ Pappelinstitut Nordrhein-Westfalen (1948) 


\section{Einleitung}

\subsection{Problemstellung}

Die stark ansteigende Nutzung der fossilen Rohstoffe Kohle, Öl und Erdgas, hervorgerufen durch die weltweite industrielle und demographische Entwicklung, ließ die $\mathrm{CO}_{2}$-Konzentration in der Atmosphäre in den letzten 100 Jahren um 120 ppm auf ca. 400 ppm ansteigen (NOAA 2012). Die Folgen dieses Anstiegs der $\mathrm{CO}_{2}$-Konzentration werden als Treibhauseffekt bezeichnet. Der hierdurch verursachte globale Temperaturanstieg beträgt bis dato ca. $0,74{ }^{\circ} \mathrm{C}$ (IPCC 2007). Die Folgen dieser Erwärmung sind weitreichend: Hierzu zählen u. a. der Anstieg des Meeresspiegels durch das Schmelzen der Polkappen, die Änderungen im Klimaregime, insbesondere in der Häufigkeit von Extremereignissen wie Dürre und Überschwemmungen, und die Verschiebung von Klimazonen (IPCC 2007). Von diesen Folgen sind große Teile der Weltbevölkerung betroffen. Gesellschaftspolitisch gesehen ist die Anpassung an die klimatischen Veränderungen weltweit eine große Herausforderung. Da die globale Nutzung fossiler Brennstoffe nicht signifikant abnimmt, sondern ansteigt, ist mit einer weiteren Erwärmung mit den damit verbundenen Folgen zu rechnen. Um dies zu verhindern und um auf die Verknappung und Verteuerung von fossilen Energieträgern vorbereitet zu sein, werden neue Energiestrategien erarbeitet. Während verbindliche globale Abkommen bisher fehlen, hat sich Deutschland bis zum Jahr 2050 das Ziel gesetzt, $80 \%$ weniger $\mathrm{CO}_{2}$ gegenüber 1990 zu emittieren, den Anteil an erneuerbaren Energien am Bruttoendenergieverbrauch auf $60 \%$ zu steigern und den Primärenergieverbrauch gegenüber 2008 um $50 \%$ zu senken (BMWi 2010).

Im Jahr 2013 wurden in Deutschland vom Endenergiebedarf ca. 23 \% für Strom, ca. 24 \% für flüssige Kraftstoffe und ca. 53 \% für Wärme benötigt (BMU 2013). Die in 2012 durch erneuerbare Quellen produzierte Energie betrug 242 TWh. Damit werden 12,2 \% des Energiebedarfes Deutschlands gedeckt (BMU 2013). Während Wind-, Wasser- und Solarkraft unter den erneuerbaren Energien flächeneffiziente Alternativen zur Stromerzeugung darstellen, spielt die Biomasse im Bereich der Wärme- und Kraftstoffbereitstellung die wichtigste Rolle (BMU und BMELV 2010). Von der Wärmeversorgung 2012 fällt der absolute Anteil an erneuerbaren Energien mit 10,4 \% (138 TWh) jedoch relativ gering aus (BMU 2013). Der erneuerbare Anteil 2012 im Kraftstoffbereich war mit 5,5\% noch niedriger (BMU 2013). Da die Rohstoffproduktion, insbesondere für die energetische aber auch für die stoffliche Nutzung aufgrund geopolitischer und nachhaltiger Kriterien als ein regionaler Markt betrachtet werden sollte, erhalten die Themen Flächenverfügbarkeit, Flächenkonkurrenz und Flächeneffizienz eine sehr wichtige Bedeutung.

Für das Erreichen der deutschen Ziele in der Klima- und Energiepolitik ist der Anbau von Kurzumtriebsplantagen (KUP) mit schnellwachsenden Baumarten eine ernstzunehmende Alternative. Hohe Massenleistungen (CHRISTERSSON 1986, FRIEDRICH 1999, HOFMANN 1999, BOELCKE 2006, RÖHRICHT 2009, BIERTÜMPFEL et al. 2012), Flächen- und Energieeffizienz (WBA 2007), aber auch ökologische Vorzüge (KROIHER et al. 2008, SCHULZ et al. 2008, LAMERSDORF und SCHULTE-BiSPING 2010) bei vergleichsweise geringem Aufwand (BURGER 2011) sind wesentliche Vorteile. Dieses Landnutzungssystem findet seinen Ursprung Mitte der 1960er Jahre in den USA (MC ALPIN et al. 1966) sowie Skandinavien (SIREN et al. 1987), wobei es sich die Wiederaustriebsfähigkeit ausgewählter Baumarten zunutze macht. In Mitteleuropa werden überwiegend Pappeln, Weiden, Robinien, Erlen und Birken angebaut (HANSEN 1991, FRIEDRICH 1999). Anfang der 1970er Jahre begann die wissenschaftliche Erfassung dieser Systeme in Deutschland. Die höchsten durchschnittlichen jährlichen Biomasseerträge (durchschnittlicher Gesamtzuwachs - dGZ) von bis zu ca. 16,0 $\mathrm{t}_{(\text {atro) }} \mathrm{ha}^{-1} \mathrm{a}^{-1}$ wurden in Deutschland mit der stockausschlagfähigen Gattung der Pappel (Populus - P.) auf guten Standorten unter optimalen Bedingungen erreicht (FRIEDRICH 1999, HOFMANN 1999, RÖHLE et al. 
2005, BoelCKE 2006 und BIERTÜMPFEL et al. 2012). Die mit Klonen der Weide (Salix - S.) auf guten Standorten erzielten dGZ reichen bis zu 15,6 $\mathrm{t}_{\text {(atro) }} \mathrm{ha}^{-1} \mathrm{a}^{-1}$ (RÖHRICHT 2009, BIERTÜMPFEL et al. 2012). Trotz der positiven Eigenschaften von KUP ist ihre Flächenausdehnung mit bis dato ca. 4.000 ha im ganzen Land eher marginal (STROHM et al. 2012).

Im Vergleich zur traditionellen Forstwirtschaft liegt der große Vorteil der KUP in den sehr hohen Erträgen (SCHIRMER 1996). Außerdem dauert es bei den verschiedenen Baumarten unterschiedlich lang, bis sie das Klimax ihrer Wachstumsleistung erreicht haben. Während Fichte, Kiefer, Buche, Eiche und Douglasie frühestens nach 40 Jahren ihre maximale Leistung erreichen, benötigen Pappeln zwischen 20 und 30 (SCHOBER 1986) und Weiden etwa vier bis sechs Jahre (WILLEBRAND et al. 1993). Gegenüber landwirtschaftlichen Kulturen für Bioenergie, wie z. B. Mais, liegt der Vorteil von KUP deutlich in ihrer positiven ökologischen Auswirkung sowie in der Effizienz der gesamten Energielinie (WBA 2007).

Für KUP konnten in der Vergangenheit wichtige Fragestellungen, wie die der Arten- und Kloneignung, der Flächenanlage (u. a. Unkrautbekämpfung, Pflanzbeetvorbereitung) sowie der Ernte, weitestgehend geklärt werden (DöHRER 1991, FRIEDRICH 1999). Im Bereich der Standortanforderungen von Pappeln wurden seit den 1950er Jahren wichtige Erfahrungen gesammelt. Die Anforderungen an den Wärme- und Wasserhaushalt sowie an bodenchemische und -physikalische Eigenschaften wurden empirisch ausgearbeitet. Es kann festgehalten werden, dass Pappeln sehr hohe Ansprüche an den Wasserhaushalt haben, lockere Böden und ausreichende Nährstoffversorgung benötigen und erst bei $\mathrm{pH}$ größer 5,0 gut gedeihen können (PAPPELINSTITUT NORDRHEIN-WESTFALEN 1948, MÜLLER 1951, WitTich 1951, MÜLler 1955, SCHMitZ-LENDERS 1956, FRÖHLICH und GROSSCURTH 1973). Der Mindestniederschlag wird mit 300 (HOFMANN 1999) bzw. $250 \mathrm{~mm}$ (DÖHRER 2010) in der Vegetationsperiode beziffert. Hinsichtlich des Wärmebedarfes empfiehlt WEISGERBER (1980) eine Mitteltemperatur von $13{ }^{\circ} \mathrm{C}$ in der Vegetationsperiode. Für die Bodenansprüche wurden Empfehlungen anhand der forstlichen Wasserstufen erteilt: frische Standorte sind für den KUP-Anbau bestens geeignet. Dagegen sind mäßig trockene bis trockene Standorte für den Anbau ungeeignet (RAU et al. 1988, HOFMANN 1999). Eine erste Quantifizierung der leistungsbeeinflussenden Standorteigenschaften und die Aufstellung eines standortbasierten Ertragsmodells von KUP mit Pappel für Sachsen wurde 2009, ein Jahr nach Beginn des Projektes, auf dem diese Arbeit basiert, veröffentlicht (ALI 2009).

Zusammengefasst kann gesagt werden, dass noch erhebliche Wissenslücken hinsichtlich der zuverlässigen Abschätzung der Ertragserwartung, bei unterschiedlichen Klima- und Bodeneigenschaften bestehen. Da diese Informationen entscheidend für den Erfolg oder Misserfolg der Kultur und Planung für den gesamten Anbau sind (AMICHEV et al. 2011), haben sie eine bedeutende Tragweite für die Einführung dieses Landnutzungssystems in der Praxis. Um eine Ertragserwartung geben zu können, ist das bisher gesammelte empirische qualitative Wissen keinesfalls ausreichend.

Um diese Wissenslücke zu schließen, entstand 2008 ein in Deutschland einmaliges Verbundvorhaben mit 24 Institutionen der landwirtschaftlichen und forstlichen Forschung. Nach einheitlichem Versuchsdesign wurden über die Bundesrepublik verteilt 38 Versuchsflächen mit jeweils drei Pappel- und zwei Weidenklonen angelegt. Die Flächen werden in dreijähriger Rotation bewirtschaftet. Durch diese große Anzahl an Versuchsflächen und ihre weite räumliche Verteilung war eine überregionale Repräsentativität gegeben. Die Projektfinanzierung erfolgte durch das Bundesministerium für Ernährung, Landwirtschaft und Verbraucherschutz über die Fachagentur Nachwachsende Rohstoffe e.V.. Die erste Projektphase wurde am 31.01.12 abgeschlossen. Die Koordination übernahm das Kompetenzzentrum HessenRohstoffe e.V.. 


\subsection{Zielstellung}

Das Hauptziel der vorliegenden Arbeit ist: die Erstellung eines überregional gültigen, empirischstatistischen standortbasierten Ertragsmodells, auf Basis der Daten aus dem Verbundvorhaben PROLOC zur Abschätzung von Ertragserwartungen von ausgewählten Pappel- und Weidenklonen auf Ackerflächen im Kurzumtrieb.

Als spezifische Ziele gelten:

1) die Erfassung der Überlebensrate, der Anzahl an Höhentriebe sowie von Durchmesser-, Höhen- und Gesamtzuwachs (Massenleistung) ausgewählter Pappel- und Weidenklone auf verschiedenen Standorte,

2) die Entwicklung von Biomassefunktionen von ausgewählten Pappel- und Weidenklonen für die Versuchsrahmenbedingungen,

3) die Identifizierung der leistungsbeeinflussenden Standorteigenschaften von ausgewählten Pappel- und Weidenklonen auf verschiedenen Standorten aus den Bereichen Klima und Boden sowie,

4) die Ermittlung standortspezifischer Ertragssteigerungsfaktoren von ausgewählten Pappelklonen für das Herleiten von Erträgen aus Folgerotationen. 


\section{Hintergrund}

Im folgenden Kapitel wird auf die Energieziele Deutschlands sowie auf die Flächen- und Nutzungskonkurrenz von KUP mit anderen Landbewirtschaftungsformen eingegangen. Darüber hinaus wird eine Einführung zum Themenkomplex KUP gegeben mit dem besonderen Schwerpunkt auf die verwendeten Baumarten Pappel und Weide. Die leistungsbeeinflussenden Faktoren von KUP werden ebenfalls erläutert, wobei der Fokus insbesondere auf den Standortanforderungen liegt. Zuletzt erfolgt eine Betrachtung der bestehenden Modelle zur Ertragsprognose von KUP.

\subsection{Energieziele Deutschlands}

Die Deckung des Energiebedarfes eines Landes ist hochkomplex. Dabei geht es um die Erhaltung des Wohlstands und der Produktivität der Gesellschaft. Angesichts des Klimawandels und der Verteuerung und Verknappung fossiler Rohstoffe ist die Suche nach Alternativen zur Deckung des Energiebedarfs unumgänglich.

Einige Zahlen zu fossilen Rohstoffen: Erdöl und Erdgas werden in Deutschland zu je $86 \%$ und $96 \%$ importiert (AGENTUR FÜR ERNEUERBARE ENERGIEN 2013). Im Jahr 2000 wurden für die gesamten Energieimporte Deutschlands 39,9 Mrd. $€$ ausgegeben, 2012 waren es bereits 92,7 Mrd. $€$ (AGENTUR FÜR ERNEUERBARE ENERGIEN 2012).

Die 2009 gewählte Bundesregierung verabschiedete im Jahr 2010 eine Strategie mit dem Namen „Energiekonzept für eine umweltschonende, zuverlässige und bezahlbare Energieversorgung“ (BMWi 2010). Hauptinhalte dieser Strategie sind:

- Reduktion der $\mathrm{CO}_{2}$-Emissionen gegenüber 1990 in 10-Jahreschritten von 2020 bis 2050: um $40 \%, 55 \%, 70 \%$ sowie um $80-95 \%$.

- Erhöhung des Anteils der erneuerbaren Energien am Bruttoendenergieverbrauch in 10-Jahreschritten von 2020 bis 2050: um $18 \%, 30 \%, 45 \%$ und $60 \%$.

- Senkung des Primärenergieverbrauchs gegenüber 2008 bis 2020 um $20 \%$ und bis 2050 um $50 \%$.

Im Strombereich sind bereits eine Vielzahl an energie-, stoff- und flächeneffizienten Technologien vorhanden. Im Wärme- und Kraftstoffbereich existieren hingegen nur wenige Alternativen zu den fossilen Energieträgern. In diesen Bereichen spielt bisher die Biomasse die wichtigste Rolle (BMU und BMELV 2010).

Im Angesicht dieser hochgesteckten Ziele stellt das flächen- und kosteneffiziente Landbewirtschaftungssystem KUP eine ernstzunehmende Alternative für die Produktion von holzigen Biomasse und Erzeugung von Energie dar. Aber wo befinden sich die Flächen und mit welchen anderen Landnutzungsformen konkurrieren KUP? Im Folgenden wird gezeigt, wo Eckpunkte der Konkurrenz von KUP mit der Forst- und Landwirtschaft bestehen.

\subsubsection{Flächen- und Nutzungskonkurrenz mit der Forstwirtschaft}

Da KUP eine Form der Ackernutzung darstellen und vom Waldbegriff ausgeschlossen sind (BUNDESWALDGESETZ § 2 WALD), besteht mit dem Wald keine Flächen- sondern eine Produktkonkurrenz. Der Anteil des energetisch genutzten Holzes innerhalb der Forstwirtschaft hat sich im Zeitraum zwischen 1987 und 2011 von ca. 13,0 Mio. $\mathrm{m}^{3}$ auf ca. 70,0 Mio. $\mathrm{m}^{3}$ ( ca. $51 \%$ ) mehr als verfünffacht (MANTAU 2012). Vom Gesamtholzauskommen betrug der energetisch genutzte Anteil 1987 ca. $28 \%$, im Jahr 2011 bereits $51 \%$. Bisher hat dies zu keiner deutlichen Erhöhung der Holzmobilisierung geführt, weil der Zuwachs derzeit noch höher als der Einschlag ist (BMELV 2012). Das Heizen mit Holz 
ist preisgünstiger als mit fossilen Rohstoffen, daher ist davon auszugehen, dass sich die Holznachfrage für die energetische Nutzung weiter erhöhen wird. Der Druck auf den Wald wird demnach zunehmen. Es hat sich zudem gezeigt, dass eine höhere Holzmobilisierung wichtige Waldfunktionen wie Erholung, Habitat und Wasserschutz beeinträchtigen kann. Wird mehr entnommen als nachwächst, ist die Substanz des Waldes gefährdet und die Prinzipien der Nachhaltigkeit werden verletzt (RöHLE 2012). Veränderungen derzeitiger Waldbaustrategien zu einer optimierten Erzeugung von Holz für die energetische Nutzung sind nur langfristig möglich (BEMMANN et al. 2010). Da KUP bereits nach einer Rotation (zum Beispiel von drei bis fünf Jahren) hohe Holzerträge produzieren, können diese eine schnell steigende Nachfrage nach Energieholz kurzfristig decken. KUP sind daher eher als Alternative zur Entlastung des Waldes zu betrachten und keine direkte Konkurrenz.

\subsubsection{Flächen- und Nutzungskonkurrenz mit der Landwirtschaft}

Obwohl die Produktion von Nahrungsmitteln eine primäre Notwendigkeit der Menschheit ist, ist die Energieerzeugung für das Funktionieren unseres gesellschaftlichen und ökonomischen Systems ebenfalls sehr wichtig. In der Landwirtschaft besteht ein großer Konflikt zwischen dem Anbau von Pflanzen als Nahrungs- und Futtermittel sowie dem Anbau für die energetische Nutzung. Weltweit hat der Anbau von zum Beispiel Mais, Weizen und Zuckerrohr für die Erstellung von Biokraftstoffen zu einer merklichen Preiserhöhung und teilweise Verknappung der Lebensmittel geführt (FAO 2013). Aus diesem Grund wird der Anbau pflanzlicher Biomasse als nachwachsender Rohstoff für die energetische aber auch für die stoffliche Nutzung, insbesondere aus Sicht der Nahrungsmittelproduzenten, kritisch betrachtet. Fakt ist jedoch, dass nachwachsende Rohstoffe sowohl für die energetische als auch stoffliche Verwendung für zukünftige Generationen essentiell sein werden.

Ein weiterer Aspekt der Konkurrenz in der Landwirtschaft, welcher nicht ausreichend beleuchtet wird, betrifft das Spannungsfeld zwischen der Futtermittelproduktion für die Erzeugung tierischen Eiweißes und die Produktion pflanzlicher Lebensmittel. Der Flächenbedarf für die Erzeugung tierischer Lebensmittel ist ca. fünf Mal höher als der für die Produktion pflanzlicher Lebensmittel (NOLEPPA und VON WITZKE 2012). In vielen Nationen wird deutlich mehr tierisches Eiweiß konsumiert als physiologisch notwendig wäre (BROwN 2012, HEINRICH BÖLL STIFTUNG et al. 2013).

In Deutschland stehen KUP natürlicherweise mit anderen zu energetischen Zwecken angebauten Kulturen in Konkurrenz. Hier kann insbesondere der Anbau von Mais zur Biogaserzeugung genannt werden. Der Maisanbau für Biogas wird subventioniert und ist somit ein profitabler Markt. Der Wissenschaftliche Beirat für Agrarpolitik des BMELV erarbeitete 2007 eine Empfehlung an die Politik für die Nutzung von Biomasse zur Energiegewinnung (WBA 2007). Eines der Hauptergebnisse der Studie

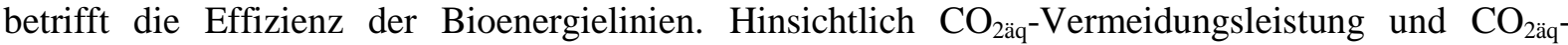
Vermeidungskosten schnitt Biogas aus Mais deutlich schlechter ab als die Verbrennung und KraftWärme-Kopplung von KUP- oder Waldrestholz. Im ERNEUERBARE-ENERGIEN-GESETZ wurden für die Stromerzeugung aus KUP-Holz höhere Vergütungssätze festgelegt. Für die Wärmeerzeugung mittels KUP-Holz sind derzeit keine Förderungen vorgesehen.

Ein weiteres Konfliktfeld stellt die Konkurrenz mit Grünland dar. Sind Landwirte an der Anlage von KUP interessiert, werden immer öfter wenig genutzte Grünlandflächen in Betracht gezogen. Dies geht einher mit einer steigenden Anzahl an Grünlandflächen, die für die Tierfütterung nicht mehr benötigt werden. Die Anlage von KUP auf Grünlandflächen ist zwar grundsätzlich möglich, aber aufgrund vieler gesetzlicher Beschränkungen insgesamt als eher problematisch zu bewerten. Grünland (Dauergrünland) ist definiert als Fläche, die durch Einsaat oder auf natürliche Weise zum Anbau von Gras genutzt wird und seit mindestens fünf Jahren nicht mehr Teil einer Fruchtfolge ist. Das Grünlanderhaltungsgebot (VO (EG) Nr. 73/2009, Artikel 6, VO (EG) Nr. 1122/2009, Artikel 3) legt für die einzelnen Bundesländer fest, dass die Grünlandgesamtfläche, bezogen auf das Jahr 2003, nicht mehr als 
5,0 \% abnehmen darf. Bei darüber hinaus gehenden Flächenverlusten soll das Land eine Verordnung erlassen, in der der Grünlandumbruch einer Genehmigungspflicht unterliegt. Ab einem Flächenverlust von mindestens 8,0\% kann das Bundesland verordnen, das umgebrochene Grünland wieder einzusäen. Bei Flächenverlusten über 10,0 \% muss das Bundesland dies verordnen.

Grünland erbringt Ökosystemdienstleistungen wie Hochwasserschutz, Kohlenstoffsenke und Habitatfunktionen. Signifikative Ökosystemleistungen können nur artenreichem Grünland zugerechnet werden. KUP können gleichwertige Ökosystemdienstleistungen wie artenreiches Grünland bei gleichzeitig hohen Flächenerträgen erbringen (BEMMANN und BÖHNISCH 2011). Seitens des Naturschutzes werden die positiven Ökosystemdienstleistungen von Grünland häufiger generalisiert. Dabei beträgt der Anteil an artenreichem Grünland an der gesamten Grünlandnutzung in Deutschland 16,8 \% (MATZDORF et al. 2010).

Synergetische Verbindungen von KUP mit dem Anbau von Ackerkulturen sowie mit dem Grünland sind in den bekannten Agroforstsystemen möglich (HERZOG 1997). Dort werden Bäume in Reihen zwischen den Ackerkulturen oder dem Grünland bewirtschaftet. Der Abstand zwischen den Reihen kann beliebig gewählt werden, zum Bespiel von fünf bis zu $100 \mathrm{~m}$ betragen. Neben der Ackerkultur oder dem Grasanbau als ein Teil der Agroforstsysteme kann zusätzlich die Holzproduktion gewährleistet werden.

Bei günstig ausgewähltem Pflanzverband ist die Reduktion der Windgeschwindigkeit eine der wichtigen ökologischen Funktionen der Baumreihen. Hierdurch werden besonders Bodenerosion und Evaporation verringert (BÄRWOLFF et al. 2011). Letzteres verbessert das Mikroklima deutlich, was sich positiv auf den Pflanzenbau auswirkt. Agroforstsysteme haben einen positiven Einfluss auf die Biodiversität, weil sie als zusätzliche Randzonen zwischen Ökotonen dienen. In Agroforstsystemen kann sowohl Wert- als auch Energieholz erzeugt werden. In den Tropen stellen sie eine bedeutsame Landbewirtschaftungsform dar (HUXLEY 1999).

\subsection{Anbau schnellwachsender Baumarten}

Neben Kiefer, Fichte, Douglasie, Eucalyptus, Ahorn, Platane, Robinie, Pawlonia, Birke und Erle zählen auch Arten der Pappel und der Weide zu den schnellwachsenden Baumarten. Der Anbau von Pappeln in Deutschland ist eine Geschichte mit vielen Hochs und Tiefs. Zu Anfang des 20. Jahrhunderts beschreibt SCHMITZ-LENDERS (1956) die Pappel als eine für die Forstwirtschaft eher exotische Baumart. In dieser Zeit wurde die Pappel nur marginal im Wald angebaut. Sie fand ihren Platz eher außerhalb des Waldes, zum Beispiel an Gewässerufern oder auf Wegen. Das Besondere an den Pappeln war damals, wie heute ihre Wuchsleistung. Schon nach 30 bis 40 Jahren ist die Produktion von Starkholz möglich.

Die erste Pappelanbauwelle entwickelte sich während des zweiten Weltkrieges aus der großen Holznot heraus (NAUMANN 1980). Die Wälder sowie auch die Feldgehölze wurden massiv übernutzt, um den Bedarf an Brennholz zu decken. Nach dem Krieg wurden große Pappelflächen angelegt, wobei die Gesamtfläche nicht mehr nachvollziehbar ist. Nach Angabe von HERBERT HESMER (in NAUMANN 1980) wurden allein in den Rheinischen Forstämtern in einem Zeitraum von vier Jahren 955.000 Pflanzen gesetzt. Wird eine Pflanzdichte von 400 Stk. ha ${ }^{-1}$ zugrunde gelegt, ergeben sich eine Fläche von ca. 2.400 ha. In dieser Zeit wurden überwiegend Schwarzpappelklone, vornehmlich Robusta, Regenerata und Serotina (allesamt $P$. nigra x P. deltoides Kreuzungen), angebaut. Im Anbau gab es sowohl große Erfolge als auch komplett misslungene Anlagen. Diese Anbauwelle erbrachte optimale Bedingungen für ein umfassendes Erforschen des Pappelanbaus. Dadurch wurden sehr detaillierte Studien zur Bewirtschaftung, Ertragskunde, Bestandespflege sowie insbesondere zu den Standortanforderungen der Pappel erstellt. 
Bedingt durch die Ölkrisen 1973 und 1980 sowie durch die kontrovers diskutierte Überproduktion von Nahrungsmitteln in den 1980er Jahren, erhielt sowohl der klassische Pappelanbau als auch die bis dato eher unbekannte Bewirtschaftung von schnellwachsenden Baumarten im Kurzumtrieb in Deutschland einen entscheidenden Impuls (KNUST 2009).

\subsubsection{Anbau im Kurzumtrieb}

Kurzumtriebsplantagen bezeichnen den Anbau von schnellwachsenden Baumarten auf Ackerflächen. KUP stellen eine innovative Form der Landnutzung dar, bei der mit hohen Flächenerträgen bei vergleichsweise geringem Arbeits- und Energieaufwand Holz erzeugt wird (BURGER 2011). Im Vordergrund steht die Maximierung der Holzerträge. Eine KUP zeichnet sich durch folgende Merkmale aus (ZSUFFA und GAMBLES 1992):

- gut vorbereitete Standorte

- gleichaltrige Individuen einer Art

- hohe Pflanzdichten

- (teilweise) Nutzung des Stockausschlags

- kurze Produktionsintervalle (Rotationen)

- hohe Standortanforderungen

- hoher Mechanisierungsgrad

KUP ähneln somit mehr einem landwirtschaftlichen als einem forstlichen System (ZsUFFA und GAMBLES 1992). In Deutschland sind für den Anbau von KUP alle Arten der Gattung der Pappeln, der Weiden, der Robinien (Robinia), der Birken (Betula) und der Erlen (Alnus), darüber hinaus der gemeinen Esche (Fraxinus excelsior), der Stieleiche (Quercus robur), der Traubeneiche (Quercus petraea) und der Roteiche (Quercus rubra) vorgesehen und erhalten eine Beihilfe. In der Praxis kommen hauptsächlich Pappel, Weide und Robinie zum Einsatz (HANSEN 1991, FRIEDRICH 1999, MAKESCHIN 1999). Für einen erfolgreichen Anbau sollte die Baumart folgende Eigenschaften besitzen (RAU et al. 1988, HOFMANN 1999, DÖHRER 2010):

- sicheres Anwuchsverhalten

- rasches Jugendwachstum

- hohe Biomasseleistung

- sehr gutes und lang anhaltendes Stockausschlags- und Regenerationsvermögen

- Früh- und Spätfrostresistenz bei optimaler Adaption an die Vegetationszeit

- Widerstandsfähigkeit gegen abiotische/biotische Schaderreger

- leichte und kostengünstige Vermehrbarkeit

- ausgeprägte Dichtestandstoleranz

- angemessene Holzqualität

Die Bezeichnung Kurzumtriebsplantage beinhaltet unterschiedlich lange Umtriebszeiten, welche aus forstwirtschaftlicher Sicht jedoch allesamt kurz sind. Die Produktionszeiträume liegen zwischen drei und 20 Jahren. MuHS (1986) klassifiziert die Umtriebszeiten nach ihrer Dauer in Mini- (2 - 5 Jahre), Midi- (6 - 10 Jahre) und Maxi-Rotation (11 - 20 Jahre). In der Mini- und Midi-Rotation wird der Stockausschlag der Gewächse ausgenutzt. In Maxi-Rotationen handelt es sich um einstämmige Bestände. Abbildung 2-1 zeigt Pappelbestände unterschiedlichen Alters.

Mit einer angepassten Wahl der Umtriebszeit kann Holz unterschiedlicher Beschaffenheit produziert werden. Im kurzen Umtrieb wird ausschließlich Energieholz, im mittleren Umtrieb Industrieholz, im langem Umtrieb Industrie- und Stammholz erzeugt. Tabelle 2-1 fasst die Anbaumöglichkeiten von KUP zusammen. 
a)
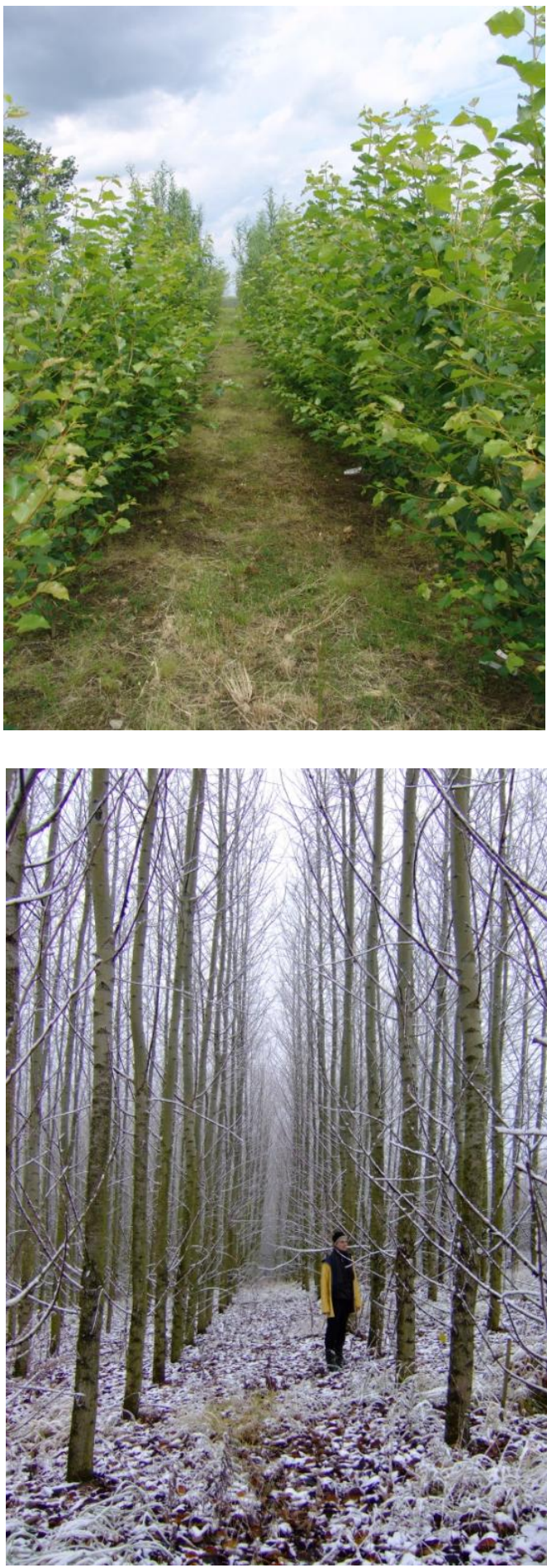

b)
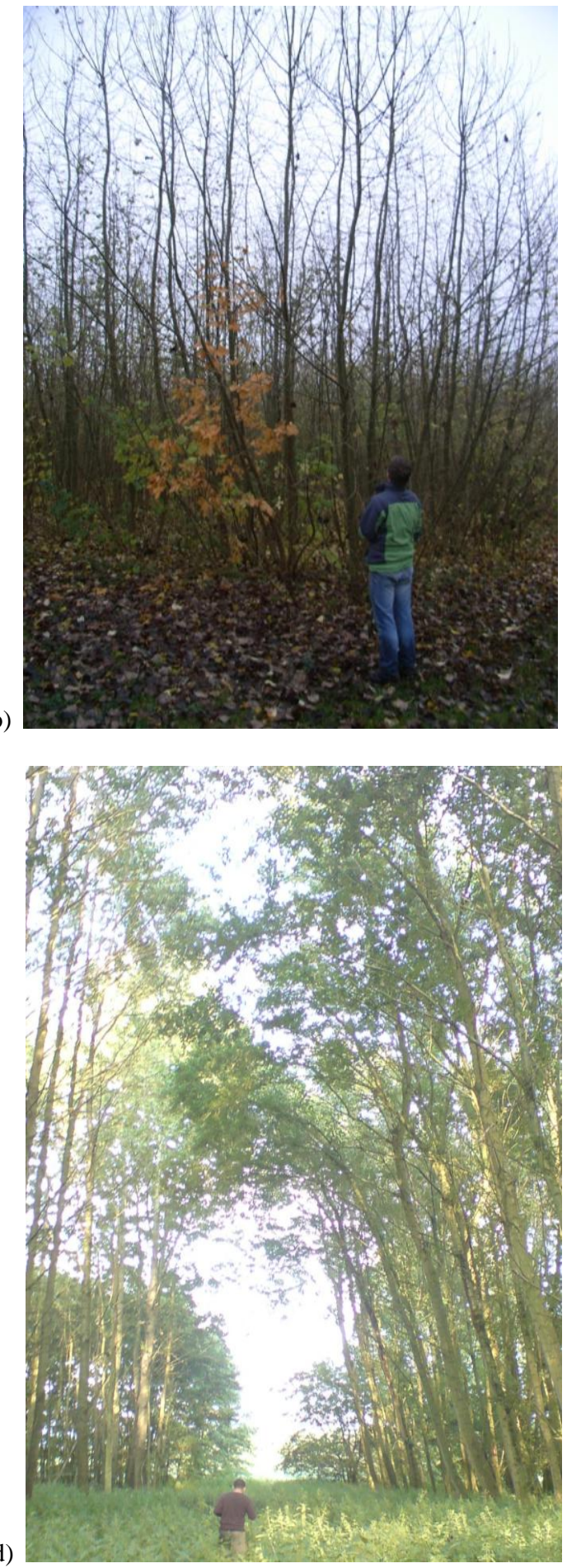

Abb. 2-1: Pappel Kurzumtriebsplantagen a) 2-jähriger Pappelbestand Pommritz b) 4-jähriger Pappelbestand Potsdam (Stockausschlag) c) 12-jähriger Pappelbestand Methau (Bild: SCHILDBACH, M.) d) 20-jähriger Pappelbestand Wildeshausen. 
Die moderne Bewirtschaftung von schnellwachsenden Baumarten in KUP hat ihre Wurzeln in der Niederwaldbewirtschaftung (PlaggenBORG 1989). Seit dem 14. Jahrhundert wurden Niederwälder genutzt, um u. a. Brennholz, Stangen und Zaunholz zu gewinnen. Dafür wurden stockausschlagsfreudige Baumarten wie zum Beispiel die Hainbuche, die Linde, die Hasselnuss, die Birke, die Erle, die Aspe und die Weide genutzt. Die Umtriebszeit betrug in der Regel 20 bis 30 Jahre. Als Pionier der modernen KUP wird oft der Platanen-Anbau in Kurzumtrieb („Silage Sycamore") in den USA genannt (MC ALPIN et al. 1966). Die Idee dazu entstand während einer Forsttagung in Savannah. Dem Vorschlag, Bambus-Arten für die Erzeugung von Biomasse in die USA einzuführen, stand die Idee, mit einer holzigen nativen Art wie der Platane zu arbeiten, gegenüber (STEINBECK 1999). Parallel zur Idee des Platanen-Anbaus in den USA begannen in Schweden die Untersuchungen zum Anbau von Weiden in KUP (SIREN et al. 1987).

Tab. 2-1: Übersicht der Anbausysteme bei den Kurzumtriebsplantagen

\begin{tabular}{|c|c|c|c|}
\hline Rotation & Mini-Rotation & Midi-Rotation & Maxi-Rotation \\
\hline Ernteintervall [a] & $2-5$ & $6-10$ & $11-20$ \\
\hline Produktziel & Energieholz & Energie- und Industrieholz & Industrie- und Stammholz \\
\hline Empfohlene Arten & $\begin{array}{c}\text { Balsampappel (sowie ihre } \\
\text { Hybride), Weide }\end{array}$ & $\begin{array}{c}\text { Balsam-, Schwarz- und } \\
\text { Zitterpappel, Robinie, Erle } \\
\text { und Birke }\end{array}$ & $\begin{array}{c}\text { Balsam-, Schwarz- und } \\
\text { Zitterpappel, Robinie, Erle } \\
\text { und Birke }\end{array}$ \\
\hline Pflanzen je Hektar & $10.000-15.000$ & $3.000-10.000$ & $400-3.000$ \\
\hline Verband $[\mathrm{m}]$ & $\begin{array}{l}\text { z. B. } 2 * 0,5-2 * 0,4 \\
\text { oder Doppelreihe }\end{array}$ & z. B. $2 * 1,5-2 * 0,5$ & z. B. $5 * 5-2 * 1,5$ \\
\hline $\begin{array}{l}\text { Maximale } d_{1,3} \text { bzw. } d_{0,1} \\
{[\mathrm{~cm}]}\end{array}$ & 8 bzw. 12 & 24 bzw. 36 & 50 bzw. 60 \\
\hline Ernte & $\begin{array}{l}\text { Agrartechnik- } \\
\text { Direkthäckseln oder } \\
\text { zweiphasig }\end{array}$ & $\begin{array}{l}\text { Forsttechnik- } \\
\text { zweiphasig oder } \\
\text { Fäller-Bündler }\end{array}$ & $\begin{array}{c}\text { Forsttechnik- } \\
\text { zweiphasig oder Vollernter }\end{array}$ \\
\hline
\end{tabular}

Wie erwähnt, wurde die KUP-Forschung in Deutschland ab den 1970er Jahren stark ausgebaut. Die Forschung wurde maßgeblich vom Forschungsinstitut für schnellwachsende Baumarten (FsB, ehemals Forschungsinstitut für Pappelwirtschaft) vorangetrieben (GROSSCURTH 1982). Die erste KUPVersuchsfläche Deutschlands - das „Haferfeld“ - wurde in der Nähe von Hannoversch Münden vom FsB im Jahr 1973 angelegt. Von 1983 bis 1997 führte das FsB zusammen mit der Bundesanstalt für Forst- und Holzwirtschaft und der Universität München große Forschungsprojekte zu KUP durch. Diese Projekte untersuchten vielseitige Fragestellungen von anbautechnischen Untersuchungen bis hin zur Bereitstellung von hochwertigem Vermehrungsgut sowie Pflanzenernährung und Standortkunde. Die Projekte wurden von den Ministerien für Forschung und Technologie sowie für Ernährung, Landwirtschaft und Verbraucherschutz gefördert. Anfang der 1990er Jahre erreichte das Thema weitere Institutionen der landwirtschaftlichen Forschung, wie zum Beispiel die Landesforschungsanstalt für Landwirtschaft und Fischerei Mecklenburg Vorpommern, das Leibniz-Institut für Agrartechnik Potsdam-Bornim e. V. und die Thüringer Landesanstalt für Landwirtschaft (TLL). Seit dem Jahre 2000 folgten größere Verbundprojekte wie Dendrom, AgroWOOD und AgroForNet, in denen KUP ganzheitlich betrachtet, die Forschung vertieft und ein integrativer Ansatz für die Einbindung der Praxis durchgeführt wurde. In dieser Phase wurden Hochschulen wie die Technische Universität Dresden, die Fachhochschule Eberswalde und die Universität Freiburg verstärkt eingebunden. Derzeit ist der Anbau von Gehölzen im Kurzumtrieb ein sehr beliebtes Forschungsobjekt. 
Deutschlandweit beträgt der kommerziell genutzte Flächenanteil von KUP, trotz der großen Anstrengungen aus der Forschung, nur ca. 4000 ha (STROHM et al. 2012). Davon befinden sich fast $40 \%$ in Brandenburg. Als Vergleich: die Flächenausdehnung beim Anbau von Mais für Bioenergie beträgt 900.000 ha (FACHVERBAND BIOGAS E.V. 2012). In Europa ist Schweden das Land mit den meisten KUP-Flächen, sein Anteil beträgt 20.000 ha (KARACIC et al. 2003). Dies kann als verhältnismäßig geringe Fläche angesehen werden.

Der Anbau von KUP bietet viele Vorteile. Im Folgenden werden einige aufgezählt:

- Sie können aufgrund ihrer hohen Ertragsleistung (siehe 2.4) maßgeblich zur Sicherstellung der Rohstoffversorgung im Land beitragen.

- Mit KUP kann innerhalb kürzester Zeit Holzbiomasse produziert werden, was in der traditionellen Forstwirtschaft aufgrund der längeren Umtriebszeiten nicht möglich ist.

- Während in der Forstwirtschaft Energieholz als Nebenprodukt der Holzernte anfällt, kann mit KUP Holz für verschiedene Zwecke produziert werden.

- Eine besondere Rolle wird Holzbiomasse in einer biobasierten Gesellschaft spielen, in der Erdölerzeugnisse für die Erstellung von beispielsweise Grundchemikalien und Kunststoffen nach und nach durch nachwachsende Rohstoffe ersetzt werden (ULBER 2010).

- Durch eine präzise Art- und Klonwahl sowie durch die Wahl der Umtriebszeit ist die Erzeugung eines qualitativ homogenen Produktes möglich.

- Gegenüber Ackerkulturen ist bei KUP die Resistenz der Gehölze gegen kühle Witterung von entscheidendem Vorteil (LEWANDOWSKI und VETTER 2000).

- Der Anbau von KUP ist aus ökologischer und naturschutzrechtlicher Sicht unbedenklicher als zum Beispiel der Maisanbau, insbesondere wenn Bestände mehr Struktur aufweisen (BfN 2012).

- Da der Einsatz von Herbiziden, Pestiziden und Dünger bei KUP selten notwendig ist, sind negative Auswirkungen auf die Schutzgüter Boden, Wasser, Luft und Habitat gegenüber Ackerkulturen deutlich verringert (TRINKAUS 1998, VANDE WALLE et al. 2007).

- KUP fördern die regionale Entwicklung: Arbeitsplätze rund um die Produktkette werden geschaffen. Das Geld, welches früher in Energieimporte ausgegeben wurde, bleibt in der Region.

Beim Anbau von KUP gibt es aber auch Risiken. Die größten Anbaurisiken sind Krankheit, inadäquate Unkrautbekämpfung bzw. fehlende Pflege sowie schlechte Standort-, Baumart- und Klonwahl (JOACHIM 1989, HANSEN 1991). Jedes der genannten Risikofaktoren kann sehr starke Schäden in einem Bestand verursachen. Unter den Krankheiten ist der Rostpilz (Melampsora spec.) hervorzuheben. Bei starkem Befall kann er eine Plantage stark schwächen (WIDIN und SCHIPPER 1981). Zusätzlich können weitere Schadfaktoren wie Schadinsekten, Frost, Wind und Hitze genannt werden. Diese haben in der Regel kein so starkes Schadausmaß als die zuerst genannten. Bei geschwächten Beständen zum Beispiel aufgrund schlechter Nährstoffversorgung oder wegen Wassermangel, ist die Intensität dieser Schäden oft erhöht.

Ein weiterer Risikoaspekt ist die stark eingeschränkte genetische Basis der Bestände. Anders als die in der Forstwirtschaft verwendeten Populationen ${ }^{1}$ werden in den KUP überwiegend Klone eingesetzt. Da oft mit wenigen Klonen gearbeitet wird, ist das Risiko von klonspezifischen Schäden biotischer und abiotischer Natur deutlich höher als bei Populationen (LIBBY 1980). Daher empfiehlt sich der Anbau von Klonmischungen, die in Reihen oder kleinen Plots gepflanzt werden. Als eine angemessene Klo-

\footnotetext{
${ }^{1}$ Als Population wird die gesamte Nachkommenschaft ausgesuchter Vater- und Mutterbäume bezeichnet, dabei handelt es sich um Individuen, welche genetisch miteinander verwandt sind. Durch den Anbau einer Population ist eine weite genetische Basis gegeben, welche einen hohen Widerstand gegenüber biotische und abiotische Schadfaktoren gewährleistet.
} 
nanzahl einer Mischung werden sieben bis 25 empfohlen (LIBBy 1980, DEBELL und HARRINGTON 1993). In Deutschland gibt es einige wenige Mehrklonsorten ${ }^{2}$. Hierzu zählen Brühl 1 bis 8 (P. trichocarpa), Tapiau 1 bis 8 ( $P$. tremula), Ahle 1 bis 20 (P. tremula $x$ P. tremula) und Münden 1 bis 20 (P. tremula $x$ P. tremuloides) (BLE 2012). In den letzten Jahren ist dennoch keine Zulassung neuer Mehrklonsorten erfolgt. Monoklonale Anlagen mit spezifischen Klonen wie bei I-214 (P. deltoides $x$ P. nigra) werden weltweit erfolgreich angebaut (FAO 1979). Jedoch gibt es auch Negativbeispiele dafür, dass Klone bei Krankheiten (zum Beispiel durch Rostpilz) komplett kapitulierten. In diesem Zusammenhang wäre der Pappelklon Beaupré ( $P$. trichocarpa $x$ P. deltoides) zu nennen (RAU et al. 1988). Daher ist die genetische Diversität der Plantagen ein ernstzunehmendes Thema.

Aus den Ergebnissen einer großen Umfragestudie von Praktikern arbeitete NeUBERT (2013) die Gründe für den (noch) ausbleibenden „Boom“ von KUP heraus und nennt drei wichtige Aspekte:

- geringe Flächenverfügbarkeit,

- geringer Profit,

- keine ausgereiften Marktstrukturen.

Darüber hinaus sollte:

- das Fehlen verlässlicher Werkzeugen zur überregionalen Ertragsprognostizierung berücksichtigt werden.

Wie im Kapitel 2.1 bereits diskutiert, sind Flächenverfügbarkeit und teilweise auch der Profit durch die Politik regelbar, wie am Beispiel Biogasmais verdeutlicht ist. Das Problem der unausgereiften Marktstrukturen kann erst beim Erreichen einer kritischen Produktmenge bzw. Anbaufläche gelöst werden. Nichtsdestotrotz liegt dem KUP-Anbau in Deutschland ein Paradoxon zugrunde: Einerseits besteht im Land ein enorm hoher Energie- und Rohstoffbedarf, andererseits wird einer der effizientesten und umweltfreundlichsten Landnutzungssysteme für die Erzeugung von Biomasse in der Praxis kaum angewandt.

\subsection{Verwendete Baumarten in Kurzumtriebsplantagen}

Im Folgenden wird auf die Taxonomie und auf die allgemeinen Eigenschaften der in KUP verwendeten Baumarten, insbesondere der im Projekt berücksichtigten Pappeln und Weiden, eingegangen.

\subsubsection{Pappel}

Die Pappel gehört, wie die Weide, zur Ordnung der Malpighiales (Malpighienartige) und zur Familie der Salicaceae (Weidengewächse). Pappeln sind auf der Nordhalbkugel beheimatet. Es werden ca. 40 Arten gezählt, wobei einige Autoren bis zu 85 Arten differenzieren (WEISGERBER 1980). Dazu gibt es eine Vielzahl an Hybriden (PlaggenbORG 1989). Aufgrund dieses Artenreichtums können Pappeln auf diversen Standorten angebaut werden und verschiedene Qualitätsansprüche erfüllen (WEISGERBER 1980, JOACHIM 1989). Pappeln sind schnellwüchsig, insbesondere im jungen Alter. Sie können Höhen von 15 bis $50 \mathrm{~m}$ und Dimensionen des $\mathrm{d}_{1,3}$ von bis zu 2,5 m erreichen. Zudem sind Pappel leicht kreuzbar und sie können vegetativ vermehrt werden (EISENREICH 1956, FRÖHLICH und GROSSCURTH 1973). Als Pionierbaumart benötigt die Pappel viel Licht, Unkrautfreiheit und lockeren Boden (HÜBENER 1989). Pappeln kulminieren, abhängig von der Standortwuchskraft, in Beständen mit geringen Pflanzdichten mit ca. 25 bis 40 Jahren (BLUME 1951). Die Systematik der Pappel ist in sechs Sektionen gegliedert. Tabelle 2-2 zeigt die aktuelle Pappelsystematik nach ECKENWALDER (1996).

\footnotetext{
${ }^{2}$ Eine Mehrklonsorte ist eine für den Markt definierte Mischung von Klonen mit gleichen Anteilen von jedem Klon.
} 
Viele Pappelarten sind in Auengebieten beheimatet. Die Mehrheit dieser Pappelarten ist den Sektionen Aigeiros (Schwarzpappel) und Tacamahaca (Balsampappel) zugeordnet. Pappeln der Sektion Leucoides sind gut an Moorstandorte angepasst. Innerhalb der Sektion Populus sind an Trockenheit angepasste Arten klassifiziert (SORIANO 1993). Die Arten der weiteren Sektionen Abaso und Turanga kommen eher in wärmeren Regionen der Tropen und Subtropen vor (HALLGREEN 1989, SORIANO 1993). Von den genannten Sektionen haben nur drei eine kommerzielle Bedeutung, die Sektionen Aigerios, Tacamahaca und Populus (RAU et al. 1988, PINON 1992, ROYLE und OSTREY 1995). Im Folgenden werden diese Sektionen weiter vertieft.

Die Pappeln der Sektion Aigeiros sind typischerweise in Nordamerika, Europa und Westasien verbreitet. Meistens finden sich die Schwarzpappeln in gemäßigten Klimaten. Mit Schwarzpappeln wie zum Beispiel $P$. nigra und $P$. deltoides werden in wärmeren Standorten sehr hohe Massenerträge erreicht. Ihr Holz ist mit ca. 300 bis $350 \mathrm{~kg} \mathrm{~m}^{-3}$ besonders leicht (BONNEMANN 1980) und kann beispielsweise als Sperr- und Papierholz sowie energetisch verwendet werden. Pappeln dieser Sektion kommen aus den Auenbereichen und können gut auf frischen Standorten angebaut werden. Die Schwarzpappel ist eine ausgesprochene Lichtbaumart (FRÖHLICH und DIETZE 1975). Sie hat hohe Ansprüche an die Wasser- und Nährstoffversorgung (WEISGERBER 1980). Schwarzpappeln verfügen über lockere Kronen, welche die Begleitflora nur schlecht unterdrücken, dafür sind sie aber relativ unempfindlich gegen Wind (WITTICH 1951). Die Schwarzpappel braucht eine lange Vegetationsperiode sowie ein warmes Frühjahr. WEISGERBER (1980) empfiehlt für die Balsampappel eine Mitteltemperatur von $14,0{ }^{\circ} \mathrm{C}$ in der forstlichen Vegetationsperiode sowie mehr als 150 Vegetationstage ${ }^{3}$. Der Pappelanbau in Deutschland war bis zu den 1960er Jahren überwiegend durch die Schwarzpappel geprägt. Seit den 1970er Jahren wurden immer mehr Balsampappeln angepflanzt, so dass deren Bestand heute größer ist als der der Schwarzpappeln (WEISGERBER 1980).

Tab. 2-2: Pappelsystematik nach ECKENWALDER (1996).

\begin{tabular}{|c|c|c|c|c|c|c|}
\hline Sektion & Abaso & Turanga & Leucoides & Aigeiros & Tacamahaca & Populus \\
\hline $\begin{array}{l}\text { Verbreit- } \\
\text { ung }\end{array}$ & Nordamerika & $\begin{array}{l}\text { Zentral und } \\
\text { Westasien, } \\
\text { Nord- Ost- und } \\
\text { Westafrika, } \\
\text { Spanien }\end{array}$ & $\begin{array}{l}\text { Ostasien, } \\
\text { Östliches } \\
\text { Nordamerika }\end{array}$ & $\begin{array}{l}\text { Europa, } \\
\text { Westasien, } \\
\text { Nordwest- } \\
\text { afrika, } \\
\text { Nordamerika }\end{array}$ & $\begin{array}{l}\text { Asien, } \\
\text { Nordamerika }\end{array}$ & $\begin{array}{l}\text { Europa, Asien, } \\
\text { Nordamerika, } \\
\text { Nordafrika }\end{array}$ \\
\hline \multirow{11}{*}{ Art } & P. mexicana & P. euphratica & P. lasiocarpa & P. nigra & P. angustifolia & P. adenopoda \\
\hline & & P. ilicifolia & P. glauca $^{\mathrm{a}}$ & P. deltoides ${ }^{\mathrm{b}}$ & P. balsamifera & P. alba \\
\hline & & P. pruinosa & P. heterophylla & P. fremontii & P. suaveolens ${ }^{\mathrm{c}}$ & P. canescens \\
\hline & & & & & P. ciliata & P. gamblei \\
\hline & & & & & P. laurifolia & P. grandidentata \\
\hline & & & & & P. simonii & P. guzmanantlensis \\
\hline & & & & & P. suaveolens & P. monticola \\
\hline & & & & & P. szechuanica & P. sieboldii \\
\hline & & & & & P. trichocarpa & P. simaroa \\
\hline & & & & & P. yunnanensis & P. tremula ${ }^{\mathrm{d}}$ \\
\hline & & & & & & P. tremuloides \\
\hline
\end{tabular}

\footnotetext{
inklusive P. wilsonii.

${ }^{\mathrm{b}}$ inklusive $P$. sargentii und $P$. wislizensii.

${ }^{c}$ inklusive $P$. cathayana, $P$. koreana und $P$. maximowiczii.

dinklusive $P$. davidiana.
}

\footnotetext{
${ }^{3}$ Tag mit einer Tagesmitteltemperatur größer $5,0{ }^{\circ} \mathrm{C}$.
} 
Die natürliche Verbreitung der Pappel der Sektion Tacamahaca ist groß. Sie kommen in ganz Nordamerika und Asien vor, bevorzugt bei kühlem und gemäßigtem Klima. Die am meisten benutzen Balsampappeln kommen aus Japan, Nordostasien ( $P$. maximowiczii) und dem westlichen Nordamerika (P. trichocarpa). Balsampappeln kommen auf einer Höhe bis um $2.000 \mathrm{~m}$ über NN natürlich vor (LÜDEMANN 1998), sie sind ertragsstark und weisen eine höhere Holzdichte als Pappeln der restlichen Sektionen auf (BONNEMANN 1980, WEISGERBER 1980). Da eine höhere Holzdichte auch einen höheren Energiegehalt je Volumeneinheit zur Folge hat, eignen sich diese Pappeln gut für die energetische Nutzung. Balsampappeln sind schattentoleranter als Pappeln der restlichen Sektionen (FRÖHLICH und DiETZE 1975, JOACHIM 1989). Dies wird in dem teilweise belaubten Stamm sichtbar sowie im Wuchsverhalten bei Beschattung. Somit sind Balsampappeln sowohl für den Engstand wie für den Weitstand geeignet (JOACHIM 1989). Nicht zu Unrecht werden Balsampappeln, speziell die Art P. trichocarpa, Waldpappeln genannt. Aufgrund ihrer starken Belaubung unterdrückt die Balsampappel effizient die Begleitflora.

Die Standortanforderungen der Balsampappel ähneln denen der Schwarzpappel. Bei ungünstigen Standorteigenschaften wie Trockenheit und Bodenverdichtung ist die Wuchsminderung von Balsampappeln geringer als die von Schwarzpappeln (FRÖHLICH und GROSSCURTH 1973). Auf schwierigeren Standorten ist daher der Anbau der Balsampappel gegenüber der Schwarzpappel vorzuziehen. Einzig die Pappeln der Sektion Populus haben geringere Standortansprüche als die Balsampappeln (WEISGERBER 1980). Klimatisch erfordert der Anbau von Balsampappeln mehr als 130 Vegetationstage mit einer mittleren Temperatur in der forstlichen Vegetationsperiode von mindestens $13,0{ }^{\circ} \mathrm{C}$ (WEISGERBER 1980). Die Balsampappel ist somit weniger kälteempfindlich, aber deutlich stärker vom Wind gefährdet als die Schwarzpappel (BOHNENS und FRIEDRICH 1990). Bis auf die Auenstandorte, in denen die Schwarzpappeln durchaus hohe Leistungen aufweisen, sind die Balsampappeln den Schwarzpappeln überlegen (JOACHIM 1989).

Pappeln der Sektion Populus wie zum Beispiel. P. tremula und P. tremuloides sowie ihre Hybriden sind sehr robust, frosthart und eine gute Wahl bei schwierigen Standortbedingungen mit beispielsweise flachgründigen nährstoffarmen Böden oder in Höhenlagen (WEISGERBER 1980). Einzig P. alba sind wärmeliebend. Zitterpappeln ( $P$. tremula und $P$. tremuloides) besitzen eine hohe Holzdichte mit 400 bis $450 \mathrm{~kg} \mathrm{~m}^{-3}$ (BONNEMANN 1980) sowie eine ebenmäßige weißliche Holzfarbe. Hinsichtlich ihrer Standortansprüche sind sie genügsamer als Schwarz- und Balsampappeln, wechselfeuchte und nasse Standorte sind auch für sie geeignet. Zudem sind sie schattentolerant. Klimatisch brauchen sie in der Vegetationsperiode einen Temperaturmittelwert größer als $12,5{ }^{\circ} \mathrm{C}$ und 120 Vegetationstage (WEISGERBER 1980). Nach LIESEBACH et al. (1999) können mit Zitterpappeln in einer zehnjährigen Rotation dGZ von bis zu 10,0 $\mathrm{t}_{\text {(atro) }} \mathrm{ha}^{-1} \mathrm{a}^{-1}$ erreicht werden.

Im Folgenden werden die Balsam- und die Schwarzpappel sowie ihre Hybriden aus Gründen der Übersichtlichkeit zusammengefasst als Pappel bezeichnet.

\subsubsection{Weide}

Weiden sind weltweit eine der größten Familien von Baumgewächsen. Außer in Australien und in der Antarktis finden sich in jedem Kontinent autochthone Weidenarten. Sie kommen in gemäßigten Zonen, in Regionen mit kühleren Temperaturen in Breitengrade größer $50^{\circ}$ sowie in tropischen und subtropischen Gebieten vor. Die meisten Weidenarten finden sich auf der Nordhalbkugel (ZASADA et al. 2008). Häufig wachsen sie in Überflutungsbereichen von Fluss- und Bachläufen, aber auch in Buschwerken und Wäldern. Die Gattung der Weide besteht aus 400 bis 500 Arten. Damit hat sie eine noch größere ökologische Amplitude als die Pappel (ARGUS et al. 1986). In der aktuell am weitesten akzeptierten Systematik werden drei Sektionen differenziert: Die Sektion Salix (Baumweiden), die Sektion Vetrix (Buschweiden) und die Sektion Chamaetia (Zwergweiden). Die Mehrheit der Weidenarten ge- 
hören zur Sektion Vetrix (AHMAN und LARSSON 1994). Tabelle 2-3 zeigt eine Auswahl an Arten jeder Sektion.

In Deutschland kommt eine Vielzahl von Weidenarten natürlich vor. Hierzu zählen beispielsweise die Salweide (S. caprea), die Silberweide (S. alba) und die Korbweide (S. viminalis). Seit dem Steinzeitalter wurde die Weide, wegen ihres sehr flexiblen Holzes und der widerstandsfähigen Rinde für Zäune, Körbe und Flechtarbeiten genutzt (KUZOVKINA und QUIGLEY 2005). Bedingt durch ihr schnelles Jugendwachstum, ihrer fast $100 \%$-igen Anwuchs- und Regenerationsfähigkeit sowie ihrer Frosthärte (PLAGGENBORG 1989, HOFMANN 1995) ist sie ein wertvolles Gewächs und besonders für den Anbau in KUP geeignet. In Kurzumtriebsplantagen werden überwiegend Hybriden der S. viminalis eingesetzt. Kreuzungspartner sind häufig S. triandra, S. schweriini und S. dasyclados. Aber auch einige Klone der S. miyabeana und S. sachalinensis sind sehr wuchsstark (LABRECQUE und TEODORESCU 2005). Fast alle in KUP verwendeten Weidenarten gehören der Sektion Vetrix an (ROYLE und OSTRY 1995, AHMAN und LARSSON 1994). Das Kulminationsalter der Weide liegt zwischen vier und sechs Jahren (WILLEBRAND et al. 1993), weshalb sie sich nur für die Mini-Rotation eignet. Die Weiden ähneln in ihren Standortansprüchen den Pappeln (LTZ 2010, PLAGGENBORG 1989).

Tab. 2-3: Auswahl von Weidenarten in den jeweiligen Sektionen (nach BlanCo 1993 und NewsHOLme 1992).

\begin{tabular}{cccl}
\hline Sektion & \multicolumn{1}{c}{ Salix } & Chametia & \multicolumn{1}{c}{ Vetrix } \\
\hline \multirow{4}{*}{ S. amygdalina } & S. reticulate & S. hastate \\
& S. alba & S. herbacea & S. caprea \\
& S. fragilis & S. retusa & S. atrocinera \\
& S. nigra & S. alpina & S. aurita \\
S. triandra & S. vestita & S. bicolour \\
& S. fragilis & S. nivalis & S. viminalis \\
& & & S. dasyclados \\
& & & S. daphnoides \\
& & & S. repens \\
& & & S. purpurea \\
\hline
\end{tabular}

\subsubsection{Anmerkung Züchtung}

Viele Pappelklone, die aktuell für den Kurzumtrieb in Deutschland verwendet werden, sind sehr alt. Beispiele sind die Geschwisterklone Androscoggin (Syn. NE-41) und Hybride 275 (Syn. H 275 ${ }^{4}$, Syn. NE-42), welche aus einer Züchtungsarbeit aus den 1920er Jahren stammen (STOUT und SCHREINER 1933). Während die Züchtung und Selektionsarbeit bei Ackerkulturen verhältnismäßig schnell vonstatten geht, ist der Züchtungsfortschritt bei den Baumgewächsen vergleichsweise langsam. Länder wie USA, China, Belgien und Italien sind Vorreiter in der Pappelzüchtung, da hier im Gegensatz zu Deutschland die Züchtung konstant betrieben wurde. Kein Klon der Pappel, welcher derzeit für den Kurzumtrieb in Deutschland verwendet wird, wurde speziell für diese Bewirtschaftungsart gezüchtet.

Weil die Weide im Gegensatz zu den Pappeln in deutlich jüngerem Alter fruktifiziert, können ihre Züchtungsprogramme schneller zu nachweisbaren Ergebnissen führen. Seit den 1990er Jahren wurden

\footnotetext{
${ }^{4}$ Im Folgenden wird dieses Synonym für die Bezeichnung von Hybride 275 verwendet.
} 
in den schwedischen und englischen Züchtungsprogrammen sehr starke Klone gezüchtet und selektiert (LARSSON et al. 2001, KARP et al. 2010).

\subsection{Leistung von Kurzumtriebsplantagen mit Pappel und Weide in zwei- bis dreijähriger Umtriebszeit}

In Europa wird der dGZ von KUP mit unterschiedlichen Spannen angegeben:

- 8,0 bis $12,0 \mathrm{t}_{\text {(atro) }} \mathrm{ha}^{-1} \mathrm{a}^{-1}$ (CANNEL 1988),

- bis zu 10,0 $\mathrm{t}_{\text {(atro) }} \mathrm{ha}^{-1} \mathrm{a}^{-1}$ (MUHS et al. 1994) sowie

- 10,0 bis $15,0 \mathrm{t}_{\text {(atro) }} \mathrm{ha}^{-1} \mathrm{a}^{-1}$ (IEA 2002).

Für Weiden-KUP werden folgende Spannen aufgeführt:

- 10,0 bis 20,0 $\mathrm{t}_{\text {(atro) }} \mathrm{ha}^{-1} \mathrm{a}^{-1}$ (CHRISTERSSON 1986),

- 2,5 und 9,0 $\mathrm{t}_{\text {(atro) }} \mathrm{ha}^{-1} \mathrm{a}^{-1}$ (JONSSON 1994 in ECKERSTEN et al. 2006) sowie

- 1,2 und 9,0 $\mathrm{t}_{\text {(atro) }} \mathrm{ha}^{-1} \mathrm{a}^{-1}$ (ALRIKSSON 1997).

Unter günstigen Bedingungen, zum Beispiel bei Bewässerung und Düngung, können dGZ über 25,0 $\mathrm{t}_{\text {(atro) }} \mathrm{ha}^{-1} \mathrm{a}^{-1}$ erreicht werden (HEILMANN und STETTLER 1985, CHRISTERSSON 1986 und ETTALA 1988). Vereinzelt wurden in Deutschland Maxima von mehr als $20 \mathrm{t}_{\text {(atro) }} \mathrm{ha}^{-1} \mathrm{a}^{-1} \mathrm{im} \mathrm{dGZ} \mathrm{erreicht}$ (BOELCKE 2006, RÖHLE 2009).

In Tabelle 2-4 sind die dGZ-Werte verschiedener Versuchsflächen in einer Umtriebszeit zwischen zwei und drei Jahren aufgeführt. Um repräsentative Aussagen treffen zu können, muss eine ausreichende Mindestbeobachtungsdauer gegeben sein (STRONG und HANSEN 1993). Daher wurden in der Tabelle nur die Versuchsflächen berücksichtigt, welche mindestens sieben Jahre beobachtet wurden. Die Erträge der P. trichocarpa x P. maximowiczii Klone Unal, Rap, Beaupré und Raspalje wurden aufgrund ihrer erhöhten Mortalitätsrate und mangelnder Rosttoleranz (PINON und FREI 2005) nicht berücksichtigt. Vorab kann festgestellt werden, dass in Deutschland KUP eine dGZ-Leistung mit einer Spanne von 3,0 bis 16,1 $\mathrm{t}_{\text {(atro) }} \mathrm{ha}^{-1} \mathrm{a}^{-1}$ bei der Pappel und von 3,9 bis 15,6 $\mathrm{t}_{\text {(atro) }}$ ha $^{-1} \mathrm{a}^{-1}$ bei der Weide aufweisen. In einer einzigen Versuchsfläche mit Zitterpappel wurden dGZ zwischen 4,9 und $7,6 \mathrm{t}_{\text {(atro) }} \mathrm{ha}^{-1} \mathrm{a}^{-1}$ registriert. 


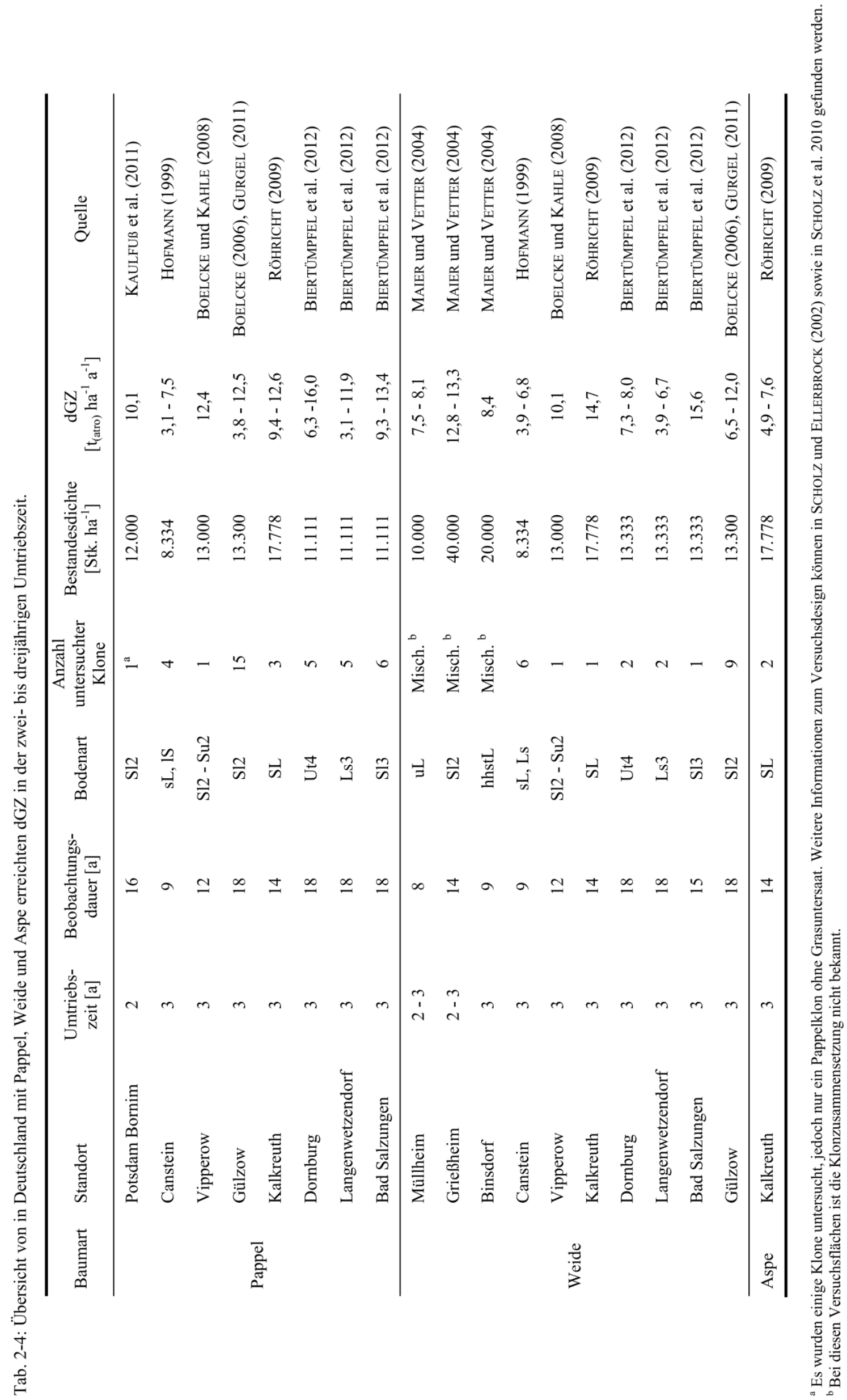




\subsection{Ertragsdynamik und leistungsbeeinflussende Faktoren von Kurzumtriebsplantagen}

Mehrere Studien belegen, dass die Wuchsdynamik von Kurzumtriebsplantagen unter Nutzung des Stockausschlags stark von den Rückschnitten bei den Erntevorgängen beeinflusst wird (WILLEBRAND et al. 1993, RiBEIRO et al. 1995, HOFMANN 1999, BOELCKE 2006). Die ertragsfördernde Wirkung des Stockausschlags ist auf die verstärkte Ausbildung des Wurzelwerks in Verbindung mit der Bildung mehrerer Höhentriebe nach jedem Rückschnitt zurückzuführen. Abbildung 2-2 zeigt die Ertragsentwicklung von der Max-Mehrklonsorte in den KUP-Versuchsflächen „Dornburg“ und „Langenwetzendorf“ sowie des Klons Max 1 auf den Versuchsflächen „Gülzow“ und „Bad Salzungen“. Die Versuchsflächen wurden zwar nicht gleichzeitig angelegt, bei einer Abweichung in der Anlage von maximal zwei Jahren weichen sie jedoch wenig von einer echten Zeitreihe ab. Entgegen der Annahme, dass bei den ersten drei bis vier Rotationen steigende Erträge zu verzeichnen sind, welche dann wieder langsam abfallen, ist nach 18 Jahren Beobachtungsdauer bei allen Flächen weiterhin eine steigende Tendenz in den Erträgen zu erkennen. Aus Abbildung 2-2 wird weiterhin ersichtlich, dass die Verläufe der Erträge sehr unterschiedlich sind. Da in diesem Vergleich die Einflussgrößen Bestandesdichte, Klon und Umtriebszeit vergleichbar sind, kann von der Standortabhängigkeit der Ertragsteigerung ausgegangen werden.

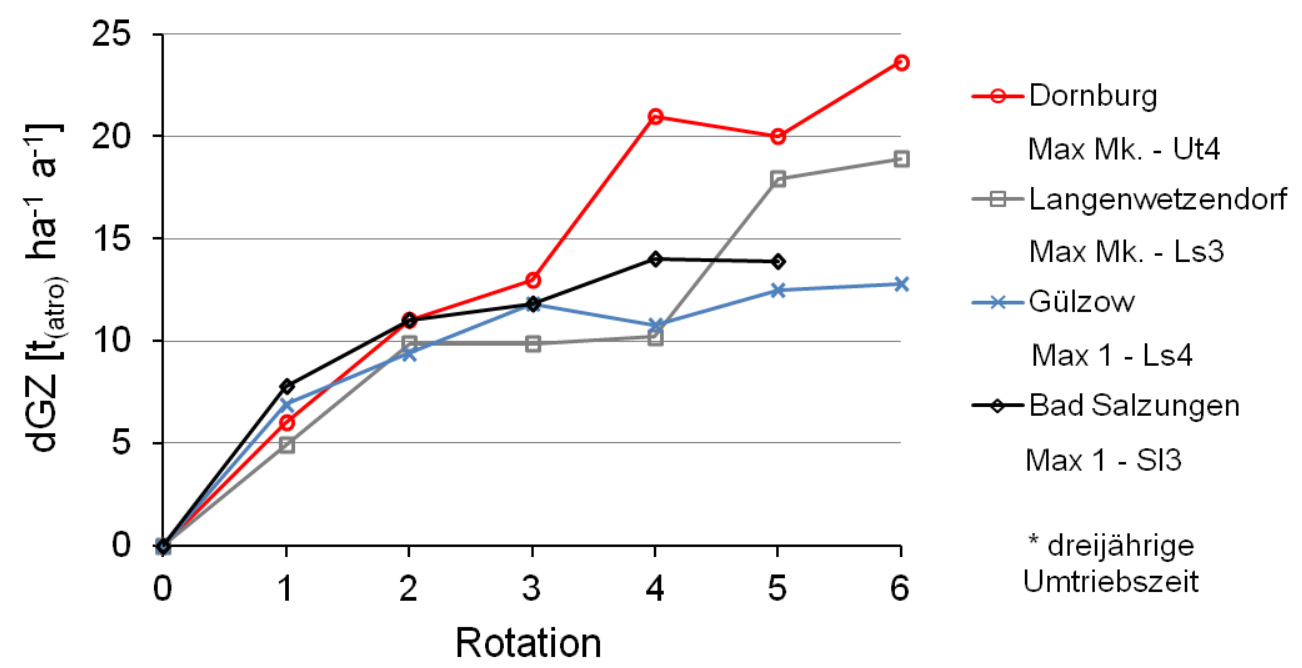

Abb. 2-2: dGZ in $\mathrm{t}_{\text {(atro) }} \mathrm{ha}^{-1} \mathrm{a}^{-1}$ von vier Langzeitbeobachtungsflächen in Thüringen und Mecklenburg-Vorpommern bei der Max-Mehrklonsorte und Max 1 in dreijähriger Rotation (Verändert nach BOELCKE 2006, GURGEL 2011 und BIERTÜMPFEL et al. 2012).

Abbildung 2-3 zeigt die dGZ-Entwicklung zweier Weidenklone gegenüber dem Klon Max 1 auf den Standorten „Bad Salzungen“ (BIERTÜMPFEL et al. 2012) und „Kalkreuth“ (RÖHRICHT 2009). Es wird ersichtlich, dass die dGZ-Entwicklung der Weidenklone Max 1 deutlich übertraf. Nach fünf Rotationen zeigten die Weiden bis $40 \%$ stärkere dGZ als Max 1. Auf beiden Versuchsflächen war der jeweils vorgestellte Weidenklon das stärkste Prüfglied. Diese Darstellung soll das Potential der Weide verdeutlichen.

Im Folgenden wird auf die leistungsbeeinflussenden Faktoren von KUP eingegangen. Als solche geIten die Baumart und der Klon, die Umtriebszeit, die Bestandesdichte sowie die Standorteigenschaften, bestehend aus Boden und Klima. Aufgrund der Zielstellung der vorliegenden Arbeit liegt der Schwerpunkt bei der Betrachtung der leistungsbeeinflussenden Faktoren bei den Standorteigenschaften. 


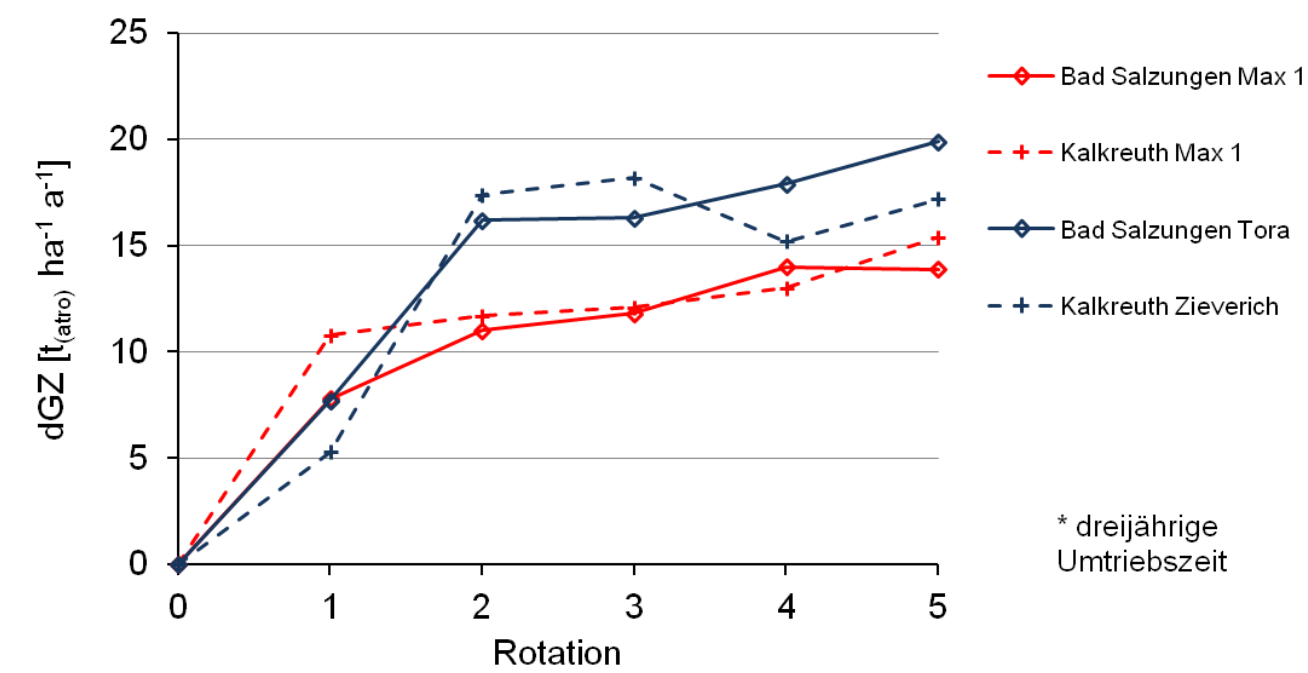

Abb. 2-3: dGZ-Entwicklung in $\mathrm{t}_{(\text {atro) }} \mathrm{ha}^{-1} \mathrm{a}^{-1}$ der Weidenklone Tora (Versuchsfläche „Bad Salzungen“) und Zieverich (Versuchsfläche „Kalkreuth“) gegenüber Max 1 in dreijähriger Rotation (Verändert nach RöHRICHT 2009 und BIERTÜMPFEL et al. 2012).

\subsubsection{Baumart und Klon}

Die Baumart- und Klonwahl ist für die gesamte Anbaukette von KUP höchst relevant, insbesondere um die Standorteigenschaften für das Wachstum optimal auszunutzen und produktangepasste Holzqualitäten zu erzeugen. Wie schon erwähnt, wiesen im kurzen Umtrieb Pappel und Weide die höchsten Leistungen auf (RAU et al. 1988, FRIEDRICH 1999, HOFMANN 1999).

Welche Baumart - Pappel oder Weide - stärkere Leistungen in KUP erbringt, kann nicht abschließend beurteilt werden. In einigen Untersuchungen waren Pappeln den Weiden stets überlegen (FRIEDRICH 1999, HofmANN 1999, LABREQUE und TeOdORESCU 2005, BoElCKe 2006). In anderen waren Weidenklone die Stärksten (RÖHRICHT 2009, BIERTÜMPFEL et al. 2012). Abbildung 2-4 zeigt Ergebnisse der deutschen KUP-Versuche mit Pappel und Weide in einer Umtriebszeit von zwei bis drei Jahren (Daten von Tabelle 2-4) ${ }^{5}$. Bei den Pappeln im Umtrieb zwischen zwei und drei Jahren variierten die dGZ zwischen 7,5 und 16,0 $\mathrm{t}_{\text {(atro) }} \mathrm{ha}^{-1} \mathrm{a}^{-1}$. In derselben Umtriebszeit hatte die Weide dGZ zwischen 6,7 und 15,6 $\mathrm{t}_{\text {(atro) }} \mathrm{ha}^{-1} \mathrm{a}^{-1}$. Die dGZ von Pappel und Weide zeigten untereinander keinen signifikanten Unterschied, wobei der mittlere dGZ der Pappel ca. 20 \% höher war. Es kann also von einer Überlegenheit der Pappel ausgegangen werden. Jedoch ist anzumerken, dass in den ausgewerteten Versuchen teilweise Weidenklone verwendet wurden, welche zur damaligen Zeit keine Wirtschaftsklone darstellten. AYLOTT et al. (2008) stellten in einer englischen großangelegten KUP-Versuchsreihe mit mehr als 40 Versuchsflächen fest, dass Weiden in der dreijährigen Rotation tendenziell höhere Erträge als Pappeln aufwiesen. In diesen Versuchen wurden die jeweils stärksten und neuesten Klone beider Gattungen verwendet.

Aktuell sind die besten Klone für die kurze Rotation bei der Pappel unangefochten die Klone der MaxGruppe. Bei den Weiden können Tora, Tordis und Zieverich empfohlen werden.

\footnotetext{
${ }^{5}$ In manchen Versuchsflächen wurden zusätzlich zu Wirtschaftsklonen sehr viele experimentelle Klone untersucht. Da diese teilweise eine deutlich schwächere Leistung als Wirtschaftsklone erreichten, wurde der Flächenmittelwert nach unten verschoben. Für die Vergleiche wurde daher statt des Mittelwerts der Versuchsfläche der beste Klon berücksichtigt.
} 


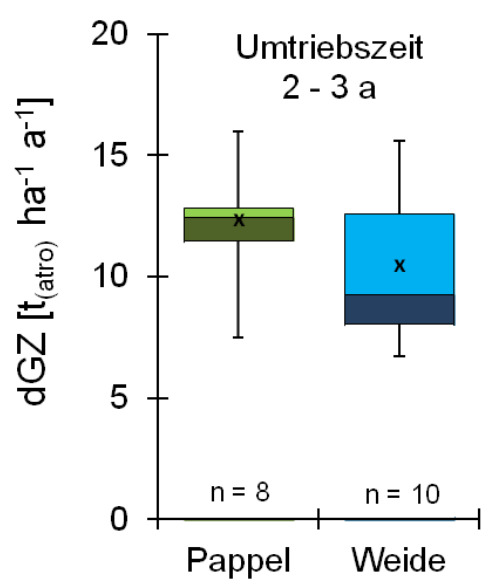

Abb. 2-4: Whiskers-Box-Plots der Leistung von Pappel und Weide in zwei bis dreijährigen Umtriebszeiten (Datengrundlage kann in Tabelle 2-4 nachgeschlagen werden).

\subsubsection{Umtriebszeit und Bestandesdichte}

Die Wahl der geeigneten Pflanzdichte und Umtriebszeit ist für die Anlage von Kurzumtriebsplantagen von zentraler Bedeutung. Diese hat eine direkte Auswirkung auf die Produktlinie. HANSEN (1991) sowie Mitchell et al. (1999) stellten fest, dass kürzere Rotationen tendenziell höhere Erträge und reduzierte Mortalitätsraten aufweisen.

Nach dem Stück-Masse-Gesetz wäre davon auszugehen, dass längere Rotationen einen höheren dGZ zur Folge haben. Ob dies wirklich zutrifft, wird im folgenden Vergleich zwischen Mini- und MaxiRotation für Pappel erläutert. Betrachtet man den dGZ nach 20 Jahren von verschiedenen Pappelertragstafeln in der ersten Bonität, ergeben sich in aufsteigender Reihenfolge 7,0 $\mathrm{t}_{\text {(atro) }} \mathrm{ha}^{-1} \mathrm{a}^{-1}$ (BAUER 1938), 7,9 $\mathrm{t}_{\text {(atro) }} \mathrm{ha}^{-1} \mathrm{a}^{-1}$ (RÄTZEL 1969), 8,7 $\mathrm{t}_{\text {(atro) }} \mathrm{ha}^{-1} \mathrm{a}^{-1}$ (GROSSCURTH 1982), 9,7 $\mathrm{t}_{\text {(atro) }} \mathrm{ha}^{-1} \mathrm{a}^{-1}$, (MITSCHERLICH und SONNTAG 1982), 12,0 $\mathrm{t}_{\text {(atro) }} \mathrm{ha}^{-1} \mathrm{a}^{-1}$ (SCHMITZ-LENDERS 1948) und $13,7 \mathrm{t}_{\text {(atro) }} \mathrm{ha}^{-1} \mathrm{a}^{-1}$ (BLUME 1951) $)^{6}$.

Weitere vier Versuchsflächen mit Umtriebszeiten zwischen 18 und 21 Jahren konnten ausgewertet werden. Diese Versuchsflächen waren „Eldena“ (JOACHIM 1989), „Groß Gerau“ (RAU et al. 1988), „Uni-Landshut“ (SCHIRMER 2012) sowie „Wanfried“ (RAU et al. 1988) (Tab. 2-5). Da von diesen Versuchsflächen nur $d_{1,3}$ vorhanden waren, erfolgte die Ermittlung des dGZ über eine für den Weitstand entwickelte Biomassefunktion nach JOHANSON und KARACIC (2011).

Tab. 2-5: Übersicht von in Deutschland mit Pappel in Maxi-Rotation erreichten dGZ.

\begin{tabular}{lcccccc}
\hline Standort & $\begin{array}{c}\text { Beobachtungs- } \\
\text { dauer [a] }\end{array}$ & Bodenart & $\begin{array}{c}\text { Anzahl } \\
\text { untersuchter } \\
\text { Klone }\end{array}$ & $\begin{array}{c}\text { Pflanzdichte } \\
\text { verbleibender } \\
\text { Bestand } \\
{\left[\text { Stk. ha }^{-1}\right]}\end{array}$ & $\begin{array}{c}\text { dGZ } \\
\text { Amplitude } \\
{\left[\mathrm{t}_{\text {(atro) }} \text { ha }^{-1} \mathrm{a}^{-1}\right]}\end{array}$ & Quelle \\
\hline Eldena & 19 & Lu & 4 & $200^{\mathrm{a}}$ & $4,2-7,7$ & JOACHIM et al. (1989) \\
Groß Gerau & 18 & $\mathrm{~T}$ & 6 & $400^{\mathrm{b}}$ & $3,3-7,6$ & RAU et al. (1988) \\
Uni - Landshut & 21 & $\mathrm{LT}$ & 5 & 312 & $2,9-9,4$ & SCHIRMER (2012) \\
Wanfried & 18 & $\mathrm{~L}$ & 13 & $400^{\mathrm{b}}$ & $2,2-13,4$ & RAU et al. (1988) \\
\hline
\end{tabular}

a Die genaue verbleibende Bestandesdichte ist unbekannt. Basierend auf die Beobachtungen von BLuME (1949) wird konservativ eine Dichte von 200 Stk. ha ${ }^{-1}$ angenommen.

${ }^{\mathrm{b}}$ Mündliche Mitteilung (SCHULZKE 2012).

\footnotetext{
${ }^{6}$ Eine Rohdichte von $400 \mathrm{~kg} \mathrm{~m}^{-3}$ (BONNEMANN 1980) sowie einen $30 \%$-igen Reisiganteil im Alter 20 (SCHMITZ-LENDERS 1956) wurden für die Expansion angenommen.
} 
Für die aufgeführten Versuchsflächen und Ertragstafeln ergaben sich dGZ zwischen 7,0 und 13,7 $\mathrm{t}_{\text {(atro) }} \mathrm{ha}^{-1} \mathrm{a}^{-1}$, der mittlere dGZ lag bei 9,7 $\mathrm{t}_{\text {(atro) }} \mathrm{ha}^{-1} \mathrm{a}^{-1}$. In der Umtriebszeit von zwei bis drei Jahren variierten die dGZ zwischen 7,5 und 16,0 $t_{\text {(atro) }}$ ha $^{-1} \mathrm{a}^{-1}$, der mittlere dGZ aller Versuchsflächen betrug $12,1 \mathrm{t}_{\text {(atro) }} \mathrm{ha}^{-1} \mathrm{a}^{-1}$. Der Unterschied zwischen den Mittelwerten war nicht signifikant. Dennoch kann festgestellt werden, dass die dGZ der kurzen Umtriebszeit von zwei bis drei Jahren tendenziell über denen der langen Umtriebszeit von 20 Jahren liegen.

Der Einfluss der Bestandesdichten auf die Biomasseleistung bei Pappel wurde in der Literatur mehrmals dokumentiert (FERM et al. 1989, ARMSTRONG et al. 1999, FANG et al. 1999, FRIEDRICH 1999, BOELCKE 2006 und BIERTÜMPFEL et al. 2009). Bei Pappel wurden signifikante Unterschiede zwischen engen und weiten Pflanzverbänden festgestellt. Höhere Erträge wurden bei höheren Pflanzdichten erzielt (STRONG und HANSEN 1993, ARMSTRONG et al. 1999, FANG et al. 1999, FRIEDRICH 1999). Für Umtriebszeiten von zwei und drei Jahren wurden optimale Bestandesdichten zwischen 10.000 und 20.000 Stk. ha ${ }^{-1}$ ermittelt (CANNEL 1980, MACPHERSON 1995).

In einer Studie aus Schweden wurden Pflanzdichten zwischen 10.000 und 40.000 Stk. ha $^{-1}$ bei Weiden untersucht. Signifikant unterschiedliche Erträge konnten nur bei der ersten Ernte von den Varianten mit kurzen Ernteintervallen (bis zu drei Jahre) verzeichnet werden (WILLEBRAND et al. 1993). Diese Ergebnisse wurden von KOPP et al. (1998) bestätigt. Für den Ertrag über mehrere Rotationen und bei längeren Ernteintervallen war eine Pflanzdichte größer 12.000 Stk. ha ${ }^{-1}$ nicht von Vorteil.

In Abbildung 2-5 ist ersichtlich, welchen Einfluss die Bestandesdichte auf die dGZ von Pappelklonen der jeweiligen Rotationen hat. Dort sind die ersten drei Rotationen von drei Versuchsflächen unterschiedlicher Bestandesdichte bei ähnlichen Standorteigenschaften und Klonwahl aufgezeigt.

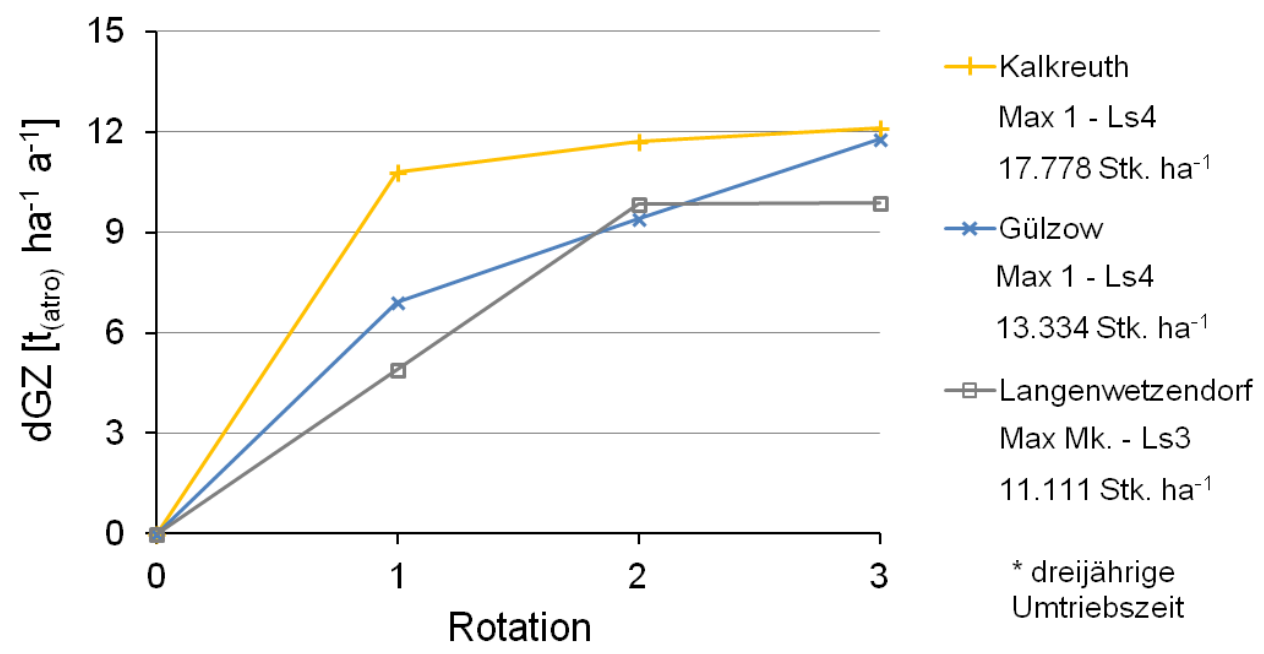

Abb. 2-5: dGZ der ersten drei Rotationen für den Klon Max 1 (Versuchsflächen „Kalkreuth“ und „Gülzow“) sowie für MaxMehrklonsorte (Versuchsfläche „Langenwetzendorf“) bei unterschiedlichen Bestandesdichten (Verändert nach BOELCKE 2006, RÖHRICHT 2009, GURGEL 2011 und BIERTÜMPFEL et al. 2012).

Es wird ersichtlich, dass der dGZ der ersten Rotation sehr verschieden ausfällt. Je höher die Bestandesdichte ist, desto größer der dGZ. Dies deckt sich mit den für Weide getroffenen Beobachtungen von WILLEBRAND et al. (1993). Die Ertragssteigerung von der ersten auf die zweite Rotation fällt in Abhängigkeit von der Bestandesdichte unterschiedlich aus. Je geringer die Bestandesdichte, umso stärker fällt die Ertragssteigerung aus. Dies wird mit der verhältnismäßig höheren Zunahme an Höhentrieben bei niedrigen Bestandesdichten erklärt. Ab der dritten Rotation blieb der dGZ, bis auf den Standort „Gülzow“, eher stabil. 


\subsubsection{Standortanforderungen}

In der Forstwirtschaft wird die Standortwuchskraft über zwei Wege erfasst, entweder direkt über die Ermittlung von Boden- und Klimacharakteristika oder indirekt über dem auf dem Standort stockenden Bestand (NAGEL 2001, MURACH et al. 2009). In der Forsteinrichtung wird die indirekte Methode verwendet, in dem der Bestand in Abhängigkeit von Alter und Höhe eingeordnet und dann in Beziehung zu den Ertragstafeln gesetzt wird. Für die Bonitierung werden häufig die Ober- oder Spitzenhöhe verwendet, da diese weniger durch die Bestandesbehandlung beeinflusst werden. Die Anwendung der indirekten Standortbonitierung anhand von Mischbeständen wird kontrovers diskutiert (NAGEL 2001), denn eine präzise Bewertung der Standortwuchskraft kann nur über die Erfassung der vorliegenden Standorteigenschaften geschehen.

Während Studien zu Standortanforderungen der Weiden dem Autor nicht bekannt sind, kann bei der Pappel auf einen großen Erfahrungsschatz aus der Mitte des 20. Jahrhunderts zurückgegriffen werden. Obwohl die Standortanforderungen der Weide nicht ausreichend beleuchtet sind, wird von einer Ähnlichkeit mit denen der Pappel berichtet (PlagGEnBORG 1997, LTZ 2010). Aus diesem Grund kann die Betrachtung der Standortanforderungen für die Pappel zunächst auch für Weide angenommen werden.

Hohe Massenleistungen bei Pappeln werden auf Standorten mit folgenden Bodeneigenschaften er-

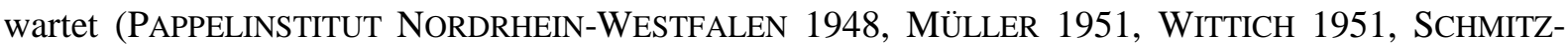
LENDERS 1956, FRÖHLICH und GROSSCURTH 1973):

- reichliche Wasserversorgung durch Niederschlag, Hangzug- oder Grundwasser,

- lockere Böden bzw. keine Bodenverdichtung,

- ausreichende Mineralstoffzufuhr,

- wachstumsangepasste Bodenreaktion.

Eine noch bessere Massenleistung wäre darüber hinaus auf Standorten mit mäßiger Luftbewegung, viel Sonneneinstrahlung und einer langen Vegetationsperiode gegeben (SCHMITZ-LENDERS 1956). Diese Ansprüche lassen sich physiologisch über die verschiedenen Eigenschaften von Pappel und Weide erklären. Im Folgenden wird auf die aufgezählten Standorteigenschaften sowie auf die Kenngrößen Wärme, Bodenschätzung - Zustandsstufe (ZS), Bodenzahl (BZ) und Ackerzahl (AZ) - und Textur eingegangen.

\subsubsection{Reichliche Wasserversorgung}

Aufgrund der hohen Transpirationsraten sind Pappel und Weide auf eine reichliche Wasserversorgung angewiesen (SCHMITZ-Lenders 1956, FAO 1979, PeTzOLD 2013). Die Transpiration der Pappel liegt etwa bei 3,3 mm pro Tag (PETZOLD 2013). Bei der Weide variiert sie zwischen 2,0 und 3,0 mm pro Tag (PERSSON und LINDROTH 1994). Das bedeutet umgerechnet auf eine fünfmonatige Vegetationsperiode eine Transpirationsrate von 300 bis $450 \mathrm{~mm}$. Der Wasserbedarf von Pappeln liegt deutlich höher als der von Fichten, Buchen, Eichen, Lärchen und Birken (FAO 1979). Diese hohe Transpiration wird durch eine sehr hohe Blattfläche (SOUCH and STEPHENS 1998, UnSELD 1999, BUNGART und HÜTTL 2004, KOOP et al. 2005), gekoppelt mit der geringen Fähigkeit, die Stomataleitfähigkeit zu steuern (GRIESE et al. 1993), verursacht. Nur wenige Klone vermögen den Wasserbedarf durch eine Stomataschließung zu optimieren, wie dies zum Beispiel bei Muhle Larsen der Fall ist (GRIESE et al. 1993). Daher können dGZ durch Bewässerung signifikant gesteigert werden (STRONG und HANSEN 1993, DiCKMANN et al. 2001). Die Bedeutung des Wasserhaushalts betonen Autoren wie RAU et al. (1988) und HOFMANN (1999) in der Beurteilung der Anbauwürdigkeit in Abhängigkeit von den forstlichen Wasserstufen. Frische Standorte eignen sich für den Anbau sehr gut, dagegen gelten mäßig trockene bis trockene Standorte als ungeeignet für den KUP-Anbau. Eine optimale Wasserversorgung ist durch ausreichende Niederschläge oder Grundwassereinfluss in 
Verbindung mit einer hohen nutzbaren Feldkapazität (nFK) gegeben (MÜLLER 1951). Da Niederschlagssummen und nFK metrisch skaliert sind, können diese Größen für die Bildung von Modellen verwendet werden.

Als ertragsfördernd werden hohe Jahresniederschlagssummen mit über $800 \mathrm{~mm}$ und eine günstige Verteilung der Niederschläge in der Vegetationsperiode angesehen (SCHMITZ-LENDERS 1956). In der Literatur werden Mindestniederschlagssummen in der Vegetationsperiode von mindestens $250 \mathrm{~mm}$ (DÖHRER 2010) bzw. 300 mm (HoFMANN 1999) genannt. Der wichtigste Zeitraum der Niederschlagsverteilung in der Vegetationsperiode stellt das Zeitfenster zwischen Mai und Juni dar (ALI 2009).

Die nFK bezeichnet die Wasserspeicherkapazität eines Bodens, die für Pflanzen nutzbar ist (SCHEFFER und SCHACHTSCHABEL 2002). Während niederschlagsfreier Perioden ist sie von großer Wichtigkeit, um das Pflanzenwachstum zu sichern. Sie wird definiert als der Wassergehalt zwischen der Feldkapazität ( $\mathrm{pF} 1,8$ bis 2,5) und dem permanenten Welkepunkt ( $\mathrm{pF} 4,2)$. Sie wird in Volumenprozent oder mm Wassersäule angegeben. Die Ad-Hoc Arbeitsgemeinschaft Boden (AG BODEN) (2005) legt für die praktische Anwendung die untere Grenze von $60 \mathrm{hPa}(\mathrm{pF} 1,8)$ der Feldkapazität zugrunde. ALRIKSSON (1997), ALI (2009) und RÖHLE und ALI (2009) betonen die ertragsfördernde Wirkung höherer nFK für KUP.

\subsubsection{Lockere Böden}

Pappel und Weide brauchen aus zwei Gründen lockere Böden:

Zum Einen erlauben lockere, unverdichtete Böden eine intensive Durchwurzelung. Das ist für das Baumwachstum günstig, insbesondere für Pionierbaumarten. Dort muss der Boden eine leichte Durchwurzelbarkeit aufweisen, damit sich ein Wurzelwerk in kurzer Zeit entwickeln kann, um das Überleben der Art zu sichern. Nach NAUMANN (1980) sucht die Pappel mit ihren Wurzeln die nährstoffreichsten und lockersten Bodenschichten. Aufgrund dieser starken Durchwurzelungsfähigkeit ist die Pappel für die Befestigung lockerer Böden bestens geeignet. Sie gilt als die beste Baumart für die Rekultivierung von Kipp- und Aufschüttungsböden. MAKESCHIN und REHFUESS (1994) sowie UNSELD (1999) nennen hohe Wurzelgehalte im Oberboden, die bis zu 3,0 g TS ${ }^{-1}$ bei der Pappel und $5,0 \mathrm{~g} \mathrm{TS}^{-1}$ bei der Weide reichen.

Zum Anderen sorgen locker gelagerte Böden für eine optimale Luft und Sauerstoffversorgung der Wurzeln. MiTCHEL et al. (1999) bezeichnete Böden mit Stauwasser oder schlecht belüftete Böden für das Baumwachstum als nachteilig, weil der Gasaustausch der Wurzel unterbundenen wird. SCHMITZLENDERS (1956) vermutete bereits in den 1950er Jahren eine hohe Wurzelatmungsrate bei Pappeln. Dass Pappeln höhere Wurzelatmungsraten als andere Baumarten aufweisen, wurde experimentell bestätigt FAO (1979). Ihre Wurzelatmungsrate ist beispielsweise fünf Mal höher als bei Lärchen (FAO 1979).

Die Lockerheit eines Bodens kann in der bodenphysikalischen Kenngröße LK ausgedrückt werden. Wie die nFK kann die LK numerisch erfasst werden. Die LK bezeichnet das Porenvolumen eines Bodens, welches bei Feldkapazität mit Luft gefüllt ist (HARTGE und HORN 1989). Sie ist neben der nFK maßgeblich für die Sauerstoffversorgung des Bodens verantwortlich. Außerdem stellt sie ein Maß für die Durchwurzelbarkeit des Bodens dar (SCHACK und HILDEBRAND 1987). Aufgrund der zunehmenden Mechanisierung in der Landwirtschaft hat die Bodenverdichtung zu- und damit einhergehend die LK abgenommen. Anhang 1 kann die Beziehung zwischen den Porenraumbereichen nFK und LK mit den Porengrößen, -durchmesser und Wasserspannung entnommen werden.

Böden mit zu hoch stehendem Grundwasser haben eine ertragsmindernde Wirkung (DANFORS et al. 1998). Wie Bodenverdichtung führt ein zu hoher Grundwasserspiegel zu einer Verringerung des durchwurzelbaren Raumes und zur Hemmung der Wurzelatmung. 
In der Literatur werden häufig Empfehlungen zur Profiltiefe für den KUP-Anbau gegeben. LEDIN und WILLEBRAND (1995) empfehlen eine Profiltiefe von 1,0 m. LEWANDOWSKI und VeTTER (2000) empfehlen für den KUP-Anbau einen durchwurzelbaren Raum von mindestens 0,6 m. Der Grund für die positive Auswirkung von tieferen Bodenprofilen ist die Vergrößerung des durchwurzelbaren Bodenraums, mit der eine Erhöhung der nFK und LK einhergeht. MÜLLER (1951) weist zudem auf die Wechselwirkung zwischen Tiefgründigkeit des Bodens und nFK der jeweiligen Bodenart hin. Ein flachgründiger Lössboden (hohe $\mathrm{nFK}$ ) ist in der Regel wuchsstärker als ein flachgründiger Sandboden (geringe $\mathrm{nFK}$ ).

\subsubsection{Ausreichende Mineralstoffzufuhr}

In der Literatur der 1940er bis 1970er Jahre wird eine ausreichende Mineralstoffzufuhr als wichtige Grundlage für den Erfolg von Pappel und Weidenanbau genannt. NIEDER et al. (2003) stellten fest, dass die Mehrheit ackerbaulich genutzter Böden über hohe Frachten pflanzenverfügbarer Nährstoffe verfügen. Diese Nährstoffmengen übersteigen bei weitem die für Bäume benötigten Mengen. Untersuchungen von MAKESCHIN und REHFUESS (1994) sowie SCHOLZ et al. (2004) auf ehemals ackerbaulich genutzten Flächen zeigten, dass Pappelklone nach der Düngung mit Stickstoff, Phosphor und Kalium keine signifikanten Ertragszunahmen aufwiesen. Sogar nach zehn Jahren Beobachtungszeit war der Ernährungszustand von Pappelbeständen optimal. Bei den Weiden hingegen wurde festgestellt, dass diese auf Stickstoffdüngung mit einer bis zu $100 \%$-igen Ertragssteigerung reagieren (MAKESCHIN und REHFUESS 1994, SchOlZ et al. 2004). Auf Braunerden und Pseudogleyen reagierten Weiden bei zunehmender N-Düngung (50 bis $100 \mathrm{~kg} \mathrm{ha}^{-1}$ ) mit Ertragssteigerungen von 70 bis zu $100 \%$.

Die Studien von JUg et al. (1999), BoELCKE und KAHLE (2008) sowie BIERTÜMPFEL et al. (2012) verweisen auf einen hohen Kalziumbedarf von Pappeln und Weiden. So wurden Kalzium-Entzüge von bis zu $120 \mathrm{~kg} \mathrm{ha}^{-1}$ durch das Erntegut festgestellt.

\subsubsection{Wachstumsangepasste Bodenreaktion}

$\mathrm{Zu}$ niedrige $\mathrm{pH}-$ Werte haben negative Auswirkungen auf das Pflanzenwachstum. Insbesondere wenn ein $\mathrm{pH}$-Wert von 4,2 unterschritten wird, ist die Grenze des Austausch-Puffers erreicht (SCHEFFER und SCHACHTSCHABEL 2002). Das Überleben der Wurzeln wird unter diesen Bedingungen schwer, denn Al(3)- und Mn(2)-Ionen wirken toxisch (Alloway 1999). Andererseits treten Mängel in der Nährstoffversorgung auf, und weitere toxische Schwermetalle werden mobil. Daher sollten Pappeln und Weiden auf Standorten mit einem $\mathrm{pH}$ von mindestens 4,5, besser 5,0, angebaut werden.

\subsubsection{Wärme}

Unter optimalen Bedingungen, das heißt bei ausreichender Wasser- und Nährstoffversorgung, hat das Wärmeangebot einen wachstumsverbessernden Einfluss (PETZOLD 2013). Oft wird eine positive Beziehung der Wärmesumme mit dem dGZ angenommen (WEISGERBER 1980). Höhere Temperaturen wirken sich auf das Wachstum positiv aus, weil sie den oberirdischen sowie unterirdischen Stoffwechsel anregen (DOWSLEY 1987).

Andererseits können höhere Temperaturen den Bodenwasserhaushalt nachteilig beeinflussen. Grund hierfür ist die Zunahme der Evapotranspiration bei höheren Temperaturen. Ist die Evapotranspiration zu hoch, trocknet der Boden sukzessive aus und kann kein Wasser mehr nachliefern. Daher können sich zu hohe Temperaturen auf die Ertragsleistung negativ auswirken, wie ALI (2009) in einer Studie zum KUP-Anbau in Sachsen feststellte.

Bei Pappel und Weide erfolgt eine C3-Photosynthese. Das Temperaturoptimum der C3-Photosynthese liegt bei $20^{\circ} \mathrm{C}$ (LEWANDOWSKI und VETTER 2000), daher verringert sich das Wachstum bei zu stark ansteigenden Temperaturen. Für den klassischen Anbau sind bieten warm-feuchte Witterung sowie 
höhere Temperaturen bei normalen Niederschlägen gute Bedingungen für das Wachstum (JOACHIM et al. 1989).

\subsubsection{Bodenschätzungskennwerte}

Bodenkenngrößen, welche großräumig vorliegen und nützlich für die Ertragsmodellierung sind, sind die Bodenwertzahlen der Reichsbodenschätzung (BROZIO et al. 2009). In der Bodenschätzung werden in der Reihenfolge die Bodenart durch Fingerprobe, ZS - Zustandsstufe - (zum Beispiel roh - reif degradiert) und die Entstehungsart (dilluvial, alluvial, Löss oder Verwitterungsboden) erfasst (AG BODEN 2005). Daraus resultiert BZ (Bodenzahl), die einen allgemeinen Wert für das natürliche Ertragspotential des Bodens darstellt. AZ (Ackerzahl) entsteht durch die zusätzliche Berücksichtigung von Standortklima, Exposition und Ausrichtung im Gelände, korrigiert mit $\mathrm{Zu}$ - oder Abschlägen auf BZ. AZ findet Anwendung in der Ertragsschätzung bzw. bei der Festsetzung steuerlicher Einheitswerte von Ackerschlägen. ALI (2009) zeigte, dass eine starke positive Beziehung zwischen AZ und nFK besteht. HoFMANN (1998) empfiehlt den Anbau auf Böden mit AZ größer 30, solange der Boden nicht verdichtet ist und eine ausreichende Wasserversorgung gewährleistet ist. Nach LEWANDOWSKI und VetTer (2000) und RÖHLE et al. (2008) ist die Nutzung der Bodenwertzahlen als alleinige Ertragsmaßzahl nicht ausreichend.

\subsubsection{Textur}

Die Textur ist für viele Bodenprozesse verantwortlich. Während die Sandfraktion weitestgehend inert ist (großer Anteil an $\mathrm{SiO}_{2}$ ) und kaum Bedeutung für den Stoffhaushalt hat, steigert sie die Luftversorgung und verringert die Wasserhaltekapazität (SCHEFFER und SCHACHTSCHABEL 2002). Die Schlufffraktion hat einen großen und positiven Einfluss auf die Wasserverfügbarkeit und Durchlüftung des Bodens (JURY et al. 1991). Die Tonfraktion hat einen relevanten Einfluss auf den Stoffhaushalt des Bodens und trägt auch zur Bodenwasserspeicherung bei (JURY et al. 1991, SCHEFFER und SCHACHTSCHABEL 2002).

\subsubsection{Standorte der Pappel}

Im Folgenden werden verschiedene Standorte hinsichtlich ihrer Bodenbeschaffenheit in Bezug auf die Anbaueignung beurteilt (hauptsächlich nach WITTICH 1951, ergänzt durch PAPPELVEREIN NORDRHEIN-WESTFALEN 1948, MÜLLER 1951, JOACHIM 1953a, EISENREICH 1956, HÜBENER 1989):

- Böden mit Grundwasseranschluss: Diese Böden, zum Beispiel Auenböden, sind ertragsstark. Am besten eignet sich ein Grundwasserstand zwischen 0,6 und 1,0 m, wobei ein Wasserstand von 2,0 m noch für gute Leistungen ausreicht. Das Grundwasser sollte nicht stagnierend sein, da sonst Sauerstoffmangel auftritt. Zudem ist es aus ernährungsphysiologischer Sicht positiv, wenn das Grundwasser kalkhaltig ist.

- Böden mit zu hohem Grundwasserspiegel: Diese Standorte versagen auf der ganzen Linie (PAPPELVEREIN NORDRHEIN-WESTFALEN 1948). Als kritische Grenze kann 0,5 m angesehen werden. Die Wurzelausbildung und die Sauerstoffversorgung werden beeinträchtigt (siehe 2.5.3.2). Der Anbau von Pappeln auf diesen Standorten ist in begrenztem Maße durch die Nutzung von Herbstrabatten oder Hügeln möglich (JOACHIM 1953b).

- Böden mit Hangwasserzugang: Diese Böden sind immer frisch. Daher sind sie exzellent für den KUP-Anbau geeignet. Zudem wirkt sich das sauerstoffreiche Hangzugwasser positiv auf das Wachstum aus.

- Lössböden: Diese Böden sind ertragsstark, da sie oft über eine gute Struktur, hohe nFK, gute Wasserleitfähigkeit sowie ausreichenden pH-Puffer durch Basen verfügen. Lössböden sind den Auenböden mindestens ebenbürtig (WITTICH 1951). Diese Standorte liefern auch bei 
Flachgründigkeit gute Erträge. Erst bei Degradation, welche sich durch Pseudovergleyung bemerkbar machen kann, nimmt die Ertragsleistung ab.

- lockere Kipp- und Aufschüttungsböden: Durch die Bodenlockerheit ist in der Regel eine optimale Luft- und Wasserversorgung gegeben. Hier kann das Wurzelwerk der Bäume den Boden bestens erschließen. Das Ergebnis ist ein gutes Wuchspotential.

- Niedermoore ${ }^{7}$ : Diese Standorte zeichnen sich durch Spitzenleistungen aus. Sie sind nährstoffreich und haben zudem eine exzellente Wasser- und Luftversorgung. Voraussetzung ist richtige Entwässerung bzw. ein ausreichender Abstand zum Grundwasser von mindestens $0,5 \mathrm{~m}$.

- durch Stauwasser beeinflusste Böden (Pseudogleye): Sind aufgrund der bereits erwähnten Unverträglichkeit von Pappel und Weide gegenüber Verdichtung und Luftmangel nicht für den Anbau geeignet. Während sich die Stauschicht im Oberboden sehr schlecht auf das Wachstum auswirkt, sind tiefer gelegene Stauschichten weniger kritisch zu bewerten.

- Letten und Tonböden: Diese Böden sind in der Regel dicht gelagert und schwer durchwurzelbar. Zudem neigen sie zu Staunässe und haben eine geringe nFK. Außerdem verfügen sie kaum über die Fähigkeit Wärme zu leiten (LANDGRAF und BöCKER 2011). Daher lassen sie für den Anbau von KUP nur schwache Wuchsleistungen erwarten.

- Lehmböden: Diese Böden sind hinsichtlich ihrer Eignung sehr indifferent zu beurteilen. Während manche Lehme bei hohem Schluffgehalt Lössböden ähneln, sind andere den Tonböden ähnlicher. Hieraus können sich sowohl sehr gute als auch sehr schlechte Wuchsbedingungen ergeben. Daher können über diese Standorte keine allgemeinen Aussagen getroffen werden. Lehme bis lehmige Sande: sind ertragreiche Standorte, wenn auch nicht vergleichbar mit den Lössstandorten. Oft anzutreffende Bodentypen sind Braunerden mit hoher Basensättigung. Ist Kalk im Boden vorhanden, ist die Leistung stärker. Lehme mit geringer Basensättigung zeigen mäßigen Ertrag. Podsolierte Lehmböden sind keine geeigneten Standorte für den KUPAnbau. Bei Lehmen mit starkem Sandanteil kann der Anbau nur bei ausreichender Wasserversorgung bedingt durch Niederschlag oder Grundwassereinfluss empfohlen werden.

- Kalkböden: Diese Böden verfügen über eine lockere Struktur und Kalkreichtum. Daher kann eine gute Leistung erwartet werden, insbesondere wenn der Boden nicht allzu flachgründig ist. Sind Kalkböden mit einer Lössschicht überdeckt, verbessert sich die Leistung der Bestände deutlich.

- Trockene Sandböden: Klassische Kiefernstandorte mit mäßiger Ertragserwartung sind ebenfalls ungeeignet. Zudem besteht auf diesen Standorten die Gefahr, dass das Wachstum irgendwann zurückbleibt. Pappeln hohen Alters können auf diesen Standorten vom Wind umgeworfen werden.

\subsection{Waldwachstumsmodellierung mit Schwerpunkt in Kurzumtriebsplantagen}

Modelle stellen ein vereinfachtes Bild von natürlichen Prozessen dar. Sie sind sozusagen Vertreter des Systems, in dem nur die wesentlichen Systembestandteile berücksichtigt werden. Sie können sowohl als Praxiswerkzeug zur Prognose von Erträgen als auch als Forschungswerkzeug verwendet werden, um Hypothesen zu stellen und Ergebnisse zu interpretieren (LANDSBERG et al. 1991). Aufdeckungen von Wissenslücken werden durch die Modellierung gefördert (PRETZSCH 2001). Die physikalisch-

\footnotetext{
${ }^{7}$ Da Moorstandorte eine wichtige C-Senke darstellen sollte die Klimawirksamkeit des Anbaus kritisch überprüft werden. Es ist anzunehmen, dass durch den hohen Wasserverbrauch von KUP die Mineralisation des Torfs voranschreitet und $\mathrm{CO}_{2}$ emittiert wird.
} 
biologischen Gesetzmäßigkeiten werden in statistischen Beziehungen ausgedrückt. Wuchs- und Ertragsmodelle stellen ein äußerst wichtiges Hilfsmittel der Wirtschaftsplanung dar.

Bei der Entwicklung von Modellen ist ökologisches Systemverständnis Voraussetzung. Gleichzeitig fördert die Modellentwicklung die Erweiterung vom Prozessverständnis. In der Forstwirtschaft sowie in der Landwirtschaft kann man Modelle in empirische und mechanistische Modelle aufteilen, wobei auch Hybridformen existieren. Im Folgenden werden diese zwei Modellarten mit dem Schwerpunkt Waldwachstum vorgestellt sowie deren Vor- und Nachteile präsentiert. Ein besonderer Aspekt liegt in der Berücksichtigung der Standorteigenschaften.

\subsubsection{Empirische Modelle}

Die empirische Modellierung kann wie folgt definiert werden: „Bei der empirischen Modellierung wird das System als Blackbox betrachtet: Die innere Struktur und Wirkungsweise des Systems wird nicht berücksichtigt, sondern die Beziehung von Input und Output wird durch eine Formel wiedergegeben, welche den in Versuchen erhobenen Daten möglichst gut entspricht" (DESCH 2011). Für die empirische Modellierung des Waldwachstums werden Variablen verwendet, welche als wachstumsbestimmend angesehen werden können (RÖHLE 1995). Sie basieren auf direkten Messungen des Systems. Jedoch hat dies zwei Seiten. Einerseits kann durch die empirische Modellentwicklung Einblick in die zugrundeliegenden physiologischen Vorgänge des Waldwachstums gegeben werden, andererseits können die Ergebnisse der Modellierung nicht kausal sein. Daher besteht die Aufgabe der Modellentwickler darin, mögliche kausale Zusammenhänge zu interpretieren bzw. zu erklären. Mathematisch werden sowohl Regressionen als auch Differentialgleichungen verwendet (RÖHLE 1995). Werden die Details der physiologischen Modelle betrachtet, sind diese in ihrem Ursprung empirisch (ISEBRANDS et al. 1990). Die meisten Modelle sind empirisch, da sie einfacher zu gestalten und zu verwenden sind. In der Entwicklung sind sie kosteneffizient und in der Verwendung praktisch.

$\mathrm{Zu}$ den empirischen Waldwachstumsmodellen zählen zum Beispiel BWINPro (NAGEL 1997) und BWINPro-S (RÖHLE et al. 2004, SCHRÖDER et al. 2005), CACTOS (WENSEL und BIGING 1987), SILVA (PRETZSCH et al. 2002) und STIM (BONNOR et al. 1995, DE JONG et al. 1995) sowie das Pappelmodell von ALI (2009). Ertragstafeln gehören ebenfalls zu dieser Modellkategorie. Empirische Ansätze für die standortbasierte Ertragsmodellierung von Pappel und Weide im Kurzumtrieb sind selten. Im Folgenden wird auf die Ansätze von ALI (2009) sowie auf die Modelle von AYLOTT et al. (2008), und von BERGANTE et al. (2010) eingegangen.

\subsubsection{Pappelmodell nach ALI (2009)}

Das Modell von ALI (2009) basiert auf sechs im Bundesland Sachsen gelegenen Versuchsarealen, in denen eine große Anzahl an Untersuchungsflächen mit variierenden Klonen und Bestandesdichten beprobt wurden. Die Modelle wurden für die Prüfgliedergruppen Max (Max 1 bis 5), Matrix (Matrix und $\mathrm{H}$ 275) sowie getrennt für die Einzelklone Androscoggin und Münden parametrisiert. Bei der Max-Gruppe wurden sechs verschiedene Bestandesdichten mit Werten zwischen 1.150 und 13.000 Stk. ha ${ }^{-1}$ berücksichtigt. Bei den restlichen Klonen wurde das Modell einzig für die Bestandesdichte 1.550 Stk. ha ${ }^{-1}$ entwickelt.

Als erster Schritt wurde die Oberhöhe $\left(\mathrm{h}_{\mathrm{dom}}\right)$ mittels Standortfaktoren ermittelt. Zur Modellentwicklung wurden die Temperaturmittelwerte und Niederschlagssummen verschiedener Zeitfenster der Monate April bis Oktober in der jeweiligen Beobachtungsdauer der Versuchsflächen herangezogen. Insgesamt wurden 15 Temperatur- und Niederschlagsvariablen geprüft. Die Mindestlänge des aggregierten Zeitraums betrug zwei Monate. Als Bodenparameter in der Modellierung fungierten AZ und nFK im effektiven Wurzelraum $\left(\mathrm{nFK}_{\mathrm{WE}}\right)$. Des Weiteren wurde der Faktor Alter berücksichtigt. Durch 
Multiplikation und Division wurden aus den bestkorrelierten Variablen aggregierte Variablen gebildet. Eine aggregierte Variable ist demnach zum Beispiel ( $\left.\mathrm{AZ} * \mathrm{nFK} \mathrm{WE}_{\mathrm{WE}}\right)$.

Das endgültige Modell beinhaltete das Alter, die Niederschlagssumme von Mai bis Juni, AZ, die Temperatursumme von April bis Juli sowie $\mathrm{nFK}_{\mathrm{WE}}$.

$\underline{\text { ALI Modell (2009) }}$

$$
\begin{aligned}
h_{\text {dom }}= & a_{1}(\text { Alter })+a_{2}\left(N_{5-6} * \text { Ackerzahl }\right)+a_{3}\left(\frac{T_{4-7}}{n F K w e}\right) \\
& \\
h_{\text {dom }}: & \text { Durchschnittlicher Gesamtzuwachs }[\mathrm{m}] \\
a_{1} \text { bis } a_{3}: & \text { Modellkoeffizienten } \\
\text { Alter }: & \text { Alter des Beastandes [a] } \\
N_{5-6}: & \text { Niederschlagssumme Mai bis Juni [mm] } \\
T_{4-7}: & \text { Temperaturmittelwert April bis Juli }\left[{ }^{\circ} \mathrm{C}\right] \\
n F K w e: & \text { nutzbare Feldkapazität im effektiven Wurzelraum [mm] }
\end{aligned}
$$

Formel 2-1

Das Modell von ALI erklärte $99 \%$ der Streuung der $\mathrm{h}_{\text {dom }}$ anhand von Standortdaten $\left(\mathrm{R}^{2}\right.$ korr $\left.=0,99\right)$. Um letztendlich den dGZ zu schätzen, wurde eine Beziehung zwischen $\mathrm{h}_{\mathrm{dom}}$ und dGZ entwickelt. Diese Beziehung hatte einen $\mathrm{R}^{2}{ }_{\text {korr }}$ von 0,92 .

Aufgrund der räumlichen Begrenzung ist das Modell in seinem Validitätsbereich limitiert. Für AZ ist das Modell zwischen 15 und 67, für nFKwe zwischen 12,6 und 20,0 cm, für $\mathrm{T}_{4-7}$ zwischen 11,3 und $15,3{ }^{\circ} \mathrm{C}$ und für $\mathrm{N}_{5-6}$ Zwischen 91 und $146 \mathrm{~mm}$ valide. Nachteilig beim Modell von ALI ist die gleichzeitige Berücksichtigung von $\mathrm{AZ}$ und Klimavariablen, denn in $\mathrm{AZ}$ ist bereits ein Klimaeinfluss enthalten (AG BODEN 2005). Auch die gleichzeitige Verwendung von $\mathrm{AZ}$ und $\mathrm{nFK}_{\mathrm{WE}}$ im Modell kann als redundant angesehen werden, schließlich korrelieren $\mathrm{BZ}$ und $\mathrm{AZ}$ eng mit der nFK (HARRACH 2008). Darüber hinaus ist die Verwendung der $\mathrm{nFK}_{\mathrm{WE}}$ umstritten, denn der effektive Wurzelraum bezieht sich standardmäßig auf Ackerkulturen und nicht auf Baumgewächse (AG BODEN 2005).

\subsubsection{Pappel- und Weidenmodell nach AyLOTT et al. (2008)}

Aus den Ergebnissen einer großangelegten Versuchsreihe in England mit insgesamt 49 Versuchsflächen wurde die Entwicklung von standortbasierten empirischen Ertragsmodellen für mehrere Pappelund Weidenklone durchgeführt. Erste Versionen der Modelle können in EvANS et al. (2006) nachgeschlagen werden. Die Hauptauswertung der empirischen Modellierung findet sich bei AYLOTT et al. (2008).

In der Modellentwicklung wurden als Bodenvariablen die Bodenwasserverfügbarkeit in $\mathrm{mm}$ nach HALL et al. (1977), der pH-Wert, die prozentuale Sand-, Schluff- und Tongehalte, die Höhe über NN und die Neigung berücksichtigt. Klimatisch gingen in das Modell die mittlere Jahres- und Monatsniederschlagssummen, die mittleren maximalen Tages- und Monatstemperaturen $\left({ }^{\circ} \mathrm{C}\right)$, Vegetationstage sowie Frosttage ein. Als abhängige Variable wurde der dGZ der jeweiligen Rotation bestimmt.

Aus diesen Eingangsgrößen wurden rotations- und klonspezifische Modelle entwickelt. In der Veröffentlichung ist die Gesamtanzahl der verwendeten Modellprädiktoren nicht ausgeführt. Angegeben sind die drei hierarchisch wichtigsten Variablen in den jeweiligen Modellen. Die Modelle waren heterogen aufgebaut. Die drei wichtigsten Prädiktoren waren manchmal nur Klimavariablen, manchmal Boden und Klimavariablen.

Das Modell des Pappelklons Trichobel der ersten Rotation beinhaltete beispielsweise die Variablen Temperaturmittelwert von Februar, sowie Neigung und Niederschlagssumme vom Juni. Die Modellierung der zweiten Rotation beinhaltete die mittlere Temperatur von Januar, Oktober und August. Die wichtigsten Eingangsgrößen im Ertragsmodell des Weidenklons Jorunn der ersten Rotation waren die Variablen $\mathrm{pH}$ in der Tiefe $25-50 \mathrm{~cm}, \mathrm{pH}$ in $0-25 \mathrm{~cm}$ sowie Höhe über NN. Im Modell der zweiten Rotation waren die Hauptvariablen Höhe über NN, Hauptbodenart und Temperaturmittelwert Januar. 
Weder auf Basis der Gattung noch auf Rotationsebene wurde eine Konsistenz in den Modellprädiktoren festgestellt. Die Modelle erklärten die Variation des dGZ mit R $^{2}$ zwischen 0,51 und 0,70.

Als Ergebnis der empirischen Modellierung zeigt sich erneut die Wasserversorgung als der am stärksten wachstumslimitierende Faktor. Frühjahrs- und Sommerniederschläge waren mit dem dGZ hochkorreliert. Bei der Bildung von Klimavariablen ist als wesentlicher Kritikpunkt dieser Studie das gewählte Zeitfenster zu sehen. Die zeitliche Ebene der Klimavariablen war lediglich ein Monat. Auf dieser Ebene kann die Unsicherheit der zeitlichen Variation sehr hoch gegenüber dem zu berücksichtigten physikalischen Prozess sein. Das heißt, ein Monatswert gibt nicht unbedingt den Standortcharakter wieder. Des Weiteren wurden in den Modellen teilweise mehrere Variablen derselben Kategorie, wie Niederschlags- oder Temperaturvariablen, berücksichtigt. Damit sind die Modelle redundant und in ihre physikalischen Validität begrenzt.

\subsubsection{Leistungsbeeinflussende Standorteigenschaften für Pappel und Weide nach BERGANTE et al. (2010)}

Mit Daten von 183 Pappel- und 102 Weidenversuchsflächen aus der Po-Ebene (Norditalien) wurde der Einfluss von standortkundlichen Variablen auf das Wachstum untersucht. Als statistische Methode wurde die schrittweise Regressionsanalyse verwendet. Als abhängige Variable wurde der dGZ nach zwei Rotationen gewählt. Tabelle 2-6 zeigt die untersuchten unabhängigen Variablen.

Die Pappeln wurden in zwei Gruppen unterteilt, die erste Gruppe setzte sich aus $P$. canadensis Klone zusammen (u. a. I 214, BL Constanzo, Clima, Luisa Avanzo, Lambro, Orion), die zweite Gruppe aus P. deltoides Klonen (zum Beispiel Lux, Dvina, Lena, Oglio, Baldo). Darüber hinaus wurde eine Weidengruppe von S. matsudana Klonen (zum Beispiel Drago und Levante) analysiert.

Der dGZ der zwei Rotationen wurde für die verschiedenen Prüfgliedergruppen durch unterschiedliche Variablenkombinationen am besten vorausgesagt. Bei der P. candensis Prüfgliedergruppe hatte das Modell CUMSEAW als einzigen Prädiktor. Das $\mathrm{R}^{2}$ korr der Regression belief sich auf 0,54. Bei $P$. deltoides waren die Prädiktoren der Regression WATER 1 und CUMSEAW. $\mathrm{R}^{2}{ }_{\text {korr }}$ betrug 0,83 . Bei der $S$. matsudana Gruppe hatte das Modell SWATER1 als einziger Prädiktor und einen $\mathrm{R}^{2}$ korr von 0,62.

Somit gilt die Wasserverfügbarkeit als die wichtigste Variable für das Pappel- und Weidenwachstum in der Po-Ebene. Weder Temperaturen noch Bodeneigenschaften oder Düngung wurden berücksichtigt. Dabei ist anzumerken, dass wichtige bodenphysikalische Kennwerte wie nFK und LK in dieser Studie nicht berücksichtigt wurden.

In Bezug auf diese Studie ergeben sich folgende wesentliche Kritikpunkte. Die Bildung von Wasserversorgungsvariablen erscheint nicht optimal, denn wenn bei zwei Rotationen mit je zwei Wuchsperioden die Daten einer einzigen Wuchsperiode oder Rotation berücksichtigt werden, ist der Bezugszeitraum der abhängigen Variablen nicht ausreichend repräsentiert. Andererseits wurde bei der Modellierung keine Begrenzung hinsichtlich der Kombination von Variablen gesetzt, welche denselben Prozess darstellen wie die Wasserversorgungsvariablen. So wurden, wie oben bereits erwähnt, bei der $P$. deltoides Klongruppe im Modell zwei Wasserversorgungsvariablen WATER 1 und CUMSEAW berücksichtigt. Dadurch ist das Modell redundant. 
Tab. 2-6: Variablen für die Identifizierung leistungsbeeinflussender Standorteigenschaften nach BERGANTE et al. (2010).

\begin{tabular}{ll}
\hline Variable & Beschreibung \\
\hline WATER1 & Jahresniederschlags- und Jahresbewässerungsmenge [mm] der ersten Wuchsperiode ${ }^{*}$ \\
WATER2 & Jahresniederschlags- und Jahresbewässerungsmenge [mm] der zweiten Wuchsperiode \\
SWATER1 & Niederschlags- und Bewässerungsmenge [mm] der ersten Vegetationsperiode ${ }^{*}$ \\
SWATER2 & Niederschlags- und Bewässerungsmenge [mm] der zweiten Vegetationsperiode \\
CUMANNW & Jahresniederschlags- und Jahresbewässerungsmenge [mm] der ersten und zweiten Wuchsperiode \\
CUMSEAW & Jahresniederschlags- und Jahresbewässerungsmenge [mm] der ersten und zweiten Vegetationsperiode \\
ANNTEMP1 & Mittlere Jahrestemperatur ${ }^{\circ} \mathrm{C}$ der ersten Wuchsperiode \\
ANNTEMP2 & Mittlere Jahrestemperatur ${ }^{\circ} \mathrm{C}$ der zweiten Wuchsperiode \\
Sand [\%] & Prozentualer Sandgehalt \\
Schluff [\%] & Prozentualer Schluffgehalt \\
Ton [\%] & Prozentualer Tongehalt \\
pH & Boden pH-Wert \\
TOT N & Gesamtstickstoff [g kg ${ }^{-1}$ ] \\
OC \% & Prozentualer organischer Kohlenstoffgehalt \\
PLD & Pflanzdichte \\
FERT & Düngung \\
\hline
\end{tabular}

* Weder die Wuchsperiode noch die Vegetationsperiode werden weiter spezifiziert.

Weitere empirische Modelle, welche direkt die Wasserversorgung als Hauptfaktor berücksichtigen, sind die Modelle von LINDROTH und BÄTH (1999) und MURACH (2009).

\subsubsection{Mechanistische Modelle}

Mechanistische Waldwachstumsmodelle, auch als ökophysiologische Modelle bekannt, basieren auf zugrundeliegenden physikalischen Prozessen - Photosynthese, Biomasseallokation, Wasseraufnahme und Transpiration - und werden häufig verwendet, um die Reaktion von Waldbeständen auf wechselnde Umweltbedingungen zu prognostizieren. Sie erklären die zu beschreibenden Systeme, da sie kausal sind. Biologische Reaktionsmechanismen und Prozesse können auch unter dynamischen Rahmenbedingungen mit diesen Modellen untersucht werden (PRETZSCH 2001). Mechanistische Modelle sind für dynamische Simulationen mit variablen Umweltbedingungen geeignet.

Mechanistische Modelle für das Baumwachstum existieren in großer Anzahl, wie zum Beispiel JABOWA-Foret (BotKIN et al. 1972), SIEVÄNEN Modell (SIEVÄNEN 1983) LINKAGES (PASTOR et al. 1987), ForGro (MOHREN 1987), BIOMASS (MCMURTRIE et al. 1989), ECOPHYS (RAUSCHER et al. 1990), FORCYTE (KRIMMINS 1990), MAESTRO (WANG und JARVIS 1990), FOREST BGC (RUNNING und GOWER 1991), TREGRO (WEINSTEIN et al. 1991), Treedyn (BOSSEL 1994), SORTIEND (PACALA et al. 1993, 1996), SOILN-FORESTSR (ECKERSTEN 1994), TRAGIC++ (HAUHS et al. 1995), FORSANA (GROTE 1997), FOREST-3PG (LANDSBERG und WARING 1997), PROMOD (SANDS et al. 2000). LINPAC (JING et al. 2012), FOREST-GROWTH-SRC (TALLIS et al. 2013) u. v. m..

Innerhalb der physiologischen Modelle wird zwischen „Bottom up“-Modellen, welche das Wachstum sehr detailliert darstellen, und „Top Down“-Modellen, welche nur Teilbereiche des Systems berücksichtigen, differenziert (ISEBRANDS und BURK 1992). Zu den „Bottom up“-Modellen zählen zum Beispiel die Modelle SOILN-FORESTSR (ECKERSTEN 1994) oder BIOMASS von MCMURTIE et 
al. (1989). Abbildung 2-6 stellt die häufig in mechanistischen Waldwachstumsmodellen berücksichtigten Prozesse dar (aus TALLIS et al. 2013).

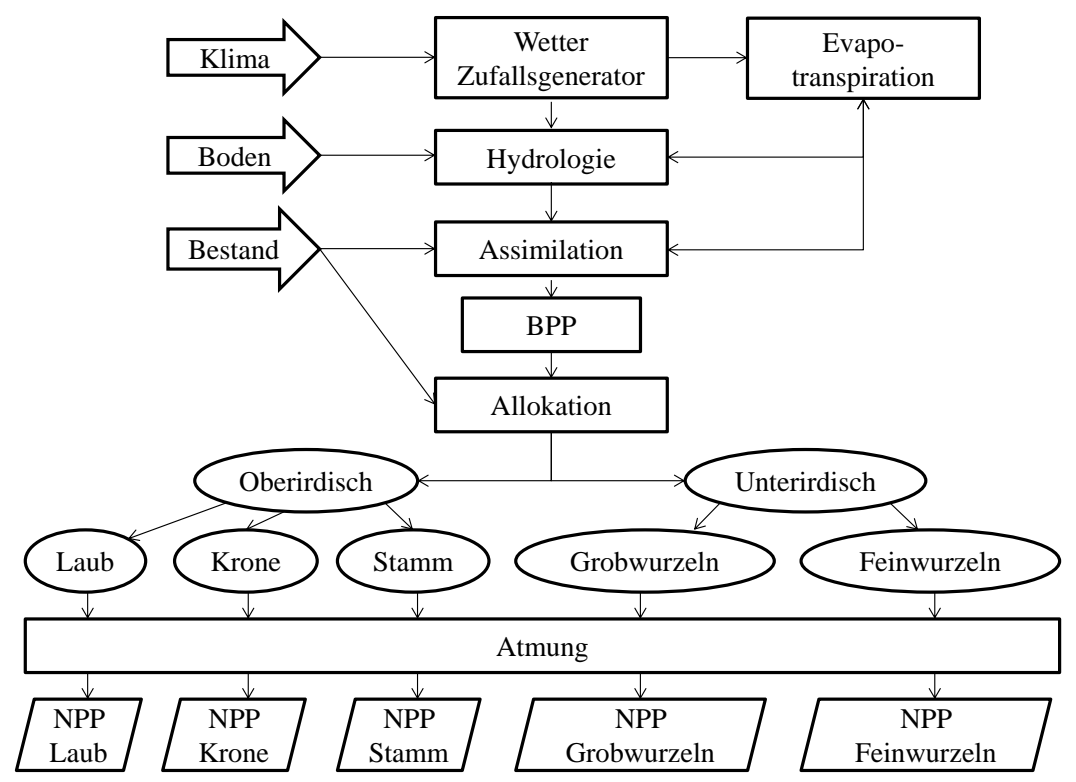

Abb. 2-6: Berücksichtigte physikalisch-biologische Waldwachstumsprozesse im mechanistischen Modell FOREST GROWTH SRC (verändert nach TALLIS et al. 2013). Mit BPP: Bruttoprimärproduktion, NPP: Nettoprimärproduktion

Das Modell FOREST-GROWTH SRC verfügt über einen Wetterzufallsgenerator. Bei der Mehrheit der mechanistischen Waldwachstumsmodelle gehen die Klimaparameter als Tages- oder Monatsdaten in die Modellierung ein.

Zu den „Top Down“-Modellen zählen zum Beispiel das SIEVÄNEN Modell (SIEVÄNEN 1983) und das Modell ECOPHYS (RAUSCHER et al 1990). Diese zwei Modelle beschränken sich auf ein Kohlenstoffmodul, welches Lichtinterzeption und Biomassebildung erfasst. Weitere Prozesse des Wasserund Stoffhaushaltes werden nicht berücksichtigt. Daher ist der Einsatz solcher Modelle für die Prognosen von Wachstum und Massenleistung ungeeignet.

Die Mehrheit der mechanistischen Modelle haben empirische Anteile. Denn manche zugrundeliegenden Prozesse des Baumwachstums, insbesondere von Biomasseallokation sind für die Forschung zurzeit noch eine „Blackbox“. Gerade Modellparameter zur Biomasseallokation in den verschiedenen Kompartimenten, sogenannte Biomassepartitionskoeffizienten, haben in mechanistischen Waldwachstumsmodellen eine hohe Sensibilität (AMICHEV et al. 2010, LING et al. 2012).

Ein Nachteil von mechanistischen Modellen sind die hohen Datenanforderungen für den Input, da sie deutlich mehr Informationen als empirische Modelle beinhalten. Zu diesen Daten zählen beispielsweise hochaufgelöste Klimadaten, bodenkundliche Charakterisierung etc.. Die Nutzung von mechanistischen Modellen wird hierdurch verkompliziert. Zudem sind bei der Entwicklung von mechanistischen Modellen Daten in sehr hoher Güte und großem Umfang erforderlich.

Einer der kritischsten Punkte in der Nutzung von mechanistischen Waldwachstumsmodellen ist jedoch deren Parametrisierung. Manche mechanistische Modelle haben sehr viele Parameter, zum Beispiel werden in FOREST 3PG über 20 Parameter benötigt. In einer Studie zur Ertragsmodellierung von Pappelklonen gaben AMICHEV et al. (2010) bei der Parametrisierung der Modelle drei verschiedene Wege an:

- durch empirische Beobachtungen der eigenen Versuchsflächen,

- aus Pappeldaten der Literatur bei ähnlicher Taxonomie,

- Grundparameter des FOREST-3PG Modells, gültig für Eucalypthus Arten. 
Ein weiterer von AMICHEV et al. (2010) vorgegebener Weg ist die einfache Variierung der restlichen Parameter, um eine gute Anpassung an die Ausgangsdaten zu erhalten.

Die Entscheidung für die Nutzung spezifischer Parameter in der Kalibrierung von Modellen, zum Beispiel für neue Baumarten, sollte fachlich fundiert sein und über eine ausreichende Genauigkeit verfügen. Mechanistische Modelle können physikalisch-biologische Vorgänge gut abbilden. Ist die Parametrisierung jedoch nicht fachgerecht, kann nicht mehr von einer Kausalität der Prognosen gesprochen werden.

Für Pappel und Weide wurden einige mechanistische Modelle entwickelt bzw. parametrisiert. Hierzu zählen u. a. das SIEVÄNEN Modell (SIEVÄNEN 1983), ECOPHYS (RAUSCHER et al. 1990), SOILNFOREST (ECKERSTEN 1994), FOREST 3PG (LANDSBERG und WARING 1997), BIOMASS (MCMurTRIE et al. 1989), SECRETS (SAMPSON und CEUlEMANS 2000, DECKMYN et al. 2004), LINPAC (LING et al. 2012) und FOREST-GROWTH SRC (TALLIS et al. 2013). Im Folgenden wird auf die Art der Berücksichtigung der Standorteigenschaften Boden und Klima eingegangen.

SIEVÄNEN (SIEVÄNEN 1983): Als klimatische Variablen werden die Lufttemperatur und die Globalstrahlung verwendet unter der Annahme, dass kein Wasserstress oder Nährstoffmangel existiert.

BIOMASS (MCMURTRIE et al. 1989): Als klimatische Eingangsgrößen werden mindestens die täglichen Niederschlagssummen und maximale und minimale Temperaturen benötigt. Dieses Modell berücksichtigt den Wasserhaushalt. Der Bodenwassergehalt wird als Steuerungsgröße für die stomatäre Leitfähigkeit des Bestandes verwendet. Das Bodenprofil kann in verschieden mächtige Schichten aufgeteilt werden.

ECOPHYS (RAUSCHER et al. 1990): Das Modell beinhaltet als klimatische Variable die Solarstrahlung und die Lufttemperatur in stündlicher Auflösung, unter der Annahme, dass kein Wasserstress oder Nährstoffmangel existiert.

SOILN-FORESTSR (ECKERSTEN 1994): Klimafaktoren sind die tägliche Sonnenscheindauer, die Tagesmittelwerte der Temperaturen von Boden und Luft sowie die Niederschlagssumme. Das Modell SOIL, ein Untermodell von SOILN-FORESTSR, ist ein vollständiges Modell des Wasserhaushaltes für das System Boden-Pflanze-Atmosphäre (JANSSON 1998). In diesem Modell werden Profiltiefe, maximale Durchwurzelungstiefe parametrisiert sowie die relevanten Prozesse Infiltration, Evapotranspiration und Versickerung simuliert (JANSSON 1991).

FOREST-3PG (LANDBERG und WARING 1997): Als klimatische Eingangsgrößen im Modell werden Niederschläge und Temperaturen, in Tages- oder Monatsauflösung, benötigt. Weitere klimatischen Variablen sind die Globalstrahlungssumme pro Monat, der Monatsmittelwert der Luftfeuchtigkeit sowie die Anzahl an Frosttagen pro Monat. Im Kompartiment Boden wird das maximale pflanzenverfügbare Bodenwasser, das heißt die nFK, parametrisiert. Mit Hilfe von klimatischen Kenngrößen wird daraus das aktuelle pflanzenverfügbare Wasser simuliert. Das Bodenwassermodul besteht aus einer einzigen Schicht. Die Dicke der Schicht ist beliebig. Zudem wird ein Bodenfruchtbarkeitsindex, welcher Werte zwischen 0 und 1 einnehmen kann, in der Modellierung berücksichtigt.

SECRETS (SAMPSON und CeulEMANS 2000): Klimatisch werden im Modell stündlich aufgelöste Daten der Globalstrahlung, des Niederschlags, der Luftfeuchte sowie der Lufttemperatur berücksichtigt. Im Boden wird die Durchwurzelungstiefe der Gewächse benötigt. Das Wassermodul war eine Anpassung vom Wassermodul aus dem Modell BIOMASS (DECKMYN 2004).

LINPAC (JING et al. 2012): Das Modell berücksichtigt Monatsmittelwerte der Globalstrahlung, der Temperatur, des Luftdrucks, der Windgeschwindigkeit und des Niederschlags. Für die Simulation des Bodenwasserhaushalts werden als Bodenparameter die Bodenart, Neigung, Profiltiefe und die nFK benötigt. 
FOREST-GROWTH SRC (TALLIS et al. 2013): Als klimatische Kenngrößen gehen ins Modell Lufttemperatur, Luftdruck, Windgeschwindigkeit, Globalstrahlung, Luftfeuchte und Niederschlag ein. Diese können sowohl in Tages- oder Monatsauflösung berücksichtigt werden. Für den Bodenwasserhaushalt wird im FOREST-GROWTH SRC das Modul „soil water balance capacity model“ (SWBCM) verwendet (EVANS et al. 1999). Das Modell ist deterministisch und simuliert Wassergehalt, Grundwasserspiegel, Versickerung, Evaporation und Transpiration.

Während die Mehrheit der „Bottom up“-Modelle klimatische Größen ausreichend berücksichtigen, ist die Berücksichtigung der Bodenhydraulik eher mäßig (PHILIPPOT 1996, LuXMOORE et al. 2008). Die Wasserverfügbarkeit kann aber erst durch die Berücksichtigung von bodenhydraulischen Parametern genau abgebildet werden. Dies war einzig bei den Modellen SOILN-FORESTSR, LINPAC und FOREST GROWTH SRC der Fall. Dasselbe gilt für die Wurzelparameter. Die Durchwurzelungstiefe, das Wurzelwachstum und die Wurzelverteilung waren in den vorgestellten Modellen entweder nicht ausreichend dokumentiert oder einfach nicht ausreichend berücksichtigt. Darüber hinaus sind die Prozesse der Photosynthese, wie die Allokation und die Respiration, schwer zugängliche Größen. In der Modellierung können diese Prozesse demnach mit großen Unsicherheiten behaftet sein (PRETZSCH 2001).

NAIR et al. (2012) stellen für Bioenergiekulturen fest, dass bezüglich der Parametrisierung und Validierung von prozessbasierten Modellen noch viel Arbeit bevorsteht. Hierzu zählen vor allem die Generierung und Verteilung von hochwertigen Felddaten für die Modellevaluierung und Validierung. AMICHEV et al. (2010) stellte ebenfalls fest, dass insbesondere im Bezug auf den Standortaspekt Optimierungsbedarf für die KUP-Modelle besteht. Für jeden Aspekt von KUPs sollten Studien durchgeführt werden. Wenn die Einflüsse auf das Wachstum verstanden werden, können physiologische Modelle (mit oder ohne empirischen Anteilen) optimal parametrisiert werden.

Je nach räumlicher und zeitlicher Dimension werden verschiedene Modelle verwendet. Im Waldbau ergeben sich aufgrund der sehr variablen Bewirtschaftungsformen, vor allem in Mischbeständen, sehr komplexe Modellformen, während diese in der Landwirtschaft verhältnismäßig einfach bleiben. In Abhängigkeit der Zielsetzung sollte die Komplexität des zu verwendenden oder zu entwickelnden Modells abgewogen werden. Wird nur der dGZ benötigt, sind Aussagen zur Biomasseallokation in Krone und Stamm überflüssig. Im Vergleich zum Hochwald kann die Modellierung von KUP aufgrund seiner Einfachheit, erkennbar an einer festgelegten genetischen Basis und kurzen Umtriebszeiten, eine wichtige Rolle bei der Optimierung der Methodik für die Entwicklung von standortbasierten Waldwachstumsmodellen spielen. 


\section{Material und Methoden}

Dieses Kapitel behandelt die Auswahl von Versuchsflächen und das Versuchsdesign. Weiterhin werden klimatische und bodenkundliche Kenngrößen sowie waldwachstumskundliche Kenngrößen erläutert. Die Methodik zur Entwicklung von standortbasierten Ertragsmodellen und standortspezifischen Ertragssteigerungsfaktoren stellt den Kern dieses Kapitels dar. Zuletzt erfolgt eine Beschreibung der angewandten statistischen Analysen sowie der verwendeten EDV.

\subsection{Flächenanlage und Versuchsdesign}

Im Frühjahr 2008 wurden im Rahmen des Verbundvorhabens PROLOC deutschlandweit insgesamt 37 Versuchsflächen nach einheitlichem Muster mit jeweils drei Pappel- und zwei Weidenklonen angelegt (Abb. 3-1). Eine weitere Versuchsfläche kam im Frühjahr 2009 dazu. Auf fünf von insgesamt 38 Standorten kamen unvorhergesehene Störungen im Anwuchsjahr oder Unplausibilitäten in der Datenübermittlung vor. Daher erfolgte die Auswertung nur auf 33 Versuchsflächen.

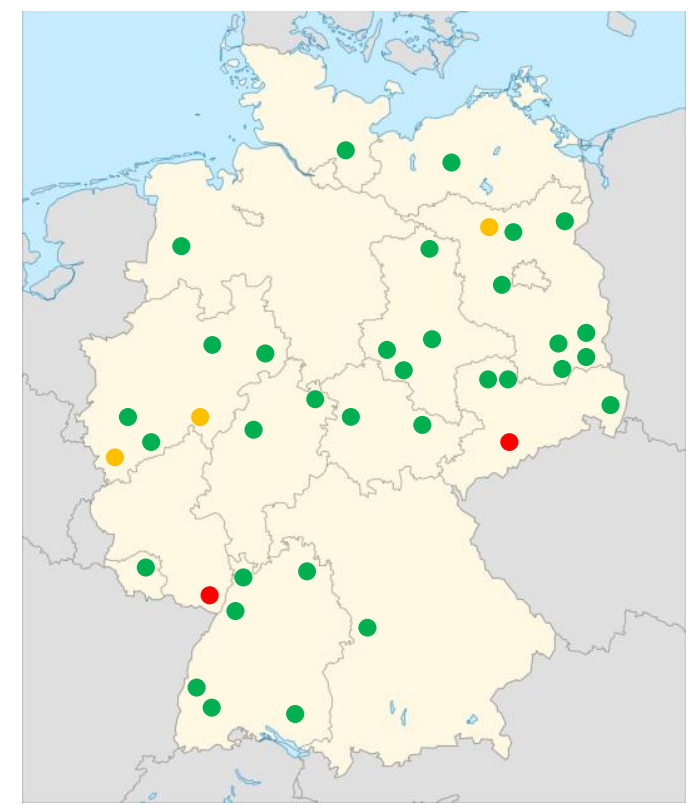

Abb. 3-1: Lage der Versuchsflächen.

Grün beschriftet: $\quad$ Versuchsflächen, die über die gesamten Versuchslaufzeit beobachtet wurden.

Rot beschriftet: $\quad$ Versuchsflächen, die nach unvorhergesehenen, massiven Störungen im Anwuchsjahr aufgegeben wurden.

Gelb beschriftet: $\quad$ Versuchsflächen, die nach unvorhergesehenen Managementproblemen sowie Unplausibilitäten in der Datenübermittlung in der zweiten Wuchsperiode aufgegeben wurden.

Im Vorhaben waren 24 Institutionen der landwirtschaftlichen und forstlichen Forschung als Projektpartner beteiligt (Tab. 3-1). Die Versuchsflächen setzen sich überwiegend aus klassischen Ackerflächen $(n=20)$, ackerbaulich genutzten Bergbaufolgelandschaften $(n=4)$, umgebrochenes Grünland $(\mathrm{n}=6)$, Baumschulflächen $(\mathrm{n}=2)$ und einer ehemaligen KUP-Fläche zusammen. Bei der Anlage der Versuchsflächen wurde eine repräsentative Verteilung angestrebt, welche unterschiedliche Klimaregionen und Bodenarten berücksichtigt. In Tabelle 3-2 sind wesentliche Eigenschaften der Versuchsflächen erläutert. 
Tab. 3-1: Übersicht der realisierten Versuchsflächen und betreuende Institution.

\begin{tabular}{|c|c|c|}
\hline $\begin{array}{l}\text { Sto. } \\
\text { Nr. }^{\text {a }}\end{array}$ & Standortname & Betreuende Institution \\
\hline 1 & Emmendingen & \multirow{2}{*}{ Forstliche Versuchsanstalt Baden-Württemberg, Abteilung Waldökologie } \\
\hline 2 & Lilienthal & \\
\hline 3 & Aulendorf & \multirow{4}{*}{$\begin{array}{l}\text { Landwirtschaftliches Technologiezentrum Augustenberg, Sachgebiet Nachwachsende } \\
\text { Rohstoffe }\end{array}$} \\
\hline 4 & Forchheim & \\
\hline 5 & Ladenburg & \\
\hline 6 & Kupferzell & \\
\hline 7 & Kaisheim & Bayerische Landesanstalt für Wald- und Forstwirtschaft \\
\hline 8 & E.Wald Welzow & \multirow{2}{*}{$\begin{array}{l}\text { Brandenburgische Technische Universität Cottbus, Lehrstuhl Bodenschutz und } \\
\text { Rekultivierung }\end{array}$} \\
\hline 9 & Löwenberg & \\
\hline 11 & Cahnsdorf & \multirow{2}{*}{ Hochschule für nachhaltige Entwicklung Eberswalde, Fachbereich Wald und Umwelt } \\
\hline 12 & Kummerow & \\
\hline 13 & Grünewalde & \multirow{2}{*}{ Forschungsinstitut für Bergbaufolgelandschaften Finsterwalde e.V. } \\
\hline 14 & Welzow Süd & \\
\hline 15 & Potsdam Bornim & $\begin{array}{l}\text { Leibniz-Institut für Agrartechnik Potsdam-Bornim e.V., Abteilung Technik der } \\
\text { Aufbereitung, Lagerung und Konservierung }\end{array}$ \\
\hline 16 & Lehmkaute & Justus Liebig Universität Gießen, Lehr- und Versuchsgut Rauischholzhausen \\
\hline 17 & Unterrieden & Universität Kassel, Fachgebiet Grünlandwissenschaft und Nachwachsende Rohstoffe \\
\hline 18 & Gülzow & $\begin{array}{l}\text { Landesforschungsanstalt für Landwirtschaft und } \\
\text { Vorpommern, Sachgebiet Nachwachsende Rohstoffe }\end{array}$ \\
\hline 19 & Werlte & Landwirtschaftskammer Niedersachsen / 3-N Niedersachsen \\
\hline 20 & Borlinghausen & \multirow{2}{*}{ Landwirtschaftskammer Nordrhein-Westfalen / Biomassehof Borlinghausen } \\
\hline 22 & Haus Düsse & \\
\hline 24 & $\begin{array}{l}\text { Königshovener } \\
\text { Höhe }\end{array}$ & RWE Power AG \\
\hline 25 & Klein Altendorf & Rheinische Friedrich-Wilhelms-Universität Bonn, Campus Klein-Altendorf \\
\hline 27 & Bärenrode & \multirow{4}{*}{$\begin{array}{l}\text { Landesanstalt für Landwirtschaft, Forsten und Gartenbau Sachsen-Anhalt } \\
\text { Koordinierungsstelle nachwachsende Rohstoffe - KoNaRo }\end{array}$} \\
\hline 28 & Bernburg & \\
\hline 29 & Hayn & \\
\hline 30 & Iden & \\
\hline 31 & Schlag Adler & $\begin{array}{l}\text { Sächsisches Landesamt für Umwelt, Landwirtschaft und Geologie, Referat } \\
\text { Pflanzenbau, Nachwachsende Rohstoffe }\end{array}$ \\
\hline 32 & Thammenhain I & \multirow{2}{*}{ Technische Universität Dresden, Institut für Waldwachstum und Forstliche Informatik } \\
\hline 33 & Thammenhain II & \\
\hline 35 & Threnthorst & Thünen-Institut für Forstgenetik \\
\hline 36 & Oberdorla & Baumschulen Oberdorla \\
\hline 37 & $\begin{array}{l}\text { Über dem } \\
\text { Erdengraben }\end{array}$ & Thüringer Landesanstalt für Landwirtschaft, Referat Nachwachsende Rohstoffe \\
\hline 38 & Heiliges Marpingen & Fachhochschule Trier, Institut für angewandtes Stoffstrommanagement \\
\hline
\end{tabular}

${ }^{a}$ Die Nummerierung der Standorte wurde über den ganzen Untersuchungszeitraum beibehalten. Aufgrund des Ausscheidens von fünf Standorten im ersten und zweiten Beobachtungsjahr ergibt sich eine lückige Durchnummerierung der Standorte. 
Tab. 3-2: Standortname, Bundesland, Höhe über NN, Exposition und genaue Koordinaten der Versuchsflächen.

\begin{tabular}{|c|c|c|c|c|c|c|}
\hline $\begin{array}{l}\text { Sto. } \\
\text { Nr. }\end{array}$ & Standortname & Bundesland & $\begin{array}{l}\text { Höhe } \\
\text { über } \mathrm{NN} \\
{[\mathrm{m}]}\end{array}$ & $\begin{array}{l}\text { Expo- } \\
\text { sition }\end{array}$ & \multicolumn{2}{|c|}{$\begin{array}{c}\text { Koordinaten } \\
{[\mathrm{N}-\mathrm{W}]}\end{array}$} \\
\hline 1 & Emmendingen & Baden-Württemberg & 330 & Südwest & $48^{\circ} 07^{\prime} 14^{\prime \prime}$ & $7^{\circ} 51^{\prime} 57^{\prime \prime}$ \\
\hline 2 & Lilienthal & Baden-Württemberg & 250 & Ost & $48^{\circ} 04^{\prime} 16^{\prime \prime}$ & $7^{\circ} 40^{\prime} 17^{\prime \prime}$ \\
\hline 3 & Aulendorf & Baden-Württemberg & 545 & n.v. & $47^{\circ} 57^{\prime} 38^{\prime \prime}$ & $9^{\circ} 38^{\prime} 54^{\prime \prime}$ \\
\hline 4 & Forchheim & Baden-Württemberg & 117 & n.v. & $48^{\circ} 58^{\prime} 55^{\prime \prime}$ & $8^{\circ} 20^{\prime} 27^{\prime \prime}$ \\
\hline 5 & Ladenburg & Baden-Württemberg & 100 & n.v. & $49^{\circ} 27^{\prime} 54^{\prime \prime}$ & $8^{\circ} 37^{\prime} 53^{\prime \prime}$ \\
\hline 6 & Kupferzell & Baden-Württemberg & 350 & Nord & $49^{\circ} 13^{\prime} 29^{\prime \prime}$ & $9^{\circ} 41^{\prime} 44^{\prime \prime}$ \\
\hline 7 & Kaisheim & Bayern & 520 & n.v. & $48^{\circ} 46^{\prime} 41^{\prime \prime}$ & $10^{\circ} 48^{\prime} 17^{\prime \prime}$ \\
\hline 8 & E.Wald Welzow & Brandenburg & 100 & n.v. & $51^{\circ} 37^{\prime} 12^{\prime \prime}$ & $14^{\circ} 19^{\prime} 20^{\prime \prime}$ \\
\hline 9 & Löwenberg & Brandenburg & 44 & n.v. & $52^{\circ} 52^{\prime} 25^{\prime \prime}$ & $13^{\circ} 09^{\prime} 07^{\prime \prime}$ \\
\hline 11 & Cahnsdorf & Brandenburg & 61 & n.v. & $51^{\circ} 51^{\prime} 32^{\prime \prime}$ & $13^{\circ} 45^{\prime} 51^{\prime \prime}$ \\
\hline 12 & Kummerow & Brandenburg & 7 & n.v. & $53^{\circ} 08^{\prime} 12^{\prime \prime}$ & $14^{\circ} 13^{\prime} 24^{\prime \prime}$ \\
\hline 13 & Grünewalde & Brandenburg & 130 & n.v. & $51^{\circ} 31^{\prime} 03^{\prime \prime}$ & $13^{\circ} 43^{\prime} 23^{\prime \prime}$ \\
\hline 14 & Welzow Süd & Brandenburg & 93 & Nord & $51^{\circ} 36^{\prime} 33^{\prime \prime}$ & $14^{\circ} 18^{\prime} 39^{\prime \prime}$ \\
\hline 15 & Potsdam Bornim & Brandenburg & 35 & n.v. & $52^{\circ} 26^{\prime} 13^{\prime \prime}$ & $13^{\circ} 00^{\prime} 58^{\prime \prime}$ \\
\hline 16 & Lehmkaute & Hessen & 175 & n.v. & $50^{\circ} 45^{\prime} 41^{\prime \prime}$ & $8^{\circ} 52^{\prime} 39^{\prime \prime}$ \\
\hline 17 & Unterrieden & Hessen & 140 & Nordwest & $51^{\circ} 20^{\prime} 40^{\prime \prime}$ & $9^{\circ} 53^{\prime} 25^{\prime \prime}$ \\
\hline 18 & Gülzow & Mecklenburg-Vorpommern & 10 & n.v. & $53^{\circ} 48^{\prime} 39^{\prime \prime}$ & $12^{\circ} 03^{\prime} 32^{\prime \prime}$ \\
\hline 19 & Werlte & Niedersachsen & 32 & n.v. & $52^{\circ} 48^{\prime} 44^{\prime \prime}$ & $7^{\circ} 39^{\prime} 47^{\prime \prime}$ \\
\hline 20 & Borlinghausen & Nordrhein-Westfalen & 308 & Nordost & $51^{\circ} 35^{\prime} 27^{\prime \prime}$ & $9^{\circ} 01^{\prime} 28^{\prime \prime}$ \\
\hline 22 & Haus Düsse & Nordrhein-Westfalen & 70 & n.v. & $51^{\circ} 38^{\prime} 08^{\prime \prime}$ & $8^{\circ} 11^{\prime} 14^{\prime \prime}$ \\
\hline 24 & Königshov. Höhe & Nordrhein-Westfalen & 108 & n.v. & $51^{\circ} 02^{\prime} 27^{\prime \prime}$ & $6^{\circ} 30^{\prime} 45^{\prime \prime}$ \\
\hline 25 & Klein Altendorf & Nordrhein-Westfalen & 210 & n.v. & $50^{\circ} 36^{\prime} 53^{\prime \prime}$ & $6^{\circ} 59^{\prime} 16^{\prime \prime}$ \\
\hline 27 & Bärenrode & Sachsen-Anhalt & 450 & n.v. & $51^{\circ} 39^{\prime} 32^{\prime \prime}$ & $10^{\circ} 59^{\prime} 25^{\prime \prime}$ \\
\hline 28 & Bernburg & Sachsen-Anhalt & 80 & n.v. & $51^{\circ} 50^{\prime} 25^{\prime \prime}$ & $11^{\circ} 41^{\prime} 25^{\prime \prime}$ \\
\hline 29 & Hayn & Sachsen-Anhalt & 441 & n.v. & $51^{\circ} 34^{\prime} 29^{\prime \prime}$ & $11^{\circ} 06^{\prime} 57^{\prime \prime}$ \\
\hline 30 & Iden & Sachsen-Anhalt & 23 & n.v. & $52^{\circ} 46^{\prime} 12^{\prime \prime}$ & $11^{\circ} 52^{\prime} 57^{\prime \prime}$ \\
\hline 31 & Schlag Adler & Sachsen & 250 & Ost & $51^{\circ} 09^{\prime} 23^{\prime \prime}$ & $14^{\circ} 33^{\prime} 48^{\prime \prime}$ \\
\hline 32 & Thammenhain I & Sachsen & 130 & Südwest & $51^{\circ} 26^{\prime} 01^{\prime \prime}$ & $12^{\circ} 50^{\prime} 48^{\prime \prime}$ \\
\hline 33 & Thammenhain II & Sachsen & 130 & Südwest & $51^{\circ} 26^{\prime} 04^{\prime \prime}$ & $12^{\circ} 50^{\prime} 50^{\prime \prime}$ \\
\hline 35 & Threnthorst & Schleswig-Holstein & 40 & n.v. & $53^{\circ} 46^{\prime} 49^{\prime \prime}$ & $10^{\circ} 31^{\prime} 28^{\prime \prime}$ \\
\hline 36 & Oberdorla & Thüringen & 230 & n.v. & $51^{\circ} 09^{\prime} 31^{\prime \prime}$ & $12^{\circ} 24^{\prime} 47^{\prime \prime}$ \\
\hline 37 & Ü. d. Erdengraben & Thüringen & 260 & Nord & $51^{\circ} 00^{\prime} 11^{\prime \prime}$ & $11^{\circ} 39^{\prime} 28^{\prime \prime}$ \\
\hline 38 & Heiliges Marpingen & Saarland & 331 & Südwest & $49^{\circ} 26^{\prime} 29^{\prime \prime}$ & $7^{\circ} 02^{\prime} 30^{\prime \prime}$ \\
\hline
\end{tabular}

Mit n.v.: nicht vorhanden.

19 der Versuchsflächen liegen in der planaren Stufe, sieben befinden sich in der kollinen Stufe, fünf in der submontanen und zwei in der montanen Stufe (tiefmontan). Eine Versuchsfläche besteht aus 20 randomisierten Einzelparzellen, besitzt eine Größe von $1.800 \mathrm{~m}^{2}$ und ist zum Schutz vor Wildschäden eingezäunt. Auf jeder Einzelparzelle existieren fünf Prüfglieder in vierfacher Wiederholung. Die Ein- 
zelparzellen besitzen eine Größe von 9,0 m mal 10,0 m. Die Versuchsfläche ist von einem 5,0 m breiten Weg umgeben (Abb. 3-2).

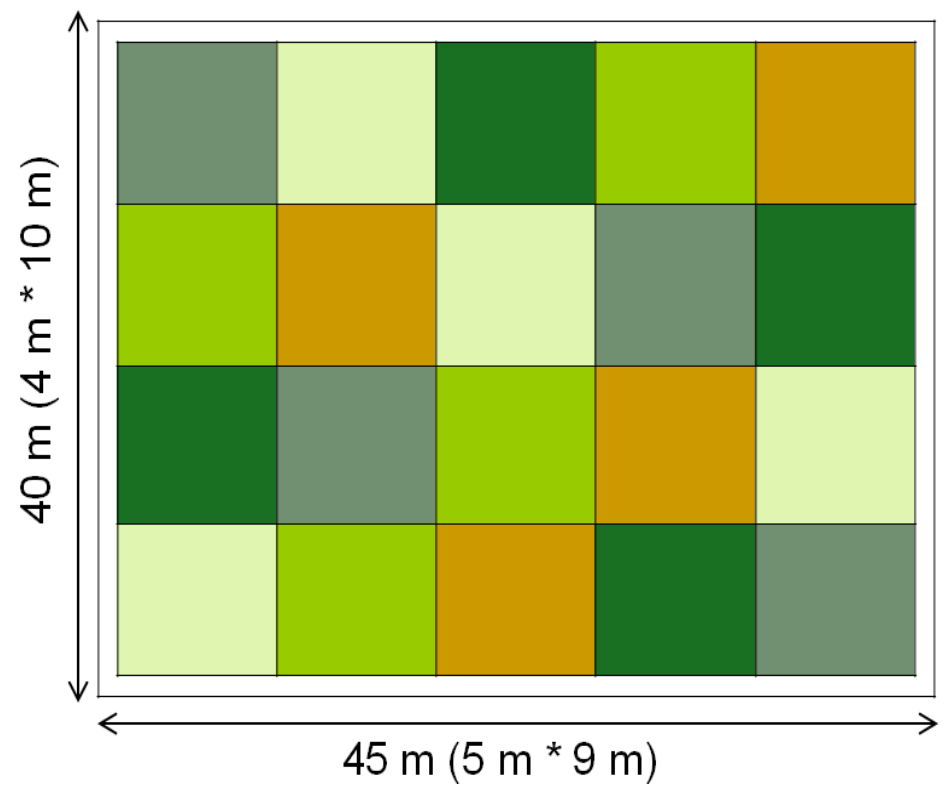

Abb. 3-2: Schematische Darstellung des Aufbaus einer Versuchsfläche, jede Farbe repräsentiert ein Prüfglied.

Pro Einzelparzelle wurden in fünf Reihen 100 Stecklinge in einem Verband von 1,8 mal 0,5 m gesteckt. Zur Vermeidung von Randeffekten wurde eine Kernparzelle definiert, in welcher die Aufnahmen des Zuwachses und der Vitalität erfolgten (Abb. 3-3). Die Kernparzelle beinhaltet die drei mittleren Baumreihen mit Ausnahme der zwei ersten und zwei letzten Pflanzplätze.

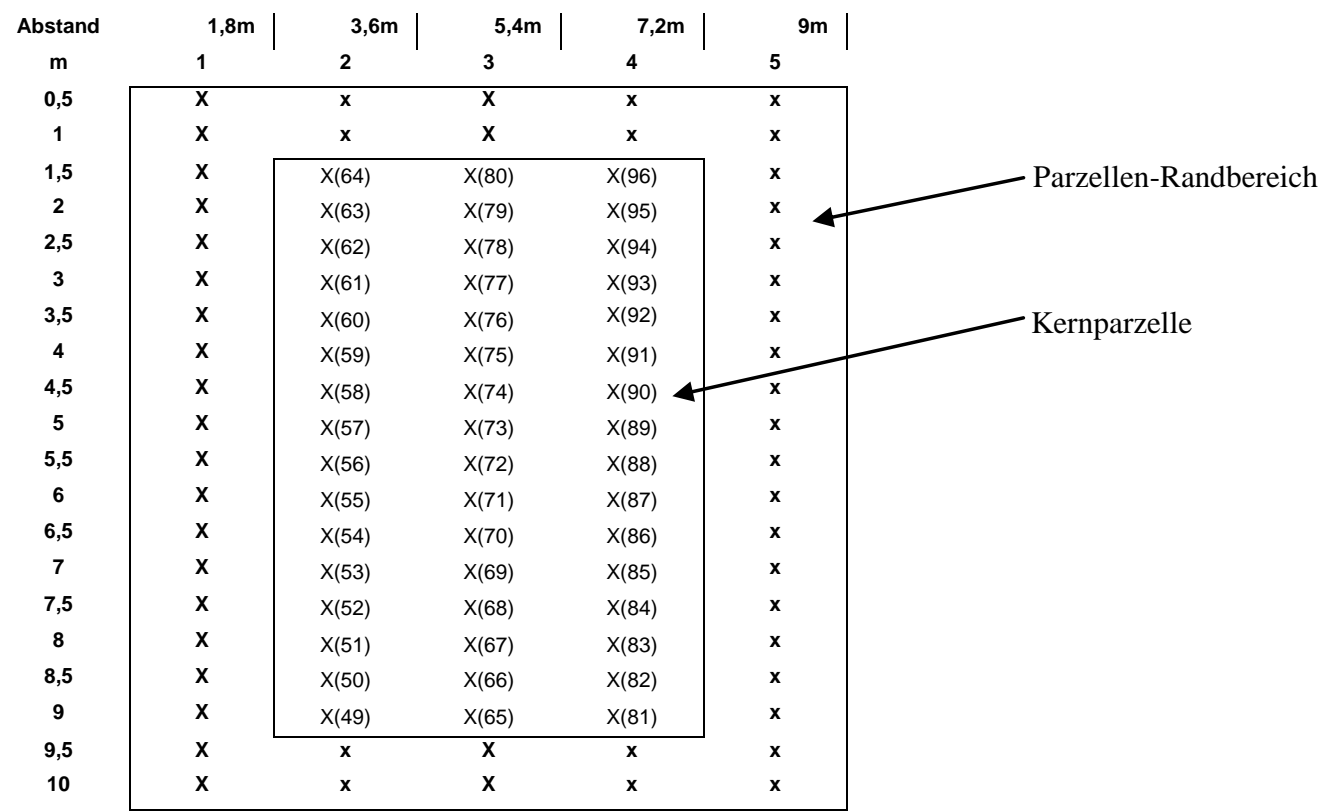

Abb. 3-3: Darstellung einer Einzelparzelle mit Kennzeichnung der Kernparzelle und des Parzellen-Randbereiches.

Der Versuch lehnt sich an das System der Mini-Rotation an (MUHS 1986) und ist als dreijährige Rotation angelegt. 


\subsubsection{Flächenvorbereitung und Pflegemaßnahmen}

Die Flächenvorbereitung erfolgte durch ortsübliche Saatbettvorbereitung, das heißt, dass die Versuchsflächen gepflügt, geeggt und anschließend mit Herbiziden behandelt wurden (Tab. 3-3).

Tab. 3-3: Übersicht zur Bodenvorbereitung und zu durchgeführten Pflegemaßnahmen bei der Flächenanlage.

\begin{tabular}{|c|c|c|c|c|c|c|c|c|}
\hline \multirow{2}{*}{$\begin{array}{l}\text { Sto. } \\
\text { Nr. }\end{array}$} & \multirow{2}{*}{$\begin{array}{l}\text { Standortname } \\
\text { Emmendingen }\end{array}$} & \multicolumn{3}{|c|}{ Bodenvorbereitung } & \multirow{2}{*}{$\begin{array}{c}\text { Vorlaufmittel } \\
-\end{array}$} & \multicolumn{2}{|c|}{$\begin{array}{c}\text { A posteriori } \\
\text { Unkrautbekämpfung }\end{array}$} & \multirow{2}{*}{$\begin{array}{c}\text { Bewässerung } \\
-\end{array}$} \\
\hline & & $\mathrm{P}$ & $\mathrm{F}$ & - & & $\mathrm{M}(2)$ & - & \\
\hline 2 & Lilienthal & $\mathrm{P}$ & $\mathrm{F}$ & - & - & M (1) & - & - \\
\hline 3 & Aulendorf & $\mathrm{D}$ & - & - & $X$ & M (1) & - & - \\
\hline 4 & Forchheim & $\mathrm{P}$ & $\mathrm{K}$ & - & - & M (5) & $\mathrm{C}(1)$ & - \\
\hline 5 & Ladenburg & $\mathrm{P}$ & $\mathrm{K}$ & - & - & M (2) & - & - \\
\hline 6 & Kupferzell & $\mathrm{P}$ & $\mathrm{K}$ & - & $X$ & M (1) & $\mathrm{C}(1)$ & - \\
\hline 7 & Kaisheim & $\mathrm{F}$ & $\mathrm{K}$ & - & - & - & - & - \\
\hline 8 & E.Wald Welzow & $\mathrm{P}$ & $\mathrm{K}$ & - & - & - & - & - \\
\hline 9 & Löwenberg & $\mathrm{F}$ & - & - & - & M (1) & - & - \\
\hline 11 & Cahnsdorf & $\mathrm{P}$ & - & - & - & M (1) & $\mathrm{C}(1)$ & - \\
\hline 12 & Kummerow & G & - & - & - & M (1) & C (1) & - \\
\hline 13 & Grünewalde & $\mathrm{P}$ & $\mathrm{F}$ & - & - & M (2) & C (3) & - \\
\hline 14 & Welzow Süd & $\mathrm{F}$ & - & - & - & - & - & - \\
\hline 15 & Potsdam Bornim & $\mathrm{P}$ & $\mathrm{K}$ & - & - & M (2) & $\mathrm{C}(1)$ & - \\
\hline 16 & Lehmkaute & $\mathrm{P}$ & $\mathrm{K}$ & - & $\mathrm{X}$ & - & $\mathrm{C}(1)$ & - \\
\hline 17 & Unterrieden & $\mathrm{D}$ & - & - & $X$ & M (1) & - & - \\
\hline 18 & Gülzow & $\mathrm{P}$ & $\mathrm{K}$ & - & - & - & $\mathrm{C}(1)$ & $X$ \\
\hline 19 & Werlte & $\mathrm{P}$ & $\mathrm{K}$ & - & - & M (4) & $\mathrm{C}(1)$ & $X$ \\
\hline 20 & Borlinghausen & $\mathrm{P}$ & $\mathrm{K}$ & - & $\mathrm{X}$ & - & - & - \\
\hline 22 & Haus Düsse & $\mathrm{P}$ & $\mathrm{K}$ & - & $X$ & M (1) & - & - \\
\hline 24 & Königshov. Höhe & $\mathrm{P}$ & G & - & $X$ & M (1) & - & - \\
\hline 25 & Klein Altendorf & $\mathrm{P}$ & $\mathrm{K}$ & - & $\mathrm{X}$ & $\mathrm{M}(2)$ & - & - \\
\hline 27 & Bärenrode & $\mathrm{F}$ & - & - & $\mathrm{X}$ & M (3) & - & $X$ \\
\hline 28 & Bernburg & $\mathrm{P}$ & $\mathrm{K}$ & - & - & M (5) & - & $X$ \\
\hline 29 & Hayn & $\mathrm{P}$ & $\mathrm{K}$ & $\mathrm{F}$ & $X$ & $M(5)$ & $\mathrm{C}(1)$ & - \\
\hline 30 & Iden & $\mathrm{P}$ & $\mathrm{K}$ & - & $X$ & M (1) & $\mathrm{C}(2)$ & $X$ \\
\hline 31 & Schlag Adler & $\mathrm{P}$ & $\mathrm{K}$ & - & $X$ & M (5) & $\mathrm{C}(1)$ & $X$ \\
\hline 32 & Thammenhain I & $\mathrm{P}$ & $\mathrm{K}$ & - & - & M (1) & - & - \\
\hline 33 & Thammenhain II & $\mathrm{P}$ & $\mathrm{K}$ & - & - & - & - & - \\
\hline 35 & Threnthorst & G & - & - & - & M (1) & - & - \\
\hline 36 & Oberdorla & $\mathrm{P}$ & $\mathrm{K}$ & - & $X$ & - & $\mathrm{C}(1)$ & $X$ \\
\hline 37 & Ü. d. Erdengraben & $\mathrm{P}$ & $\mathrm{K}$ & - & - & M (3) & $\mathrm{C}(2)$ & - \\
\hline 38 & Heiliges Marpingen & $P$ & $\mathrm{~K}$ & - & - & - & - & - \\
\hline
\end{tabular}

Mit P: Pflügen F: Fräsen $\quad$ D: Direktstecken $\quad \mathrm{K}$ : Kreiseln $\quad$ G: Grubbern

M: Mechanisch, C: Chemisch, in Klammer angegeben ist die Behandlungshäufigkeit 
Mit Ausnahme der Standorte Nr. 2 „Lilienthal“, Nr. 7 „Kaisheim“ und Nr. 27 „Bärenrode“ konnte der Unkrautdruck auf einem unkritischen Niveau gehalten werden (Abb. 3-4). Am Sto. Nr. 2 „Lilienthal“ wurde ein sehr hoher Unkrautbefall festgestellt, welcher besonders die Weidenprüfglieder betraf. Der Sto. Nr. 7 „Kaisheim“ zeigte einen generalisierten Unkrautbefall. Der Sto. Nr. 27 „Bärenrode“ wies ebenfalls eine sehr hohe Unkrautschicht auf. Bei der Flächenbegründung wurden anstatt des mit Pflug und Egge üblichen Vollumbruches Streifen gefräst. Dies führte zu einer starken Verunkrautung des Standortes.
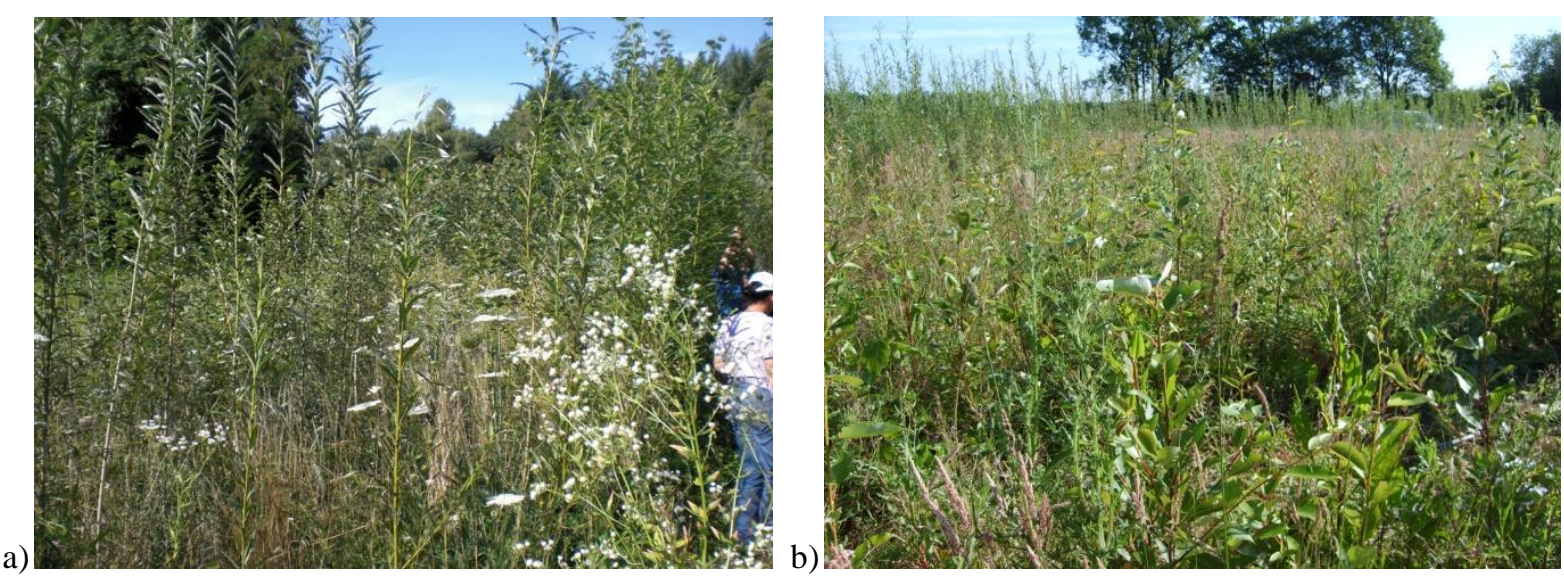

Abb. 3-4: Versuchsflächen mit hoher Verunkrautung in der zweiten Wuchsperiode, Jahr 2009 a) Standort Nr. 2 „Lilienthal“ b) Standort Nr. 7 „Kaisheim“.

Aufgrund der ausgeprägten Frühjahrstrockenheit im Anpflanzjahr 2008 mussten einige Versuchsflächen unterschiedlich oft und stark bewässert werden, um sie vor einem Komplettausfall zu bewahren. Die Bewässerungsmenge wurde genau dokumentiert, um die Auswertbarkeit der Ergebnisse zu gewährleisten.

Trotz der Pflegemaßnahmen war der Anwuchs auf vielen Flächen nicht optimal. Um lückenlose Bestände zu erhalten, die für die Homogenität der Einzelbaumdimensionen von Bedeutung ist, wurden im Frühjahr 2009 Nachbesserungen auf den Versuchsflächen durch die Projektpartner durchgeführt (Tab. 3-4). Die Standorte mit niedrigen Anwuchsraten aller Prüfglieder waren: Sto. Nr. 5, „Ladenburg“, Sto. Nr. 6 „Kupferzell“, Sto. Nr. 11 „Cahnsdorf“, Sto. Nr. 32 „Thammenhain I“ und Sto. Nr. 33 „Thammenhain II“. Auf Standorten mit Ausfällen kleiner $50 \%$ erfolgte eine pflanzplatzweise Nachbesserung, dagegen auf Standorten mit Ausfällen größer $50 \%$ eine flächige Nachbesserung. Bei einer flächigen Nachbesserung war es wichtig, dass die nachgebesserten Prüfglieder über ein einheitliches Alter verfügten. Da das Herausgraben oder Fräsen der einjährigen Pflanzplätze sich als sehr zeitund arbeitsaufwendig erwies, wurden diese zurück geschnitten. Die flächige Nachbesserung einzelner Prüfglieder einer Versuchsfläche führte dazu, dass innerhalb einer Versuchsfläche Bestände unterschiedlichen Alters vorliegen.

Tab. 3-4: Nachgebesserte Prüfglieder nach Standort und Art des Pflanzmaterials (Zahlen zeigen Standortnummern, Zahl in Klammer zeigt die Gesamtanzahl der nachgebesserten Standorte).

\begin{tabular}{|c|c|c|c|c|c|}
\hline \multirow{2}{*}{$\begin{array}{l}\text { Art der } \\
\text { Nachbesserung }\end{array}$} & \multicolumn{5}{|c|}{ Nachgebesserter Standort (Nr.) } \\
\hline & $\operatorname{Max} 1$ & Н 275 & $\mathrm{AF} 2$ & Inger & Tordis \\
\hline $\begin{array}{l}\text { Flächige } \\
\text { Nachbesserung } \\
\text { mit Stecklingen }\end{array}$ & $\begin{array}{c}11,32,33 \\
(\mathrm{n}=3)\end{array}$ & $\begin{array}{c}5,6,11,16,19 \\
32,33,36,37 \\
(\mathrm{n}=9)\end{array}$ & $\begin{array}{c}11,27,32,33 \\
(\mathrm{n}=4)\end{array}$ & $\begin{array}{c}11,32,33 \\
(\mathrm{n}=3)\end{array}$ & $\begin{array}{c}5,6,11,32,33(\mathrm{n} \\
=5)\end{array}$ \\
\hline $\begin{array}{l}\text { Punktuelle } \\
\text { Nachbesserung } \\
\text { mit Steckruten }\end{array}$ & $\begin{array}{c}2,3,4,5,6,7,8 \\
9,10,15,16,18 \\
19,27,28,29,30 \\
36 \\
(\mathrm{n}=18)\end{array}$ & $\begin{array}{c}1,2,3,4,7,8,9 \\
12,15,17,18,20 \\
22,27,28,29,30 \\
35 \\
(\mathrm{n}=18)\end{array}$ & $\begin{array}{c}1,2,4,5,6,8,9 \\
12,15,16,19,20 \\
22,29,30,36 \\
(\mathrm{n}=16)\end{array}$ & $\begin{array}{c}4,5,6,8,9,15 \\
19,22,22,27,30 \\
(\mathrm{n}=11)\end{array}$ & $\begin{array}{c}1,2,4,8,9,12 \\
15,16,19,22,27 \\
28,29,30 \\
(\mathrm{n}=14)\end{array}$ \\
\hline
\end{tabular}




\subsubsection{Prüfglieder}

Die im Verbundvorhaben PROLOC verwendeten Prüfglieder gehören zu den ertragsstärksten Weidenund Pappelklonen in Deutschland, die nach aktuellem Stand darüber hinaus die höchste Resistenz gegen Pathogene aufweisen. Zu den Pappelprüfgliedern zählen die altbewährten Klone Max 1 (P. nigrax P. maximowiczii) und $\mathrm{H} 275$ (P. maximowiczii $x$ P. trichocarpa) sowie der italienische Klon AF 2 (P. nigra $x$ P. deltoides). Mit jeweils AF 2 und H 275 sind die interspezifischen Hybriden der Sektionen Aigeiros und Tacamahaca vertreten. Die Berücksichtigung intrasektioneller Hybride erfolgte über den Klon Max 1. Zu den ausgewählten Weidenprüfgliedern zählen die schwedischen Züchtungen Inger (S. triandra $x$ S. viminalis) und Tordis ((S. viminalis $x S$. schwerinii) $x$ S. viminalis). Mit diesen Klonen waren die Sektionen Vetrix und Salix vertreten.

\subsubsection{Pappelklon Max 1}

Klon Max 1 (P. nigra x P. maximowiczii) stammt aus Japan. Er wurde zusammen mit Max 2 bis 5 im Jahr 1966 nach Deutschland vom FsB eingeführt. Es handelt sich dabei um Selektionen natürlicher Hybride, die vom Oji Institute for Forest Tree Improvement in Kuriyama ausgesucht wurden (RAU et al. 1988). Max 1 zeichnet sich durch einen sehr guten Zuwachs aus, besonders in Umtriebszeiten von bis zu fünf Jahren (FRIEDRICH 1999). Außerdem weist er eine exzellente Wiederaustriebsfähigkeit auf. Die Überlebensrate von Max 1 liegt nach mehrmaliger Ernte in der Regel über 90 \% (FRIEDRICH 1999, HOFMANN 1999, BOELCKE 2006). Er zeigt eine gute Toleranz gegenüber Pappelkrebs durch Xanthomonas (Ridé) (KECHEL 1984) sowie eine gute bis sehr gute Rosttoleranz (RAU et al. 1988, HOFMANN und LIPPELT 2008). Kritisch zu bewerten sind die nur mittelmäßigen Formeigenschaften sowie die Neigung zur Marssonina-Krankheit. Max 1, 3 und 4 wurden als Mehrklonsorte im Jahr 1981 zugelassen. Mittlerweile ist die Mehrklonsorte vergriffen, so dass Max 1, 3 und 4 nur noch als Einzelklone auf dem Markt erhälttich sind (BLE 2012). Die Darrdichte beträgt 0,45 $\mathrm{g} \mathrm{cm}^{-3}$ und der Wassergehalt bei der Ernte liegt bei durchschnittlich 54,6 \% (AMTHAUER GALLARDO und SEYMOUR 2011, Anhang 2). Abbildung 3-5 zeigt zweijährige Max 1 Bäume.

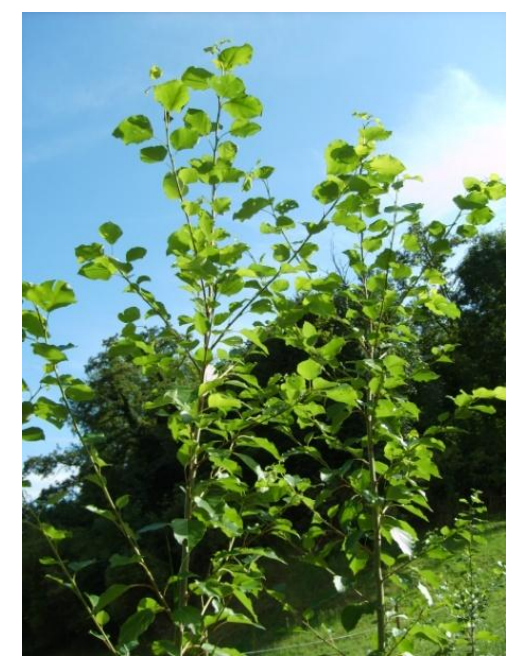

Abb. 3-5: Pappelklon Max 1.

\subsubsection{Pappelklon H 275}

Klon Hybride 275 (H 275) (P. maximowiczii x P. trichocarpa), ursprünglich als NE-42 benannt, stammt aus einer gelenkten Kreuzung von STOUT und SCHREINER (1933) aus den 1920er Jahren und ist ein männlicher Klon (Abb. 3-6). H 275 wurde im Jahr 1966 vom FsB über einen Umweg aus dem Arboretum Kórnik in Polen eingeführt (ANONYMOUS 1973, zitiert in HoFMANN 2005). Seitdem wird dieser auf Versuchs- und Wirtschaftsflächen verwendet. Positive Eigenschaften sind seine gute Rost- 
toleranz (RAU et al. 1988), hervorragende Zuwächse sowohl in kurzen als auch in langen Rotationen (RAU et al. 1988, JOACHIM et al. 1989, FRIEDRICH 1999, HOFMANN 1999) und eine gute Resistenz gegenüber Pappelkrebs und Rindenbrand (BÖDEN und KECHEL 1984, KECHEL 1984). H 275 gilt als geradwüchsig. Er bildet eine mittelbreite Krone (JoACHIM et al. 1989). Die Dauer der Wachstumsperiode ist im Vergleich zu anderen Balsampappelhybriden länger (TGL 37868 1987). Dieser Klon ist zwar nicht in Deutschland, dafür aber in der Tschechischen Republik zugelassen (UHLMANN 2013). Das Holz vom H 275 ist im Verhältnis zu Klonen anderer Sektionen schwer. Die Darrdichte beträgt $0,51 \mathrm{~g} \mathrm{~cm}^{-3}$. Der Wassergehalt bei der Ernte liegt bei durchschnittlich 50,8 \% (siehe Anhang 2).

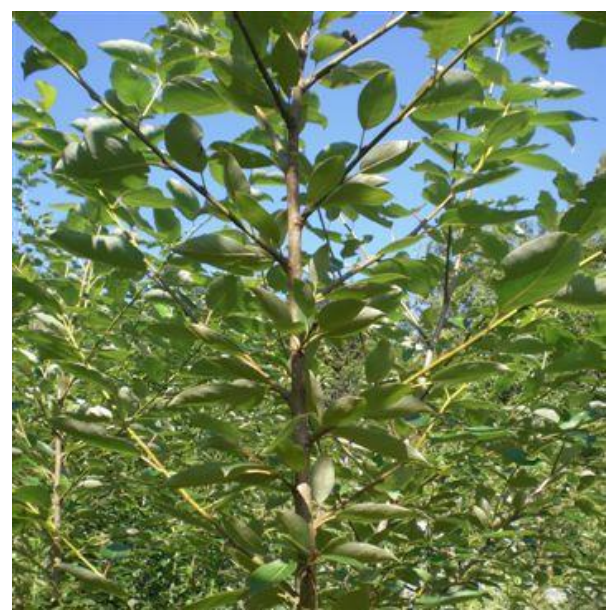

Abb. 3-6: Pappelklon H 275.

\subsubsection{Pappelklon AF 2}

Der männliche Klon AF 2 ( $P$. deltoides $x$ P. nigra) entstammt einer gelenkten Kreuzung von Alasia New Clones S. r. 1. (Italien) aus dem Jahre 1994 (Abb. 3-7). Die Mutter ist P. deltoides 145-86 aus Illinois (USA). Der Vater ist ein P. nigra Klon aus Piemont (Italien). Nach Angaben der Züchterinstitution ist der Klon gegenüber vielen Krankheiten hochtolerant. Hierzu zählen die Triebspitzenkrankheit, der Blattrost, die Marssonina-Krankheit sowie der Rindenbrand und der Pappel-Mosaikvirus. AF 2 ist geradschaftig und bildet eine für Schwarzpappeln lockere charakteristische Krone aus. Die hohe Lichtdurchlässigkeit fördert eine erhöhte Krautschichtausbildung. In wärmebegünstigten Gebieten werden sehr hohe Erträge erzielt, welche bis zu 22,0 $t_{(\text {atro) }}$ ha $^{-1} \mathrm{a}^{-1}$ erreichen können (MAYER 2009). In Deutschland wird AF 2 erst seit wenigen Jahren auf Versuchsflächen und im Praxisanbau verwendet. Nachteilig sind seine sehr geringe Darrdichte von $0,37 \mathrm{~g} \mathrm{~cm}^{-3}$ und ein sehr hoher Wassergehalt bei der Ernte von 56,7 \% (siehe Anhang 2).

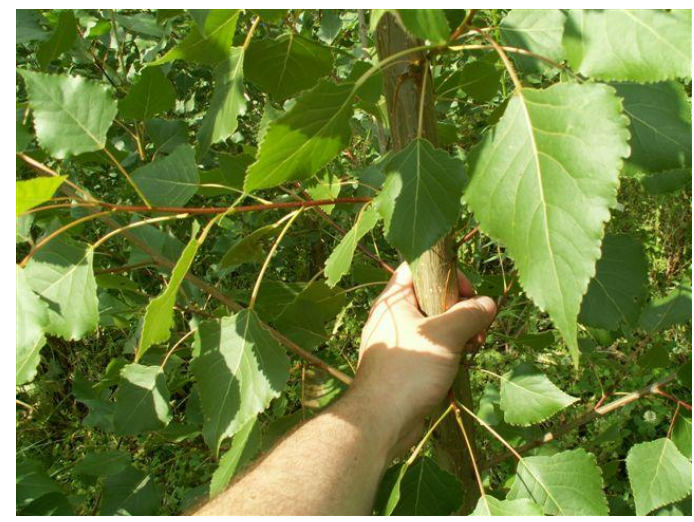

Abb. 3-7: Pappelklon AF 2. 


\subsubsection{Weidenklon Inger}

Der Klon Inger (S. triandra $x$ S. viminalis) stammt aus einer Kreuzung des Klons Jorr mit einem russischen Klon der Korbweide (Abb. 3-8). Obwohl Rost- und Frosttoleranz nur als mäßig bis gut eingestuft werden, zeigt dieser Klon sehr große Zuwächse (NEUMEISTER 2009) und eine gute Eignung für den Anbau auf trockenen Standorten. Er besitzt eine besondere Empfindlichkeit gegenüber dem Befall durch Blattwespenlarven (Nematus pavidus) (PERNY und STEYRER 2009). Bei Mischanbau von Inger, Tora und Tordis zeigten die Blattwespenlarven eine Präferenz für Inger. Während die anderen Klone unversehrt blieben, wurde die gesamte Inger-Fläche kahlgefressen (PERNY und STEYRER 2009). Andere Untersuchungen zur Toleranz von Inger gegenüber weiteren biotischen Schadfaktoren sind dem Autor nicht bekannt. Die Holzdarrdichte beträgt $0,51 \mathrm{~g} \mathrm{~cm}^{-3}$. Der Wassergehalt des Ernteguts liegt mit $51,4 \%$ in der Regel bis zu 5,0 \% niedriger als bei Pappelholz (siehe Anhang 2).

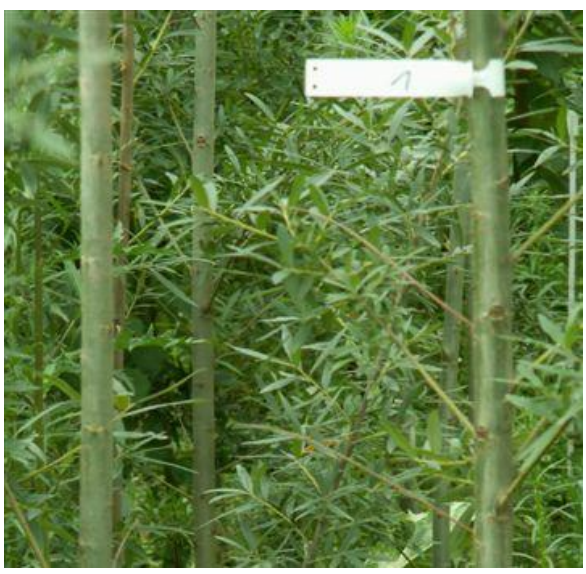

Abb. 3-8: Weidenklon Inger.

\subsubsection{Weidenklon Tordis}

Wie Inger gehört Tordis ((S. viminalis $x$ S. schwerinii) $x$ S. viminalis) zu den im Rahmen des schwedischen Weidenzüchtungsprogramms entwickelten Klonen. Tordis ist eine Kreuzung der Klone Tora und Ulv. Wie der Klon Tora zeichnet sich Tordis durch eine geringere Anzahl an Höhentrieben bei gleichzeitigem stärkeren Dickenwachstum aus (Abb. 3-9). Reine Klone der S. schwerinii werden von JOACHIM et al. (1989) als stark wachsend, dicht belaubt und sehr gut geeignet für feuchte Standorte beschrieben. Darüber hinaus besitzt Tordis zusätzlich eine gute Toleranz gegen Trockenheit. Nach Angaben von Lantmännen Agroenergi ist Tordis absolut resistent gegen Blattrost. Bei diesem Klon werden sehr hohe Erträge verzeichnet (NEUMEISTER 2009). Die Holzdichte beziffert sich auf $0,50 \mathrm{~g} \mathrm{~cm}^{-3}$ und der Wassergehalt des Ernteguts auf 52,5\% (siehe Anhang 2).

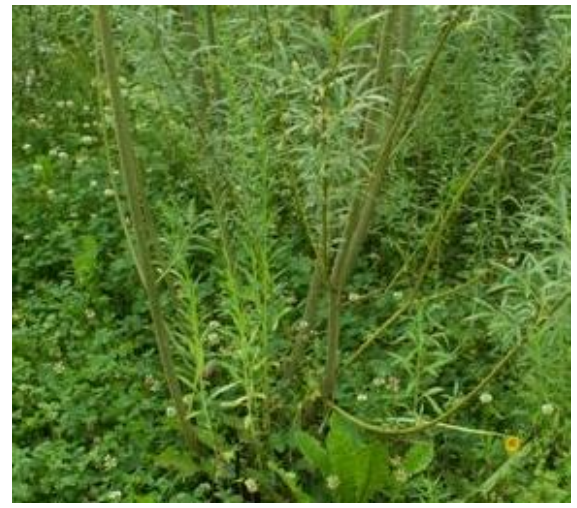

Abb. 3-9: Weidenklon Tordis. 


\subsection{Klimatische Standortcharakterisierung}

Von primärer Bedeutung für eine Modellentwicklung ist die Aufnahme des Klimas in den Untersuchungsgebieten. Durch die weite räumliche Verteilung der Standorte ist eine Erfassung der wichtigsten Klimabereiche Deutschlands gegeben (Abb. 3-1). Berücksichtigt wurden maritim und kontinental beeinflusste Bereiche sowie Mittelgebirgslagen und Niederungen. Die Daten wurden von Stationen des deutschen Wetterdienstes bezogen. Die maximale Entfernung der Klimastationen zum Standort beträgt ca. $29 \mathrm{~km}$, wobei die meisten Stationen sich in einem Umkreis von $10 \mathrm{~km} \mathrm{zu}$ den Versuchsflächen befinden (Tab. 3-5). Die aufgenommenen Daten umfassten Tagesmittelwerte der Temperatur und Tagessummen des Niederschlages ${ }^{8}$. Aus den einzelnen Tagesdaten wurden Monatssummen bzw. -mittelwerte gebildet. Des Weiteren wurden Trockenheitsindizes nach DE MARTONNE (1926), um die Wechselwirkung zwischen Temperatur und Niederschlag abzubilden, nach Formel 3-1 bestimmt:

Trockenheitsindex

$$
T I=\frac{N}{(T+10)}
$$

$$
\begin{array}{ll}
T I: & \text { Trockenheitsindex } \\
N: & \text { Niederschlagssumme des ausgewählten Zeitfensters }[\mathrm{mm}] \\
T: & \text { Temperaturmittelwert des ausgewählten Zeitfensters }\left[{ }^{\circ} \mathrm{C}\right]
\end{array}
$$

Tabelle 3-5 zeigt einen Überblick der langjährigen Klimaverhältnisse der verschiedenen Standorte, differenziert für das Jahr und die Vegetationsperiode ${ }^{9}$. Das langjährige Klima bezieht sich in dieser Arbeit auf das Zeitfenster von 1990 bis 2010. Die langjährigen Werte der mittleren Jahrestemperaturen schwankten zwischen 7,6 (Sto. Nr. 3 „Aulendorf“, Sto. Nr. 27 „Bärenrode“ und Sto. Nr. 29 „Hayn“) und 11,6 ${ }^{\circ} \mathrm{C}$ (Sto. Nr. 2 „Lilienthal“) (Tab. 3-5). Der minimale Temperaturdurchschnitt betrug während der forstlichen Vegetationsperiode $12,7^{\circ} \mathrm{C}$, (Sto. Nr. 3 „Aulendorf") und der maximale $18,4{ }^{\circ} \mathrm{C}$ (Sto. Nr. 2 „Lilienthal“). Die Jahresniederschlagssummen bewegen sich zwischen 504 (Sto. Nr. 36 „Oberdorla“) und 923 mm (Sto. Nr. 38 „Heiliges Marpingen“). Die Niederschlagssummen schwankten während der Vegetationsperiode zwischen 255 (Sto. Nr. 36 „Oberdorla“) und 456 mm (Sto. Nr. 2 „Lilienthal").

\footnotetext{
${ }^{8}$ Freilandniederschlag.

${ }^{9}$ In dieser Arbeit wird die Vegetationsperiode als die forstliche Vegetationsperiode definiert. Diese beinhaltet den Zeitraum von Mai bis September.
} 
Tab. 3-5: Durchschnittliche langjährige Werte (1990 - 2010) der Niederschlagssummen, der Temperatur- und der gemittelten Trockenheitsindizes für die Bezugszeiträume Jahr und Vegetationsperiode.

\begin{tabular}{|c|c|c|c|c|c|c|c|c|c|}
\hline \multirow{2}{*}{$\begin{array}{l}\text { Sto. } \\
\text { Nr. }\end{array}$} & \multirow{2}{*}{ Standortname } & \multirow{2}{*}{ Klimastation $^{\text {a }}$} & \multirow{2}{*}{$\begin{array}{c}\text { Entf. }^{b} \\
{[\mathrm{~km}]}\end{array}$} & \multicolumn{3}{|c|}{ Jahr } & \multicolumn{3}{|c|}{ Vegetationsperiode } \\
\hline & & & & $\mathrm{T}\left[{ }^{\circ} \mathrm{C}\right]$ & $\mathrm{N}[\mathrm{mm}]$ & TI & $\mathrm{T}\left[{ }^{\circ} \mathrm{C}\right]$ & $\mathrm{N}[\mathrm{mm}]$ & $\mathrm{TI}$ \\
\hline 1 & Emmendingen & Emmendingen Mün. & 3 & 10,5 & 826 & 3,6 & 17,0 & 440 & 3,3 \\
\hline 2 & Lilienthal & Freiburg & 13 & 11,6 & 864 & 3,6 & 18,4 & 456 & 3,3 \\
\hline 3 & Aulendorf & Pfullendorf & 26 & 7,6 & 841 & 4,4 & 12,7 & 449 & 4,0 \\
\hline 4 & Forchheim & Rheinstetten & 2 & 11,2 & 734 & 3,3 & 18,2 & 346 & 2,5 \\
\hline 5 & Ladenburg & Heidelberg & 5 & 11,5 & 693 & 3,0 & 18,2 & 339 & 2,4 \\
\hline 6 & Kupferzell & Öhringen & 13 & 10,1 & 764 & 3,7 & 16,8 & 354 & 2,7 \\
\hline 7 & Kaisheim & Osterweiler & 6 & 8,7 & 735 & 3,6 & 15,8 & 421 & 3,3 \\
\hline 8 & E.Wald Welzow & Cottbus & 18 & 9,8 & 553 & 2,6 & 17,0 & 297 & 2,2 \\
\hline 9 & Löwenberg & Zehdenik & 16 & 9,2 & 537 & 2,7 & 16,3 & 273 & 2,1 \\
\hline 11 & Cahnsdorf & Lübben Blumenfelde & 12 & 9,5 & 543 & 2,7 & 16,7 & 288 & 2,2 \\
\hline 12 & Kummerow & Angermünde & 19 & 9,1 & 518 & 2,5 & 16,1 & 294 & 2,3 \\
\hline 13 & Grünewalde & Klettwitz & 12 & 9,3 & 605 & 3,0 & 16,4 & 322 & 2,5 \\
\hline 14 & Welzow Süd & Cottbus & 18 & 9,8 & 553 & 2,6 & 17,0 & 297 & 2,2 \\
\hline 15 & Potsdam-Bornim & Potsdam & 7 & 9,5 & 564 & 2,8 & 16,6 & 300 & 2,3 \\
\hline 16 & Lehmkaute & Coelbe & 13 & 9,8 & 620 & 3,0 & 16,5 & 308 & 2,3 \\
\hline 17 & Unterrieden & Göttingen & 18 & 9,3 & 616 & 3,0 & 15,6 & 308 & 2,4 \\
\hline 18 & Gülzow & Teterow & 32 & 8,9 & 524 & 2,6 & 15,5 & 273 & 2,2 \\
\hline 19 & Werlte & Löningen & 12 & 9,6 & 684 & 3,2 & 15,8 & 339 & 2,7 \\
\hline 20 & Borlinghausen & Warburg & 12 & 9,1 & 635 & 3,1 & 15,6 & 317 & 2,5 \\
\hline 22 & Haus Düsse & Börkenförde & 15 & 10,2 & 789 & 3,6 & 16,3 & 375 & 2,9 \\
\hline 24 & Königshov. Höhe & Heinsberg-Schleiden & 29 & 10,4 & 672 & 3,0 & 16,1 & 329 & 2,5 \\
\hline 25 & Klein Altendorf & Ahrweiler & 11 & 10,4 & 618 & 2,8 & 16,6 & 305 & 2,3 \\
\hline 27 & Bärenrode & Stiege & 7 & 7,6 & 570 & 3,2 & 14,1 & 287 & 2,4 \\
\hline 28 & Bernburg & Bernburg & 3 & 9,8 & 514 & 2,4 & 16,8 & 286 & 2,2 \\
\hline 29 & Hayn & Harzgerode & 8 & 7,6 & 570 & 3,2 & 14,1 & 287 & 2,4 \\
\hline 30 & Iden & Seehausen & 17 & 9,4 & 554 & 2,7 & 16,2 & 282 & 2,2 \\
\hline 31 & Schlag Adler & Kubschütz & 5 & 9,4 & 621 & 3,1 & 16,4 & 337 & 2,6 \\
\hline 32 & Thammenhain I & Klitzschen & 10 & 9,5 & 561 & 2,7 & 16,5 & 297 & 2,3 \\
\hline 33 & Thammenhain II & Klitzschen & 10 & 9,5 & 561 & 2,7 & 16,5 & 297 & 2,3 \\
\hline 35 & Threnthorst & Lübeck-Blankensee & 12 & 9,0 & 676 & 3,4 & 15,4 & 327 & 2,6 \\
\hline 36 & Oberdorla & Mühlhausen-Görmar & 8 & 9,0 & 504 & 2,6 & 16,1 & 255 & 2,0 \\
\hline 37 & Ü. d. Erdengraben & Jena (Sternwarte) & 9 & 10,0 & 592 & 2,7 & 16,8 & 321 & 2,4 \\
\hline 38 & Heiliges Marpingen & Tholey & 3 & 9,4 & 923 & 5,0 & 15,9 & 367 & 2,9 \\
\hline
\end{tabular}

${ }^{a}$ Für einige Standorte mussten Daten aus einer anderen Klimastation herangezogen werden, um eine möglichst lückenfreie Datenlage zu erzeugen. Eine Liste mit diesen Stationen befindet sich im Anhang 3.

${ }^{\mathrm{b}}$ Entf.: Entfernung von der Klimastation zum Versuchsstandort.

Wie bereits in Kapitel 3.1 ausgeführt, wurden aufgrund des Niederschlagsmangels einige Versuchsflächen während des Anpflanzjahres bewässert. Tabelle 3-6 kann die Bewässerungsmenge pro Monat 
und Versuchsfläche entnommen werden. Für die Variablenbildung im Rahmen der Modellierung wurden Bewässerungsmenge und die jeweilige Monatsniederschlagssumme aufaddiert, um den Bewässerungseffekt in der Gesamtauswertung zu berïcksichtigen.

Tab. 3-6: Applizierte Bewässerungsmenge im Anpflanzjahr [mm].

\begin{tabular}{llccc}
\hline \multirow{2}{*}{$\begin{array}{l}\text { Sto. } \\
\text { Nr. }\end{array}$} & \multirow{2}{*}{ Standortname } & \multicolumn{3}{c}{ Bewässerungsmenge [mm] } \\
\cline { 3 - 5 } & & April & Mai & Juni \\
\hline 18 & Gülzow & 11,0 & - & 11,0 \\
19 & Werlte & - & 20,0 & 10,0 \\
27 & Bärenrode & - & 2,2 & - \\
28 & Bernburg & - & 0,9 & - \\
30 & Iden & - & 2,2 & - \\
31 & Schlag Adler & - & 5,0 & - \\
36 & Oberdorla & 5,0 & - & - \\
\hline
\end{tabular}

Im Folgenden wird die Witterung im Untersuchungszeitraum (2008 - 2010) betrachtet. Der Schwerpunkt liegt dabei auf der Variation gegenüber dem langjährigen Klimamittel.

\subsubsection{Temperatur}

Bei den Jahrestemperaturen wurde bei Betrachtung aller Standorte eine unerwartete jährliche Variation festgestellt (Abb. 3-10). Die Jahresmitteltemperatur 2010 aller Versuchsflächen lag ca. $15 \%$ unter dem langjährigen Mittel. Im Gegensatz dazu waren die Jahresmitteltemperaturen 2009 annähernd deckungsgleich mit dem langjährigen Mittel. Die Werte von 2008 zeigten eine leichte Überlegenheit in Höhe von ca. 5 \% gegenüber dem langjährigen Mittel. Die Variation der Temperaturen der Vegetationsperiode der einzelnen Jahre 2008 bis 2010 ist im Vergleich zu den Jahreswerten geringer.
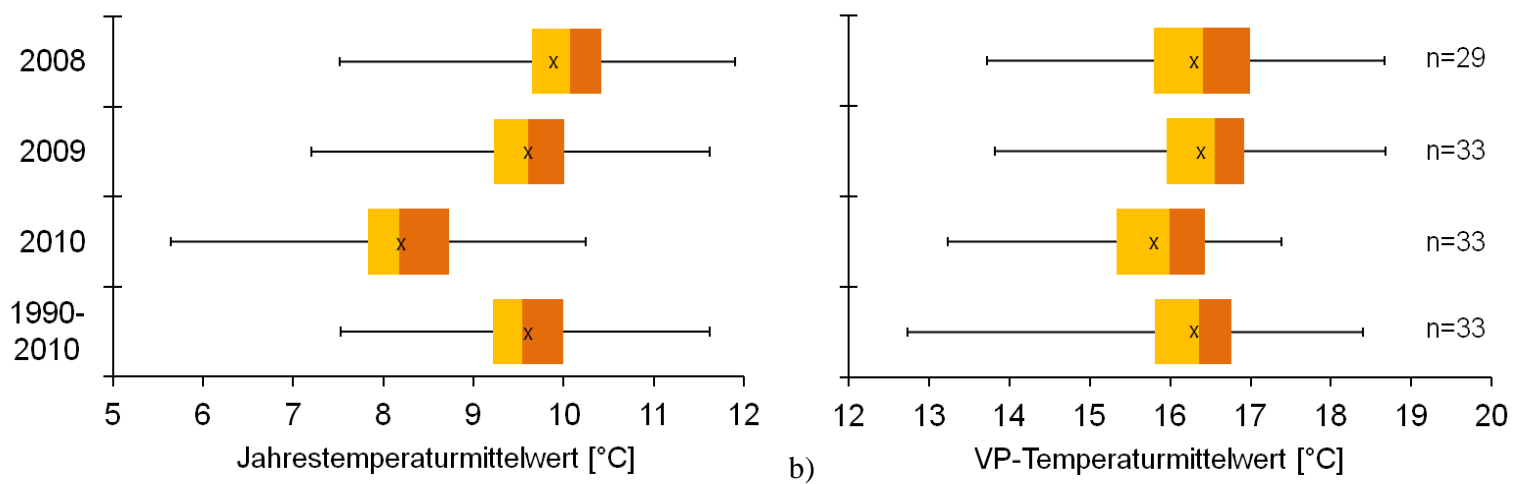

Abb. 3-10: Box-Whiskers-Plots der mittleren Temperatur 2008, 2009, 2010 sowie für den Zeitraum von 1990 bis 2010 über alle Standorte für die Bezugszeitfenster a) Jahr und b) VP.

Für die Modellanwendung zur Ertragsprognose mittels empirischer Modelle werden langjährige Klimawerte als Input verwendet. Daher ist eine Betrachtung der Abweichung der Mittelwerte und Summen im Untersuchungszeitraum in Bezug zum langjährigen Mittel von großer Bedeutung. Zur Veranschaulichung wurde eine deutschlandweite, räumlich repräsentative Auswahl an Standorten bzw. Klimastationen getroffen (Abb. 3-11). 


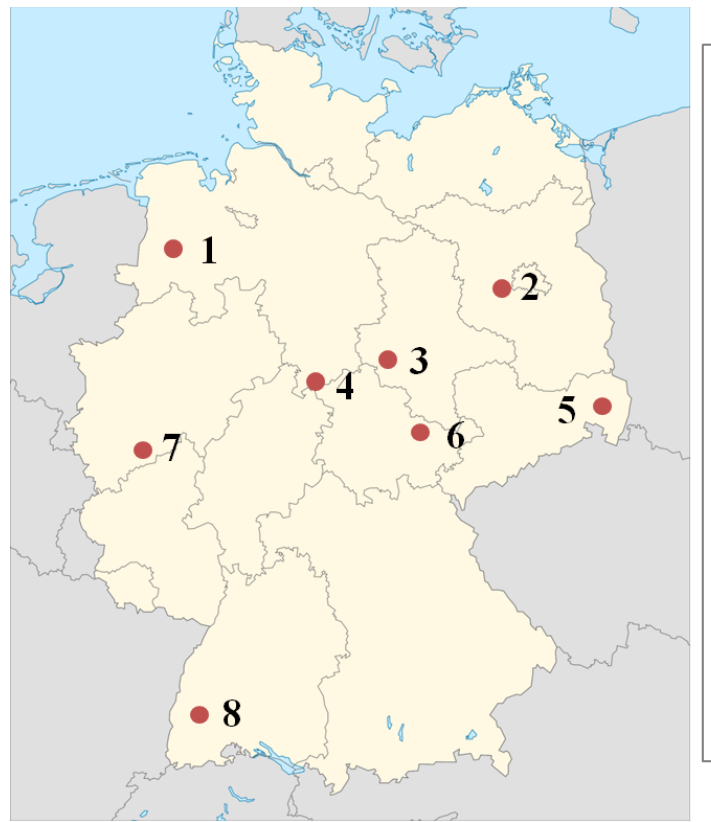

1 Sto. Nr. 19 ,Werlte

Kl-St. Löningen

2 Sto. Nr. 15 „Potsdam Bornim“

Kl-St. Potsdam

3 Sto. Nr. 29 „Hayn“

Kl-St. Harzgerode

4 Sto. Nr. 17 ,Unterrieden“ Kl-St. Göttingen

5 Sto. Nr. 31 ,Schlag Adler Kl-St. Kubschütz

6 Sto. Nr. 37 ,ü. d. Erdengraben

Kl-St. Jena Sternwarte

7 Sto. Nr. 25 ,Klein Altendorf“ Kl-St. Neuenahr, Bad Ahrweiler

8 Sto. Nr. 1,Emmendingen

Kl-St. Emmendingen-Mündingen

Abb. 3-11: Lage der Vergleichsstandorte mit relevanten Klimastationen (Kl-St.) zur Betrachtung des Klimas der ersten Rotation.

Bei Betrachtung aller Beobachtungsjahre war die Variation der Jahreswerte und Werte der Vegetationsperioden gegenüber den langjährigen Werten sehr gering (Tab. 3-7). Im Untersuchungszeitraum waren die Mittelwerte der Jahrestemperaturen stets den langjährigen Werten unterlegen. Bei den Mittelwerten der Vegetationsperiode hingegen wurden sowohl positive als auch negative Abweichungen von den langjährigen Temperaturmittelwerten festgestellt. Besonders warm waren die Monate April, Juli und November. Die restlichen Monate zeigten niedrigere Temperaturen als im langjährigen Mittel.

Tab. 3-7: Mittlere Monats-, Vegetationsperiode- und Jahrestemperaturen im Untersuchungszeitraum 2008 - 2010 von acht ausgewählten Standorten. Relative Vergleiche zum langjährigen Mittel wurden mittels Farbgebung gekennzeichnet.

\begin{tabular}{|c|c|c|c|c|c|c|c|c|c|}
\hline \multirow[b]{2}{*}{ Zeitraum } & \multicolumn{8}{|c|}{ Temperaturmittelwert $\left[{ }^{\circ} \mathrm{C}\right]$} & \multirow[b]{2}{*}{$\begin{array}{l}\text { Abweichung vom } \\
\text { langjährigen } \\
\text { Mittelwert }\end{array}$} \\
\hline & $\begin{array}{l}\text { Sto. Nr. 1 } \\
\text { "Emmen- } \\
\text { dingen" }\end{array}$ & $\begin{array}{l}\text { Sto. Nr. } 15 \\
\text { "Potsdam } \\
\text { Bornim" }\end{array}$ & $\begin{array}{l}\text { Sto. Nr. } 17 \\
\text { "Unterrie- } \\
\text { den" }\end{array}$ & $\begin{array}{l}\text { Sto. Nr. } 19 \\
\text { "Werlte" }\end{array}$ & $\begin{array}{l}\text { Sto. Nr. } 25 \\
\text { "Klein- } \\
\text { Altendorf" }\end{array}$ & $\begin{array}{l}\text { Sto. Nr. } 29 \\
\text { "Hayn" }\end{array}$ & $\begin{array}{l}\text { Sto. Nr. } 31 \\
\text { "Schlag } \\
\text { Adler" }\end{array}$ & $\begin{array}{l}\text { Sto. Nr. } 37 \\
\text { "ü. d. Erd- } \\
\text { engraben" }\end{array}$ & \\
\hline Januar & 0,6 & $-1,2$ & $-0,5$ & 1,1 & $-1,0$ & $-2,2$ & $-1,4$ & $-0,2$ & $+[>30 \%]$ \\
\hline Februar & 2,8 & 1,5 & 1,7 & 2,3 & 3,2 & $-0,3$ & 1,6 & 2,2 & $+[20-30 \%]$ \\
\hline März & 6,0 & 4,8 & 4,8 & 5,3 & 6,0 & 2,7 & 4,5 & 5,5 & $+[10-20 \%]$ \\
\hline April & 10,6 & 10,5 & 9,4 & 10,1 & 10,4 & 7,6 & 9,9 & 10,4 & $+[5-10 \%]$ \\
\hline Mai & 15,1 & 13,7 & 12,6 & 13,1 & 11,4 & 10,9 & 13,1 & 13,9 & $+[0-5 \%]$ \\
\hline Juni & 18,0 & 17,2 & 15,7 & 16,0 & 16,9 & 14,0 & 16,5 & 17,3 & $-[0-5 \%]$ \\
\hline Juli & 19,9 & 20,3 & 18,6 & 18,7 & 19,4 & 17,1 & 19,6 & 20,2 & $-[5-10 \%]$ \\
\hline August & 18,8 & 18,8 & 17,7 & 17,3 & 18,2 & 16,1 & 18,8 & 18,7 & - [10 - $20 \%]$ \\
\hline September & 14,1 & 13,9 & 13,3 & 13,3 & 13,7 & 11,6 & 13,8 & 13,8 & $-[20-30 \%]$ \\
\hline Oktober & 9,7 & 8,3 & 8,7 & 9,4 & 9,7 & 6,9 & 8,4 & 9,1 & - [>30\%] \\
\hline November & 7,0 & 5,8 & 6,4 & 6,8 & 7,6 & 4,3 & 6,1 & 6,6 & \\
\hline Dezember & 1,6 & $-1,2$ & $-0,6$ & 0,0 & 0,8 & $-2,6$ & $-0,9$ & $-0,4$ & \\
\hline Jahr & 10,3 & 9,4 & 9,0 & 9,5 & 9,7 & 7,2 & 9,2 & 9,7 & \\
\hline VP & 17,2 & 16,8 & 15,6 & 15,7 & 15,9 & 14,0 & 16,4 & 16,8 & \\
\hline
\end{tabular}

Beispiel: Die mittlere Temperatur am Sto. Nr. 1 „Emmendingen“ war im November mit 7,0 ${ }^{\circ} \mathrm{C}$ um 20 bis $30 \%$ wärmer als das langjährige Mittel. 
Spätfrostereignisse traten im Untersuchungszeitraum nur selten auf ${ }^{10}$. Einzig die beiden Standorte im Harz (Nr. 27 „Bärenrode und Nr. 29 „Hayn“) wiesen häufiger Spätfröste auf, vor allem im Jahr 2008. Des Weiteren wurde am 02. Mai 2010 an zehn Standorten im Nordosten und in der Mitte Deutschlands ein Spätfrostereignis verzeichnet, mit einer Temperatur von etwa $-3,0{ }^{\circ} \mathrm{C}$ jedoch keine nennenswerten Schäden an den Beständen verursachte.

\subsubsection{Niederschlag}

Die Niederschläge im Untersuchungszeitraum zeigten für alle Standorte eine hohe Variation. Tendenziell wurden im Jahr 2008 und 2009 geringere Niederschlagssummen als im langjährigen Mittel gemessen, sowohl in der Vegetationsperiode als auch im Jahreswert. Die Abweichung der Niederschläge in den Jahren 2008 und 2009 gegenüber dem langjährigen Mittelwert betrug ca. $10 \%$. Überdurchschnittliche Niederschlagssummen zeigte das Jahr 2010. Die Abweichung der Jahresniederschlagssummen 2010 zu den langjährigen Summen lag um 20 \% höher. Bei den Niederschlagssummen in der Vegetationsperiode belief sich die Variation zum langjährigen Mittel auf ca. $40 \%$. Abbildung 3-12 zeigt die Verteilungen der Niederschlagssummen über alle Standorte hinweg sowohl für die Vegetationsperiode als auch für das jeweilige Jahr. Als Vergleich dienen die langjährigen Mittelwerte (1990 2010).
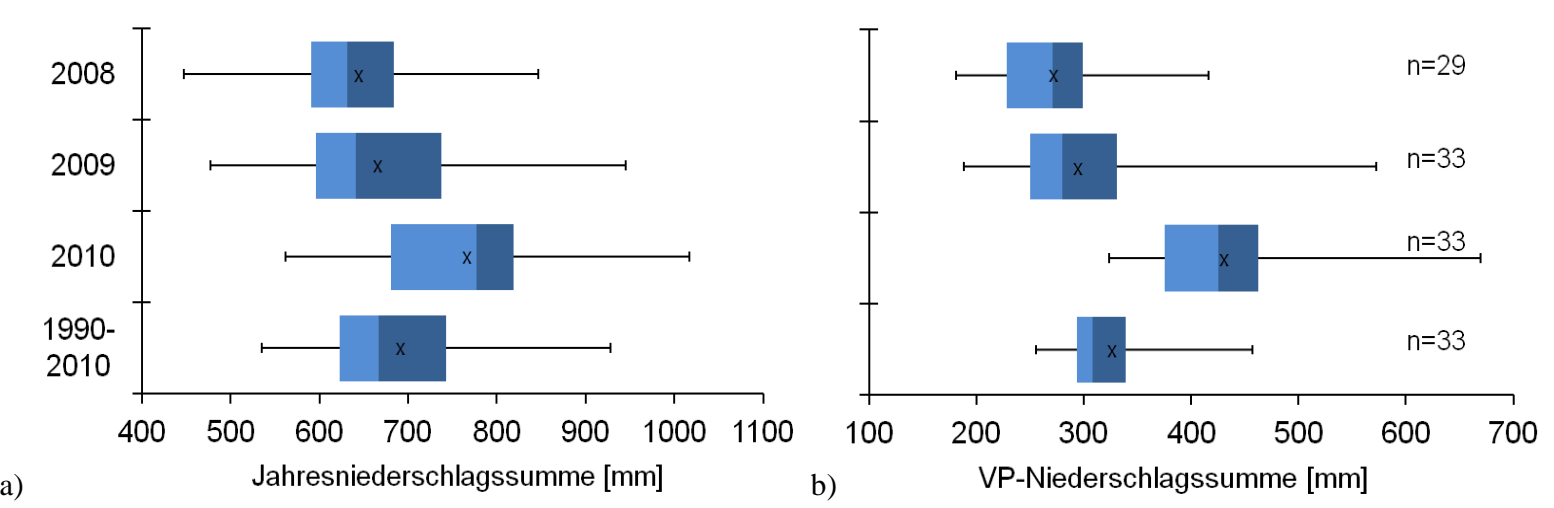

Abb. 3-12: Box-Whiskers-Plots der Niederschlagssummen 2008, 2009, 2010 sowie für den Zeitraum von 1990 bis 2010 über alle Standorte für die Bezugszeitfenster a) Jahr und b) VP.

Räumlich betrachtet wurden die langjährigen Klimaverhältnisse bestätigt. Die Verteilung des Niederschlags war jedoch in jedem Jahr unterschiedlich. Im Anlagejahr 2008 war der Monat April überdurchschnittlich regenreich (ca. $30 \%$ Abweichung zum langjährigen Wert). An den meisten Standorten wies der Monat Mai extrem geringe Niederschläge auf, mit 40 bis $80 \%$ weniger Niederschlag als im langjährigen Mittel. Die Folgemonate Juni und Juli waren bei der Mehrzahl der Standorte ebenfalls regenarm, jedoch in geringerem Maße als im Mai. Besonders trocken war der Frühling im nördlichen Baden-Württemberg, im nördlichen Brandenburg sowie in Mecklenburg Vorpommern. Der einzige Bereich Deutschlands, welcher von der Frühjahrstrockenheit nicht betroffen war, war das Gebiet im südwestlichen Nordrhein-Westfalen.

Die Niederschlagsverteilung 2009 war günstiger als 2008. Besonders die Monate Mai, Juni und Juli waren überdurchschnittlich regenreich (bis zu $30 \%$ mehr Niederschlag als im langjährigen Mittel). August und September waren hingegen sehr regenarm (bis zu $80 \%$ Abweichung zum langjährigen Wert). Dieser Trend bestätigte sich an allen Standorten, mit Ausnahme der Standorte in Sachsen, Südbrandenburg, Hessen und Thüringen. Extrem hohe Niederschläge wurden im August 2010 auf fast allen Versuchsflächen gemessen. Hier war die Abweichung zum langjährigen Mittel weit über $100 \%$ und im extremsten Fall bis zu $180 \%$. Der Monat Mai 2010 wies ebenfalls überdurchschnittliche Niederschläge auf. Im April und Juni hingegen wurden kaum Niederschläge gemessen. Zusammenfassend

\footnotetext{
${ }^{10} \mathrm{Ab}$ dem 01. Mai auftretende Fröste.
} 
war 2010 ein sehr regenreiches Jahr, sowohl in der Jahressumme als auch in der Vegetationsperiode. Insgesamt kann die jährliche Abweichung der Niederschläge als hoch angesehen werden.

Tabelle 3-8 zeigt die mittleren Monats-, Vegetationsperioden- und Jahresniederschlagssummen im Untersuchungszeitraum sowie den relativen Vergleich zu den langjährigen Werten der ausgewählten Standorte. Die mittleren Jahresniederschlagssummen sowie die Niederschlagssummen in der Vegetationsperiode des Untersuchungszeitraumes waren, mit Ausnahme der Jahressumme am Sto. Nr. 25 „Klein Altendorf“, immer höher als die langjährigen Werte. Die Abweichung betrug maximal $32 \%$ in der Vegetationsperiode und $28 \%$ im Jahreswert (beide gemessen am Sto. Nr. 31 „Schlag Adler“). Im Osten Deutschlands, anhand des Sto. Nr. 15 „Potsdam Bornim“ ersichtlich, war in der Vegetationsperiode eine schlechte Wasserversorgung zu verzeichnen.

Tab. 3-8: Mittlere Monats-, Vegetationsperiode- und Jahresniederschlagssummen im Untersuchungszeitraum 2008 - 2010 von acht ausgewählten Standorten. Relative Vergleiche zum langjährigen Mittel wurden mittels Farbgebung gekennzeichnet.

\begin{tabular}{|c|c|c|c|c|c|c|c|c|c|}
\hline \multirow[b]{2}{*}{ Zeitraum } & \multicolumn{8}{|c|}{ Niederschlagssumme [mm] } & \multirow[b]{2}{*}{$\begin{array}{c}\text { Abweichung vom } \\
\text { langjährigen Mittelwert }\end{array}$} \\
\hline & $\begin{array}{l}\text { Sto. Nr. } 1 \\
\text { "Emmen- } \\
\text { dingen" }\end{array}$ & $\begin{array}{l}\text { Sto. Nr. } 15 \\
\text { "Potsdam } \\
\text { Bornim" }\end{array}$ & $\begin{array}{c}\text { Sto. Nr. } 17 \\
\text { "Unterrie- } \\
\text { den" }\end{array}$ & $\begin{array}{l}\text { Sto. Nr. } 19 \\
\text { "Werlte" }\end{array}$ & $\begin{array}{l}\text { Sto. Nr. } 25 \\
\text { "Klein- } \\
\text { Altendorf" }\end{array}$ & $\begin{array}{l}\text { Sto. Nr. } 29 \\
\text { "Hayn" }\end{array}$ & $\begin{array}{c}\text { Sto. Nr. } 31 \\
\text { "Schlag } \\
\text { Adler" }\end{array}$ & $\begin{array}{l}\text { Sto. Nr. } 37 \\
\text { "ü. d. Erd- } \\
\text { engraben" }\end{array}$ & \\
\hline Januar & 31 & 46 & 34 & 67 & 26 & 33 & 48 & 25 & $+[>40 \%]$ \\
\hline Februar & 45 & 30 & 42 & 51 & 38 & 36 & 33 & 34 & $+[30-40 \%]$ \\
\hline März & 66 & 43 & 52 & 75 & 76 & 46 & 57 & 45 & $+[20-30 \%]$ \\
\hline April & 57 & 25 & 34 & 25 & 38 & 44 & 33 & 64 & $+[10-20 \%]$ \\
\hline Mai & 80 & 60 & 57 & 44 & 57 & 75 & 81 & 61 & $+[0-10 \%]$ \\
\hline Juni & 50 & 37 & 60 & 39 & 66 & 49 & 60 & 36 & $-[0-10 \%]$ \\
\hline Juli & 102 & 50 & 81 & 89 & 63 & 70 & 92 & 90 & $-[10-20 \%]$ \\
\hline August & 127 & 57 & 68 & 86 & 80 & 59 & 129 & 76 & $-[20-30 \%]$ \\
\hline September & 68 & 72 & 56 & 54 & 49 & 65 & 73 & 53 & $-[30-40 \%]$ \\
\hline Oktober & 72 & 58 & 43 & 71 & 33 & 48 & 62 & 43 & $-[>40 \%]$ \\
\hline November & 53 & 62 & 68 & 70 & 44 & 74 & 62 & 69 & \\
\hline Dezember & 80 & 61 & 45 & 43 & 49 & 65 & 65 & 71 & \\
\hline Jahr & 831 & 600 & 640 & 714 & 618 & 664 & 795 & 667 & \\
\hline VP & 427 & 276 & 321 & 313 & 315 & 317 & 435 & 318 & \\
\hline
\end{tabular}

Beispiel: Der mittlere Niederschlag im November am Sto. Nr. 1 war mit 53 mm um 20 bis $30 \%$ niedriger als das langjährige Mittel.

Die Monate Juni, Juli und August waren niederschlagsärmer als der Durchschnitt. Die submontane Versuchsfläche im Harz (Sto. Nr. 29 „Hayn“) und der Standort in der sächsischen Schweiz (Sto. Nr. 31 „Schlag Adler“) zeigten hingegen überdurchschnittliche Monatsniederschlagssummen auf. Die Monate Mai und Juni waren, mit Ausnahme der Standorte Nr. 29 „Hayn“ und Nr. 31 „Schlag Adler“, im Mittel regenarm. Die größte Abweichung des Juniniederschlags wurde mit ca. $45 \%$ in Sto. Nr. 1 „Emmendingen“ gemessen. Bei den meisten Standorten fielen die höchsten Niederschläge in den Monaten Juli und August. Diese lagen zumeist über dem langjährigen Mittel.

Sowohl bei der Betrachtung aller Standorte als auch bei der spezifischen Standortauswahl ähnelte die Variation der Trockenheitsindices mehr dem Niederschlag als den Temperaturen (siehe Anhang 4 und 5). Dies bedeutet, dass in deutschen Klimaverhältnissen der Trockenheitsindex deutlich sensibler auf Veränderungen des Niederschlages reagiert als auf Änderungen der Temperatur.

\subsection{Bodenkundliche Standortcharakterisierung}

Im Rahmen der bodenkundlichen Standortcharakterisierung wurde an allen Standorten eine Profilansprache durchgeführt sowie Bodenproben für physikalische und chemische Analysen genommen. Die 
bodenphysikalischen Analysen umfassten Korngrößenverteilung und Trockenrohdichte (TRD) mit anschließender Herleitung der nFK sowie der LK. Zu den chemischen Analysen zählten die Messung des pH-Werts, des Gehaltes an organischem $\left(\mathrm{C}_{\text {org }}\right)$ und anorganischem Kohlenstoff (Carbonat), des Gesamtstickstoffs $\left(\mathrm{N}_{\mathrm{t}}\right)$ und des pflanzenverfügbaren Phosphors $(\mathrm{P})$, Kaliums $(\mathrm{K})$ und Magnesiums $(\mathrm{Mg})$. Zur Identifizierung leistungsbeeinflussender Standorteigenschaften und damit verbundener standortbasierter Ertragsmodellierung wurde nur eine Auswahl an bodenkundlichen Kenngrößen herangezogen (siehe 3.5.1) Die Standortbeprobung für die bodenkundlichen Charakterisierungen fand überwiegend in 2009 während der zweiten Vegetationsperiode statt. 17 der 33 Standorte wurden im Rahmen des Teilprojekts Bodenkunde der Universität Kassel ${ }^{11}$ beprobt, während die bodenkundlichen Charakterisierungen der restlichen Standorte durch die HeRo-Arbeitsgruppe durchgeführt wurden.

\subsubsection{Profilansprache und allgemeine Standortinformationen}

Die Profilansprache erfolgte nach den Vorgaben der AG BODEN (2005). Im Detail wurden die Bodenart, der Bodentyp, das Ausgangssubstrat für die Bodenbildung sowie die unterschiedlichen Horizonte angesprochen und definiert. In den Horizonten wurden charakteristische Horizontmerkmale wie Oxidation und Reduktion, Lessivierung, Verbraunung, Podsolierung sowie Anreicherung an organischer Substanz bestimmt. Ergänzend wurden die Gesamtprofiltiefe, anzutreffende Bodenlebewesen, der Skelettgehalt sowie die Höhe über NN am Standort erfasst.

\subsubsection{Durchführung der Probennahme}

Die Probeentnahme fand in allen vier Einzelparzellen eines definierten Prüfglieds statt. Je Einzelparzelle und Tiefe wurde eine Mischprobe gebildet. Die Proben wurden aus dem Randbereich der Einzelparzelle entnommen (Abb. 3-13).

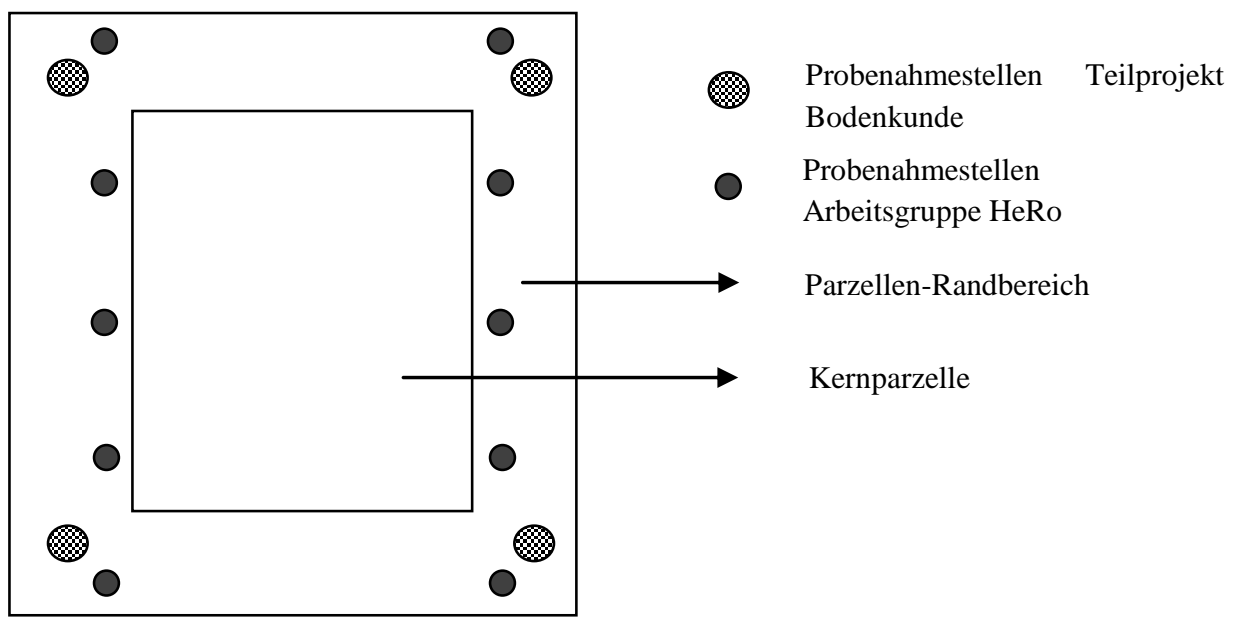

Abb. 3-13: Probenahmeschema Teilprojekt Bodenkunde und HeRo-Arbeitsgruppe.

Im Rahmen des Teilprojekts Bodenkunde bestand die Mischprobe aus dem Material von vier Bohrungen mit einem Rammkern von 8,2 cm Durchmesser. Die Proben umfassten Tiefen von $0-5 \mathrm{~cm}$, $5-30 \mathrm{~cm}$ und $30-60 \mathrm{~cm}$. Die Mischproben, welche von der HeRo-Arbeitsgruppe gewonnen wurden, setzten sich aus zehn $\mathrm{N}_{\text {min }}$-Bohrer-Einstichen aus den Tiefen 0 - 30 und $30-60 \mathrm{~cm}$ zusammen. Um die Vergleichbarkeit der Daten beider Probenahmen zu gewährleisten, wurde aus den Ergebnissen der Tiefen 0 - 5 und 5 - $30 \mathrm{~cm}$ vom Teilprojekt Bodenkunde jeweils ein Wert für die gesamte Tiefe 0 - 30 durch die Bildung gewichteter Mittelwerte errechnet. Um die TRD sowie weitere bodenphysikalische

${ }^{11}$ Ein wichtiges Teilprojekt im Verbundvorhaben ProLoc zur Bodenkunde und Kohlenstoffdynamik von KUP führte die Universität Kassel, Fachgebiet für Bodenbiologie und Pflanzenernährung durch. 
Kenngrößen zu ermitteln, wurden Stechzylinderproben gewonnen. Im Teilprojekt Bodenkunde wurden vier Stechzylinderproben je Untersuchungstiefe und Parzelle an einem Profil gewonnen. Die Profile wurden im Bereich zwischen der Kern- und der Randparzelle gegraben. Die HeRo-Arbeitsgruppe entnahm je Untersuchungstiefe und Parzelle, analog zum Vorgehen des Teilprojektes Bodenkunde, vier Stechzylinderproben. Abweichend zum Vorgehen im Teilprojekt Bodenkunde wurden $250 \mathrm{~cm}^{-3}$ Stechzylinder verwendet und die vier Proben in zwei Profile statt in einem verteilt. Mit der Wahl der Tiefe zwischen 0 und $60 \mathrm{~cm}$ ist die bodenkundliche Erfassung des Standorts gewährleistet (siehe 2.5.3.2).

\subsubsection{Bodenphysikalische Untersuchungen}

$\mathrm{Zu}$ den bodenphysikalischen Untersuchungen zählten die Korngrößenanalyse mit Berücksichtigung des Skelettanteils, die Bestimmung der TRD sowie die Herleitung von Porenraumkenngrößen wie nFK und LK. Tabelle 3-9 zeigt die verwendeten Methoden der bodenphysikalischen Analysen.

Tab. 3-9: Methodik der bodenphysikalischen Analysen (verändert nach HEYN und WACHENDORF 2011).

\begin{tabular}{lll}
\hline Kenngröße & Methode & Quelle \\
\hline $\begin{array}{l}\text { Bodenart } \\
\text { Korngrößenverteilung) }\end{array}$ & $\begin{array}{l}\text { Aufschluss durch Humuszerstörung mit Wasserstoffperoxid und } \\
\text { Carbonatzerstörung mit 2,0 M Salzsäure, Dispergierung in } \\
\text { Natrium-Pyrophosphat, Korngrößenbestimmung durch } \\
\text { Nasssiebung und Pipettmethode nach KÖHN. }\end{array}$ & $\begin{array}{l}\text { DI998) } \\
\text { Trockenrohdichte }\end{array}$ \\
& $\begin{array}{l}\text { Entnahme ungestörter Bodenproben durch Stechzylinder mit } \\
\text { einem Volumen von 100 (Teilprojekt Bodenkunde) bzw. }\end{array}$ & DIN ISO 11272 \\
& $250 \mathrm{~cm}^{3}$ (HeRo Arbeitsgruppe), Trocknung der entnommenen \\
& Bodenprobe bei 105 ${ }^{\circ} \mathrm{C}$, anschließend Rückwaage. & \\
Skelett & Bestimmung durch Siebung auf 2 mm. & \\
\hline
\end{tabular}

Da die direkte Bestimmung von Porenraumkenngrößen zeit-, material- und kostenintensiv ist ${ }^{12}$, wurden in der vorliegenden Arbeit die nFK und die LK für die Tiefen 0 - 30 und 30 - $60 \mathrm{~cm}$ nach den Tabellen von RENGER et al. (2008) indirekt ermittelt. Die Herleitung erfolgte mit Hilfe der TRD, der Bodenart, des Gehaltes an organischer Substanz, des Skelettgehaltes sowie der Porosität.

\subsubsection{Grundwassereinfluss}

Die numerische Abschätzung des Einflusses vom Grundwasser auf das Wasserangebot im Boden kann über die Ermittlung des kapillaren Aufstiegs ${ }^{13}$ erfolgen. Dies wurde in der vorliegenden Arbeit aufgrund folgender Tatsachen ausgelassen. Erstens erfolgte keine dynamische in-situ-Messung des Grundwasserspiegels auf den jeweiligen Standorten, was für eine ordnungsgemäße Ermittlung des kapillaren Aufstiegs Voraussetzung ist. Stattdessen wurden 2009 einmalig Werte aus der Umgebung von dem jeweiligen Flächenbetreuer herangezogen, um einen praxisnahen Eindruck des Grundwasserspiegels zu dokumentieren. Zweitens ist die Messung erst interpretierbar, wenn der effektive Wurzelraum der Gewächse bekannt ist. Diese Messgröße wurde im Rahmen des Verbundvorhabens PROLOC nicht erfasst. Es gibt zwar Annahmen aus der Literatur, diese beziehen sich aber nur auf Ackerkulturen. Allgemeingültige Studien zur Erschließung des Wurzelraums von Pappel und Weide sind dem Autor nicht bekannt. Drittens ist anzunehmen, dass der effektive Wurzelraum der KUP auch stark vom

\footnotetext{
${ }^{12}$ Für die direkte Bestimmung der nFK sowie der LK ist die Ermittlung der Wasserspannungs-Wassergehalts-Beziehung für den jeweiligen Boden notwendig. Als anerkannte direkte Methoden gelten Entwässerungs- oder Evaporationsexperimente.

${ }^{13}$ Per Definition das Wasser, welches sich durch große Gradienten in der Bodenwasserspannung aus feuchten bzw. teilweise wassergesättigten Bereichen in deutlich trockenere aufwärts bewegt. Der kapillare Aufstieg ist ein sehr komplexer Vorgang. Er ist abhängig von den zeitlichen Schwankungen der Bodenfeuchte bzw. der Bodenwasserspannung.
} 
Wuchsstadium (Rotations- bzw. Ernteanzahl) abhängig und nicht nur auf die Bodenart und TRD zurückzuführen ist. Eine vereinfachte Annahme des effektiven Wurzelraumes ohne Berücksichtigung des Alters bzw. der Dimensionen der Aufwüchse, führt zur Verzerrung der realen Verhältnisse. Dennoch wurden die grundbeeinflussten Standorte von den nicht grundwasserbeeinflussten Standorten über eine binäre Dummyvariable getrennt und analysiert, um den Einfluss des Grundwassers auf den dGZ festzustellen.

\subsubsection{Bodenchemische Untersuchungen}

Eine Übersicht der ermittelten bodenchemischen Kenngrößen und der dafür angewandten Methodik kann Tabelle 3-10 entnommen werden ${ }^{14}$.

Tab. 3-10: Methodik der bodenchemischen Analysen (verändert nach HEYN und WACHENDORF 2011).

\begin{tabular}{|c|c|c|}
\hline Kenngröße & Methode & Literatur \\
\hline Carbonatgehalt & Gasvolumetrisch mit einer SCHEIBLER-Apparatur. & $\begin{array}{l}\text { DIN ISO } 10693 \\
(1997)\end{array}$ \\
\hline $\begin{array}{l}\text { Gesamtgehalt an Stickstoff und } \\
\text { Kohlenstoff }\end{array}$ & $\begin{array}{l}\text { Hochtemperaturverbrennung bei } 1150^{\circ} \mathrm{C} \text { und nachfolgende } \\
\text { Gasanalyse durch Kohlenstoff/Stickstoff/Wasserstoff- } \\
\text { Makroelementaranalysator VarioMax der Firma Elementar. }\end{array}$ & $\begin{array}{l}\text { DIN ISO } 10694 \\
(1997) \\
\text { DIN ISO } 11261 \\
(1997)\end{array}$ \\
\hline Organischer Kohlenstoff & $\begin{array}{l}\text { Bestimmung des Carbonatgehaltes. } \\
\text { Bestimmung des Gesamtkohlenstoffs. } \\
\text { Bestimmung des organischen Kohlenstoffs durch } \\
\text { Differenzverfahren aus Gesamtkohlenstoff und } \\
\text { Kohlenstoffgehalt aus Carbonat. }\end{array}$ & \\
\hline Organische Substanz & $\begin{array}{l}\text { Multiplikation des Gehaltes an organischem Kohlenstoff }\left[\mathrm{mg} \mathrm{g}^{-1}\right] \\
\text { mit dem Faktor } 1,724 \text {. }\end{array}$ & ROWELL 1997 \\
\hline $\begin{array}{l}\text { Pflanzenverfügbarer Kalium und } \\
\text { Phosphor }\end{array}$ & $\begin{array}{l}\text { Erstellung eines Eluats in Calciumacetatlactat Auszug } \\
\text { anschließend photometrische Bestimmung. }\end{array}$ & VDLUFA (1991) \\
\hline Pflanzenverfügbares Magnesium & $\begin{array}{l}\text { Erstellung eines Eluats in Calciumchlorid Auszug }(0,00125 \mathrm{M}) \\
\text { anschließend photometrische Bestimmung. }\end{array}$ & VDLUFA (1991) \\
\hline pH-Wert & $\begin{array}{l}\text { Elektrochemische Bestimmung der Wasserstoff- Ionenaktivität } \\
\text { in Bodensuspensionen ( } 10 \mathrm{~g} \text { Feinboden }+25 \mathrm{ml} \mathrm{0,01} \mathrm{M} \\
\text { Calciumchlorid Lösung) mit einer } \mathrm{pH} \text {-Elektrode. }\end{array}$ & $\begin{array}{l}\text { DIN ISO } 10390 \\
(1997)\end{array}$ \\
\hline
\end{tabular}

\subsubsection{Bodenschätzungskennwerte}

Die Bodenschätzungen der jeweiligen Standorte wurden von der zuständigen Institution beim örtlichen Finanzamt angefragt und an die HeRo-Arbeitsgruppe weitergeleitet. Für ehemalige Grünlandstandorte wurde die Schätzung einer nahegelegenen Ackerfläche herangezogen, um eine Vergleichbarkeit zu gewährleisten. Bei Renaturierungsflächen, für die in der Regel keine Schätzungen vorlagen, erfolgte die Schätzung gutachterlich durch erfahrene Bodenschätzer. Für Baumschulstandorte konnten Ackerschätzungen herangezogen werden. Wenn sich bei einer Fläche mehrere Bodenschätzungen fanden bzw. überschnitten, wurde diejenige mit dem größten Flächenanteil berücksichtigt. Ein Beispiel für eine Bodenschätzung würde sich aus einer Bodenart (zum Beispiel L) eine ZS (zum Beispiel 3) eine Entstehungsart (zum Beispiel Lö), eine BZ (zum Beispiel 77) und eine AZ (zum Beispiel 89) zusammensetzten. Das zusammengesetzte Beispiel ergibt die Schätzung L 3 Lö 77/89.

14 Die Bestimmung bodenchemischer Kenngrößen wurde überwiegend im Labor des Fachgebiets Bodenbiologie und Pflanzenernährung der Universität Kassel durchgeführt. 


\subsubsection{Zusammenfassende Betrachtung der bodenkundlichen Charakteris- tika}

Tabellen 3-11 und 3-12 geben einen Überblick aller Versuchsflächen und deren bodenkundliche Charakteristika. Da die bodenkundlichen Daten als Grundlage für die Variablenbildung der Modelle dienen, fungieren diese als Material für diese Arbeit und werden nicht, wie in anderen Studien, als Ergebnis präsentiert. In Kapitel 3.5 können die Kenngrößen nachgeschlagen werden, die zur Modellbildung und Identifizierung leistungsbeeinflussender Standorteigenschaften ausgewählt wurden. Der verbleibende Teil der bodenkundlichen Daten dient der allgemeinen Standortcharakterisierung. 
Tab. 3-11: Ehemalige Bewirtschaftung, Bodenausgangssubstrat, Bodentyp und Bodenschätzung der Versuchsflächen.

\begin{tabular}{|c|c|c|c|c|c|}
\hline $\begin{array}{l}\text { Sto. } \\
\text { Nr. }\end{array}$ & Standortname & $\begin{array}{l}\text { Eh. Bewirt- } \\
\text { schaftung a }\end{array}$ & Ausgangssubstrat der Bodenbildung & Bodentyp & Bodenschätzung \\
\hline 1 & Emmendingen & Baumschule & Löß & Schwarzerde & L3 Lö 77/89 \\
\hline 2 & Lilienthal & Grünland & Löß und Decklehm & Parabraunerde & L 3 Lö 72/76 \\
\hline 3 & Aulendorf & Grünland & Niedermoortorf & Niedermoor & Mo 2 46/46 \\
\hline 4 & Forchheim & Acker & Flugsand aus Fluvisand & Braunerde & sL 4 Dg 26/30 \\
\hline 5 & Ladenburg & Acker & Auelehm aus Schwemmlöß & Gley & L 4 AL 66/78 \\
\hline 6 & Kupferzell & Acker & Tonsteinverwitt. (Keuper) & Pseudogley & LT 5 V 50/45 \\
\hline 7 & Kaisheim & KUP & Lößlehm auf Kalkstein (Kreide) & Parabraunerde & L 5 Lö 64/62 \\
\hline 8 & E.Wald Welzow & Bergbaufol. & Kiesfüh. Kippkalklehmsand & Lockersyrosem & S1 7 Al 14/14 \\
\hline 9 & Löwenberg & Grünland & Niedermoortorf über Fluvisand & Humusgley & Mo 4 36/36 \\
\hline 11 & Cahnsdorf & Acker & Fluvioglazialer Sand & Braunerde & S 4 D 28/27 \\
\hline 12 & Kummerow & Acker & Flugsand aus Flüssesand & Braunerde & S 6 D 14/12 \\
\hline 13 & Grünewalde & Bergbaufol. & Tertiärer Kippkohlelehmsand & Regosol & S1 3 Al 38/38 \\
\hline 14 & Welzow Süd & Bergbaufol. & Quartärer Kipp-Reinsand & Lockersyrosem & S 7 Al 13/9 \\
\hline 15 & Potsdam-Bornim & Acker & Flugsand & Parabraunerde & S1 3 D 36/35 \\
\hline 16 & Lehmkaute & Acker & Löß & Parabraunerde & L 3 Lö 80/85 \\
\hline 17 & Unterrieden & Acker & Sandsteinverw. (Buntsandstein) & Braunerde & Sl 3 D 39/39 \\
\hline 18 & Gülzow & Acker & Lehmsand auf Geschiebemergel & Braunerde & SL 3 D 36/36 \\
\hline 19 & Werlte & Acker & Flug- und Fluvisand & Parabraunerde & S 3 D 33/37 \\
\hline 20 & Borlinghausen & Acker & Tonsteinverwitt. (Jura) & Pseudogley & T 6 V 34/28 \\
\hline 22 & Haus Düsse & Grünland & Löß über Lößlehm & Pseudogley & L 4 Lö 72/73 \\
\hline 24 & Königshov. Höhe & Bergbaufol. & Löß & Lockersyrosem & L 4 Lö 66/71 \\
\hline 25 & Klein Altendorf & Acker & Löß über Lößlehm & Parabraunerde & L 4 Lö 72/76 \\
\hline 27 & Bärenrode & Acker & Tonsteinverwitterung & Pseudogley & SL 4 V 55/44 \\
\hline 28 & Bernburg & Grünland & Löß & Schwarzerde & L 1 Lö 100/96 \\
\hline 29 & Hayn & Acker & Löß auf Kalkstein-Frostschutt & Parabraunerde & L 4 V 50/42 \\
\hline 30 & Iden & Acker & Niederungssand & Gley & S 5 Al 19/22 \\
\hline 31 & Schlag Adler & Acker & Lösslehm kiesführend & Braunerde & LS 5 DV 34/35 \\
\hline 32 & Thammenhain I & Acker & Lösslehm über Kiesesand & Gley & sL 5 Lö 48/49 \\
\hline 33 & Thammenhain II & Acker & Lösslehm über Kiesesand & Gley & sL 5 Lö 48/49 \\
\hline 35 & Threnthorst & Grünland & Geschiebelehm & Gley & L 5 D 55/53 \\
\hline 36 & Oberdorla & Baumschule & Löß auf Kalkstein (Kreide) & Parabraunerde & L 5 V 50/50 \\
\hline 37 & Ü. d. Erdengraben & Acker & Löß auf Kalkstein (Kreide) & Parabraunerde & L 5 Lö 62/58 \\
\hline 38 & Heiliges Marpingen & Acker & Tonsteinverw. (Rotliegende) & Regosol & sL 6 V 38/31 \\
\hline
\end{tabular}

${ }^{\mathrm{a}}$ Eh. Bewirtschaftung: Ehemalige Bewirtschaftung. 
Tab. 3-12: Bodenphysikalische und -chemische Eigenschaften der Versuchsflächen (Die bodenphysikalischen Werte beziehen sich auf die Gesamttiefe $0-60 \mathrm{~cm}$, die bodenchemischen Werte auf die Tiefe $0-30 \mathrm{~cm}$ ).

\begin{tabular}{|c|c|c|c|c|c|c|c|c|c|c|c|c|}
\hline \multirow{3}{*}{$\begin{array}{l}\text { Sto. } \\
\text { Nr. }\end{array}$} & \multirow{3}{*}{$\begin{array}{l}\text { Standortname } \\
\text { Emmendingen }\end{array}$} & \multirow{3}{*}{$\begin{array}{l}\mathrm{GW}^{\mathrm{a}} \\
{[\mathrm{m}]} \\
1,5\end{array}$} & \multirow{3}{*}{$\begin{array}{l}\text { Boden- } \\
\text { art }\end{array}$} & $\mathrm{nFK}$ & LK & TRD & $\mathrm{pH}$ & $\mathrm{C}_{\text {org }}$ & $\mathrm{N}_{\mathrm{t}}$ & $\mathrm{P}_{2} \mathrm{O}_{5}$ & $\mathrm{~K}_{2} \mathrm{O}$ & $\mathrm{Mg}$ \\
\hline & & & & \multicolumn{2}{|c|}{$[\mathrm{cm}]$} & \multicolumn{2}{|c|}{$\left[\mathrm{g} \mathrm{cm}^{-3}\right]$} & \multicolumn{2}{|c|}{$\left[\mathrm{mg} \mathrm{g}_{-}{ }^{1}\right]$} & \multicolumn{3}{|c|}{$\left[\mathrm{mg} 100 \mathrm{~g}^{-1}\right]$} \\
\hline & & & & 13,8 & 6,3 & 1,42 & 6,5 & 17,5 & 1,5 & 26,1 & 19,5 & 14,7 \\
\hline 2 & Lilienthal & $>10,0$ & Ut3 & 16,2 & 10,5 & 1,25 & 7,2 & 22,7 & 2,1 & 2,4 & 8,3 & 13,9 \\
\hline 3 & Aulendorf & 10,0 & Org. ${ }^{b}$ & 24,0 & 18,0 & 0,47 & 7,0 & 134,8 & 12,9 & 7,0 & 4,0 & 38,6 \\
\hline 4 & Forchheim & 10,0 & $\mathrm{~S} 12$ & 8,4 & 10,8 & 1,55 & 4,8 & 8,1 & 0,7 & 15,7 & 9,7 & 4,3 \\
\hline 5 & Ladenburg & 8,0 & Lt3 & 7,5 & 3 & 1,59 & 6,4 & 11,2 & 1,3 & 22,0 & 15,7 & 15,7 \\
\hline 6 & Kupferzell & 15,0 & Lt3 & 9,6 & 4,8 & 1,40 & 7,2 & 22,3 & 2,2 & 6,4 & 20,6 & 18,7 \\
\hline 7 & Kaisheim & 1,2 & Ut4 & 12,3 & 5,4 & 1,45 & 6,5 & 14,5 & 1,3 & 26,5 & 23,6 & 12,6 \\
\hline 8 & E.Wald Welzow & $>10,0$ & Lts & 8,4 & 5,1 & 1,66 & 5,9 & 1,7 & 0,2 & 2,1 & 8,7 & 25,6 \\
\hline 9 & Löwenberg $^{c}$ & 1,2 & $\mathrm{~S} 13$ & 16,8 & 13,2 & 0,61 & 5,9 & 102,9 & 8,6 & 12,0 & 17,9 & 24,8 \\
\hline 11 & Cahnsdorf & $>10,0$ & $\mathrm{Su} 2$ & 10,5 & 8,1 & 1,68 & 5,4 & 6,1 & 0,6 & 6,8 & 8,8 & 7,0 \\
\hline 12 & Kummerow & 1,8 & St2 & 6,6 & 22,2 & 1,35 & 5,0 & 7,1 & 0,6 & 10,5 & 3,4 & 10,2 \\
\hline 13 & Grünewalde & 8,0 & St2 & 12,3 & 13,5 & 1,37 & 5,4 & $42,5^{\mathrm{d}}$ & 2,0 & 8,0 & 12,3 & 27,2 \\
\hline 14 & Welzow Süd & 30,0 & S14 & 9,6 & 13,2 & 1,62 & 7,1 & 1,7 & 0,2 & 5,2 & 2,7 & 6,3 \\
\hline 15 & Potsdam-Bornim & 8,0 & $\mathrm{Su} 2$ & 9,6 & 10,5 & 1,54 & 7,1 & 12,0 & 1,2 & 27,6 & 14,8 & 6,4 \\
\hline 16 & Lehmkaute & 5,0 & $\mathrm{Lu}$ & 10,5 & 5,7 & 1,50 & 5,5 & 11,3 & 1,2 & 12,0 & 18,9 & 20,2 \\
\hline 17 & Unterrieden & 3,0 & $\mathrm{~S} 13$ & 9,9 & 10,2 & 1,51 & 5,8 & 8,0 & 0,9 & 9,3 & 18,1 & 11,4 \\
\hline 18 & Gülzow & 2,0 & Ss & 9,6 & 14,4 & 1,50 & 6,5 & 6,4 & 0,6 & 13,1 & 9,1 & 12,2 \\
\hline 19 & Werlte & 6,0 & $\mathrm{Su} 2$ & 11,4 & 10,8 & 1,62 & 5,6 & 18,4 & 1,5 & 30,7 & 16,0 & 9,4 \\
\hline 20 & Borlinghausen & $>10,0$ & Lt3 & 9,3 & 5,1 & 1,38 & 5,6 & 17,2 & 1,8 & 2,7 & 13,9 & 25,6 \\
\hline 22 & Haus Düsse & 2,0 & Ut4 & 13,8 & 4,8 & 1,51 & 7,2 & 8,4 & 0,9 & 10,6 & 5,8 & 9,2 \\
\hline 24 & Königshov.Höhe & $>10,0$ & $\mathrm{Uu}$ & 15,3 & 4,2 & 1,47 & 7,5 & 8,2 & 0,4 & 2,9 & 7,3 & 18,1 \\
\hline 25 & Klein Altendorf & 20,0 & $\mathrm{Lu}$ & 11,4 & 4,8 & 1,50 & 6,9 & 9,6 & 1,2 & 24,3 & 19,5 & 11,6 \\
\hline 27 & Bärenrode & $>10,0$ & $\mathrm{Lu}$ & 11,1 & 6,3 & 1,47 & 6,0 & 16,0 & 1,9 & 7,2 & 13,9 & 17,2 \\
\hline 28 & Bernburg & 60,0 & Ut4 & 14,4 & 8,1 & 1,29 & 7,4 & 13,9 & 1,3 & 14,2 & 17,6 & 15,7 \\
\hline 29 & Hayn & 2,0 & $\mathrm{Lu}$ & 11,7 & 7,8 & 1,38 & 6,1 & 18,6 & 2,0 & 1,7 & 2,3 & 12,9 \\
\hline 30 & Iden & 2,0 & Ss & 9,6 & 12,3 & 1,58 & 3,8 & 9,5 & 0,9 & 22,9 & 5,1 & 3,2 \\
\hline 31 & Schlag Adler & $>10,0$ & $\mathrm{~S} 12$ & 9,3 & 8,7 & 1,54 & 5,9 & 8,7 & 0,9 & 10,2 & 9,3 & 13,6 \\
\hline 32 & Thammenhain I & 1,5 & Su3 & 13,5 & 9,3 & 1,50 & 5,0 & 22,1 & 2,0 & 10,1 & 31,1 & 19,8 \\
\hline 33 & Thammenhain II & 4,5 & $\mathrm{Su} 3$ & 14,1 & 9,3 & 1,50 & 5,0 & 22,1 & 1,7 & 15,4 & 23,3 & 13,2 \\
\hline 35 & Threnthorst & 1,5 & Ls2 & 9,3 & 4,5 & 1,60 & 6,4 & 12,7 & 1,2 & 14,8 & 14,6 & 26,7 \\
\hline 36 & Oberdorla & 1,4 & Ut4 & 12,9 & 4,2 & 1,55 & 7,0 & 14,8 & 1,3 & 12,3 & 9,7 & 20,4 \\
\hline 37 & Ü. d. Erdengraben & $>10,0$ & $\mathrm{Tu} 4$ & 10,8 & 3,9 & 1,58 & 6,5 & 9,7 & 1,1 & 11,3 & 9,8 & 22,5 \\
\hline 38 & H. Marpingen & $>10,0$ & Ls2 & 9,9 & 7,2 & 1,27 & 4,4 & 15,1 & 1,5 & 12,0 & 19,4 & 8,1 \\
\hline
\end{tabular}

${ }^{a}$ Grundwasserflurabstand: bei diesen Werten handelt es sich um keine in-Situ-Pegelmessungen, sondern um Werte benachbarter Brunnen welche nur als Orientierungswerte gelten.

${ }^{\mathrm{b}}$ org.: Organische Bodenart; Moor.

${ }^{c}$ Am Sto. Nr. 9 „Löwenberg“ ist der Boden bei manchen Messungen als semiterrestrischer, bei anderen als organischer Boden klassifiziert worden.

'Am Sto. Nr. 13 „Grünewalde“ ist ein Anteil C org kohlebürtig. 
Die im Rahmen des Verbundvorhabens PROLOC angelegten Versuchsflächen variieren in den Ausgangssubstraten für die Bodenbildung. Geordnet nach ihrer Häufigkeit handelt es sich um folgende Bodensubstrate: Löße, Lößlehme und Sandlöße $(\mathrm{n}=15)$, äolisch, fluviatil und glazial abgelagerte Sandsubstrate $(n=10)$, tonig verwitternde Festgesteine $(n=4)$, Torfsubstrate (inklusiv eines stark anmoorigen Sandes, $n=2)$ sowie Geschiebelehm $(n=1)$ und sandig verwitterndes Festgestein $(n=1)$.

Die Versuchsflächen weisen folgende Bodentypen auf: Rohböden, wie Lockersyroseme und Regosole $(n=5)$, bis hin zu entwickelten Bodentypen, wie Braunerden $(n=6)$, Parabraunerden $(n=9)$ und Schwarzerden $(n=2)$. Grund- und stauwasserbeeinflusste Bodentypen wie Gleye $(n=6)$ bzw. Pseudogleye $(n=4)$ sowie Moorböden $(n=1)$ sind ebenfalls vertreten. Die AZ der Standorte schwanken zwischen 12 und 98. Die Anzahl der Standorte mittlerer Güte (AZ zwischen 20 bis 60) beträgt im Verbundvorhaben 24.

Acht Standorte weisen Grundwasserflurabstände kleiner 2,0 $\mathrm{m}$ auf und gelten daher als grundwasserbeeinflusst (Tab. 3-10). Weitere vier Standorte zeigen Grundwasserflurabstände zwischen 2,0 und 4,0 m. Die restlichen 21 Standorte besitzen einen Grundwasserflurabstand größer 4,0 m.

Die prozentuale $\mathrm{nFK}$ der terrestrischen und semiterrestrischen Standorte für die Gesamttiefe von 0 bis $60 \mathrm{~cm}$ betrug zwischen 6,6 (Sto. Nr. 11 „Cahnsdorf“) und 16,2 cm (Sto. Nr. 2 „Lilienthal“), lediglich die Standorte organischem Substrates Nr. 3 „Aulendorf“ und Nr. 9 „Löwenberg“ wiesen höhere nFK als die terrestrischen und semiterrestrischen Standorte mit bis zu 24,0 cm auf.

Bei der LK verhielt es sich anders. Bei den terrestrischen und semiterrestrischen Böden mit sandigen Substraten waren die höchsten Werte festzustellen (Maximum 22,2 cm am Sto. Nr. 12 „Kummerow“), während schwere Böden die niedrigsten LK aufwiesen. Das Minimum wurde mit $3,0 \mathrm{~cm}$ am Sto. Nr. 5 „Ladenburg“ festgestellt. In der Gesamttiefe von 0 - $60 \mathrm{~cm}$ wurden beim mittleren TRD der terrestrischen und semi-terrestrischen Standorte Werte zwischen 1,25 und 1,68 $\mathrm{g} \mathrm{cm}^{-3}$ ermittelt. Der sandige Sto. Nr. 11 „Cahnsdorf“ wies den Maximalwert auf. Bei den bindigen Böden hatte Sto. Nr. 5 „Ladenburg“ mit einer TRD von $1,59 \mathrm{~g} \mathrm{~cm}^{-3}$ den höchsten Wert.

Die pH-Werte der Versuchsflächen variierten zwischen 3,8 und 7,5. Die Mehrheit der Versuchsflächen $(n=31)$ zeigte jedoch $\mathrm{pH}$-Werte zwischen 4,8 und 7,4, was eine Einstufung von mäßig sauer bis leicht alkalisch bedeutet. Die $\mathrm{C}_{\text {org }}$-Gehalte der terrestrischen und semiterrestrischen Böden lagen zwischen 1,7 und $22,3 \mathrm{mg} \mathrm{g}^{-1}$. Tendenziell wiesen Sandsubstrate sowie ehemalige Bergbaufolgelandschaften die geringsten $\mathrm{C}_{\text {org }}$-Gehalte auf.

Auf den terrestrischen und semiterrestrischen Standorten bewegte sich der Nt-Gehalt zwischen 0,2 und $2,1 \mathrm{mg} \mathrm{g}^{-1}$. Die geringsten Werte wurden, analog wie beim $\mathrm{C}_{\text {org }}$, bei den Sandsubstraten nachgewiesen. Die Nt-Gehalte der Standorte Nr. 9 „Löwenberg“ und Nr. 3 „Aulendorf“ lagen mit je 8,6 und $12,9 \mathrm{mg} \mathrm{g}^{-1}$ deutlich über dem Niveau anderer Standorte.

Die pflanzenverfügbare Diphosphorpentoxidgehalte $\left(\mathrm{P}_{2} \mathrm{O}_{5}\right)$ variierten $\quad$ zwischen 1,7 und 30,7 mg $100 \mathrm{~g} \mathrm{~g}^{-1}$ Boden. Nach der Einteilung von KERSCHBERGER et al. (1997) wiesen sechs Standorte eine niedrige (A), fünf eine mittlere (B), 15 eine hohe (C) und sieben eine sehr hohe Phosphorversorgung (D) auf. Somit verfügen die meisten Standorte im Verbundvorhaben über eine gute Phosphorversorgung.

Die Kaliumversorgung kann über alle Standorte hinweg ebenfalls als gut bewertet werden. Die Ka-

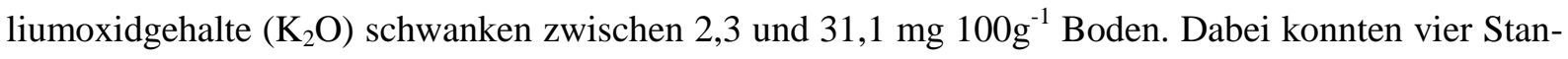
dorte in der Nährstoffklasse A, 15 in B, 12 in C und jeweils einer in D bzw. E eingestuft werden (KUNTZE et al. 1994). Die Verteilung der Magnesium-Nährstoffklassen der Versuchsflächen war mit in A zwei, in B sechs, in C 17, in D sechs und in E zwei ähnlich der anderen Nährstoffe. Der Schwan-

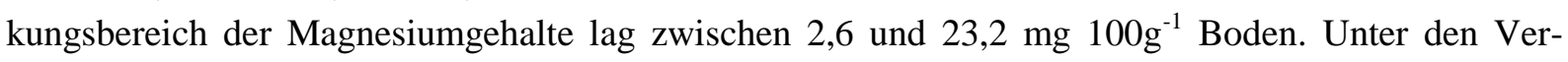


suchsflächen zeigten die Standorte Nr. 2 „Lilienthal“ und Nr. 27 „Hayn“ die niedrigsten Gehalte an pflanzenverfügbaren Nährstoffen.

\subsection{Bestandesaufnahmen und Ernte}

$\mathrm{Zu}$ den Bestandesaufnahmen gehörten die Bestimmung der Anwuchs-/Überlebensrate, die Messung der Baumhöhe, des Baumdurchmessers in 0,1 $\left(\mathrm{d}_{0.1}\right)$ und 1,3 $\mathrm{m}\left(\mathrm{d}_{1,3}\right)$ Stammhöhe sowie die Erfassung der Anzahl an Höhentrieben. Die Ernte erfolgte nach der dritten bzw. bei nachgebesserten Flächen oder einzelnen Prüfgliedern nach der zweiten Vegetationsperiode. Für die Orientierung in der Versuchsfläche und zur korrekten Durchführung der Aufnahmen erhielt jeder einzelne Pflanzplatz der Versuchsfläche eine Prüfglied- und Baumnummer.

\subsubsection{Anwuchs- und Überlebensrate}

Die prozentuale Überlebensrate wurde einmal jährlich im belaubten Zustand auf den Versuchsflächen aufgenommen. Bei der Erfassung der Überlebensrate wurden die vitalen Pflanzplätze einer Einzelparzelle gezählt. Vitale Pflanzplätze wurden definiert als Pflanzen mit sichtbarem Blattaustrieb. Die Überlebensrate wird errechnet, indem man die Anzahl der aufgenommenen vitalen Pflanzplätze durch die Gesamtanzahl der Pflanzplätze dividiert. Die Anwuchsrate bezeichnet die Überlebensrate im Anwuchsjahr.

\subsubsection{Anzahl an Höhentrieben}

Die Zählung der Höhentriebe wurde im blattlosen Zustand durchgeführt. Der Höhentrieb ist als aufstrebender Trieb definiert, der mit zunehmendem Alter eine eigene Krone ausbildet. Die Bonitur fand an jedem zweiten Pflanzplatz in der Kernparzelle statt.

\subsubsection{Höhe}

Die Messung der Baumhöhe wurde an jedem sechsten Pflanzplatz im blattlosen Zustand mittels einer Messlatte auf einen Dezimeter genau durchgeführt. Die Baumhöhe wurde als der höchste Punkt in der Lotrechten definiert. Dadurch wurden je Versuchsfläche 32 Höhenmessungen pro Klon durchgeführt.

\subsubsection{Durchmesser}

An jedem zweiten Pflanzplatz einer Kernparzelle wurde die systematische Durchmessermessung während der Winterbonitur im blattlosen Zustand durchgeführt. Mittels einer Messschieblehre wurden der $\mathrm{d}_{1,3}$ und der $\mathrm{d}_{0,1}$ auf den Millimeter genau erhoben. Traten abnorme Schaftformen an den definierten Messstellen auf, wurden die Durchmesser jeweils ober- und unterhalb gemessen und die beiden Messergebnisse arithmetisch gemittelt. Bei Verzwieselungen erfolgten Messung und Kennzeichnung der einzelnen Höhentriebe getrennt.

\subsubsection{Ertragsbestimmung}

Als Ertragsmessgröße wurde in dieser Arbeit der dGZ, welcher als der durchschnittliche jährliche Biomasseertrag definiert ist, verwendet. Im Verbundvorhaben PROLOC erfolgte die Ertragsbestimmung durch zwei unterschiedliche Methoden: Die dGZ der ersten und zweiten Wuchsperiode wurden indirekt und zerstörungsfrei über Biomassefunktionen, auch bekannt als Regressionsmethode, geschätzt (RÖHLE et al. 2006). Die Ermittlung des dGZ zum Ende der ersten Rotation fand über eine systematische Teilbeerntung der Kernparzelle statt. Im Folgenden werden beide Methoden zur Ertragsermittlung näher erläutert. 


\subsubsection{Biomassefunktionen}

Mithilfe von Biomassefunktionen wird die Beziehung zwischen ausgewählten Baumdimensionen und Baumgewicht dargestellt. Sie werden für die Ertragsbestimmung verwendet, wenn destruktive Erntemethoden zu arbeitsintensiv und kostspielig werden oder diese schlicht nicht verwendet werden können. Die Beziehung zwischen $\mathrm{d}_{1,3}$ und Gewicht wird am häufigsten verwendet, weil sie eine sehr gute Korrelation mit dem Ertrag aufweist (RöHLE et al. 2006). Für die Herleitung der Biomassefunktion wird zunächst eine Bonitur der Dimensionen des Bestandes benötigt. Dabei sind Minima und Maxima des $d_{1,3}$ und/oder der Höhe zu identifizieren. Im Anschluss werden je Klon oder Sorte die Triebe innerhalb des gesamten Durchmesserspektrums entnommen und ihr Frischgewicht bestimmt. Für die Biomassebestimmung muss eine Zuordnung der Triebdimensionen gewährleistet sein. Anschließend ist der Wasser- bzw. Trockensubstanzgehalt des Holzes im Labor auf eine Temperatur von $105{ }^{\circ} \mathrm{C}$ bis zur Gewichtskonstanz zu ermitteln. Sind alle Daten vorhanden, kann die Anpassung der Biomassefunktion vorgenommen werden. Dafür können unterschiedliche Arten der Datenanpassung verwendet werden (RÖHLE et al. 2006). Abbildung 3-14 zeigt jeweils eine Biomassefunktion mit allometrischem und exponentiellem Ansatz.

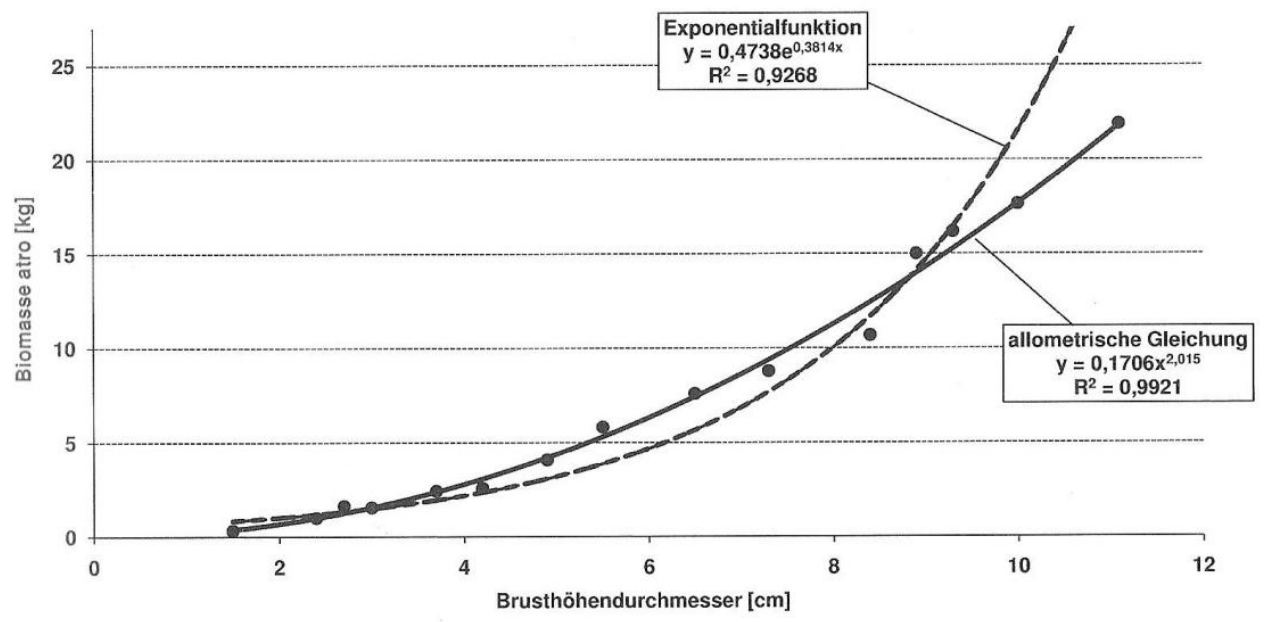

Abb. 3-14: Biomassefunktion für Pappel (aus RöHLE et al. 2010).

Bei der Bildung von Biomassefunktionen zeigt die allometrische Anpassung die durchweg besten Bestimmtheitsmaße (RÖHLE et al. 2006, HARTMANN 2010). Einflussgrößen für die Funktionskoeffizienten sind vor allem der Pflanzverband und die durchschnittliche Höhe (HARTMANN 2010). Im Rahmen des Verbundvorhaben PROLOC wurden Biomassefunktionen an dreijährigen Beständen für alle Klone entwickelt. Dabei wurde ausschließlich der allometrische Ansatz verwendet. Mithilfe der Biomassefunktionen wird das Pflanzplatzgewicht anhand der vermessenen $\mathrm{d}_{1,3}$ indirekt ermittelt. Anschließend wird der Ertrag pro Hektar (Gesamtwuchsleistung) durch die Multiplikation des durchschnittlichen Pflanzplatzgewichtes mit der Pflanzdichte je Hektar errechnet. Wird die Gesamtwuchsleistung durch das Alter geteilt, ergibt sich der dGZ.

\subsubsection{Systematische Teilbeerntung der Kernparzelle}

Obwohl indirekte Methoden der Ertragsbestimmung bereits etabliert sind, ist eine direkte Wägung des Ernteguts die sicherste Ertragsermittlung. Die Durchführung der Ernte der Versuchsflächen im Winter 2010 bis 2011 sah die systematische Beerntung von 24 Pflanzplätzen pro Einzelparzelle und Klon vor. Dabei wurde das gleiche Raster wie bei der Erfassung der Bestandesdimensionen angewandt. Pro Einzelparzelle erfolgte die Bestimmung des Trockensubstanzgehaltes an drei Pflanzplätzen. Aus dem frischen Material aller Triebbereiche wurden für jede Einzelparzelle eine 2,0 kg schwere Mischprobe gewonnen und anschließend im Labor bis zur Gewichtskonstanz bei $105{ }^{\circ} \mathrm{C}$ getrocknet. Nach dem 
berechneten Trockensubstanzgehalt der Biomasse wurde ein Faktor ermittelt. Mit Hilfe des Faktors wurden sodann die frischen Pflanzplatzgewichte auf atro-Gewichte umgerechnet. Aus der Formel 3-2 kann der Berechnungsweg für den dGZ mittels der vorgestellten Methode entnommen werden.

Durchschnittlicher Gesamtzuwachs Teilbeerntung

$$
\begin{array}{cl}
d G Z\left[t_{\text {(atro) }} h^{-1} \mathrm{a}^{-1}\right]=\frac{\sum P P K P * P D}{A Z P P K P * A} & \\
& \\
d G Z: & \text { Durchschnittlicher Gesamtzuwachs }\left[\mathrm{t}_{\text {(atro) }} \mathrm{ha}^{-1} \mathrm{a}^{-1}\right] \\
P P K P: & \text { Gewicht entnommener Kernparzellen-Pflanzplatz [ } \left.\mathrm{t}_{\text {(atro) }}\right] \\
P D: & \text { Pflanzdichte [Stk. ha } \left.{ }^{-1}\right] \\
A Z P P K P: & \text { Anzahl entnommener Pflanzplätze je Kernparzelle [Stk.] } \\
A: & \text { Alter des Bestandes [a] }
\end{array}
$$

\subsection{Identifizierung von leistungsbeeinflussenden Standorteigenschaften und Entwicklung standortbasierter Ertragsmodelle}

Das Ziel der Ertragsmodellierung war es, entweder mit univariaten oder multivariaten Verfahren das höchste Bestimmtheitsmaß der Regression für die abhängige Variable dGZ der dritten Wuchsperiode aus den Standortvariablen zu erreichen.

Die Modellentwicklung beinhaltete mehrere Schritte. Im ersten Schritt erfolgte eine Korrelationsanalyse (a) abhängig von der Variablenkategorie (ordinal bzw. metrisch skaliert) nach Pearson oder Spearman (Abb. 3-15). Im zweiten Schritt wurden univariate lineare $\left(b_{1}\right)$ sowie nicht-lineare Regressionen $\left(b_{2}\right)$ berechnet. Schritt eins und zwei werden im Folgenden als die Auswertung zur Identifizierung leistungsbeeinflussender Eigenschaften definiert und sind in dezidierter Form im Ergebniskapitel dargestellt. Ziel der Analyse war es, den Einfluss verschiedener Indikatoren des Baumwachstums auf den dGZ zu quantifizieren. Die Analyse wurde getrennt nach Standortclustern vorgenommen (siehe 3.5.2). Der dritte Schritt in der Modellierung war die Anwendung multipler linearer Regressionen $\left(b_{3}\right)$ nach den Methoden „vorwärts schrittweise“ und „best subsets“. Der vierte Schritt stellte die multiple nicht-lineare Regression $\left(\mathrm{b}_{4}\right)$ dar. Als Optimierungsschritte wurden $\mathrm{c}_{1}$ ) Variablentransformation/aggregierung sowie $c_{2}$ ) Analysen getrennt nach Standortcluster verwendet.

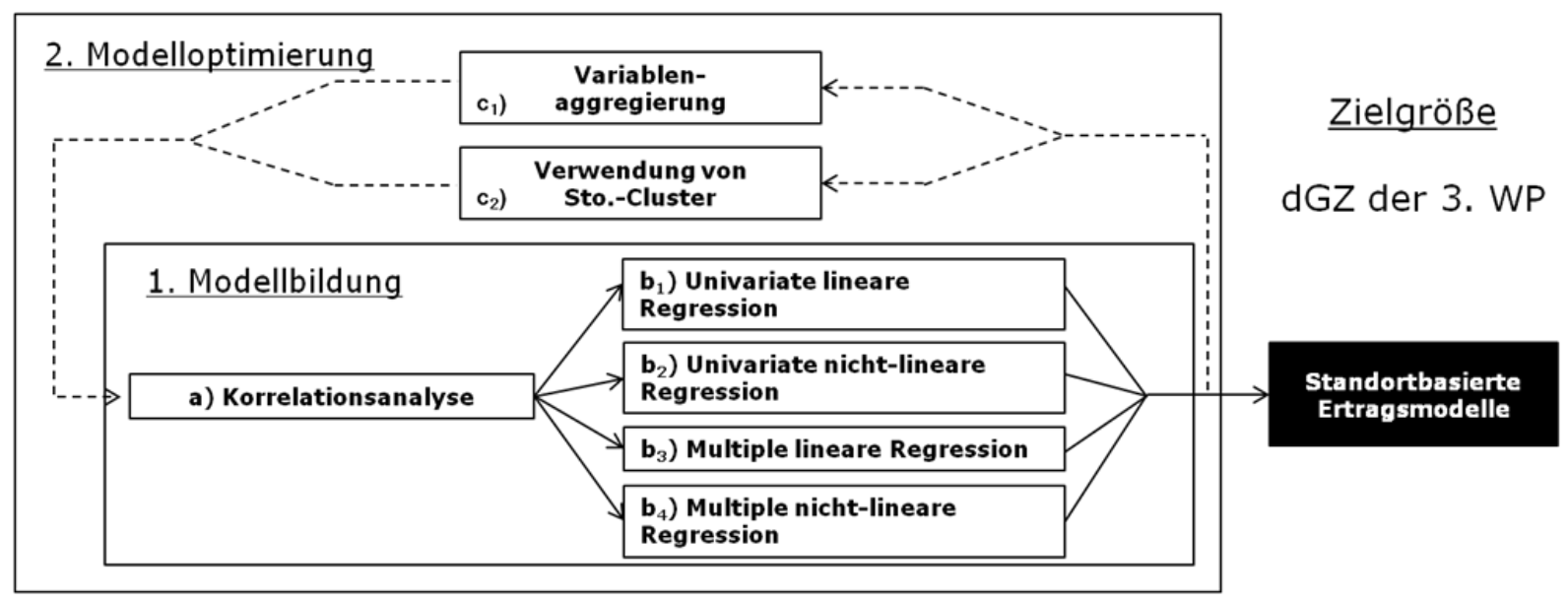

Abb. 3-15: Schematische Darstellung der Arbeitsschritte der Modellentwicklung.

Da die Mortalität nach der Nachbesserung auf einem unkritischen Niveau gehalten werden konnte, wurde diese in der Modellierung nicht berïcksichtigt. Es ist anzunehmen, dass in Folgerotationen die Mortalität eine wichtige Rolle in der Ertragsmodellierung spielen wird. Eine tiefer gehende Erläuterung der statistischen Methoden kann im Kapitel 3.8 nachvollzogen werden. 


\subsubsection{Variablenwahl und -bildung}

Als Indikatoren für das Baumwachstum können Kennwerte des Temperaturhaushalts, des Niederschlagsregimes, der Bodenfruchtbarkeit und des Bodenporenraums betrachtet werden (PRETZSCH 2002, Petzold et al. 2010). Aus diesen Indikatoren wurden Variablen definiert, um leistungsbeeinflussende Standorteigenschaften zu identifizieren und Eingangsgrößen für das Modell festzulegen (Tab. 3-13). Diese Eingangsgrößen, welche als unabhängige Variablen im Modell verwendet werden, wurden im Rahmen der Standortcharakterisierung aufgenommen und dokumentiert.

Tab. 3-13: Ausgewählte Indikatoren des Baumwachstums und deren Variablen zur Identifizierung der leistungsbeeinflussenden Standorteigenschaften und zur Modellentwicklung.

\begin{tabular}{llll}
\hline Klima & Bodenfruchtbarkeit & Porenraum & Korngrößenverteilung $^{\mathrm{b}}$ \\
\hline Temperatur & Zustandsstufe $^{\mathrm{a}}$ & Luftkapazität & Sandgehalt \\
Niederschlag & Bodenzahl & Nutzbare Feldkapazität & Schluffgehalt \\
Trockenheitsindex & Ackerzahl & Trockenrohdichte & Tongehalt
\end{tabular}

${ }^{a}$ Die Zustandsstufe kann als nicht metrisch skalierte Variable in der Modellierung nicht verwendet werden, sie wird jedoch im Kapitel leistungsbeeinflussende Standorteigenschaften in der Korrelationsanalyse berücksichtigt.

${ }^{\mathrm{b}}$ Stark grusige Standorte waren im Verbundvorhaben nicht vorhanden. Daher findet der Skelettgehalt keine Verwendung als Variable. Der Einfluss des Skeletts wurde jedoch wohl in der Ermittlung der Porenraumvariablen berücksichtigt.

Weitere unabhängige Faktoren wie zum Beispiel der Nährstoffspiegel des Bodens, wurden nicht in der Modellierung berücksichtigt. Dies wird mit den geringen Nährstoffansprüchen der Pappel begründet (siehe 2.5.3.3). Einzig für das Weidenwachstum war der $\mathrm{N}_{\min }$-Gehalt des Bodens relevant. Da in der vorliegenden Arbeit kein $\mathrm{N}_{\text {min }}$-Gehalt im Boden untersucht wurde, scheidet die Berücksichtigung dieses Faktors aus.

\subsubsection{Klima}

Zur Bildung der Klimavariablen wurden Niederschläge, Temperaturen und Trockenheitsindices herangezogen. Die klimatischen Variablen wurden aus mittleren Monatssummen bzw. Monatsmittelwerten aus den drei Beobachtungsjahren (2008 bis 2010), die in unterschiedlich langen Zeitfenstern aggregiert wurden, gebildet. Die Mehrheit der Zeitfenster fiel in die Vegetationsperiode (Mai bis September), andererseits beinhalteten manche Variablen Monate vor der Vegetationsperiode wie Januar, Februar, März und April. Für die Nomenklatur wurde unter dem Variablennamen das Zeitfenster angegeben. Zum Beispiel wird eine Niederschlagsvariable für das Zeitfenster von Januar bis September mit $\mathrm{N}_{1,9}$ bezeichnet.

Die Tabelle 3-14 zeigt die Niederschlagsvariablen. Die Variablen stellen die Niederschlagssumme der angegebenen Zeitfenster in Millimetern dar. Die Monate Februar, März und April gehören nicht zur forstlichen Vegetationsperiode. Weil jedoch eine zeitverzögerte Auswirkung auf das Wasserangebot in der Vegetationsperiode eintreten kann, wurden diese Monate bei der Summenbildung berücksichtigt.

Der Faktor Temperatur wird über die Summe von Temperaturmittelwerten in ${ }^{\circ} \mathrm{C}$ verschiedener Monate berïcksichtigt. Der Zeitrahmen zur Variablenbildung war zwischen März und September (Tab. 3-15).

Tab. 3-14: Niederschlagsvariablen, dargestellt in unterschiedlich langen Zeitfenstern. Zahlen indizieren die Monatsnummer (Januar 1, Februar 2, usw., mit J: Jahr, VP: Vegetationsperiode).

\begin{tabular}{lllllll}
\hline $\mathrm{N}_{2,3}$ & $\mathrm{~N}_{2,4}$ & $\mathrm{~N}_{2,5}$ & $\mathrm{~N}_{2,6}$ & $\mathrm{~N}_{2,7}$ & $\mathrm{~N}_{2,8}$ & $\mathrm{~N}_{2,9}$ \\
$\mathrm{~N}_{3,4}$ & $\mathrm{~N}_{3,5}$ & $\mathrm{~N}_{3,6}$ & $\mathrm{~N}_{3,7}$ & $\mathrm{~N}_{3,8}$ & $\mathrm{~N}_{3,9}$ & $\mathrm{~N}_{4,5}$ \\
$\mathrm{~N}_{4,6}$ & $\mathrm{~N}_{4,7}$ & $\mathrm{~N}_{4,8}$ & $\mathrm{~N}_{4,9}$ & $\mathrm{~N}_{5,6}$ & $\mathrm{~N}_{5,7}$ & $\mathrm{~N}_{5,8}$ \\
$\mathrm{~N}_{\mathrm{VP}}$ & $\mathrm{N}_{6,7}$ & $\mathrm{~N}_{6,8}$ & $\mathrm{~N}_{6,9}$ & $\mathrm{~N}_{7,8}$ & $\mathrm{~N}_{7,9}$ & $\mathrm{~N}_{\mathrm{J}}$ \\
\hline
\end{tabular}


Tab. 3-15: Temperaturvariablen, dargestellt in unterschiedlich langen Zeitfenstern. Zahlen indizieren die Monatsnummer (Januar 1, Februar 2, usw., mit J: Jahr, VP: Vegetationsperiode).

\begin{tabular}{ccccccc}
\hline $\mathrm{T}_{1,2}$ & $\mathrm{~T}_{1,3}$ & $\mathrm{~T}_{2,3}$ & $\mathrm{~T}_{3,4}$ & $\mathrm{~T}_{3,5}$ & $\mathrm{~T}_{3,6}$ & $\mathrm{~T}_{3,7}$ \\
$\mathrm{~T}_{3,8}$ & $\mathrm{~T}_{3,9}$ & $\mathrm{~T}_{4,5}$ & $\mathrm{~T}_{4,6}$ & $\mathrm{~T}_{4,7}$ & $\mathrm{~T}_{4,8}$ & $\mathrm{~T}_{4,9}$ \\
$\mathrm{~T}_{5,6}$ & $\mathrm{~T}_{5,7}$ & $\mathrm{~T}_{5,8}$ & $\mathrm{~T}_{\mathrm{VP}}$ & $\mathrm{T}_{6,7}$ & $\mathrm{~T}_{6,8}$ & $\mathrm{~T}_{6,9}$ \\
$\mathrm{~T}_{7,8}$ & $\mathrm{~T}_{7,9}$ & $\mathrm{~T}_{\mathrm{J}}$ & & & & \\
\hline
\end{tabular}

Die Darstellung der Wechselwirkung von Temperatur und Niederschlag in einer kombinierten Variablen erfolgt über den Trockenheitsindex nach DE MARTONNE (1926) (siehe 3.2). Wie bei den Niederschlagsvariablen wurden auch hier die Summen unterschiedlich langer Zeitfenster im Zeitrahmen zwischen Februar und Oktober berücksichtigt (Tab. 3-16). Für die statistische Analyse der Klimavariablen wurde oft die Variable für das Zeitfenster der Vegetationsperiode als eine Referenzvariable, das heißt, für den Vergleich mit anderen Zeitfenstern, verwendet.

Tab. 3-16: Variablen des Trockenheitsindizes dargestellt in unterschiedlich langen Zeitfenstern. Zahlen indizieren die Monatsnummer (Januar 1, Februar 2, usw., mit J: Jahr, VP: Vegetationsperiode).

\begin{tabular}{ccccccc}
\hline $\mathrm{TI}_{2,3}$ & $\mathrm{TI}_{2,4}$ & $\mathrm{TI}_{2,5}$ & $\mathrm{TI}_{2,6}$ & $\mathrm{TI}_{2,7}$ & $\mathrm{TI}_{2,8}$ & $\mathrm{TI}_{2,9}$ \\
$\mathrm{TI}_{3,4}$ & $\mathrm{TI}_{3,5}$ & $\mathrm{TI}_{3,6}$ & $\mathrm{TI}_{3,7}$ & $\mathrm{TI}_{3,8}$ & $\mathrm{TI}_{3,9}$ & $\mathrm{TI}_{4,5}$ \\
$\mathrm{TI}_{4,6}$ & $\mathrm{TI}_{4,7}$ & $\mathrm{TI}_{4,8}$ & $\mathrm{TI}_{4,9}$ & $\mathrm{TI}_{5,6}$ & $\mathrm{TI}_{5,7}$ & $\mathrm{TI}_{5,8}$ \\
$\mathrm{TI}_{\mathrm{VP}}$ & $\mathrm{TI}_{6,7}$ & $\mathrm{TI}_{6,8}$ & $\mathrm{TI}_{6,9}$ & $\mathrm{TI}_{7,8}$ & $\mathrm{TI}_{7,9}$ & $\mathrm{TI}_{\mathrm{J}}$ \\
\hline
\end{tabular}

\subsubsection{Bodenschätzung}

Im Rahmen der Identifizierung leistungsbeeinflussender Standorteigenschaften wurden ZS (Zustandsstufe), BZ (Bodenzahl) und AZ (Ackerzahl) als Variablen berücksichtigt. Weil AZ bereits eine Klimakomponente durch die Berücksichtigung der Jahreswerte der Temperatur und des Niederschlags enthält, wurde in der vorliegenden Arbeit ausschließlich BZ als Variable in der Modellbildung verwendet. Diese stellt eine rein bodenbezogene Variable dar und gibt die allgemeine Bodenfruchtbarkeit wieder. Die Berücksichtigung des Klimas für die Modellbildung erfolgt in dieser Arbeit über die bereits ausgeführten Klimavariablen (Tab. 3-14 bis 3-16).

\subsubsection{Textur}

Als Texturvariablen werden die prozentualen Gehalte von Sand, Schluff und Ton berücksichtigt. Diese Gehalte gehen als Mittelwerte der beiden Tiefen 0 - 30 und 30 - $60 \mathrm{~cm}$ ein (Tab. 3-17).

Tab. 3-17: Texturvariablen.

\begin{tabular}{lll}
\hline $\mathrm{S}_{[\%]}$ & $\mathrm{U}_{[\%]}$ & $\mathrm{T}_{[\%]}$ \\
\hline
\end{tabular}

\subsubsection{Porenraum}

Für die indirekte Ermittlung von Porenraumkenngrößen ist die Bodenkundliche Kartieranleitung - KA 5 - (AG BODEN 2005) die in den letzten Jahren meistverwendete Quelle. RENGER et al. (2008) stellten jedoch gravierende Mängel für die Herleitung der bodenphysikalischen Kennwerte nFK und LK fest. Diese Mängel betrafen hauptsächlich die Schätzung und Klassifikation der TRD sowie die $\mathrm{Zu}$ - und Abschläge für die jeweiligen Kennwerte in Abhängigkeit der Humusgehalte. Die Schwächen 
aus der KA 5 wurden in der Arbeit von RENGER et al. (2008) behoben in dem neue Auswertungen mit einer größeren und qualitativ hochwertigeren Datenbasis vorgenommen wurden.

Darüber hinaus merkten RENGER et al. (2008) an, dass die statische Betrachtung der Feldkapazität beim $\mathrm{pF}$ 1,8 eine Ungenauigkeit in der Ermittlung von $\mathrm{nFK}$ und LK darstelle. Vielmehr empfehlen die Autoren die Nutzung des Feuchtigkeitsäquivalenten (FÄ) ${ }^{15}$ nach VETTERLEIN (1983) als Grenze zwischen die Porenraumbereiche LK und nFK.

Tabelle 3-18 zeigt die für die Modellierung ausgewählten Porenraumvariablen. Die in der Modellentwicklung verwendeten nFK und LK beziehen sich auf eine Gesamttiefe von 0 bis $60 \mathrm{~cm}$ und sind in Zentimeter angegeben. Weil die TRD das größte Gewicht an der Bestimmung der Gesamtporenvolumen besitzt, kann deren Auswirkung mit dem Gesamtporenvolumen gleichgesetzt werden. Wie bei den prozentualen Gehalten an Sand, Schluff und Ton wurde bei der TRD (in $\mathrm{g} \mathrm{cm}^{-3}$ angegeben) der durchschnittliche Wert der Gesamttiefe 0 - $60 \mathrm{~cm}$ verwendet.

Tab. 3-18: Porenraumvariablen.

\begin{tabular}{ccc}
\hline nFK & LK & TRD \\
nFK FÄ & LK FÄ & nFK + LK \\
\hline
\end{tabular}

\subsubsection{Aggregierte Variablen}

Unter Einbehaltung von definierten Rahmenbedingungen die keine Kombination von

- Temperatur- oder Niederschlags- mit Trockenheitsindexvariablen,

- Textur- und Porenraumvariablen sowie,

- Variablen, die mit r größer 0,8 miteinander korrelierten

erlaubten, sind mehrere aggregierte Variablen gebildet worden. Diese Begrenzungen waren erforderlich, um größere Überlagerungseffekte im Modell zu vermeiden. Für die Bildung aggregierter Variablen wurden die bestkorrelierten Variablen aus den jeweiligen Variablenkategorien verwendet. Die aggregierten Variablen wurden durch Division und Multiplikation der einzelnen Bestandteile gebildet (Abb. 3-16).

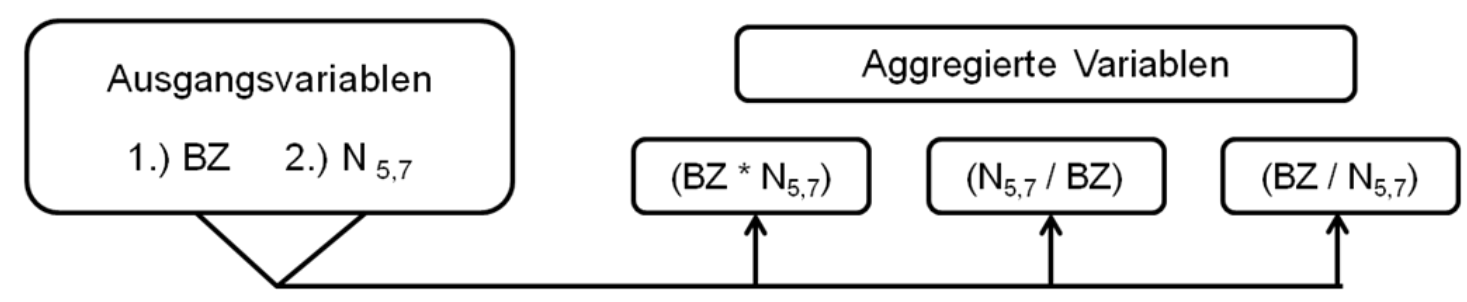

Abb. 3-16: Beispiel zur Bildung aggregierter Variablen.

\subsubsection{Weitere Einflussgrößen}

Metrisch skalierte Variablen erlauben die Verwendung der Regressionsanalyse für die Modellbildung. Weiterhin war es wichtig, den Einfluss von nominal skalierten Variablen wie Vornutzung, Bodentyp, Bodenart und Grundwasser auf den dGZ zu untersuchen. Die unterschiedlichen Ausprägungen der Variablen Vornutzung, Bodentyp und Bodenart (Hauptbodenart) wurden daher numerisch kodiert. Um den Einfluss des Grundwassers auf den dGZ zu ermitteln, wurde eine binäre Dummyvariable gebildet, welche grundwasserbeeinflusste Versuchsflächen von nicht grundwasserbeeinflussten Versuchsflä-

\footnotetext{
${ }^{15}$ Das Feuchtigkeitsäquivalent kann als eine in-Situ bodenartspezifische Ermittlung der Feldkapazität verstanden werden (VETTERLEIN 1983). Die Ergebnisse liegen häufig zwischen pF 1,8 und 2,5.
} 
chen unterteilte. Als Grenze wurde 2,0 m Flurabstand festgelegt (MÜLLER 1951, siehe 2.5.3.6) Die Analyse des Einflusses des Grundwasserflurabstandes ist bei der vorliegenden Datengrundlage mit Unsicherheiten behaftet (siehe 3.3.4).

\subsubsection{Abhängige Variable}

Hauptzielvariable des Modells war der durchschnittliche Gesamtzuwachs in $\mathrm{t}_{(\mathrm{atro})} \mathrm{ha}^{-1} \mathrm{a}^{-1}$ der dritten Wuchsperiode.

\subsubsection{Standortcluster}

Durch die Bildung von Standortclustern war eine spezifischere Auswertung möglich (Tab. 3-19). Unter den nominal skalierten Variablen, die einen signifikanten Einfluss auf dem dGZ aufwiesen, wurde die Hauptbodenart als Clusterungsvariable gewählt. Dies erfolgte aufgrund zwei wichtiger Tatsachen. Zum einen zeigen Hauptbodenarten ähnliche bodenphysikalische Eigenschaften, zum anderen hätte die Wahl einer anderen Variablen (Vornutzung, Bodentyp, etc.) sehr kleine Stichprobenumfänge in den Clustern zur Folge gehabt. Letzteres hätte die Entwicklung standortbasierter Ertragsmodelle erschwert.

Für die genaue Definition der Standortcluster nach der Hauptbodenart wurde die Einteilung nach AG BODEN (2005) verwendet. Dabei wurde zwischen den Hauptbodenarten Sand (Standortcluster S), Schluff (Standortcluster U) und Lehm (Standortcluster L) differenziert. Der einzig im Projekt aufgenommene Tonboden wurde dem Standortcluster L zugeteilt. Die genaue Zusammensetzung der Standortcluster kann in Tabelle 3-19 nachgeschlagen werden.

Tab. 3-19: Berücksichtigte Bodenarten bei den verschiedenen Standortclustern der deskriptiven Ergebniskapitel.

\begin{tabular}{ll}
\hline Standortcluster & Bodenarten \\
\hline ALL & Alle untersuchten Standorte \\
S & Ss, S12, S13, S14, Su2, Su3, St2 \\
U & Ut4, Ut3, Uu \\
L & Lt3, Lts, Lu, Ls2, Tu4 \\
\hline
\end{tabular}

Die Standorte Nr. 3 „Aulendorf“ (Niedermoor) und Nr. 9 „Aulendorf“" (stark anmooriger Standort) wurden für einige Abbildungen im Bereich der waldwachstumskundlichen Ergebnisse unter Standortcluster M zusammengefasst.

\subsection{Datenbasis der standortbasierten Ertragsmodellierung}

Für die deskriptive Ergebnisbeschreibung (Überlebensraten, Anzahl der Hohentriebe, Bestandesdimensionen sowie Wuchsleistung) wurden alle Daten, die zur Verfügung standen, verwendet, das heißt, alle 33 Standorte und alle drei Wuchsperioden. Bei der Entwicklung standortbasierter Modelle musste jedoch die Nutzung der Daten kritisch beurteilt werden. Um die leistungsbeeinflussenden Standorteigenschaften richtig identifizieren und physikalisch signifikante Modelle ohne Überlagerung der Hauptfaktoren durch unerwünschte Flächeneigenschaften oder Managementeinflüsse entwickeln zu können, konnten in der vorliegenden statistischen Auswertung nicht alle Standorte berücksichtigt werden (Tab. 3-20). Für die Modellierung kamen einzig Daten der praxisrelevanten dritten Wuchsperiode zum Einsatz. 
Tab. 3-20: Nicht berücksichtigte Standorte in der statistischen Analyse zur Ermittlung leistungsbeeinflussender Standorteigenschaften und Entwicklung standortbasierter Ertragsmodelle.

\begin{tabular}{ll}
\hline Standort & $\begin{array}{l}\text { Grund } \\
\text { Nichtberücksichtigung }\end{array}$ \\
\hline Nr. 8 „,E.Wald Welzow“ & $\begin{array}{l}\text { Flärchenvornutzung } \\
\text { Bergbaufolgelandschaft }\end{array}$ \\
Nr. 14 „Welzow Süd“ & Organischer Boden \\
Nr. 24 „Königshov. Höhe“ & Stark saurer Boden-pH \\
\hline Nr. 3 „,Aulendorf“ & \\
Nr. 9 „Löwenberg“ & Massiver Unkrautdruck \\
\hline Nr. 30 „Iden“ & \\
\hline Nr. 2 „Lilienthal“ & \\
Nr. 7 „Kaisheim“ & \\
Nr. 27 „Bärenrode“ & Dritte Wuchsperiode nicht \\
\hline Nr. 11 „Cahnsdorf“ & erreicht \\
Nr. 32 „Thammenhain I“ & \\
Nr. 33 „Thammenhain II“ & \\
Nr. 38 „Heiliges Marpingen“ &
\end{tabular}

Nicht berücksichtigte Standorte sind solche mit einer Vornutzung als Bergbaufolgelandschaft aufgrund deutlich abweichender Stoffdynamik und Bodenleben gegenüber landwirtschaftlich genutzten Böden (KNOCHE 2001). Einzig der Sto. Nr. 13 „Grünewalde“, der seit fast 60 Jahren als renaturierte Bergbaufolgelandschaft dokumentiert ist, wurde in die Modellentwicklung mit einbezogen.

Die Modellentwicklung wurde nur an terrestrischen und semiterrestrischen Böden durchgeführt. Organische Standorte, wie Nr. 3 „Aulendorf“ und Nr. 9 „Löwenberg“, konnten in diesem Modell nicht berücksichtigt werden. Aufgrund ihrer geringen Repräsentanz $(n=2)$ war keine eigene Modellentwicklung durchführbar. Sto. Nr. 30 „Iden“ konnte aufgrund des pH-Wertes ${ }^{16}$ im stark sauren Bereich nicht ausgewertet werden.

Bei den meisten Standorten war der Unkrautdruck unkritisch. Allerdings gab es drei Standorte mit massivem Unkrautdruck und damit einhergehenden Ertragseinbußen (siehe 3.1.1), welche deshalb aus der Modellentwicklung komplett ausschieden. Dies waren die Standorte Nr. 2 „Lilienthal“, Nr. 7 „Kaisheim“ und Nr. 27 „Bärenrode“.

Standorte mit einer flächigen Nachbesserung im Jahr 2009 erreichten keine dritte Wuchsperiode. Da die Identifizierung leistungsbeeinflussender Standorteigenschaften und die Modellentwicklung anhand der dGZ aus der praxisrelevanten dritten Wuchsperiode durchgeführt wurde, scheiden die Sto. Nr. 11 „Cahnsdorf“, Nr. 32 „Thammenhain I“, Nr. 33 „Thammenhain II“ sowie Nr. 38 „Heiliges Marpingen“ aus. Die berücksichtigten Bodenarten in den Standortclustern für den Bereich der Modellierung ${ }^{17}$ sind in Tabelle 3-21 zu finden.

\footnotetext{
${ }_{17}^{16} \mathrm{pH}$-Werte im stark sauren Bereich sind phytotoxisch und daher als stark wachstumshemmend zu bewerten.

17 Aufgrund ausbleibender Berücksichtigung mancher Standorte bei der Modellierung (Tab. 3-19) war die Zusammensetzung der Standortcluster der ursprünglichen Standortclusterung unterschiedlich.
} 
Tab. 3-21: Berücksichtigte Bodenarten bei den verschiedenen Standortclustern zur Ermittlung leistungsbeeinflussender Standorteigenschaften und Entwicklung standortbasierter Ertragsmodelle.

\begin{tabular}{ll}
\hline Standortcluster & Bodenarten \\
\hline ALL & Alle untersuchten Standorte \\
$\mathrm{S}$ & $\mathrm{Ss}, \mathrm{S} 12, \mathrm{~S} 13, \mathrm{Su} 2, \mathrm{St} 2$ \\
$\mathrm{U}$ & $\mathrm{Ut} 4$ \\
$\mathrm{~L}$ & $\mathrm{Lt} 3, \mathrm{Lu}, \mathrm{Ls} 2, \mathrm{Tu} 4$ \\
\hline
\end{tabular}

Eine Übersicht der berücksichtigten Standorte getrennt nach Standortclustern kann Tabelle 3-22 entnommen werden.

Tab. 3-22: Zusammensetzung der Standortcluster zur Ermittlung leistungsbeeinflussender Standorteigenschaften und Entwicklung standortbasierter Ertragsmodelle.

\begin{tabular}{lll}
\hline Standortcluster S $(\mathrm{n}=8)$ & Standortcluster U $(\mathrm{n}=5)$ & Standortcluster L (n=7) \\
\hline Sto. Nr. 4 „Forchheim“ & Sto. Nr. 1 „Emmendingen“ & Sto. Nr. 5 „Ladenburg“ \\
Sto. Nr. 12 „Kummerow“ & Sto. Nr. 22 „Haus Düsse“ & Sto. Nr. 6 „Kupferzell“ \\
Sto. Nr. 13 „Grünewald““ & Sto. Nr. 28 „Bernburg“ & Sto. Nr. 16 „Rauischholzhausen“ \\
Sto. Nr. 15 „Potsdam Bornim“ & Sto. Nr. 36 „Oberdorla“ & Sto. Nr. 20 „Borlinghausen“ \\
Sto. Nr. 17 „Unterrieden“ & Sto. Nr. 37 „Über dem Erdengraben“ & Sto. Nr. 25 „Klein-Altendorf“ \\
Sto. Nr. 18 „Gülzow“ & & Sto. Nr. 29 „Hayn“ \\
Sto. Nr. 19 „Werlte“ & & Sto. Nr. 35 „Threnthorst“ \\
Sto. Nr. 31 „Schlag Adler“ & & \\
\hline
\end{tabular}

Aufgrund der flächigen Nachbesserung einzelner Klone einer Versuchsfläche (siehe 3.1.1, Tab. 3-4) ergeben sich verschiedene Wiederholungsanzahlen für die verschiedenen Klone in den jeweiligen Standortclustern (Tab. 3-23). Modelle, welche stark abweichende Wiederholungsanzahlen aufweisen, sind nicht vergleichbar. Wie in Tabelle 3-23 zu sehen ist, kann aus diesem Grund für den Klon H 275 die Modellierung in den Standortclustern ALL, U und L nicht vorgenommen werden. Bei Tordis blieb die Modellierung im Standortcluster L aus.

Tab. 3-23: Wiederholungsanzahl (n) der standortbasierten Ertragsmodelle bei den verschiedenen Klonen und Standortclustern (+: ausgewertetes Modell, -: nicht ausgewertetes Modell).

\begin{tabular}{|c|c|c|c|c|c|c|c|c|c|c|}
\hline \multirow{2}{*}{$\begin{array}{l}\text { Standort- } \\
\text { cluster }\end{array}$} & \multicolumn{2}{|c|}{ Max 1} & \multicolumn{2}{|c|}{ Н 275} & \multicolumn{2}{|c|}{ AF 2} & \multicolumn{2}{|c|}{ Inger } & \multicolumn{2}{|c|}{ Tordis } \\
\hline & $\mathrm{n}$ & & $\mathrm{n}$ & & $\mathrm{n}$ & & $\mathrm{n}$ & & $\mathrm{n}$ & \\
\hline ALL & 20 & + & 14 & - & 20 & + & 20 & + & 18 & + \\
\hline$S$ & 8 & + & 7 & + & 8 & + & 8 & + & 8 & + \\
\hline $\mathrm{U}$ & 5 & + & 3 & - & 5 & + & 5 & + & 5 & + \\
\hline $\mathrm{L}$ & 7 & + & 4 & - & 7 & + & 7 & + & 5 & - \\
\hline
\end{tabular}

\subsection{Ertragssteigerungsfaktor}

Ertragssteigerungsfaktoren werden verwendet, wenn Erträge über die erste Rotation auf mehrere Folgerotationen vorausgesagt werden sollen. In der vorliegenden Arbeit wurde ein generalisierter Ansatz für die Ertragssteigerung von der ersten $\left(\mathrm{dGZ}_{1}\right)$ zur zweiten Rotation $\left(\mathrm{dGZ}_{2}\right)$ verwendet (HoRN et al. 2013, siehe Formel 3-3). 
Ertragssteigerungsfaktor nach HoRN et al. (2013)

$$
\begin{aligned}
& \operatorname{ESF} d G Z_{1,2}=1,2408+\left(\frac{2,9356}{d G Z_{1}}\right) \quad \text { (Formel 3-3) } \\
& \text { ESF } d G Z_{1,2}: \quad \text { Ertragssteigerungsfaktor vom } \mathrm{dGZ}_{1} \text { auf } \mathrm{dGZ}_{2} \\
& d G Z_{l}: \quad \text { dGZ der ersten Rotation }\left[\mathrm{t}_{\text {(atro }} \text { ha }^{-1} \mathrm{a}^{-1}\right]
\end{aligned}
$$

Um den dGZ ab der dritten Rotation herleiten zu können, wurden neue Ertragssteigerungsfaktoren entwickelt. Die Ertragssteigerung ist unter anderem von der Bodenbeschaffenheit abhängig (JOACHIM 1989). Aus diesem Grund wurden bodenartspezifische Ertragssteigerungsfaktoren entwickelt. Als Grundlage wurden Daten der KUP-Versuchsflächen der TLL verwendet. Die TLL legte Mitte der 1990er Jahre drei Versuchsflächen an, welche „Dornburg“ (Anlagejahr 1994), „Langenwetzendorf“ (Anlagejahr 1994) und „Bad Salzungen“ (Anlagejahr 1996) genannt wurden. In diesen Versuchsflächen wurden verschiedene Pappel- und Weidenklone bzw. Klonmischungen in der ein-, drei- und fünfjährigen Rotation untersucht. Für die ein- und dreijährige Rotation betrug die Parzellengröße $30 \mathrm{~m}^{2}$ und für die Fünfjährige $70 \mathrm{~m}^{2}$. Der Versuch wurde in vierfacher Wiederholung angelegt. Mit einer derzeitigen 19-jährigen („Dornburg“ und „Langenwetzendorf") bzw. 17-jährigen („Bad Salzungen“) Beobachtungsdauer zählen diese Versuchsflächen zu den am längsten wissenschaftlich beobachteten KUP-Versuchen in Deutschland. Die in dreijähriger Rotation geführten Pappelprüfglieder wurden, analog der PROLOC-Versuchsflächen, mit einer Pflanzdichte von 11.111 Stk. ha ${ }^{-1}$ angelegt. Daher sind die entwickelten Ertragssteigerungsfaktoren optimal in Verbindung mit den standortbasierten Modellen anwendbar. Des Weiteren repräsentiert jeweils eine der TLL-Versuchsflächen eine Hauptbodenart. „Dornburg“ repräsentiert die Bodenart stark toniger Schluff (Hauptbodenart U), „Langenwetzendorf“ einen sandigen Lehm (Hauptbodenart L) und „Bad Salzungen“ einen lehmigen Sand (Hauptbodenart S). In „Bad Salzungen“ wurden bislang die dGZ von fünf Rotationen aufgenommen. Um eine einheitliche Ertragssteigerung bis in die sechste Rotation zu gestatten wurde der Ertragssteigerungsfaktor auf die sechste Rotation extrapoliert. Die Datengrundlage kann den Publikationen von BIERTÜMPFEL et al. 2009 und 2012 entnommen werden.

Die geprüften Max-Klone übertrafen in ihrer Ertragsentwicklung deutlich die restlichen Prüfglieder auf den TLL-Versuchsflächen. Daher wurde die Entwicklung der Ertragssteigerungsfaktoren nach Prüfgliedergruppen getrennt durchgeführt. Die Zusammensetzung der Prüfgliedergruppen Balsampappelklone (BP) und Max-Klone (Max) kann Tabelle 3-24 entnommen werden.

Tab. 3-24: Zusammensetzung der Klongruppen Balsampappel (BP) und Max-Klone (Max) von den Ertragssteigerungsfaktoren an den Standorten „Bad Salzungen“", „Dornburg“ und „Langenwetzendorf“.

\begin{tabular}{cccc}
\hline \multirow{2}{*}{ Klongruppe } & \multicolumn{3}{c}{ Klon-Zusammensetzung } \\
\cline { 2 - 4 } & Bad Salzungen & Dornburg & Langenwetzendorf \\
\hline BP & Androscoggin, H 275 & Muhle Larsen, Androscoggin & Muhle Larsen, Androscoggin \\
Max & Max 1, Max 3 & Max Mehrkl., Max 2 & Max Mehrkl., Max 2 \\
\hline
\end{tabular}

Bei der Entwicklung von Ertragssteigerungsfaktoren wurde, wie im Kapitel 2.3 erwähnt, auf die Verwendung der belgischen P. trichocarpa $x$ P. deltoides Klone (Unal, Raspalje, Beaupré und Donk) verzichtet.

Die Modellanpassung erfolgte mit dem sigmoidalen Ansatz (siehe 3.8.3.1, Formel 3-12). Diesem Ansatz liegt eine konservative Ertragssteigerung zu Grunde. Die Ertragssteigerung ist von vielen Faktoren abhängig, daher stellen die Ertragssteigerungsfaktoren eine „riskante Art“ der Voraussage der Erträge von Folgerotationen dar. Die Datengrundlage ist zudem mit einer Versuchsfläche für jedes 
Standortcluster begrenzt. Basierend auf den derzeit in Deutschland vorhandenen Datengrundlagen, ist es dennoch ein fundierter Ansatz.

\subsection{EDV und Statistik}

Die Bearbeitung von Rohdaten sowie graphische Darstellungen wurden mittels der Software Excel 2007 durchgeführt. Statistische Analysen erfolgten mit der Software SPSS für Windows (Version 19.0) sowie Minitab (Version 15.0). Es wurden Methoden der deskriptiven Statistik wie die Bestimmung von Lage- und Streuungsmaßen verwendet. Als Streuungsmaß wurde die Standardabweichung berechnet. Zur Darstellung von Lagemaßen der untersuchten Größen kamen Box-Whiskers-Plots ${ }^{18}$ zum Einsatz. Außerdem kamen bei der Auswertung Methoden der beurteilenden Statistik wie Varianz-, Korrelations- und Regressionsanalysen zur Anwendung. Auf diese wird im Folgenden näher eingegangen.

Für die verschiedenen statistischen Analysen wurden Irrtumswahrscheinlichkeiten auf vier Niveaus festgelegt. Mit $\mathrm{p}<0,10:^{(*)}$ wurde ein annähernd signifikanter Trend dargelegt. Für echte Signifikanzen wurden die Irrtumswahrscheinlichkeiten von $\mathrm{p}<0,05:{ }^{*} ; \mathrm{p}<0,01:{ }^{* *} ; \mathrm{p}<0,001$ : $^{* * *}$ zugrunde gelegt und gekennzeichnet ${ }^{19}$. In den deskriptiven Kapiteln wurde, wenn nichts anderes angegeben, die Irrtumswahrscheinlichkeit $\mathrm{p}<0,05$ als Signifikanzschwelle verwendet. Die Signifikanz einer Regression wurde mit der vorgestellten Nomenklatur dem $\mathrm{R}^{2}$ korr beigefügt. Grundsätzlich wurde mit der zweiseitigen Signifikanz gearbeitet.

\subsubsection{Varianzanalyse}

Die Varianzanalyse ist eine statistische Methode, mit der Unterschiede zwischen zentralen Werten wie Mittelwerten oder Mediane ermittelt werden können. Je nach Datenbeschaffenheit werden parametrische oder nicht parametrische Verfahren angewandt. Die Nutzung parametrischer Verfahren setzt eine Normalverteilung der Residuen voraus. Daher erfolgte zuerst ein Normalitätstest der Residuen der Stichproben nach Shapiro-Wilk (SHAPIRO und WILK 1965). Da die Mehrheit der untersuchten Größen die Hauptvoraussetzung, Normalverteilung der Residuen, für die Verwendung parametrischer Verfahren nicht erfüllten, kamen nicht-parametrische Verfahren zum Einsatz. Mit Hilfe der Stichprobenanzahl sowie der ermittelten Rangsumme der untersuchten Stichproben werden die Testgrößen ermittelt. Bei nicht-parametrischen Tests ist die Datenverteilung irrelevant (STRECK 2004). Die Nullhypothese lautet, dass die Mediane gleich sind. Nichtparametrische Tests werden auch als robuste Analyseverfahren bezeichnet.

Je nachdem, ob zwei oder mehr unabhängige Stichproben untersucht wurden, kamen entweder der U-Test nach Mann-Whitney (MANN und WHITNEY 1947) oder der H-Test nach Kruskal-Wallis (KRUSKAL und WALLIS 1952) zum Einsatz. Ab drei Stichproben fungierte der Mann-Whitney-Test nach dem Kruskal-Wallis als post-hoc-Test. Beide Tests basieren auf einem Rangsummenvergleich.

Die Varianzanalyse soll die Frage nach Unterschieden zentraler Tendenzen der waldwachstumskundlichen Kenngrößen und der Koeffizienten von Biomassefunktionen beantworten. Des Weiteren wurden Varianzanalysen eingesetzt, um den Einfluss der Vornutzung, des Bodentyps, der Bodenart und des Grundwasserabflusses auf den dGZ zu prüfen. Für die Varianzanalyse wurden Einzeldaten anstatt Parzellenwerte verwendet, um eine hohe Genauigkeit zu erzielen. Zur besseren Übersicht wurden in den Abbildungen die Anzahl der untersuchten Standorte mit angegeben. Je Standort und Klon waren an Messgrößen ca. $96\left(\mathrm{~d}_{1,3}, \mathrm{~d}_{0,1}\right)$ oder ca. 24 (Höhe) Messungen vorhanden.

\footnotetext{
${ }^{18}$ Abbildungsform, welche die Kennwerte Median, erstes und drittes Quartil, Maximum und Minimum beinhaltet.

${ }^{19}$ Alle Signifikanzen wurden mit Fettdruck zusätzlich gekennzeichnet.
} 


\subsubsection{Korrelationsanalyse}

Mit Hilfe dieser Analysemethode kann ein stochastischer linearer Zusammenhang zwischen zwei Variablen untersucht werden. Der Korrelationskoeffizient $r$ stellt die Stärke des linearen Zusammenhangs dar (Formel 3-4). Dabei wurden zwei Arten der Korrelationsanalyse verwendet: die PearsonMaßkorrelation für mindestens metrisch skalierte Variablen bzw. die Spearman-Rangkorrelation bei ordinal skalierten Variablen (wie zum Beispiel ZS).

$\underline{\text { Korrelationskoeffizient } \mathrm{r}}$

$$
r_{\mathrm{xy}}=\frac{S_{\mathrm{xy}}}{S_{\mathrm{x}} * S_{\mathrm{y}}}=\frac{\sum_{i=1}^{n}\left(x_{\mathrm{i}}-\bar{x}\right) *\left(y_{\mathrm{i}}-\bar{y}\right)}{\sqrt{\sum_{i=1}^{n}\left(x_{\mathrm{i}}-\bar{x}\right)^{2 *} \sum_{i=1}^{n}\left(y_{\mathrm{i}}-\bar{y}\right)^{2}}}
$$

$\begin{array}{ll}r_{x y}: & \text { Korrelationskoeffizient der Merkmale } \mathrm{x} \text { und } \mathrm{y} \\ S_{x y}: & \text { Empirische Kovarianz der Merkmale } x \text { und } y \\ S_{x}: & \text { Standardabweichung des Merkmals } x \\ S_{y}: & \text { Standordabweichung des Merkmals } y \\ x_{i}, y_{i}: & \text { Merkmalausprägung i der Merkmale } x \text { und } y \\ \bar{x}, \bar{y}: & \text { Mittelwert der Merkmale } x \text { und } y\end{array}$

Zur Bewertung und besseren Erläuterung der Zusammenhänge wurde die so genannte COHENKonvention (COHEN 1988) herangezogen. Diese Konvention definiert bei verschiedenen r-Werten unterschiedlich starke statistische Zusammenhänge:

- $0,1<\mathrm{r}<0,3=$ kleiner bzw. schwacher Zusammenhang;

- $0,3<\mathrm{r}<0,5=$ mittlerer bzw. mäßiger Zusammenhang;

- $r>0,5=$ großer bzw. starker Zusammenhang.

Darüber hinaus wurden Korrelationen mit Werten für $r$ größer 0,8 im Rahmen dieser Arbeit als sehr große bzw. sehr starke Zusammenhänge bewertet. Zusätzlich zur Signifikanz einer Korrelation stellen die Stärke und die Richtung der Korrelation inhaltlich relevante Informationen dar. Daher werden in dieser Arbeit sowohl signifikante als auch nicht signifikante Korrelationen gezeigt, sofern dies als notwendig erachtet wird.

Statistische Korrelationen haben mit kausalen Zusammenhängen prinzipiell nichts zu tun. Da in der vorliegenden Arbeit Standortvariablen aus Indikatoren des Baumwachtums als unabhängige Variablen und die Standortleistung in Form des dGZ als abhängige Variable fungierten, können die ermittelten Zusammenhänge als Effekte der Standortvariablen auf den dGZ interpretiert werden. Die Frage nach der Kausalität der Beobachtung kann nun einerseits mit weiteren Methoden, wie der Regressionsanalyse eruiert oder im Rahmen der Diskussion mit den Erkenntnissen aus der Literatur verglichen werden.

Die Korrelationsanalyse wurde angewandt, um einerseits relevante Wechselwirkungen zwischen Klima- und Bodenvariablen und dem dGZ abzugrenzen und andererseits den Zusammenhang zwischen Standortvariablen zu untersuchen. Für die Korrelationsanalyse wurde der mittlere Standort-dGZ je Klon sowie der Mittelwert der jeweiligen Standortvariablen in der Analyse herangezogen.

\subsubsection{Regressionsanalyse}

Die Regressionsanalyse beschreibt die stochastische Abhängigkeit zwischen abhängiger (y) und unabhängiger(n) (x) (eine oder mehrere) Variablen. In der vorliegenden Arbeit wurden die einfache lineare (lin, Formel 3-5), die multiple lineare (mlin, Formel 3-6) sowie die nicht-lineare Regression (siehe 3.8.3.1) verwendet. Im Folgenden wird auf die linearen Methoden eingegangen.

Die Schätzung der Regression erfolgt mit der Methode der kleinsten Quadrate (OLS, vom englischen „ordinary last squares“). Diese Methode sieht eine Minimierung der Summe der quadrierten Residuen 
(beobachteter minus geschätzter Wert) vor (SACHS und HEDDERICH 2009). Die Güte der Anpassung einer Regression wird durch das Bestimmtheitsmaß $\left(\mathrm{R}^{2}\right)$ abgebildet (Formel 3-7). $\mathrm{R}^{2}$ ist der Quotient der erklärten Streuung (SSE) geteilt durch die Gesamtstreuung (SST). Somit ist das $\mathrm{R}^{2}$ ein Maß dafür, wie gut die unabhängige(n) Variable(n) die Streuung der abhängigen erklärt bzw. erklären. $R^{2}$ stellt das Verhältnis beider Größen in Form einer Fraktion dar, die Werte zwischen 0 und 1 annehmen kann. Oft wird $\mathrm{R}^{2}$ in Prozent für die Erklärungsgüte angegeben.

Mit zunehmender Anzahl von Prädiktoren bei gleichzeitig kleinen Stichproben nimmt das $\mathrm{R}^{2}$ der Regression zu. Daher wird, um zu einer realistischen Abschätzung der Regressionsgüte zu kommen, das korrigierte Bestimmtheitsmaß ( $\mathrm{R}^{2}{ }_{\text {korr }}$, Formel 3-8) eingesetzt. Dadurch wird einer Überparametrisierung der Regression entgegengewirkt, und nur die wichtigen Prädiktoren werden im Modell berücksichtigt. $\mathrm{R}_{\text {korr }}$ wurde in dieser Arbeit bevorzugt als Gütemaß der Regressionen verwendet.

Lineare Regression

$$
\begin{array}{cl}
y=a_{0}+a_{1} * x & \\
& \\
y: & \text { Zielgröße } \\
a_{0} \text { und } a_{1}: & \text { Regressionskoeffizient } \\
x: & \text { Prädiktor }
\end{array}
$$$$
\text { (Formel 3-5) }
$$

Multiple Lineare Regression

$$
\begin{array}{cl}
y=a_{0}+a_{1} * x_{1}+a_{2} * x_{2}+\cdots+ & a_{\mathrm{n}} * x_{\mathrm{n}} \\
y: & \text { Zielgröße } \\
a_{0 \ldots a_{n}:} & \text { Regressionskoeffizient } \\
x_{1 \ldots x_{n}:} & \text { Prädiktoren }
\end{array}
$$

(Formel 3-6)

$\underline{\text { Bestimmtheitsmaß } \mathrm{R}^{2}}$

$$
\begin{array}{cl}
R^{2}=1-\frac{S S E}{S S T}=1-\frac{\sum_{i=1}^{n}\left(y_{\mathrm{i}}-\hat{y}_{\mathrm{i}}\right)^{2}}{\sum_{i=1}^{n}\left(y_{\mathrm{i}}-\bar{y}_{\mathrm{i}}\right)^{2}} & \\
R^{2}: & \text { Bestimmtheitsmaß } \\
S S E: & \text { Erklärte Streuung } \\
S S T: & \text { Gesamtstreuung } \\
y_{i}: & \text { Merkmalsausprägung } i \text { des beobachteten Wertes } y \\
\hat{y}_{\mathrm{i}}: & \text { Merkmalsausprägung } i \text { des geschätzten Wertes } \hat{y} \\
\bar{y} & \text { Mittelwert beobachtet }
\end{array}
$$

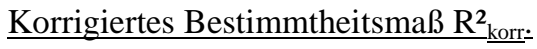

$$
\begin{array}{cl}
R_{\text {korr }}^{2}=R^{2}-\frac{k *\left(1-R^{2}\right)}{n-k-1} & \\
R^{2}{ }_{\text {korr }}: & \text { Korrigiertes Bestimmtheitsmaß } \\
R^{2}: & \text { Unkorrigiertes Bestimmtheitsmaß } \\
k: & \text { Anzahl an Prädiktoren } \\
n: & \text { Stichprobenumfang }
\end{array}
$$

(Formel 3-8)

Die lineare Regression wurde „vorwärts schrittweise“ und mittels „best subsets“ durchgeführt. Durch die Methode der „vorwärts schrittweisen“ Regression werden signifikante Variablen in Abhängigkeit von ihrem Erklärungsbeitrag automatisch ausgewählt bzw. die beste Prädiktorengruppe in die Regression einbezogen (WÖSTEN et al. 2001). Als erstes wird die Variable mit dem höchsten Erklärungsbeitrag gewählt, die zweite Modellvariable ist diejenige, welche die höchste Zunahme an $\mathrm{R}^{2}$ korr zur Folge hat. Die Prozedur wird so lange wiederholt, bis keine zusätzlichen signifikanten Prädiktoren eine Zunahme der Regressionsgüte zur Folge haben. In der „best subsets“ Methode wird die Regression mit möglichst wenigen unabhängigen Variablen bei maximalen $\mathrm{R}^{2}{ }_{\text {korr }}$ angestrebt. Alle beliebigen 
Prädiktor-Kombinationen werden getestet, jedoch wird nur diejenige mit der höchsten Erklärungsrate gewählt. Die auf Basis der „best subsets“ Methode ermittelten Regressionen können eine andere Prädiktorenzusammensetzung aufweisen, als die berechneten Regressionen mittels der schrittweisen Methode.

\subsubsection{Nicht-lineare Regression}

Zur Verbesserung der Modellanpassung wurde die Eignung acht nicht-linearer Ansätze geprüft. Dazu zählen der logarithmische (ln, Formel 3-9), der inverse (inv, Formel 3-10), der compound (comp, Formel 3-11), der allometrische (allo, Formel 3-12), der sigmoidale (sig, Formel 3-13) sowie zwei weitere exponentielle Ansätze exponentiell 1 (exp1, Formel 3-14) und exponentiell 2, (exp2, Formel 3-15). Weil polynomische Ansätze wie dem quadratischen oder kubischen bei ihrer Anpassung physikalisch und biologisch unplausible Kurvenverläufe mit hoher Güte hervorbringen können, wurde auf deren Verwendung verzichtet.

Logarithmischer Ansatz

$$
y=a_{0}+a_{1} \ln (x)
$$

Inverser Ansatz

$$
y=a_{0}+\frac{a_{1}}{x}
$$

Compound Ansatz

$$
y=a_{0} a_{1}^{x}
$$

Allometrischer Ansatz

$$
y=a_{0} x^{a_{1}}
$$

$\underline{\text { Sigmoidaler Ansatz }}$

$$
y=e^{\left(a_{0}+\frac{a_{1}}{x}\right)}
$$

Exponentieller Ansatz 1

$$
y=e^{\left(a_{0}+a_{1} * x\right)}
$$

Exponentieller Ansatz 2

$$
y=a_{0} e^{\left(a_{1} * x\right)}
$$

(Formel 3-15)

$\begin{array}{ll}y: & \text { Zielgröße } \\ a_{0}, a_{l}: & \text { Modellkoeffizienten } \\ l n: & \text { Natürlicher Logarithmus } \\ x: & \text { Prädiktor } \\ e & \text { Eulersche Zahl }\end{array}$

Alle nicht-linearen Regressionen wurden mit der OLS-Methode errechnet. Mit Ausnahme des logarithmischen und inversen Ansatzes sah die Berechnung der Regression mittels OLS in SPSS eine Linearisierung der Modelle durch logarithmische Transformation vor. Zur Beurteilung der Schätzgenauigkeit wurde, wie bei den linearen Methoden, das korrigierte Bestimmtheitsmaß $\mathrm{R}^{2}{ }_{\text {korr }}$ herangezogen. Die Modelle wurden erst berücksichtigt, wenn sowohl das Gesamtmodell als auch die einzelnen Koeffizienten mindestens auf einem p-Niveau $<0,05$ signifikant waren. Eine wichtige Voraussetzung für die Gültigkeit der Modelle ist zudem, dass die Residuen zufällig auftreten und einer Normalverteilung entsprechen (BROSIUS 1998). Die Prüfung dieser Voraussetzungen erfolgte dadurch, dass die Residuen der entwickelten Modelle einem Shapiro-Wilk-Test unterzogen wurden.

Des Weiteren wurden multiple nichtlineare Modelle verwendet (siehe Formel 3-16 bis 3-24). Die multiplen nichtlinearen Modellansätze 1a bis 1c orientierten sich an dem inversen Ansatz (siehe 
Formel 3-10), die Modelle 2a bis 2c an dem compound Ansatz (siehe Formel 3-11) und die Modelle $3 \mathrm{a}$ bis $3 \mathrm{c}$ an dem allometrischen Ansatz (siehe Formel 3-12). Die Modelle mit dem Suffix a, b und c unterschieden sich in der Verwendung der Konstante, welche entweder als Summand (a) oder Multiplikator (b) fungierte oder nicht verwendet wurde (c).

Multiple nicht-lineare Modelle

$$
y=a_{0}+\left(\frac{1 \mathrm{a}}{x_{1}}\right)+\left(\frac{a_{2}}{x_{2}}\right)
$$

(Formel 3-16)

$\underline{2 \mathrm{a}}$

$$
y=a_{0}+\left(a_{1}^{x_{1}}\right)+\left(a_{2}^{x_{2}}\right)
$$

(Formel 3-19)

$\underline{3 \mathrm{a}}$

$$
y=a_{0}+\left(x_{1}{ }^{a_{1}}\right)+\left(x_{2}{ }^{a_{2}}\right)
$$

(Formel 3-22)

$$
y=a_{0} *\left(\left(\frac{a_{1}}{x_{1}}\right)+\left(\frac{a_{2}}{x_{2}}\right)\right)
$$

(Formel 3-17)

$\underline{2 \mathrm{~b}}$

$$
y=a_{0} *\left(\left(a_{1}^{x_{1}}\right)+\left(a_{2}^{x_{2}}\right)\right)
$$

(Formel 3-20)

$\underline{3 \mathrm{~b}}$

$$
y=a_{0} *\left(\left(x_{1}{ }^{a_{1}}\right)+\left(x_{2}{ }^{a_{2}}\right)\right)
$$

(Formel 3-23)

$$
y=\left(\frac{\underline{a_{1}}}{x_{1}}\right)+\left(\frac{a_{2}}{x_{2}}\right)
$$

(Formel 3-18)

$$
y=\left(a_{1}^{x_{1}}\right)+\left(a_{2}^{x_{2}}\right)
$$

(Formel 3-21)

$$
y=\left(x_{1}^{a_{1}}\right)+\left(x_{2}{ }^{a_{2}}\right)
$$

(Formel 3-24)

$$
\begin{array}{ll}
y: & \text { Zielgröße } \\
a_{0}, a_{1,} a_{2}: & \text { Modellkoeffizienten } \\
x_{1}, x_{2}: & \text { Prädiktoren }
\end{array}
$$

Weitere multiple nichtlineare Modelle wurden in Form von Kombinationsmodellen ermittelt. Diese stellten die Verbindung der Ansätze linear, invers, allometrisch sowie compound dar. Die Kombinationsmodelle 1a bis 1c (siehe Formel 3-25 bis 3-27) stellen beispielhaft die Zusammenführung des allometrischen mit dem inversen Ansatz dar. Insgesamt wurden 48 Kombinationsmodelle geprüft.

Beispiel Kombinationsmodell

$$
\begin{aligned}
& \underline{1 \mathrm{a}} \\
& y=a_{0}+\left(x_{1}{ }^{a_{1}}\right)+\left(\frac{a_{2}}{x_{2}}\right) \\
& \text { (Formel 3-25) } \\
& y=a_{0} *\left(\left(x_{1}^{a_{1}}\right)+\left(\frac{a_{2}}{x_{2}}\right)\right) \\
& \begin{array}{ll}
y: & \text { Zielgröße } \\
a_{0}, a_{1,} a_{2}: & \text { Modellkoeffizienten } \\
x_{1}, x_{2}: & \text { Prädiktoren }
\end{array}
\end{aligned}
$$

Bei den multiplen nicht-linearen Modellen kam als Iterationsmethode der Levenberg-Marquart-Ansatz zur Anwendung (MARQUARDT 1963). Als Anfangswert für die Schätzung der Modellkoeffizienten wurde standardmäßig 1,0 gewählt.

\subsubsection{Evaluierungsgrößen}

Als Modellevaluierungsgrößen wurden Bias (Formel 3-28), Prognosestreuung (Formel 3-29) und Treffgenauigkeit (Formel 3-18) und das „root-mean-square error“ (RMSE, Formel 3-30) verwendet (PRETZSCH 2001). Des Weiteren erfolgte eine Evaluierung anhand linearer Regressionen zwischen beobachteten und geschätzten Daten. 
Bias/Verzerrung

$$
\bar{e}=\frac{\sum_{i=1}^{n}\left(\hat{y}_{\mathrm{i}}-y_{\mathrm{i}}\right)}{n}
$$

(Formel 3-28)

Prognosestreuung

$$
S_{\mathrm{e}}=\sqrt{\frac{\sum_{i=1}^{n}\left(\hat{y}_{\mathrm{i}}-\bar{e}-y_{\mathrm{i}}\right)^{2}}{n-1}}
$$

$\underline{\text { Treffgenauigkeit }}$

$$
m_{\mathrm{x}}=\sqrt{S_{\mathrm{e}}{ }^{2}+\bar{e}}
$$

(Formel 3-30)

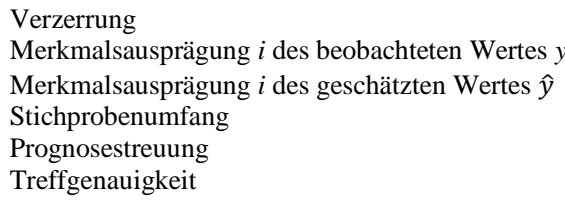

Die Relativwerte für Bias, Prognosestreuung oder Treffgenauigkeit lassen sich wie folgt berechnen (Formel 3-31).

Prozentuale Bias, Prognosestreuung und Treffgenauigkeit

$$
\begin{array}{cl}
\overline{\mathrm{e}}[\%]\left(S_{e}[\%], m_{X}[\%]\right)=\frac{\overline{\mathrm{e}}\left(s_{e}, m_{\mathrm{x}}\right) * 100}{\bar{y}} & \\
& \\
\bar{e}[\%]: & \text { Prozentuale Bias } \\
S_{e}[\%]: & \text { Prozentuale Prognosestreuung } \\
m_{x}[\%]: & \text { Prozentuale Treffenauigkeit } \\
\bar{y} & \text { Mittelwert beobachtet }
\end{array}
$$$$
\text { (Formel 3-31) }
$$

Darüber hinaus wurde der RMSE ${ }^{20}$ nach folgender Formel berechnet (Formel 3-32).

Root mean square error RMSE

$$
\begin{array}{cl}
R M S E=\sqrt{\frac{S S E}{n-1}} & \\
R M S E: & \text { Root mean square error } \\
S S E: & \text { Erklärte Streuung } \\
n: & \text { Stichprobenumfang }
\end{array}
$$

\footnotetext{
${ }^{20}$ Der RMSE ist analog zum Standardfehler des Mittelwertes der geschätzten Werte.
} 


\section{Ergebnisse}

Das vorliegende Kapitel stellt die Ergebnisse der waldwachstumskundlichen Kennwerte dar. Zusätzlich werden Aspekte der Ertragsermittlung in Form von Biomassefunktionen näher erläutert. Der Hauptteil des Kapitels enthält die wesentlichen Ergebnisse, welche sich mit der Identifizierung leistungsbeeinflussender Standorteigenschaften sowie mit der standortbasierten Ertragsmodellierung befassen. Im letzten Kapitel werden Ertragssteigerungsfaktoren behandelt, und es wird aufgezeigt, wie diese angewandt werden, um den dGZ von Folgerotationen herzuleiten.

\subsection{Waldwachstumskundliche Ergebnisse}

In diesem Kapitel werden die Ergebnisse von der Anwuchs- und Überlebensrate, die Anzahl an Höhentrieben, die Bestandesdimensionen Höhe, $\mathrm{d}_{0,1}, \mathrm{~d}_{1,3}$ sowie der durchschnittliche Gesamtzuwachs vorgestellt. Die gesonderte Auswertung von Biomassefunktionen zur Ertragsermittlung kann im letzten Teil dieses Kapitels nachvollzogen werden.

\subsubsection{Anwuchs- und Überlebensrate}

Wie im Kapitel 2.1.1 bereits erwähnt, war im Anlagejahr 2008 ein erfolgreicher Anwuchs der Bestände nicht überall gegeben (Abb. 4-1). Für alle Standorte sind im Jahr 2008 Anwuchsraten zwischen 5 und $100 \%$ registriert worden. Die mittleren Anwuchsraten betrugen aufsteigend für $\mathrm{H} 27560 \%$, für AF $281 \%$, für Tordis $83 \%$, für Max $184 \%$ und für Inger $89 \%$. Die Anwuchsleistung fiel somit, abgesehen von H 275, insgesamt hoch aus. Die Weiden zeigten mit $86 \%$ eine im Mittel ca. $10 \%$ höhere signifikante Anwuchsleistung als die Klone der Pappel. Eine Betrachtung der einzelnen Prüfglieder bestätigt, dass der Klon Max 1 die beste Überlebensrate unter den Pappeln besitzt und teilweise das Niveau der Weidenklone erreicht. Der Pappelklon H 275 wies durchgehend die signifikant niedrigste Anwuchsrate auf, wobei er auf einem Viertel der Standorte eine Anwuchsrate von $40 \%$ nicht überstieg. Trotz Bewässerung im Anwuchsjahr konnte H 275 am Sto. Nr. 19 „Werlte“ nicht etabliert werden, die Überlebensrate betrug an diesem Standort lediglich $6 \%$. AF 2 zeigte auffällig niedrige Anwuchsraten am Sto. Nr. 27 „Bärenrode“. Dort musste er komplett nachgebessert werden.

WP 1 (Anwuchsjahr)

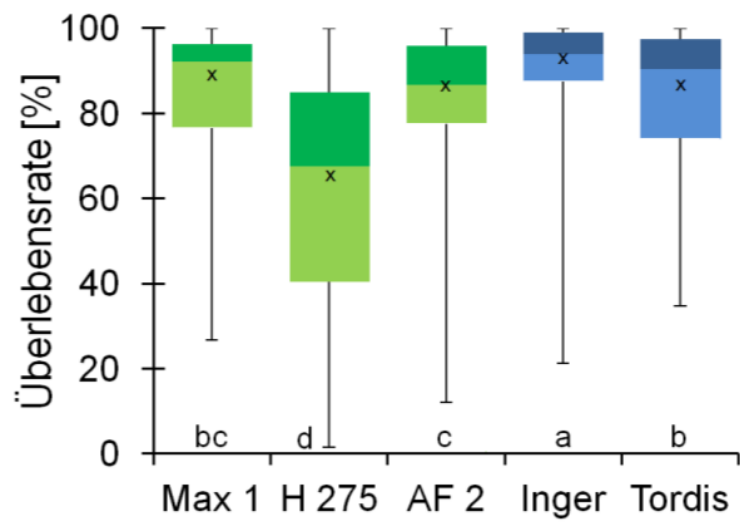

Klon
WP 2 (mit Nachbesserung)

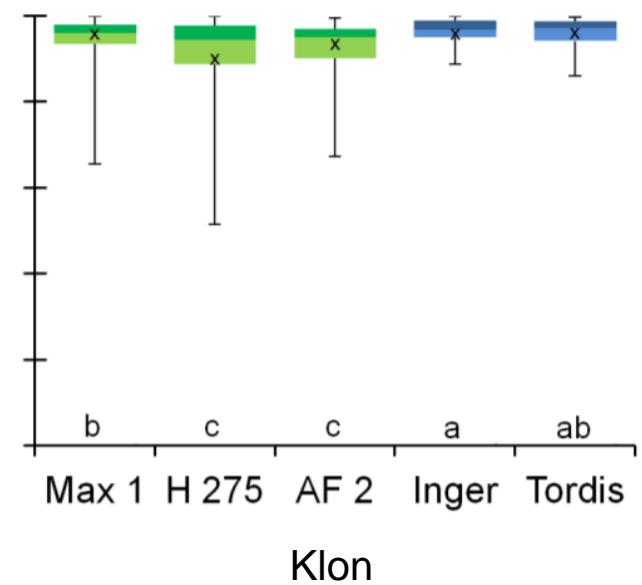

Abb. 4-1: Überlebensraten [\%] der Pappelklone Max 1, H 275 und AF 2 sowie der Weidenklone Inger und Tordis über alle Standorte a) im Anwuchsjahr sowie b) im Folgejahr nach durchgeführter Nachbesserung dargestellt mittels Box-WhiskersPlots ( $\mathrm{n}=33$, ungleiche Buchstaben indizieren signifikante Unterschiede auf dem p-Niveau < 0,05).

Bei den Weiden Inger und Tordis ist bis auf die Standorte Nr. 5 „Ladenburg“ und Nr. 6 „Kupferzell“ eine fast identische, durchgehend hohe Anwuchsrate registriert worden. An den genannten Standorten, 
welche durch schwere Bodenarten und teilweise verdichteten Untergrund gekennzeichnet sind, wurde eine signifikant höhere Überlebensrate des Klons Inger gegenüber Tordis beobachtet. Eine Übersicht der erzielten Anwuchsraten sowie der Überlebensraten in der zweiten Wuchsperiode kann den Anhängen 6 und 7 entnommen werden.

Nach der Nachbesserung wurde eine gute Bestockung der Flächen erreicht. In aufsteigender Reihenfolge waren die mittleren Überlebensraten nun für H 275 89\%, für AF $292 \%$, für Max $195 \%$ sowie jeweils für Inger und Tordis $96 \%$. Die Minima der Weiden lagen mit $86 \%$ bei Tordis und mit $89 \%$ bei Inger noch im hohen Wertebereich. Bei den Pappeln lagen die Minima mit Ausnahme von H 275 (Minimum von $52 \%$ ) über $60 \%$. Eine Abnahme der Überlebensrate von der zweiten auf die dritte Wuchsperiode war vereinzelt vorhanden und wurde überwiegend beim Schwarzpappelklon AF 2 beobachtet. Betroffene Standorte waren die Nr. 12 „Kummerow, Nr. 18 „Gülzow“ sowie Nr. 31 „Schlag Adler“. Diese Standorte zeichneten sich entweder durch sehr niedrige Wintertemperaturen und/oder geringes Wasserangebot in der Vegetationsperiode aus.

Je nach Hauptbodenart wurde nach einem kausalen Zusammenhang zwischen Anwuchserfolg und Standorteigenschaften gesucht (Abb. 4-2 und 4-3).

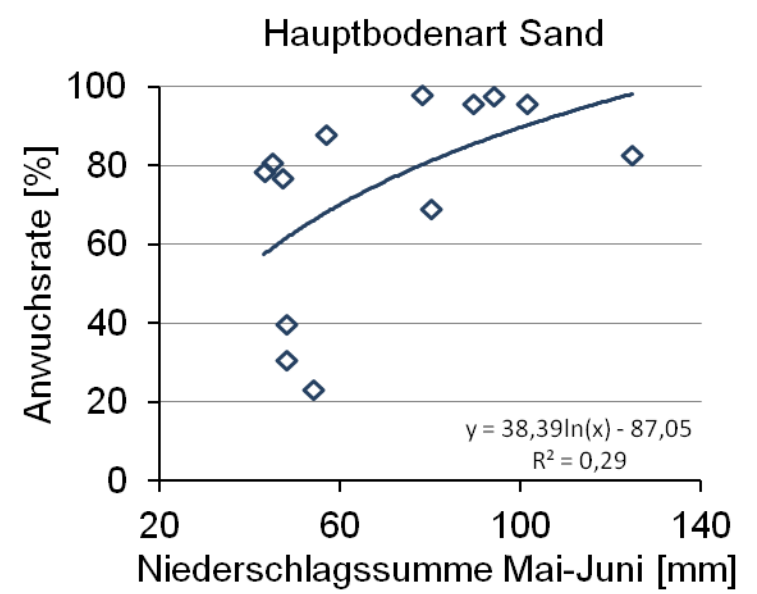

Abb. 4-2: Mittlere Anwuchsraten [\%] aller Prüfglieder in Abhängigkeit der Niederschlagssumme der Monate Mai und Juni bei der Hauptbodenart Sand.

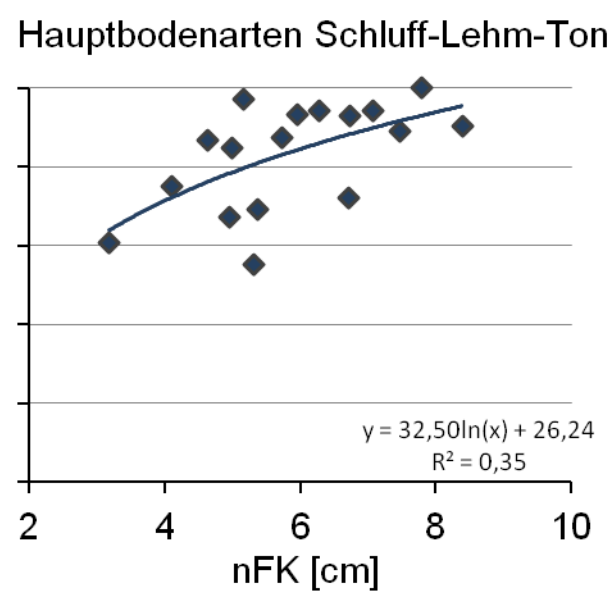

Abb. 4-3: Mittlere Anwuchsraten [\%] aller Prüfglieder in Abhängigkeit der nFK der Bodenoberschicht $(0$ - 30 $\mathrm{cm}$ ) bei den Hauptbodenarten Schluff, Lehm und Ton.

Die Standorte mit dem geringsten Anwuchserfolg waren Böden mit hohen Sand- oder Tongehalten, was in der Regel auf eine niedrige nFK hinweist. Anwuchsraten kleiner $50 \%$ sind bei den Sandböden erst bei Niederschlagssummen kleiner $60 \mathrm{~mm}$ in den Monaten Mai und Juni, im Anschluss zur Pflanzung, erfasst worden (Abb. 4-2). Innerhalb der Hauptbodenart Sand war ein Zusammenhang zwischen Anwuchsraten und nFK nicht zu erkennen. Hingegen konnte bei den anderen Bodenarten (Schluffe, Lehme, Ton), bei einer Bodentiefe von 0 bis $30 \mathrm{~cm}$, eine positive Beziehung $\left(R^{2}=0,35\right)$ zwischen nFK und Anwuchsrate beobachtet werden.

\subsubsection{Anzahl an Höhentrieben}

Die Anzahl an Höhentrieben je Klon und Gattung erwies sich bereits in der ersten Rotation als variabel. Die mittlere Anzahl der Höhentriebe betrug in aufsteigender Reihenfolge für H 275 1,2, für AF 2 1,3, für Max 1,4, für Inger 1,8 und für Tordis 1,9. Sowohl Klon als auch Gattung hatten einen signifikanten Einfluss auf die Anzahl an Höhentrieben, wobei die Weiden signifikant mehr Höhentriebe produzierten als die Pappeln. Innerhalb der Weiden war die Anzahl der Höhentriebe beider Klone nicht signifikant unterschiedlich, während bei den Pappeln H 275 eine signifikant geringere Anzahl an Höhentrieben aufwies (Abb. 4-4). 


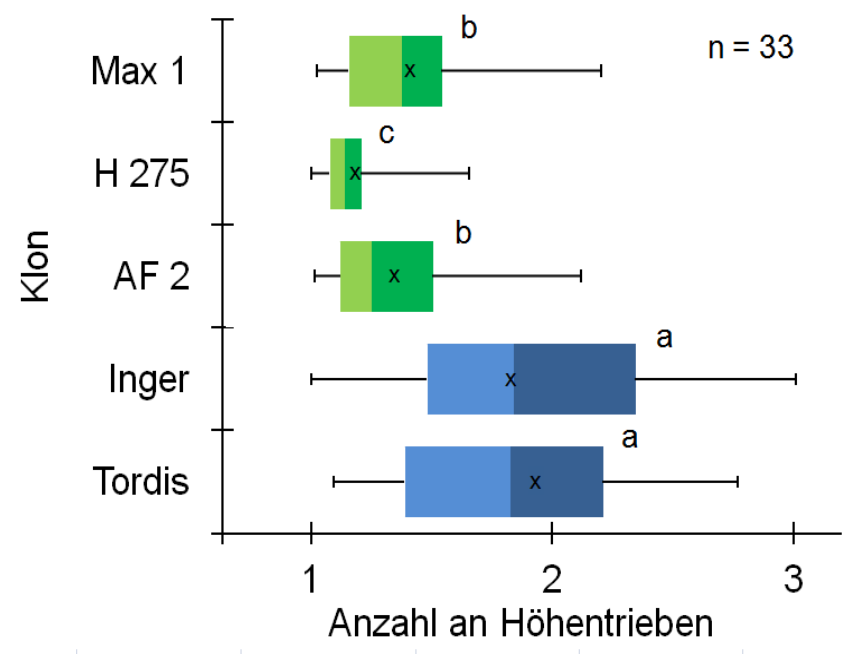

Abb. 4-4: Mittlere Anzahl an Höhentrieben der Pappelklone Max 1, H 275 und AF 2 sowie der Weidenklone Inger und Tordis über alle Standorte aus der zweiten Wuchsperiode dargestellt mittels Box-Whisker-Plots ( $\mathrm{n}=33$, ungleiche Buchstaben indizieren signifikante Unterschiede auf dem p-Niveau $<0,05)$.

\subsection{3 Übersicht Bestandesdimensionen und Wuchsleistung}

Mess- und Ertragsgrößen für die dritte Wuchsperiode sind für jeden Standort exemplarisch in Tabelle 4-1 aufgeführt.

Auf die Ergebnisse der Bestandesdimensionen Höhe, $\mathrm{d}_{1,3}, \mathrm{~d}_{0,1}$ sowie -wuchsleistung aller drei Wuchsperioden wird in den folgenden Kapiteln eingegangen. 
Tab. 4-1: Mittlere Höhe [m], $\mathrm{d}_{1,3}[\mathrm{~cm}]$ und dGZ $\left[\mathrm{t}_{(\text {atro) }} \mathrm{ha}^{-1} \mathrm{a}^{-1}\right]$ der Pappelklone Max 1, H 275 und AF 2 sowie der Weidenklone Inger und Tordis in der dritten Wuchsperiode ${ }^{\mathrm{a}}$ für alle untersuchten Standorte.

\begin{tabular}{|c|c|c|c|c|c|c|c|c|c|c|c|c|c|c|c|c|}
\hline \multirow{2}{*}{$\begin{array}{l}\text { Sto } \\
\text { Nr. }\end{array}$} & \multirow{2}{*}{ Standortname } & \multirow[b]{2}{*}{ 肴 } & \multicolumn{3}{|c|}{$\begin{array}{l}\text { Höhe } \\
{[\mathrm{m}]}\end{array}$} & \multirow[b]{2}{*}{ 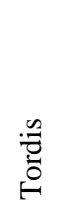 } & \multirow[b]{2}{*}{$\bar{x}$} & \multicolumn{3}{|c|}{$\begin{array}{l}\mathrm{d}_{1,3} \\
{[\mathrm{~cm}]}\end{array}$} & \multirow[b]{2}{*}{$\stackrel{0}{?}$} & \multirow[b]{2}{*}{$\bar{x}$} & \multicolumn{3}{|c|}{$\begin{array}{c}\mathrm{dGZ} \\
{\left[\mathrm{t}_{\text {(atro) }} \mathrm{ha}^{-1} \mathrm{a}^{-1}\right]}\end{array}$} & \multirow[b]{2}{*}{$\stackrel{\mathscr{0}}{0}$} \\
\hline & & & 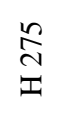 & 至 & $\begin{array}{l}\dot{\bar{D}} \\
\stackrel{\infty}{\Xi}\end{array}$ & & & $\begin{array}{l}\stackrel{n}{N} \\
\underset{I}{I}\end{array}$ & 要 & $\stackrel{\dot{\Phi}}{\Xi}$ & & & $\begin{array}{l}\stackrel{n}{N} \\
\stackrel{I}{I}\end{array}$ & $\stackrel{N}{2}$ & $\stackrel{\bar{\Delta}}{\Xi}$ & \\
\hline 1 & Emmendingen & 5,9 & 6,4 & 6,4 & 6,7 & 7,1 & 3,7 & 3,7 & 3,7 & 3,2 & 2,9 & 7,6 & 5,2 & 6,3 & 7,1 & 7,8 \\
\hline 2 & Lilienthal & 5,4 & 3,5 & 6,2 & 3,9 & 3,9 & 3,4 & 2,4 & 3,8 & 1,6 & 1,6 & 6,1 & 2,7 & 6,4 & 1,6 & 1,5 \\
\hline 3 & Aulendorf & 6,9 & 5,9 & 6,9 & 7,2 & 6,9 & 4,7 & 3,8 & 4,6 & 3,6 & 3,1 & 10,0 & 5,6 & 8,4 & 7,9 & 7,2 \\
\hline 4 & Forchheim & 5,0 & 4,5 & 4,9 & 4,0 & 3,4 & 3,2 & 2,9 & 3,5 & 2,0 & 1,5 & 5,5 & 4,4 & 4,5 & 2,8 & 2,2 \\
\hline $5^{\mathrm{a}}$ & Ladenburg & 2,3 & 1,9 & 3,5 & 2,9 & 1,6 & 0,9 & 0,5 & 2,0 & 0,9 & 0,5 & 0,8 & 0,3 & 2,2 & 0,8 & 0,2 \\
\hline $6^{\mathrm{a}}$ & Kupferzell & 4,9 & 2,7 & 4,7 & 5,0 & 3,5 & 2,7 & 1,2 & 3,1 & 2,1 & 1,2 & 3,8 & 0,6 & 3,8 & 2,8 & 0,9 \\
\hline 7 & Kaisheim & 2,2 & 3,0 & 3,7 & 3,9 & 4,3 & 1,1 & 1,7 & 2,4 & 2,0 & 1,9 & 0,7 & 1,6 & 2,6 & 2,4 & 2,3 \\
\hline 8 & E.Wald Welzow & 1,5 & 1,6 & 1,7 & 1,6 & 1,5 & 0,6 & 0,8 & 0,7 & 0,6 & 0,5 & 0,4 & 0,5 & 0,5 & 0,3 & 0,1 \\
\hline 9 & Löwenberg & 5,5 & 4,5 & 5,1 & 5,1 & 4,4 & 3,3 & 2,5 & 3,5 & 2,5 & 2,1 & 5,6 & 2,8 & 4,0 & 2,6 & 2,0 \\
\hline $11^{\mathrm{a}}$ & Cahnsdorf & 1,8 & 2,4 & 2,3 & 2,8 & 3,1 & 0,9 & 1,2 & 1,2 & 1,3 & 1,3 & 0,4 & 0,8 & 0,8 & 1,2 & 1,2 \\
\hline 12 & Kummerow & 2,5 & 2,6 & 2,2 & 3,0 & 2,9 & 1,7 & 1,7 & 1,5 & 1,6 & 1,5 & 2,0 & 2,0 & 0,8 & 1,9 & 1,7 \\
\hline 13 & Grünewalde & 5,0 & 4,7 & 4,8 & 4,5 & 4,8 & 3,0 & 2,7 & 3,2 & 2,2 & 2,2 & 4,6 & 3,9 & 3,6 & 3,4 & 4,7 \\
\hline 14 & Welzow Süd & 1,1 & 1,5 & 1,4 & 1,6 & 1,6 & 0,5 & 0,8 & 0,7 & 0,6 & 0,6 & 0,2 & 0,2 & 0,2 & 0,2 & 0,1 \\
\hline 15 & PotsdamBornim & 4,4 & 4,0 & 5,4 & 5,2 & 5,2 & 2,6 & 2,3 & 3,7 & 2,4 & 2,3 & 4,5 & 3,0 & 5,9 & 3,8 & 3,5 \\
\hline $16^{\mathrm{a}}$ & Lehmkaute & 4,7 & 2,6 & 4,5 & 4,9 & 4,5 & 2,9 & 1,2 & 3,5 & 2,5 & 2,1 & 3,3 & 1,1 & 4,8 & 4,7 & 4,7 \\
\hline 17 & Unterrieden & 7,3 & 6,7 & 7,1 & 7,3 & 7,6 & 4,3 & 4,1 & 4,5 & 3,4 & 3,3 & 9,9 & 10,2 & 8,8 & 9,1 & 10,0 \\
\hline 18 & Gülzow & 2,3 & 1,6 & 2,4 & 2,5 & 2,5 & 1,7 & 0,9 & 1,5 & 1,5 & 1,2 & 1,4 & 0,6 & 0,8 & 1,4 & 0,8 \\
\hline $19^{\mathrm{a}}$ & Werlte & 5,4 & 2,4 & 5,2 & 5,3 & 5,6 & 3,5 & 1,3 & 4,0 & 3,4 & 3,1 & 6,4 & 1,2 & 7,3 & 9,2 & 9,1 \\
\hline 20 & Borlinghausen & 3,4 & 3,4 & 3,7 & 4,0 & 4,6 & 1,9 & 1,8 & 2,0 & 1,8 & 1,7 & 2,1 & 1,5 & 1,5 & 1,8 & 2,0 \\
\hline 22 & Haus Düsse & 5,1 & 5,3 & 5,8 & 4,7 & 6,9 & 2,9 & 3,1 & 3,6 & 2,1 & 2,9 & 5,0 & 5,3 & 5,8 & 3,2 & 7,9 \\
\hline 24 & Königshov. Höhe & 6,2 & 4,8 & 6,1 & 6,8 & 6,4 & 3,9 & 2,8 & 4,2 & 3,7 & 3,3 & 7,1 & 3,7 & 7,0 & 9,5 & 9,4 \\
\hline 25 & Klein Altendorf & 6,9 & 6,8 & 7,3 & 6,6 & 7,1 & 4,2 & 4,4 & 5,0 & 3,4 & 3,4 & 9,6 & 9,1 & 9,2 & 7,0 & 8,0 \\
\hline $27^{\mathrm{a}}$ & Bärenrode & 2,0 & 1,9 & 1,9 & 2,9 & 2,1 & 0,8 & 0,9 & 0,7 & 1,3 & 0,7 & 1,0 & 0,9 & 0,6 & 1,5 & 1,1 \\
\hline 28 & Bernburg & 6,4 & 6,6 & 6,7 & 6,9 & 6,9 & 3,7 & 4,1 & 4,3 & 3,3 & 3,0 & 10,1 & 8,0 & 11,1 & 10,6 & 10,0 \\
\hline 29 & Hayn & 5,4 & 5,7 & 5,2 & 6,1 & 6,0 & 3,2 & 3,2 & 3,0 & 2,9 & 2,7 & 8,4 & 7,5 & 6,9 & 8,3 & 8,1 \\
\hline 30 & Iden & 1,0 & 1,1 & 2,0 & 2,2 & 1,8 & 0,4 & 0,7 & 0,9 & 0,9 & 0,6 & 0,4 & 0,5 & 1,5 & 0,8 & 0,4 \\
\hline 31 & Schlag „Adler“ & 6,4 & 6,5 & 6,2 & 6,8 & 6,9 & 3,7 & 4,0 & 3,9 & 3,3 & 3,1 & 7,4 & 8,9 & 6,6 & 8,1 & 9,1 \\
\hline $32^{\mathrm{a}}$ & Thammenhain I & 3,1 & 3,2 & 3,6 & 4,1 & 4,1 & 1,5 & 1,8 & 2,2 & 1,8 & 1,7 & 1,2 & 1,7 & 1,7 & 1,6 & 1,6 \\
\hline $33^{\mathrm{a}}$ & Thammenhain II & 1,9 & 1,5 & 2,2 & 2,3 & 2,1 & 0,8 & 0,7 & 1,1 & 0,9 & 0,9 & 0,5 & 0,3 & 0,5 & 0,4 & 0,4 \\
\hline 35 & Threnthorst & 2,9 & 2,7 & 2,2 & 2,6 & 3,6 & 1,6 & 1,5 & 1,2 & 1,4 & 1,8 & 1,5 & 0,3 & 0,8 & 1,9 & 0,9 \\
\hline $36^{\mathrm{a}}$ & Oberdorla & 5,4 & 3,4 & 6,0 & 5,5 & 5,9 & 3,3 & 1,5 & 4,3 & 2,7 & 2,5 & 4,9 & 1,9 & 7,1 & 7,0 & 6,7 \\
\hline $37^{\mathrm{a}}$ & Ü. d. Erdengraben & 5,9 & 4,2 & 5,1 & 5,8 & 6,3 & 3,7 & 2,5 & 3,6 & 3,1 & 3,0 & 7,4 & 4,8 & 5,8 & 6,7 & 7,3 \\
\hline $38^{\mathrm{a}}$ & H. Marpingen & 0,9 & 1,0 & 1,2 & 1,1 & 1,1 & 0,5 & 0,4 & 0,6 & 0,4 & 0,6 & 0,0 & 0,0 & 0,0 & 0,0 & 0,1 \\
\hline
\end{tabular}

a Aufgrund flächenspezifischer Nachbesserungen einzelner oder aller Prüfglieder (siehe markierte Versuchsflächen) werden Werte aus der zweiten Wuchsperiode statt aus der dritten Wuchsperiode in kursiver Form und mit grauem Hintergrund hervorgehoben. 


\subsubsection{Höhe}

Für alle Standorte und Wuchsperioden waren die Höhenleistungen erwartungsgemäß sehr variabel. Die mittleren Bestandeshöhen variierten in der ersten Wuchsperiode zwischen 0,4 m (Sto. Nr. 30 „Iden“, Klon H 275) und 3,3 m (Sto. Nr. 1 „Emmendingen“, Klon Inger). In der zweiten Wuchsperiode schwankten die mittleren Höhen der Einzelstandorte zwischen 0,7 m (Sto. Nr. 30 „Iden“, Klon Max 1) und 5,9 m (Sto. Nr. 17 „Unterrieden“, Klon Tordis). In der dritten Wuchsperiode bewegten sich die Höhenwerte zwischen 1,0 m (Sto. Nr. 30 „Iden“, Klon Max 1) und 7,6 m (Sto. Nr. 17 „Unterrieden“, Klon Tordis). In den ersten zwei Wuchsperioden zeigten die Weiden signifikant größere Höhen als die Pappeln (Tab. 4-2). Der relative mittlere Höhenunterschied zwischen den Gattungen belief sich auf ca. 5 bis $10 \%$ in der ersten Wuchsperiode und auf 10 bis $15 \%$ in der zweiten Wuchsperiode. In der dritten Wuchsperiode zeigten erneut die Weiden die höchsten Werte, der relative Unterschied zu den Pappeln war aber mit rund 5 bis $10 \%$ analog zur ersten Wuchsperiode gering. AF 2 wies stets die signifikant höheren Werte unter den Pappelklonen auf. In der dritten Wuchsperiode wies der Pappelklon AF 2 ein vergleichbares Höhenniveau wie das der Weiden auf und war sogar geringfügig (aber signifikant) stärker als der Weidenklon Inger. Innerhalb der Weidenklone wurden nur in der dritten Wuchsperiode signifikante Unterschiede festgestellt. Dabei stellte sich Tordis als die wuchsstärkere Weide heraus.

Tab. 4-2: Mittlere Höhen [m] der Pappelklone Max 1, H 275 und AF 2 sowie der Weidenklone Inger und Tordis in den Wuchsperioden eins bis drei unter Berücksichtigung aller Standorte (Standardabweichung in Klammern; ungleiche Buchstaben zeigen signifikante Unterschiede innerhalb der Wuchsperiode auf dem p-Niveau <0,05).

\begin{tabular}{|c|c|c|c|c|c|c|c|c|c|c|c|c|c|}
\hline \multirow{3}{*}{ Gattung } & \multirow{3}{*}{ Klon } & \multicolumn{12}{|c|}{ Mittlere Höhe [m] } \\
\hline & & \multicolumn{4}{|c|}{ WP 1} & \multicolumn{4}{|c|}{ WP 2} & \multicolumn{4}{|c|}{ WP 3} \\
\hline & & $\mathrm{n}$ & $\varnothing$ & s.a. & & $\mathrm{n}$ & $\varnothing$ & s.a. & & $\mathrm{n}$ & $\varnothing$ & s.a. & \\
\hline \multirow{3}{*}{ Pappel } & Max 1 & 29 & 1,2 & $(0,9)$ & $\mathrm{c}$ & 31 & 3,1 & $(1,7)$ & $\mathrm{c}$ & 28 & 4,7 & $(2,1)$ & $\mathrm{c}$ \\
\hline & H 275 & 29 & 1,2 & $(0,8)$ & $\mathrm{c}$ & 31 & 2,8 & $(1,6)$ & d & 23 & 4,4 & $(2,1)$ & d \\
\hline & AF 2 & 29 & 1,4 & $(0,9)$ & $\mathrm{b}$ & 31 & 3,3 & $(1,5)$ & $\mathrm{b}$ & 27 & 5,1 & $(1,9)$ & a \\
\hline \multirow{2}{*}{ Weide } & Inger & 29 & 1,6 & $(1,0)$ & $\mathrm{a}$ & 31 & 3,6 & $(1,7)$ & $\mathrm{a}$ & 28 & 4,9 & $(1,9)$ & $b$ \\
\hline & Tordis & 29 & 1,5 & $(1,0)$ & $\mathrm{a}$ & 31 & 3,6 & $(1,8)$ & $\mathrm{a}$ & 26 & 5,2 & $(2,1)$ & $\mathrm{a}$ \\
\hline
\end{tabular}

Abbildung 4-5 zeigt die Höhen getrennt nach Klon, Wuchsperiode und Bodenartcluster mittels BoxWhiskher-Plots. Die Ursachen der Variation in der Wiederholungsanzahl zwischen verschiedenen Klonen und Wuchsperioden wurde bereits im Kapitel 3.1.1 ausgeführt. Die Varianzanalyse (Signifikanzniveau $\mathrm{p}<0,05$ ) ergab für alle Klone und Wuchsperioden einen signifikanten Einfluss der Hauptbodenart auf die mittlere Bestandeshöhe. Anhand von Abbildung 4-5 wird ersichtlich, dass die Höhenentwicklung im Standortcluster L am langsamsten verlief. Innerhalb des Standortclusters U waren durchweg die größten Bestandeshöhen zu verzeichnen. Diese Überlegenheit erwies sich für alle untersuchten Fälle (Klon und Wuchsperiode) als signifikant. Die Standortcluster L und S zeigten in der ersten Wuchsperiode signifikant unterschiedliche Verteilungen. Dabei wies stets der Standortcluster S größere Höhen als der Standortcluster L auf. In der zweiten Wuchsperiode bot sich ein anderes Bild. Die Standortcluster S und L waren einzig bei den Klonen Max 1 und H 275 signifikant unterschiedlich, während bei den restlichen Klonen die Höhenwerte der benannten Bodenarten vergleichbar waren. Hier waren die Standortcluster S und L einzig bei den Klonen Max 1 und H 275 signifikant unterschiedlich. Bei ihnen waren die Höhenwerte der benannten Bodenarten vergleichbar. In der dritten Wuchsperiode holten die Lehmböden gegenüber den Sandböden auf, jedoch konnten keine signifikanten Unterschiede zwischen den Standortclustern gefunden werden. 

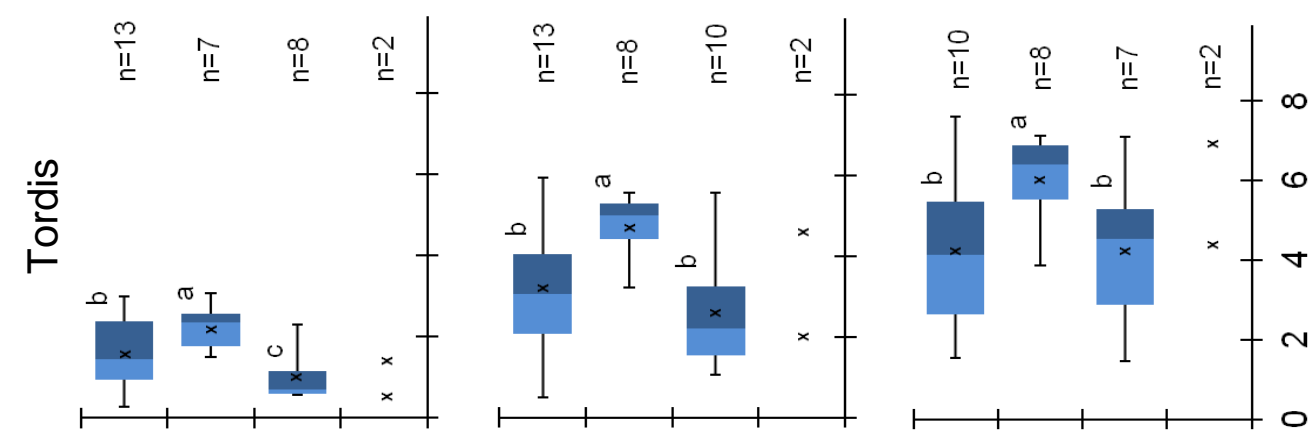

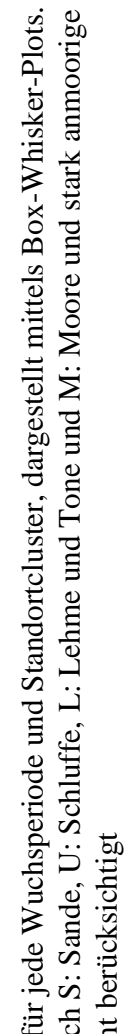
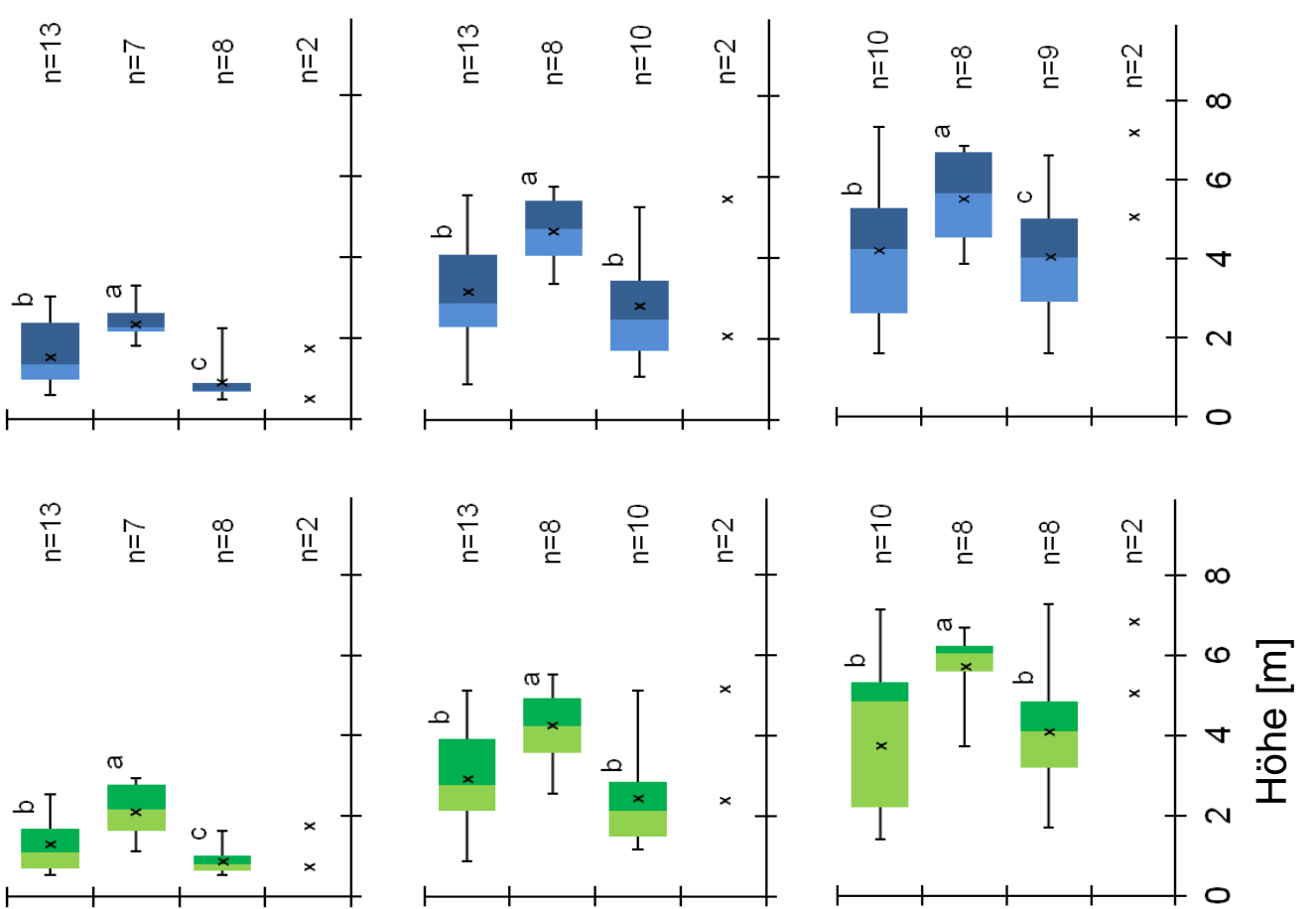

氞

$\sim$
$\stackrel{4}{4}$
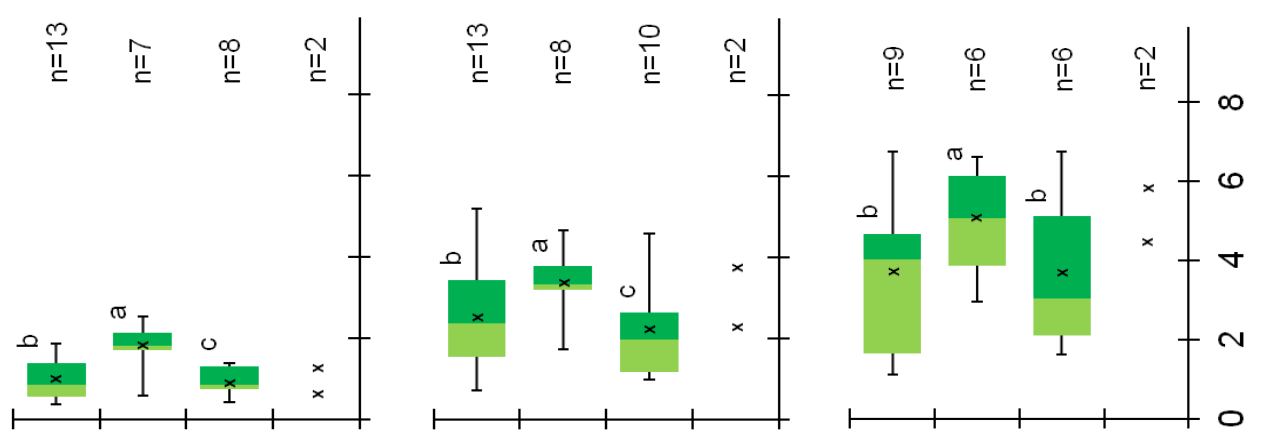

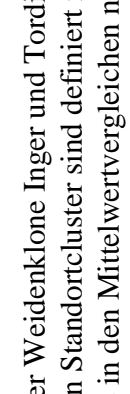

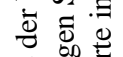

흘 훙

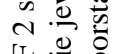

崖吕

离:

命

I

局 霆

$\sum_{0}$ 츨
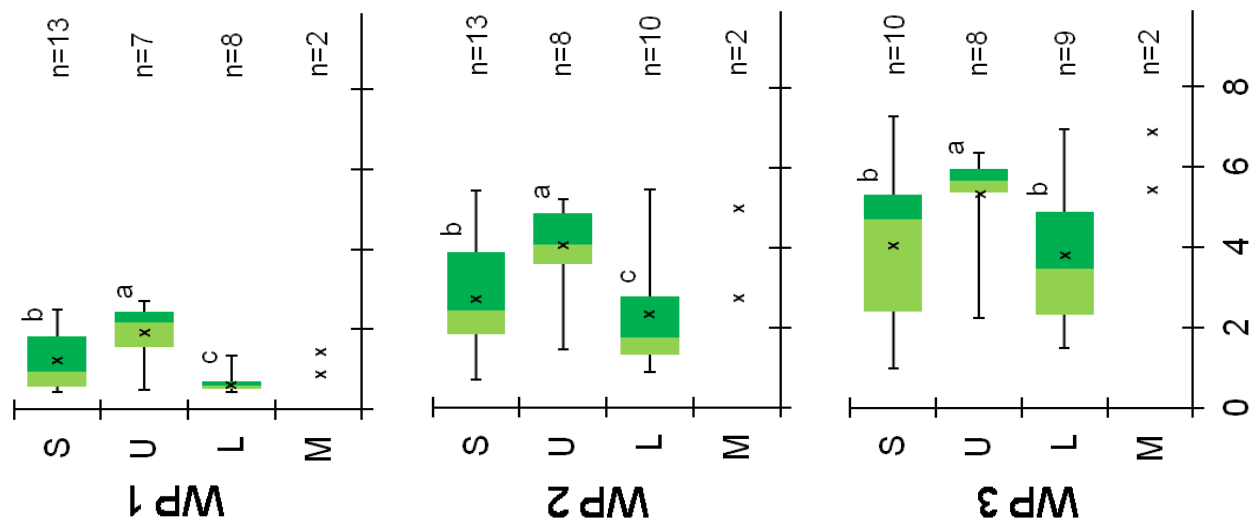

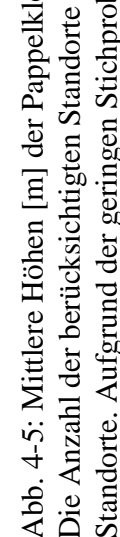


Interessanterweise hatten die zwei schwächeren Standortcluster S und L ab der zweiten Wuchsperiode ähnliche Maxima wie der Standortcluster U. Innerhalb dieser schwächeren Bodenartengruppen konnten sowohl wuchsschwache als auch wuchsstarke Standorte identifiziert werden.

Die Moorböden, die aufgrund einer deutlich geringeren Wiederholungsanzahl $(\mathrm{n}=2)$ nicht in der Varianzanalyse berücksichtigt werden konnten, wiesen bemerkenswerte Höhenleistungen auf. Der anmoorige Sto. Nr. 9 „Löwenberg“ im nördlichen Brandenburg hatte in der dritten Wuchsperiode, je nach Klon, Höhen zwischen 4,0 m und 5,5 m. Am Niedermoorstandort Nr. 3 Aulendorf waren die Höhen in der dritten Wuchsperiode mit Werten zwischen 6,0 $\mathrm{m}$ und 7,0 $\mathrm{m}$ im Mittel $30 \%$ höher als am Sto. Nr. 9 „Löwenberg“. Damit gehörte der Sto. Nr. 3 „Aulendorf“ zu einem der wuchsstärksten Standorte im Projekt. Anhang 8 kann die Höhenentwicklung für die Klone getrennt nach Bodenartcluster sowie für die Cluster getrennt nach Klon entnommen werden.

\subsubsection{Durchmesser}

Analog zu den Höhen variierten zwischen den Klonen und Standorten auch die Durchmesser stark. In der ersten Wuchsperiode wurden mittlere $\mathrm{d}_{0,1}$ zwischen 0,4 cm (Sto. Nr. 30 „Iden“, Klon H 275) und 2,6 cm (Sto. Nr. 13 „Grünewalde“, Klon AF 2) gemessen. Der stärkste Klon war AF 2, gefolgt von Inger, Max 1, Tordis und schließlich H 275 (Tab. 4-3). Bis auf Max 1 und Tordis, welche im Mittel auf demselben Niveau lagen, waren alle restlichen Klone untereinander signifikant unterschiedlich. In der ersten Wuchsperiode schwankte der $\mathrm{d}_{1,3}$ zwischen 0,2 cm (Sto. Nr. 33 „Thammenhain II“, Klon H 275) und 1,7 cm (Sto. Nr. 1 „Emmendingen“, Klon AF 2). Es muss angemerkt werden, dass bei elf Standorten mindestens ein Klon die Höhe von 1,3 m nicht erreichte und deswegen keine Messung möglich war. Über alle Standorte hinweg betrug der mittlere $d_{1,3}$ in aufsteigender Reihenfolge $0,8 \mathrm{~cm}$ bei $\mathrm{H}$ 275, 0,9 $\mathrm{cm}$ bei je Inger und Tordis, $1,0 \mathrm{~cm}$ bei Max 1 und 1,2 $\mathrm{cm}$ bei AF 2 .

In der zweiten Wuchsperiode schwankte der $\mathrm{d}_{0,1}$ zwischen 0,4 cm (Sto. Nr. 14 „Welzow Süd“, Klon Tordis) und 4,6 cm (Sto. Nr. 3 „Aulendorf“, Klon AF 2). Über alle Standorte hinweg betrug der $\mathrm{d}_{0,1}$-Mittelwert in absteigender Reihenfolge für AF $22,7 \mathrm{~cm}$, für Max $12,4 \mathrm{~cm}$, für H $2752,3 \mathrm{~cm}$, für Inger 2,3 cm und für Tordis $2,1 \mathrm{~cm}$. AF 2 wies die signifikant stärksten $\mathrm{d}_{0,1}$ auf, gefolgt von Max 1 und Inger. Tordis und $\mathrm{H} 275$ wiesen die signifikant niedrigsten $\mathrm{d}_{0,1}$ auf. Beim $\mathrm{d}_{1,3}$ variierten die Werte zwischen 0,4 cm (Sto. Nr. 14 „Welzow Süd“, Klon Max 1) und 3,5 cm (Sto. Nr. 3 „Aulendorf“, Klon AF 2). Über alle Standorte hinweg schwankte der mittlere $d_{1,3}$ zwischen $1,6 \mathrm{~cm}$ (Tordis) und $2,1 \mathrm{~cm}$ (AF 2). Zwischen den Klonen in der zweiten Wuchsperiode bot sich bei den statistischen Mittelwertvergleichen des $d_{1,3}$ ein weitestgehend ähnliches Bild wie in der ersten Wuchsperiode.

In der dritten Wuchsperiode bewegten sich die durchschnittlichen standortspezifischen $\mathrm{d}_{0,1}$ zwischen $1,0 \mathrm{~cm}$ (Sto. Nr. 14 „Welzow Süd“, Klon Tordis) und 6,0 cm (Sto. Nr. 3 „Aulendorf“, Klon Max 1) (Tab. 4-4). Dabei wiesen die Pappeln stets signifikant höhere Werte als die Weiden auf. Innerhalb der Gattungen waren die Unterschiede zwischen den Klonen signifikant. Die Mittelwerte betrugen in absteigender Reihenfolge für AF 2 4,1 cm, für Max $13,8 \mathrm{~cm}$, für H $2753,6 \mathrm{~cm}$, für Inger 3,2 cm und für Tordis 3,1 cm. Die Werte des $d_{1,3}$ bewegten sich standortspezifisch für Max 1 zwischen 0,4 und $4,7 \mathrm{~cm}$, für $\mathrm{H} 275$ zwischen 0,7 und 4,4 cm, für AF 2 zwischen 0,7 und $5,0 \mathrm{~cm}$, für Inger zwischen 0,6 und 3,7 cm und für Tordis zwischen 0,5 und 3,4 cm. Der Pappelklon AF 2 war mit einem mittleren $\mathrm{d}_{1,3}$ von 3,2 cm signifikant stärker, gefolgt von Max 1 mit 2,8 cm und schließlich H 275 mit 2,6 cm. Die Werte des $\mathrm{d}_{1,3}$ der Weiden unterschieden sich signifikant von denen der Pappeln. Der mittlere $\mathrm{d}_{1,3}$ der Weiden betrug für Inger 2,4 cm und für Tordis $2,3 \mathrm{~cm}$. Sie lagen statistisch auf demselben Niveau. 
Tab. 4-3: Mittlere $\mathrm{d}_{0,1}[\mathrm{~cm}]$ der Pappelklone Max 1, H 275 und AF 2 sowie der Weidenklone Inger und Tordis in den Wuchsperioden eins bis drei unter Berücksichtigung aller Standorte (Standardabweichung in Klammern, ungleiche Buchstaben zeigen innerhalb der Wuchsperiode signifikante Unterschiede auf dem p-Niveau < 0,05).

\begin{tabular}{|c|c|c|c|c|c|c|c|c|c|c|c|c|c|}
\hline \multirow{3}{*}{ Gattung } & \multirow{3}{*}{ Klon } & \multicolumn{12}{|c|}{ Mittlerer $\mathrm{d}_{0,1}[\mathrm{~cm}]$} \\
\hline & & \multicolumn{4}{|c|}{ WP 1} & \multicolumn{4}{|c|}{ WP 2} & \multicolumn{4}{|c|}{ WP 3} \\
\hline & & $\mathrm{n}$ & $\varnothing$ & s.a. & & $\mathrm{n}$ & $\varnothing$ & s.a. & & $\mathrm{n}$ & $\varnothing$ & s.a. & \\
\hline \multirow{3}{*}{ Pappel } & Max 1 & 29 & 1,1 & $(0,7)$ & $\mathrm{c}$ & 31 & 2,4 & $(1,3)$ & $\mathrm{b}$ & 28 & 3,8 & $(1,7)$ & $\mathrm{b}$ \\
\hline & Н 275 & 29 & 1,0 & $(0,7)$ & d & 31 & 2,3 & $(1,4)$ & $\mathrm{c}$ & 23 & 3,6 & $(1,7)$ & $\mathrm{c}$ \\
\hline & $\mathrm{AF} 2$ & 29 & 1,4 & $(0,7)$ & $\mathrm{a}$ & 31 & 2,7 & $(1,4)$ & $\mathrm{a}$ & 27 & 4,1 & $(1,7)$ & $\mathrm{a}$ \\
\hline \multirow{2}{*}{ Weide } & Inger & 29 & 1,1 & $(0,5)$ & $\mathrm{b}$ & 31 & 2,3 & $(1,1)$ & $\mathrm{b}$ & 28 & 3,2 & $(1,3)$ & $\mathrm{d}$ \\
\hline & Tordis & 29 & 1,1 & $(0,6)$ & $\mathrm{c}$ & 31 & 2,1 & $(1,0)$ & $\mathrm{d}$ & 26 & 3,1 & $(1,3)$ & $\mathrm{e}$ \\
\hline
\end{tabular}

Tab. 4-4: Mittlere $\mathrm{d}_{1,3}[\mathrm{~cm}]$ der Pappelklone Max 1, H 275 und AF 2 sowie der Weidenklone Inger und Tordis in den Wuchsperioden eins bis drei unter Berücksichtigung aller Standorte (Standardabweichung in Klammern, ungleiche Buchstaben zeigen innerhalb der Wuchsperiode signifikante Unterschiede auf dem p-Niveau < 0,05).

\begin{tabular}{|c|c|c|c|c|c|c|c|c|c|c|c|c|c|}
\hline \multirow{3}{*}{ Gattung } & \multirow{3}{*}{ Klon } & \multicolumn{12}{|c|}{ Mittlerer $\mathrm{d}_{1,3}[\mathrm{~cm}]$} \\
\hline & & \multicolumn{4}{|c|}{ WP 1} & \multicolumn{4}{|c|}{ WP 2} & \multicolumn{4}{|c|}{ WP 3} \\
\hline & & $\mathrm{n}$ & $\varnothing$ & s.a. & & $\mathrm{n}$ & $\varnothing$ & s.a. & & $\mathrm{n}$ & $\varnothing$ & s.a. & \\
\hline \multirow{3}{*}{ Pappel } & $\operatorname{Max} 1$ & 29 & 1,0 & $(0,4)$ & b & 31 & 1,8 & $(1,1)$ & $\mathrm{b}$ & 28 & 2,8 & $(1,5)$ & $\mathrm{b}$ \\
\hline & Н 275 & 29 & 0,8 & $(0,5)$ & d & 31 & 1,6 & $(1,0)$ & d & 23 & 2,6 & $(1,4)$ & $\mathrm{c}$ \\
\hline & $\mathrm{AF} 2$ & 29 & 1,2 & $(0,5)$ & $\mathrm{a}$ & 31 & 2,1 & $(1,2)$ & $\mathrm{a}$ & 27 & 3,2 & $(1,5)$ & $\mathrm{a}$ \\
\hline \multirow{2}{*}{ Weide } & Inger & 29 & 0,9 & $(0,4)$ & $\mathrm{c}$ & 31 & 1,7 & $(0,8)$ & $\mathrm{b}$ & 28 & 2,4 & $(1,1)$ & d \\
\hline & Tordis & 29 & 0,9 & $(0,4)$ & $\mathrm{c}$ & 31 & 1,6 & $(0,8)$ & $\mathrm{c}$ & 26 & 2,3 & $(1,0)$ & d \\
\hline
\end{tabular}

Über alle Wuchsperioden hinweg entwickelten die Pappeln stärkere $\mathrm{d}_{0,1}$ und $\mathrm{d}_{1,3}$ als die Weiden. Der Klon AF 2 hatte stets signifikant die höchsten Werte, gefolgt von Max 1 und H 275. Die Weidenklone Inger und Tordis zeigten untereinander weder in den $\mathrm{d}_{0,1}$ noch im $\mathrm{d}_{1,3}$ signifikante Unterschiede. Das mittlere jährliche Durchmesserwachstum $\left(\mathrm{d}_{1,3}\right)$ der Pappeln betrug ca. 1,0 cm, das der Weiden hingegen 0,7 bis $0,9 \mathrm{~cm}$. Der Unterschied zwischen dem Durchmesserwachstum von $\mathrm{d}_{1,3}$ und $\mathrm{d}_{0,1}$ liegt bei 20 bis $40 \%$, wobei das $d_{0,1}$-Wachstum höher ausfiel als das $d_{1,3}$-Wachstum.

Abbildungen 4-6 und 4-7 zeigen, differenziert nach Klon, Wuchsperiode und Standortclustern die $\mathrm{d}_{0,1}$ und $\mathrm{d}_{1,3}$-Verteilungen. Das standortabhängige Verhalten, welche bereits bei den Höhen analysiert wurde, findet sich bei den $\mathrm{d}_{0,1^{-}}$und $\mathrm{d}_{1,3}$-Dimensionen weitestgehend wieder. Anhänge 9 und $10 \mathrm{kann}$ die $\mathrm{d}_{0,1^{-}}$und $\mathrm{d}_{1,3}$-Entwicklung für die Klone getrennt nach Bodenartcluster sowie für die Bodenartcluster getrennt nach Klon entnommen werden. Eine Übersicht der Verhältnisse Höhen $/ \mathrm{d}_{1,3}, \mathrm{Höhe} / \mathrm{d}_{0,1}$ sowie $\mathrm{d}_{1,3} / \mathrm{d}_{0,1}$ in Abhängigkeit der durchschnittlichen Bestandeshöhe kann im Anhang 11 bis 13 gefunden werden. 

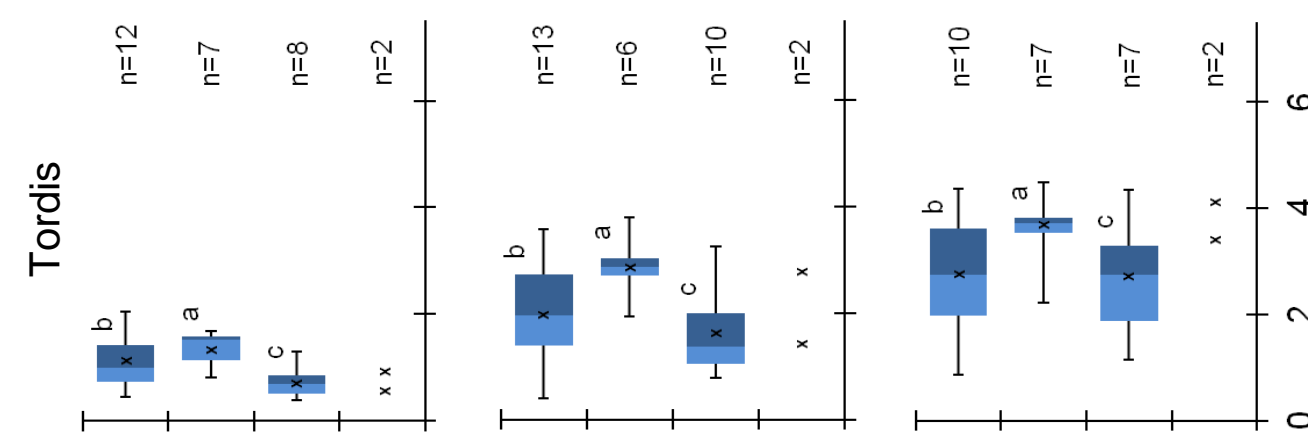

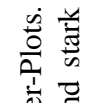
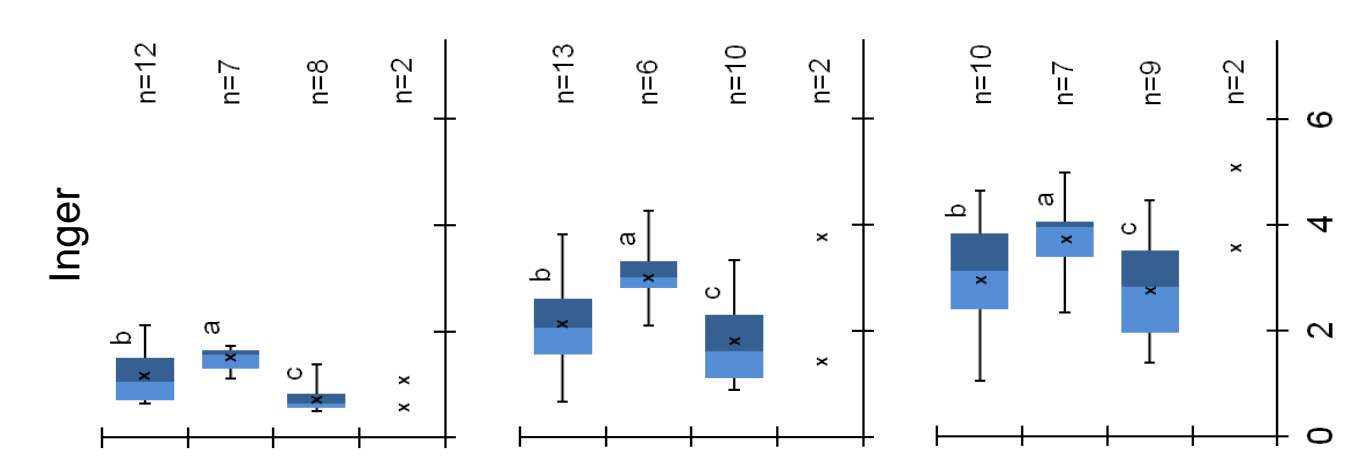

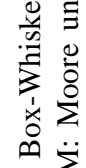

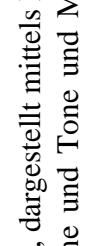

离总

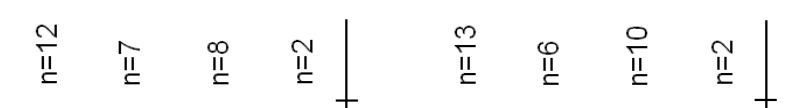

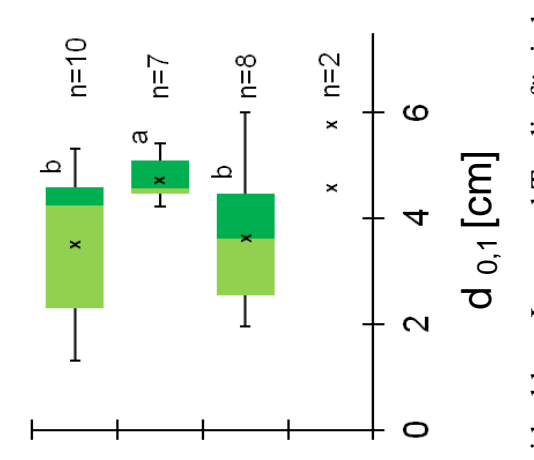

渮

要要

응
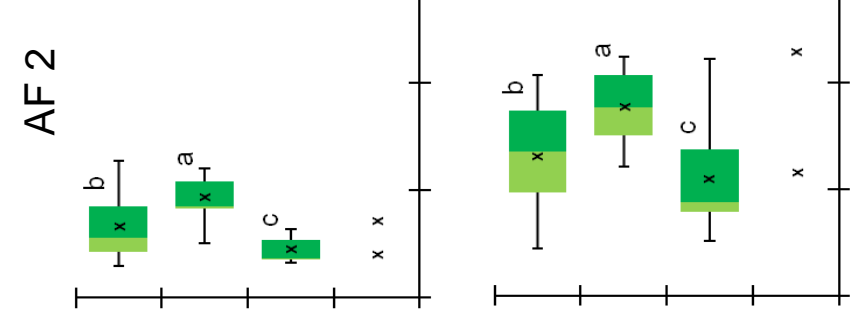

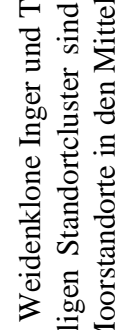

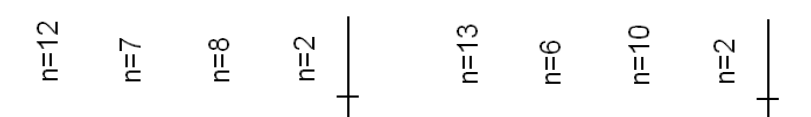

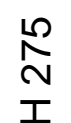
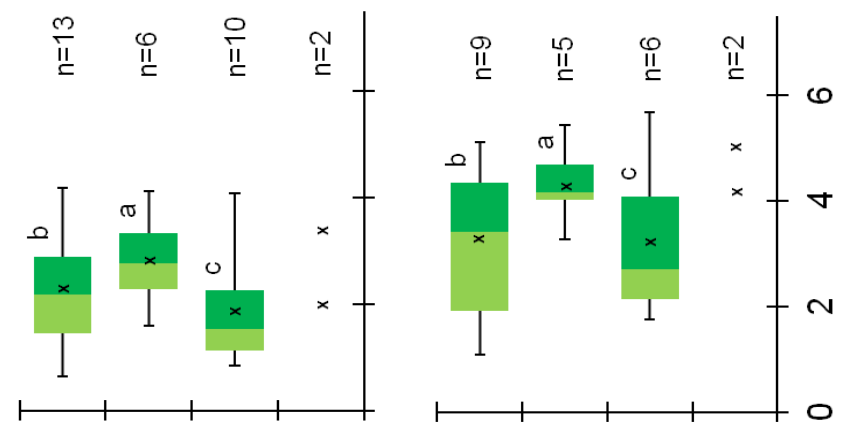

$\overline{\mathrm{s}} \overline{\mathrm{s}}$

?.

空

至宕产

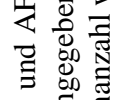

命

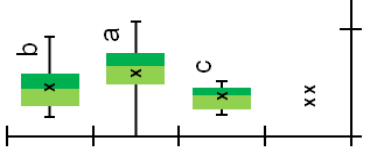

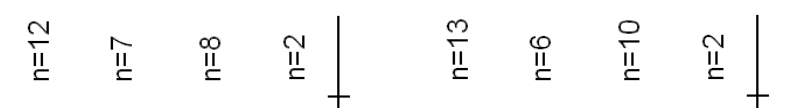
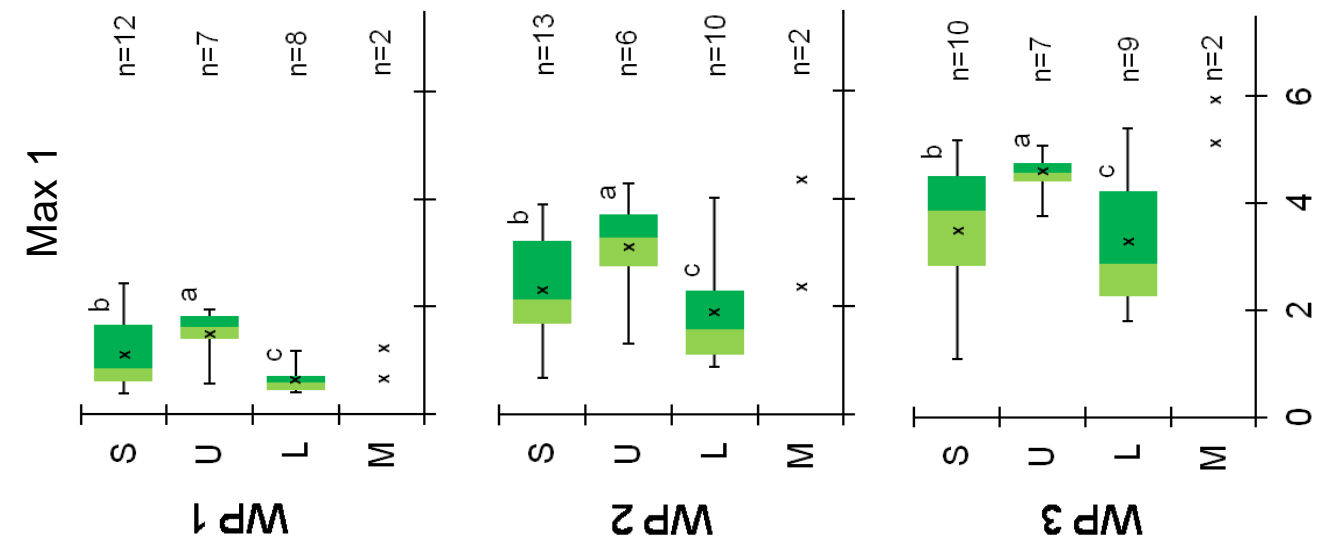

王

政

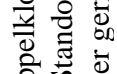

क्षे के

氜总总

割:

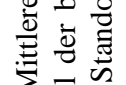

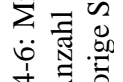

䢓品 言 


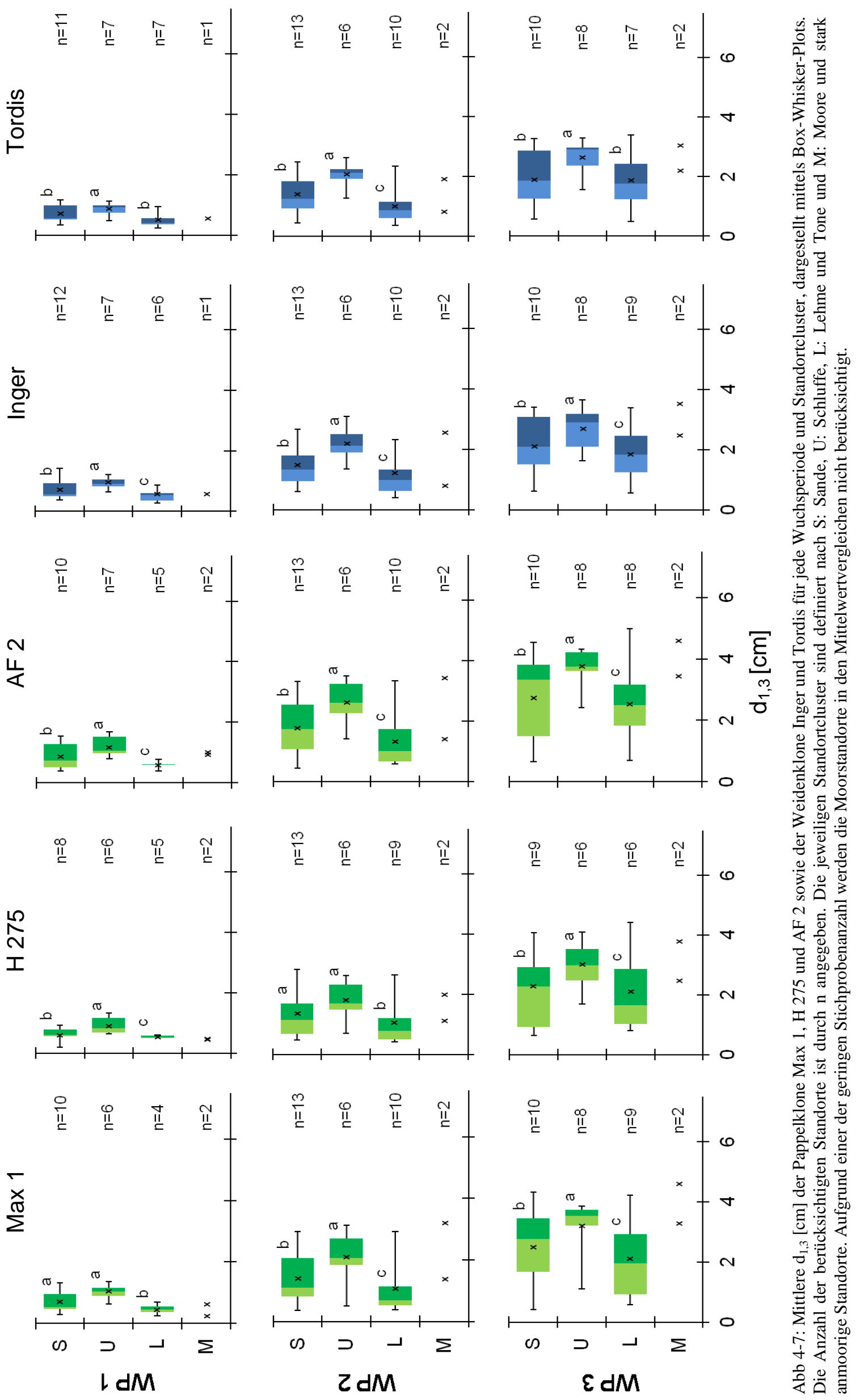




\subsubsection{Durchschnittlicher Gesamtzuwachs}

In den verschiedenen Wuchsperioden und entlang der Klone gestaltete sich der Zuwachs unterschiedlich (Tab. 4-5, Abb. 4-8). In der ersten Wuchsperiode wurde der Höchstwert am Sto. Nr. 19 „Werlte“ für den Klon Tordis mit einem dGZ von 6,9 $\mathrm{t}_{(\text {atro) }} \mathrm{ha}^{-1} \mathrm{a}^{-1}$ registriert. Auf einigen Standorten war in der ersten Wuchsperiode die Massenleistung mit $<0,1 \mathrm{t}_{\text {(atro) }} \mathrm{ha}^{-1} \mathrm{a}^{-1}$ marginal. Über alle Versuchsflächen hinweg betrug der dGZ in der ersten Wuchsperiode, differenziert nach Klon, im Mittel zwischen 0,6 und 1,2 $\mathrm{t}_{\text {(atro) }} \mathrm{ha}^{-1} \mathrm{a}^{-1}$. Dabei war die Leistung des Pappelklons H 275 signifikant am niedrigsten und die von Inger am höchsten. Insgesamt wiesen die Weiden den höchsten dGZ in der ersten Wuchsperiode auf. In der zweiten Wuchsperiode schwankten die dGZ einzelner Klone zwischen $0,1 \mathrm{t}_{\text {(atro) }} \mathrm{ha}^{-1} \mathrm{a}^{-1}$ (u. a. Sto. Nr. 38, ,Heiliges Marpingen“) und 10,1 $\mathrm{t}_{\text {(atro) }} \mathrm{ha}^{-1} \mathrm{a}^{-1}$. Der mittlere dGZ über alle Standorte schwankte zwischen 2,0 und 3,8 $\mathrm{t}_{\text {(atro) }} \mathrm{ha}^{-1} \mathrm{a}^{-1}$. Der Weidenklon Tordis hatte den höchsten dGZ, gefolgt von Inger und AF 2. Zusammen bildeten diese drei Klone eine homogene Untergruppe (Tab. 4-5). Max 1 und H 275 waren dagegen die schwächsten Prüfglieder. Die Ertragssteigerung von der ersten zur zweiten Wuchsperiode fiel mit bis zu $350 \%$ sehr hoch aus.

Tab. 4-5: Mittlere dGZ $\left[\mathrm{t}_{\text {(atro) }} \mathrm{ha}^{-1} \mathrm{a}^{-1}\right]$ der Pappelklone Max 1, H 275 und AF 2 sowie der Weidenklone Inger und Tordis in den Wuchsperioden eins bis drei unter Berücksichtigung aller Standorte (Standardabweichung in Klammern, ungleiche Buchstaben indizieren signifikante Unterschiede innerhalb der Wuchsperiode auf dem p-Niveau $<0,05$ ).

\begin{tabular}{|c|c|c|c|c|c|c|c|c|c|c|c|c|c|}
\hline \multirow{3}{*}{ Gattung } & \multirow{3}{*}{ Klon } & \multicolumn{12}{|c|}{ Mittlerer dGZ $\left[\mathrm{t}_{(\mathrm{atro})} \mathrm{ha}^{-1} \mathrm{a}^{-1}\right]$} \\
\hline & & \multicolumn{4}{|c|}{ WP 1} & \multicolumn{4}{|c|}{ WP 2} & \multicolumn{4}{|c|}{ WP 3} \\
\hline & & $\mathrm{n}$ & $\varnothing$ & s.a. & & $\mathrm{n}$ & $\varnothing$ & s.a. & & $\mathrm{n}$ & $\varnothing$ & s.a. & \\
\hline \multirow{3}{*}{ Pappel } & Max 1 & 29 & 0,9 & $(1,3)$ & d & 31 & 2,5 & $(2,7)$ & $\mathrm{b}$ & 28 & 4,7 & $(3,5)$ & $\mathrm{b}$ \\
\hline & Н 275 & 29 & 0,6 & $(1,4)$ & $\mathrm{e}$ & 31 & 2,0 & $(2,3)$ & $\mathrm{c}$ & 23 & 3,7 & $(3,3)$ & $\mathrm{c}$ \\
\hline & AF 2 & 29 & 1,1 & $(1,3)$ & $\mathrm{c}$ & 31 & 3,1 & $(3,1)$ & $\mathrm{a}$ & 27 & 4,8 & $(3,2)$ & a \\
\hline \multirow{2}{*}{ Weide } & Inger & 29 & 1,2 & $(1,5)$ & $\mathrm{a}$ & 31 & 3,1 & $(3,0)$ & $\mathrm{a}$ & 28 & 4,4 & $(3,4)$ & $\mathrm{b}$ \\
\hline & Tordis & 29 & 1,2 & $(1,9)$ & $\mathrm{b}$ & 31 & 3,8 & $(4,2)$ & $\mathrm{a}$ & 26 & 4,8 & $(3,8)$ & $\mathrm{a}$ \\
\hline
\end{tabular}

In der dritten Wuchsperiode sind Wuchsleistungen von 0,1 bis $11,1 \mathrm{t}_{\text {(atro) }} \mathrm{ha}^{-1} \mathrm{a}^{-1}$ ermittelt worden. Die niedrigsten dGZ wurden in den Bergbaufolgelandschaften „Energiewald Welzow“ (Sto. Nr. 8) und „Welzow Süd“ (Sto. Nr. 14) sowie am stark versauerten ( $\mathrm{pH}=3,8)$ Sto. Nr. 30 „Iden“ beobachtet. Zu den leistungsstärksten Standorten gehörten gleichermaßen die Lößböden (zum Beispiel Sto. Nr. 28. „Bernburg“), unter optimalen Klimabedingungen die lehmigen Sande (zum Beispiel Sto. Nr. 17 „Unterrieden“) sowie die Niedermoorstandorte (zum Beispiel Sto. Nr. 3 „Aulendorf"). Über alle Standorte hinweg bewegten sich in der dritten Wuchsperiode die mittleren dGZ, differenziert nach Klon, zwischen 4,1 und5, $0 \mathrm{t}_{\text {(atro) }}$ ha $^{-1} \mathrm{a}^{-1}$. Die ertragsstärksten Klone waren Tordis, Max 1 und AF 2. Zwar wurde nachgewiesen, dass AF 2 die signifikant größten Dimensionen (Höhe, $d_{1,3}$ und $d_{0,1}$ ) aufwies, jedoch überschritten dessen dGZ nicht immer die der anderen Pappelklone.

Die zeitliche Entwicklung der dGZ wird in Abbildung 4-8 anhand der Mittelwerte je Cluster und Klon für jede Wuchsperiode dargestellt. In Abbildung 4-9 sind Whiskers-Box-Plots der erzielten dGZ für alle Klone, Standortclustern und Wuchsperioden zu finden. Die Ertragssteigerung von der zweiten auf die dritte Wuchsperiode fiel, für alle Standorte gleichzeitig betrachtet, deutlich mäßiger aus im Vergleich zur Steigerung der ersten auf die zweite Wuchsperiode. Erstere betrug bei der Pappel bis zu $100 \%$, bei der Weide lag sie dagegen zwischen 10 bis $50 \%$. Auf einigen Standorten blieb der dGZ von der zweiten auf die dritte Wuchsperiode bei den Weiden annähernd unverändert.

Während einige Klone in der zweiten Wuchsperiode im Standortcluster S bei den Bestandesdimensionen dem Standortclusters L glichen, konnte dies beim dGZ nicht bestätigt werden. Der dGZ der zwei- 
ten Wuchsperiode war bei allen Klonen im Standortcluster S den schweren Böden im Standortcluster L signifikant überlegen. Die Ertragssteigerung von der zweiten auf die dritte Wuchsperiode war im Standortcluster L am höchsten. Erst in dieser Wuchsperiode erreichten die Klone, mit Ausnahme von AF 2, die Leistung des Standortclusters S.

Auf den Schluffböden wurden die stärksten dGZ-Zunahmen von der ersten auf die zweite Wuchsperiode gemessen. Der dGZ von Tordis blieb sogar von der zweiten auf die dritten quasi unverändert, für Inger wurde eine ca. $15 \%$-ige dGZ-Zunahme registriert. Die Ertragssteigerung verlief beim Standortcluster L am langsamsten, mit einem für alle Klone eher exponentiellen Verlauf. Dagegen war im Standortcluster S die dGZ-Entwicklung näher an einem linearen Verlauf, insbesondere bei den Weiden.

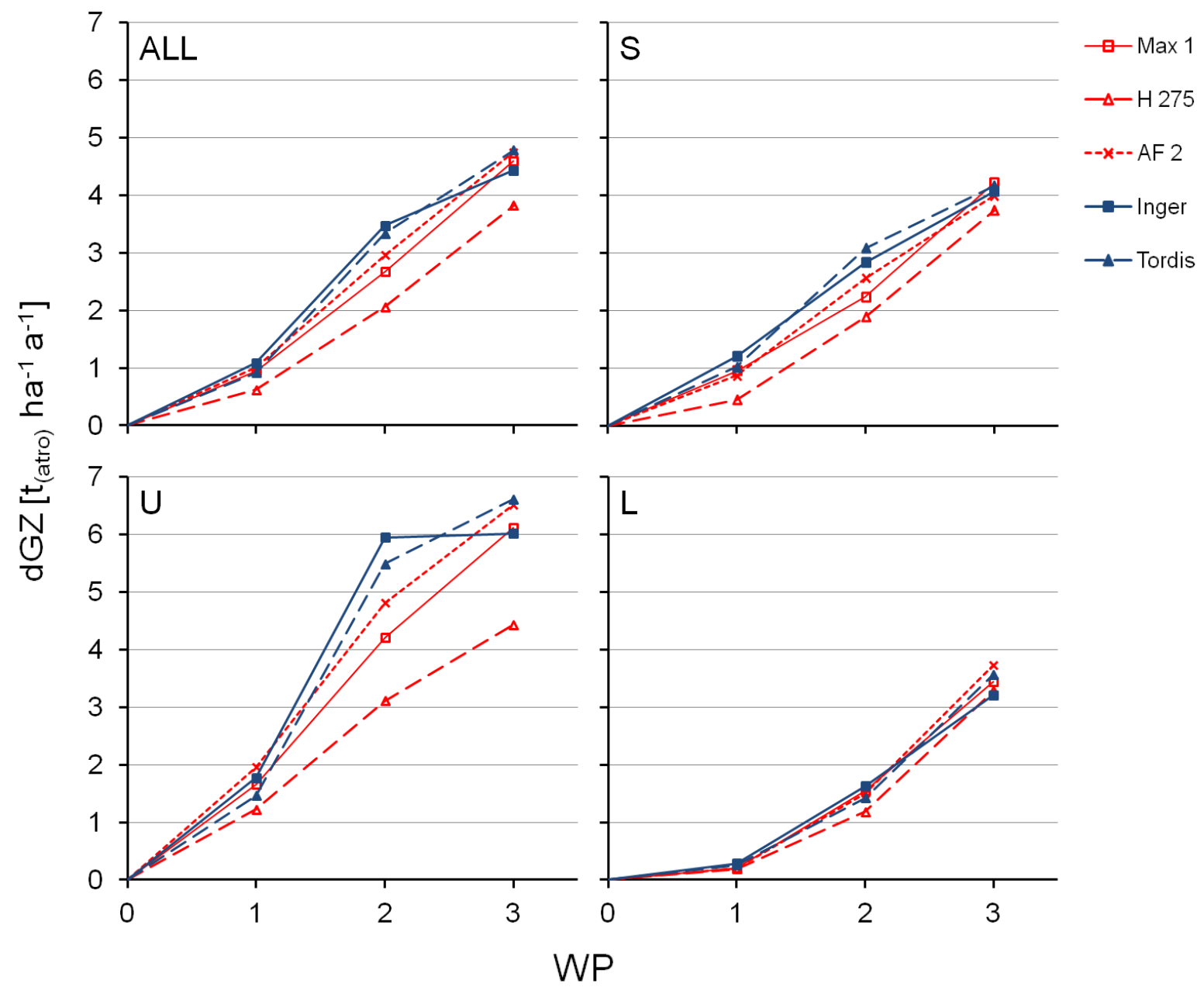

Abb. 4-8: dGZ-Entwicklung der Pappelklone Max 1, H 275 und AF 2 sowie der Weidenklone Inger und Tordis in den jeweiligen Standortclustern. 

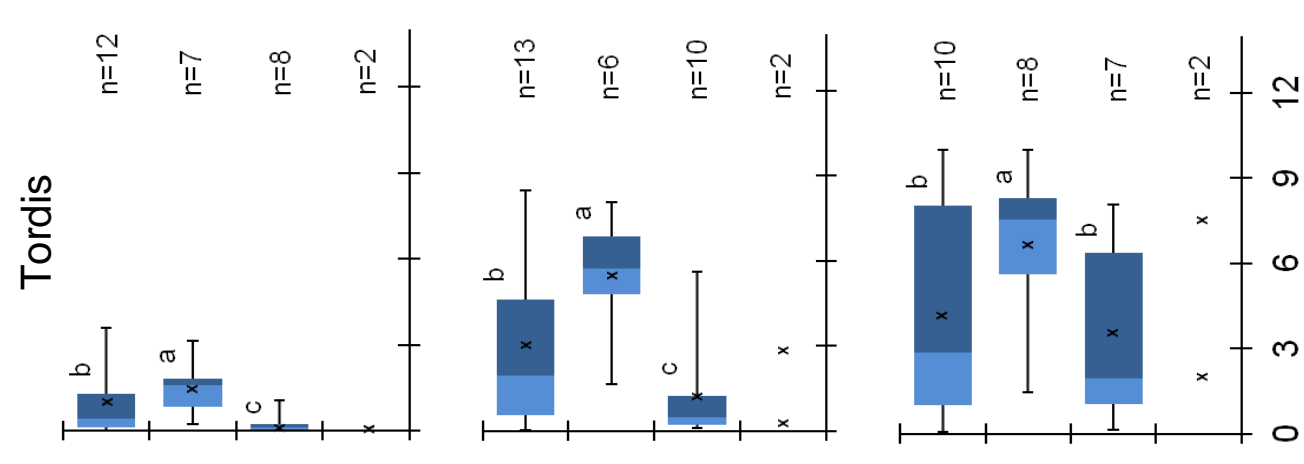

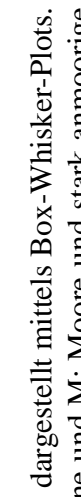
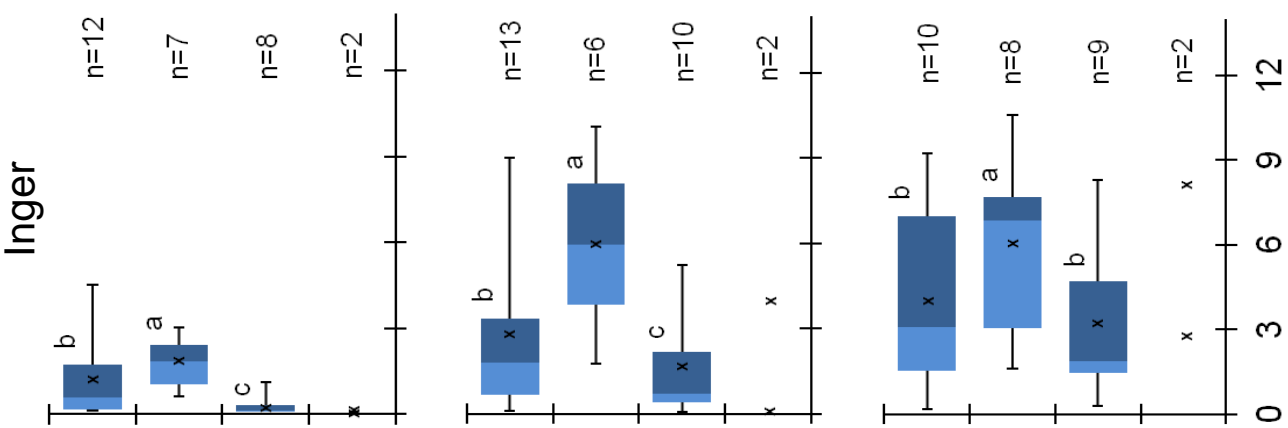

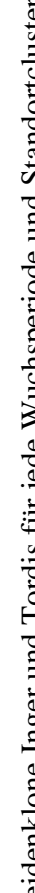

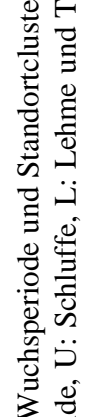

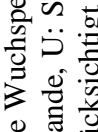

o जी

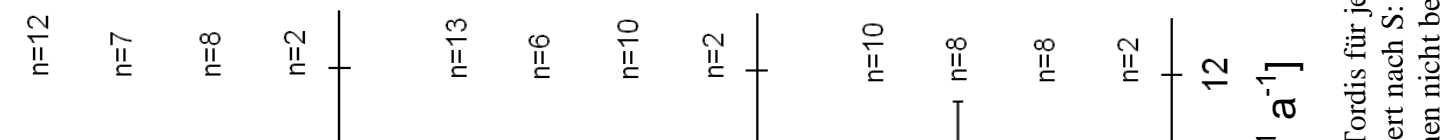
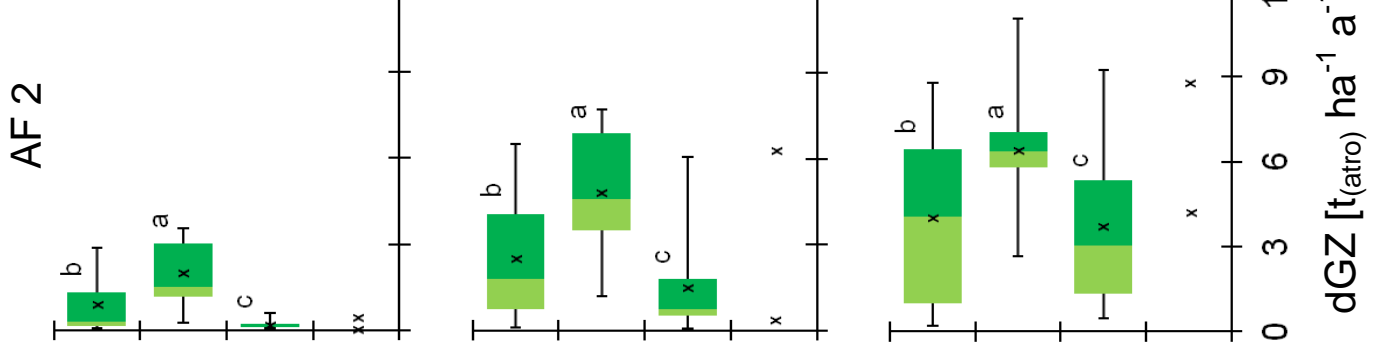

焉

总 $\overrightarrow{0}$

它

क्ष

㱐
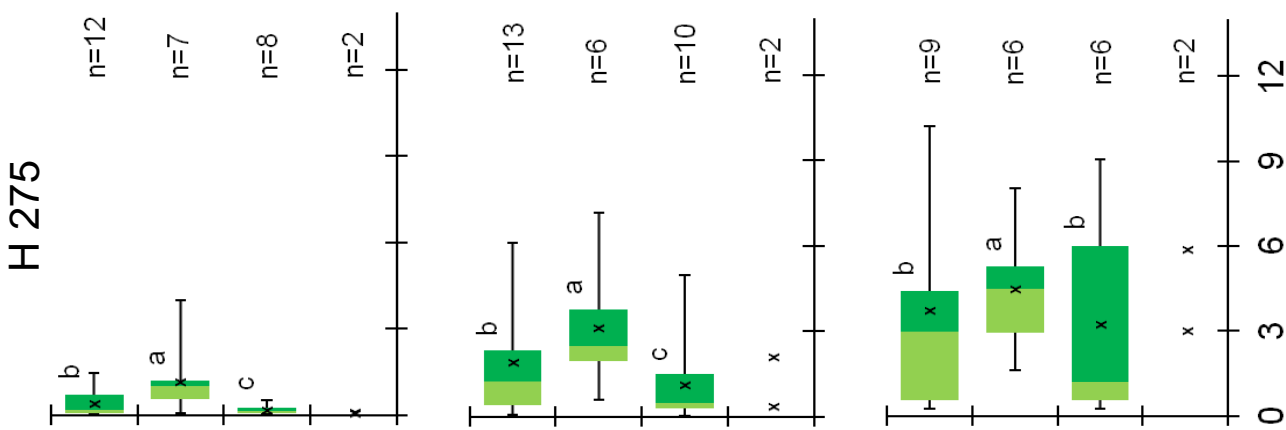

क्षे

을

ㄱ.

泶虽亯

音宁:

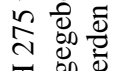

I

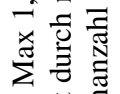

语

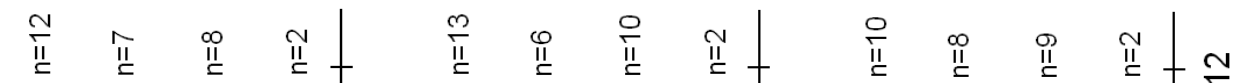

잉
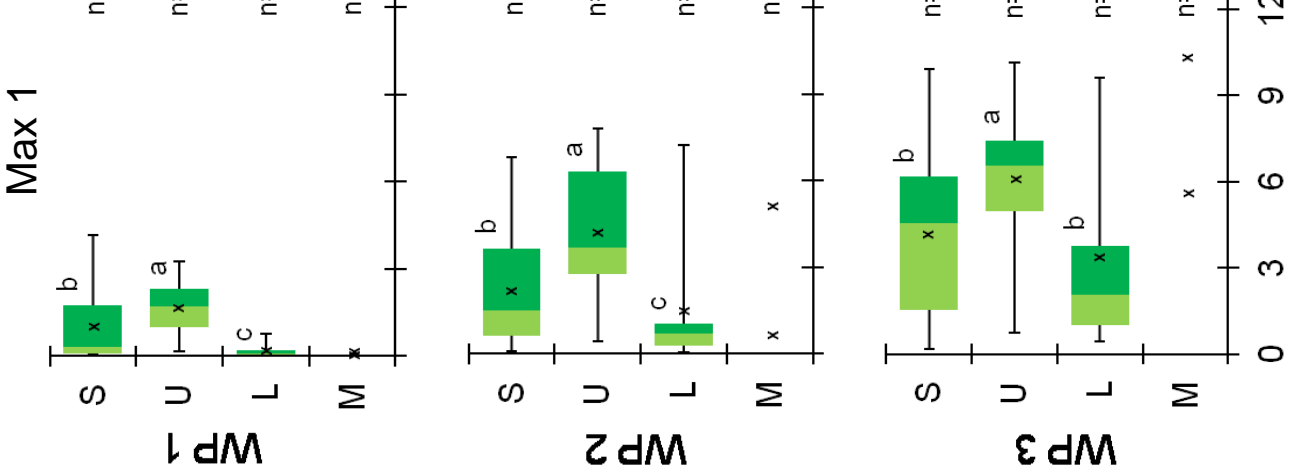

可

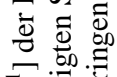

$7 \frac{100}{5}$

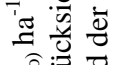

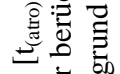

N仓웡

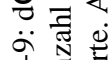

+

榇 
Zwischen den Klonen in den unterschiedlichen Standortclustern wurden Varianzanalysen durchgeführt (Tab. 4-6). Einige Standorte konnten in den Standortclustern für die Modellierung nicht berücksichtigt werden (siehe 3.6), daher sind die Ergebnisse der Varianzanalyse bei Berücksichtigung der Standortcluster für die Modellierung extra in Tabelle 4-6 aufgeführt. Die Ergebnisse der Varianzanalyse zeigen, dass der Faktor Klon einen signifikanten Einfluss in der Ertragsbildung in den Standortclustern U und L (nur Modellierungscluster) hat. Beim Standortcluster U waren Tordis und AF 2 den weiteren Klonen überlegen. Beim Standortcluster U der Modellierung war einzig Tordis stärker als die anderen Klone. Im Standortcluster L der Modellierung wurden zwei homogene Gruppen festgestellt: die stärkere Gruppe mit Max 1 und AF 2 und die schwächere Gruppe mit Max 1 und Inger.

Tab. 4-6: Mittlerer dGZ der Pappelklone Max 1, H 275, AF 2 und der Weidenklone Inger und Tordis im Standortcluster S, U und $\mathrm{L}$ für alle sowie für die in der Modellierung berücksichtigten Standorte (ungleiche Buchstaben zeigen signifikante Unterschiede innerhalb der Prüfglieder eines Standort auf dem p-Niveau < 0,05, H 275 wurde aufgrund einer kleineren Wiederholungsanzahl gegenüber den restlichen Prüfgliedern nicht in der Varianzanalyse mit Mittelwertvergleich berücksichtigt).

\begin{tabular}{|c|c|c|c|c|c|c|c|c|c|c|c|c|c|}
\hline \multirow{4}{*}{ Gattung } & \multirow{4}{*}{ Klon } & \multicolumn{12}{|c|}{$\mathrm{dGZ}\left[\mathrm{t}_{(\mathrm{atro})} \mathrm{ha}^{-1} \mathrm{a}^{-1}\right]$} \\
\hline & & \multicolumn{6}{|c|}{ Standortcluster } & \multicolumn{6}{|c|}{ Standortcluster Modellierung } \\
\hline & & \multicolumn{2}{|c|}{ S } & \multicolumn{2}{|c|}{$\mathrm{U}$} & \multicolumn{2}{|c|}{$\mathrm{L}$} & \multicolumn{2}{|c|}{$S$} & \multicolumn{2}{|c|}{$\mathrm{U}$} & \multicolumn{2}{|c|}{$\mathrm{L}$} \\
\hline & & $\mathrm{n}$ & $\varnothing$ & $\mathrm{n}$ & $\varnothing$ & $\mathrm{n}$ & $\varnothing$ & $\mathrm{n}$ & $\varnothing$ & $\mathrm{n}$ & $\varnothing$ & $\mathrm{n}$ & $\varnothing$ \\
\hline \multirow{2}{*}{ Pappel } & Max 1 & 10 & $4,22 \mathrm{a}$ & 8 & $6,11 \mathrm{~b}$ & 9 & $3,44 \mathrm{a}$ & 8 & $5,34 \mathrm{a}$ & 5 & $7,14 \mathrm{~b}$ & 7 & $5,25 a b$ \\
\hline & AF 2 & 10 & $3,99 \mathrm{a}$ & 8 & $6,52 \mathrm{a}$ & 8 & - & 8 & $5,22 \mathrm{a}$ & 5 & $7,54 \mathrm{~b}$ & 7 & $5,34 \mathrm{a}$ \\
\hline \multirow{2}{*}{ Weide } & Inger & 10 & $4,07 \mathrm{a}$ & 8 & $6,02 \mathrm{~b}$ & 9 & $3,21 \mathrm{a}$ & 8 & $5,10 \mathrm{a}$ & 5 & $7,08 \mathrm{~b}$ & 7 & $4,79 \mathrm{~b}$ \\
\hline & Tordis & 10 & $4,16 \mathrm{a}$ & 8 & $6,82 \mathrm{a}$ & 5 & - & 8 & $5,31 \mathrm{a}$ & 5 & $8,33 \mathrm{a}$ & 5 & - \\
\hline
\end{tabular}

- Mittelwerte, welche gegenüber dem Rest der Prüfglieder auf einer geringeren Stichprobenanzahl basieren, wurden in dieser Darstellung nicht berücksichtigt.

Anhang 14 bis 16 können Ergebnisse einer Varianzanalyse für den dGZ der dritten Wuchsperiode für jeden Standort in den jeweiligen Standortcluster entnommen werden.

\subsubsection{Biomassefunktionen}

Für die Ermittlung des dGZ nach der ersten und zweiten Wuchsperiode wurden Biomassefunktionen verwendet. In diesem Kapitel sind detaillierte Ausführungen zu den entwickelten Funktionen zu finden. Wie im Kapitel 3.4.5.1 erwähnt, wurden ausschließlich Funktionen mit allometrischem Ansatz verwendet.

\subsubsection{Wahl der unabhängigen Variablen}

Zuerst wurde die Verwendung des $\mathrm{d}_{0,1}$ oder des $\mathrm{d}_{1,3}$ als unabhängige Variable in der Funktionsbildung untersucht. Tendenziell wiesen die $\mathrm{d}_{0,1}$-Funktionen geringere $\mathrm{R}^{2}$ korr als die $\mathrm{d}_{1,3}$-Funktionen auf. Dieser Unterschied war statistisch jedoch nicht signifikant. Innerhalb der $\mathrm{d}_{1,3}$-Funktionen konnte beobachtet werden, dass mit zunehmender Bestandesmittelhöhe das Bestimmtheitsmaß zunahm (Abb. 4-10). Die $\mathrm{R}_{\text {korr }}^{2}$ der $\mathrm{d}_{1,3}$-Funktionen in Beständen mit mittleren Höhen größer 3,0 $\mathrm{m}$ waren häufig geringfügig größer als jene der $\mathrm{d}_{0,1}$-Funktionen. Die statistischen Kennwerte der einzelnen Biomassefunktionen können den Anhängen 17 bis 26 entnommen werden. 
a)

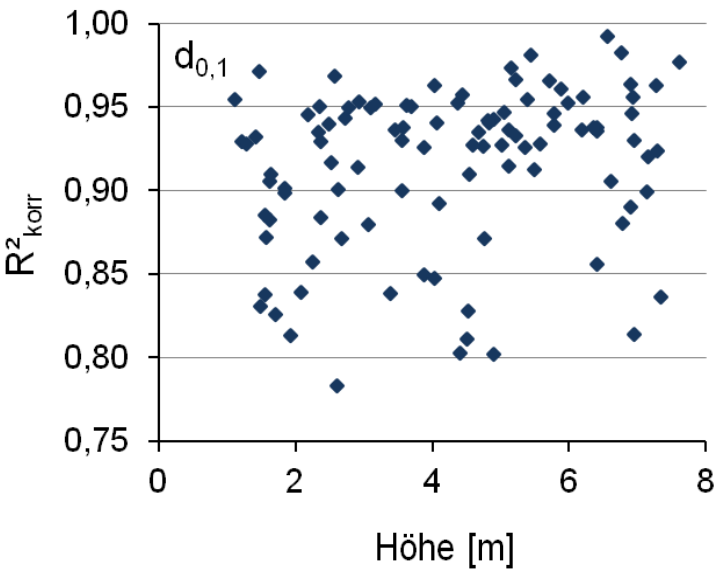

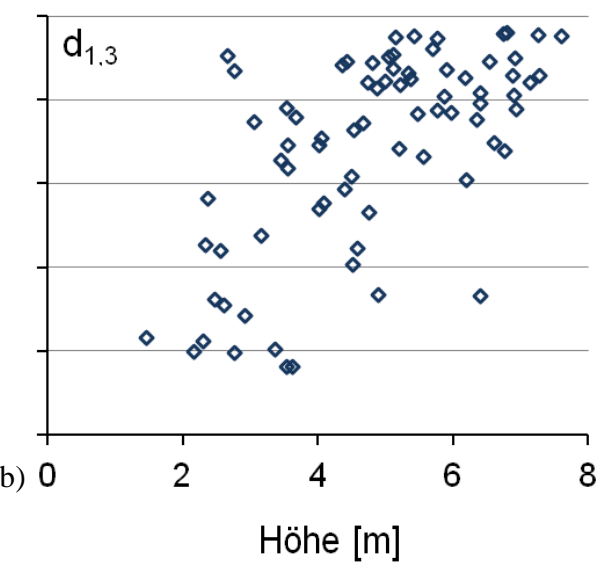

Höhe [m]

Abb. 4-10: Gegenüberstellung der mittleren Höhe [m] mit dem $\mathrm{R}^{2}{ }_{\text {korr }}$ der Biomassefunktionen aller untersuchten Prüfglieder mit jeweils a) $d_{0,1}$ und b) $d_{1,3}$ als unabhängige Variable.

\subsubsection{Einfluss der Gattung und des Klons}

Zwischen Pappel- und Weidenbiomassefunktionen konnten keine statistisch signifikanten Unterschiede festgestellt werden. Aufgrund der physiologischen Unterschiede wurden jedoch beide Prüfgliedergruppen für weitere Auswertungen nach Gattungen getrennt analysiert. Unter den Pappelklonen wurde ein Effekt des Klons beim Koeffizient $\mathrm{a}_{0}$ festgestellt (Tab. 4-7). Nachgeschaltete Tests ergaben einen signifikanten Unterschied beim $\mathrm{a}_{0}$ zwischen AF 2 und den restlichen zwei Pappelklonen, Max 1 und H 275. Obwohl die Biomassefunktion des Weidenklon Tordis einen optisch gut erkennbaren, steileren Verlauf im Vergleich zum Inger hatte, konnte kein signifikanter Unterschied festgestellt werden. Der berechnete $\mathrm{p}$-Wert der Varianzanalyse von $a_{1}$ war mit 0,065 jedoch sehr gering (Tab. 4-7).

Tab. 4-7: Varianzanalyse der Funktionskoeffizienten $\mathrm{a}_{0}$ und $\mathrm{a}_{1}$ für die Testgrößen Klon, Gattung, Klon in der jeweiligen Gattung sowie zwischen den einzelnen Pappelklonen, dargestellt durch den p-Wert.

\begin{tabular}{lcc}
\hline \multirow{2}{*}{ Testgröße } & \multicolumn{2}{c}{$\mathrm{p}$-Wert } \\
\cline { 2 - 3 } & $\mathrm{a}_{0}$ & $\mathrm{a}_{1}$ \\
\hline Klon & $\mathbf{0 , 0 0 2}$ & 0,146 \\
Gattung & 0,696 & 0,113 \\
Gattung Pappel * Klon & $\mathbf{0 , 0 0 1}$ & 0,591 \\
Gattung Weide * Klon & 0,479 & 0,065 \\
\hline Max 1* H 275 & 0,471 & 0,577 \\
Max 1* AF 2 & $\mathbf{0 , 0 0 3}$ & 0,522 \\
H 275 * AF 2 & $\mathbf{0 , 0 0 1}$ & 0,356 \\
\hline
\end{tabular}

\subsubsection{Einfluss der Bestandesmittelhöhe}

Mittels Regressionsanalyse wurde die Wechselwirkung von der Bestandesmittelhöhe mit den Funktionskoeffizienten untersucht. Der Abbildung 4-11 können sowohl die Zusammenhänge zwischen $\mathrm{a}_{0}$ und $\mathrm{a}_{1}$ als auch die Zusammenhänge zwischen mittlerer Bestandeshöhe und den Funktionskoeffizienten der $\mathrm{d}_{1,3}$-Funktionen, am Beispiel der Klone AF 2 und Inger entnommen werden. 


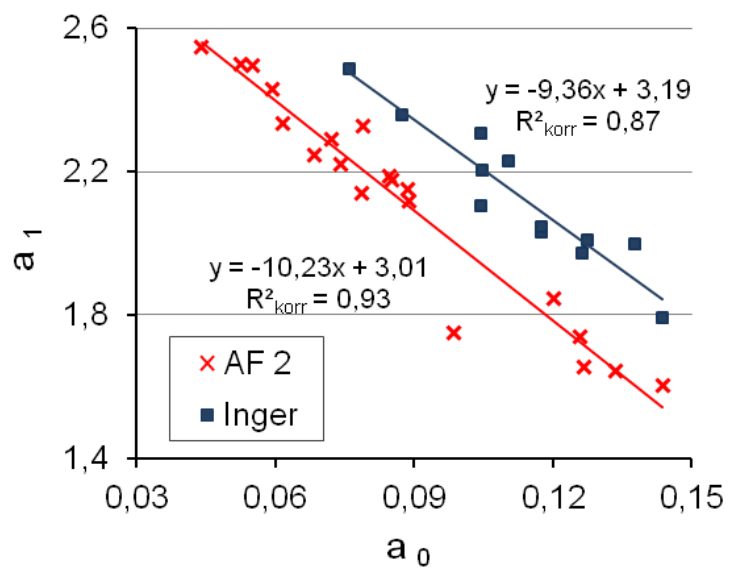

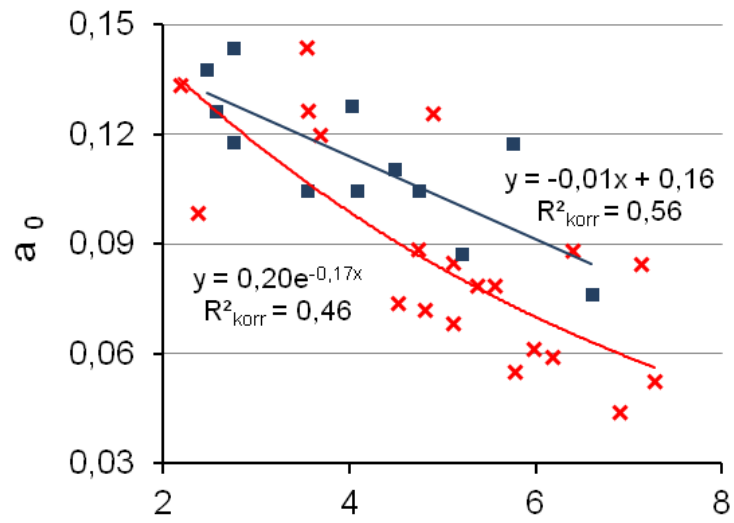

b)

Höhe $[\mathrm{m}]$

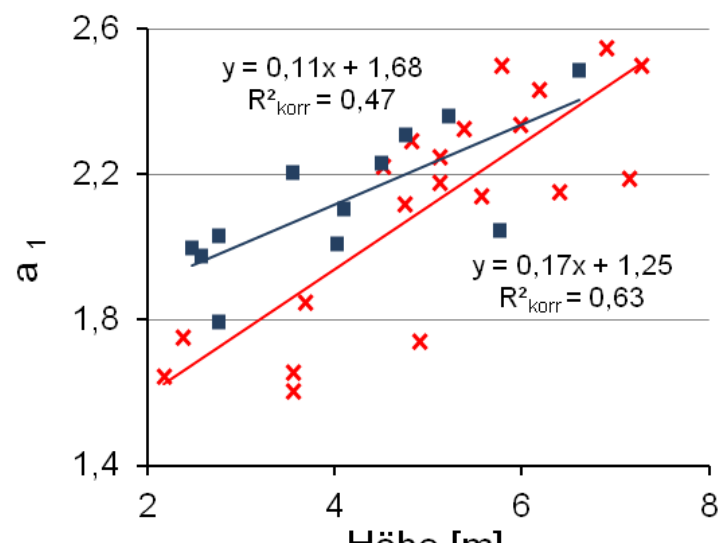

c)

Höhe [m]

Abb. 4-11: Regressionsanalyse zwischen den Funktionskoeffizienten $\mathrm{a}_{0}$ und $\mathrm{a}_{1}$ (a) sowie zwischen der mittleren Höhe und den jeweiligen Funktionskoeffizienten (b und c) für die Klone AF 2 und Inger.

Die Beziehung zwischen den Koeffizienten $\mathrm{a}_{0}$ und $\mathrm{a}_{1}$ ist mit $\mathrm{R}^{2}$ korr von über 0,87 sehr straff. Bei beiden Klonen war die lineare Regression diejenige Anpassung mit dem höchsten korrigierten Bestimmtheitsmaß. Mit zunehmendem $\mathrm{a}_{0}$ nimmt $\mathrm{a}_{1} \mathrm{ab}$. Die Regression zwischen Höhe und den Koeffizienten $\mathrm{a}_{0}$ und $\mathrm{a}_{1}$ wies $\mathrm{R}^{2}{ }_{\text {korr }}$ größer 0,46 auf, wobei mit zunehmender Bestandesmittelhöhe $\mathrm{a}_{1}$ zu- und $\mathrm{a}_{0}$ abnahm. Diese Beobachtungen konnten bei weiteren Prüfgliedern bestätigt werden. Auch hier wurde sichtbar, dass die Funktionskoeffizienten von AF 2 stets kleiner als die von Inger waren.

Mit Hilfe der mittels Regressionsfunktionen errechneten Zusammenhänge (Beziehungen zwischen $\mathrm{a}_{0}$ und $\mathrm{a}_{1}$, Höhe und $\mathrm{a}_{0}$, Höhe und $\mathrm{a}_{1}$ ) konnten höhengestufte Biomassefunktionen für alle Klone hergeleitet werden. Je nach Klon und Güte der Anpassung wurde als erstes einer der beiden Koeffizienten, $a_{0}$ oder $a_{1}$, aus der Höhe hergeleitet. Bei AF 2 wurde zuerst $a_{1}$, bei Inger zuerst $a_{0}$ hergeleitet. Der fehlende Koeffizient wurde im Anschluss mit Hilfe der sehr straffen Regression zwischen $a_{0}$ und $a_{1}$ oder $a_{1}$ und $a_{0}$ hergeleitet. Die Höhenstufencluster, die zur Modellierung der Biomassefunktionen dienten, sind jeweils für einen Höhenbereich von einem vollen Meter valide. Für die Höhenstufe 3 (H3) der Modellierung gelten somit Bestände mit einer mittleren Höhe zwischen 2,5 und 3,5 m. Weitere Höhenstufen wurden in der gleichen Art und Weise definiert. Eine Übersicht des Einflusses der mittleren Höhe auf die Form der Kurven der jeweiligen Höhenstufen ist anhand Abbildung 4-12 für AF 2 und Inger dargestellt. 

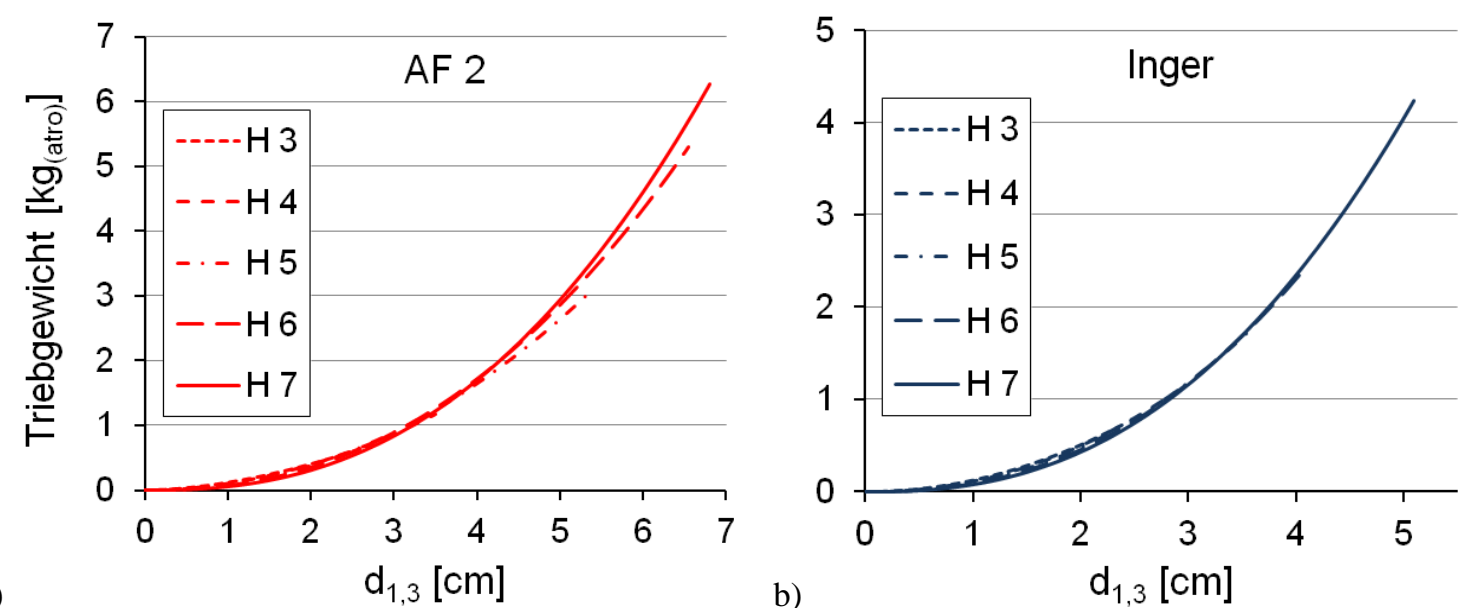

Abb. 4-12: Modellierte Biomassefunktionen nach Höhencluster sowie Hauptfunktion a) beim Pappelklon AF 2 und b) beim Weidenklon Inger. Mit H3: Funktion der Höhenstufe 3, usw..

Abbildung 4-12 kann entnommen werden, dass erkennenswerte Abstufungen nach Höhenclustern einzig beim AF 2 festzustellen waren. Mit zunehmender Bestandeshöhe können Triebe gleichen Durchmessers um bis zu $8 \%$ schwerer sein. Bei den weiteren Pappelklonen Max 1 und H 275 waren die Unterschiede zwischen den höhenspezifischen Biomassefunktionen noch weniger ausdifferenziert als bei AF 2. Beim Weidenklon Inger wie auch beim Weidenklon Tordis ist der Effekt der mittleren Höhe auf die Biomassefunktion so gut wie nicht vorhanden. Hier verlaufen alle höhengestuften Funktionen weitestgehend identisch.

\subsubsection{Allgemeingültige Biomassefunktionen}

Ein relevanter Einfluss der durchschnittlichen Bestandeshöhe auf dem Verlauf der Biomassefunktion war kaum zu erkennen. Daher wurden allgemeingültige (höhenunspezifische) Funktionen für jeden Klon ermittelt (Abb. 4-13, Tab. 4-8). Die Funktionsbildung erfolgte mit Hilfe einer großen Anzahl an Daten (ca. 600 Datenpaare bei den Pappeln, ca. 200 Datenpaare bei den Weiden). Die klonspezifischen Biomassefunktionen sind für eine Bestandesdichte von 11.111 Stk. ha ${ }^{-1}$ valide.

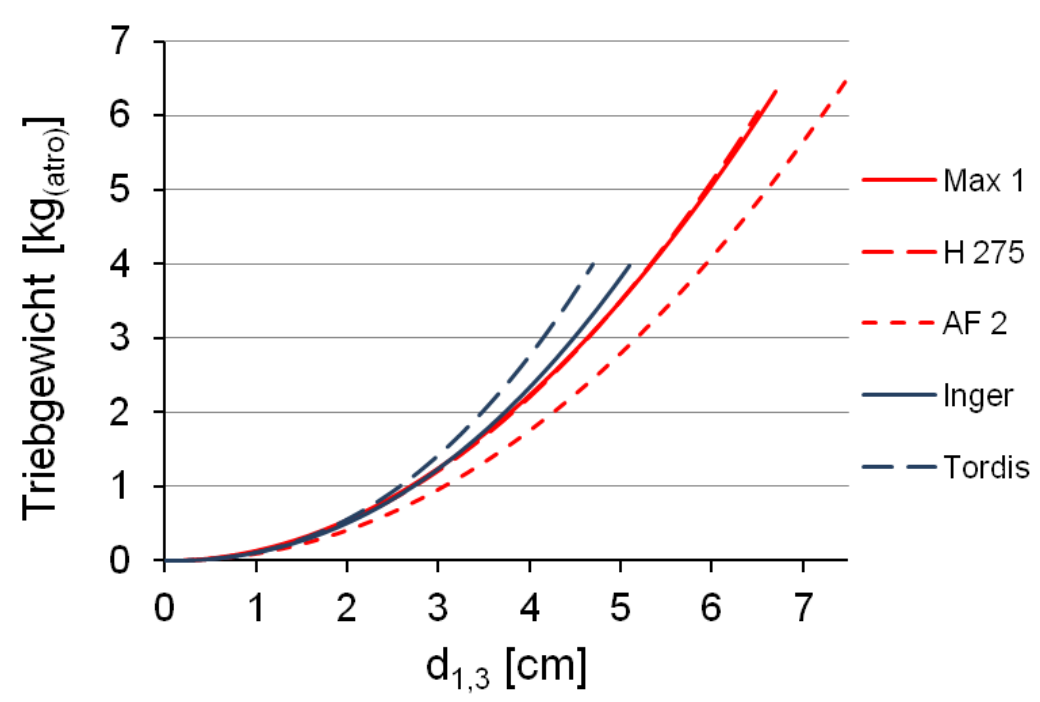

Abb. 4-13: Allgemeingültige Biomassefunktionen für die Bestandesdichte 11.111 Stk. ha ${ }^{-1}$ der Pappelklone Max 1, H 275, AF 2 sowie der Weidenklone Inger und Tordis.

Aus Abbildung 4-13 der allgemeingültigen Funktionen wird eine Klon- bzw. Gattungsabhängigkeit der Verläufe ersichtlich. Tendenziell weisen die Weiden bei gleichen Durchmessern höhere Gewichte auf als die Pappeln. Unter den Weidenklonen zeigt die Tordis-Funktion den steilsten Anstieg auf. Bei 
den Pappeln ist ein fast identischer Kurvenverlauf der Max 1- und H 275-Funktionen zu beobachten. Hingegen weist der Pappelklon AF 2 einen deutlich flacheren Kurvenverlauf als die anderen Pappelklone auf. Bei gleichen $d_{1,3}$-Werten aller Pappelklone sind die Triebgewichte von AF 2 zwischen 20 und $30 \%$ geringer als die anderen Pappelklone.

Tabelle 4-8 beinhaltet ergänzende Informationen zu den in Abbildung 4-13 dargestellten Biomassefunktionen. Hierzu zählen die genauen Funktionskoeffizienten, die Validitätsbereiche sowie statistische Abweichungs- und Streuungsangaben der gebildeten Funktionen.

Tab. 4-8: Statistische Kennwerte der Biomassefunktionen der Pappelklone Max 1, H 275, AF 2 sowie der Weidenklone Inger und Tordis.

\begin{tabular}{|c|c|c|c|c|c|c|c|c|c|c|c|c|}
\hline \multirow{2}{*}{ Gattung } & \multirow{2}{*}{ Klon } & \multirow{2}{*}{$\mathrm{n}$} & \multirow{2}{*}{ n Sto. ${ }^{a}$} & Min & Max & \multirow{2}{*}{$\mathrm{R}_{\text {korr }}^{2}$} & \multirow{2}{*}{$\mathrm{a}_{0}$} & \multirow{2}{*}{$a_{1}$} & \multirow{2}{*}{$\begin{array}{c}\text { RMSE } \\
{[\mathrm{kg}]}\end{array}$} & $\mathrm{e}$ & $\mathrm{S}_{\mathrm{e}}$ & $\mathrm{m}_{\mathrm{x}}$ \\
\hline & & & & \multicolumn{2}{|c|}{$[\mathrm{cm}]$} & & & & & \multicolumn{3}{|c|}{ [\%] } \\
\hline \multirow{3}{*}{ Pappel } & Max 1 & 612 & 17 & 1,0 & 6,7 & $0,97^{* * * *}$ & $0,136^{* * * *}$ & $2,018^{* * * *}$ & 0,18 & 2,65 & 19,83 & 20,00 \\
\hline & Н 275 & 594 & 16 & 1,0 & 6,5 & $0,96^{* * * *}$ & $0,125^{* * * *}$ & $2,072^{* * * *}$ & 0,19 & 1,60 & 15,78 & 15,86 \\
\hline & AF 2 & 682 & 20 & 1,0 & 7,5 & $0,96^{* * * *}$ & $0,097^{* * * *}$ & $2,088^{* * * *}$ & 0,20 & 2,83 & 17,31 & 17,54 \\
\hline \multirow{2}{*}{ Weide } & Inger & 225 & 12 & 1,0 & 5,1 & 0,96 ***** & $0,111^{* * *}$ & $2,197^{* * * *}$ & 0,19 & 1,95 & 17,13 & 17,24 \\
\hline & Tordis & 205 & 11 & 1,0 & 4,7 & $0,97^{* * * *}$ & $0,113^{* * * *}$ & $2,303^{* * *}$ & 0,16 & 0,94 & 13,42 & 13,45 \\
\hline
\end{tabular}

${ }^{a}$ n Sto. bezeichnet die Anzahl an Standorten, welche für die Funktionsermittlung verwendet wurden. Die Anzahl an Wertepaaren wird durch n dargestellt.

Für die Ertragsbestimmung der Pappel- und Weidenbestände in der ersten und zweiten Wuchsperiode wurden allgemeingültige, höhenunspezifische Biomassefunktionen verwendet. In Beständen mit einer durchschnittlichen Höhe kleiner 2,5 m kamen $\mathrm{d}_{0,1}$-Funktionen zum Einsatz (siehe Anhang 27 und 28). In Beständen mit einer durchschnittlichen Höhe größer 2,5 $\mathrm{m}$ wurden allgemeingültige $\mathrm{d}_{1,3}$-Funktionen zur Kalkulation herangezogen. Modellierte, höhengestufte Funktionen fanden keine Verwendung in der Ertragsermittlung.

\subsection{Leistungsbeeinflussende Standorteigenschaften}

Einer der Schwerpunkte der Arbeit ist die Suche nach leistungsbeeinflussenden Standorteigenschaften für den Anbau von Pappel und Weide auf Ackerflächen. Wie bereits im Kapitel 3.5 erwähnt, wurden für das Baumwachstum Indikatoren aus den Bereichen Klima und Boden ausgewählt.

\subsubsection{Temperatur}

Die Klimavariablen zeigten erwartungsgemäß eine relevante Beziehung zur Ertragsleistung auf. Bei den Temperaturen zeigten sich, abhängig von Standortcluster und Klon bzw. der Gattung, unterschiedlich gerichtete Effektstärken der Korrelationen auf den dGZ (Tab. 4-9).

Bei gleichzeitiger Berücksichtigung aller Standorte (Standortcluster ALL) waren die besten Korrelationen $\left(\mathrm{V}_{1}\right)$ aller Klone stets schwach mit r-Werten zwischen -0,33 (Inger) und -0,13 (AF 2) (Tab. 4-9). Mit wenigen Ausnahmen waren Temperaturvariablen, gebildet aus spezifischen Zeitfenstern innerhalb der Vegetationsperiode oder des Jahres, stärker mit dem dGZ korreliert als die Referenzvariable $\mathrm{T}_{\mathrm{VP}}$. Die p-Werte der Korrelationen erreichten maximal 0,06 und übertrafen somit die Irrtumswahrscheinlichkeitsgrenze von $\mathrm{p}<0,05$ nicht.

Im Standortcluster S konnten drei Zusammenhänge beobachtet werden, welche nach Gattung unterschiedlich stark waren. Schwache bis mäßige Korrelationen zwischen Wintertemperaturen, ausgedrückt mittels der Variablen $\mathrm{T}_{1,2}, \mathrm{~T}_{1,3}$ und $\mathrm{T}_{2,3}$ und dem dGZ stellten den ersten Zusammenhang dar. 
Tab. 4-9: Korrelationsanalyse zwischen Temperaturvariablen und dem dGZ für die untersuchten Klone in den Standortclustern ALL, S, L und U. Dargestellt sind abnehmend die r-Werte der bestkorrelierten Variablen $\mathrm{V}_{1}$ bis $\mathrm{V}_{3}$ sowie der Referenzvariablen $\left(\mathrm{V}_{\mathrm{Ref}}\right)$.

\begin{tabular}{|c|c|c|c|c|c|c|c|c|c|c|c|}
\hline $\begin{array}{l}\text { Standort- } \\
\text { cluster }\end{array}$ & Gattung & Klon & $\mathrm{n}$ & $\mathrm{V}_{1}$ & $\mathrm{r}$ & $\mathrm{V}_{2}$ & $\mathrm{r}$ & $\mathrm{V}_{3}$ & $r$ & $\mathrm{~V}_{\text {ref }}$ & $\mathrm{r}$ \\
\hline \multirow{4}{*}{ ALL } & \multirow{2}{*}{ Pappel } & Max 1 & 20 & $\mathrm{~T}_{7,9}$ & $-0,20$ & $\mathrm{~T}_{7,8}$ & $-0,18$ & $\mathrm{~T}_{\mathrm{VP}}$ & $-0,16$ & $-{ }^{a}$ & - \\
\hline & & AF 2 & 20 & $\mathrm{~T}_{7,9}$ & $-0,13$ & $\mathrm{~T}_{7,8}$ & $-0,11$ & $\mathrm{~T}_{\mathrm{VP}}$ & $-0,08$ & $-{ }^{a}$ & - \\
\hline & \multirow{2}{*}{ Weide } & Inger & 20 & $\mathrm{~T}_{7,9}$ & $-0,33$ & $\mathrm{~T}_{7,8}$ & $-0,31$ & $\mathrm{~T}_{6,9}$ & $-0,29$ & $\mathrm{~T}_{\mathrm{VP}}$ & $-0,28$ \\
\hline & & Tordis & 18 & $\mathrm{~T}_{7,9}$ & $-0,15$ & $\mathrm{~T}_{7,8}$ & $-0,14$ & $\mathrm{~T}_{6,9}$ & $-0,09$ & $\mathrm{~T}_{\mathrm{VP}}$ & $-0,07$ \\
\hline \multirow{5}{*}{$S$} & \multirow{3}{*}{ Pappel } & $\operatorname{Max} 1$ & 8 & $\mathrm{~T}_{1,2}$ & 0,34 & $\mathrm{~T}_{1,3}$ & 0,33 & $\mathrm{~T}_{2,3}$ & 0,32 & $\mathrm{~T}_{\mathrm{VP}}$ & $-0,08$ \\
\hline & & H 275 & 7 & $\mathrm{~T}_{1,2}$ & 0,17 & $\mathrm{~T}_{2,3}$ & 0,15 & $\mathrm{~T}_{1,3}$ & 0,15 & $\mathrm{~T}_{\mathrm{VP}}$ & $-0,11$ \\
\hline & & AF 2 & 8 & $\mathrm{~T}_{1,2}$ & 0,39 & $\mathrm{~T}_{1,3}$ & 0,38 & $\mathrm{~T}_{2,3}$ & 0,35 & $\mathrm{~T}_{\mathrm{VP}}$ & $-0,07$ \\
\hline & \multirow{2}{*}{ Weide } & Inger & 8 & $\mathrm{~T}_{7,8}$ & $-0,54$ & $\mathrm{~T}_{7,9}$ & $-0,54$ & $\mathrm{~T}_{6,9}$ & $-0,46$ & $\mathrm{~T}_{\mathrm{VP}}$ & $-0,37$ \\
\hline & & Tordis & 8 & $\mathrm{~T}_{7,9}$ & $-0,56$ & $\mathrm{~T}_{7,8}$ & $-0,55$ & $\mathrm{~T}_{6,9}$ & $-0,48$ & $\mathrm{~T}_{\mathrm{VP}}$ & $-0,39$ \\
\hline \multirow{4}{*}{$\mathrm{U}$} & \multirow{2}{*}{ Pappel } & Max 1 & 5 & $\mathrm{~T}_{7,9}$ & $0,84^{(*)}$ & $\mathrm{T}_{7,8}$ & $0,83^{(*)}$ & $\mathrm{T}_{6,9}$ & 0,75 & $\mathrm{~T}_{\mathrm{VP}}$ & 0,66 \\
\hline & & AF 2 & 5 & $\mathrm{~T}_{7,9}$ & 0,27 & $\mathrm{~T}_{7,8}$ & 0,24 & $\mathrm{~T}_{6,9}$ & 0,12 & $\mathrm{~T}_{\mathrm{VP}}$ & 0,01 \\
\hline & \multirow{2}{*}{ Weide } & Inger & 5 & $\mathrm{~T}_{7,8}$ & 0,51 & $\mathrm{~T}_{7,9}$ & 0,46 & $\mathrm{~T}_{6,8}$ & 0,41 & $\mathrm{~T}_{\mathrm{VP}}$ & 0,33 \\
\hline & & Tordis & 5 & $\mathrm{~T}_{7,9}$ & 0,57 & $\mathrm{~T}_{7,8}$ & 0,47 & $\mathrm{~T}_{6,9}$ & 0,40 & $\mathrm{~T}_{\mathrm{VP}}$ & 0,28 \\
\hline \multirow{3}{*}{$\mathrm{L}$} & \multirow{2}{*}{ Pappel } & Max 1 & 7 & $\mathrm{~T}_{4,5}$ & $-0,55$ & $\mathrm{~T}_{1,2}$ & $-0,52$ & $\mathrm{~T}_{5,7}$ & $-0,52$ & $\mathrm{~T}_{\mathrm{VP}}$ & $-0,52$ \\
\hline & & AF 2 & 7 & $\mathrm{~T}_{1,2}$ & $-0,35$ & $\mathrm{~T}_{7,9}$ & $-0,30$ & $\mathrm{~T}_{\mathrm{VP}}$ & $-0,20$ & $-{ }^{a}$ & - \\
\hline & Weide & Inger & 7 & $\mathrm{~T}_{1,2}$ & $-0,71^{(*)}$ & $\mathrm{T}_{7,9}$ & $-0,66$ & $\mathrm{~T}_{\mathrm{VP}}$ & $-0,65$ & $-{ }^{a}$ & - \\
\hline
\end{tabular}

${ }^{a}$ Beim Standortcluster ALL Klone Max 1 und AF 2 sowie beim Standortcluster L, Klone AF 2 und Inger war die Variable $\mathrm{T}_{\mathrm{VP}}$ die drittbeste, daher wurde diese nicht als Referenzvariable berücksichtigt.

Die am besten korrelierte Variable mit dem dGZ war $\mathrm{T}_{1,2}$. Die Korrelation wurde beginnend vom Klon AF 2 mit einem r-Wert von 0,39 über die Klone Max 1, Inger, Tordis schwächer. Der Klon H 275 zeigte die schwächste Korrelation mit einem r-Wert von 0,17. Auch die Frühjahrstemperaturen, ausgedrückt in den Variablen $T_{3,4}, T_{4,5}$ und $T_{3,5}$ waren bei den Pappelklonen positiv mit dem dGZ korreliert. Die errechneten $r$-Werte waren geringfügig kleiner als die der Variablen $T_{1,2} T_{1,3}$ und $T_{2,3}$. Bei den Weiden wurden hier bereits sehr schwache negative Korrelationen mit dem dGZ beobachtet.

Ein weiterer Zusammenhang bildete die negative Korrelation zwischen den Sommertemperaturen $\left(\mathrm{T}_{7,8}, \mathrm{~T}_{7,9}, \mathrm{~T}_{6,9}\right.$ und $\left.\mathrm{T}_{6,8}\right)$ und dem dGZ. Hier gab es zwischen den Gattungen deutliche Abweichungen, was die Stärke des Zusammenhangs betraf. Die r-Werte der Variablen $\mathrm{T}_{7,8}$ lagen bei den Pappeln gleichmäßig bei $-0,27$ und stellten somit eine schwache Korrelation dar. Bei der Weide konnten starke Korrelationen mit einem $r$-Wert von $-0,54$ bei Inger und $-0,56$ bei Tordis festgestellt werden. Somit war der negative Einfluss der höheren Sommertemperaturen auf den dGZ bei den Weidenklonen deutlich ausgeprägter als bei den Pappelklonen. Die Referenzvariable $\mathrm{T}_{\mathrm{VP}}$ war bei den Pappelklonen mit einem maximalen r-Wert von -0,11 negativ korreliert, was als eine sehr schwache Korrelation interpretiert werden kann. Dagegen wurden für die Weidenklone negative mäßige Korrelationen zwischen $\mathrm{T}_{\mathrm{VP}}$ und dem dGZ ermittelt.

Im Standortcluster $U$ waren die Sommertemperaturen (Variablen $T_{7,8}, T_{7,9}, T_{6,8}$ und $T_{6,9}$ ) mit dem dGZ positiv schwach bis stark korreliert. Die am stärksten korrelierte Temperaturvariable hatte $r$-Werte von 0,84 für Max $1\left(\mathrm{~T}_{7,9}\right)$, 0,57 für Tordis ( $\left.\mathrm{T}_{7,9}\right)$, 0,51 für Inger $\left(\mathrm{T}_{7,8}\right)$ und 0,27 für AF 2 ( $\left.\mathrm{T}_{7,9}\right)$ (Tab. 4-9). Somit war die Schwankung zwischen den Klonen sehr hoch. Durchschnittlich betrugen die r-Werte der Korrelation von $\mathrm{T}_{7,9}$ mit dem dGZ für Pappel 0,55 und für Weide 0,45. Die Referenzvariable $\mathrm{T}_{\mathrm{VP}}$ hatte, wie die bestkorrelierten Variablen $\mathrm{T}_{7,8}$ und $\mathrm{T}_{7,9}$, eine positiv gerichtete Korrelation mit dem dGZ, 
nur mit reduzierten r-Werten. Die r-Werte der Korrelationen aus dem Frühjahr waren deutlich niedriger als die des Hochsommers.

Beim Standortcluster L waren alle Temperaturvariablen mit dem dGZ negativ korreliert. Die ermittelten r-Werte variierten nur wenig unter den Variablen. Verglichen mit anderen Standortclustern, in denen unter den Klonen ähnliche Tendenzen zwischen dGZ und den bestkorrelierten Temperaturvariablen existierten, bot sich bei den Lehmen ein eher heterogenes Bild (Tab. 4-9). $T_{1,2}$ war oft die am stärksten mit dem dGZ korrelierte Temperaturvariable (AF 2 und Inger), bzw. unter den am stärksten korrelierten Temperaturvariablen (Max 1). $\mathrm{T}_{7,9}$ war bei AF 2 und Inger am zweitstärksten mit dem dGZ korreliert. Bei Max 1 verhielt es sich anders, neben $\mathrm{T}_{1,2}$ waren $\mathrm{T}_{4,5}, \mathrm{~T}_{5,7}$ und $\mathrm{T}_{\mathrm{VP}}$ mit dem dGZ stark korreliert. Da keine signifikanten Regressionen, weder lineare noch nicht-lineare, für die Klone differenziert nach Standortclustern ermittelt wurden, wird auf eine tabellarische bzw. graphische Darstellung verzichtet.

\subsubsection{Niederschlag}

In Abhängigkeit von Klon und Standortcluster zeigten die Analysen von Niederschlagsvariablen und dGZ mäßige bis sehr stark positive Korrelationen der verschiedenen Zeitfenster (Tab. 4-10).

Tab. 4-10: Korrelationsanalyse zwischen Niederschlagsvariablen und dem dGZ für die untersuchten Klone in den Standortclustern ALL, S, L und U. Dargestellt sind abnehmend die r-Werte der bestkorrelierten Variablen $\mathrm{V}_{1}$ bis $\mathrm{V}_{3}$ sowie der Referenzvariablen $\left(\mathrm{V}_{\mathrm{Ref}}\right)$.

\begin{tabular}{|c|c|c|c|c|c|c|c|c|c|c|c|}
\hline $\begin{array}{l}\text { Standort- } \\
\text { cluster }\end{array}$ & Gattung & Klon & $\mathrm{n}$ & $\mathrm{V}_{1}$ & $\mathrm{r}$ & $\mathrm{V}_{2}$ & $\mathrm{r}$ & $\mathrm{V}_{3}$ & $\mathrm{r}$ & $\mathrm{V}_{\text {Ref }}$ & $\mathrm{r}$ \\
\hline \multirow{4}{*}{ ALL } & \multirow{2}{*}{ Pappel } & Max 1 & 20 & $\mathrm{~N}_{6,7}$ & $0,42^{(*)}$ & $\mathrm{N}_{5,7}$ & $0,42^{(*)}$ & $\mathrm{N}_{4,7}$ & $0,38^{(*)}$ & $\mathrm{N}_{\mathrm{VP}}$ & 0,28 \\
\hline & & AF 2 & 20 & $\mathrm{~N}_{6,7}$ & 0,30 & $\mathrm{~N}_{5,7}$ & 0,29 & $\mathrm{~N}_{4,7}$ & 0,24 & $\mathrm{~N}_{\mathrm{VP}}$ & 0,12 \\
\hline & \multirow{2}{*}{ Weide } & Inger & 20 & $\mathrm{~N}_{6,7}$ & 0,28 & $\mathrm{~N}_{5,7}$ & 0,27 & $\mathrm{~N}_{4,7}$ & 0,20 & $\mathrm{~N}_{\mathrm{VP}}$ & 0,17 \\
\hline & & Tordis & 18 & $\mathrm{~N}_{6,7}$ & $0,57^{*}$ & $\mathrm{~N}_{5,7}$ & $0,52^{*}$ & $\mathrm{~N}_{4,7}$ & $0,48^{*}$ & $\mathrm{~N}_{\mathrm{VP}}$ & 0,36 \\
\hline \multirow{5}{*}{ S } & \multirow{3}{*}{ Pappel } & Max 1 & 8 & $\mathrm{~N}_{6,7}$ & $\mathbf{0 , 8 1} 1^{*}$ & $\mathrm{~N}_{5,7}$ & $0,63^{(*)}$ & $\mathrm{N}_{4,7}$ & 0,57 & $\mathrm{~N}_{\mathrm{VP}}$ & 0,47 \\
\hline & & Н 275 & 7 & $\mathrm{~N}_{6,7}$ & $0,87^{*}$ & $\mathrm{~N}_{5,7}$ & $0,75^{*}$ & $\mathrm{~N}_{4,7}$ & $0,69^{(*)}$ & $\mathrm{N}_{\mathrm{VP}}$ & 0,61 \\
\hline & & AF 2 & 8 & $\mathrm{~N}_{6,7}$ & $0,64^{(*)}$ & $\mathrm{N}_{5,7}$ & 0,44 & $\mathrm{~N}_{3,7}$ & 0,41 & $\mathrm{~N}_{\mathrm{VP}}$ & 0,31 \\
\hline & \multirow{2}{*}{ Weide } & Inger & 8 & $\mathrm{~N}_{6,7}$ & $0,71^{*}$ & $\mathrm{~N}_{6,9}$ & 0,47 & $\mathrm{~N}_{5,7}$ & 0,45 & $\mathrm{~N}_{\mathrm{VP}}$ & 0,38 \\
\hline & & Tordis & 8 & $\mathrm{~N}_{6,7}$ & $0,76^{*}$ & $\mathrm{~N}_{6,9}$ & 0,59 & $\mathrm{~N}_{7,9}$ & 0,55 & $\mathrm{~N}_{\mathrm{VP}}$ & 0,48 \\
\hline \multirow{4}{*}{$\mathrm{U}$} & \multirow{2}{*}{ Pappel } & Max 1 & 5 & $\mathrm{~N}_{4,5}$ & 0,75 & $\mathrm{~N}_{5,6}$ & 0,70 & $\mathrm{~N}_{4,6}$ & 0,64 & $\mathrm{~N}_{\mathrm{VP}}$ & 0,25 \\
\hline & & $\mathrm{AF} 2$ & 5 & $\mathrm{~N}_{5,6}$ & 0,39 & $\mathrm{~N}_{4,5}$ & 0,13 & $\mathrm{~N}_{4,6}$ & 0,03 & $\mathrm{~N}_{\mathrm{VP}}$ & $-0,24$ \\
\hline & \multirow{2}{*}{ Weide } & Inger & 5 & $\mathrm{~N}_{5,6}$ & 0,61 & $\mathrm{~N}_{4,5}$ & 0,45 & $\mathrm{~N}_{4,6}$ & 0,37 & $\mathrm{~N}_{\mathrm{VP}}$ & $-0,13$ \\
\hline & & Tordis & 5 & $\mathrm{~N}_{5,6}$ & 0,45 & $\mathrm{~N}_{4,5}$ & 0,36 & $\mathrm{~N}_{4,6}$ & 0,24 & $\mathrm{~N}_{\mathrm{VP}}$ & 0,16 \\
\hline \multirow{3}{*}{$\mathrm{L}$} & \multirow{2}{*}{ Pappel } & $\operatorname{Max} 1$ & 7 & $\mathrm{~N}_{5,6}$ & 0,36 & $\mathrm{~N}_{6,7}$ & 0,33 & $\mathrm{~N}_{4,6}$ & 0,27 & $\mathrm{~N}_{\mathrm{VP}}$ & $-0,12$ \\
\hline & & $\mathrm{AF} 2$ & 7 & $\mathrm{~N}_{5,6}$ & 0,60 & $\mathrm{~N}_{4,6}$ & 0,47 & $\mathrm{~N}_{6,7}$ & 0,40 & $\mathrm{~N}_{\mathrm{VP}}$ & $-0,11$ \\
\hline & Weide & Inger & 7 & $\mathrm{~N}_{5,6}$ & 0,44 & $\mathrm{~N}_{4,6}$ & 0,27 & $\mathrm{~N}_{5,7}$ & 0,15 & $\mathrm{~N}_{\mathrm{VP}}$ & $-0,30$ \\
\hline
\end{tabular}

Bei Berücksichtigung aller Standorte konnten schwache bis mittlere Korrelationen zwischen dem dGZ und den Niederschlagsvariablen identifiziert werden. Die Variablen mit den höchsten r-Werten waren, sowohl für die Gattungen als auch für die einzelnen Pappel- und Weidenklone, in absteigender Reihenfolge $\mathrm{N}_{6,7}, \mathrm{~N}_{5,7}$ und $\mathrm{N}_{4,7}$. Bei der Korrelation der Variablen $\mathrm{N}_{6,7}$ mit dem dGZ variierten die r-Werte zwischen 0,57 (Tordis) und 0,28 (Inger). Lediglich beim Weidenklon Tordis waren die Korrelationen signifikant. Im Vergleich zeigte die Variable $\mathrm{N}_{\mathrm{VP}}$ deutlich geringere r-Werte gegenüber $\mathrm{N}_{5,7}$ und $\mathrm{N}_{6,7}$. Tabelle 4-11 sowie Abbildung 4-14 zeigen die Ergebnisse der Regressionsanalyse im Über- 
blick. Für Max 1, AF 2 und Tordis wurden signifikante Regressionen mit dem inversen Ansatz (Max 1) oder mit dem allometrischen Ansatz (AF 2, Tordis) hergeleitet. Die $\mathrm{R}^{2}$ korr der Anpassungen waren niedrig, für Max 1 kann die Varianz des dGZ nur mit $15 \%$, für Tordis dagegen mit $31 \%$ erklärt werden.

Tab. 4-11: Regressionsanalyse für den dGZ in Abhängigkeit von unterschiedlichen Niederschlagsvariablen im Standortcluster ALL. Ausgewählt sind die klonspezifisch besten Anpassungen.

\begin{tabular}{llllcc}
\hline Gattung & Klon & $\mathrm{n}$ & Variable & Anpassung & $\mathrm{R}^{2}{ }_{\text {korr }}$ \\
\hline \multirow{2}{*}{ Pappel } & Max 1 & 20 & $\mathrm{~N}_{5,7}$ & inv & $\mathbf{0 , 1 5}^{*}$ \\
& AF 2 & 20 & $\mathrm{~N}_{6,7}$ & allo & $\mathbf{0 , 1 5}^{*}$ \\
\multirow{2}{*}{ Weide } & Inger & 20 & $\mathrm{~N}_{6,7}$ & comp & 0,04 \\
& Tordis & 18 & $\mathrm{~N}_{6,7}$ & allo & $\mathbf{0 , 3 1}$ \\
\hline
\end{tabular}

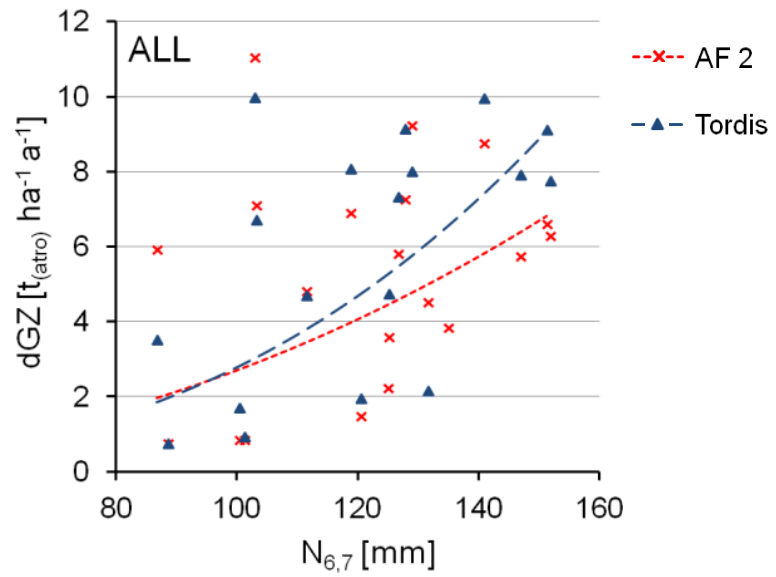

Abb. 4-14: Regressionsverlauf für den dGZ in Abhängigkeit von $\mathrm{N}_{6,7}$ und im Standortcluster ALL, dargestellt am Beispiel der Klone AF 2 und Tordis.

Im Standortcluster S fielen die Korrelationen zwischen dem dGZ und den verschiedenen Niederschlagsvariablen mäßig bis sehr stark aus. Wie bereits im Standortcluster ALL beobachtet, ist die Korrelation des dGZ mit den Niederschlagssummen zum Anfang bzw. zur Mitte der Vegetationsperiode $\left(\mathrm{N}_{5,7}, \mathrm{~N}_{6,7}\right)$ höher als im Vergleich mit der Korrelation von dGZ und Summe der gesamten Vegetationsperiode $\left(\mathrm{N}_{\mathrm{VP}}\right)$ (Tab. 4-10). Dabei zeigte stets die Variable $\mathrm{N}_{6,7}$ die stärkste Beziehung zum dGZ. Die r-Werte schwankten zwischen 0,87 (H 275) und 0,64 (AF 2). Mit Ausnahme von AF 2 waren alle Korrelationen signifikant. Darauf aufbauend wurden für alle Klone signifikante Kurvenanpassungen vorgenommen (Tab. 4-12). Für die Anpassung wurden logarithmische und compound Modelle verwendet. Die Erklärungskraft der Funktionen kann bei $\mathrm{R}^{2}$ korr mit Werten von 0,46 bis 0,66 als hoch bewertet werden. Im Folgenden sind die logarithmischen Modelle mit den höchsten $\mathrm{R}^{2}$ korr der Klone Max 1 und H 275 abgebildet (Abb. 4-15).

Tab. 4-12: Regressionsanalyse für den dGZ in Abhängigkeit von $\mathrm{N}_{6,7}$ im Standortcluster S. Ausgewählt sind die klonspezifisch besten Anpassungen.

\begin{tabular}{|c|c|c|c|c|c|}
\hline Gattung & Klon & $\mathrm{n}$ & Variable & Anpassung & $\mathrm{R}_{\text {korr }}^{2}$ \\
\hline \multirow{3}{*}{ Pappel } & Max 1 & 8 & $\mathrm{~N}_{6,7}^{\mathrm{a}}$ & $\ln$ & $\mathbf{0 , 5 7}{ }^{*}$ \\
\hline & Н 275 & 7 & $\mathrm{~N}_{6,7}$ & $\ln$ & $0,66^{*}$ \\
\hline & $\mathrm{AF} 2$ & 8 & $\mathrm{~N}_{6,7}$ & comp & 0,33 \\
\hline \multirow{2}{*}{ Weide } & Inger & 8 & $\mathrm{~N}_{6,7}$ & comp & $0,46^{*}$ \\
\hline & Tordis & 8 & $\mathrm{~N}_{6,7}$ & comp & $\mathbf{0 , 4 7 ^ { * }}$ \\
\hline
\end{tabular}

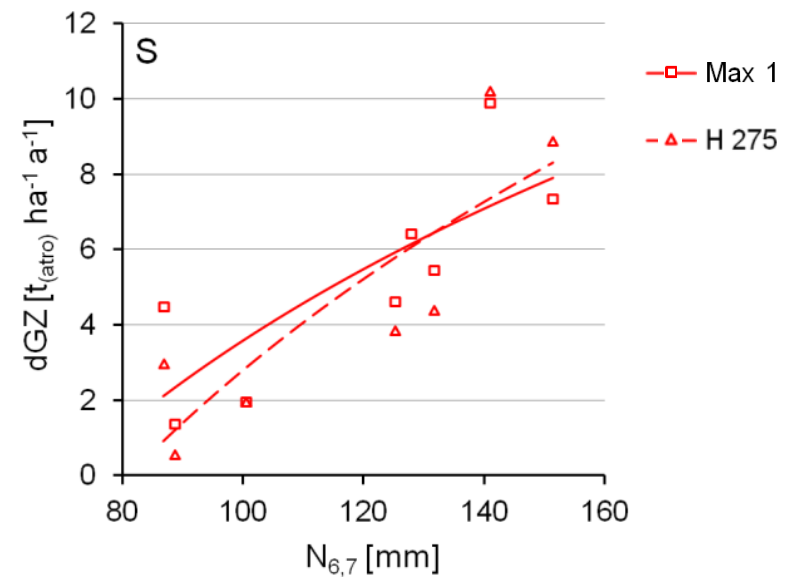

Abb. 4-15: Regressionsverlauf für den dGZ in Abhängigkeit von $\mathrm{N}_{6,7}$ im Standortcluster $\mathrm{S}$, dargestellt am Beispiel der Klone Max 1 und $\mathrm{H} 275$. 
Beim Bodenartcluster $\mathrm{U}$ waren bedeutsame Niederschlagsvariablen $\mathrm{N}_{5,6}, \mathrm{~N}_{4,5}$ und $\mathrm{N}_{4,6}$. Für AF 2, Inger und Tordis wurden die besten Korrelationen mit der Variablen $\mathrm{N}_{5,6}$ erzielt. Bei Max 1 war $\mathrm{N}_{4,5}$ am stärksten korreliert. Bezüglich der einzelnen Klone schwankten die r-Werte zwischen 0,61 (Inger) und 0,39 (AF 2). Obwohl die r-Werte im mittleren bis starken Bereich lagen, konnten keine signifikanten Kurvenanpassungen ermittelt werden.

Bei den Lehmböden wurden die stärksten Korrelationen zu Beginn der Vegetationsperiode festgestellt. Dies betraf insbesondere den Zeitraum Mai bis Juni $\left(\mathrm{N}_{5,6}\right)$ sowie April bis Juni $\left(\mathrm{N}_{4,6}\right)$. Die r-Werte der stärksten Korrelationen der verschiedenen Klone betrugen für Max 1 0,36, für Inger 0,44 und für AF 2 0,60. Im Vergleich zu den aggregierten Monatssummen zeigten die Jahressumme $\left(\mathrm{N}_{\mathrm{J}}\right)$ und die Summe der Vegetationsperiode $\left(\mathrm{N}_{\mathrm{VP}}\right)$, betrachtet als größere Zeitfenster, geringere Korrelationsstärken. $\mathrm{N}_{\mathrm{VP}}$ und $\mathrm{N}_{\mathrm{J}}$ korrelierten sogar negativ mit dem dGZ. Mittels Regressionsanalyse konnte eine signifikante Funktion für den Klon AF 2 mit der Variablen $\mathrm{N}_{5,6}$ errechnet werden (Tab. 4-13, Abb. 4-16). Es handelte sich dabei um den compound Ansatz, der ermittelte $\mathrm{R}^{2}$ korr betrug in diesem Falle 0,50.

Tab. 4-13: Regressionsanalyse für den dGZ in Abhängigkeit von unterschiedlichen Niederschlagsvariablen im Standortcluster L. Ausgewählt sind die klonspezifisch besten Anpassungen.

\begin{tabular}{llcccc}
\hline Gattung & Klon & $\mathrm{n}$ & Variable & Anpassung & $\mathrm{R}_{\text {korr }}^{2}$ \\
\hline Mappel & Max 1 & 7 & - & - & - \\
& AF 2 & 7 & $\mathrm{~N}_{5,6}$ & comp & $\mathbf{0 , 5 0}$ \\
Weide & Inger & 7 & $\mathrm{~N}_{6,7}$ & $\ln$ & 0,05 \\
\hline $\begin{array}{l}{ }^{\mathrm{a}} \text { Für Max 1 } \\
\text { ermittelt werden }\end{array}$ &
\end{tabular}

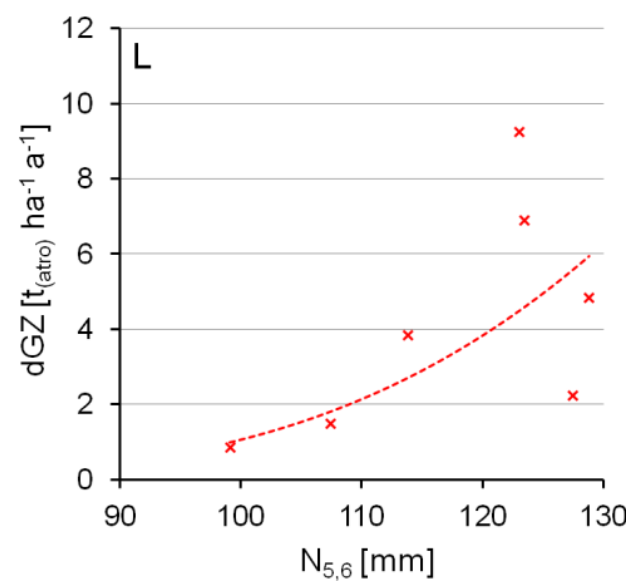

$--x--A F 2$

Abb. 4-16: Regressionsverlauf für den dGZ in Abhängigkeit von $\mathrm{N}_{5,6}$ und im Standortcluster L, dargestellt am Beispiel des Klons AF 2.

\subsubsection{Trockenheitsindex}

Bei der Korrelationsanalyse von Trockenheitsindexvariablen und dGZ, differenziert nach Klon und Standortcluster, zeigte sich ein ähnliches Bild wie bei den Niederschlagsvariablen (Tab. 4-14). Es wurden zahlreiche starke bis sehr starke positive Korrelationen bei den verschiedenen Standortclustern ermittelt. Gegenüber der Niederschlagsvariablen wurden bei den Trockenheitsindexvariablen mit einer einzigen Ausnahme, stets höhere Korrelationskoeffizienten festgestellt. Die Verbesserung der r-Werte betrug zwischen 5 und $50 \%$.

Im Standortcluster ALL wurden mehr signifikante Korrelationen zwischen Trockenheitsindexvariablen und dGZ festgestellt als zwischen Niederschlagsvariablen und dGZ. Die bestkorrelierte Variable beim Standortcluster ALL für jeden Klon war $\mathrm{TI}_{5,7}$. Danach folgten die Variablen $\mathrm{TI}_{6,7}$ und $\mathrm{TI}_{4,7} \mathrm{bzw}$. $\mathrm{TI}_{\mathrm{VP}}$. Für die Korrelationen zwischen $\mathrm{TI}_{5,7}$ und dGZ variierten dier-Werte zwischen 0,54 (Tordis) und 0,33 (AF 2). Die Korrelationsstärke war somit als mäßig bis stark zu bewerten. Im Bereich der Regressionen konnten zwei signifikante Funktionen für Max 1 und Tordis mit jeweils $\mathrm{TI}_{5,7}$ und $\mathrm{TI}_{6,7}$ ermittelt werden (Tab. 4-15, Abb. 4-17). Die ermittelten $\mathrm{R}^{2}$ korr waren für Max $121 \%$ und für Tordis $27 \%$. Bei den Kurvenanpassungen waren, differenziert nach Klon, entweder die Niederschlags- oder Trockenheitsindexvariablen die mit der besten Anpassung.

Die r-Werte der Korrelationen zwischen der bestkorrelierten Variable $\mathrm{TI}_{6,7}$ und dGZ schwankten zwischen 0,90 (H 275) und 0,66 (AF 2) (Tab. 4-16). Nur im Falle von Klon AF 2 waren die Korrelationen 
nicht signifikant. Die bestkorrelierten Trockenheitsindexvariablen zeigten eine 3- bis $15 \%$-ige Verbesserung des r-Werts gegenüber der bestkorrelierten Niederschlagsvariablen. Durch die Kurvenanpassung konnten, bis auf den Klon AF 2, signifikante Funktionen für jeden Klon ermittelt werden. Dabei war stets $\mathrm{TI}_{6,7}$ der beste Prädiktor (Tab. 4-16, Abb. 4-18). Die $\mathrm{R}_{\text {korr }}$ schwankten zwischen 0,76 (H 275) und 0,44 (Inger). Die dazu verwendeten Ansätze waren der inverse (Max 1 und Tordis), der lineare (H 275 und AF 2) und der sigmoidale (Inger). Im Vergleich zu den Niederschlagsvariablen wurden je nach Klon teilweise bessere, teilweise schlechtere $\mathrm{R}^{2}$ korr erzielt.

Tab. 4-14: Korrelationsanalyse zwischen Trockenheitsindexvariablen und dem dGZ für die untersuchten Klone in den Standortclustern ALL, S, L und U. Dargestellt sind abnehmend die r-Werte der bestkorrelierten Variablen $\mathrm{V}_{1}$ bis $\mathrm{V}_{3}$ sowie der Referenzvariablen $\left(\mathrm{V}_{\mathrm{Ref}}\right)$.

\begin{tabular}{|c|c|c|c|c|c|c|c|c|c|c|c|}
\hline $\begin{array}{l}\text { Standort- } \\
\text { cluster }\end{array}$ & Gattung & Klon & $\mathrm{n}$ & $\mathrm{V}_{1}$ & $\mathrm{r}$ & $\mathrm{V}_{2}$ & $\mathrm{r}$ & $\mathrm{V}_{3}$ & $\mathrm{r}$ & $V_{\text {Ref }}$ & $\mathrm{r}$ \\
\hline \multirow{4}{*}{ ALL } & \multirow{2}{*}{ Pappel } & Max 1 & 20 & $\mathrm{TI}_{5,7}$ & $0,50^{*}$ & $\mathrm{TI}_{6,7}$ & $0,46^{*}$ & $\mathrm{TI}_{4,7}$ & $0,45^{*}$ & $\mathrm{TI}_{\mathrm{VP}}$ & 0,30 \\
\hline & & AF 2 & 20 & $\mathrm{TI}_{5,7}$ & 0,33 & $\mathrm{TI}_{6,7}$ & 0,31 & $\mathrm{TI}_{4,7}$ & 0,26 & $\mathrm{TI}_{\mathrm{VP}}$ & 0,18 \\
\hline & \multirow{2}{*}{ Weide } & Inger & 20 & $\mathrm{TI}_{5,7}$ & 0,37 & $\mathrm{TI}_{6,7}$ & 0,34 & $\mathrm{TI}_{\mathrm{VP}}$ & 0,28 & - & - \\
\hline & & Tordis & 18 & $\mathrm{TI}_{6,7}$ & $\mathbf{0 , 5 8 *}$ & $\mathrm{TI}_{5,7}$ & $0,54^{*}$ & $\mathrm{TI}_{4,7}$ & $0,48^{*}$ & $\mathrm{TI}_{\mathrm{VP}}$ & 0,40 \\
\hline \multirow{5}{*}{ S } & \multirow{3}{*}{ Pappel } & Max 1 & 8 & $\mathrm{TI}_{6,7}$ & $\mathbf{0 , 8 3}{ }^{*}$ & $\mathrm{TI}_{5,7}$ & $0,63^{(*)}$ & $\mathrm{TI}_{4,7}$ & 0,55 & $\mathrm{TI}_{\mathrm{VP}}$ & 0,49 \\
\hline & & Н 275 & 7 & $\mathrm{TI}_{6,7}$ & $0,90^{* *}$ & $\mathrm{TI}_{5,7}$ & $0,79^{*}$ & $\mathrm{TI}_{4,7}$ & $0,72^{(*)}$ & $\mathrm{TI}_{\mathrm{VP}}$ & 0,64 \\
\hline & & AF 2 & 8 & $\mathrm{TI}_{6,7}$ & $0,66^{(*)}$ & $\mathrm{TI}_{5,7}$ & 0,42 & $\mathrm{TI}_{6,9}$ & 0,40 & $\mathrm{TI}_{\mathrm{VP}}$ & 0,33 \\
\hline & \multirow{2}{*}{ Weide } & Inger & 8 & $\mathrm{TI}_{6,7}$ & $0,77^{*}$ & $\mathrm{TI}_{6,9}$ & 0,52 & $\mathrm{TI}_{6,8}$ & 0,51 & $\mathrm{TI}_{\mathrm{VP}}$ & 0,43 \\
\hline & & Tordis & 8 & $\mathrm{TI}_{6,7}$ & $\mathbf{0 , 8 2 ^ { * }}$ & $\mathrm{TI}_{6,9}$ & $0,63^{(*)}$ & $\mathrm{TI}_{6,8}$ & 0,59 & $\mathrm{TI}_{\mathrm{VP}}$ & 0,55 \\
\hline \multirow{4}{*}{$\mathrm{U}$} & \multirow{2}{*}{ Pappel } & Max 1 & 5 & $\mathrm{TI}_{5,6}$ & 0,81 & $\mathrm{TI}_{4,5}$ & 0,78 & $\mathrm{TI}_{4,6}$ & 0,70 & $\mathrm{TI}_{\mathrm{VP}}$ & 0,28 \\
\hline & & AF 2 & 5 & $\mathrm{TI}_{5,6}$ & 0,57 & $\mathrm{TI}_{4,5}$ & 0,17 & $\mathrm{TI}_{4,6}$ & 0,08 & $\mathrm{TI}_{\mathrm{VP}}$ & $-0,19$ \\
\hline & \multirow{2}{*}{ Weide } & Inger & 5 & $\mathrm{TI}_{5,6}$ & 0,72 & $\mathrm{TI}_{4,5}$ & 0,47 & $\mathrm{TI}_{4,6}$ & 0,41 & $\mathrm{TI}_{\mathrm{VP}}$ & $-0,10$ \\
\hline & & Tordis & 5 & $\mathrm{TI}_{5,6}$ & 0,61 & $\mathrm{TI}_{5,7}$ & 0,34 & $\mathrm{TI}_{4,6}$ & 0,31 & $\mathrm{TI}_{\mathrm{VP}}$ & 0,21 \\
\hline \multirow{3}{*}{$\mathrm{L}$} & \multirow{2}{*}{ Pappel } & Max 1 & 7 & $\mathrm{TI}_{6,7}$ & $0,68^{(*)}$ & $\mathrm{TI}_{5,6}$ & 0,66 & $\mathrm{TI}_{5,7}$ & 0,56 & $\mathrm{TI}_{\mathrm{VP}}$ & 0,51 \\
\hline & & AF 2 & 7 & $\mathrm{TI}_{5,6}$ & $0,70^{(*)}$ & $\mathrm{TI}_{6,7}$ & 0,60 & $\mathrm{TI}_{4,6}$ & 0,59 & $\mathrm{TI}_{\mathrm{VP}}$ & 0,28 \\
\hline & Weide & Inger & 7 & $\mathrm{TI}_{5,6}$ & $\mathbf{0 , 8 0 ^ { * }}$ & $\mathrm{TI}_{4,6}$ & 0,63 & $\mathrm{TI}_{5,7}$ & 0,56 & $\mathrm{TI}_{\mathrm{VP}}$ & 0,49 \\
\hline
\end{tabular}

Tab. 4-15: Regressionsanalyse für den dGZ in Abhängigkeit von unterschiedlichen Trockenheitsindexvariablen im Standortcluster ALL. Ausgewählt sind die klonspezifisch besten Anpassungen.

\begin{tabular}{llcccc}
\hline Gattung & Klon & $\mathrm{n}$ & Variable & Anpassung & $\mathrm{R}^{2}{ }_{\text {korr }}$ \\
\hline \multirow{2}{*}{ Pappel } & Max 1 & 20 & $\mathrm{TI}_{5,7}$ & inv & $\mathbf{0 , 2 1 ^ { * }}$ \\
& AF 2 & 20 & $\mathrm{TI}_{6,7}$ & allo & 0,14 \\
\multirow{2}{*}{ Weide } & Inger & 20 & $\mathrm{TI}_{5,7}$ & lin & 0,09 \\
& Tordis & 18 & $\mathrm{TI}_{6,7}$ & sig & $\mathbf{0 , 2 7}$ \\
\hline
\end{tabular}

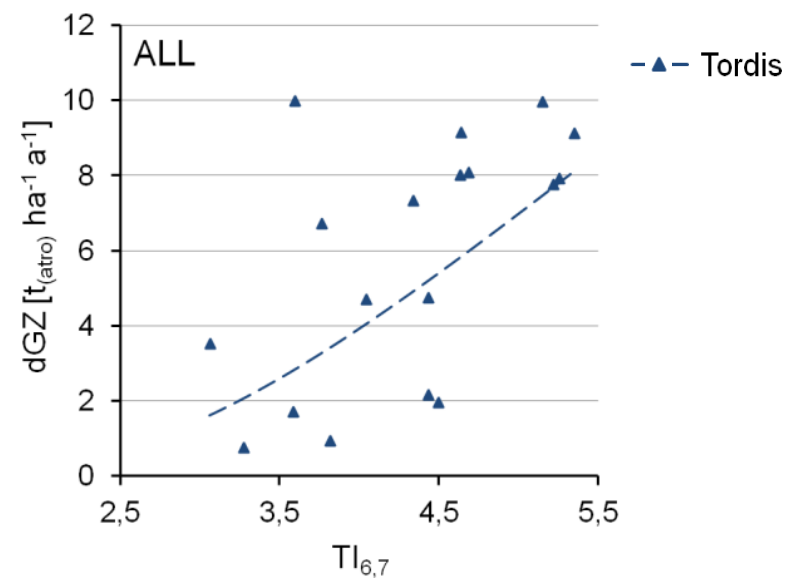

Abb. 4-17: Regressionsverlauf für den dGZ in Abhängigkeit von $\mathrm{TI}_{6,7}$ im Standortcluster ALL, dargestellt am Beispiel des Klon Tordis. 
Tab. 4-16: Regressionsanalyse für den dGZ in Abhängigkeit von $\mathrm{TI}_{6,7}$ im Standortcluster S. Ausgewählt sind die klonspezifisch besten Anpassungen.

\begin{tabular}{lllccc}
\hline Gattung & Klon & $\mathrm{n}$ & Variable & Anpassung & $\mathrm{R}^{2}$ korr \\
\hline \multirow{4}{*}{ Pappel } & Max 1 & 8 & $\mathrm{TI}_{6,7}$ & inv & $\mathbf{0 , 5 4}^{*}$ \\
& $\mathrm{H} 275$ & 7 & $\mathrm{TI}_{6,7}$ & lin & $\mathbf{0 , 7 6}^{*}$ \\
& AF 2 & 8 & $\mathrm{TI}_{6,7}$ & lin & 0,34 \\
Weide & Inger & 8 & $\mathrm{TI}_{6,7}$ & sig & $\mathbf{0 , 4 4}^{*}$ \\
& Tordis & 8 & $\mathrm{TI}_{6,7}$ & inv & $\mathbf{0 , 5 1}$ \\
\hline
\end{tabular}

$\mathrm{TI}_{6,7}$ wies unter den Trockenheitsindexvariablen im Standortcluster $\mathrm{S}$ stets die klonspezifisch besten Anpassungen für den dGZ auf.

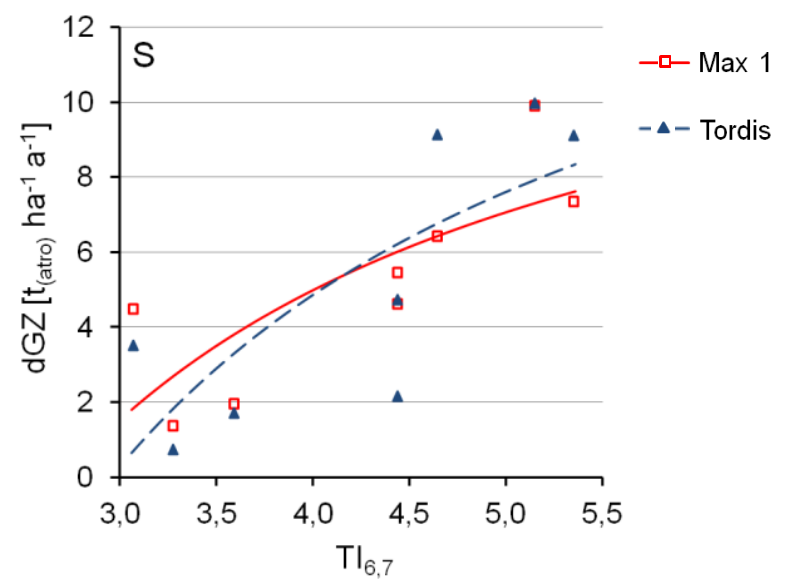

Abb. 4-18: Regressionsverlauf für den dGZ in Abhängigkeit von $\mathrm{TI}_{6,7}$ im Standortcluster S dargestellt am Beispiel der Klone Max 1 und Tordis.

Beim Standortcluster $\mathrm{U}$ waren $\mathrm{TI}_{5,6}, \mathrm{TI}_{4,5}$ und $\mathrm{TI}_{4,6}$ die mit dem dGZ am besten korrelierten Variablen. Die höchsten $\mathrm{r}$-Werte wurden bei der Variablen $\mathrm{TI}_{5,6}$ beobachtet. Sie schwankten zwischen 0,81 (Max 1) und 0,57 (AF 2). Die Referenzvariable $\mathrm{TI}_{\mathrm{VP}}$ wies deutlich niedrigere $\mathrm{r}$-Werte auf im Vergleich zu den Trockenheitsindexvariablen spezifischer Zeitfenster. Ähnlich wie bei den Niederschlagsvariablen konnten im Standortcluster U weder signifikante Korrelationen noch Regressionen für Trockenheitsindexvariablen ermittelt werden.

Beim Standortcluster L wurden folgende r-Werte zwischen der am stärksten korrelierten Trockenheitsindexvariable $\left(\mathrm{TI}_{5,6}\right)$ und dem dGZ beobachtet: für Inger 0,80, für AF 2 0,70 und für Max 1 0,66. Somit waren die r-Werte von $\mathrm{TI}_{5,6}$ gegenüber $\mathrm{N}_{5,6}$ um bis zu $50 \%$ erhöht. Zwei signifikante Funktionen konnten angepasst werden und zwar für den Klon AF 2 mit dem sigmoidalen Ansatz und $\mathrm{TI}_{4,6}$ als Prädiktor sowie für den Klon Inger mit dem logarithmischen Ansatz und $\mathrm{TI}_{5,6}$ als Prädiktor (Tab. 4-17). Abbildung 4-19 zeigt die Kurvenanpassung für den Klon Inger.

Tab. 4-17: Regressionsanalyse für den dGZ in Abhängigkeit von unterschiedlichen Trockenheitsindices im Standortcluster L. Ausgewählt sind die klonspezifisch besten Anpassungen.

\begin{tabular}{llcccc}
\hline Gattung & Klon & $\mathrm{n}$ & Variable & Anpassung & $\mathrm{R}^{2}{ }_{\text {korr }}$ \\
\hline \multirow{2}{*}{ Pappel } & Max 1 & 7 & $\mathrm{TI}_{5,6}$ & lin & 0,32 \\
& AF 2 & 7 & $\mathrm{TI}_{4,6}$ & sig & $\mathbf{0 , 5 3}^{*}$ \\
Weide & Inger & 7 & $\mathrm{TI}_{5,6}$ & ln & $\mathbf{0 , 5 5}^{*}$ \\
\hline
\end{tabular}

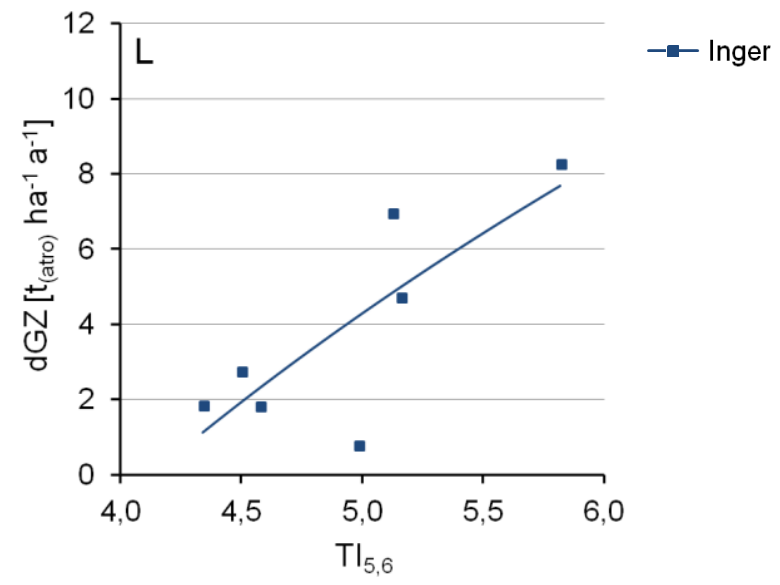

Abb. 4-19: Regressionsverlauf für den dGZ in Abhängigkeit von $\mathrm{TI}_{5,6}$ im Standortcluster L, dargestellt am Beispiel des Klons Inger.

\subsubsection{Bodenschätzungskennwerte}

Die drei hier geprüften Variablen ZS, BZ und AZ zeigten bei allen Standortclustern überwiegend mäBig bis starke Korrelationen mit dem dGZ auf (Tab. 4-18). AZ und BZ waren positiv, ZS erwartungsgemäß negativ mit dem dGZ korreliert. 
Tab. 4-18: Korrelationsanalyse zwischen Bodenschätzungsvariablen und dem dGZ für die untersuchten Klone in den Standortclustern ALL, S, L und U. Dargestellt sind die r-Werte.

\begin{tabular}{|c|c|c|c|c|c|c|}
\hline \multirow{2}{*}{$\begin{array}{l}\text { Standort- } \\
\text { cluster }\end{array}$} & \multirow{2}{*}{ Gattung } & \multirow{2}{*}{ Klon } & \multirow{2}{*}{$\mathrm{n}$} & \multicolumn{3}{|c|}{$\mathrm{r}$} \\
\hline & & & & $\mathrm{ZS}$ & $\mathrm{BZ}$ & $\mathrm{AZ}$ \\
\hline \multirow{4}{*}{ ALL } & \multirow{2}{*}{ Pappel } & Max 1 & 20 & $-0,43^{(*)}$ & 0,33 & 0,29 \\
\hline & & AF 2 & 20 & $-0,53^{*}$ & $0,46^{*}$ & $0,43^{(*)}$ \\
\hline & \multirow{2}{*}{ Weide } & Inger & 20 & $-0,44^{(*)}$ & 0,32 & 0,28 \\
\hline & & Tordis & 18 & $-0,39$ & $0,46^{(*)}$ & $0,46^{(*)}$ \\
\hline \multirow{5}{*}{ S } & \multirow{3}{*}{ Pappel } & $\operatorname{Max} 1$ & 8 & $-0,23$ & 0,46 & 0,52 \\
\hline & & Н 275 & 7 & 0,00 & 0,36 & 0,40 \\
\hline & & AF 2 & 8 & $-0,35$ & 0,52 & 0,58 \\
\hline & \multirow{2}{*}{ Weide } & Inger & 8 & $-0,19$ & 0,42 & 0,49 \\
\hline & & Tordis & 8 & $-0,17$ & 0,46 & 0,50 \\
\hline \multirow{4}{*}{$\mathrm{U}$} & \multirow{2}{*}{ Pappel } & $\operatorname{Max} 1$ & 5 & $-0,81^{(*)}$ & $0,82^{(*)}$ & 0,73 \\
\hline & & AF 2 & 5 & $-0,82^{(*)}$ & 0,72 & 0,56 \\
\hline & \multirow{2}{*}{ Weide } & Inger & 5 & $-0,65$ & 0,53 & 0,43 \\
\hline & & Tordis & 5 & $-0,94^{*}$ & $0,97^{* *}$ & $0,84^{(*)}$ \\
\hline \multirow{3}{*}{$\mathrm{L}$} & \multirow{2}{*}{ Pappel } & Max 1 & 7 & $-0,32$ & 0,18 & 0,05 \\
\hline & & AF 2 & 7 & $-0,55$ & 0,44 & 0,32 \\
\hline & Weide & Inger & 7 & $-0,49$ & 0,25 & 0,09 \\
\hline
\end{tabular}

ZS zeigte unter den untersuchten Variablen die höchsten r-Werte bei der Korrelation mit dem dGZ. Die r-Werte schwankten dabei zwischen -0,53 (AF 2) und -0,39 (Tordis). Sowohl für BZ als auch für AZ waren die Korrelationen mit dem dGZ allesamt mäßiger Stärke. BZ war bei allen Klonen geringfügig besser mit dem dGZ korreliert als AZ. Prozentual betrug der Unterschied der r-Werte zwischen den Korrelationen von BZ und AZ mit dem dGZ 0,5\% bis $18 \%$. Im Standortcluster ALL waren nur zwei Korrelationen signifikant: ZS sowie BZ jeweils mit dem dGZ beim Klon AF 2 (Tab. 4-18). Für die Klone AF 2 und Tordis wurden mit AZ als Prädiktor die besten Regressionen mit dem dGZ ermittelt (Tab. 4-19, Abb. 4-20).

Tab. 4-19: Regressionsanalyse für den dGZ in Abhängigkeit von AZ im Standortcluster ALL. Ausgewählt sind die klonspezifisch besten Anpassungen.

\begin{tabular}{llcccc}
\hline Gattung & Klon & $\mathrm{n}$ & Variable & Anpassung & $\mathrm{R}^{2}$ korr \\
\hline \multirow{2}{*}{ Pappel } & Max 1 & 20 & $\mathrm{AZ}$ & inv & 0,06 \\
& AF 2 & 20 & AZ & sig & $\mathbf{0 , 2 2}^{*}$ \\
\multirow{2}{*}{ Weide } & Inger & 20 & AZ & inv & 0,05 \\
& Tordis & 18 & AZ & ln & $\mathbf{0 , 2 0}$
\end{tabular}

AZ wies unter den Bodenschätzungsvariablen im Standortcluster ALL stets die klonspezifischen besten Anpassungen für den dGZ auf.

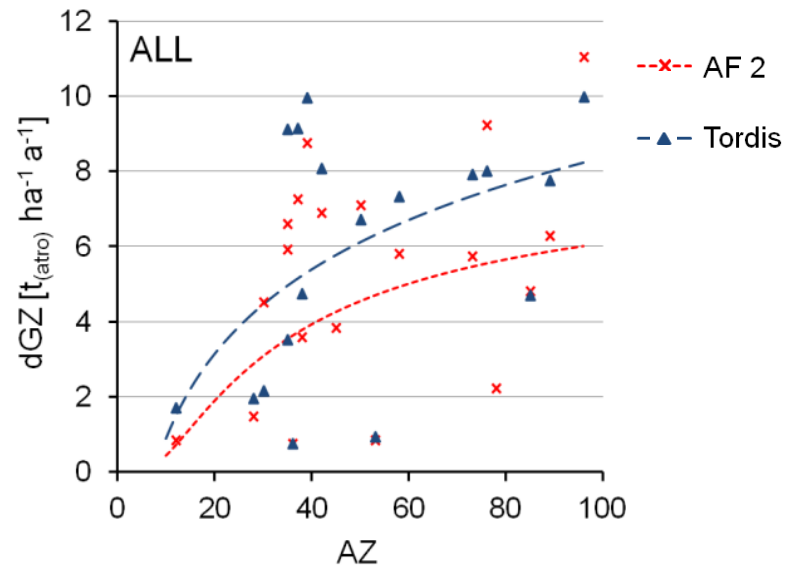

Abb. 4-20: Regressionsverlauf für den dGZ in Abhängigkeit von AZ im Standortcluster ALL dargestellt am Beispiel der Klone AF 2 und Tordis. 
Die $\mathrm{R}^{2}$ korr der Funktionen bezifferten sich auf 0,22 bei AF 2 und auf 0,20 bei Tordis. Aufgrund der Ordinalskalierung der Variablen ZS ist die Verwendung der Regression, trotz der hohen r-Werte, statistisch nicht durchführbar.

Im Standortcluster S verhielt sich die Stärke der Korrelationen der untersuchten Variablen mit dem dGZ wie folgt zueinander: ZS war am dritthöchsten, BZ am zweithöchsten, AZ am höchsten mit dem dGZ korreliert. Die Korrelationen bei ZS zeigten r-Werte in einem Schwankungsbereich zwischen 0,00 und $-0,35$. Bei BZ und AZ hingegen waren die Korrelationen mäßig bis stark mit r-Werten zwischen 0,58 (AZ - AF 2) und 0,36 (BZ - H 275). Im Standortcluster S waren alle Korrelationen und Regressionen nicht signifikant.

Beim Standortcluster U wurden die stärksten Korrelationen mit dem dGZ bei der Variablen ZS ermittelt, gefolgt von BZ und AZ. Die Korrelationsstärke war bei allen untersuchten Variablen stark bis sehr stark. Die einzelnen r-Werte schwankten bei ZS zwischen -0,94 (Tordis) und -0,65 (Inger), bei BZ zwischen 0,97 (Tordis) und 0,53 (Inger) sowie bei AZ zwischen 0,84 (Tordis) und 0,43 (Inger). AF 2 zeigte durchweg die niedrigsten, Tordis die höchsten r-Werte. Signifikante Korrelationen mit dem dGZ wurden für Tordis für ZS und BZ nachgewiesen. Bei der Kurvenanpassung wurde für den Klon Tordis eine signifikante Regression mit dem compound Ansatz ermittelt (Tab. 4-20, Abb. 4-21). Der erzielte $\mathrm{R}^{2}$ korr war mit $0,95 \mathrm{sehr}$ hoch.

Tab. 4-20: Regressionsanalyse für den dGZ in Abhängigkeit von BZ im Standortcluster U. Ausgewählt sind die klonspezifisch besten Anpassungen.

\begin{tabular}{llcccc}
\hline Gattung & Klon & $\mathrm{n}$ & Variable & Anpassung & $\mathrm{R}^{2}{ }_{\text {korr }}$ \\
\hline \multirow{2}{*}{ Pappel } & Max 1 & 5 & $\mathrm{BZ}^{\mathrm{a}}$ & lin & 0,57 \\
& $\mathrm{AF} \mathrm{2}$ & 5 & $\mathrm{BZ}$ & lin & 0,36 \\
Weide & Inger & 5 & $\mathrm{BZ}$ & lin & 0,05 \\
& Tordis & 5 & $\mathrm{BZ}$ & comp & $\mathbf{0 , 9 5}^{*}$ \\
\hline $\begin{array}{l}\text { a BZ wies unter den Bodenschätzungsvariablen im Standort- } \\
\text { cluster U stets die klonspezifisch besten Anpassungen für den } \\
\text { dGZ auf. }\end{array}$
\end{tabular}

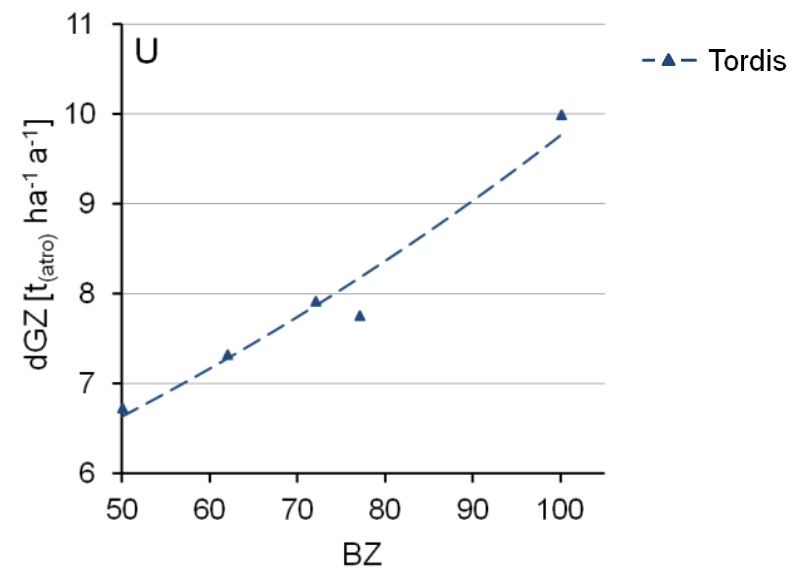

Abb. 4-21: Regressionsverlauf für den dGZ in Abhängigkeit von BZ im Standortcluster U, dargestellt am Beispiel des Klons Tordis.

Beim Standortcluster L waren die Korrelationen von Bodenschätzungsvariablen und dem dGZ deutlich niedriger als bei den restlichen Standortclustern. Die besten Korrelationen mit dem dGZ wurden bei ZS erzielt mit r-Werten zwischen -0,32 (Max 1) und -0,55 (AF 2). Dagegen waren die Korrelationen zwischen BZ und dem dGZ schwach bis mäßig. Die r-Werte variierten zwischen 0,44 (AF 2) und 0,18 (Max 1). Bei der Korrelation zwischen AZ und dGZ betrugen die r-Werte für Max 1 0,05, für AF 20,32 und für Inger 0,09. Somit waren sie deutlich niedriger als die Korrelation zwischen BZ und dGZ.

\subsubsection{Textur}

Im Bereich der Textur wurden schwache bis sehr starke Korrelationen mit dem dGZ festgestellt (Tab. 4-21). Unter den Texturvariablen beim Standortcluster ALL war $\mathrm{U}_{[\%]}$ am besten mit dem dGZ korreliert (Tab. 4-21).

Bei den einzelnen Klonen lagen die errechneten r-Werte zwischen 0,46 (Tordis) und 0,33 (Max 1). Die beiden anderen Texturvariablen, $\mathrm{S}_{[\%]}$ und $\mathrm{T}_{[\%]}$, waren mit dem dGZ überwiegend schwach korreliert. In 
diesem Standortcluster war keine Korrelation signifikant. Signifikante Regressionen wurden für die Klone AF 2 und Tordis mit $\mathrm{U}_{[\%]}$ als Prädiktor angepasst (Tab. 4-22, Abb. 4-22). Hierbei wurde der sigmoidale Ansatz verwendet, der $\mathrm{R}_{\text {korr }}^{2}$ betrug für AF 20,35 und für Tordis 0,33.

Tab. 4-21: Korrelationsanalyse zwischen Texturvariablen und dem dGZ für die untersuchten Klone in den Standortclustern ALL, S, L und U. Dargestellt sind die r-Werte.

\begin{tabular}{|c|c|c|c|c|c|c|}
\hline \multirow{2}{*}{$\begin{array}{l}\text { Standort- } \\
\text { cluster }\end{array}$} & \multirow{2}{*}{ Gattung } & \multirow{2}{*}{ Klon } & \multirow{2}{*}{$\mathrm{n}$} & \multicolumn{3}{|c|}{$\mathrm{r}$} \\
\hline & & & & $\mathrm{S}_{[\%]}$ & $\mathrm{U}_{[\%]}$ & $\mathrm{T}_{[\%]}$ \\
\hline \multirow{4}{*}{ ALL } & \multirow{2}{*}{ Pappel } & Max 1 & 20 & $-0,15$ & 0,33 & $-0,23$ \\
\hline & & $\mathrm{AF} 2$ & 20 & $-0,25$ & $0,43^{(*)}$ & $-0,17$ \\
\hline & \multirow{2}{*}{ Weide } & Inger & 20 & $-0,16$ & 0,35 & $-0,25$ \\
\hline & & Tordis & 18 & $-0,35$ & $0,46^{(*)}$ & 0,00 \\
\hline \multirow{5}{*}{ S } & \multirow{3}{*}{ Pappel } & Max 1 & 8 & $-0,81^{*}$ & $0,75^{*}$ & 0,47 \\
\hline & & Н 275 & 7 & $-0,85^{*}$ & $\mathbf{0 , 7 7 ^ { * }}$ & $0,68^{(*)}$ \\
\hline & & $\mathrm{AF} 2$ & 8 & $-0,82^{*}$ & $0,83^{*}$ & 0,22 \\
\hline & \multirow{2}{*}{ Weide } & Inger & 8 & $-0,79^{*}$ & $0,80^{*}$ & 0,23 \\
\hline & & Tordis & 8 & $-0,83^{*}$ & $0,79^{*}$ & 0,38 \\
\hline \multirow{4}{*}{$\mathrm{U}$} & \multirow{2}{*}{ Pappel } & Max 1 & 5 & 0,52 & $-0,59$ & $-0,04$ \\
\hline & & $\mathrm{AF} 2$ & 5 & 0,53 & $-0,50$ & $-0,17$ \\
\hline & \multirow{2}{*}{ Weide } & Inger & 5 & 0,30 & $-0,18$ & $-0,21$ \\
\hline & & Tordis & 5 & 0,77 & $-0,89^{*}$ & $-0,05$ \\
\hline \multirow{3}{*}{$\mathrm{L}$} & \multirow{2}{*}{ Pappel } & Max 1 & 7 & $-0,18$ & $0,79^{*}$ & $-0,50$ \\
\hline & & $\mathrm{AF} 2$ & 7 & $-0,25$ & $\mathbf{0 , 8 7 ^ { * }}$ & $-0,51$ \\
\hline & Weide & Inger & 7 & $-0,14$ & $0,89^{* *}$ & $-0,62$ \\
\hline
\end{tabular}

Tab. 4-22: Regressionsanalyse für den dGZ in Abhängigkeit von $\mathrm{U}_{[\%]}$ im Standortcluster ALL. Ausgewählt sind die klonspezifisch besten Anpassungen.

\begin{tabular}{|c|c|c|c|c|c|}
\hline Gattung & Klon & $\mathrm{n}$ & Variable & Anpassung & $\mathrm{R}_{\text {korr }}^{2}$ \\
\hline \multirow{2}{*}{ Pappel } & Max 1 & 20 & $\mathrm{U}_{[\%]}^{\mathrm{a}}$ & inv & 0,11 \\
\hline & AF 2 & 20 & $\mathrm{U}_{[\%]}$ & sig & $0,35^{* *}$ \\
\hline \multirow{2}{*}{ Weide } & Inger & 20 & $\mathrm{U}_{[\%]}$ & sig & 0,13 \\
\hline & Tordis & 18 & $\mathrm{U}_{[\%]}$ & sig & $\mathbf{0 , 3 3}{ }^{* *}$ \\
\hline
\end{tabular}

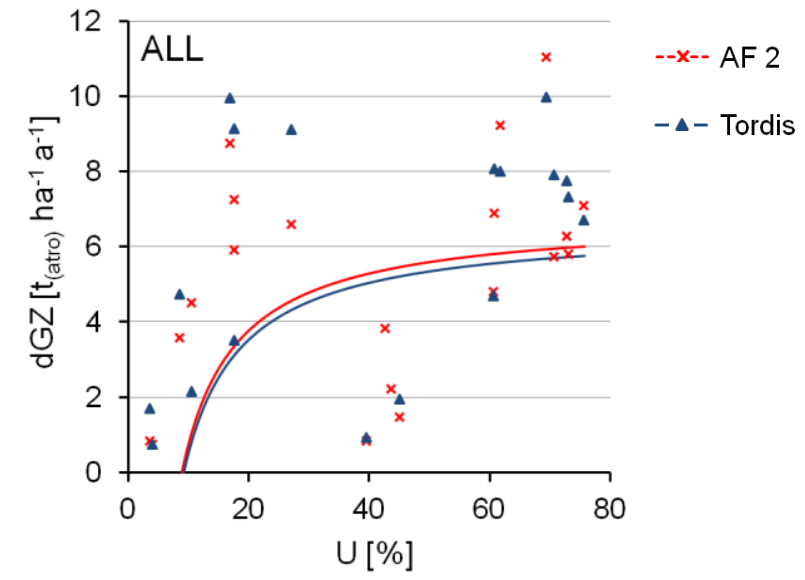

Abb. 4-22: Regressionsverlauf für den dGZ in Abhängigkeit von $\mathrm{U}_{[\%]}$ im Standortcluster ALL dargestellt am Beispiel der Klone AF 2 und Tordis.

Beim Standortcluster $\mathrm{S}$ waren die dGZ überwiegend sehr stark negativ mit $\mathrm{S}_{[\%]}$ korreliert. Es sei vermerkt, dass die sehr hohe negative Korrelation mit $S_{[\%]}$ eine im gleichen Maß sehr starke aber positiv gerichtete Korrelation mit dem Feinbodenanteil (Summe von $\mathrm{U}_{[\%]}$ und $\mathrm{T}_{[\%]}$ ) impliziert. Die r-Werte der allesamt signifikanten Korrelationen schwankten zwischen -0,85 (H 275) und -0,79 (AF 2). Damit ist die Variation der r-Werte der Klone als gering einzustufen. Des Weiteren wurden starke bis sehr starke 
positive Korrelationen zwischen $\mathrm{U}_{[\%]}$ und dem dGZ festgestellt. Deren r-Werte bewegten sich zwischen 0,83 (AF 2) und 0,75 (Max 1). Im Mittel waren die r-Werte bei der Korrelation zwischen $\mathrm{U}_{[\%]}$ und dem dGZ geringfügig kleiner als bei der Korrelation zwischen $S_{[\%]}$ und dem dGZ. Für jeden Klon konnten zwischen den Variablen $\mathrm{U}_{[\%]}$ und dem dGZ signifikante Kurvenanpassungen hergeleitet werden (Tab. 4-23, Abb. 4-23a). Bis auf Inger wurde hierfür der sigmoidale Ansatz verwendet. Die $\mathrm{R}^{2}{ }_{\text {korr }}$ variierten zwischen 0,95 (AF 2) und 0,57 (H 275). Zwischen $S_{[\%]}$ und dem dGZ sind ebenfalls signifikante Funktionen ermittelt worden (Tab. 4-23, Abb. 4-23b). Dabei wurde überwiegend die exponentielle Anpassung (1) verwendet. Die $\mathrm{R}^{2}$ korr der Anpassungen mit $\mathrm{S}_{[\%]}$ als Prädiktor waren durchschnittlich geringer als die der Anpassungen mit $\mathrm{U}_{[\%]}$ als Prädiktor. Insbesondere bei AF 2 war die Anpassung der Variable $\mathrm{U}_{[\%]}$ deutlich stärker als die von $\mathrm{S}_{[\%]}$. Bei den Klonen H 275 und Tordis war die Beziehung der Variablen $\mathrm{S}_{[\%]}$ mit dem dGZ bis zu $10 \%$ genauer als die der Variablen $\mathrm{U}_{[\%]}$ mit dem dGZ.

Tab. 4-23: Regressionsanalyse für den dGZ in Abhängigkeit von den Texturvariablen im Standortcluster S. Ausgewählt sind die klonspezifisch besten Anpassungen für $\mathrm{S}_{[\%]}$ sowie für $\mathrm{U}_{[\%]}$.

\begin{tabular}{|c|c|c|c|c|c|}
\hline Gattung & Klon & $\mathrm{n}$ & Variable & Anpassung & $\mathrm{R}^{2}{ }_{\text {korr }}$ \\
\hline \multirow{6}{*}{ Pappel } & \multirow{2}{*}{ Max 1} & \multirow{2}{*}{8} & $\mathrm{~S}_{[\%]}$ & exp1 & $0,64^{*}$ \\
\hline & & & $\mathrm{U}_{[\%]}$ & $\operatorname{sig}$ & $0,81^{* *}$ \\
\hline & \multirow{2}{*}{ Н 275} & \multirow{2}{*}{7} & $\mathrm{~S}_{[\%]}$ & $\operatorname{lin}$ & $0,66^{*}$ \\
\hline & & & $\mathrm{U}_{[\%]}$ & sig & $0,57^{*}$ \\
\hline & \multirow{2}{*}{ AF 2} & \multirow{2}{*}{8} & $\mathrm{~S}_{[\%]}$ & $\exp 1$ & $0,63^{*}$ \\
\hline & & & $\mathrm{U}_{[\%]}$ & sig & $0,95^{* * * *}$ \\
\hline \multirow{4}{*}{ Weide } & \multirow{2}{*}{ Inger } & \multirow{2}{*}{8} & $\mathrm{~S}_{[\%]}$ & $\exp 1$ & $0,69^{* *}$ \\
\hline & & & $\mathrm{U}_{[\%]}$ & $\exp 2$ & $0,68^{*}$ \\
\hline & \multirow{2}{*}{ Tordis } & \multirow{2}{*}{8} & $\mathrm{~S}_{[\%]}$ & $\exp 1$ & $0,67^{* *}$ \\
\hline & & & $\mathrm{U}_{[\%]}$ & sig & $0,62^{* *}$ \\
\hline
\end{tabular}
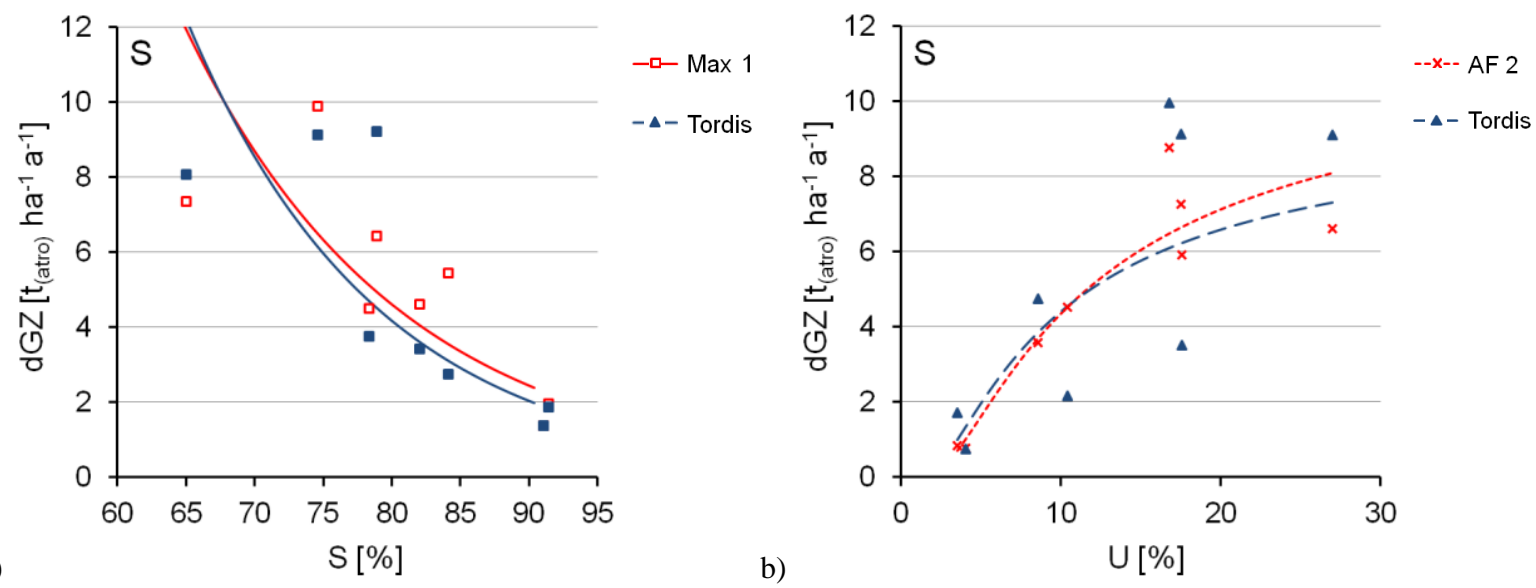

Abb. 4-23: Regressionsverlauf für den dGZ in Abhängigkeit von Texturvariablen im Standortcluster S. Mit a) $S_{[\%]}$ dargestellt am Beispiel der Klone Max 1 und Inger und b) $\mathrm{U}_{[\%]}$ dargestellt am Beispiel der Klone AF 2 und Tordis.

Beim Standortcluster $\mathrm{U}$ wurden positive, mäßige bis starke Korrelationen von $\mathrm{S}_{[\%]}$, negative schwache bis sehr starke Korrelationen von $\mathrm{U}_{[\%]}$ sowie sehr schwache bis schwache Korrelationen von $\mathrm{T}_{[\%]}$ mit dem dGZ ermittelt. Im Standortcluster U waren die Korrelationen nicht signifikant, mit Ausnahme der Korrelation bei Tordis von $\mathrm{U}_{[\%]}$ mit dem dGZ. Dabei ist zu ergänzen, dass abweichend von den bisher untersuchten Variablen bei den Standorten im Standortcluster $U$ die absolute Variation von $S_{[\%]}$, $U_{[\%]}$ und $\mathrm{T}_{[\%]}$ sehr gering ist. Die Texturvariablen variierten wie folgt: $\mathrm{S}_{[\%]}$ zwischen je 3,8 und 9,3\%, $\mathrm{U}_{[\%]}$ 
zwischen 69,2 und 75,5\% sowie $\mathrm{T}_{[\%]} \mathrm{zwischen} 18,9$ und 24,3\%. Damit ist die Aussagekraft der Korrelationen zwischen Texturvariablen und dem dGZ in diesem Standortcluster begrenzt.

Bei den Lehmböden war $\mathrm{U}_{[\%]}$ die mit dem dGZ am besten korrelierte Texturvariable. Bei den einzelnen Klonen waren die Korrelationen mit r-Werten zwischen 0,89 (Inger) und 0,79 (Max 1) stets stark bis sehr stark (Tab. 4-21). Des Weiteren zeigte $\mathrm{T}_{[\%]}$ eine starke negative Beziehung mit dem dGZ. Die $r$-Werte bei den einzelnen Klonen variierten zwischen -0,62 (Inger) und -0,50 (Max 1). Im Rahmen der Regressionsanalyse erfolgten bei allen Klonen signifikante Anpassungen zwischen Texturvariablen und dGZ (Tab. 4-24, Abb. 4-24). Bei Max 1 handelte es sich um die logarithmische Anpassung, bei den restlichen Klonen um die lineare Anpassung.

Tab. 4-24: Regressionsanalyse für den dGZ in Abhängigkeit von $\mathrm{U}_{[\%]}$ im Standortcluster L. Ausgewählt sind die klonspezifisch besten Anpassungen.

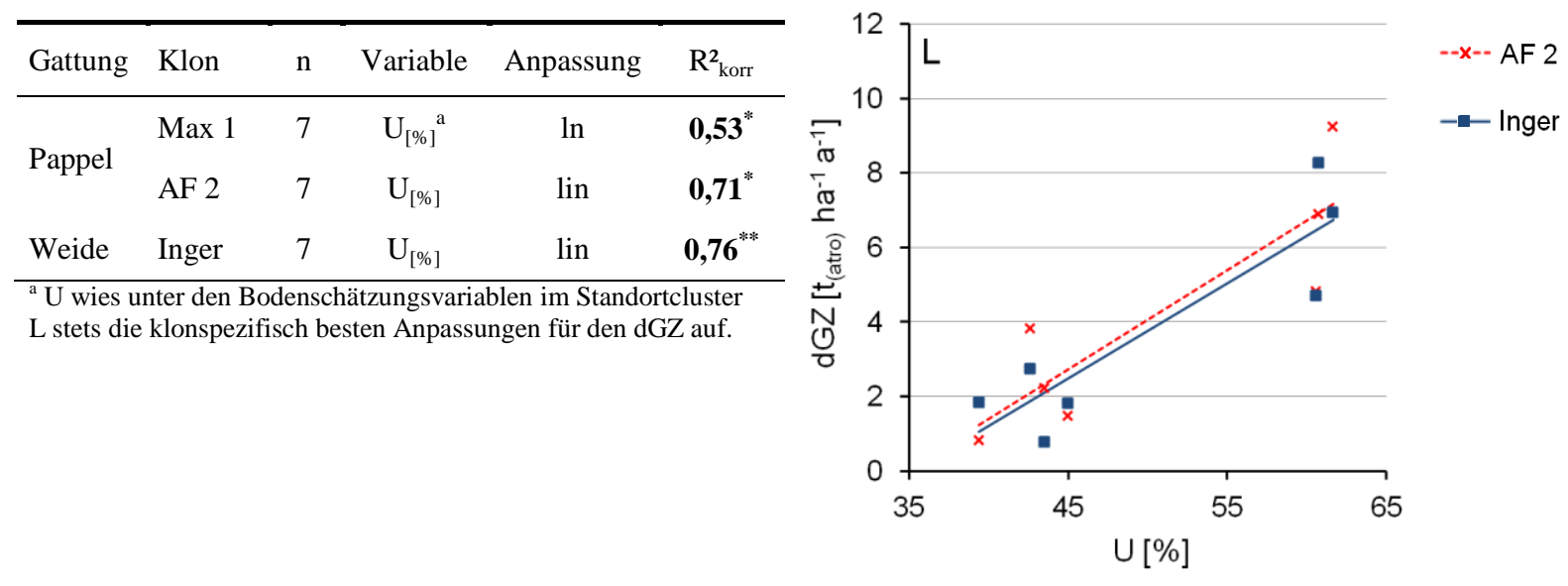

Abb. 4-24: Regressionsverlauf für den dGZ in Abhängigkeit von $\mathrm{U}_{[\%]}$ im Standortcluster L, dargestellt am Beispiel der Klone AF 2 und Inger.

\subsubsection{Porenraum}

Im Standortcluster ALL hatte die Korrelationsanalyse zwischen nFK FÄ und LK FÄ mit dem dGZ höhere r-Werte ergeben als bei der Korrelation zwischen dem dGZ mit nFK und LK (siehe Anhang 29). In den weiteren Standortclustern S, U und L gab es keine signifikative Verbesserung der r-Werte. Aus diesem Grund sowie aufgrund der bisher marginalen Verwendung in der Praxis der Porenraumkenngrößen beim FÄ wird im Fortlauf dieser Arbeit ausschließlich mit den Variablen nFK sowie LK nach Standardfeldkapazität bei $\mathrm{pF} 1,8$ gearbeitet.

Bei den Variablen des Porenraums konnten starke bis sehr starke Korrelationen mit dem dGZ beschrieben werden. Je nach Standortcluster veränderten sich die bestkorrelierten Variablen. Bei Berücksichtigung aller Standorte zeigte die nFK unter den Porenraumvariablen den größten Zusammenhang mit dem dGZ auf (Tab. 4-25). Die r-Werte der einzelnen Klone variierten zwischen 0,61 (Tordis) und 0,52 (Max 1). Dabei waren alle Korrelationen signifikant. Die Korrelationen der Variablen LK und TRD mit dem dGZ waren im Standortcluster ALL allesamt sehr schwach bis schwach. Signifikante Funktionen konnten für jeden Klon mit nFK als Prädiktor angepasst werden (Tab. 4-26, Abb. 4-25). Die besten $\mathrm{R}^{2}$ korr wurden für Max 1 mit dem allometrischen Ansatz, für AF 2 und Tordis mit dem logarithmischen Ansatz sowie für Inger mit dem sigmoidalen Ansatz ermittelt. 
Tab. 4-25: Korrelationsanalyse zwischen Porenraumvariablen und dem dGZ für die untersuchten Klone in den Standortclustern ALL, S, U und L. Dargestellt sind die r-Werte.

\begin{tabular}{|c|c|c|c|c|c|c|}
\hline \multirow{2}{*}{$\begin{array}{l}\text { Standort- } \\
\text { cluster }\end{array}$} & \multirow{2}{*}{ Gattung } & \multirow{2}{*}{ Klon } & \multirow{2}{*}{$\mathrm{n}$} & \multicolumn{3}{|c|}{$\mathrm{r}$} \\
\hline & & & & nFK & LK & TRD \\
\hline \multirow{4}{*}{ ALL } & \multirow{2}{*}{ Pappel } & Max 1 & 20 & $0,52^{*}$ & $-0,12$ & $-0,15$ \\
\hline & & $\mathrm{AF} 2$ & 20 & $0,61^{* *}$ & $-0,22$ & $-0,05$ \\
\hline & \multirow{2}{*}{ Weide } & Inger & 20 & $0,56^{*}$ & $-0,08$ & $-0,07$ \\
\hline & & Tordis & 18 & $0,61^{* *}$ & $-0,31$ & $-0,01$ \\
\hline \multirow{5}{*}{ S } & \multirow{3}{*}{ Pappel } & Max 1 & 8 & 0,23 & $-0,66^{(*)}$ & 0,47 \\
\hline & & H 275 & 7 & 0,08 & $-0,56$ & 0,38 \\
\hline & & AF 2 & 8 & 0,31 & $-0,69^{(*)}$ & 0,64 \\
\hline & \multirow{2}{*}{ Weide } & Inger & 8 & 0,36 & $-0,49$ & 0,56 \\
\hline & & Tordis & 8 & 0,41 & $-0,52$ & 0,43 \\
\hline \multirow{4}{*}{$\mathrm{U}$} & \multirow{2}{*}{ Pappel } & Max 1 & 5 & 0,25 & $0,81^{(*)}$ & $-0,79$ \\
\hline & & AF 2 & 5 & 0,59 & $0,84^{(*)}$ & $-0,83^{(*)}$ \\
\hline & \multirow{2}{*}{ Weide } & Inger & 5 & 0,21 & 0,69 & $-0,69$ \\
\hline & & Tordis & 5 & 0,67 & $0,92^{*}$ & $-0,90^{*}$ \\
\hline \multirow{3}{*}{$\mathrm{L}$} & \multirow{2}{*}{ Pappel } & Max 1 & 7 & $0,86^{*}$ & 0,60 & $-0,39$ \\
\hline & & AF 2 & 7 & $0,84^{*}$ & 0,49 & $-0,27$ \\
\hline & Weide & Inger & 7 & $0,93^{* * *}$ & $\mathbf{0 , 8 0 ^ { * }}$ & $-0,39$ \\
\hline
\end{tabular}

Tab. 4-26: Regressionsanalyse für den dGZ in Abhängigkeit von nFK im Standortcluster ALL. Ausgewählt sind die klonspezifisch besten Anpassungen.

\begin{tabular}{llcccc}
\hline Gattung & Klon & $\mathrm{n}$ & Variable & Anpassung & $\mathrm{R}^{2}{ }_{\text {korr }}$ \\
\hline \multirow{2}{*}{ Pappel } & Max 1 & 20 & $\mathrm{nFK}^{\mathrm{a}}$ & allo & $\mathbf{0 , 2 6}^{*}$ \\
& AF 2 & 20 & $\mathrm{nFK}$ & ln & $\mathbf{0 , 3 3}^{\text {*** }}$ \\
\multirow{2}{*}{ Weide } & Inger & 20 & $\mathrm{nFK}$ & sig & $\mathbf{0 , 3 3}^{\text {*** }}$ \\
& Tordis & 18 & $\mathrm{nFK}$ & ln & $\mathbf{0 , 3 4}^{\text {*** }}$ \\
\hline a nFK wies unter den Texturvariablen im Standortcluster ALL \\
stets die klonspezifisch besten Anpassungen für den dGZ auf
\end{tabular}

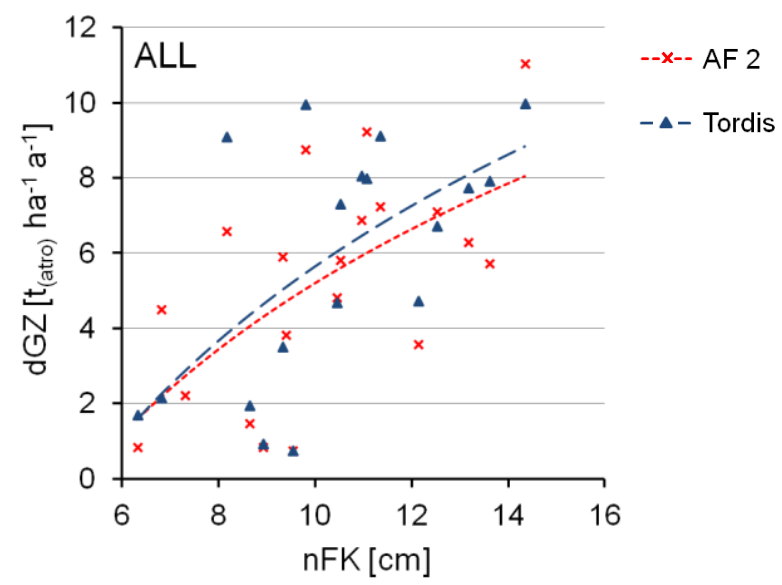

Abb. 4-25: Regressionsverlauf für den dGZ in Abhängigkeit von nFK im Standortcluster ALL, dargestellt am Beispiel der Klone AF 2 und Tordis.

Beim Standortcluster $\mathrm{S}$ wurden unerwartet nur schwache bis mäßige Korrelationen zwischen nFK und dem dGZ nachgewiesen. Die r-Werte bewegten sich zwischen 0,41 (Tordis) und 0,08 (H 275). Bei den Sandböden war die Korrelation zwischen LK und dem dGZ negativ und stark ausgeprägt. Die r-Werte schwankten zwischen -0,69 (AF 2) und -0,49 (Inger). Für Max 1 und AF 2 resultierten signifikante Regressionen mit LK als unabhängige Variable mittels des exponentiellen Ansatzes (1) (Tab. 4-27, Abb. 4-26). 
Tab. 4-27: Regressionsanalyse für den dGZ in Abhängigkeit von LK im Standortcluster S. Ausgewählt sind die klonspezifisch besten Anpassungen.

\begin{tabular}{lllccc}
\hline Gattung & Klon & $\mathrm{n}$ & Variable & Anpassung & $\mathrm{R}^{2}{ }_{\text {korr }}$ \\
\hline \multirow{4}{*}{ Pappel } & Max 1 & 8 & LK $^{\mathrm{a}}$ & $\operatorname{exp1}$ & $\mathbf{0 , 4 8}^{*}$ \\
& H 275 & 7 & LK & sig & 0,39 \\
& AF 2 & 8 & LK & exp1 & $\mathbf{0 , 6 0}^{*}$ \\
\multirow{2}{*}{ Weide } & Inger & 8 & LK & allo & 0,26 \\
& Tordis & 8 & LK & sig & 0,26 \\
\hline
\end{tabular}

${ }^{a}$ LK wies unter den Bodenschätzungsvariablen im Standortcluster $\mathrm{S}$ stets die klonspezifisch besten Anpassungen für den dGZ auf

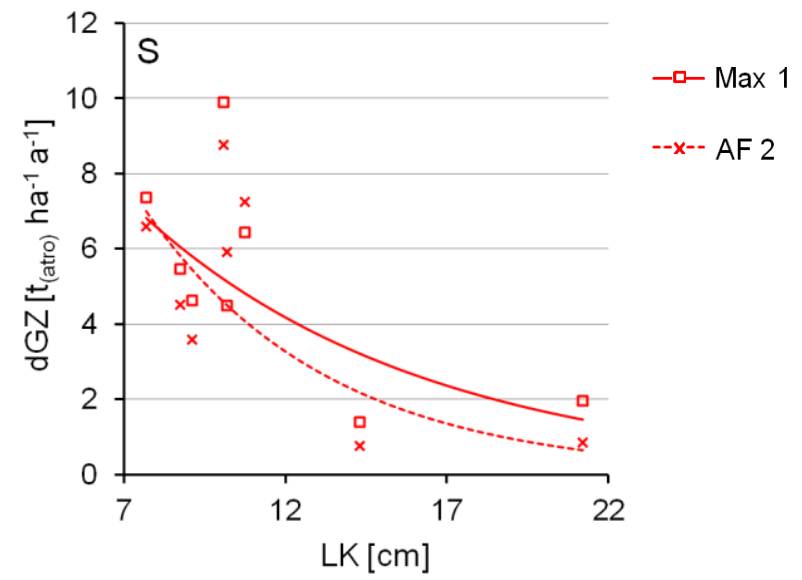

Abb. 4-26: Regressionsverlauf für den dGZ in Abhängigkeit von LK im Standortcluster S, dargestellt am Beispiel der Klone Max 1 und AF 2.

Während beim Standortcluster U die Korrelation zwischen nFK und dem dGZ mäßig bis stark ausfiel, wurden bei LK und TRD mit dem dGZ starke bis sehr starke Korrelationen erfasst (Tab. 4-25). Dabei war LK mit dem dGZ negativ korreliert. Für die genannte Korrelation wurden r-Werte zwischen -0,92 (Inger) und -0,69 (AF 2) ermittelt. Bei der Korrelation zwischen TRD und dGZ schwankten die r-Werte zwischen 0,90 (Tordis) und 0,69 (Inger).

Die nFK war im Standortcluster L signifikant, stark bis sehr stark positiv mit dem dGZ korreliert. In diesem Standortcluster war der Effekt der nFK auf dem dGZ gegenüber dem Textureffekt (bestkorrelierte Variable $\mathrm{U}_{[\%]}$ ) deutlicher. Bei den Lehmböden war die Korrelation zwischen LK und dGZ positiv. Bei den einzelnen Klonen schwankten die r-Werte zwischen 0,80 (Inger) und 0,49 (AF 2), wobei nur die Korrelation bei Inger signifikant war. Zwischen Texturvariablen und dGZ wurden die besten Kurvenanpassungen mit der Variablen nFK als Prädiktor ermittelt (Tab. 4-28, Abb. 4-27). Für Max 1 und Inger wurde der exponentielle Ansatz (1) gewählt, dagegen für AF 2 der lineare Ansatz. Die $\mathrm{R}^{2}$ korr lagen zwischen 0,98 (Inger) und 0,64 (AF 2).

Tab. 4-28: Regressionsanalyse für den dGZ in Abhängigkeit von nFK im Standortcluster L. Ausgewählt sind die klonspezifisch besten Anpassungen.

\begin{tabular}{|c|c|c|c|c|c|}
\hline Gattung & Klon & $\mathrm{n}$ & Variable & Anpassung & $\mathrm{R}_{\text {korr }}^{2}$ \\
\hline \multirow{2}{*}{ Pappel } & Max 1 & 7 & $\mathrm{nFK}^{\mathrm{a}}$ & exp1 & $0,87^{* *}$ \\
\hline & $\mathrm{AF} 2$ & 7 & $\mathrm{nFK}$ & $\operatorname{lin}$ & $0,64^{*}$ \\
\hline Weide & Inger & 7 & $\mathrm{nFK}$ & exp1 & $0,98^{* * * *}$ \\
\hline
\end{tabular}

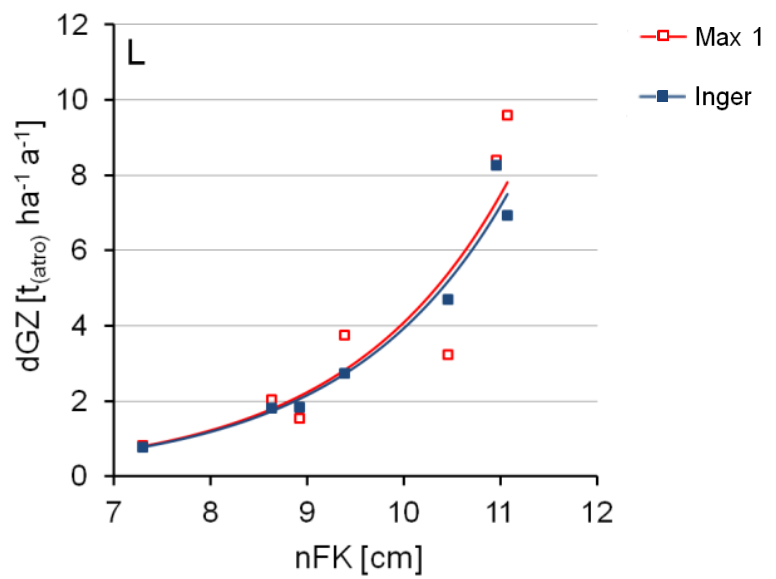

Abb. 4-27: Regressionsverlauf für den dGZ in Abhängigkeit von nFK im Standortcluster L dargestellt am Beispiel der Klone Max 1 und Inger. 


\subsubsection{Korrelation zwischen unabhängigen Variablen}

Eine sehr starke Korrelation zwischen Bodenvariablen im Standortcluster ALL wiesen die Variablen $\mathrm{U}_{[\%]}$ und $\mathrm{BZ}(\mathrm{r}=0,80)$, im Standortcluster $\mathrm{S}$ die Variablen $\mathrm{S}_{[\%]}$ und $\mathrm{U}_{[\%]}(\mathrm{r}=-0,97)$, im Standortcluster U die Variablen LK und TRD $(r=0,99)$, LK und BZ $(r=0,94)$ sowie TRD und BZ $(r=-0,92)$ sowie im Standortcluster L die Variablen $\mathrm{U}_{[\%]}$ und $\mathrm{nFK}(\mathrm{r}=0,84)$ auf.

Bei den Klimavariablen derselben Kategorie wie, Temperatur, Niederschlag oder Trockenheitsindexvariablen wurden oft sehr starke Korrelationen beobachtet. Insbesondere traf dies zu, wenn ähnliche Zeiträume vorlagen, wie zum Beispiel die Summe von Mai bis Juli $(5,7)$ sowie die Summe von Juni bis Juli $(6,7)$. Im Standortcluster ALL zeigten $\mathrm{N}_{5,7}$ und $\mathrm{N}_{6,7}, \mathrm{~N}_{5,7}$ und $\mathrm{TI}_{5,7}$ sowie $\mathrm{N}_{6,7}$ und $\mathrm{TI}_{6,7}$ eine sehr starke Korrelation. Im Standortcluster $\mathrm{S}$ wurden sehr starke Korrelationen zwischen $\mathrm{T}_{7,8}$ und $\mathrm{T}_{7,9}$ sowie $\mathrm{N}_{6,7}$ und $\mathrm{TI}_{6,7}$ festgestellt. Eher auffällige Korrelationen wurden im Standortcluster U erfasst. Hier waren die Variablen $\mathrm{T}_{7,8}, \mathrm{~T}_{7,9}, \mathrm{~N}_{4,5}$, sowie $\mathrm{TI}_{4,5}$ mit einem $\mathrm{r}$-Wert größer 0,90 korreliert. Darüber hinaus wiesen im Standortcluster U die Niederschlags- und Trockenheitsindexvariablen des Zeitfensters Mai bis Juni Korrelationen mit r-Werten größer 0,80 auf. Beim Standortcluster L waren die Variablen $\mathrm{T}_{\mathrm{VP}}$ und $\mathrm{T}_{7,9}$ mit $\mathrm{r}$-Wert von 0,98 miteinander korreliert.

Ebenfalls wurden sehr starke Korrelationen zwischen Boden- und Klimavariablen festgestellt. Im Standortcluster $\mathrm{L}$ waren die Variable LK mit je $\mathrm{T}_{7,9}$ und $\mathrm{T}_{\mathrm{VP}}$ sowie $\mathrm{U}_{[\%]}$ und $\mathrm{TI}_{5,6}$, im Standortcluster $\mathrm{U}$ $\mathrm{TI}_{5,6}$ mit je LK und TRD miteinander sehr stark korreliert. Weder beim Standortcluster ALL noch beim Standortcluster S wurden sehr starke Korrelationen zwischen Boden- und Klimavariablen identifiziert. Eine tabellarische Übersicht der Korrelationen zwischen den Standorteigenschaften, Bodenund Klimavariablen ist im Anhang (30 bis 33) zu finden.

\subsubsection{Aggregierte Variablen}

In diesem Kapitel werden die Variablen präsentiert, die zur Bildung von aggregierten Variablen fungierten. Darüber hinaus werden die Ergebnisse der Korrelationsanalyse zwischen aggregierten Variablen und dem dGZ vorgestellt.

Im Standortcluster ALL sind die Variablen $\mathrm{nFK}, \mathrm{BZ}, \mathrm{U}_{[\%]}, \mathrm{N}_{5,7}, \mathrm{TI}_{5,7}, \mathrm{~N}_{6,7}$ und $\mathrm{TI}_{6,7}$ zur Bildung aggregierter Variablen verwendet worden. Tabelle 4-29 können die für Standortcluster ALL durchgeführten Kombinationen entnommen werden. Wie im Kapitel 3.5.1.5 erwähnt, wurden die aggregierten Variablen mittels Multiplikation und Division gebildet.

Tab. 4-29:Variablenkombinationen im Standortcluster ALL.

\begin{tabular}{ccccc}
\hline $\mathrm{nFK}-\mathrm{BZ}$ & $\mathrm{U}_{[\%]}-\mathrm{N}_{5,7}$ & $\mathrm{U}_{[\%]}-\mathrm{N}_{6,7}$ & $\mathrm{U}_{[\%]}-\mathrm{TI}_{5,7}$ & $\mathrm{U}_{[\%]}-\mathrm{TI}_{6,7}$ \\
$\mathrm{nFK}-\mathrm{N}_{5,7}$ & $\mathrm{nFK}-\mathrm{N}_{6,7}$ & $\mathrm{nFK}-\mathrm{TI}_{5,7}$ & $\mathrm{nFK}-\mathrm{TI}_{6,7}$ & $\mathrm{BZ}-\mathrm{N}_{5,7}$ \\
$\mathrm{BZ}-\mathrm{N}_{6,7}$ & $\mathrm{BZ}-\mathrm{TI}_{5,7}$ & $\mathrm{BZ}-\mathrm{TI}_{6,7}$ & & \\
\hline
\end{tabular}

In der Tabelle 4-30 sind (maximal vier) aggregierte Variablen nach r-Wert geordnet dargestellt sowie eine Referenzvariable. Als Referenzvariable wurde die mit dem dGZ am stärksten korrelierte nicht aggregierte Variable herangezogen. Die aggregierten Variablen wurden erst in der Abbildung berücksichtigt wenn sie einen höheren r-Wert als die Korrelation zwischen der Referenzvariablen und dem dGZ aufwiesen. Es wird ersichtlich, dass die Hinzunahme von aggregierten Variablen in der Korrelationsanalyse deren r-Werte erheblich steigert. Dabei ist zu ergänzen, dass die vier in Tabelle 4-30 abgebildeten aggregierten Variablen lediglich eine Auswahl darstellen. Je nach Klon und Standortcluster konnten bis zu zehn aggregierte Variablen identifiziert werden, welche einen höheren r-Wert in der Korrelation mit dem dGZ als die Referenzvariable aufwiesen. 
Tab. 4-30: Korrelationsanalyse der aggregierten Variablen und dem dGZ im Standortcluster ALL. Dargestellt sind die rWerte der aggregierten Variablen $\mathrm{V}_{\mathrm{A} 1}$ bis $\mathrm{V}_{\mathrm{A} 4}$ (mit dem dGZ erst- bis viertbestkorrelierter aggregierter Variablen) sowie der Referenzvariablen $\mathrm{V}_{\text {ref }}$ (mit dem dGZ bestkorrelierter, nicht aggregierter Variablen).

\begin{tabular}{|c|c|c|c|c|c|c|c|c|}
\hline & \multicolumn{2}{|c|}{ Max 1} & \multicolumn{2}{|l|}{$\mathrm{AF} 2$} & \multicolumn{2}{|l|}{ Inger } & \multicolumn{2}{|c|}{ Tordis } \\
\hline & Variable & $\mathrm{r}$ & Variable & $\mathrm{r}$ & Variable & $\mathrm{r}$ & Variable & $\mathrm{r}$ \\
\hline $\mathrm{V}_{\mathrm{A} 1}$ & $\left(\mathrm{nFK} * \mathrm{TI}_{5,7}\right)$ & $0,69^{* * * *}$ & $\left(\mathrm{nFK} * \mathrm{TI}_{5,7}\right)$ & $0,68^{* *}$ & $\left(\mathrm{nFK} * \mathrm{TI}_{5,7}\right)$ & $0,65^{* *}$ & $\left(\mathrm{nFK} * \mathrm{TI}_{5,7}\right)$ & $0,76^{* * * *}$ \\
\hline $\mathrm{V}_{\mathrm{A} 2}$ & $\left(\mathrm{nFK} * \mathrm{~N}_{5,7}\right)$ & $0,65^{* *}$ & $\left(\mathrm{nFK} * \mathrm{~N}_{5,7}\right)$ & $0,65^{* *}$ & $\left(\mathrm{nFK} * \mathrm{~N}_{5,7}\right)$ & $0,60^{* *}$ & $\left(\mathrm{nFK} * \mathrm{~N}_{5,7}\right)$ & $0,75^{* * *}$ \\
\hline $\mathrm{V}_{\mathrm{A} 3}$ & $\left(\mathrm{nFK} * \mathrm{TI}_{6,7}\right)$ & $0,59^{* *}$ & & & & & $\left(\mathrm{nFK} * \mathrm{TI}_{6,7}\right)$ & $0,73^{* * * *}$ \\
\hline $\mathrm{V}_{\mathrm{A} 4}$ & $\left(\mathrm{nFK} * \mathrm{~N}_{6,7}\right)$ & $\mathbf{0 , 5 8} 8^{* *}$ & & & & & $\left(\mathrm{nFK} * \mathrm{~N}_{6,7}\right)$ & $\mathbf{0 , 7 3} 3^{* * * *}$ \\
\hline $\mathrm{V}_{\text {Ref }}$ & nFK & $0,52^{*}$ & $\mathrm{nFK}$ & $0,61^{* *}$ & $\mathrm{nFK}$ & $0,56^{*}$ & $\mathrm{nFK}$ & $0,61^{*}$ \\
\hline
\end{tabular}

Für alle Klone war die aggregierte Variable $\left(\mathrm{nFK} * \mathrm{TI}_{5,7}\right)$ die mit der besten Korrelation mit dem dGZ. Weitere Variablen, die eine höhere Korrelationsstärke als die Referenzvariable zeigten, waren für alle untersuchten Klone $\left(\mathrm{nFK} * \mathrm{~N}_{5,7}\right)$ sowie für die Klone Max 1 und Tordis zusätzlich $\left(\mathrm{nFK} * \mathrm{TI}_{6,7}\right)$ und $\left(n F K * \mathrm{~N}_{6,7}\right)$. Bei der Korrelation zwischen $\left(\mathrm{nFK}^{*} \mathrm{TI}_{5,7}\right)$ und dem dGZ wurden r-Werte zwischen 0,76 (Tordis) und 0,65 (Inger) festgestellt. Demnach konnte eine Verbesserung beim r-Wert gegenüber der Korrelation zwischen nFK und dGZ für Max 1 von $40 \%$, für AF 2 von $12 \%$, für Inger von $20 \%$ und für Tordis von $25 \%$ erzielt werden.

Beim Standortcluster $S$ wurden insgesamt fünf Klimavariablen $\left(\mathrm{T}_{7,8}, \mathrm{~T}_{7,9}, \mathrm{~N}_{6,7}\right.$ und $\left.\mathrm{TI}_{6,7}\right)$ mit je vier Bodenvariablen $\left(\mathrm{S}_{[\%]}, \mathrm{U}_{[\%]}\right.$, LK und BZ) kombiniert (Tab. 4-31). Darüber hinaus wurden die Temperaturvariablen $\mathrm{T}_{7,8}$ und $\mathrm{T}_{7,9}$ mit der Niederschlagsvariablen $\mathrm{N}_{6,7}$ kombiniert.

Tab. 4-31: Variablenkombinationen im Standortcluster S.

\begin{tabular}{rllll}
\hline $\mathrm{S}_{[\%]}-\mathrm{T}_{7,9}$ & $\mathrm{U}_{[\%]}-\mathrm{T}_{7,9}$ & $\mathrm{LK}-\mathrm{T}_{7,9}$ & $\mathrm{BZ}-\mathrm{T}_{7,9}$ & $\mathrm{~T}_{7,9}-\mathrm{N}_{6,7}$ \\
$\mathrm{~S}_{[\%]}-\mathrm{T}_{7,8}$ & $\mathrm{U}_{[\%]}-\mathrm{T}_{7,8}$ & $\mathrm{LK}-\mathrm{T}_{7,8}$ & $\mathrm{BZ}-\mathrm{T}_{7,8}$ & $\mathrm{~T}_{7,8}-\mathrm{N}_{6,7}$ \\
$\mathrm{~S}_{[\%]}-\mathrm{N}_{6,7}$ & $\mathrm{U}_{[\%]}-\mathrm{N}_{6,7}$ & $\mathrm{LK}-\mathrm{N}_{6,7}$ & $\mathrm{BZ}-\mathrm{N}_{6,7}$ & $\mathrm{~S}_{[\%]}-\mathrm{TI}_{6,7}$ \\
$\mathrm{U}_{[\%]}-\mathrm{TI}_{6,7}$ & $\mathrm{LK}-\mathrm{TI}_{6,7}$ & $\mathrm{BZ}-\mathrm{TI}_{6,7}$ & & \\
\hline
\end{tabular}

Bei der Korrelationsanalyse wies eine Vielzahl von aggregierten Variablen eine stärkere Korrelation mit dem dGZ auf als die Referenzvariable (Tab. 4-32). Beim Vergleich der besten Korrelation zwischen aggregierten Variablen und dem dGZ zur Korrelation zwischen Referenzvariablen und dem dGZ bei Max 1 wurde eine ca. $5 \%$-ige Verbesserung im r-Wert erzielt. Die bestkorrelierte aggregierte Variable war $\left(\mathrm{S}_{[\%]} / \mathrm{TI}_{6,7}\right)$ mit einem r-Wert von -0,88. Bei H 275 war ebenfalls ( $\left.\mathrm{S}_{[\%]} / \mathrm{TI}_{6,7}\right)$ die bestkorrelierte Variable, ihr Vorsprung gegenüber der Referenzvariablen $\left(\mathrm{TI}_{6,7}\right)$ war jedoch sehr gering. Beim Klon AF 2 wurden für mehrere aggregierte Variablen bessere Korrelationen zum dGZ festgestellt als bei den Referenzvariablen. Die beste Korrelation mit dem dGZ wies die Variable $\left(\mathrm{T}_{7,8} / \mathrm{U}_{[\%]}\right)$ mit einem r-Wert von -0,92 auf. Bei Inger und Tordis waren sechs Variablen besser mit dem dGZ korreliert als die Referenzvariable. Die stärkste Korrelation erreichten beide Klone mit $\left(\mathrm{S}_{[\%]} * \mathrm{~T}_{7,8}\right)$, der $r$-Wert betrug für Inger $-0,90$ und für Tordis $-0,93$. 
Tab. 4-32: Korrelationsanalyse der aggregierten Variablen und dem dGZ im Standortcluster S. Dargestellt sind die r-Werte der aggregierten Variablen $\mathrm{V}_{\mathrm{A} 1}$ bis $\mathrm{V}_{\mathrm{A} 4}$ (mit dem dGZ erst- bis viertbestkorrelierter aggregierter Variablen) sowie der Referenzvariablen $\mathrm{V}_{\text {ref }}$ (mit dem dGZ bestkorrelierter, nicht aggregierter Variablen).

\begin{tabular}{|c|c|c|c|c|c|c|c|c|c|c|}
\hline & \multicolumn{2}{|c|}{ Max 1} & \multicolumn{2}{|c|}{ Н 275} & \multicolumn{2}{|c|}{ AF 2} & \multicolumn{2}{|l|}{ Inger } & \multicolumn{2}{|c|}{ Tordis } \\
\hline & Variable & $\mathrm{r}$ & Variable & $\mathrm{r}$ & Variable & $\mathrm{r}$ & Variable & $\mathrm{r}$ & Variable & $\mathrm{r}$ \\
\hline $\mathrm{V}_{\mathrm{A} 1}$ & $\left(\mathrm{~S}_{[\%]} / \mathrm{TI}_{6,7)}\right.$ & $-0,88^{* *}$ & $\left(\mathrm{~S}_{[\%]} / \mathrm{TI}_{6,7}\right)$ & $-0,92^{* *}$ & $\left(\mathrm{~T}_{7,8} / \mathrm{U}_{[\%]}\right)$ & $-0,92^{* * *}$ & $\left(\mathrm{~S}_{[\%]} * \mathrm{~T}_{7,8}\right)$ & $-0,90^{* * *}$ & $\left(\mathrm{~S}_{[\%]} * \mathrm{~T}_{7,8}\right)$ & $-0,93^{* * *}$ \\
\hline $\mathrm{V}_{\mathrm{A} 2}$ & $\left(\mathrm{~S}_{[\%]} / \mathrm{N}_{6,7)}\right.$ & $-0,87^{* *}$ & $\left(\mathrm{~S}_{[\%]} / \mathrm{N}_{6,7}\right)$ & $-0,91^{* *}$ & $\left(\mathrm{~T}_{7,9} / \mathrm{U}_{[\%]}\right)$ & $-0,92^{* *}$ & $\left(\mathrm{~S}_{[\%]} * \mathrm{~T}_{7,9}\right)$ & $-0,89^{* *}$ & $\left(\mathrm{~S}_{[\%]} * \mathrm{~T}_{7,9}\right)$ & $-0,93^{* *}$ \\
\hline $\mathrm{V}_{\mathrm{A} 3}$ & $\left(\mathrm{~N}_{6,7} / \mathrm{T}_{7,8}\right)$ & $0,84^{* *}$ & - & - & $\left(\mathrm{N}_{6,7} / \mathrm{U}_{[\%]}\right)$ & $-0,89^{* *}$ & $\left(\mathrm{U}_{[\%]} * \mathrm{TI}_{6,7}\right)$ & $0,84^{* *}$ & $\left(\mathrm{~S}_{[\%]} / \mathrm{TI}_{6,7}\right)$ & $-0,86^{* * *}$ \\
\hline $\mathrm{V}_{\mathrm{A} 4}$ & $\left(\mathrm{~N}_{6,7} / \mathrm{T}_{7,9)}\right.$ & $0,84^{* *}$ & - & - & $\left(\mathrm{TI}_{6,7} / \mathrm{U}_{[\%]}\right)$ & $-0,89^{* *}$ & $\left(\mathrm{U}_{[\%]} / \mathrm{T}_{7,8}\right)$ & $0,83^{*}$ & $\left(\mathrm{U}_{[\%]} * \mathrm{TI}_{6,7}\right)$ & $\mathbf{0 , 8 5 ^ { * * }}$ \\
\hline$V_{\text {Ref }}$ & $\mathrm{TI}_{6,7}$ & $0,83^{*}$ & $\mathrm{TI}_{6,7}$ & $0,90^{* *}$ & $\mathrm{U}_{[\%]}$ & $0,83^{*}$ & $\mathrm{U}_{[\%]}$ & $0,80^{*}$ & $\mathrm{~S}_{[\%]}$ & $-0,83^{*}$ \\
\hline
\end{tabular}

Beim Standortcluster U wurden aufgrund der niedrigen Korrelationen zwischen den nicht aggregierten Variablen und den dGZ mehr Variablen zur Bildung aggregierter Variablen gewählt als bei den restlichen Standortclustern. Als Bodenvariablen wurden LK, BZ, und TRD, als Klimavariablen $\mathrm{T}_{7,9}, \mathrm{~N}_{5,6}$, $\mathrm{N}_{4,5}, \mathrm{TI}_{4,5}$ sowie $\mathrm{T}_{7,8}$ zur Kombination herangezogen (Tab. 4-33).

Tab. 4-33: Variablenkombinationen im Standortcluster U.

\begin{tabular}{ccccc}
\hline LK $-\mathrm{T}_{7,9}$ & LK $-\mathrm{N}_{5,6}$ & $\mathrm{BZ}-\mathrm{T}_{7,9}$ & $\mathrm{BZ}-\mathrm{N}_{5,6}$ & $\mathrm{BZ}-\mathrm{TI}_{5,6}$ \\
$\mathrm{~T}_{7,9}-\mathrm{N}_{5,6}$ & $\mathrm{BZ}-\mathrm{N}_{4,5}$ & $\mathrm{BZ}-\mathrm{TI}_{4,5}$ & $\mathrm{BZ}-\mathrm{T}_{7,8}$ & TRD $-\mathrm{T}_{7,9}$ \\
$\mathrm{TRD}-\mathrm{T}_{7,8}$ & $\mathrm{TRD}-\mathrm{N}_{5,6}$ & $\mathrm{TRD}-\mathrm{N}_{4,5}$ & $\mathrm{TRD}-\mathrm{TI}_{4,5}$ & LK $-\mathrm{N}_{4,5}$ \\
$\mathrm{LK}-\mathrm{TI}_{4,5}$ & $\mathrm{LK}-\mathrm{T}_{7,8}$ & & & \\
\hline
\end{tabular}

Im Standortcluster U wiesen aggregierte Variablen oft bessere Korrelationen auf als die Referenzvariablen (Tab. 4-34). Die mit dem dGZ bestkorrelierte aggregierte Variable war für Max $1\left(\mathrm{BZ} * \mathrm{TI}_{4,5}\right)$, für AF $2\left(\mathrm{LK} / \mathrm{T}_{7,9}\right)$, für Inger (TRD / $\left.\mathrm{TI}_{5,6}\right)$ und für Tordis $\left(\mathrm{BZ} / \mathrm{T}_{7,9}\right)$. Für jeden Klon existierte somit eine andere aggregierte Variable, die mit dem dGZ am besten korrelierte.

Tab. 4-34: Korrelationsanalyse der aggregierten Variablen und dem dGZ im Standortcluster U. Dargestellt sind die r-Werte der aggregierten Variablen $\mathrm{V}_{\mathrm{A} 1}$ bis $\mathrm{V}_{\mathrm{A} 4}$ (mit dem dGZ erst- bis viertbestkorrelierter aggregierter Variablen) sowie der Referenzvariablen $\mathrm{V}_{\text {ref }}$ (mit dem dGZ bestkorrelierter, nicht aggregierter Variablen).

\begin{tabular}{ccccccccc}
\hline & \multicolumn{2}{c}{ Max 1} & \multicolumn{2}{c}{$\mathrm{AF} 2$} & \multicolumn{2}{c}{ Inger } & \multicolumn{2}{c}{ Tordis } \\
\cline { 2 - 8 } & Variable & $\mathrm{r}$ & Variable & $\mathrm{r}$ & Variable & $\mathrm{r}$ & Variable & $\mathrm{r}$ \\
\hline $\mathrm{V}_{\mathrm{A} 1}$ & $\left(\mathrm{TI}_{4,5} * \mathrm{BZ}\right)$ & $\mathbf{0 , 9 3}^{*}$ & $\left(\mathrm{LK} / \mathrm{T}_{7,9}\right)$ & $0,85^{(*)}$ & $\left(\mathrm{TRD} / \mathrm{TI}_{5,6}\right)$ & $-0,75$ & $\left(\mathrm{BZ} / \mathrm{T}_{7,9}\right)$ & $\mathbf{0 , 9 7}^{* *}$ \\
$\mathrm{~V}_{\mathrm{A} 2}$ & $\left(\mathrm{BZ} * \mathrm{~N}_{4,5}\right)$ & $\mathbf{0 , 9 3}^{*}$ & $(\mathrm{LK} * \mathrm{BZ})$ & $0,85^{(*)}$ & $\left(\mathrm{LK} * \mathrm{TI}_{5,6}\right)$ & 0,74 & & \\
$\mathrm{~V}_{\mathrm{A} 3}$ & $\left(\mathrm{LK} * \mathrm{TI}_{4,5}\right)$ & $\mathbf{0 , 9 2}$ & $\left(\mathrm{LK} / \mathrm{T}_{7,8}\right)$ & $0,85^{(*)}$ & $\left(\mathrm{TRD} / \mathrm{T}_{7,8}\right)$ & $-0,72$ & & \\
$\mathrm{~V}_{\mathrm{A} 4}$ & $\left(\mathrm{LK} * \mathrm{~N}_{4,5}\right)$ & $\mathbf{0 , 9 1}$ & & & $\left(\mathrm{LK}^{*} \mathrm{~N}_{5,6}\right)$ & 0,72 & & \\
\hline $\mathrm{V}_{\mathrm{Ref}}$ & $\mathrm{T}_{7,9}$ & 0,84 & $\mathrm{LK}$ & 0,84 & $\mathrm{TI}_{5,6}$ & 0,72 & $\mathrm{BZ}$ & $\mathbf{0 , 9 7}^{* *}$ \\
\hline
\end{tabular}

Bei den Lehmböden wurden insgesamt zwölf Kombinationen durchgeführt. Zur Bildung aggregierter Variablen wurden beim Standortcluster $\mathrm{L}$ die Bodenvariablen $\mathrm{nFK}$, LK und $\mathrm{U}_{[\%]}$ sowie die Klimavariablen $\mathrm{N}_{5,6}, \mathrm{TI}_{5,6}, \mathrm{~T}_{7,9}$ und $\mathrm{T}_{\mathrm{VP}}$ herangezogen (Tab. 4-35). 
Tab. 4-35: Variablenkombinationen im Standortcluster L.

\begin{tabular}{ccccc}
\hline $\mathrm{nFK}-\mathrm{LK}$ & $\mathrm{U}_{[\%]}-\mathrm{N}_{5,6}$ & $\mathrm{nFK}-\mathrm{N}_{5,6}$ & $\mathrm{LK}-\mathrm{N}_{5,6}$ & $\mathrm{nFK}-\mathrm{TI}_{5,6}$ \\
$\mathrm{LK}-\mathrm{TI}_{5,6}$ & $\mathrm{U}_{[\%]}-\mathrm{T}_{\mathrm{VP}}$ & $\mathrm{nFK}-\mathrm{T}_{\mathrm{VP}}$ & $\mathrm{U}_{[\%]}-\mathrm{T}_{7,9}$ & $\mathrm{nFK}-\mathrm{T}_{7,9}$ \\
$\mathrm{~T}_{\mathrm{VP}}-\mathrm{N}_{5,6}$ & $\mathrm{~T}_{7,9}-\mathrm{N}_{5,6}$ & & & \\
\hline
\end{tabular}

Die mit dem dGZ bestkorrelierten aggregierten Variablen waren für Max $1\left(\mathrm{nFK} * \mathrm{TI}_{5,6}\right)$ mit einem r-Wert von 0,87, für AF $2\left(\mathrm{nFK} * \mathrm{~N}_{5,6}\right)$ mit einem $\mathrm{r}$-Wert von 0,91 sowie für Tordis $\left(\mathrm{nFK} * \mathrm{TI}_{5,6}\right)$ mit einem r-Wert von 0,99. Abweichend von den restlichen Standortclustern wiesen hier wenige aggregierte Variablen einen höheren r-Wert auf als die Referenzvariable (Tab. 4-36).

Tab. 4-36: Korrelationsanalyse der aggregierten Variablen und dem dGZ im Standortcluster L. Dargestellt sind die r-Werte der aggregierten Variablen $\mathrm{V}_{\mathrm{A} 1}$ bis $\mathrm{V}_{\mathrm{A} 4}$ (mit dem dGZ erst- bis viertbestkorrelierter aggregierter Variablen) sowie der Referenzvariablen $\mathrm{V}_{\text {ref }}$ (mit dem dGZ bestkorrelierter, nicht aggregierter Variablen).

\begin{tabular}{|c|c|c|c|c|c|c|}
\hline & \multicolumn{2}{|c|}{$\operatorname{Max} 1$} & \multicolumn{2}{|c|}{$\mathrm{AF} 2$} & \multicolumn{2}{|c|}{ Inger } \\
\hline & Variable & $\mathrm{r}$ & Variable & $\mathrm{r}$ & Variable & $\mathrm{r}$ \\
\hline $\mathrm{V}_{\mathrm{A} 1}$ & $\left(\mathrm{nFK} * \mathrm{TI}_{5,6}\right)$ & $0,87^{*}$ & $\left(\mathrm{nFK} * \mathrm{~N}_{5,6}\right)$ & $0,91^{* *}$ & $\left(\mathrm{nFK} * \mathrm{TI}_{5,6}\right)$ & $0,99^{* * * *}$ \\
\hline $\mathrm{V}_{\mathrm{A} 2}$ & & & & & $\left(\mathrm{U}_{[\%]} / \mathrm{T}_{7,9}\right)$ & $0,94^{* *}$ \\
\hline $\mathrm{V}_{\mathrm{A} 3}$ & & & & & $\left(\mathrm{nFK} / \mathrm{T}_{7,9}\right)$ & $0,94^{* *}$ \\
\hline $\mathrm{V}_{\text {Ref }}$ & $\mathrm{nFK}$ & $0,86^{*}$ & $\mathrm{U}_{[\%]}$ & $0,87^{*}$ & $\mathrm{nFK}$ & $0,93^{* *}$ \\
\hline
\end{tabular}

\subsubsection{Weitere Einflussgrößen}

Im Folgenden werden die Ergebnisse der Varianzanalyse von den nominal skalierten Variablen Vornutzung, Bodentyp und Grundwasser auf den dGZ am Beispiel des Klons Max 1 vorgestellt. Der Einfluss der Bodenart kann unter anderem dem Kapitel 4.1 entnommen werden.

\subsubsection{Vornutzung}

Die untersuchten Versuchsflächen wiesen vier verschiedene Vornutzungen auf. Diese waren Acker $(n=14)$, Baumschule $(n=2)$, Grünland $(n=3)$ und Bergbaufolgelandschaft $(n=1)$. Dem Ergebnis der Varianzanalyse nach hatte die Vornutzung einen signifikanten Einfluss auf den dGZ. Auf die Bildung homogener Untergruppen wurde aufgrund stark abweichender Wiederholungsanzahlen verzichtet.

Abbildung 4-28 zeigt die dGZ der Standorte unterschiedlicher Vornutzung für den Klon Max 1. Die Standorte mit Vornutzung Acker deckten die gesamte Spanne an dGZ von 0,8 bis hin zu $10,0 \mathrm{t}_{\text {(atro) }} \mathrm{ha}^{-1} \mathrm{a}^{-1} \mathrm{ab}$. In den Standorten mit Vornutzung Baumschule waren dGZ-Werte im Bereich von 5,0 bis 8,0 $\mathrm{t}_{\text {(atro) }}$ ha $^{-1} \mathrm{a}^{-1}$ festzustellen. Bei komplett umgebrochenen ehemaligen Grünlandstandorten wurden sowohl niedrige als auch sehr hohe dGZ-Werte (bis über 10,0 $\mathrm{t}_{\text {(atro) }} \mathrm{ha}^{-1} \mathrm{a}^{-1}$ ) erzielt. Bei den Bergbaufolgelandschaften werden teilweise extrem niedrige aber auch hohe dGZ beobachtet. Die einzige Versuchsfläche mit Vornutzung KUP wies einen sehr geringen dGZ auf. 


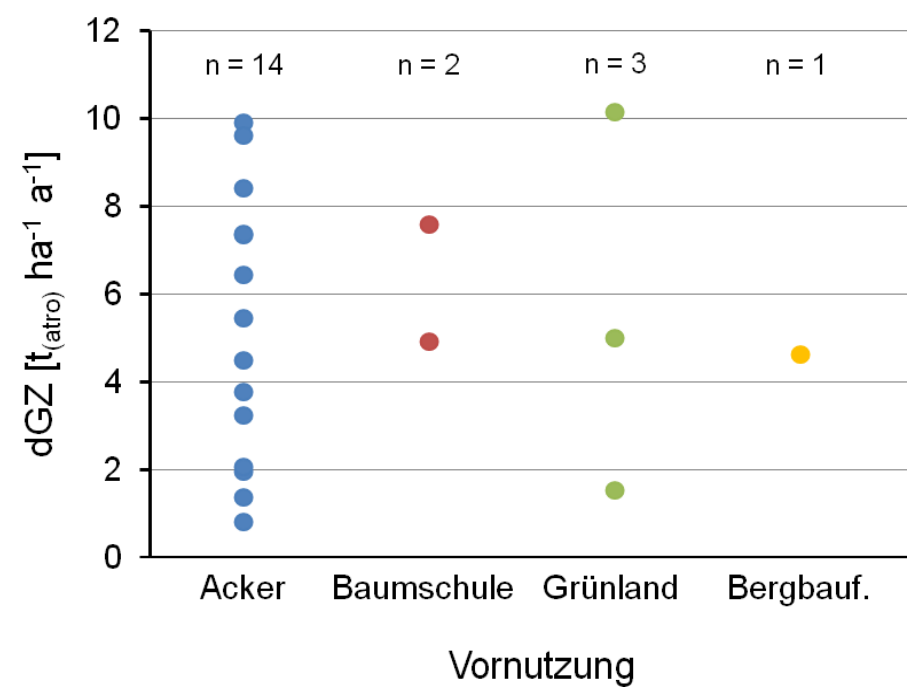

Abb. 4-28: dGZ des Klons Max 1 bei Standorten unterschiedlicher Vornutzung in der dritten Wuchsperiode.

\subsubsection{Bodentyp}

Der Bodentyp hatte einen signifikanten Einfluss auf den dGZ. Bodentypen, die ausschließlich hohe dGZ aufwiesen, waren ein Moor und die Schwarzerden (Abb. 4-29). Hier konnten dGZ größer 7,5 $\mathrm{t}_{\text {(atro) }} \mathrm{ha}^{-1} \mathrm{a}^{-1}$ beobachtet werden. Bei den Braunerden sowie Parabraunerden wurden sowohl niedrige - um 1,0 $\mathrm{t}_{\text {(atro) }} \mathrm{ha}^{-1} \mathrm{a}^{-1}$ - als auch sehr hohe - bis 10,0 $\mathrm{t}_{\text {(atro) }} \mathrm{ha}^{-1} \mathrm{a}^{-1}$ - dGZ erreicht. Gleye und Pseudogleye zeigten schwache bis mäßige dGZ auf, diese lagen stets unter 6,0 $t_{\text {(atro) }}$ ha $^{-1} \mathrm{a}^{-1}$. Beim Regosol am Sto. Nr. 13 „Grünewalde“ wurde ein dGZ von $4,6 \mathrm{t}_{\text {(atro) }}$ ha $^{-1} \mathrm{a}^{-1}$ ermittelt.

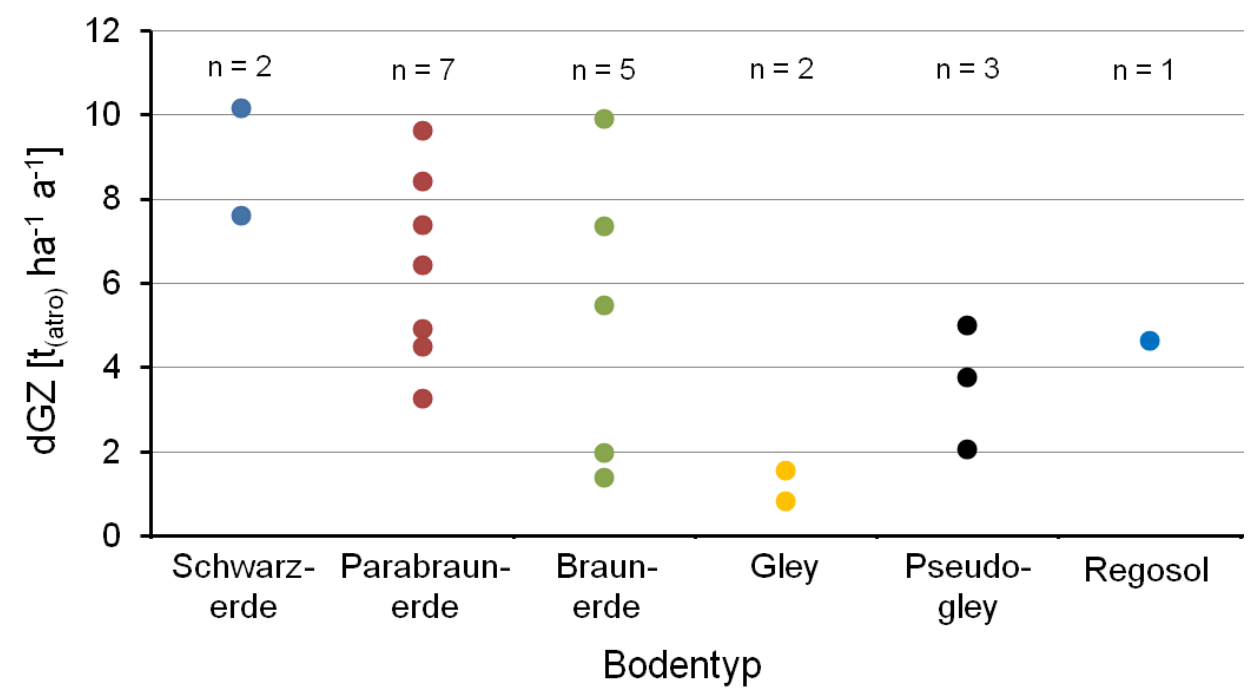

Abb. 4-29: dGZ des Klons Max 1 bei Standorten unterschiedlichen Bodentyps in der dritten Wuchsperiode.

Abbildungen mit Berücksichtigung aller Standorte welche drei Wuchsperioden erreichten und teilweise in der Modellierung nicht berücksichtigt werden konnten $(n=29)$ sind den Anhänge 34 und 35 zu entnehmen.

\subsubsection{Grundwasser}

Eine Varianzanalyse zwischen beiden Gruppen auf grundwassernahen und grundwasserfernen Standorten, ergab einen signifikanten Einfluss des Grundwasserflurabstandes auf den dGZ. Dies traf auf alle Klone zu. Die grundwasserbeeinflussten Standorte zeigten höhere dGZ. 


\subsection{Standortbasierte Ertragsmodellierung}

Die Ergebnisse der standortbasierten Ertragsmodellierung wurden mittels der einfachen linearen, der multiplen linearen sowie der nicht-linearen Regression errechnet. Die Ergebnisdarstellung begrenzt sich überwiegend auf die einfache sowie auf die univariate nicht-lineare Regression, weil für keinen Klon und Standortcluster signifikante Modelle mit mehr als einen Prädiktor ermittelt werden konnten. Die multiplen nicht-linearen Modelle führten teilweise zu einer Steigerung der Modellgenauigkeit (siehe Anhang 36), bei keinem der Modelle war jedoch das komplette Parameterset signifikant.

Unter den errechneten Modellen werden im Folgenden je Klon und Bodenartcluster die Modelle mit der besten Anpassung in Form des $\mathrm{R}_{\text {korr }}$ dargestellt. Dabei wurde nach dem genausten Modell je Klon gesucht. Die Anpassung eines „gemeinsamen Modells“ wurde bevorzugt, wenn zwei oder mehrere Klone bei einer definierten Modellanpassung (gleicher Prädiktor und identische Anpassung) eine geringe relative Abweichung im $\mathrm{R}_{\text {korr }}(<10 \%$ ) zueinander aufwiesen. Das „gemeinsame Modell“ hat den Vorteil, die ertragsbildenden Prozesse besser darzustellen und ist zudem anwendungsfreundlicher.

Wachstumsprozesse zeichnen sich oft durch sigmoidale Verläufe aus (RICHARDS 1959). Im niedrigen Wertebereich ist der Verlauf exponentiell, dann wechselt die Kurve in einen asymptotischen Verlauf über. Mit zunehmender Beobachtungsdauer bzw. bei größer werdenden Werten der unabhängigen Variablen (zum Beispiel Umweltfaktoren) tendieren die Modellverläufe dazu asymptotisch zu werden. In der vorliegenden Arbeit stand die Optimierung des $\mathrm{R}^{2}$ korr im Vordergrund. Nicht selten wurden aufgrund der vorliegenden Datenbasis exponentielle oder linear verlaufende Modelle angepasst. In diesem Zusammenhang ist darauf hinzuweisen, dass diese Modelle keinesfalls extrapolierbar sind.

\subsubsection{Standortcluster ALL}

Im Standortcluster ALL wurden mit der aggregierten Variablen $\left(\mathrm{nFK} * \mathrm{TI}_{5,7}\right)$ als Prädiktor die besten Modelle für die Klone Max 1, Inger und Tordis ermittelt. Für den Klon AF 2 fungierte die aggregierte Variable $\left(\mathrm{nFK} * \mathrm{~N}_{5,7}\right)$ als der beste Prädiktor bei der Modellierung. Bei Max 1, Inger und Tordis wurde in der Modellierung der inverse Ansatz (Modell Nr. 1, Formel 4-1), bei AF 2 der sigmoidale Ansatz (Modell Nr. 2, Formel 4-2) verwendet.

Modell Nr. 1 (Standortcluster ALL - inv - Max 1, Inger, Tordis)

$$
d G Z\left[t_{(\text {atro })} \mathrm{ha}^{-1} \mathrm{a}^{-1}\right]=a_{0}+\frac{a_{1}}{\left(n F K * T I_{5,7}\right)}
$$

(Formel 4-1)

Modell Nr. 2 (Standortcluster ALL - sig - AF 2)

$$
\begin{array}{cl}
d G Z\left[t_{\text {(atro })} h^{-1} \mathrm{a}^{-1}\right]=e^{\left(a_{0}+\frac{a_{1}}{\left(n F K * N_{5,7}\right)}\right)} & \\
& \\
d G Z: & \text { Durchschnittlicher Gesamtzuwachs }\left[\mathrm{t}_{\text {(arro) }} \mathrm{ha}^{-1} \mathrm{a}^{-1}\right] \\
a_{0} \text { und } a_{l}: & \text { Modellkoeffizienten } \\
n F K: & \text { Nutzbare Feldkapazität in der Tiefe } 0-60 \mathrm{~cm}[\mathrm{~cm}] \\
T I_{5,7}: & \text { Trockenheitsindexsumme des Zeitfensters Mai bis Juli } \\
N_{5,7}: & \text { Niederschlagssumme des Zeitfensters Mai bis Juli [mm] }
\end{array}
$$

Tabelle 4-37 gibt eine Übersicht der ermittelten Modelle mit ihren statistischen Kennwerten wieder. $\mathrm{Zu}$ diesen Kennwerten zählen der $\mathrm{R}^{2}$ korr, der $\mathrm{p}$-Wert des Modells, die geschätzten Koeffizienten $\mathrm{a}_{0}$ und $\mathrm{a}_{1}$ sowie RMSE, prozentuale Bias, Präzision und Treffgenauigkeit. Die $\mathrm{R}^{2}{ }_{\text {korr }}$ der Modelle im Standortcluster ALL betrugen für Max 1 0,47, für AF 2 0,58, für Inger 0,47 und für Tordis 0,64. Alle Modelle sowie Modellkoeffizienten waren auf dem p-Niveau $<0,001$ hochsignifikant. Die Werte für RMSE betrugen in absteigender Reihenfolge für Inger 2,17 $\mathrm{t}_{\text {(atro) }} \mathrm{ha}^{-1} \mathrm{a}^{-1}$, für Max $12,05 \mathrm{t}_{\text {(atro) }} \mathrm{ha}^{-1} \mathrm{a}^{-1}$, für AF 2 $1,99 \mathrm{t}_{\text {(atro) }} \mathrm{ha}^{-1} \mathrm{a}^{-1}$ und für Tordis $1,83 \mathrm{t}_{\text {(atro) }} \mathrm{ha}^{-1} \mathrm{a}^{-1}$. Die Modelle waren kaum verzerrt ( $\overline{\mathrm{e}} \%$ war negativ 
im $10^{-6}$ Bereich) und zeigten eine Prognosestreuung von 32,5\% (Tordis) bis 39,6\% (Max 1). Aufgrund des sehr geringen Bias war der Unterschied zwischen prozentualer Prognosestreuung und Treffgenauigkeit minimal.

Abbildung 4-30 zeigt die standortbasierten Ertragsmodelle im Cluster ALL. Dargestellt sind die gemessenen Datenpaare der aggregierten Variablen mit dem dGZ samt Modellanpassung. Es wird zunächst sichtbar, dass der Modellverlauf stark von der Art der Anpassung abhängt. Die mit dem inversen Ansatz berechneten Modelle verlaufen asymptotisch, diejenigen mit dem sigmoidalen Ansatz berechneten Modell nahezu linear.

Tab. 4-37: Statistische Kennwerte der standortbasierten Ertragsmodelle im Standortcluster ALL für die Pappelklone Max 1 und AF 2 sowie für die Weidenklone Inger und Tordis.

\begin{tabular}{|c|c|c|c|c|c|c|c|c|c|c|c|}
\hline \multirow{2}{*}{ Gattung } & \multirow{2}{*}{ Klon } & \multirow{2}{*}{$\mathrm{n}$} & \multirow{2}{*}{ Prädiktor } & \multirow{2}{*}{$\begin{array}{l}\text { Anpas- } \\
\text { sung }\end{array}$} & \multirow{2}{*}{$\mathrm{R}_{\text {korr }}^{2}$} & \multirow[b]{2}{*}{$\mathrm{a}_{0}$} & \multirow[b]{2}{*}{$a_{1}$} & \multirow{2}{*}{$\begin{array}{c}\mathrm{RMSE} \\
{\left[\mathrm{t}_{\text {(atro) }} \mathrm{ha}^{-1} \mathrm{a}^{-1}\right]}\end{array}$} & $\overline{\mathrm{e}}$ & $\mathrm{Se}$ & $\mathrm{Mx}$ \\
\hline & & & & & & & & & \multicolumn{3}{|c|}{ [\%] } \\
\hline \multirow{2}{*}{ Pappel } & Max 1 & 20 & $\left(\mathrm{nFK} * \mathrm{TI}_{5,7}\right)$ & inv & $0,47^{* * * *}$ & $13,52^{* * * *}$ & $-563,40^{* * *}$ & 2,05 & $-7,7 * 10^{-7}$ & 39,6 & 39,6 \\
\hline & $\mathrm{AF} 2$ & 20 & $\left(\mathrm{nFK} * \mathrm{~N}_{5,7}\right)$ & sig & $0,58^{* * * *}$ & $3,87^{* * * *}$ & $-4365,43^{* * * *}$ & 1,99 & $-4,1 * 10^{-6}$ & 37,5 & 37,5 \\
\hline \multirow{2}{*}{ Weide } & Inger $^{\mathrm{a}}$ & 20 & $\left(\mathrm{nFK} * \mathrm{TI}_{5,7}\right)$ & inv & $0,45^{* * * *}$ & $13,41^{\text {**** }}$ & $-571,35^{* * *}$ & 2,17 & $-1,8^{*} 10^{-6}$ & 43,8 & 43,8 \\
\hline & Tordis & 18 & $\left(\mathrm{nFK} * \mathrm{TI}_{5,7}\right)$ & inv & $0,64^{* * * *}$ & $15,81^{* * * *}$ & $-694,16^{* * * *}$ & 1,83 & $-2,7 * 10^{-7}$ & 32,5 & 32,5 \\
\hline
\end{tabular}

${ }^{a}$ Die sigmoidale Anpassung hatte einen geringfügigen besseren $\mathrm{R}^{2}$ korr, er war mit 0,47 nur $4 \%$ höher als das $\mathrm{R}^{2}$ korr des ,gemeinsamen Modells“, daher wurde bei Inger das ,gemeinsame Modell“ bevorzugt.

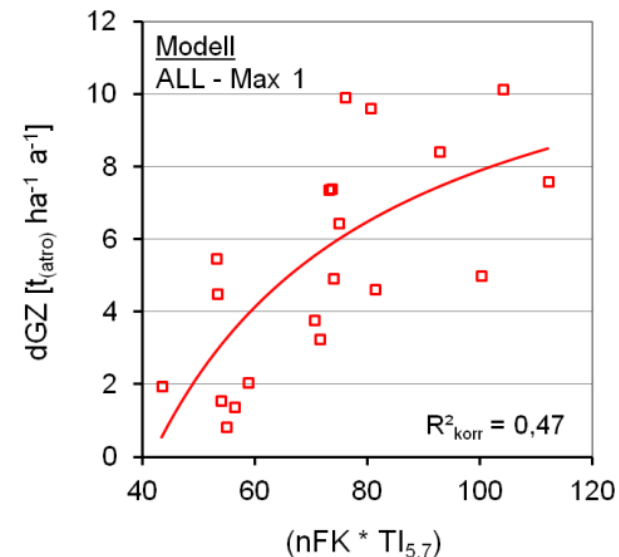

a)

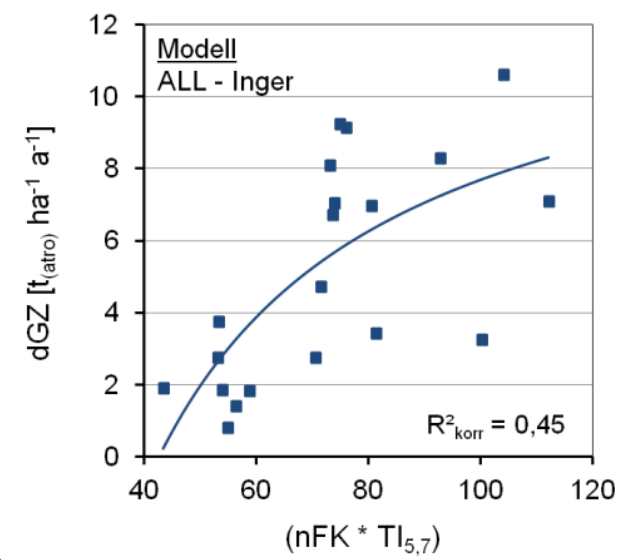

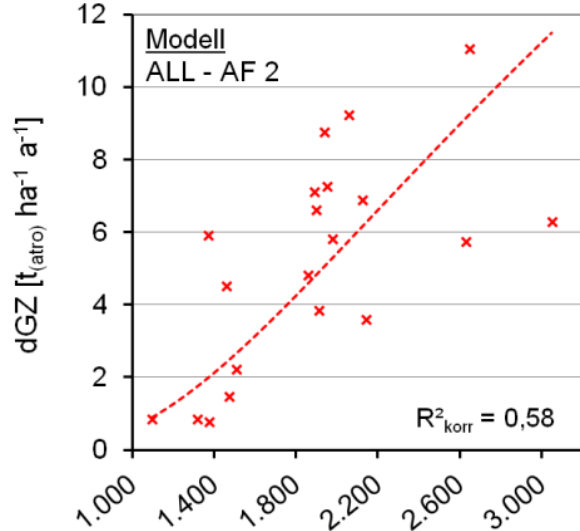

b)

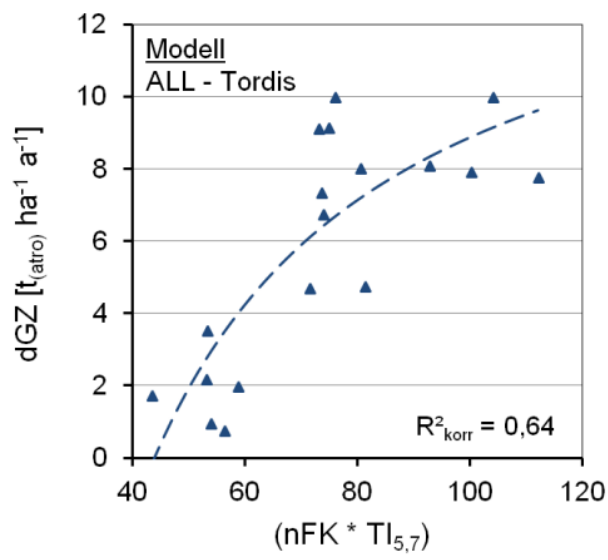

d)

Abb. 4-30: Standortbasierte Ertragsmodelle im Standortcluster ALL für a) Max 1 b) AF 2 c) Inger und d) Tordis.

Zur besseren Interpretation der Modelle, speziell des Einflusses der einzelnen Bestandteile der aggregierten Variablen auf den dGZ, kommen dreidimensionale Flächendiagramme zum Einsatz (Abb. 4-31). In den Abbildungen sind die nicht-aggregierten Variablen, die als Bestandteile der aggre- 
gierten Variablen fungieren, in den Abzissen, der dGZ in der Ordinate zu finden. Die Modelle sind für den Validitätsbereich (Min-Max Abstand) der nicht aggregierten Variablen dargestellt. Abbildung 431 zeigt die Modelle für die Klone Max 1 und AF 2 nach Aufteilung des aggregierten Prädiktors in die zwei nicht-aggregierten Variablen.
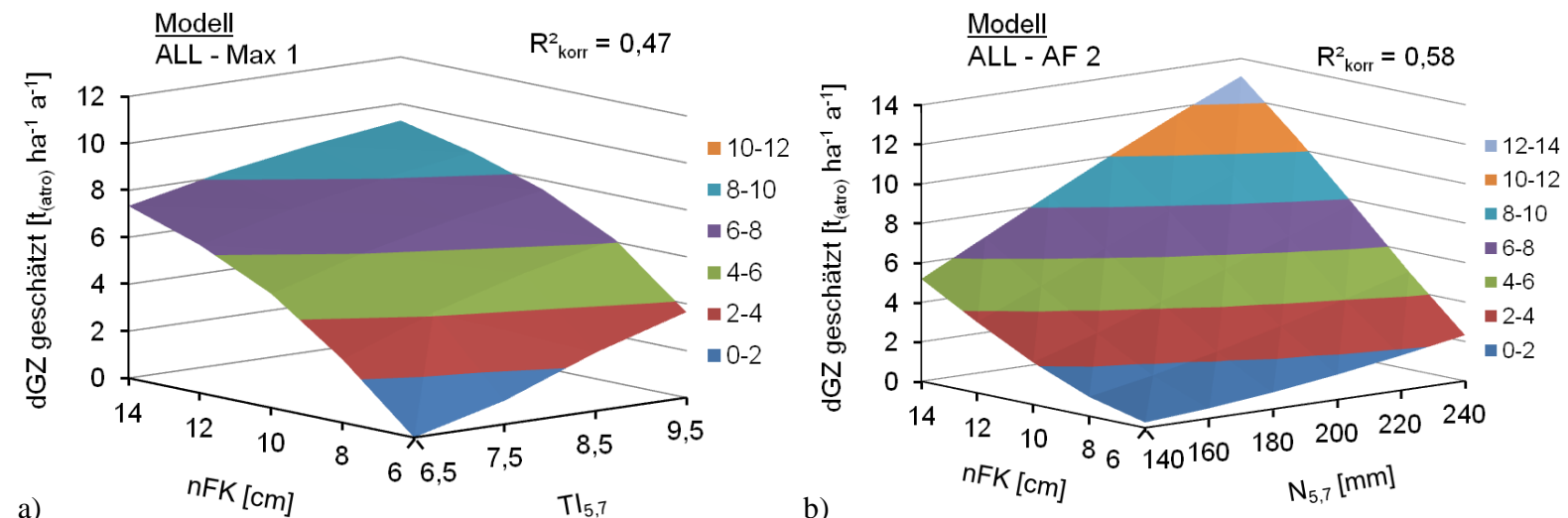

Abb. 4-31: Dreidimensionale Darstellung der standortbasierten Ertragsmodelle im Standortcluster ALL. Dargestellt ist der dGZ in Abhängigkeit der zwei nicht aggregierten Variablen aus denen sich der aggregierte Modellprädiktor zusammensetzt, für a) Max 1 und b) AF 2.

Die Modelle wurden je mit dem inversen und sigmoidalen Ansatz berechnet. Im Max 1-Modell ist das Modell erst ab einem $\mathrm{TI}_{5,7}$ größer 6,5 dargestellt, weil das Modell im Trockenheitsindex-Bereich kleiner 6,5 bei niedrigen nFK negative dGZ-Werte zur Folge hatte. Für das Max 1-Modell wird sichtbar, dass die nFK die größte Rolle für den Zuwachs spielt. Diese Beobachtung basiert auf der stärkeren dGZ-Zunahme bei steigendem $\mathrm{nFK}$ im Vergleich mit der dGZ-Zunahme mit steigendem $\mathrm{TI}_{5,7}$. Dies kann am besten an den Modellrändern beobachtet werden. Die Klimavariable $\mathrm{TI}_{5,7}$ und die Bodenvariable $\mathrm{nFK}$ stehen bezüglich ihres Einflusses auf den Zuwachs in dynamischer Beziehung. So war die durch höher werdende Trockenheitsindex-Werte verursachte dGZ-Zunahme im geringen nFK-Bereich deutlich stärker ausgeprägt als im höheren nFK-Bereich. Diese Beobachtungen fanden sich auch in den Modellen für Inger und Tordis wieder, welche ebenfalls mit dem inversen Ansatz berechnet wurden.

Im ermittelten Modell für AF 2, basierend auf sigmoidalem Ansatz, stieg der dGZ stark an, wenn gleichzeitig ein Anstieg von $\mathrm{nFK}$ und $\mathrm{N}_{5,7} \mathrm{zu}$ verzeichnen war. Das inverse Modell (Max 1, Inger und Tordis) unterschätzte die dGZ vor allem im oberen Wertebereich, während das AF 2-Modell die dGZ tendenziell überschätzte. Der Einfluss der nFK auf den dGZ hat in beiden Modellen, sigmoidal und invers, hohes Gewicht.

Tabelle 4-38 zeigt die Ergebnisse des Tests auf Normalverteilung für die Modellresiduen. Es wird sichtbar, dass alle Modelle normalverteilte Residuen aufweisen.

Tab. 4-38: Test auf Normalverteilung der Modellresiduen im Standortcluster ALL für die Pappelklone Max 1, AF 2 sowie für die Weidenklone Inger und Tordis. Eine Normalverteilung ist bei p-Werte $<0,05$ nicht gegeben.

\begin{tabular}{lll}
\hline Gattung & Klon & p-Wert \\
\hline \multirow{2}{*}{ Pappel } & Max 1 & 0,083 \\
& AF 2 & 0,618 \\
\multirow{2}{*}{ Weide } & Inger & 0,695 \\
& Tordis & 0,403 \\
\hline
\end{tabular}




\subsubsection{Standortcluster $S$}

Bei Max 1 und AF 2 fungierte die aggregierte Variable $\left(\mathrm{U}_{[\%]} * \mathrm{~N}_{6,7}\right)$ als Prädiktor im Modell. Dafür wurde der sigmoidale Ansatz (Modell Nr. 3, Formel 4-3) gewählt. Die Modelle erreichten $\mathrm{R}^{2}$ korr in Höhe von 0,89 für Max 1 und 0,97 für AF 2 (Tab. 4-39). Für H 275 wurde das genaueste Modell mit dem logarithmischen Ansatz und die Variable $\left(\mathrm{S}_{[\%]} / \mathrm{TI}_{6,7}\right)$ als Prädiktor entwickelt (Modell Nr. 4, Formel 4-4). Dieses Modell wies ein $\mathrm{R}^{2}$ korr von 0,84 auf. Die für die Weidenklone ermittelten Modelle bauten auf die unabhängige Variable $\left(\mathrm{S}_{[\%]} * \mathrm{~T}_{7,8}\right)$ auf. Die beste $\mathrm{R}^{2}$ korr konnten durch den linearen Ansatz abgebildet werden (Modell Nr. 5, Formel 4-5). Das Inger-Modell wies ein $\mathrm{R}^{2}$ korr von 0,77, das Tordis-Modell wies ein $\mathrm{R}^{2}$ korr von 0,85 auf.

Modell Nr. 3 (Standortcluster S - sig - Max 1, AF 2)

$$
d G Z\left[t_{(\text {atro })} \mathrm{ha}^{-1} \mathrm{a}^{-1}\right]=e^{\left(a_{0}+\frac{a_{1}}{(U[\%] * N 6,7)}\right)}
$$

(Formel 4-3)

Modell Nr. 4 (Standortcluster S - ln - H 275)

$$
d G Z\left[t_{(\text {atro })} \mathrm{ha}^{-1} \mathrm{a}^{-1}\right]=a_{0}+a_{1} * \ln \left(\left[\mathrm{S}_{[\%]} / T I_{6,7}\right) \quad\right. \text { (Formel 4-4) }
$$

Modell Nr. 5 (Standortcluster S - lin - Inger, Tordis)

$$
\begin{array}{cl}
d G Z\left[t_{\text {(atro) }} \mathrm{ha}^{-1} \mathrm{a}^{-1}\right]=a_{0}+a_{1} *\left(S_{[\%]} * T_{7,8}\right) & \text { (Formel 4-5) } \\
& \\
d G Z: & \text { Durchschnittlicher Gesamtzuwachs }\left[\mathrm{t}_{\text {(atro) }} \mathrm{ha}^{-1} \mathrm{a}^{-1}\right] \\
e: & \text { Eulersche Zahl } \\
a_{0} \text { und } a_{l}: & \text { Modellkoeffizienten } \\
U_{[\%]}: & \text { Schluffgehalt in der Tiefe } 0-60 \mathrm{~cm}[\%] \\
N_{6,7}: & \text { Niederschlagssumme des Zeitfensters Juni bis Juli [mm] } \\
S_{[\%]}: & \text { Sandgehalt in der Tiefe } 0-60 \mathrm{~cm}[\%] \\
T I_{6,7}: & \text { Trockenheitsindexsumme des Zeitfensters Juni bis Juli } \\
T_{7,8}: & \text { Temperatursumme des Zeitfensters Juli bis August }\left[{ }^{\circ} \mathrm{C}\right]
\end{array}
$$

Die Modelle waren für Max 1, AF 2 und Tordis auf dem p-Niveau < 0,001, die von H 275 und Inger auf dem p-Niveau < 0,01 signifikant (Tab. 4-39). Die RMSE schwankten zwischen 0,14 und $1,36 \mathrm{t}_{\text {(atro) }} \mathrm{ha}^{-1} \mathrm{a}^{-1}$. Die Modelle zeigten eine Prognosestreuung zwischen 12,3 (AF 2) und 27,5\% (H 275). Die Modelle wiesen kaum Verzerrungen auf. Abbildung 4-32 können graphische Darstellungen der Modelle entnommen werden. Die für Max 1 und AF 2 errechneten Modelle verlaufen asymptotisch. Mit zunehmenden $\left(\mathrm{U}_{[\%]} * \mathrm{~N}_{6,7}\right)$ nimmt der dGZ zu. Das H 275-Modell verläuft deutlich gleichmäßiger als die Max 1- und AF 2-Modelle. Mit zunehmenden Werten für $\left(\mathrm{S}_{[\%]} / \mathrm{TI}_{6,7}\right)$ nimmt der

\begin{tabular}{|c|c|c|c|c|c|c|c|c|c|c|c|}
\hline \multirow{2}{*}{ Gattung } & \multirow{2}{*}{ Klon } & \multirow{2}{*}{$\mathrm{n}$} & \multirow{2}{*}{ Prädiktor } & \multirow{2}{*}{$\begin{array}{l}\text { Anpas- } \\
\text { sung }\end{array}$} & \multirow{2}{*}{$\mathrm{R}_{\text {korr }}^{2}$} & \multirow{2}{*}{$a_{0}$} & \multirow{2}{*}{$a_{1}$} & \multirow{2}{*}{$\begin{array}{c}\mathrm{RMSE} \\
{\left[\mathrm{t}_{\text {(atro) }} \mathrm{ha}^{-1} \mathrm{a}^{-1}\right]}\end{array}$} & $\mathrm{e}$ & $\mathrm{Se}$ & $\mathrm{Mx}$ \\
\hline & & & & & & & & & \multicolumn{3}{|c|}{ [\%] } \\
\hline \multirow{3}{*}{ Pappel } & Max 1 & 8 & $\left(\mathrm{U}_{[\%]} * \mathrm{~N}_{6,7}\right)$ & sig & $0,89^{* * * *}$ & $2,16^{* * * *}$ & $-594,13^{* * * *}$ & 1,23 & $-2,5 * 10^{-14}$ & 13,79 & 13,79 \\
\hline & Н 275 & 7 & $\left(\mathrm{~S}_{[\%]} / \mathrm{TI}_{6,7}\right)$ & $\ln$ & $0,84^{* *}$ & $36,30^{* * *}$ & $-10,62^{* *}$ & 1,20 & $-2,3 * 10^{-14}$ & 27,50 & 27,50 \\
\hline & AF 2 & 8 & $\left(\mathrm{U}_{[\%]} * \mathrm{~N}_{6,7}\right)$ & sig & $0,97^{* * *}$ & $2,28^{* * * *}$ & $-885,98^{* * * *}$ & 0,95 & $-1,3 * 10^{-14}$ & 12,29 & 12,29 \\
\hline \multirow{2}{*}{ Weide } & Inger $^{a}$ & 8 & $\left(\mathrm{~S}_{[\%]} * \mathrm{~T}_{7,8}\right)$ & $\operatorname{lin}$ & $0,77^{* * *}$ & $30,29^{* *}$ & $-0,008^{* *}$ & 1,36 & $9,4 * 10^{-14}$ & 23,39 & 29,39 \\
\hline & Tordis & 8 & $\left(\mathrm{~S}_{[\%]} * \mathrm{~T}_{7,8}\right)$ & $\operatorname{lin}$ & $0,85^{* * *}$ & $34,94^{* * *}$ & $-0,010^{* * *}$ & 1,27 & $2,2 * 10^{-3}$ & 26,43 & 26,43 \\
\hline
\end{tabular}
$\mathrm{dGZ}$ ab. Die Inger- und Tordis-Modelle zeigen einen abnehmenden dGZ bei höher werdenden Werten für $\left(\mathrm{S}_{[\%]} * \mathrm{~T}_{7,8}\right)$.

Tab. 4-39: Statistische Kennwerte der standortbasierten Ertragsmodelle im Standortcluster S für die Pappelklone Max 1, H 275, AF 2 sowie für die Weidenklone Inger und Tordis.

${ }^{a}$ Bei Inger wurde mit dem exponentiellen Ansatz (1) ein $\mathrm{R}^{2}$ korr von 0,81 erreicht, da der Unterschied zum Lin-Modell nur $5 \%$ betrug und das exponentielle Modell (1) bis zu 60 \% am oberen Modellrand überschätzte, wurde das ,gemeinsame Modell“ bevorzugt. 


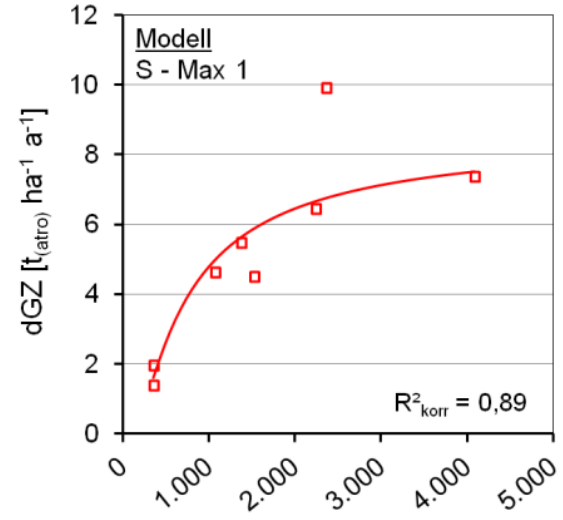

a)

$$
\left(\mathrm{U}_{[\%]}{ }^{*} \mathrm{~N}_{6,7}\right)
$$

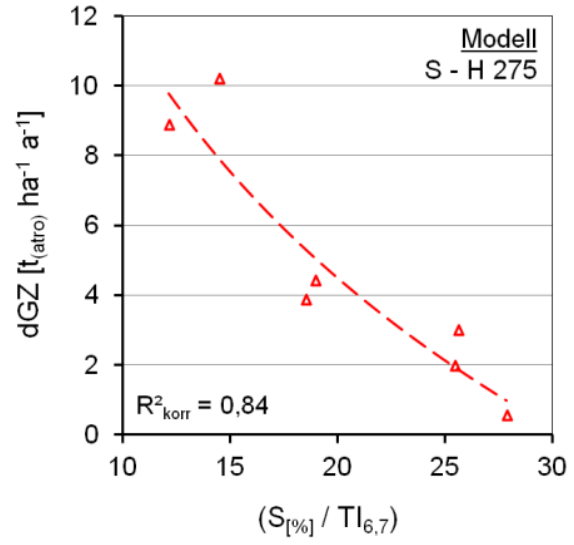

b)

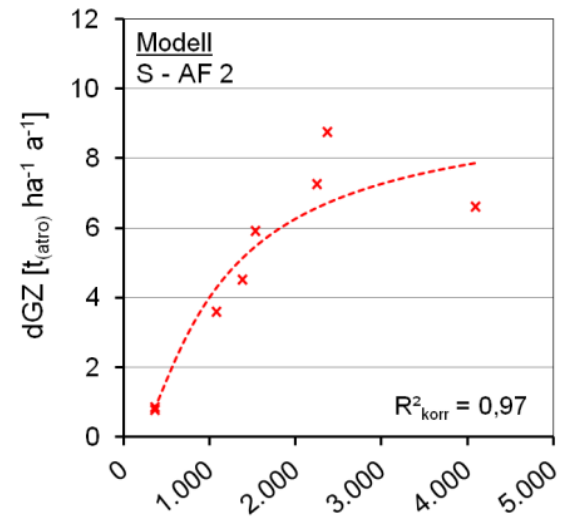

c)

$$
\left(U_{[\%]}{ }^{*} N_{6,7}\right)
$$

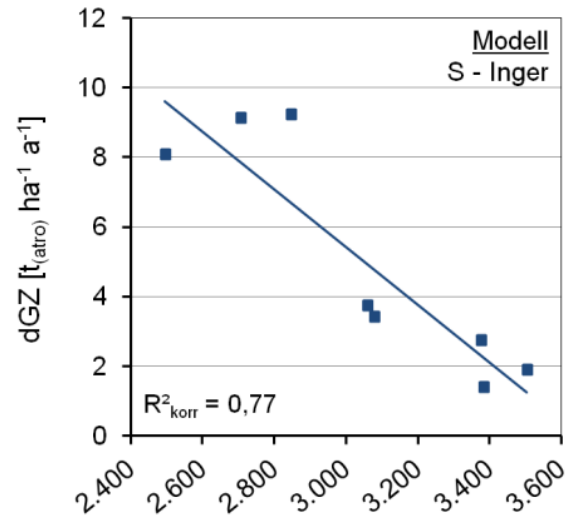

d)

$$
\left(S_{[\%]}{ }^{*} T_{7,8}\right)
$$

e)

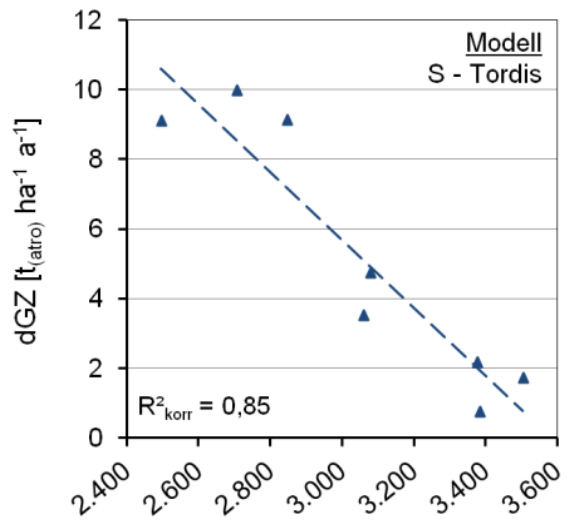

$\left(\mathrm{S}_{[\%]}{ }^{*} \mathrm{~T}_{7,8}\right)$

Abb. 4-32: Standortbasierte Ertragsmodelle im Standortcluster S für a) Max 1 b) H 275 c) AF 2 d) Inger und e) Tordis.

Da die Klone keine signifikanten Unterschiede untereinander aufwiesen, wurden die Gattungen zusammen als Prüfglied in der Modellierung untersucht. Bei den Weiden wird ersichtlich, dass mit weiterer Berücksichtigung von unabhängigen Variablen die Genauigkeit der Modelle deutlich zunimmt (Tab. 4-40). Das beste Modell wurde mit der multiplen linearen Regression mit den Prädiktoren $\mathrm{U}_{[\%]}$, $\mathrm{T}_{7,8}$ und $\mathrm{N}_{6,7}$ erreicht. Für die Pappel wurde mit der multiplen nicht-linearen Regression keine Verbesserung der Bestimmtheitsmaße erzielt.

Für die Veranschaulichung der Effekte der nicht-aggregierten Variablen in den univariaten klonspezifischen Modellen werden im Folgenden dreidimensionale Graphiken für die Klone H 275, AF 2 und Tordis dargestellt (Abb. 4-33). 
Tab. 4-40: Beste Modellanpassungen für die Weide im Standortcluster S mit je univariater und multipler Regression.

\begin{tabular}{|c|c|c|c|c|c|c|c|c|c|}
\hline $\mathrm{n}$ & $\begin{array}{c}\text { Anzahl } \\
\text { Präd. }\end{array}$ & Prädiktor & $\begin{array}{l}\text { Anpas- } \\
\text { sung }\end{array}$ & $\mathrm{R}_{\text {korr }}^{2}$ & $\mathrm{a}_{0}$ & $\mathrm{a}_{1}$ & $\mathrm{a}_{2}$ & $a_{3}$ & $\begin{array}{c}\text { RMSE } \\
{\left[t_{\text {(arto })} h a^{-1} a^{-1}\right]}\end{array}$ \\
\hline 16 & 1 & $\left(\mathrm{~S}_{[\%]} * \mathrm{~T}_{7,8}\right)$ & pow & $\mathbf{0 , 8 2 ^ { * * * * }}$ & $32,62^{* * * *}$ & $-0,009^{* * * *}$ & - & - & 1,34 \\
\hline 16 & 2 & $\mathrm{~S}_{[\%]}, \mathrm{T}_{7,8}^{\mathrm{a}}$ & mlin & $0,87^{* * *}$ & $76,76^{* *}$ & $-0,312^{* *}$ & $-1,229^{* *}$ & & 1,11 \\
\hline 16 & 3 & $\mathrm{U}_{[\%]}, \mathrm{T}_{7,8}, \mathrm{~N}_{6,7}$ & mlin & $\mathbf{0 , 9 3} *^{*}$ & $41,52^{* *}$ & $0,254^{* *}$ & $-1,198^{* *}$ & $0,047^{* *}$ & 0,75 \\
\hline
\end{tabular}

Beide Weidenklone, Inger und Tordis, sind in der vorliegenden Berechnung aggregiert

${ }^{a}$ Das Modell mit $\mathrm{U}_{[\%]}$ als bodenbezogene Variable hatte annähernd dasselbe $\mathrm{R}^{2}{ }_{\text {korr }}$ wie das Modell mit $\mathrm{S}_{[\%]}$ als bodenbezogene Variable
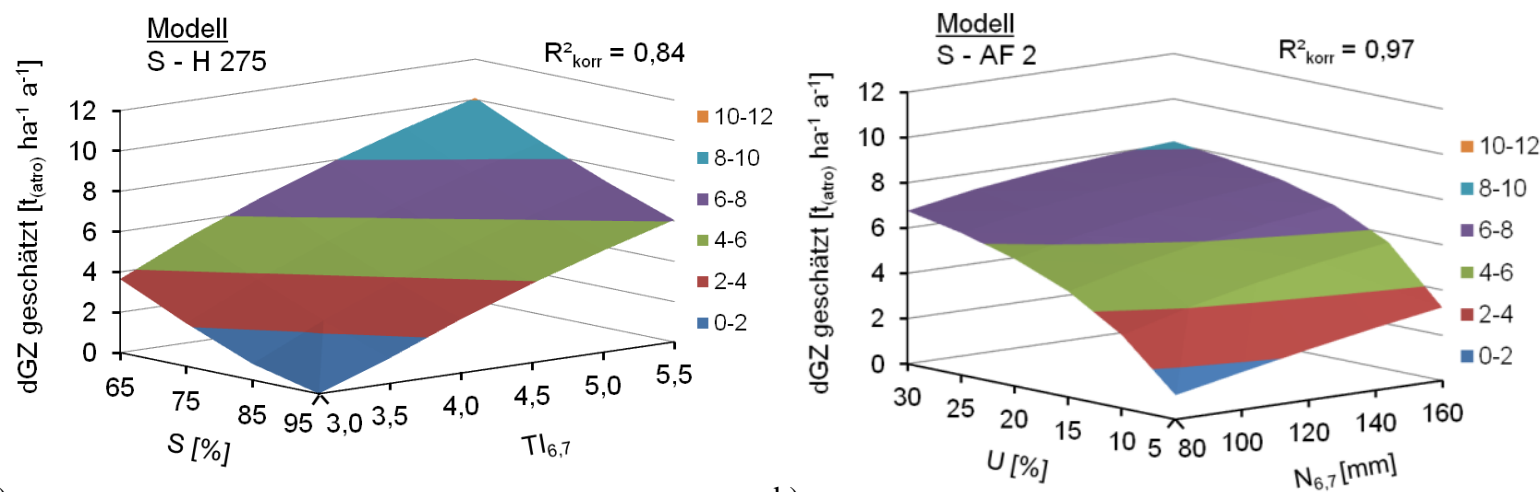

a)

b)

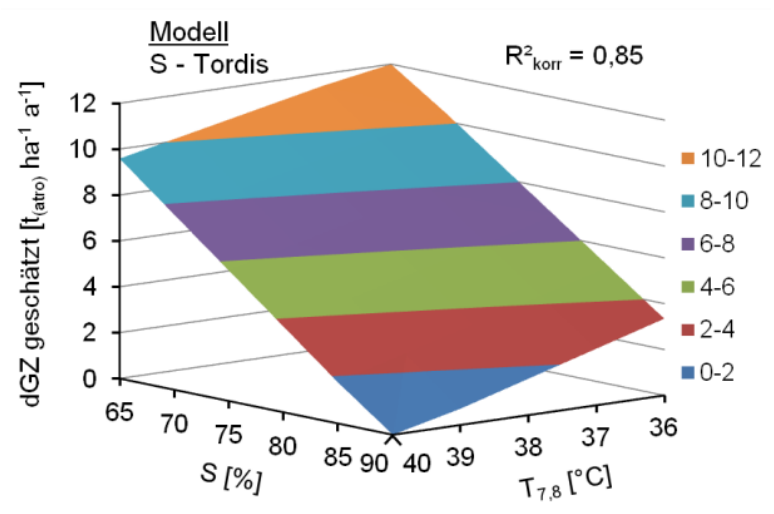

Abb. 4-33: Dreidimensionale Darstellung der standortbasierten Ertragsmodelle im Standortcluster S. Dargestellt ist der dGZ in Abhängigkeit der zwei nicht aggregierten Variablen, aus denen sich der aggregierte Modellprädiktor zusammensetzt für a) $\mathrm{H} 275$ b) AF 2 und c) Tordis.

Das Modell des Klons H 275, mittels sigmoidaler Anpassung berechnet, weist einen deutlich höheren Einfluss des klimabezogenen Anteils des Prädiktors $\left(\mathrm{TI}_{6,7}\right)$ auf als der bodenbezogene $\left(\mathrm{S}_{[\%]}\right)$ auf die Ertragsbildung. Der modellierte dGZ steigt beim höchsten Wert für $\mathrm{S}_{[\%]}$ mit zunehmenden $\mathrm{TI}_{6,7}$ auf ca. 6,0 $\mathrm{t}_{\text {(atro) }} \mathrm{ha}^{-1} \mathrm{a}^{-1}$, dementsprechend steigt der modellierte dGZ beim kleinsten Wert für $\mathrm{TI}_{6,7}$ mit abnehmenden $S_{[\%]}$ auf ca. 4,0 $t_{(\text {atro) }}$ ha $^{-1} \mathrm{a}^{-1}$. Diese Wechselwirkung zwischen den Einzelbestandteilen des Prädiktors bleibt über den gesamten Validitätsbereich des Modells annähernd konstant. Bei der Teilabbildung für das AF 2-Modell zeigt sich ein anderes Bild als bei H 275. Hier hat der bodenbezogene Teil des Prädiktors $U_{[\%]}$ eine größere Bedeutung in der Ertragsbildung als der klimabezogene Teil des Prädiktors $\mathrm{N}_{6,7}$. Je nach Wert für $\mathrm{U}_{[\%]}$ ist der Klimaeffekt unterschiedlich stark. Im niedrigen Wertebereich von $\mathrm{U}_{[\%]}$ hat der $\mathrm{N}_{6,7}$ einen stärkeren Einfluss auf den $\mathrm{dGZ}$ als im hohen Wertebereich. Mit zunehmenden $U_{[\%]}$ lässt die ertragssteigernde Wirkung höher werdender $\mathrm{N}_{6,7}$ nach. Die hier erläuterten Zusammenhänge sind für den Klon Max 1 übertragbar.

Beide Weidenmodelle nutzen die aggregierten Variablen $\left(\mathrm{S}_{[\%]} * \mathrm{~T}_{7,8}\right)$ als Prädiktor. Somit ist keine Klimavariable mit einem direkten Bezug auf den Wasserhaushalt, wie Niederschlags- oder Trocken- 
heitsindexvariablen, im Modell berücksichtigt. Wie bei Max 1 und AF 2 trägt der bodenbezogene Anteil des Prädiktors, in diesem Fall $S_{[\%]}$, den größten Anteil zur Ertragsbildung bei. Die bereits im Kapitel 4.4.1 beschriebene negative Korrelation zwischen $\mathrm{T}_{7,8}$ und dGZ spiegelt sich in der Regression wider. Dieser Effekt ist beim Modell im hohen Wertebereich von $\mathrm{S}_{[\%]}$ am stärksten ausgeprägt. Bis auf das Max 1-Modell waren die Residuen der Modelle normalverteilt (Tab. 4-41).

Tab 4-41: Test auf Normalverteilung der Modellresiduen im Standortcluster S für die Pappelklone Max 1, H 275, AF 2 sowie für die Weidenklone Inger und Tordis. Eine Normalverteilung ist bei $\mathrm{p}$-Werten $<0,05$ nicht gegeben.

\begin{tabular}{llc}
\hline Gattung & Klon & p-Wert \\
\hline \multirow{3}{*}{ Pappel } & Max 1 & $\mathbf{0 , 0 0 6}$ \\
& H 275 & 0,689 \\
& AF 2 & 0,733 \\
Weide & Inger & 0,341 \\
& Tordis & 0,440 \\
\hline
\end{tabular}

Eine Gegenüberstellung der beobachteten und prognostizierten dGZ der Ertragsmodelle ist Abbildung 4-34 zu entnehmen. Die Modelle zeigen eine hohe Genauigkeit und nur geringe Abweichungen zwischen beobachteten und geschätzten dGZ. Lediglich beim Klon Max 1 wird der höchste beobachtete Wert im Vergleich zum geschätzten Wert um etwa $30 \%$ unterschätzt.
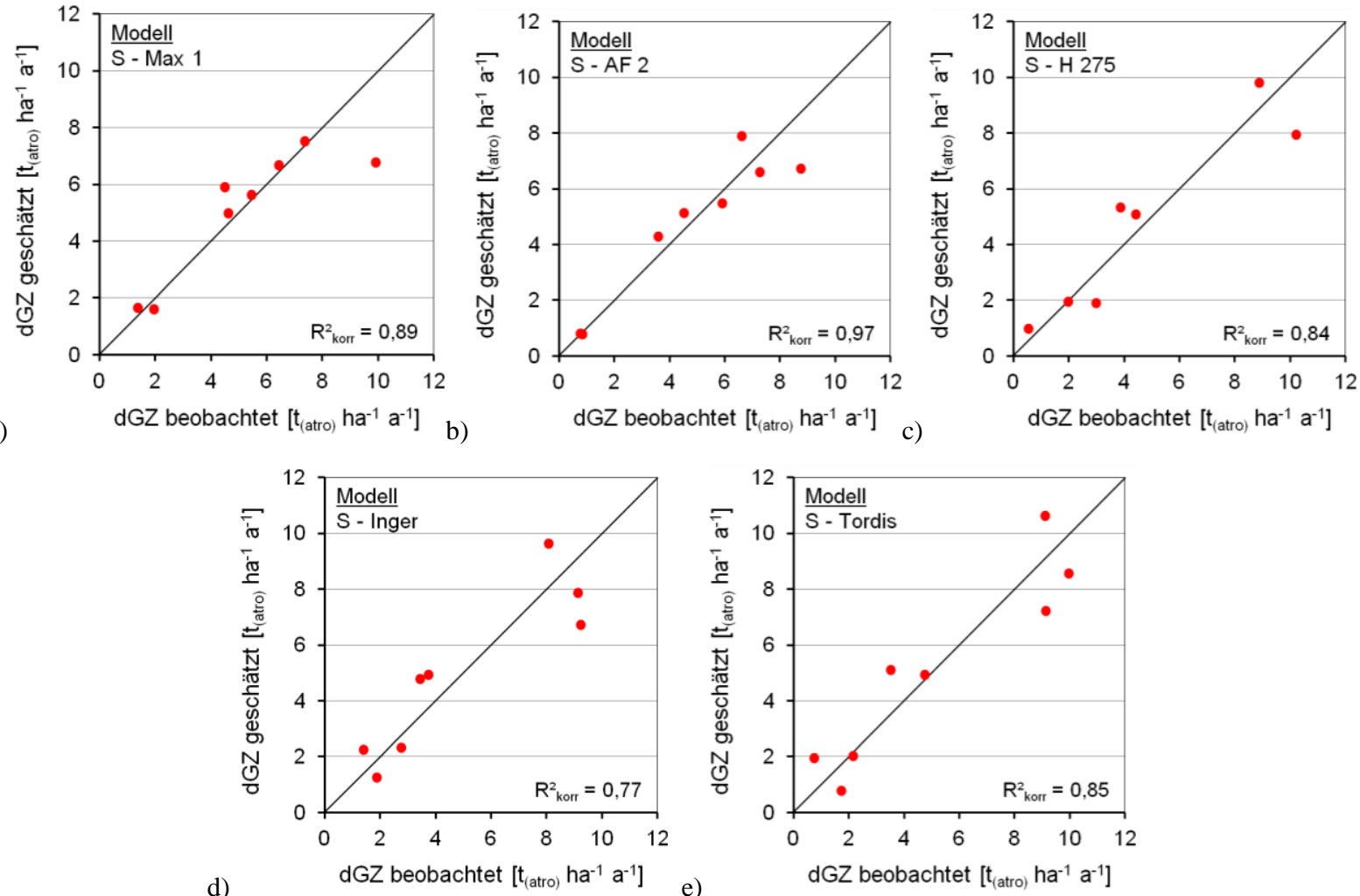

Abb. 4-34: Vergleich beobachtete und geschätzte dGZ für die Modelle im Standortcluster S für die Modelle der Klone a) Max 1 b) H 275 c) AF 2 d) Inger e) Tordis.

\subsubsection{Standortcluster $U$}

Beim Standortcluster U konnten nur signifikante Modelle für die Klone Max 1 und Tordis ermittelt werden. Für die Klone kamen jeweils die Variablen $\left(\mathrm{BZ} * \mathrm{TI}_{4,5}\right)$ sowie $(\mathrm{BZ})$ zum Einsatz. Beide Modelle wurden mithilfe des compound Ansatzes entwickelt (Modell Nr. 6, Formel 4-6, Modell Nr. 7, Formel 4-7). 
Modell Nr. 6 (Standortcluster U- comp - Max 1)

$$
d G Z\left[t_{(\text {atro })} \mathrm{ha}^{-1} \mathrm{a}^{-1}\right]=a_{0} * a_{1}\left(B Z * T I_{4,5}\right) \quad \text { (Formel 4-6) }
$$

Modell Nr. 7 (Standortcluster U - comp - Tordis)

$$
\begin{aligned}
& d G Z\left[t_{(\text {atro })} \mathrm{ha}^{-1} \mathrm{a}^{-1}\right]=a_{0} * a_{1}{ }^{(B Z)} \quad \text { (Formel 4-7) } \\
& d G Z \text { : Durchschnittlicher Gesamtzuwachs [ } \mathrm{t}_{(\text {atro) }} \text { ha }^{-1} \mathrm{a}^{-1} \text { ] } \\
& a_{0} \text { und } a_{l} \text { : Modellkoeffizienten } \\
& \text { BZ: } \quad \text { Bodenzahl } \\
& T_{4,5} \text { : } \quad \text { Trockenheitsindexsumme des Zeitfensters April bis Mai }
\end{aligned}
$$

Tabelle 4-42 zeigt eine Übersicht der errechneten Modelle mit ihren statistischen Kennwerten. Die $\mathrm{R}^{2}{ }_{\text {korr }}$ betrugen für das Max 1-Modell 0,84 und für das Tordis-Modell 0,95. Das Max 1-Modell war auf dem p-Niveau $<0,05$ und das Tordis-Modell auf dem p-Niveau $<0,01$ signifikant. Die Modellkoeffizienten waren bis auf $\mathrm{a}_{0}$ beim Max 1-Modell auf dem p-Niveau $<0,001$ signifikant. Beim Standortcluster $U$ waren sehr geringe Prognosestreuungen zu verzeichnen. Sie betrugen für Max $15,6 \%$ und für Tordis $1,4 \%$.

Tab. 4-42: Statistische Kennwerte der standortbasierten Ertragsmodelle im Standortcluster U für den Pappelklon Max 1 sowie für den Weidenklon Tordis.

\begin{tabular}{lccccccccccc}
\hline Gattung & \multirow{2}{*}{ Klon } & $\mathrm{n}$ & Prädiktor & $\begin{array}{c}\text { Anpas- } \\
\text { sung }\end{array}$ & $\mathrm{R}^{2}{ }_{\text {korr }}$ & $\mathrm{a}_{0}$ & $\mathrm{a}_{1}$ & $\begin{array}{c}\mathrm{RMSE} \\
{\left[\mathrm{t}_{\text {arro }} \mathrm{ha}^{-1} \mathrm{a}^{-1}\right]}\end{array}$ & $\mathrm{e}$ & $\mathrm{Se}$ & $\mathrm{Mx}$ \\
\hline Pappel & Max 1 & 5 & $\left(\mathrm{BZ}^{*} \mathrm{TI}_{4,5}\right)$ & comp & $\mathbf{0 , 8 4}^{*}$ & $\mathbf{3 , 2 9}^{* *}$ & $\mathbf{1 , 0 0 1 9 1}^{* * *}$ & 0,62 & $4,5^{*} 10^{-7}$ & 5,61 & 5,61 \\
Weide & Tordis & 5 & $(\mathrm{BZ})$ & comp & $\mathbf{0 , 9 5}^{* *}$ & $\mathbf{4 , 5 0}^{* *}$ & $\mathbf{1 , 0 0 7 7 7}^{* * *}$ & 0,22 & $2,6^{*} 10^{-13}$ & 1,44 & 1,44 \\
\hline
\end{tabular}

Abbildung 4-35 zeigt die Max 1 und Tordis Modelle. Bei Max 1 nimmt der dGZ mit steigenden Werten von $\left(\mathrm{BZ} * \mathrm{TI}_{4,5}\right)$, bei Tordis mit steigenden Werten von (BZ) zu. Ersichtlich ist, dass beim Klon Tordis deutlich höhere dGZ erzielt wurden als bei Max 1. Aus diesem Grund verläuft das TordisModell viel flacher als das Max 1-Modell. Im Tordis-Modell spielt alleine die BZ die größte Rolle, der Gradient zwischen den erzielten Werten ist jedoch sehr flach.

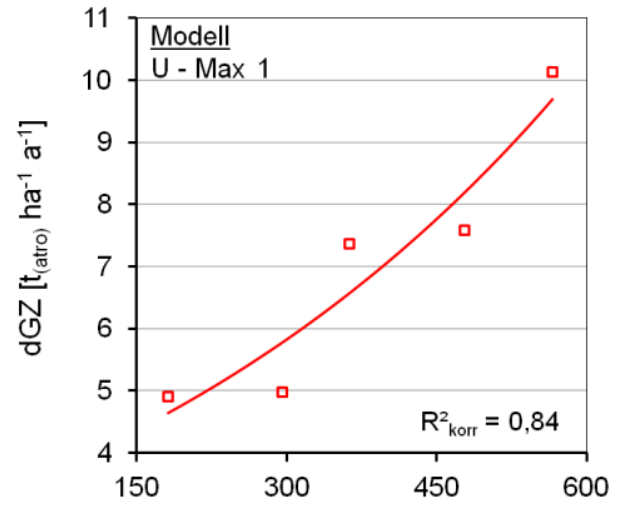

a)

$\left(\mathrm{BZ} * \mathrm{Tl}_{4,5}\right)$

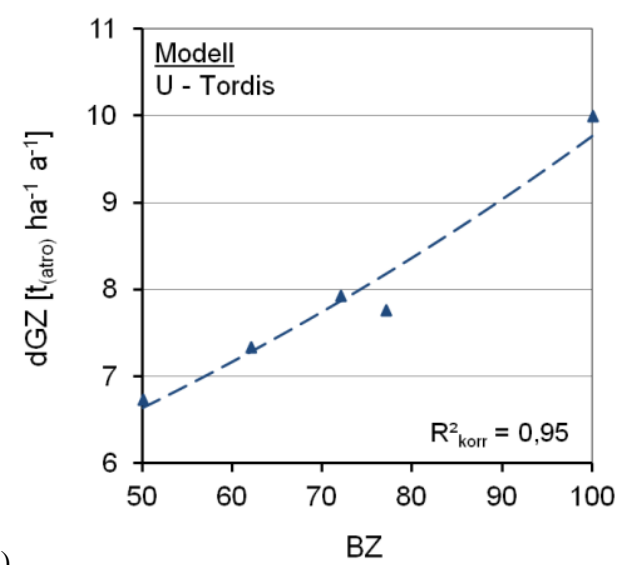

b)

$\mathrm{BZ}$

Abb. 4-35: Standortbasierte Ertragsmodelle im Standortcluster U für a) Max 1 und b) Tordis.

Die Wechselwirkung zwischen den beiden Bestandteilen des aggregierten Prädiktors beim Max 1Modell und den geschätzten dGZ ist in Abbildung 4-36 zu finden. Es kann beobachtet werden, dass beide Variablen BZ und $\mathrm{TI}_{4,5}$ ähnlich viel zur dGZ-Entwicklung beitragen. Steigende Werte von beiden Prädiktorbestandteilen führen zur dGZ-Zunahme. 


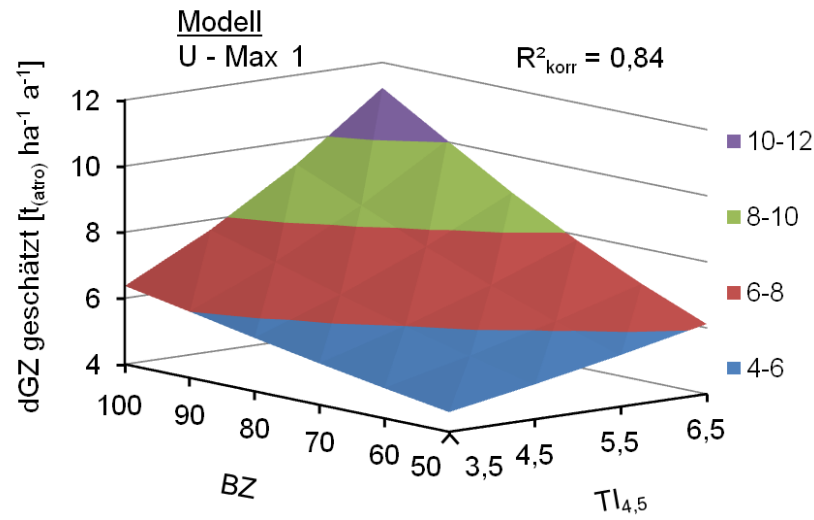

Abb. 4-36: Dreidimensionale Darstellung des standortbasierten Ertragsmodells im Standortcluster U. Dargestellt ist der dGZ in Abhängigkeit der zwei nicht aggregierten Variablen aus denen sich der aggregierte Modellprädiktor für den Klon Max 1 zusammensetzt.

Die Modellresiduen beider Klone sind normalverteilt (Tab. 4-43). Zudem weichen die modellierten dGZ nur geringfügig von den gemessenen Werten ab, wie Abbildung 4-37 zeigt.

Tab. 4-43: Test auf Normalverteilung der Modellresiduen im Standortcluster U für den Pappelklon Max 1 sowie für den Weidenklon Tordis. Eine Normalverteilung ist bei p-Werten $<0,05$ nicht gegeben.

\begin{tabular}{llc}
\hline Gattung & Klon & $\mathrm{p}$-Wert \\
\hline Pappel & Max 1 & 0,458 \\
Weide & Tordis & 0,085 \\
\hline
\end{tabular}

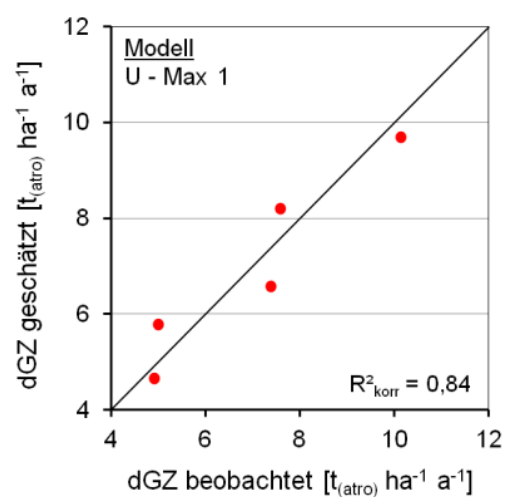

a)

Abb. 4-37: Vergleich beobachteter und geschätzter dGZ für die Modelle im Standortcluster U für die Modelle der Klone a) Max 1 und b) Tordis.

\subsubsection{Standortcluster $L$}

Die im Standortcluster L errechneten Modelle erreichten eine sehr hohe Genauigkeit (Tab. 4-44). Für Max 1 und Inger war alleine die nFK für die Ertragsbildung verantwortlich (Modell Nr. 8, Formel 4-8). $\mathrm{R}^{2}$ korr betrug betrug für Max 10,87 und für Tordis 0,98. Für das Modell wurde die exponentielle Funktion (1) verwendet. Beim AF 2-Modell wurde die beste Anpassung mit den aggregierten Variablen $\left(\mathrm{nFK} * \mathrm{~N}_{5,6}\right.$ ) erreicht (Modell Nr. 9, Formel 4-9). Das Modell wurde mit dem sigmoidalen Ansatz errechnet, sein $\mathrm{R}^{2}$ korr betrug 0,86 . 
Modell Nr. 8 (Standortcluster L - exp 1 - Max 1, Inger)

$$
d G Z\left[t_{(\text {atro })} h^{-1} \mathrm{a}^{-1}\right]=e^{\left(a_{0}+a_{1} *(n F K)\right)}
$$

Modell Nr. 9 (Standortcluster L - sig - AF 2)

$$
\begin{array}{cl}
d G Z\left[t_{\text {(atro })} \text { ha }^{-1} \mathrm{a}^{-1}\right]=e^{\left(a_{0}+\frac{a_{1}}{(n F K * N 5,6)}\right)} & \text { (Formel 4-9) } \\
& \\
d G Z: & \text { Durchschnittlicher Gesamtzuwachs }\left[\mathrm{t}_{\text {(atro) }} \mathrm{ha}^{-1} \mathrm{a}^{-1}\right] \\
e: & \text { Eulersche Zahl } \\
a_{0} \text { und } a_{1}: & \text { Modellkoeffizienten } \\
n F K: & \text { Nutzbare Feldkapazität in der Tiefe } 0-60 \mathrm{~cm} \mathrm{[cm]} \\
N_{5,6}: & \text { Niederschlagssumme des Zeitfensters Mai bis Juni [mm] }
\end{array}
$$

\begin{tabular}{|c|c|c|c|c|c|c|c|c|c|c|c|}
\hline \multirow{2}{*}{ Gattung } & \multirow{2}{*}{ Klon } & \multirow{2}{*}{$\mathrm{n}$} & \multirow{2}{*}{ Prädiktor } & \multirow{2}{*}{$\begin{array}{c}\text { Anpas- } \\
\text { sung }\end{array}$} & \multirow{2}{*}{$\mathrm{R}_{\text {korr }}$} & \multirow{2}{*}{$\mathrm{a}_{0}$} & \multirow{2}{*}{$a_{1}$} & \multirow{2}{*}{$\begin{array}{c}\text { RMSE } \\
{\left[\mathrm{t}_{\text {(atro) }} \mathrm{ha}^{-1} \mathrm{a}^{-1}\right]}\end{array}$} & $\mathrm{e}$ & $\mathrm{Se}$ & Mx \\
\hline & & & & & & & & & \multicolumn{3}{|c|}{ [\%] } \\
\hline \multirow{2}{*}{ Pappel } & Max 1 & 7 & $(\mathrm{nFK})$ & $\exp 1$ & $0,87^{* *}$ & $-4,63^{* *}$ & $0,6045^{* *}$ & 1,21 & $-4,9 * 10^{-7}$ & 26,25 & 26,25 \\
\hline & $\mathrm{AF} 2$ & 7 & $\left(\mathrm{nFK} * \mathrm{~N}_{5,6}\right)$ & sig & $\mathbf{0 , 8 6}{ }^{* *}$ & $5,29^{* * * *}$ & $-4490,09^{* *}$ & 1,19 & $-9,7 * 10^{-6}$ & 25,66 & 25,66 \\
\hline Weide & Inger & 7 & $(\mathrm{nFK})$ & $\exp 1$ & $0,98^{* * * *}$ & $-4,65^{* * * *}$ & $0,6022^{* * * *}$ & 0,56 & $-4,4 * 10^{-6}$ & 8,90 & 8,90 \\
\hline
\end{tabular}

Die Max 1- und AF 2-Modelle waren auf dem p-Niveau < 0,01, das Inger-Modell auf dem p-Niveau $<0,001$ signifikant. Die RMSE-Werte schwankten zwischen 0,09 und 0,28 $\mathrm{t}_{\text {(atro) }} \mathrm{ha}^{-1} \mathrm{a}^{-1}$. Die Prognosestreuung betrug für Max $126,3 \%$, für AF $225,7 \%$ und für Inger 8,9 \%. Die errechneten Bias waren negativ und sehr gering, daher war der Unterschied zwischen Prognosestreuung und Treffgenauigkeit nur minimal.

Tab. 4-44: Statistische Kennwerte der standortbasierten Ertragsmodelle im Standortcluster L für die Pappelklone Max 1, AF 2 sowie den Weidenklon Inger.

Abbildung 4-38 zeigt die ermittelten Modelle für das Standortcluster L. Die Max 1- und IngerModelle basieren einzig auf der Variablen nFK, wobei der dGZ mit zunehmenden nFK steigt. Das AF 2-Modell hingegen verwendet die aggregierte Variable $\left(\mathrm{nFK} * \mathrm{~N}_{5,6}\right)$ als Prädiktor im Modell. Der dGZ steigt mit zunehmenden Werten für $\left(\mathrm{nFK} * \mathrm{~N}_{5,6}\right)$.

Abbildung 4-39 zeigt den dreidimensionalen Verlauf des AF 2-Modells. Im AF 2-Modell hat der bodenbezogene Teil des Prädiktors (nFK) an der dGZ-Zunahme einen größeren Einfluss als der klimabezogene Anteil $\left(\mathrm{N}_{5,6}\right)$. Die Wechselwirkung zwischen den nicht aggregierten Variablen ist im unteren Wertebereich wenig ausgeprägt, jedoch nimmt der dGZ mit zunehmenden Werten von $\mathrm{nFK}$ und $\mathrm{N}_{5,6}$ exponentiell zu. 

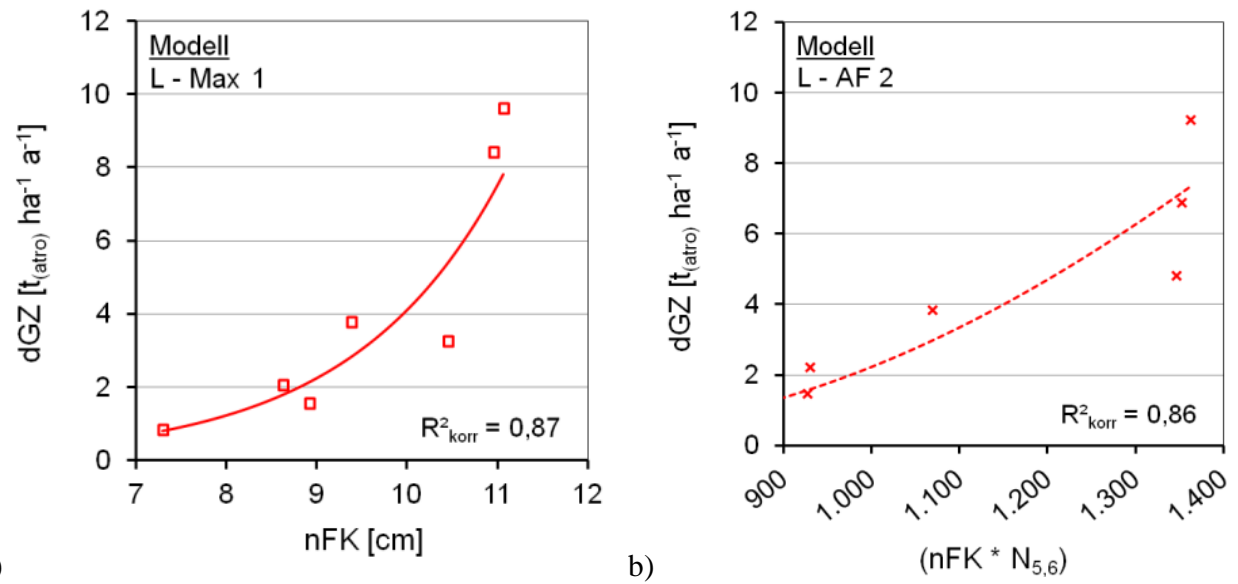

a)

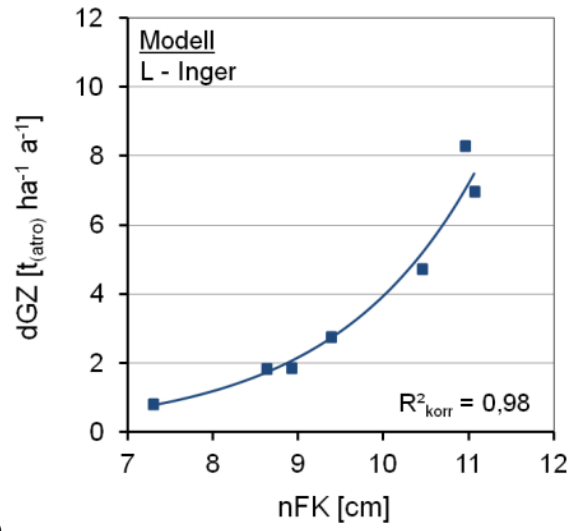

c)

Abb. 4-38: Standortbasierte Ertragsmodelle im Standortcluster L für a) Max 1 b) AF 2 und c) Inger.

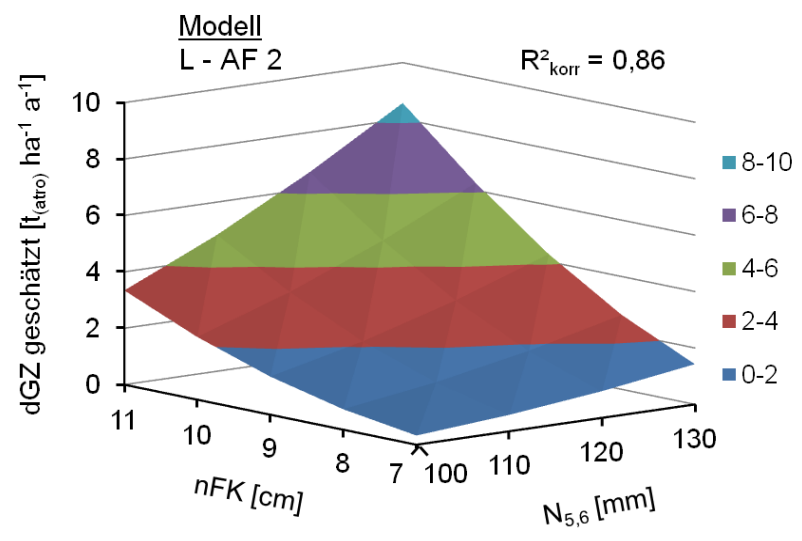

Abb. 4-39: Dreidimensionale Darstellung des standortbasierten Ertragsmodells im Standortcluster L. Dargestellt ist der dGZ in Abhängigkeit der zwei nicht aggregierten Variablen, aus denen sich der aggregierte Modellprädiktor für den Klon AF 2 zusammensetzt.

Die Residuen aller ermittelten Modelle im Standortcluster L sind normalverteilt (Tab. 4-45). Abbildung 4-40 enthält eine Gegenüberstellung der beobachteten und geschätzten dGZ. Die Max 1- und Inger-Modelle zeigen eine gute Übereinstimmung. Beim AF 2-Modell, insbesondere im höheren Wertebereich, kommt es jedoch zu größeren Abweichungen zwischen den beobachteten und geschätzten dGZ. 
Tab. 4-45: Test auf Normalverteilung der Modellresiduen im Standortcluster L für die Pappelklone Max 1, AF 2 sowie den Weidenklon Inger. Eine Normalverteilung ist bei p-Werten $<0,05$ nicht gegeben.

\begin{tabular}{lll}
\hline Gattung & Klon & p-Wert \\
\hline \multirow{2}{*}{ Pappel } & Max 1 & 0,769 \\
& AF 2 & 0,791 \\
Weide & Inger & 0,057 \\
\hline
\end{tabular}

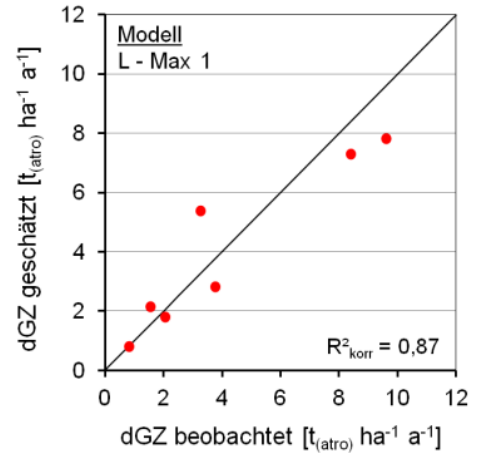

a)
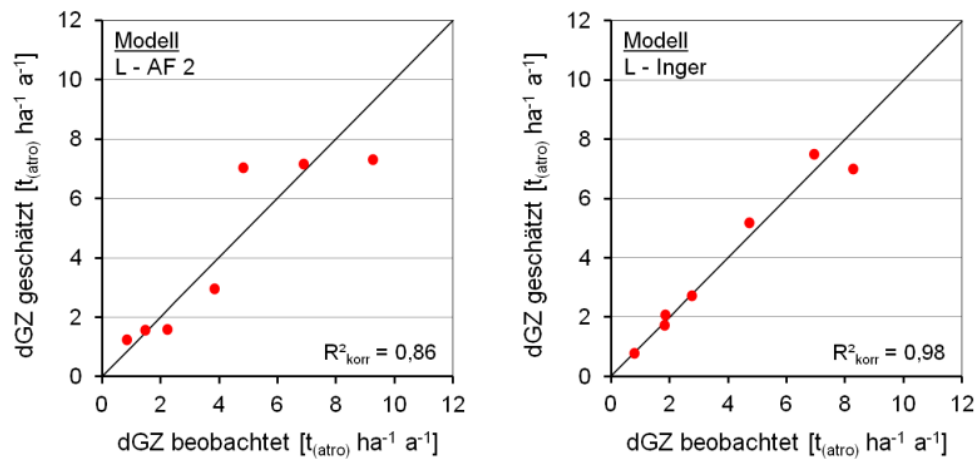

c)

Abb. 4-40: Vergleich beobachteter und geschätzter dGZ für die Modelle im Standortcluster L für die Modelle der Klone a) Max 1 b) AF 2 und c) Inger.

Eine Übersicht der Validitätsbereiche der präsentierten standortbasierten Ertragsmodelle können den Anhängen 37 und 38 entnommen werden.

\subsection{Ertragssteigerungsfaktoren und Ertragssteigerung in Folgerotationen}

Die Entwicklung von Ertragssteigerungsfaktoren erfolgte über mehrere Schritte. Im ersten Schritt erfolgte eine nicht-lineare Kurvenanpassung der dGZ-Entwicklung mehrerer Rotationen im dreijährigen Umtrieb. Zu diesem Zweck wurde der sigmoidale Ansatz verwendet. Wie im Kapitel 3.7 erwähnt, stammen die Daten aus den in Thüringen gelegenen Versuchsflächen der TLL (BIERTÜMPFEL et al. 2009 und 2012). Abbildung 4-41 zeigt die dGZ-Entwicklung der Klon-Gruppen BP und Max für die Standorte „Bad Salzungen“, „Dornburg“ und „Langenwetzendorf“. Es wird ersichtlich, dass die Ertragssteigerung der Max-Klone deutlich über die der Balsampappel liegt. Zudem ist ein standortspezifisches Verhalten zu erkennen. Bei den Max-Klonen weist der Schluffstandort den stärksten Anstieg im dGZ auf. Der Verlauf des dGZ in den Sand- und Lehmstandorten erscheint ähnlich, jedoch besteht ein entscheidender Unterschied im Anfangsbereich der Kurve. Der Sandstandort weist einen deutlich höheren dGZ der ersten Rotation auf als der Lehmboden. Die Ertragssteigerung verläuft danach beim Lehmstandort steiler als beim Sandstandort. Nach sechs Rotationen liegen beide auf einem ähnlichen Niveau, wobei der dGZ beim Sandstandort geringfügig höher ist. Bei den Balsampappeln zeigt sich ein anderes Bild. Hier hat der Sandstandort die höchste Ertragssteigerung, gefolgt von Schluff- und Lehmstandort. 

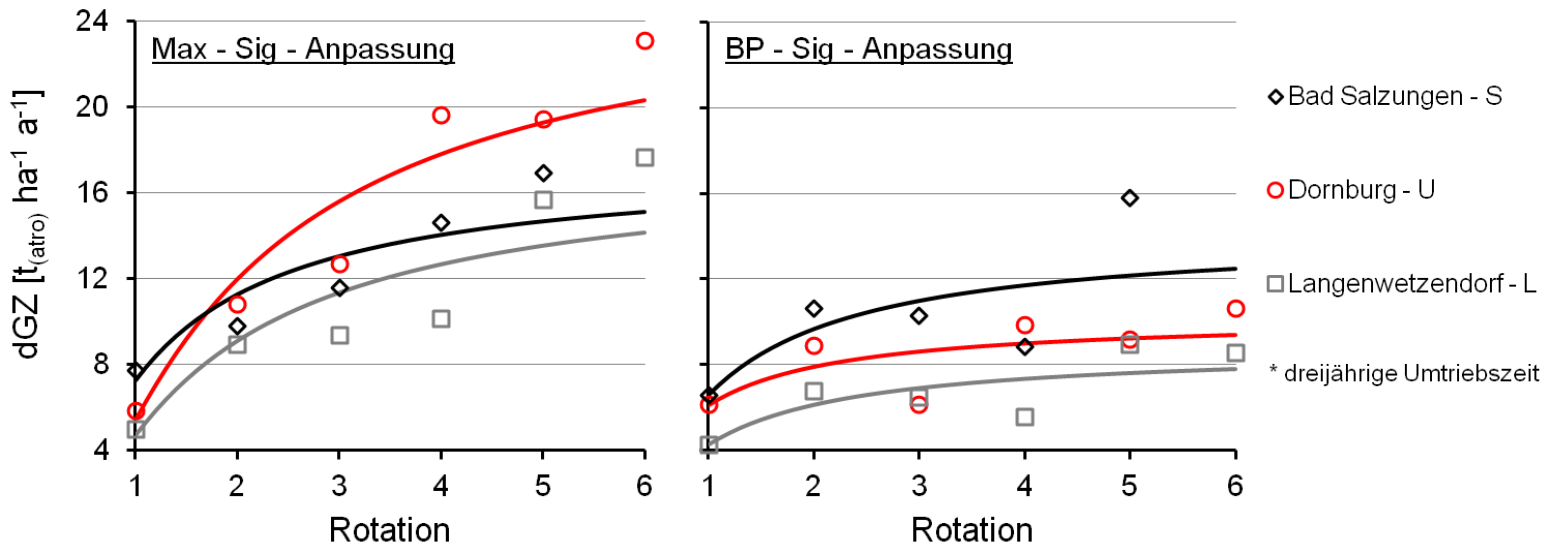

Abb. 4-41: dGZ Entwicklung der Klongruppen Max und BP in den Standorten „Bad Salzungen“ (Hauptbodenart S), „Dornburg“ (Hauptbodenart U) und „Langenwetzendorf“ (Hauptbodenart L).

Tabelle 4-46 können statistische Kennwerte der angepassten Funktionen entnommen werden. Während bei den Max-Klonen stets signifikante Anpassungen erzielt wurden, war bei den BP-Klonen einzig die Kurvenanpassung der Ertragssteigerung in „Langenwetzendorf“ signifikant. Bei den MaxKlonen betrug das $\mathrm{R}^{2}$ korr 0,79 in „Bad Salzungen“, 0,92 in „Dornburg“ und 0,80 in „Langenwetzendorf".

Tab. 4-46: Statistische Kennwerte der Modellanpassung mittels sigmoidalem Ansatz der dGZ-Entwicklung für die Klongruppen Max und BP an den Standorten „Bad Salzungen“, „Dornburg“ und „Langenwetzendorf“.

\begin{tabular}{ccccc}
\hline Standort / Bodenart & Klongruppe & $\mathrm{R}^{2}$ korr & $\mathrm{a}_{0}$ & $\mathrm{a}_{1}$ \\
\hline \multirow{2}{*}{ Bad Salzungen / S } & Max & $\mathbf{0 , 7 9}^{*}$ & $\mathbf{2 , 8 6 3}^{* * *}$ & $\mathbf{- 0 , 8 8 4}^{*}$ \\
& $\mathrm{BP}$ & 0,47 & $\mathbf{2 , 6 5 2}^{* * * *}$ & $-0,763$ \\
\hline \multirow{2}{*}{ Dornburg / U } & Max & $\mathbf{0 , 9 2}^{* *}$ & $\mathbf{3 , 2 7 5}^{* * *}$ & $\mathbf{- 1 , 5 8 4}^{* *}$ \\
& BP & 0,33 & $\mathbf{2 , 3 2 6}^{* * *}$ & $-0,520$ \\
\hline \multirow{2}{*}{ Langenwetzendorf / L } & Max & $\mathbf{0 , 8 0}^{* *}$ & $\mathbf{2 , 8 7 1}^{* * *}$ & $\mathbf{- 1 , 3 2 6}^{* *}$ \\
& BP & $\mathbf{0 , 5 9}^{*}$ & $\mathbf{2 , 1 7 6}^{* * *}$ & $\mathbf{- 0 , 7 2 4}^{*}$ \\
\hline
\end{tabular}

Mit den modellierten Daten wurden die standortspezifischen Ertragssteigerungsfaktoren gebildet. Das hat den Vorteil der Bereinigung der saisonalen Variation des dGZ (zum Beispiel durch Klimaeinflüsse). Formel 4-10 zeigt exemplarisch die Berechnung für den Ertragssteigerungsfaktor von der zweiten auf die dritte Rotation $\left(\mathrm{dGZ}_{2,3}\right)$. Der Ertragssteigerungfaktor wird ermittelt, indem $\mathrm{dGZ}_{3}$ durch $\mathrm{dGZ}_{2}$ dividiert wird. Diese Vorgehensweise wird für die Ermittlung der Ertragssteigerungsfaktoren weiterer Rotationen wiederholt. Wie in Kapitel 3.7 erläutert, wurde der Ertragssteigerungsfaktor $\mathrm{dGZ}_{1,2}$ aus der Studie von HoRN et al. (2013) verwendet.

Beispielberechnung Ertragssteigerungsfaktor

$E S F d G Z_{2,3}=\frac{d G Z_{3}}{d G Z_{2}}$

(Formel 4-10)

Mit:

$\begin{array}{ll}\text { ESF } d G Z_{2,3}: & \text { Ertragssteigerungsfaktor vom } d G Z_{2} \text { auf } d G Z_{3} \\ d G Z_{2}: & \text { dGZ der zweiten Rotation }\left[\mathrm{t}_{\text {(atro) }} \mathrm{ha}^{-1} \mathrm{a}^{-1}\right] \\ d G Z_{3}: & \text { dGZ der dritten Rotation }\left[\mathrm{t}_{(\text {(atro) }} \mathrm{ha}^{-1} \mathrm{a}^{-1}\right]\end{array}$


Abbildung 4-42 und Tabelle 4-47 zeigen die ermittelten Ertragssteigerungsfaktoren bei den Bodenarten $\mathrm{S}, \mathrm{U}$ und $\mathrm{L}$ für die dritte, vierte, fünfte und sechste Rotation $\left(\mathrm{dGZ}_{2,3}, \mathrm{dGZ}_{2,4}, \mathrm{dGZ}_{2,5}, \mathrm{dGZ}_{2,6}\right)$ für die Klongruppe Max.

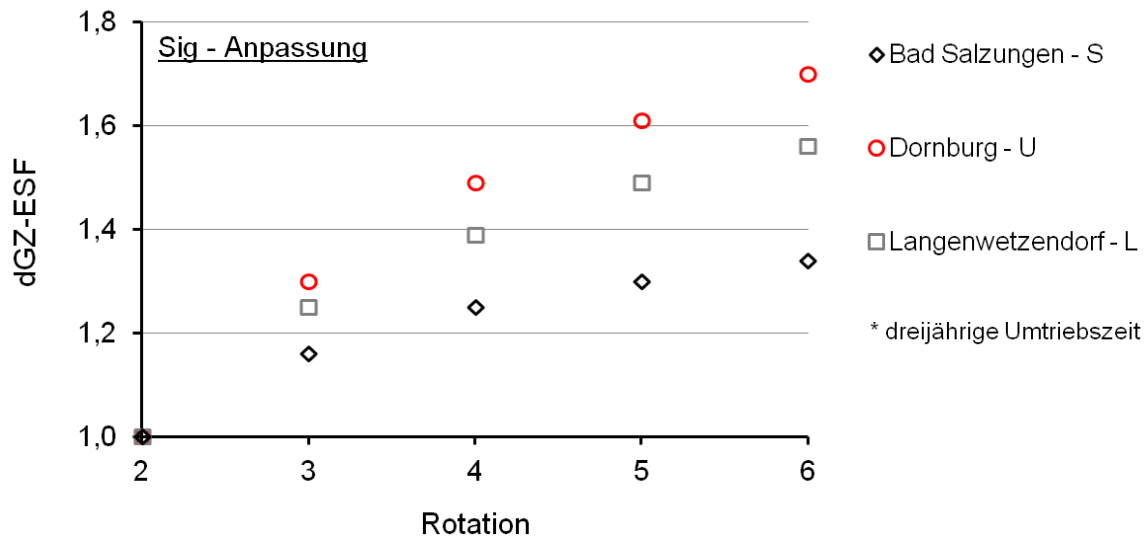

Abb. 4-42: Standortspezifische Ertragssteigerungsfaktoren auf Basis der Referenz-dGZ der zweiten Rotation.

Tab. 4-47: Standortspezifische Ertragssteigerungsfaktoren auf Basis der Referenz-dGZ der zweiten Rotation für die Klongruppe Max.

\begin{tabular}{lcccc}
\hline \multirow{2}{*}{$\begin{array}{l}\text { Standort / } \\
\text { Hauptbodenart }\end{array}$} & \multicolumn{4}{c}{ Ertragssteigerungsfaktor } \\
\cline { 2 - 5 } & $\mathrm{dGZ}_{2,3}$ & $\mathrm{dGZ}_{2,4}$ & $\mathrm{dGZ}_{2,5}$ & $\mathrm{dGZ}_{2,6}$ \\
\hline Bad Salzungen / S & 1,16 & 1,25 & 1,30 & 1,34 \\
Dornburg / U & 1,30 & 1,49 & 1,61 & 1,70 \\
Langenwetzendorf / L & 1,25 & 1,39 & 1,49 & 1,56 \\
\hline
\end{tabular}

Die Herleitung der dGZ der sechs Rotationen für den Klon Max 1 gestaltet sich wie folgt: zunächst werden die dGZ der ersten Rotation mit den in Kapitel 4.3 präsentierten standortbasierten Ertragsmodellen geschätzt. Danach wird der generalisierte Ertragssteigerungsfaktor $\mathrm{dGZ}_{1,2}$ von HoRN et al. (2013) auf die modellierten $\mathrm{dGZ}_{1}$ angewandt. Mit den dann modellierten $\mathrm{dGZ}_{2}$ werden anschließend unter Nutzung der im vorliegenden Kapitel ermittelten standortspezifischen Ertragssteigerungsfaktoren $\mathrm{dGZ}_{3}$ bis $\mathrm{dGZ}_{6}$ errechnet. Abbildung 4-43 zeigt die Ertragssteigerung differenziert nach Bodencluster für den Klon Max 1. Dabei ist zu ergänzen, dass die Residuen des Max 1-Modells im Standortcluster S nicht normalverteilt sind und daher seine Anwendung begrenzt ist.

Die Ertragssteigerung von $\mathrm{dGZ}_{1}$ auf $\mathrm{dGZ}_{2}$ ist am stärksten. Nach den ermittelten Modellen haben die Lehme das geringste Ertragssteigerungspotential. Am stärksten ist die Ertragsentwicklung bei den Schluffböden. Bei Betrachtung der halbtransparenten Linie, welche den mittleren dGZ der sechs Rotationen darstellt, wird ersichtlich, dass die oft angenommenen 10,0 bzw. 12,0 $\mathrm{t}_{\text {(atro) }} \mathrm{ha}^{-1} \mathrm{a}^{-1}$ nur auf ertragsstarken Standorten innerhalb der Standortcluster zu erreichen sind. Über die gesamte Schätzdauer von sechs Rotationen liegen die maximalen dGZ im Standortcluster S bei ca. 12,5, im Standortcluster $U$ bei 16,5 sowie im Standortcluster L bei ca. 13,0 $\mathrm{t}_{\text {(atro) }} \mathrm{ha}^{-1} \mathrm{a}^{-1}$.

In der Berechnung der Ertragssteigerung in Folgerotationen sollte beachtet werden, dass die Auswirkung der Umweltfaktoren in einer längeren Beobachtungszeit (bei fortschreitender Rotationsanzahl) einen anderen Kurvenverlauf als den in dieser Arbeit ermittelten hervorrufen können. 


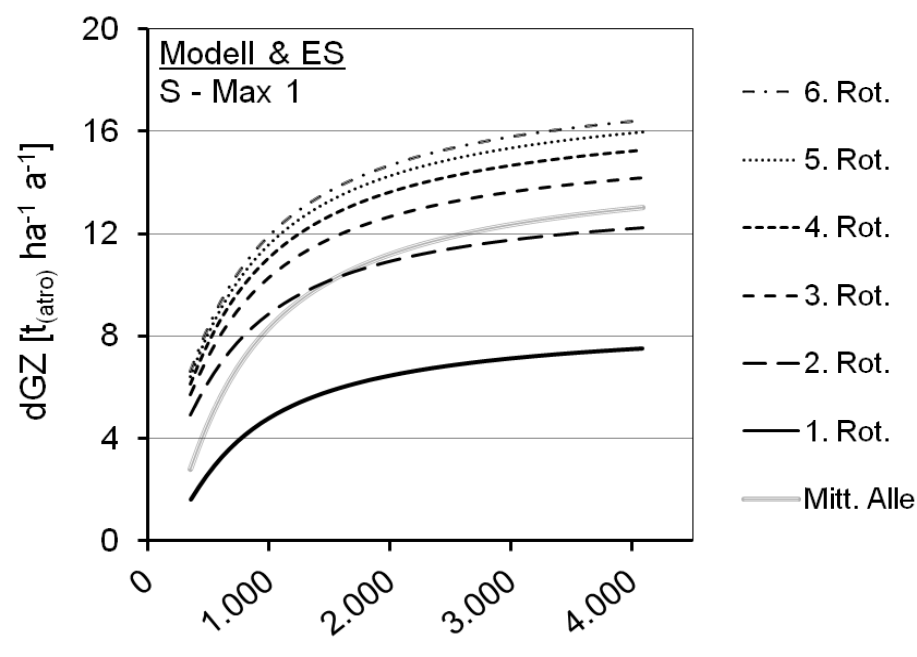

a)

$$
\left(\mathrm{U}_{[\%]}{ }^{*} \mathrm{~N}_{6,7}\right)
$$

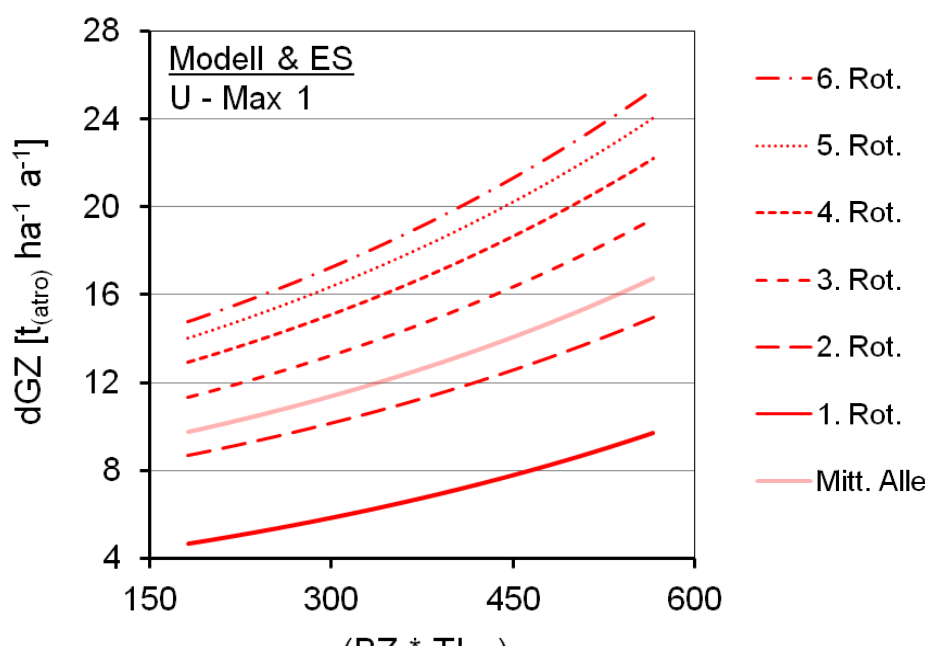

b)

$\left(\mathrm{BZ} * \mathrm{TI}_{4,5}\right)$

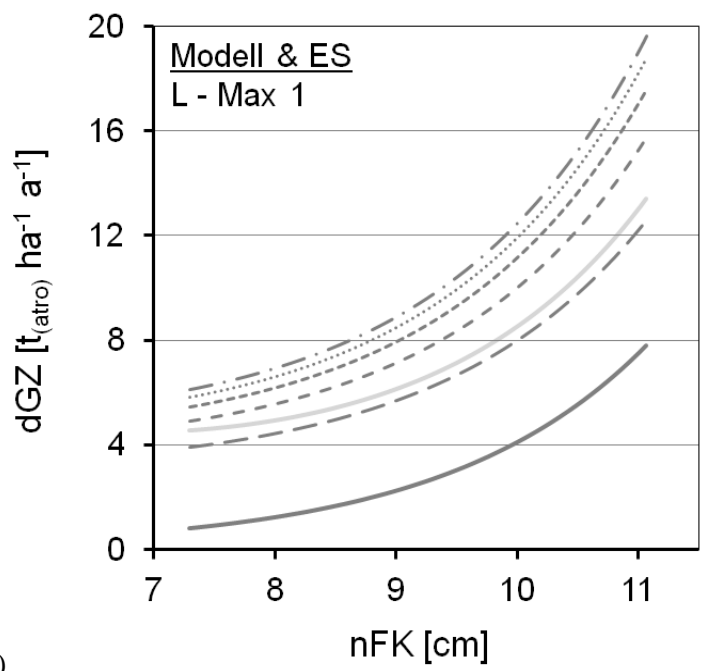

$-\cdot-6$. Rot.

5. Rot

----- 4. Rot.

- - - 3. Rot.

--2 . Rot.

-1. Rot.

Mitt. Alle

c)

Abb. 4-43: Standortbasierte modellierte dGZ der ersten Rotation sowie mittels Faktoren ermittelte Ertragssteigerungen von fünf Folgerotationen für den Klon Max 1 in den Standortclustern a) S b) U und c) L (Die halbtransparente Linie „Mitt. Alle“ stellt den dGZ der gesamten Schätzdauer von sechs Rotationen á drei Jahre, insgesamt 18 Jahre, dar). Mit ES - Ertragssteigerung. 


\section{Diskussion}

Im Folgenden werden Material und Methodik, waldwachstumskundliche Ergebnisse, leistungsbeeinflussenden Standorteigenschaften, standortbasierte Modellierung und Ertragssteigerungsfaktoren diskutiert.

Zu erwähnen ist, dass für die Diskussion in der Literatur keine Versuchsreihe mit vergleichbarem Umfang, Material und Methodik wie jene des Verbundvorhabens PROLOC vorhanden ist. Vielmehr sind die bereits publizierten Veröffentlichungen zu KUP in ihren Anbaubedingungen sehr heterogen und schwer auf die Rahmenbedingungen der vorliegenden Arbeit übertragbar. Dies betrifft die Baumarten, die Klone, die Rotationsdauer, die Bestandesdichte und die Standorte.

Selten sind direkte Vergleiche zwischen Pappel und Weide zu finden. Viele Studien fokussieren sich nur auf eine Gattung. Es wurde festgestellt, dass in den Veröffentlichungen die Standortbedingungen oft nur unzureichend dargestellt sind. Üblicherweise ist nur die Hauptbodenart angegeben.

Je nach vorhandener Literatur wurde die Diskussion der kausalen Bereiche dieser Arbeit (siehe 5.3 und 5.4) auf der Ebene der Klone, der Gattung oder des allgemeinen Waldwachstums vorgenommen. Insbesondere zum Einfluss von Bodeneigenschaften auf das Wachstum gibt es kaum pappel- oder weidenspezifische Untersuchungen.

\subsection{Material und Methodik}

Im Folgenden werden die Vor- und Nachteile des verwendeten Materials, der angewandten Methodik sowie die methodischen Unsicherheiten diskutiert.

\subsubsection{Versuchsflächen}

Der größte Vorzug der vorliegenden Arbeit ist ohne Frage die hohe Gesamtanzahl an untersuchten Versuchsflächen. Diese erlauben die Erarbeitung fundierter Ergebnisse.

Aufgrund des Versuchsdesigns und der Managementmaßnahmen bergen die im Projekt erhobenen Daten einige Unsicherheiten, deren Wirkung nur schwer einzuschätzen ist. Hierzu zählt u. a. die Bewässerung einiger Versuchsflächen im Frühjahr des Anlagejahrs in Folge starker Trockenheit. Mithilfe von dosierten Wassermengen wurden viele Versuchsflächen vor einem Komplettausfall bewahrt (siehe 3.1 und 3.2). Der Einfluss dieses Eingriffs ist leider in dieser Arbeit nicht quantifizierbar. Zwar kann angenommen werden, dass sich der Bewässerungseffekt der Flächen nach drei Vegetationsperioden abgemildert hat, er bleibt aber auf jeden Fall ein kritischer Faktor für die Auswertung. Diese Beobachtungen verdeutlichen die große Bedeutung der Wasserversorgung im Anlagejahr, insbesondere in der Zeit im Anschluss zur Pflanzung. Weitere Autoren wie zum Beispiel SCHILDBACH et al. (2008) und STOLL (2011), bestätigen dies.

Ein weiterer Unsicherheitsfaktor stellt die Heterogenität der Vornutzungen bei der Flächenauswahl dar. Nach einer Prüfung der Vergleichbarkeit der Vornutzungen musste von der Vornutzung der Bergbaufolgelandschaften für die Modellierung abgesehen werden (siehe 3.6). Wie im Kapitel 4.2.8.1 erläutert, hatte die Vornutzung einen signifikanten Effekt auf den dGZ. Für das Erreichen der Projektziele wäre eine einheitliche Vornutzung von großem Vorteil gewesen. Weitere Ausführungen zur Bedeutung der Vornutzung können in Kapitel 3.6 gefunden werden.

Die inhomogen durchgeführten Flächenbehandlungen in Form der Bodenbearbeitung sowie der Unkrautbekämpfung zählen ebenfalls zu den Unsicherheitsfaktoren (siehe 3.1.1). Es gab Standorte, welche aufgrund einer ausbleibenden Unkrautbekämpfung nicht ausgewertet werden konnten, wie 
zum Beispiel Sto. Nr. 7 „Kaisheim“. Andere Standorte mit erhöhtem Unkrautdruck, wie zum Beispiel Sto. Nr. 20 „Borlinghausen“, hätten trotz Vorlaufmittelapplikation vermutlich einen anderen Wuchsverlauf gezeigt, wenn dort nachträglich eine mechanische oder chemische Unkrautbekämpfung durchgeführt worden wäre.

Ein weiterer Aspekt, welcher oft in der Literatur als Unsicherheit von experimentellen Versuchsflächen angesehen wird, ist die Größe der Versuchsfläche bzw. der Parzelle. CANNEL und SMITH (1980), HANSEN (1991) und Mitchell (1999) gehen davon aus, dass in kleinen Versuchsflächen höhere Erträge als in größeren erzielt werden. Folgende Gründe werden dafür angegeben:

- hoher Einfluss von wuchssteigernden Randeffekten (Licht),

- häufige Auswahl von Standorten mit hoher Wuchskraft in Versuchsflächen und damit höhere Wahrscheinlichkeit einer positiven Klon-Standort-Wechselwirkung,

- bessere und intensivere Pflege von kleinen gegenüber großen Versuchsflächen.

Randeffekte wurden durch die Definition einer Kernparzelle vermieden (siehe 3.1). Beobachtungen der untersuchten Versuchsflächen zeigten keine nennenswerte Variation im Wachstum zwischen Parzellen aus dem Zentrum der Versuchsflächen gegenüber Parzellen, die am Rand liegen. Für die untersuchten Flächen scheint daher der Einfluss der wuchssteigernden Randeffekte nicht zuzutreffen. In Bezug auf b) kann folgendes erwähnt werden: Im Verbundvorhaben PROLOC wurden Standorte verschiedener Wuchskraft von sehr niedrig (zum Beispiel Sto. Nr. 12 „Kummerow“) bis sehr hoch (zum Beispiel Sto. Nr. 28 „Bernburg“) ausgewählt. Die Pflege der Versuchsflächen begrenzte sich auf das erste Jahr und war daher mit der praxisüblichen Vorgehensweise vergleichbar.

Die Anlage und Betreuung von Versuchsflächen ist kosten- und zeitintensiv. Daher müssen Kosten und Nutzen bei der Wahl der Flächengröße genau erwogen werden. Für standortkundliche Untersuchungen bieten kleine Versuchsflächen den entscheidenden Vorteil einer geringen Variabilität in Bezug auf die Bodenparameter.

\subsubsection{Modellierung und Variablenbildung}

Die Daten, welche in der Bildung von Modellvariablen verwendet wurden, sind mit Unsicherheiten behaftet. Im Bereich Klima kann die Entfernung der Klimastation zum Standort, insbesondere bei den räumlich sehr variablen Niederschlägen (O’LOUGHLIN et al. 1996, WEILGUNI 2006), als ein Unsicherheitsfaktor angesehen werden. Die Installation einer Klimastation auf jeder Versuchsfläche war aus Kostengründen nicht möglich.

Eine weitere Unsicherheitsquelle betraf die Bestimmung der Porenraumvariablen. Die Herleitung von nFK und LK stützte sich auf indirekte Methoden (siehe 3.3.3.1). Diese sind ökonomisch sinnvoll, aber deren Genauigkeit ist für Modellierungszwecke nicht optimal (FINKE et al. 1996). Aus Kostengründen konnten keine genaueren Messungen vorgenommen werden.

Die Nutzung von Kennwerten der Bodenschätzung für die Modellierung birgt auch einige Unsicherheiten. Eine Grenze ist ihre niedrige Auflösung bzw. hohe Aggregierung. Die Aufnahmen sind qualitativer Natur und bauen aufeinander auf (siehe 2.5.3.6). Eine entscheidende Größe bei der Bodenschätzung ist die Bestimmung der Bodenart. Diese erfolgt mittels Fingerprobe. Diese Bestimmung erlaubt keine hohe Genauigkeit und darf keinesfalls mit Laboruntersuchungen verglichen werden. Eine weitere Unsicherheit der Bodenschätzung ist die Reproduzierbarkeit, welche nicht als $100 \%$-ig angesehen werden kann. Sie wird zwar nach einer festgelegten Methodik ermittelt, wird aber durch eine Person durchgeführt und ist, wie oben bereits erwähnt, qualitativer Natur. Schließlich stellt das Alter der Schätzungen eine weitere Unsicherheit dar. Die ersten Bodenschätzungen stammen aus den 1930er Jahren, wobei die Mehrheit der Schätzungen ca. 70 Jahre alt ist (RUST 2006). Sie können daher keine 
Auskunft über Verdichtungsschäden geben, welche im Zuge der Mechanisierung der Landwirtschaft der letzten Jahrzehnte entstanden sind.

Eine weitere Unsicherheit stellten nach der Standortclusterung bei den bodenartspezifischen Modellen die geringen Besetzungszahlen der Cluster dar. Eine höhere Wiederholungsanzahl hätte die signifikante Berücksichtigung weiterer Faktoren oder überhaupt die Signifikanz von Modellen erst ermöglicht, wie im Standortcluster der Schluffe. Die Robustheit der Modelle wäre ebenfalls stärker, wenn sie sich auf mehr Daten stützen würde.

Eine Validierung der Modelle mit Hilfe unabhängiger Datensätze konnte nicht durchgeführt werden. Die Validierung war anhand von Daten der eigenen Versuchsflächen vorgesehen. Aufgrund des Ausbleibens vieler Versuchsflächen wurden jedoch alle zur Verfügung stehenden Daten für die Entwicklung von Modellen verwendet. Die Diskussion der leistungsbeeinflussenden Standorteigenschaften und der Modelle fokussiert sich daher auf die physikalisch-biologische Plausibilität der Ergebnisse.

Die Unsicherheiten bei der Entwicklung und Nutzung der Ertragssteigerungsfaktoren wurden bereits im Kapitel 3.7 erläutert.

\subsection{Waldwachstumskundliche Ergebnisse}

Im Folgenden wird auf die Diskussion der waldwachstumskundlichen Ergebnisse Anwuchs- und Überlebensrate, Dimension- und dGZ-Entwicklung sowie auf die Biomassefunktionen eingegangen.

\subsubsection{Anwuchs- und Überlebensrate}

Im Etablierungsjahr 2008 sind teilweise sehr niedrige Anwuchsraten aufgenommen worden. Dies hing vermutlich mit den unterdurchschnittlichen Niederschlägen in den Monaten nach der Pflanzung sowie mit den relativ späten Pflanzzeitpunkten der Versuchsflächen zusammen. TRNKA et al. (2008) beobachteten ein ähnliches Phänomen in der Tschechischen Republik. Plantagen, welche im Trockenjahr 2003 gepflanzt wurden, hatten massive Ausfälle zu verzeichnen. Viele Flächen mussten komplett neu begründet werden. Die Trockenheit im Anschluss zur Pflanzung erschwert das Gedeihen der Stecklinge, die eine langsamere Wuchsphase aufweisen als krautige Pflanzen (SCHILDBACH 2008, STOLL 2011). Aus diesem Grund werden viele Pappelplantagen, insbesondere in den USA und in Italien, in den ersten Jahren nach der Pflanzung bewässert (COYLE et al. 2005, ZALESNY et al. 2009).

Verglichen mit den Pappelklonen zeigten die Weidenklone höhere Anwuchsraten. Die Ergebnisse dieser Arbeit decken sich mit denen aus der Literatur, die über exzellente Anwuchsraten und über die Regenerationsfähigkeit der Weiden berichteten (HOFMANN 1995, VANDE WALLE et al. 1997, BOELCKE 2006). Die unter den Pappeln beobachtete hohe Überlebensrate des Klons Max 1 deckt sich mit den Ergebnissen von FRIEDRICH (1999), HOFMANN (1999), BOELCKE (2006), und BIERTÜMPFEL (2008). Die sehr niedrige Anwuchsrate von H 275 kann mit Erkenntnissen aus der Literatur nicht bestätigt werden. Dort werden zwar niedrigere Anwuchsraten von Balsampappelhybriden im Vergleich mit den intersektionellen Max-Klonen berichtet, jedoch wurde keine auffällige Minderleistung von $\mathrm{H}$ 275 festgestellt (FrIEDRICH 1999, HOFMANN 1999, BOELCKE 2006 und BIERTÜMPFEL 2008). Die Probleme mit H 275 hingen vermutlich mit Qualitätsmängel im Pflanzgut zusammen. Dies wurde am Sto. Nr. 19 „Werlte“ sichtbar, der trotz Bewässerung H 275 nicht anwuchs. Die höhere Mortalität von AF 2 auf dem im Harz gelegenen Sto. Nr. 27 „Bärenrode“ deutet auf eine Kälteempfindlichkeit hin. Die Phänologie der AF 2 könnte Aufschluss für die Beobachtung geben. AF 2 hat einen sehr späten Austrieb, was auf eine generelle Kälte- sowie Spätfrostempfindlichkeit hindeutet (SCHIRMER 2011). Die Kälteempfindlichkeit von Schwarzpappelklonen ist aus der Literatur bekannt (WEISGERBER 1980). 
Bei den Anwuchs- und Überlebensraten wurde ein standortabhängiges Verhalten beobachtet. Die Ergebnisse einer Regressionsanalyse zeigten bei den Sandböden einen Zusammenhang zwischen geringen Anwuchsraten und geringen Niederschlägen. Dies kann mit der niedrigen $\mathrm{nFK}$ und der hohen Leitfähigkeit der Sandböden (AG BODEN 2005, RENGER et al. 2008) erklärt werden. Wenn der Boden nicht genügend Wasser speichert, muss alternativ die Wasserversorgung in Form ausreichender Niederschläge erfolgen. Bei Schluff-, Lehm- und Tonböden war ein positiver Zusammenhang zwischen nFK und Anwuchsrate vorhanden. Da die nFK das pflanzenverfügbare Wasser darstellt (LÖSCH 2001), ist dies ein logischer Zusammenhang. Aufgrund seines niedrigen $\mathrm{R}^{2}$ kann dieser eher nur als Tendenz interpretiert werden.

\subsubsection{Anzahl an Höhentrieben}

Obwohl diese Studie in der ersten Rotation einer KUP durchgeführt wurde, das heißt ohne Förderung des Stockausschlags durch einen Erntevorgang, gab es bei der Anzahl an Höhentrieben signifikante Unterschiede zwischen den Klonen. Die höhere Anzahl an Höhentrieben von Weide gegenüber Pappel deckt sich mit den Beobachtungen u. a. von BOHNENS und FRIEDRICH (1990), HOFMANN (1999) sowie RÖHRICHT und KIESEWALTER (2008) auf deutschen Versuchsflächen. Unter den Pappeln hatten Max 1 und AF 2 durchschnittlich mehr Höhentriebe als H 275. Dies wurde in der Literatur nicht beobachtet (HOFMANN 1999, RÖHRICHT und KIESEWALTER 2008) und kann daher in der vorliegenden Studie eventuell auf die verminderte Qualität des Pflanzguts von H 275 zurückzuführen sein.

\subsubsection{Höhe}

Die ermittelten Höhen befinden sich im Rahmen der bekannten Werte aus der Literatur. Im Vergleich der Höhenentwicklung zwischen Weiden- und Pappelklonen wurden in der Literatur einerseits stärkere Leistungen der Weide (HOFMANN 1999, RÖHRICHT 2008), andererseits auch schwächere Höhenleistungen von Weidenklonen registriert (FRIEDRICH 1999, SCHOLZ 1999). Der Grund für die unterschiedlichen Beobachtungen kann in den geprüften Klonen gesucht werden. Während FRIEDRICH (1999) und SCHOLZ (2008) Versuchsklone, deren Wachstumseigenschaften nicht bekannt waren, prüften, untersuchten HOFMANN (1999) und RÖHRICHT (2008) etablierte Klone wie Zieverich und weitere wuchsstarke schwedische Klone. Des Weiteren zeigte HofMANN (1999) in der Versuchsfläche „Wildeshausen“ bei der Gegenüberstellung des Weidenklons Zieverich mit den wüchsigen Pappelklonen Rap und 7/83 eine ähnliche Beobachtung der Wuchsdynamik wie in der vorliegenden Arbeit. Und zwar war die Höhe Zieverichs in den ersten zwei Wuchsperioden den Pappeln überlegen, in der dritten Wuchsperiode wurde, anders als bei den Ergebnissen dieser Arbeit, der Weidenklon von den Pappelklonen überholt.

Dass AF 2 zu den wuchsstärksten Schwarzpappelklonen gehört, wurde bereits von MAYER (2008) gezeigt. Verglichen zu anderen Schwarzpappelklonen hat AF 2 eine überlegene Wuchsleistung, wie bereits auch FILAT et al. (2010) in einer Untersuchung in Rumänien aufzeigten. Höhenvergleiche zwischen AF 2 und den Klonen H 275 und Max 1 konnten in der Literatur nicht gefunden werden. Hingegen wurde in der Literatur häufig erwähnt, dass H 275 ein schwächeres Höhenwachstum als Max 1 oder andere Max-Klone aufweist (BOHNENS und FRIEDRICH 1995, RÖHRICHT und KIESEWALTER 2008, BURGER 2011). Einzig BUnGART und HÜTTL (2004) konnten eine höhere Höhenleistung von H 275 gegenüber den Max-Klonen nachweisen.

Warum Tordis der stärkere Weidenklon war, lässt sich aus den Ergebnissen der vorliegenden Arbeit nicht herleiten. Die Gründe für die Überlegenheit eines Klons können mit einigen physiologischen Ursachen zusammenhängen, zum Beispiel mit einer besseren Durchwurzelungsfähigkeit (CURLE und TRUELOVE 1986, STIMM et al. 2013) oder mit einer erhöhten Umwandlungseffizienz der photosynthetisch aktiven Strahlung (AMICHEV et al. 2010). Eine höhere Entwicklung der Blattfläche kann auch zu 
einer erhöhten Biomassebildung führen (FANG et al. 1999). Solche physiologischen Untersuchungen sind selten und für die in der vorliegenden Arbeit verwendeten Klone nicht zu finden.

Die Moorböden hatten sehr hohe Wuchsleistungen, welche mit den stärksten der anderen Bodenartcluster vergleichbar waren. Dies kann vor allem mit seiner guten Durchwurzelbarkeit sowie einem i. d. R. sehr hohen Wasserangebot erklärt werden (AG BODEN 2005, MÜLLER 1951, WITTICH 1951). Die guten Standorteigenschaften der Moore für den Pappel- und Weidenanbau wurden bereits in Kapitel 2.5.3.6 ausführlich erläutert.

Die Dynamik der Dimensionsentwicklung bzw. der Massenleistung in den verschiedenen Bodenartclustern wird nicht an dieser Stelle, sondern im Kapitel 5.2.5 diskutiert.

\subsubsection{Durchmesser}

Die in Kapitel 4.1.5 vorgestellte Differenzierung des Durchmesserwachstums zwischen Pappel und Weide stimmt mit den Beobachtungen von FRIEDRICH (1999), HOFMANN (1999), RÖHRICHT und KIESEWALTER (2008) überein. Ob die Weidenklone in der ersten Wuchsperiode vergleichbare Durchmesser wie die Pappelklone aufwiesen, war klonspezifisch. Im Durchmesserwachstum konnten in den ersten Wuchsperioden einzig starke Weidenklone mit den Pappeln mithalten.

Das stärkste Durchmesserwachstum unter den Pappeln wies AF 2 auf, gefolgt von Max 1 und H 275. In der einzig vorhandenen Quelle von MAYER (2008) aus Österreich zeigt die AF 2 nach zwei Jahren mindestens $20 \%$ stärkere Durchmesser als geprüfte Max-Klone. In deutschen Versuchsflächen wurden in der ersten Umtriebszeit häufig höhere Dimensionen von AF 2 gegenüber anderen Pappelklonen beobachtet (SCHLEPPHORST 2013). Die Überlegenheit der Max Klone gegenüber H 275 wurde, wie im Kapitel 5.2.3 bereits erwähnt, mehrfach beobachtet und zwar auch für die Durchmesserleistung.

Das jährliche absolute Durchmesserwachstum der Pappel war stärker als das der Weide. Wie bereits beobachtet, waren die absoluten Durchmesser der Weide niedriger als die der Pappel. Daher sind die jährlichen Durchmesserzuwächse automatisch niedriger.

\subsubsection{Durchschnittlicher Gesamtzuwachs}

Der dGZ hatte eine klonabhängige Entwicklung für jede Wuchsperiode. In der ersten und zweiten Wuchsperiode dominierten die Weidenklone. Dies deutet auf eine sehr schnelle Erschließung des Standortes durch die Weide in der Etablierungsphase hin. Eventuell führen die Weiden eine schnellere Durchwurzelung des Bodens als die Pappel durch. Belegt ist, dass Weiden deutlich mehr Fein- und Grobwurzelbiomasse im Oberboden bilden (MAKESCHIN und REHFUESS 1995). Die Annahme, dass die Wurzelbildung von Weiden schneller vonstatten geht als jene der Pappel, erscheint daher plausibel. Im Anwuchsjahr sowie in der zweiten Wuchsperiode investierten die Pappeln sehr viel Energie in die Bildung von Laub (HEYN und WACHENDORF 2012). Die Weide hingegen entwickelt eine geringere Blattfläche im Vergleich zur Pappel (KoOP et al. 2005). Daraus lässt sich (unabhängig von der Stammzahl) ableiten, dass die Weide eine höhere Assimilatenbildung je Einheit Blattfläche aufweist. Außerdem ist denkbar, dass sie die Allokation von Assimilaten stärker in den Stamm richtet. Leider ist mit der verfügbaren Literatur keine Differenzierung möglich. Die Weidenklone wiesen trotz signifikant schwächerer Durchmesserentwicklung gegenüber der Pappel zum Teil höhere dGZ auf. Dies ist durch eine höhere Anzahl an Höhentrieben, ein höheres Höhen/Durchmesserverhältnis sowie eine höhere Holzdichte begründet (siehe Anhang 2).

In der letzten der drei Wuchsperioden gab es keine Überlegenheit einer Gattung. Die stärksten Klone waren über alle Standorte hinweg AF 2 und Tordis. MAYER (2008) beobachtete in Österreich sehr hohe Erträge für AF 2, die über denen der Max Klone lagen. Bei den Weiden finden sich widersprüchliche Aussagen in der Literatur. Einerseits fand VALENTINE (2007) für irische Standortbedin- 
gungen die Überlegenheit von Tordis gegenüber Inger. SEVEL et al. (2012) zeigten jedoch in einer dänischen Untersuchung, dass Inger geringfügig ertragsstärker als Tordis war. In der vorliegenden Studie kann aufgrund der hohen Anzahl der berücksichtigten Standorte die Überlegenheit Tordis gegenüber Inger in der ersten dreijährigen Rotation als gesichert angesehen werden. Weitere veröffentlichte Klonvergleiche zwischen Inger und Tordis oder gar zwischen Inger, Tordis, Max 1, H 275 und AF 2 sind dem Autor nicht bekannt.

Einige Standorte zeigten in der ersten Wuchsperiode sehr niedrige dGZ, teilweise kleiner als $0,1 t_{(a t r o)} h^{-1} a^{-1}$. Davon entwickelten sich einige in der zweiten bzw. dritten Wuchsperiode sehr gut, wie zum Beispiel der Sto. Nr. 20 „Borlinghausen“. Dieser Verlauf war, wie bereits erwähnt, bei schwereren Bodenarten gegeben. Andererseits gab es Standorte, welche durchgängig $0,1 \mathrm{t}_{\text {(atro) }} \mathrm{ha}^{-1} \mathrm{a}^{-1}$ nicht überschritten. Dies waren extrem schwierige Standorte, in welchen mehrere problematische Standortbedingungen gleichzeitig auftraten (siehe 2.5.3). Beispielsweise zeigte der Sto. Nr. 30 „Iden“ neben einem reinen Sandsubstrat (Ss) mit der damit gekoppelten sehr niedrigen nFK einen pH-Wert kleiner 4,0. Der Sto. Nr. 38 „Heiliges Marpingen“ hatte ebenfalls einen stark sauren pH-Wert. Darüber hinaus war der Boden flachgrundig und mit seiner Lage an einem Südhang der Wasserhaushalt zusätzlich von höheren Evapotranspirationsraten belastet. Die Bergbaufolgelandschaften am Tagebau Welzow (Sto. Nr. 8 „Energiewald Welzow“ und Sto. Nr. 14 „Welzow Süd“) zeigten ebenfalls äußerst niedrige dGZ. Diese sind mit dem groben Bodensubstrat und der damit einhergehenden niedrigen $\mathrm{nFK}$ sowie dem nicht ausgereiften Stoffhaushalt (KNOCHE 2003) zu begründen. Die Maxima wurden für alle Klone zum größten Teil auf den Lössstandorten registriert (zum Beispiel Sto. Nr. 28 „Bernburg“). Dies deckt sich mit den Ausführungen in Kapitel 2.5.3.6.

Interessanterweise waren die Maxima aller drei Standortcluster ähnlich. Die Maxima der Sand- und Lehmcluster wurden jeweils bei den Böden mit den höchsten Schluffgehalten registriert, was erstmals verdeutlicht, wie wichtig der Schluffgehalt für das Wachstum ist (siehe 3.5.1.3). Andererseits müssen bei den Sand- und Lehmböden weiteren wuchssteigernden Eigenschaften vorhanden sein, um die Leistung von Schluffböden zu erreichen. Vermutlich spielt bei den Sanden die höhere Luftverfügbarkeit und Durchwurzelbarkeit des Bodens ebenfalls eine wuchssteigernde Rolle (siehe 3.5.1.3 sowie AG BODEN 2005, RENGER et al. 2008). Bei den Lehmen könnte zum Beispiel die lange Verweilzeit des Bodenwassers im Zuge einer niedrigen gesättigten sowie ungesättigten Leitfähigkeit (AG BODEN 2005, RENGER et al. 2008) bei gleichzeitig akzeptablen Werten der nFK einen Vorteil darstellen.

Die Leistung der Klone in der dritten Wuchsperiode wurde getrennt nach Hauptbodenart analysiert. Auf den Sandstandorten gab es keinen Einfluss des Klons auf den Zuwachs, das heißt, alle Klone lagen auf demselben Ertragsniveau. Auf den Sandstandorten „Bad Salzungen“ (BIERTÜMPFEL et al. 2008) und „Gülzow“ (GURGEL 2011) wurden in der ersten Rotation keine größeren Abweichungen zwischen den stärksten Klonen registriert. Obwohl keine Varianzanalyse durchgeführt wurde, spricht die geringe Abweichung für das hier gezeigte Ergebnis.

Bei den Schluffböden wurde der allgemeine Trend, AF 2 und Tordis als stärkste Klone, bestätigt. Daraus kann man folgern, dass Tordis und AF 2 unter besten Standortbedingungen eine höhere Ausnutzung der Umweltressourcen als die restlichen Klone aufweisen. Das bedeutet, dass jene über das größte Ertragspotential unter den Klonen verfügen. Bei AF 2 könnte die Überlegenheit durch die größere räumliche ober- sowie unterirdische Ausbreitung, sichtbar in durchweg signifikant größeren Dimensionen (siehe 4.1.7.4), erklärt werden. Durch signifikant größere Dimensionen ist eine stärkere Ausdehnung des Wurzelsystems anzunehmen. Auf diese Weise können die Umweltressourcen in gröBerem Umfang zur Biomassebildung verwendet werden.

Bei den Lehmen zeigte AF 2 die signifikant besten Leistungen. Dies könnte mit dem Ausdehnungsgebiet der Schwarzpappel zusammenhängen. Sowohl $P$. nigra als auch $P$. deltoides kommen aus dem Bereich der Auen (siehe 2.3.1). In Auenbereichen werden als Substrat häufig Auenlehme gefunden. Es 
ist daher anzunehmen, dass AF 2 eine höhere Anpassung an schwere Böden aufweist. Die oben erwähnte signifikant stärkere Dimensionsentwicklung kommt AF 2 bei diesen schwer erschließbaren Bodenarten ebenfalls zugute.

Während Höhen und Durchmesser durchschnittlich linear und mit geringer Steigung zunahmen, nahmen die dGZ deutlich steiler und teilweise nicht-linear zu. Höhen- und Durchmesserzuwächse erwecken den Eindruck einer Gleichmäßigkeit im Wachstum. Das absolute Wachstum wird jedoch im dGZ ausgedrückt. Aus diesem Grund wird die Wuchsdynamik je Klon und Standortcluster anhand des dGZ diskutiert. Die dGZ-Steigerung hat sich, gattungsunabhängig, von der ersten auf die zweite Wuchsperiode im Mittel verdreifacht. Dies kann wie folgt begründet werden: das Wachstum in der ersten Wuchsperiode ist aus vielfachen Gründen begrenzt. Einer der Gründe ist, dass in der ersten Wuchsperiode alle Organe, Wurzeln, Laub und Stamm erst gebildet werden müssen. Die Nutzung der Umweltressourcen zur Biomassebildung fängt deshalb deutlich verlangsamt an. Bedingt durch diese langsame Entwicklung ist die effektive Länge der ersten Vegetationsperiode kürzer als die der Folgenden. In der zweiten Wuchsperiode kann der Baum auf vorhandene Organe zurückgreifen, daher ist das Wachstum bzw. die Allokation von Energie optimiert. Das heißt, in dieser Phase sind die Bäume etabliert und gehen stark in die Biomassebildung. Aus dem großen Unterschied zwischen den dGZ aus der ersten und zweiten Wuchsperiode entsteht die große Steigerungsrate.

Interessanterweise differenziert sich in der dritten Wuchsperiode die dGZ-Entwicklung zwischen den Gattungen und Standortclustern. Während sich bei der Pappel der dGZ von der zweiten auf die dritte Wuchsperiode fast verdoppelt, fällt die Steigerung bei den Weiden mit 10 bis $50 \%$ deutlich geringer aus. Die Niederschläge der dritten Vegetationsperiode waren $50 \%$ höher als in der ersten und zweiten Vegetationsperiode. Sie waren daher nicht in der zweiten, sondern in der dritten Wuchsperiode am günstigsten. Höchstwahrscheinlich ist der Wachstumsklimax der untersuchten Weiden durch den Wuchshabitus bestimmt. Es sei hier daran erinnert, dass die verwendeten Weidenarten zu den Buschweiden gehören, deren maximaler Wuchs deutlich früher begrenzt ist als der der Baumweiden (ARGUS 1986). Die geringste Zunahme im dGZ bei beiden Gattungen wurde bei den stärksten Standorten, den Schluffböden, beobachtet.

Bei den Schluffen lag die stärkste Ertragssteigerung zwischen der ersten und zweiten Wuchsperiode, der Verlauf war eher asymptotisch. Die Ertragssteigerung verlief bei den Lehmen am steilsten (exponentiell), bei den Sanden war sie eher gleichmäßig (linear). Schluffböden, in der Regel Lösssubstrate, bieten optimale bodenphysikalische sowie -chemische Eigenschaften. LK und $\mathrm{nFK}$ befinden sich beide in einem für das Pflanzenwachstum optimalen Bereich (KUNTZE et al. 1994). Das begünstigt eine schnelle Erschließung des Standorts. Das sich langsam einstellende Wachstum bei den Lehmböden kann physiologisch über die niedrige Durchwurzelbarkeit dieser Böden erklärt werden (AG BODEN 2005). Mit zunehmender Erschließung des Wurzelraums nimmt der Zuwachs bei den Lehmen zu. Sandstandorte zeigen eine zu Beginn starke Leistung, geprägt von einer schnellen Durchwurzelung des Bodens infolge hoher LK (SCHACK und HILDEBRAND 1987). Danach verläuft das Wachstum gleichmäßig.

\subsubsection{Biomassefunktionen}

Auf die Frage, ob $d_{0,1}$ oder $d_{1,3}$ als unabhängige Variablen bei der Bildung von Biomassefunktionen fungierten, gab es keine signifikanten Indizien. RÖHLE et al. (2006) sowie HARTMANN (2010) fanden in einer Untersuchung zur Aufstellung von Biomassefunktionen ebenfalls heraus, dass die Durchmesser $\mathrm{d}_{0,1}, \mathrm{~d}_{0,6}$ und $\mathrm{d}_{1,3}$ keinen signifikanten Einfluss in der Güte der Biomassefunktion ausmachten.

Die Funktionen der Weiden waren gegenüber jenen der Pappel erkennbar steiler. Dies war zwar nicht statistisch belegt, aber visuell klar erkennbar. Dies kann sicherlich auf die verschiedenen Wuchshabite der Gattungen zurückgeführt werden. Die Pappel weist niedrigere Höhen-Durchmesser-Verhältnisse 
auf als die Weide (siehe Anhang 14 und 15, sowie HofMANN 1999). Bei gleichem Durchmesser hat die Pappel demnach ein niedrigeres Stammvolumen als die Weide. Zudem weist die Weide eine höhere Holzdichte auf als die Pappel (Bonemmann 1980, Amthauer Gallardo und Seymour 2011). AF 2 hatte einen statistisch belegten deutlich flacheren Verlauf als die anderen beiden Pappelklone, was sehr wahrscheinlich mit der Holzdichte zusammenhängt. Diese ist bei AF 2 ca. $20 \%$ geringer als die von Max 1 und H 275 (siehe Anhang 2). Die Unterschiede zwischen der Inger- und der Tordisfunktion, welche nicht statistisch aber visuell erkennbar waren, können ebenfalls in der Holzdichte gesucht werden, welche bei Tordis ca. $15 \%$ höher liegt als bei Inger (siehe Anhang 2).

Die Beziehungen zwischen Bestandesmittelhöhe gegen $\mathrm{a}_{0}$ sowie $\mathrm{a}_{1}$ hatten $\mathrm{R}_{\text {korr }}$ von 0,46 bis 0,63 . Diese Werte sprechen für eine mäßige Ausprägung eines Höheneffektes. Ähnliches beobachtete HARTMANN (2010) in seiner Studie zur Entwicklung eines Ertragsschätzers für Pappeln. Dabei wurden Beziehungen zwischen der Bestandesmittelhöhe und $\mathrm{a}_{0}$ sowie $\mathrm{a}_{1}$ mit $\mathrm{R}^{2}$ zwischen 0,55 und 0,64 ermittelt. Der Einfluss der Bestandesdichte auf die Funktionskoeffizienten war deutlich geringer als jener der Höhe ( $\mathrm{R}^{2}$ zwischen 0,27 und 0,34).

Überhaupt ergaben sich bei den höhengestuften modellierten Funktionen sehr geringe Unterschiede in der Funktionsform. Bei den Weiden waren diese Abstufungen nicht vorhanden, was ein sehr interessantes Ergebnis darstellt. Der Grund des marginalen Einflusses der Höhe in den Biomassefunktionen liegt darin, dass wenn $\mathrm{a}_{0}$ als Multiplikator mit zunehmender Höhe abnahm, nahm der Exponent $\mathrm{a}_{1} \mathrm{zu}$. Für die untersuchte Datenbasis dieser Studie hoben sich die Effekte von $a_{0}$ und $a_{1}$ mit der Höhe zu nahezu $100 \%$ gegenseitig auf.

\subsection{Leistungsbeeinflussende Standorteigenschaften}

Im Folgenden werden die klimatischen leistungsbeeinflussenden Standorteigenschaften Temperatur, Niederschlag und Trockenheitsindex sowie die bodenkundlichen leistungsbeeinflussenden Standorteigenschaften Bodenzahlen, Textur und Porenraumkenngrößen diskutiert. Die Ergebnisse zur Korrelation zwischen unabhängigen Variablen wurde einzeln im Kapitel 4.2.7 präsentiert. In der Diskussion werden diese nicht einzeln diskutiert, sondern in die relevanten Kapitel 5.3.1 bis 5.3.6 integriert.

\subsubsection{Temperatur}

Aus den Korrelationen zwischen dGZ und Temperaturen, welche öfters nicht signifikant und nicht von allzu großer Stärke waren, kann man folgern, dass die Schwankungen in den Temperaturen weder zu großen Wuchssteigerungen noch zu markanten Wuchsdepressionen führten. Die getesteten Pappelund Weidenklone eignen sich demnach für unsere Temperaturzone gut. Es ist jedoch an dieser Stelle anzumerken, dass keines der Beobachtungsjahre 2008 bis 2010 ein klimatisches Extremjahr war (siehe 3.2.1). Ist eine mehrjährige Versuchsreihe von Klimaextrema betroffen, ist die Übertragbarkeit der Aussagen auf mittlere Klimaverhältnisse schwer möglich.

Über alle Standorte hinweg wurden schwache negative Beziehungen zwischen der Temperatur und dem dGZ herausgefunden. Dies beobachtete auch ALI (2009) in einer Studie in Sachsen. Die negative Korrelation zwischen der Temperatur und dem dGZ erklärte ALI indirekt mit einer negativen Korrelation zwischen der Temperatur und dem Niederschlag. In der vorliegenden Arbeit hatten die Temperaturen und Niederschläge jedoch eine schwache positive Korrelation. Da die Analyse der Beziehungen zwischen Temperatur und dem dGZ in dieser Arbeit nach der Hauptbodenart unterschiedliche Ergebnisse zeigte, wird die Diskussion der Ergebnisse auf deren Basis geführt.

Die negativen Korrelationen zwischen dGZ und Temperaturen in der Vegetationsperiode (oder Teilen davon) sowie der Sand- und Lehmböden deuten auf einen negativen Einfluss höher werdender Tempe- 
ratur auf den Wasserhaushalt in Form von erhöhter Transpiration und Evaporation hin. Ein wachstumsfördernder Wärmeeffekt würde sich in einer positiven Korrelation ausdrücken. Da die benannten Bodenarten eine begrenzte Wasserverfügbarkeit zeigen, wirkten sich erhöhte Wasserverluste durch Temperatureinwirkung besonders kritisch auf den Wasserhaushalt und demnach auf das Wachstum aus.

Des Weiteren ist die negative Wirkung höherer Temperaturen im Hochsommer auf die Weidenklone in den Sandböden hervorzuheben. Bedenkt man die kühleren Herkünfte der verwendeten Weidenklone aus Russland und Schweden (LARSSON et al. 2001) gegenüber den Pappelklonen, könnten die negativen Korrelationen auf einen echten Hitzestress bei den Weiden hindeuten. Diese Beobachtungen lieBen sich anhand der Korrelationen zwischen dem dGZ und den Niederschlägen (siehe 4.2.2), die unabhängig von der Gattung waren, stützen. Betrachtet man aber die Literatur zum Wasserverbrauch von Weiden im Vergleich zu Pappeln, lässt sich eine andere Interpretation aufstellen. GUIDI et al. (2008) und PISTOCCHI et al. (2009) stellten in Freilandversuchen mit Großlysimeter eine bis zu $50 \%$ höhere Evapotranspiration bei Weiden gegenüber Pappeln fest. Damit wäre der Temperatureffekt der Weiden möglicherweise, wie auch schon bei den Pappeln, über die negative Beeinflussung des Wasserhaushalts zu erklären.

Die Zeiträume, in denen die höchsten Korrelationen auftreten, sind nicht verwunderlich. Die höchsten Temperaturen während der Vegetationsperiode wurden immer im selben Zeitfenster, Juli bis August bzw. Juli bis September, registriert. Diese sehr hohen Temperaturen in Verbindung mit einer voll entfalteten Blattfläche bewirken, insbesondere bei den Sandböden, eine starke Verringerung des Transpirationswasserangebotes (MEIRESONNE et al. 1999, ROBINSON et al. 2004, PETZOLD 2013). Die benötigte Blattkühlung durch Transpiration sowie der Saftfluss sind begrenzt; Hitzeschäden und einhergehende Wuchsdepressionen können vermehrt auftreten (LÖSCH 2001).

In den Lehm- und Sandböden wurden schwache inverse Korrelationen zwischen Wintertemperaturen und dem dGZ festgestellt, und zwar vermehrt bei den Pappeln. Dies spricht für die aus der Literatur bekannte Empfindlichkeit der Pappel gegenüber Winterkälte (SCHMITZ-LENDERS 1956, WEISGERBER 1980, BOHNENS und FRIEDRICH 1990).

Die zu erwartende positive Beeinflussung des Wachstums durch höhere Temperaturen in der Vegetationsperiode wurde einzig bei den Schluffböden sowie zeitlich begrenzt bei den Sandböden beobachtet. Während bei den Schluffen die Hochsommertemperaturen sich am positivsten auf das Wachstum auswirkten, taten dies die Frühjahrstemperaturen bei den Sanden, und zwar nur bei den Pappeln. Bei den Schluffböden kann von einer idealen Wasser- und Nährstoffversorgung ausgegangen werden (siehe 2.5.3.6 und 3.5.1.3). Ihr solider Wasserhaushalt, gekennzeichnet durch sehr hohe nFK, wurde anscheinend von steigenden Temperaturen nicht negativ beeinflusst, vielmehr konnten die erhöhten Temperaturen in der Vegetationsperiode ihre bekannte stoffwechselaktivierende Wirkung durch eine Zuwachssteigerung zeigen (DOBBERTIN und GIOGGIOLA 2006). Der Effekt höherer Temperaturen im Frühjahr bei den Sanden kann mit der Bodenerwärmung in Beziehung gebracht werden. Erst durch die Zunahme der Temperaturen im Frühjahr wird der Pflanzenstoffwechsel und somit das Wachstum aktiviert. Sandböden wärmen sich deutlich schneller und intensiver auf als Lehm- und Schluffböden (JURY et al. 1991). Warum die Pappeln von den höheren Temperaturen mehr profitieren als die Weiden, wurde bereits anhand der Wintertemperaturen erläutert.

\subsubsection{Niederschlag}

Wie zu erwarten, hatten Niederschlagsvariablen bei allen Klonen und Bodenclustern stets eine positive Auswirkung auf das Wachstum. Dies hängt selbstverständlich mit dem hohen Wasserbedarf von Pappel- und Weidenplantagen zusammen (MÜLLER 1951, FAO 1979, PETZOLD et al. 2010). 
Die positive Beziehung von Niederschlagsvariablen mit dem dGZ war bei den Sandböden am stärksten. Dies ist leicht zu erklären, da Sandböden die niedrigsten nFK und gleichzeitig die höchsten hydraulischen Leitfähigkeiten unter den untersuchten Bodenarten aufweisen (AG BODEN 2005, RENGER et al. 2008). Aus diesem Grund ist der Niederschlag für das Wachstum am wichtigsten. Bei den Lehmen, welche auch über niedrige nFK verfügen, ist die hydraulische Leitfähigkeit deutlich geringer als die der Sande (JURY et al. 1991, RENGER et al. 2008). Deswegen ist das in der nFK gespeicherte Wasser deutlich länger verfügbar.

Für alle Cluster waren die Niederschläge der ersten drei Monate in der Vegetationsperiode Mai bis Juli am bedeutsamsten. Die Begründung dafür kann mit folgenden physiologischen Vorgängen zusammenhängen: Einerseits scheint die Interzeption bzw. die Interzeptionevaporation, welche einen saisonal abhängigen Verlauf hat, eine Rolle in der Bedeutung verschiedener Zeitfenster zu spielen. Und zwar hindert die Interzeption eine größere Menge des Niederschlags daran, im Boden zu versickern. Die Blattfläche ist je nach Klima im Zeitraum zwischen Juni und Mitte Juli voll ausgebildet, ab diesem Zeitpunkt bleibt sie mehr oder weniger konstant (ISEBRANDS et al. 1990, MEIRESONNE et al. 1999, ROBINSON et al. 2004, PETZOLD 2013).

Die Interzeption in der Vegetationsperiode lag bei einer sechs- bis achtjährigen Pappelplantage und voll ausgebildeter Blattfläche nach UNSELD (1999) im Mittel bei ca. 40 \%. Je höher der Sommerniederschlag war, desto höher der Kronendurchlass. Bei Monatsniederschlagssummen bis zu $25 \mathrm{~mm}$ wurden nur $20 \%$ des Niederschlags durchgelassen, bis $150 \mathrm{~mm}$ waren es $65 \%$. Tabelle 5-1 veranschaulicht den Zusammenhang zwischen Interzeption in Abhängigkeit der Höhe der Einzelniederschläge und des Blattflächenindex für Ackerkulturen. Je größer die Blattfläche und je niedriger die Niederschläge sind, umso größere Anteile des Niederschlags bleiben als Interzeption im Bestand hängen. In Wäldern werden noch höhere Interzeptionsraten als bei Ackerkulturen erreicht (RUTTER et al. 1971). Nach FRÖHLICH (2009) wurden in einem 80-jährigen Fichtenbestand erst ab einem Niederschlagsereignis von mindestens $10 \mathrm{~mm}$ signifikante Änderungen im Bodenwassergehalt registriert.

Tab. 5-1: Errechnete Interzeption nach dem Modell von HoynINGEN-HuENE (1983) für unterschiedliche Blättflächenindizes und Freilandniederschläge für Ackerkulturen.

\begin{tabular}{cccccccc}
\hline \multirow{2}{*}{$\begin{array}{c}\text { LAI } \\
{\left[\mathrm{m}^{2} \mathrm{~m}^{-2}\right]}\end{array}$} & \multicolumn{6}{c}{ Niederschlagshöhe im Freiland in [mm] (Einzelniederschläge) } \\
\cline { 2 - 7 } & 1 & 2 & 4 & 8 & 12 & 16 & 20 \\
\hline 1 & 0,1 & 0,4 & 0,7 & 1,2 & 1,3 & 1,3 & 1,3 \\
2 & 0,3 & 0,6 & 1 & 1,6 & 1,9 & 1,9 & 1,9 \\
4 & 0,6 & 1 & 1,5 & 2,3 & 2,8 & 3 & 3 \\
8 & 0,9 & 1,4 & 2,1 & 3,5 & 4,4 & 5 & 5 \\
\hline
\end{tabular}

Insgesamt kann stark davon ausgegangen werden, dass im Zeitraum zwischen Juli und September ein beachtlicher Teil der Niederschläge nicht den Boden erreicht. Die Ergebnisse von ALI (2009) stützen diese Beobachtungen. Dort hatte die Niederschlagsvariable $\mathrm{N}_{5,6}$, welche auch die Frühphase der Vegetationsperiode repräsentiert, die höchste Beziehung zum dGZ.

Der Grund für die ungenaueren Korrelationen und Regressionen mit den Niederschlagsvariablen aus der zweiten Hälfte der Vegetationsperiode liegt vermutlich in der LAI-Entwicklung der verschiedenen Bestände. Die wuchsstärksten Standorte im Projekt hatten höhere Laubmassen und demnach höhere LAI als die wuchsschwächsten (HEYN und WACHENDORF 2012). Demzufolge war die Interzeption an den wuchsstärksten Standorten am höchsten. Gleichzeitig war der Freilandniederschlag bei den stärksten Standorten am höchsten, sowohl in der ersten als auch in der zweiten Hälfte der Vegetationsperiode. Allerdings bewirkt der durch den Niederschlag bestärkte Wuchs eine höhere Interzeption. Damit 
ist die Differenz der Freilandniederschläge zu den Bestandesniederschlägen erhöht. Dies beeinflusste die positiv gerichtete Beziehung zwischen Freilandniederschlägen und dGZ für diese Zeitfenster genau in die entgegengesetzte Richtung und verminderte somit ihre Genauigkeit.

Bei den Zeitfenstern der bestkorrelierten Niederschlagsvariablen gab es klare Unterschiede zwischen den Sandböden und den bindigeren Schluff- und Lehmböden. So war die $\mathrm{N}_{6,7}$ bei den Sanden die am besten korrelierte Variable, wobei $\mathrm{N}_{5,6}$ sowohl bei den Lehmen als auch bei den Schluffen mit dem $\mathrm{dGZ}$ am besten korrelierte. Warum es zwischen den Bodenarten zu diesen Abweichungen kam, hängt mit der Verweilzeit des Wassers im Boden zusammen. Wie bereits erwähnt, erreicht die Plantage ihren maximalen Wasserverbrauch in der Zeit der maximalen Blattfläche und höchsten Temperaturen, das heißt, im Zeitraum zwischen Juli und September. Eine optimale Wasserversorgung in dieser Zeit wird, abhängig von der Wasserleitfähigkeit des Bodens, durch Niederschläge unterschiedlicher Zeiträume gewährleistet. Sandböden haben unter den untersuchten Böden die höchste Leitfähigkeit (JURY et al. 1991). Daher ist anzunehmen, dass der wichtigste Zeitraum für die Wasserinfiltration während oder kurz vor der Phase des maximalen Wasserverbrauchs ist. Dies spiegelt sich in der Variablen $\mathrm{N}_{6,7}$ wieder. Demzufolge ist bei den Schluff- und Lehmböden, bedingt durch die geringeren Leitfähigkeiten, der relevante Zeitraum vorher. Dies drückt sich in der Niederschlagsvariablen $\mathrm{N}_{5,6}$ aus.

Mittels der Korrelations- und Regressionsanalyse wurde kein erhöhter Wasserbedarf eines bestimmten Klons oder der Gattungen festgestellt, alle scheinen ähnliche Anforderungen an das Wasser zu haben.

\subsubsection{Trockenheitsindex}

In den Beziehungen zwischen Trockenheitsindex und dGZ zeigte sich außer bei den Lehmböden eine nur geringe Verbesserung der $r$-Werte und $\mathrm{R}_{\text {korr }}^{2}$ gegenüber der Beziehung zwischen den Niederschlägen und dem dGZ. In einigen Fällen verschlechterte sich sogar die Beziehung zum dGZ geringfügig. Die stärkste Verbesserung der $r$ - und $\mathrm{R}_{\text {korr }}^{2}$-Werte wurde bei den Lehmböden beobachtet. Sie hängt mit den größeren Korrelationskoeffizienten der Temperatur mit dem dGZ in den relevanten Zeiträumen der Wasserversorgung von April bis Mai, von Mai bis Juni sowie von Juni bis Juli (siehe 4.2.1) zusammen.

Ein wichtiges Ergebnis in der Analyse von klimatischen Größen war die Aufteilung der Vegetationsperiode in spezifische Zeitfenster. Damit wurde eine erhöhte Genauigeit in den Korrelation- und Regressionsanalyse erreicht. Dies traf auf alle Klimavariablen, Klone und Standortcluster zu. Der Grund für dieses Phänomen liegt sicherlich in der zeitlich spezifischen Entwicklungsphase der Pflanze. Vor allem die Entwicklung der Blattfläche, welche eine erhöhte Relevanz auf Prozesse wie zum Beispiel Transpiration oder Interzeption hat, zeigt eine markante zeitliche Dynamik (MEIRESONNE et al. 1999). Die Vegetationsperiode ist daher kein zuverlässiges Indikatorzeitfenster für das Wachstum und kann aus diesem Grund nur begrenzt Auskunft geben über die Eignung verschiedener Standorte für die Nutzung als KUP.

Ein weiteres wichtiges Ergebnis, welches sich über alle Indikatoren hinweg zeigt, ist der positive Einfluss der Clusterung auf die Güte der Korrelationen und Regressionen.

\subsubsection{Bodenschätzungskennwerte}

Vor der eigentlichen Diskussion der Variablen aus dem Bereich des Bodens ist es wichtig, sich der Wechselwirkung zwischen ihnen bewusst zu werden. Alle drei Typen von Bodenvariablen - die Bodenschätzungs-, die Textur-, und die Porenraumvariablen - bedingen sich gegenseitig. Das heißt, hier werden durch die verschiedenen Variablen teilweise ähnliche Prozesse angesprochen. Die Bodenzahlen sind das Resultat der Texturansprache, der Entstehungsart sowie der ZS, welche maßgeblich vom Gehalt organischer Substanz abhängt. Zur Bildung der Porenraumvariablen gehen die Einfluss- 
größen Textur, Gehalt an organischer Substanz sowie TRD in aggregierter Form ein. Porenraumvariablen und Bodenschätzungsvariablen beinhalten somit ähnliche Eingangsinformationen. Die weiteren Variablen sind die Texturvariablen. Die hier beinhalteten Informationen werden sowohl bei den Bodenschätzungsvariablen als auch bei den Porenraumvariablen berücksichtigt. Aus den genannten Gründen erfolgt die Diskussion der Bodenvariablen besonders vorsichtig.

Für alle Standorte erreichten die Kennwerte der Bodenschätzung eine mäßige Korrelation mit dem Wachstum. Überraschenderweise zeigte ZS, mit Ausnahme des Sandclusters, die höchste Bedeutung für das Wachstum. ZSgibt Auskunft über die Profilentwicklung bzw. Profiltiefe, über den Gehalt und Verteilung im Profil der organischen Substanz sowie über die Bodenreife bzw. Bodenentwicklung. Alle diese Kennwerte haben einen maßgeblichen Einfluss auf die Ertragsfähigkeit der mit KUP bewirtschafteten Böden (siehe 2.5.3). Insbesondere der Gehalt an organischer Substanz hat einen sehr positiven Einfluss den Stoff- und Wasserhaushalt des Bodens (TIESSEN et al. 1994, TISDALL und OADES 2006). ALI (2009) untersuchte unter den Bodenschätzungskennwerten nur die Bedeutung von AZ für das Wachstum. Für die verschiedenen Alterstufen der Pappel auf den Versuchsflächen wurden Regressionen zwischen $\mathrm{AZ}$ und der Oberhöhe mit $\mathrm{R}^{2}$ von 0,58 bis 0,98 ermittelt.

Bei den Sand- und Lehmböden hatten die Bodenschätzungskennwerte eine mittlere bis starke Bedeutung für die Ertragsbildung. Bei den Sanden zeigte AZ die besseren Werte. Wie bereits in den vorigen Kapiteln diskutiert, haben klimatische Kenngrößen eine große Bedeutung für das Wachstum in den Sandböden. Da AZ das in-situ Klima (Temperatur und Niederschlag) in Form von Zu- oder Abschlägen berücksichtigt (siehe 3.5.1.2), ergibt sich ein Vorsprung in der Aussagekraft von AZ für das Wachstum gegenüber den anderen Bodenschätzungsvariablen. Es sei jedoch angemerkt, dass ZS bei den Sanden eine ungünstige Verteilung der Datenpaare hatte. Von den acht berücksichtigten Versuchsflächen zeigten fünf einen ZS-Wert von „Drei“. Aus diesem Grund war die Ermittlung stärkerer Korrelationen nicht möglich.

Der Effekt der Kennwerte der Bodenschätzung war bei den Schluffböden am stärksten. Die vorliegenden Schluffböden wurden nach der Bodenschätzung allesamt als Bodenart „L“, Entstehungsart „Lö“, klassifiziert. Daher waren BZ und AZ maßgeblich von ZS bestimmt. Die Bedeutung von ZS auf das Wachstum wurde im oberen Passus bereits diskutiert. Wie Kapitel 4.2.7 entnommen werden kann, sind einige Bodenvariablen bei den Schluffböden sehr hoch miteinander korreliert, und zwar LK, TRD, ZS, BZ und AZ. Der hohe Zusammenhang zwischen den Bodenschätzungsvariablen und den Variablen des Porenraums erschwert die Diffenzierung in der Interpretation der Effekte der genannten Variablen auf den dGZ enorm. Die hohe Variation der r-Werte kann mit der bereits erläuterten allgemeinen Unsicherheit der Daten bei den Schluffböden erklärt werden (siehe 5.1).

Wie im Kapitel 2.5.3.5 erwähnt, reichen die Bodenschätzungsvariablen alleine nicht als Indikator für das Pappel- und Weidenwachstum. Sie können aber für die Bildung von Modellen nützlich sein (ALI 2009).

\subsubsection{Textur}

Bei der Betrachtung der Texturvariablen wurde sichtbar, dass der Schluffgehalt die wichtigste Texturvariable für das Wachstum in allen Standortclustern war. Dies kann mit ihrer Bedeutung für den Wasserhaushalt erklärt werden (siehe 2.5.3.7, JURY et al. 1991 und HILLEL 1998).

Beim Sandbodencluster wurden die stärksten Beziehungen zum dGZ mit den prozentualen Schluffund Sandgehalten festgestellt. Beide Variablen waren miteinander fast exakt korreliert. Dies spricht für eine sehr geringe Variation des Tongehaltes. Die Werte schwankten zwischen 3,5 und 9,0\%. Der Haupteffekt für die günstigen Auswirkungen auf das Wachstum kann somit überwiegend dem Schluffanteil zugerechnet werden. Die Höhe der Beziehung zwischen den genannten Bodeneigenschaften 
und dem Wachstum war fast genauso hoch wie bei den geprüften Klimavariablen Niederschlag und Trockenheitsindex.

Bei den Schluffen waren die Beziehungen oftmals komplex. Der Schluffgehalt korreliert beispielsweise negativ mit dem dGZ, was physikalisch unplausibel ist. Dieses Ergebnis hing höchstwahrscheinlich mit der sehr geringen Variation der Textur-Werte zusammen. Die einzige physikalisch plausible positive Korrelation war die zwischen dem Sandgehalt und dem dGZ. Diese könnte auf eine Verbesserung der Luftverfügbarkeit im Boden zurückzuführen sein (JURY et al. 1991).

Bei den Lehmen war allein der Schluffgehalt entscheidend für das Wachstum. Der Grund für die Bedeutung des Schluffes für das Wachstum wurde bereits im obersten Passus erläutert. Anhand der Korrelations- und Regressionsanalyse wurde eine geringe klonspezifische Variation in den untersuchten Prozessen bzw. Variablen festgestellt.

\subsubsection{Porenraum}

Für alle Standorte gleichzeitig betrachtet, hatte nFK die höchste Bedeutung für das Wachstum. Dieses Ergebnis war zu erwarten und ist aus der Literatur bekannt (ALRIKSSON 1997, ALI 2009). ALI (2009) ermittelte für verschiedene Alterstufen lineare Regressionen zwischen der Oberhöhe und der $\mathrm{nFK}_{\mathrm{WE}}$ mit $\mathrm{R}^{2}$ zwischen 0,73 und 0,91. Diese Werte lagen weit über den im Standortcluster ALL ermittelten Regressionen, welche je nach Klon $\mathrm{R}^{2}$ korr $\mathrm{zwischen} 0,26$ bis 0,34 zeigten.

Unerwartet wurden bei den Sandböden eher mäßige Beziehungen mit den Porenraumvariablen, insbesondere der nFK mit dem dGZ, festgestellt. Das ist ein unerwartetes Ergebnis, denn bei den Sandböden wurde stark angenommen, dass hohe $\mathrm{nFK}$ auch zu hohen dGZ führen. Seltsamerweise deutet die sehr starke Korrelation zwischen dem prozentualen Schluffgehalt und dem dGZ nur indirekt auf eine starke Beziehung zwischen $\mathrm{nFK}$ und Wachstum hin. Die Begründung für den ausbleibenden Zusammenhang der nFK mit dem Wachstum in den Sandböden kann in der geringen Genauigkeit der Ermittlung von Porenraumkenngrößen gesucht werden (siehe 3.3.3.1 und 5.1). Insbesondere bei den Sandböden, in denen die Variation der $\mathrm{nFK}$ sehr gering ist, sind die Messungen ungenau ging durch da die indirekte Methode angewandt wurde (RENGER et al. 2008).

Die LK hingegen hatte bei den Sandböden einen höheren Einfluss auf den dGZ und war wie erwartet invers. Vermutlich ist dies auf den Zusammenhang zwischen Leitfähigkeit und LK zurückzuführen. Je höher LK desto höher ist die Leitfähigkeit des Bodens (JURY et al. 1991). Wie schon anhand des Klimas diskutiert (siehe 5.3.2), führt eine hohe Leitfähigkeit zur geringen Verweilzeit des Wassers im Boden, was mit einem Ertragsrückgang einhergeht. Die TRD oder, besser gesagt, die Gesamtporosität hatte einen positiven Einfluss auf den dGZ. Dies ist leicht zu erklären, denn je größer ein Porenvolumen ist, umso bessere Wachstumsmöglichkeiten im Wurzelraum sind gegeben (JURY et al. 1991).

Bei den Schluffböden hatten die LK und die TRD eine deutlich stärkere Rolle in der Ertragsbildung als die nFK. Ersteres bedeutet, dass das Luftangebot sich besonders positiv auf das Wachstum auswirkte (SCHMITZ-LENDERS 1956, FAO 1979). Letzteres bedeutet, dass der Gesamtporenraum und eine lockere Lagerung sich in Folge einer leichten und umfassenden Durchwurzelung auf das Wachstum positiv auswirkten (HARTGE und HORN 1989). Obwohl LK und TRD fast exakt miteinander korreliert waren, können die Effekte beider Kenngrößen als plausibel angesehen werden. Eine Erklärung für die geringe Korrelation der $\mathrm{nFK}$ mit dem dGZ kann in den durchweg hohen Werten der $\mathrm{nFK}$ in den Schluffböden gesucht werden, da somit optimale Bedingungen für den Zuwachs in dem Beobachtungszeitraum gegeben waren. Mit einer längeren Beobachtungszeit kann eventuell die nFK besser ausgenutzt werden, und Standortunterschiede werden deutlicher. 
Bei den Lehmböden hatten sowohl nFK als LK sehr starke Beziehungen zum dGZ. Die nFK war bei diesen Böden die wichtigste Variable, die Gründe für die positive Beeinflussung des Wachstums durch nFK und LK wurde im vorigen Passus erwähnt.

\subsubsection{Aggregierte Variablen}

Die aggregierten Variablen brachten erwartungsgemäß eine große Verbesserung der Korrelationskoeffizienten für jeden Klon und Cluster. Die Kombinationen mit den größten Verbesserungen setzten sich aus je einer Boden- und einer Klimavariablen zusammen. Das ist kein überraschendes Ergebnis, denn aus beiden Bereichen sind die wesentlichen Einflüsse für das Wachstum herzuleiten (siehe 3.5.1).

Bei der Betrachtung aller Standorte bildeten die bestkorrelierte aggregierte Variable nFK und entweder die Niederschlags- oder die Trockenheitsindexsumme des Zeitraumes zwischen Mai und Juli. Diese Variablen waren jeweils die bestkorrelierten Variablen aus dem jeweiligen Bereich. ALI (2009) hat mehrere aggregierte Variablen ermittelt und für alle Altersstufen zusammen dargestellt. Dort waren deutlich geringere $\mathrm{R}^{2}$ zu verzeichnen als bei Betrachtung der Beziehung zwischen Standortvariablen und $\mathrm{h}_{\mathrm{dom}}$ getrennt nach Altersstufen. Die beste aggregierte Variable stimmt mit den im Kapitel 2.5.3.1 erwähnten Zusammenhang überein. Dort wird erläutert, dass die für das Wachstum entscheidende Wasserversorgung ein Wechselspiel von Niederschlag mit der Wasserhaltekapazität des Bodens ist. Die Wasserversorgung wird von AYLOTT et al. (2008) als den am stärksten wachstumslimitierenden Faktor für das Pappel- und Weidenwachstum angesehen.

Bei den Sandböden setzte sich bis auf AF 2 die bestkorrelierte aggregierte Variable aus den bestkorrelierten Einzelvariablen der jeweiligen Bereiche Boden und Klima zusammen. Bei AF 2 wurde in der bestkorrelierten aggregierten Variablen die Temperatur anstelle von Trockenheitsindex oder Niederschlag zusätzlich zur Bodenvariablen berücksichtigt. Das ist insofern verwunderlich, da bei einem Schwarzpappelhybrid die ertragsmindernde Wirkung höherer Temperaturen nicht so ausgeprägt sein sollte wie bei Pappeln anderer Sektionen (FAO 1979).

Im Cluster der Schluffböden wurde bis auf Max 1 keine nennenswerte Variation durch die Berücksichtigung der aggregierten Variablen gegenüber den bestkorrelierten Einzelvariablen erzielt. Die bestkorrelierten Variablen waren unter den Klonen nicht einheitlich. Für beide Beobachtungen kann die Erklärung in der kleinen Wiederholungsanzahl gesucht werden. Des Weiteren waren die aggregierten Variablen auch, sehr variabel, insbesondere im Bereich des Bodens. Hierbei spielt auch die hohe Korrelation zwischen den Bodenvariablen eine Rolle (siehe 4.2.7). Die Ergebnisse waren bei den restlichen Cluster deutlich einheitlicher.

Im Lehmbodencluster wurden nur geringe Verbesserungen der aggregierten Variablen gegenüber den besten nicht aggregierten Variablen festgestellt. Die Berücksichtigung der Trockenheitsindexvariablen aus dem Zeitraum Mai bis Juni brachte die größten Verbesserungen im r-Wert. Der Grund für den geringen Klimaeinfluss in den Kombinationsvariablen kann unter anderem mit dem geringeren Gradient der Ausgangswerte des Niederschlags sowohl in den Variablen $\mathrm{N}_{5,6}$ wie $\mathrm{N}_{6,7}$ gesucht werden. Die Spannweite der Werte bei den Niederschlagsvariablen des gleichen Zeitfensters war bei den Lehmen fast halb so groß wie bei den Sanden. Er betrug $30 \mathrm{~mm}$ bei den Lehmen und $60 \mathrm{~mm}$ bei den Sanden. Dieser Umstand, gekoppelt mit der längeren Verweilzeit des Wassers im Boden, schwächt den Klimaeffekt noch zusätzlich.

Der Anteil an Erklärung der einzelnen Variablen an den aggregierten Variablen kann nicht ermittelt werden. Der Grund hierfür war, dass die Variablenaggregierung multiplikativ oder divisorisch erfolgte. Die Korrelationsanalyse der aggregierten Variablen stellte einen Zwischenschritt zur Entwicklung standortbasierter Modelle dar. 


\subsubsection{Weitere Einflussgrößen}

Es wurde zwar statistisch ein Effekt der Vornutzung auf dem dGZ festgestellt, aufgrund der sehr geringen Wiederholungsanzahl der Vornutzungen Baumschule, Grünland und Bergbaufolgelandschaft gilt diese jedoch als nicht gesichert. Der Grund des Effektes von Vornutzungen auf den dGZ kann aus verschiedenen Perspektiven betrachtet werden. Insbesondere Grünlandstandorte haben durch die regelmäßige Befahrung tendenziell dichter gelagerte Böden (SCHMEER et al. 2009) und eine deutlich stärkere Verunkrautungsgefahr als andere Vornutzungen. Baumschulen werden in der Regel wie Ackerböden intensiv bewirtschaftet, regelmäßig gedüngt und mechanisch bearbeitet. Hier kann keine große Abweichung erwartet werden. Über die großen Unterschiede zwischen Bergbaufolgelandschaften mit ackerbaulich genutzten Flächen wurde bereits in 2.2.2. berichtet.

In der vorliegenden Arbeit wurde ein Einfluss des Bodentyps auf das Wachstum festgestellt. Die sehr fruchtbaren Schwarzerden waren die ertragsstärksten Bodentypen. Dies ist nicht verwunderlich, zumal sie auch bei Ackerkulturen aufgrund ihres sehr hohen Schluffgehaltes sowie dem Gehalt an organischer Substanz höchste Erträge erwarten lassen (ZECH und HINTERMAIER-ERHARD 2002). Auch Moorstandorte wiesen sehr starke Leistungen auf. Die positiven Eigenschaften von Moorböden für das KUP-Wachstum sind in Kapitel 2.5.3.6 bereits erläutert. Braunerden und Parabraunerden hatten ein großes Spektrum an dGZ. Daher lassen sich deren Leistungen nicht generalisieren. Wie wuchsstark solch ein Bodentyp ist, hängt sicherlich mit den spezifischen klimatischen und bodenkundlichen Standortgegebenheiten zusammen. Gleye und Pseudogleye hatten schwache bis mittlere Massenleistungen. Erstaunlicherweise hatten Gleye in der vorliegenden Arbeit deutlich niedrigere Massenleistungen als die Pseudogleye. Dieses Ergebnis war nicht zu erwarten, denn es wurde angenommen, dass grundwasserbeeinflusste Böden durch größere Profile und nicht verdichtete Horizonte höhere dGZ aufweisen als stauwasserbeeinflusste. Dass dies nicht zutraf, mag mit den sehr schweren Bodensubstraten der untersuchten Gleye zusammenhängen: Das Bodensubstrat war am Sto. Nr. 5 „Ladenburg“ ein Auenlehm und am Standort Nr. 35 „Threnthorst“ ein Geschiebelehm. Diese Standorte zeichneten sich durch sehr niedrige nFK und LK aus (Tab. 3-12). Dies wirkte sich, wie bereits im Kapitel 5.3.6 erwähnt, schlecht auf das Wachstum aus. Auf Gleyen mit leichten Bodensubstraten wie zum Beispiel lehmigen Sanden wären sicherlich hohe dGZ zu erwarten gewesen (siehe 2.5.3.1). Die im Kapitel 2.5.3.2 benannte negative Auswirkung von Pseudovergleyung auf das Pappel- und Weidenwachstum wird mit den vorliegenden Ergebnissen bestätigt. Die dGZ von Pseudogleyen können aber auch variieren. Der sehr stark verdichtete schwere Boden am Standort Nr. 6 „Kupferzell“ hatte die niedrigsten dGZ zur Folge. Pseudogleye mit einer besseren Bodenstruktur in Folge von höheren $\mathrm{C}_{\text {org- }}$ und geringeren Tongehalten wie es an den Sto. Nr. 20 „Borlinghausen“ und Sto. Nr. 22 „Haus Düsse“ der Fall war, hatten höhere dGZ zur Folge.

Anders als angenommen wurde am Regosol vom Sto. Nr. 13 „Grünewalde“ ein sehr hoher dGZ festgestellt. Dies hängt vermutlich mit der Anreicherung des Substrats mit Kohle, die zu einer Verbesserung der bodenchemischen und -physikalischen Eigenschaften beiträgt, zusammen (JIEN und WANG 2013).

Der Grundwasserspiegel hatte einen signifikanten positiven Einfluss auf das Wachstum. Die Gründe für die positive Auswirkung auf das Wachstum wurden bereits im Kapitel 2.5.3.1 ausführlich erläutert.

\subsection{Standortbasierte Ertragsmodellierung}

Alle ermittelten Modelle waren univariat, und mittels der multiplen Regressionsansätze ergaben sich keine signifikanten Modelle auf Ebene der Klone. Dies ist teilweise auf den geringen Stichprobenumfang zurückzuführen. Es konnte aber gezeigt werden, dass bei einem größeren Stichprobenumfang, nach Aggregierung der Gattung Weide im Standortcluster S, multiple lineare Regressionsmodelle sig- 
nifikant waren. Multiple nicht-lineare Modelle waren aber auch dort nicht signifikant, allerdings ist davon auzugehen, dass auch hier eine höhere Stichprobenanzahl für die Signifikanz der Modelle nützlich gewesen wäre.

Bei der Mehrheit der ermittelten Modelle war der gewählte Prädiktor eine aggregierte Variable, häufig waren die Modelle nicht-linear. Es wurde sichtbar, dass durch die Nutzung mathematischer Ressourcen wie die der nicht-linearen Regression und der Variablenaggregierung sehr hohe Bestimmtheitsmaße erreicht werden konnten. Dasselbe betrifft die Standortclusterung: Erst nach der Clusterung erreichten die Modelle eine bessere Genauigkeit.

\subsubsection{Standortcluster ALL}

Die Wahl des Prädiktors im Modell basiert auf den Beobachtungen im Bereich der leistungsbeeinflussenden Eigenschaften (siehe 4.2.1 bis 4.2.6) und aggregierten Variablen (siehe 4.2.9) und wird hier nicht weiter ausgeführt. Ein interessanter Vergleich kann jedoch mit dem Modell VON ALI gezogen werden. Das Modell von ALI beinhaltete für Ermittlung von $\mathrm{h}_{\text {dom }}$ zusätzlich zum Prädiktor Alter zwei aggregierte Prädiktoren $\left(\mathrm{N}_{5,6} * \mathrm{AZ}\right)$ sowie $\left(\mathrm{T}_{4,7} / \mathrm{nFK}\right)$. Das Modell hatte einen $\mathrm{R}_{\text {korr }}$ von 0,99 . Zusätzlich ermittelte ALI aber auch ein Modell mit nur zwei Prädiktoren, dies waren das Alter und die aggregierte Variable $\left(\mathrm{N}_{5,6} * \mathrm{AZ}\right)$. Es ergab sich eine hohe Aussagekraft mit einem $\mathrm{R}_{\text {korr }}$ 0,97. Die Berücksichtigung der weiteren Standortvariablen $\left(\mathrm{T}_{4,7} / \mathrm{nFK}_{\mathrm{WE}}\right)$ brachte nur eine $2 \%$-ige Verbesserung im $\mathrm{R}_{\text {korr }}$. Aus diesem Ergebnis lässt sich demnach herleiten, dass die wichtigsten Faktoren für das Pappelwachstum in Sachsen die $\mathrm{N}_{5,6}$ und AZ sind. In der vorliegenden Arbeit hingegen waren die $\mathrm{nFK}$ und $\mathrm{N}_{5,7}$ die wichtigsten Variablen. Der Grund für diesen Unterschied lag wahrscheinlich in der weitaus größeren Standortanzahl im Verbundvorhaben PROLOC als in der Arbeit von ALI (2009).

Eine interessante Interpretation kann anhand der Form des Modells durchgeführt werden. Die Anpassungen, welche je nach dem exponentiell, linear oder asymptotisch verlaufen, können Auskunft darüber geben, wie die Umweltressourcen genutzt wurden und ob sich noch weiteres Wachstumspotential herleiten lässt. Bei Betrachtung alle Standorte verliefen alle Modelle bis auf das der AF 2 asymptotisch. Das könnte für ein insgesamt höheres Wuchspotential der AF 2 gegenüber den anderen Pappeln und Weiden sprechen. Dass AF 2 ein stärkeres Wuchspotential als die anderen Pappelprüfglieder haben könnte, wurde bereits in Kapitel 5.2.5 diskutiert.

Der bodenbezogene Anteil der Modelle, die nFK, hatte sichtbar eine größere Bedeutung als der klimabezogene Anteil, unabhängig davon, ob die Modelle einen exponentiellen oder asympthotischen Verlauf hatten. Im jungen Alter erschließen sich die Bäume den Boden durch die Bildung des Wurzelwerks. Erst durch diese Erschließung des Bodens können die Gewächse die Boden- und Klimaressourcen optimal nutzen. Der Einfluss des Bodens ist grundlegend und bedingt die anderen. Ein Beispiel: die nFK gibt Auskunft über den nutzbaren Porenraum für Wassersaufnahme. Ohne eine hohe nFK können hohe Niederschläge gar nicht in vollem Umfang ausgenutzt werden. Anders ausgedrückt, wenn die nFK niedrig ist, ist die Durchwurzelung, die Wasser- und Stoffaufnahme begrenzt. ALI (2009) bestätigt diese Beobachtung. Bei der Betrachtung der Einzelvariablen auf das Wachstum zeigte er, dass Bodenkenngrößen wie nFK und AZ einen deutlich stärkeren Einfluss als die Klimavariablen darstellten. Es ist denkbar, dass, wenn der Boden im späteren Alter vom Bestand weitestgehend erschlossen ist, Klimavariablen eine größere Rolle spielen.

Da wenige empirische Modelle in der Literatur vorhanden sind, kann an dieser Stelle dieses Phänomen nicht weiter diskutiert werden. Für die Validierung dieser Beobachtungen wären Sensitivitätsanalysen mit Hilfe von Waldwachstumsmodellen mit einem umfassenden Wasserhaushaltsmodul vonnöten. 


\subsubsection{Standortcluster $S$}

Die Wahl des Hauptprädiktors baut auf den in Kapitel 4.2 erläuterten Ergebnissen auf und ist bereits in Kapitel 5.3 diskutiert. Auffällig bei den Modellen in den Sandböden ist, dass mal die Variable $\mathrm{U}_{[\%]}$ oder $\mathrm{S}_{[\%]}$ als bodenbezogener Teilprädiktor im Modell fungierten.

Anders als bei allen anderen Klonen hatte das H 275-Modell einen stärkeren klimabezogenen Teil. Dies deckt sich mit den Ergebnissen aus den Kapiteln 4.2.2 und 4.2.3, welche für H 275 eine hohe Bedeutung des Klimas zeigten. Ob das Ergebnis des Modells physikalisch plausibel ist, ist unklar. In der Regel haben Schwarzpappeln einen stärkeren Wasserbedarf als Balsampappeln, der Effekt kann aber auch klonspezifisch sein. Da H 275 in den anderen Clustern nicht geprüft werden konnte, kann diese Aussage für die weiteren Cluster nicht überprüft werden.

Die Residuen vom Max 1-Modell waren als einzige von allen ermittelten Modellen nicht normal verteilt. Die Begründung hierzu kann in einem Ausreißer gesucht werden (siehe 4.3.2, Abb. 4-32). Demnach wird eine überdurchschnittlich große Residue ermittelt und dies verursacht die nicht normale Verteilung (SACHS und HEDDERICH 2009).

\subsubsection{Standortcluster $\mathrm{U}$}

Viele Modelle waren im Bodenartcluster, obwohl die Korrelation aggregierter Variablen signifikante Ergebnisse zeigte, nicht signifikant. Wie mehrfach erwähnt, ist die geringe Wiederholungsanzahl höchstwahrscheinlich für die nicht signifikanten Beziehungen verantwortlich. Des Weiteren spielt die insgesamt geringe Variation des dGZ eine Rolle.

BZ hat offensichtlich eine sehr hohe Erklärungsrate für den dGZ bei den Schluffböden, schließlich hatte das Tordis-Modell BZ als einzigen Prädiktor mit einer sehr hohen Erklärungsrate. Es muss aber angemerkt werden, dass bei anderen Klonen auch andere leistungsbeeinflussende Eigenschaften eine Rolle spielten. Das Max 1-Modell beinhaltete beispielsweise zwei Komponenten: BZ und $\mathrm{TI}_{4,5}$. Bei der Betrachtung der Einzelkomponenten wurde deutlich, dass sie in etwa gleiche Anteile in der Ertragsbildung hatten. Die Modelle waren beide exponentiell und zeigten anders als bei den Sanden noch Wachstumspotential. BIERTÜMPFEL et al. (2012) zeigten die Entwicklung des Ertrages auf drei verschiedenen Standorten. Dort wurde sichtbar, dass der Ertrag auf dem Lössstandort kontinuierlich zunahm und dieser unter den drei geprüften Standorten das höchste Potential aufwies (siehe 4.4 Abb. 441).

\subsubsection{Standortcluster $L$}

Zwei der drei ermittelten Modelle beinhalteten als einzigen Prädiktor nFK. Hierdurch entsteht der Eindruck, dass das Klima in den Lehmböden keine Bedeutung hat. Dies kann aber keinesfalls generalisiert werden (siehe 4.2.9). Das AF 2 Modell berücksichtigte eine Klimavariable, $\mathrm{N}_{5,6}$, zusätzlich zur nFK. Man könnte daraus schließen, dass AF 2 auf eine höhere Wasserversorgung als die anderen Pappelklone angewiesen ist. Diese Beobachtung bestätigt sich aber in den anderen Clustern nicht. Im Bodenartcluster der Schluffe berücksichtigte das Max 1-Modell sowohl eine Boden- als auch eine Klimavariable.

\subsubsection{Vergleich mit anderen Modellen}

Die $\mathrm{R}^{2}{ }_{\text {korr }}$ der in der vorliegenden Arbeit ermittelten Modelle für die jeweiligen Bodenartcluster schwankten zwischen 0,77 und 0,98 mit einem Mittelwert von 0,88. Diese Genauigkeit ist im Rahmen der Literatur zur Modellierung von Pappel- und Weidenerträgen in KUP als hoch anzusehen. Im empirischen Bereich meldete ALI (2009) den höchsten $R^{2}$ korr für die Schätzung der $h_{\text {dom }}$ mit 0,99. Für die 
Schätzung des dGZ aus der $\mathrm{h}_{\text {dom }}$ betrug der $\mathrm{R}^{2}{ }_{\text {korr }}$ 0,92. Damit liegt die Gesamtgenauigkeit des Modell von ALI bei 0,91. BERGANTE et al. (2010) ermittelte Modelle mit einem $\mathrm{R}^{2}{ }_{\text {korr }}$ zwischen 0,54 und 0,62. Beim Modell von AYLOTT et al. (2008) variierten die $\mathrm{R}^{2}$ der Modelle je nach Klon zwischen 0,51 und 0,70. ECKERSTEN et al. (2006) stellte $\mathrm{R}^{2}$ zwischen 0,82 und 0,86 fest. Im mechanistischen Bereich lagen die $\mathrm{R}^{2}$ zwischen 0,75 und 0,98 (AMICHEV et al. 2010), zwischen 0,85 bis 0,91 (TALLIS et al. 2013) sowie bei 0,95 (JING et al. 2013).

Bei den vorgestellten bodenartspezifischen Modellen wurden RMSE zwischen 0,22 und $1,36 \mathrm{t}_{\text {(atro) }} \mathrm{ha}^{-1} \mathrm{a}^{-1}$ festgestellt. Im Mittel für die bodenartspezifischen Modelle betrug der RMSE $0,98 \mathrm{t}_{\text {(atro) }} \mathrm{ha}^{-1} \mathrm{a}^{-1}$. Im Kontext der Literatur zu Modellierung von Pappel und Weide im Kurzumtrieb sind die Ergebnisse des RMSE sehr gut angesiedelt. Bei einem empirischen Modell wurden RMSE zwischen 1,37 und 2,09 $\mathrm{t}_{\text {(atro) }}$ ha $^{-1} \mathrm{a}^{-1}$ registriert (AYLOTT et al. 2008). Bei den mechanistischen Modellen wurden RMSE zwischen 1,46 und 1,53 $\mathrm{t}_{\text {(atro) }} \mathrm{ha}^{-1} \mathrm{a}^{-1}$ (TALLIS et al. 2013), 1,0 $\mathrm{t}_{\text {(atro) }} \mathrm{ha}^{-1} \mathrm{a}^{-1}$ (JING et al. 2013) sowie 2,3 $t_{\text {(atro) }} h^{-1} a^{-1}$ (WERNER et al. 2012) festgestellt. Vergleiche zu Bias, Prognosestreuung sowie Treffgenauigkeit mit anderen Pappel- und Weidenmodellen wurden aufgrund schlechter Informationslage in der Literatur nicht durchgeführt.

Der interessantere Vergleich der hier präsentierten Modellen ist den Modellen von ALI (2009) gegenübergestellt (Tab. 5-2).

Tab. 5-2: Vergleich der Modelle dieser Arbeit mit den Modellen von Ali (2009) in ihren Rahmenbedingungen für die Anwendungen.

\begin{tabular}{lll}
\hline Merkmal & Diese Arbeit & ALI (2009) \\
\hline Baumart/Klon & $\begin{array}{l}\text { Pappel: Max 1, H 275, AF 2 } \\
\text { Weide: Inger und Tordis }\end{array}$ & $\begin{array}{l}\text { Pappel: Max 1, Androscoggin, Matrix, } \\
\text { Münden }\end{array}$ \\
Umtriebszeit & dreijährige & drei- bis neunjährige \\
Betandesdichte & $11.111 \mathrm{Stk}^{-1} \mathrm{ha}^{-1}$ & 1.550 bis 13.000 Stk. ha ${ }^{-1}$ für Max 1 \\
& Beispielshaft für Max 1 über & 1.550 Stk. ha ${ }^{-1}$ für die weitere Klone \\
Stockausschlag & Ertragssteigerungsfaktoren & - \\
Validitätsbereich & Überregional & Sachsen \\
Besonderheiten & Bodenartspezifisch & Variierende Umtriebszeiten und \\
\hline
\end{tabular}

Die wesentlichen Vorteile der hier präsentierten Modelle gegenüber denen von ALI (2009) liegen in der Berücksichtigung von Weidenklonen, im überregionalen Validitätsbereich sowie in der bodenartspezifischen Aussage der Modelle. Die Vorteile der Modelle von ALI stellen offensichtlich die variablen Bestandesdichten und Umtriebszeiten. Der entscheidende Nachteil beider Modelle ist, dass sie keine Bewirtschaftung im Stockausschlag berücksichtigen. Die für Max 1 errechnete Ertragssteigerung (siehe 4.4) ist eine grobe Annährung, welche aufgrund vieler Annahmen und Unsicherheiten (siehe 3.7) noch verifiziert werden muss. Sie ist aber die derzeit einzig vorhandende Informationsquelle für einen repräsentativen Zeitraum der Bewirtschaftung und kann für theoretische Modellberechnungen wie Ökobilanzen oder betriebswirtschaftliche Berechnungen von Nutzen sein.

Obwohl diese Modelle aus der Zeit der Etablierung von KUP entwickelt wurden, die Bestandteile der Modelle in Teilen ähnlich mit denen von ALI (2009), der größere Zeiträume (bis zu neun Jahre) in ihre Arbeit einfließen ließ. Dies ließe die Annahme zu, dass die hier gezeigten leistungsbeeinflussenden Standorteigenschaften nicht nur für die Etablierungsphase gelten, sondern das Pappel- und Weidenwachstum getreu wiedergeben. Dies bedarf auf jeden Fall einer Verifizierung. 


\subsection{Ertragssteigerungsfaktoren und Ertragsteigerung in Folgerotationen}

Die Ertragssteigerung verlief je nach Bodenarten unterschiedlich. Bei den Sandböden war sie zu Beginn sehr schnell, war aber danach von allen Bodenarten die flachste. Dies hängt mit der leichten Erschließung des Bodens durch hohe LK zusammen. Geringe bis mittlere nFK erklären die schwache bis mittlere Ertragsleistung. JOACHIM (1953) beobachtete bei Sandböden ein starkes Jugendwachstum, das später jedoch abklingt. Der Schluffboden hatte die stärkste Ertragssteigerung und das stärkste absolute Ertragspotential. Dies lässt sich mit seinen sehr positiven Eigenschaften für das Wachstum erklären, hierzu zählen u. a. die gute Luft- und Wasserversorgung (JURY et al. 1991). Die Ertragssteigerung verlief beim Lehm zum Anfang hin langsam und wurde mit jeder Rotation stärker, sogar stärker als beim Sandboden. Warum die Ertragssteigerung so verläuft, hängt vermutlich mit der schweren Durchwurzelbarkeit des Bodens zusammen. Mit zunehmender Erschließung des Wurzelraums erhöht sich auch der Zuwachs.

Die Erklärung für die Ertragssteigerung durch wiederholte Erntevorgänge kann in der Entwicklung der Stammzahl gesucht werden (HoRN et al. 2013). Nach jeder Rotation steigt sie an und erlaubt eine optimale Ausnutzung des Standraums. Zudem hat das progressive Wurzelwachstum von Rotation zu Rotation eine ertragsfördernde Wirkung (MAKESCHIN und REHFÜESS 1994).

An den Ergebnissen der Ertragssteigerung wurde sichtbar, dass mittlere dGZ über $12 \mathrm{t}_{\text {(atro) }} \mathrm{ha}^{-1} \mathrm{a}^{-1}$ bei einer Laufzeit von 18 Jahren einzig auf Lössböden erreicht werden. Das heißt, dass ohne einen Züchtungsfortschritt oder Verbesserung durch Management oder Standortmelioration der oft angenommene mittlere Ertrag von 10,0 $\mathrm{t}_{\text {(atro) }} \mathrm{ha}^{-1} \mathrm{a}^{-1}$ nicht möglich ist. Es ist anzumerken, dass diese Aussagen nur den Klon Max 1 betreffen. Die Ertragsentwicklung der kommerziellen starken Weidenklone ist vielversprechend und sollte weiterhin beobachtet werden (siehe $2.5 \mathrm{Abb}$. 2-3).

Die größte Unsicherheit in der Nutzung der bodenartspezifischen standortbasierten Ertragsmodelle mit den Ertragssteigerungsfaktoren betrifft die Gültigkeit der Klimavariablen. Die zugrundeliegenden Klimadaten in den vorgestellten Modellen basieren auf drei Jahren. Insbesondere die Niederschläge weichen stark vom langjährigen Mittelwert ab (siehe 3.2.2) Die echten Witterungs-Zuwachsbeziehungen von Langzeitversuchsflächen sind im Einzelnen nicht bekannt. 


\section{Schlussfolgerungen und Ausblick}

Das Ziel der vorliegenden Arbeit war die Erstellung überregional gültiger, empirisch-statistischer standortbasierter Ertragsmodelle ausgewählter Pappel- und Weidenklone auf Ackerflächen im Kurzumtrieb. Als spezifische Ziele galten: (1) das Wuchsverhalten verschiedener Pappel- und Weidenklone auf vielen Standorten zu erfassen, (2) die Entwicklung von Biomassefunktionen für die Versuchsrahmenbedingungen, (3) die Identifizierung der leistungsbeeinflussenden Standorteigenschaften aus den Bereichen Klima und Boden, sowie (4) die Erstellung überregional gültiger standortbasierter Ertragsmodelle, inklusive Ertragsteigerungsfaktoren.

Da es sich beim Verbundvorhaben PROLOC um die erste überregionale Versuchsreihe zu KUP in kurzer Rotation in Deutschland handelt, haben die erzielten Ergebnisse eine große Tragweite. Die Validität der Ergebnisse ist hoch und geht deutlich über die eines Trends aus einzelnen Versuchsflächen hinaus.

Im Bereich der waldwachstumskundlichen Kennwerte wurde beobachtet, dass die Klone untereinander signifikante Unterschiede im Höhen- und Durchmesserwachstum sowie in der dGZ-Entwicklung in den verschiedenen Standortclustern aufweisen. AF 2 und Tordis waren die stärksten Klone. Die standortspezifischen Unterschiede zwischen den Klonen waren gering. Demnach können die geprüften Klone als standortplastisch bezeichnet werden. Standortspezifische Charakteristika im Wachstum der jeweiligen Pappelklone in Abhängigkeit ihrer Sektionszugehörigkeit konnten, bis auf die Überlegenheit von AF 2 auf schweren Bodenarten, nicht festgestellt werden. Die relativ neuen schwedischen Weidenklone waren den Pappeln in dieser Untersuchungsreihe ebenbürtig.

Im Bereich der Biomassefunktionen wurde festgestellt, dass der Funktionsverlauf von AF 2 deutlich flacher ist als die der anderen Klone. Der Einfluss der Bestandesmittelhöhe auf die Form der Funktion war bei den Pappeln marginal, bei den Weiden fehlte sie völlig. Demnach wurden allgemeingültige Funktionen für die Pflanzdichte von 11.111 Stk. ha ${ }^{-1}$ entwickelt.

Bei der Untersuchung zur Identifizierung der leistungsbeeinflussenden Standorteigenschaften wurde die Mehrheit der Erfahrungen aus der Vergangenheit bestätigt. Entscheidend bei dieser Arbeit war die genaue und bodenartspezifische Quantifizierung der Effekte. Bei den klimatischen Variablen wurde das Prozessverständnis hinsichtlich des Einflusses von Klimagrößen auf das Wachstum, bezogen auf den gegenwärtigen Stand des Wissens, deutlich erweitert. Nach aktuellem Kenntnisstand haben höhere Temperaturen einen positiven Einfluss auf das Wachstum. In der vorliegenden Arbeit wurde aufgezeigt, dass unter Freilandbedingungen oft das Gegenteil der Fall ist. Höhere Sommertemperaturen korrelierten negativ mit dem dGZ. Dieser Effekt war bei den Weidenklonen stärker ausgeprägt als bei den Pappelklonen und traf bei den Lehm- und Sandböden zu. Die Ergebnisse beim Niederschlag und beim Trockenheitsindex bestätigten den hohen Wasserbedarf von KUP wieder und verdeutlichten die Relevanz der verschiedenen Zeitfenster in der Wuchsperiode. Abweichend von den Annahmen aus Literaturquellen, welche der Jahresniederschlagssumme oder der Niederschlagssumme der Vegetationsperiode eine hohe Bedeutung für das Wachstum beimessen, war der Zeitraum der ersten Hälfte der Vegetationsperiode am wichtigsten. Im Bereich des Bodens spiegelten die Merkmale nFK und LK die Wasser- sowie die große Luftbedürftigkeit der Pappeln und Weiden getreu wieder. Die Kennwerte der Bodenschätzung sowie der Textur erwiesen sich als ebenfalls hilfreich für die Modellierung.

Die standortbasierten Ertragsmodelle stellen die Synthese der leistungsbeeinflussenden Standorteigenschaften dar. Je nach Klon und Standortcluster werden die Faktoren Durchwurzelung (Einflussgrößen: $\mathrm{nFK}, \mathrm{LK}, \mathrm{S}_{[\%]}, \mathrm{U}_{[\%]}$ ), Wasserversorgung (Einflussgrößen: nFK, Niederschlag, Trockenheitsindex, Temperatur) und Bodengüte (Einflussgrößen: BZ) berücksichtigt. Die Modelle setzen sich mehrheitlich aus aggregierten Variablen zusammen. Dieser methodische Schritt war offensichtlich für die Mo- 
dellierung sehr wichtig. Die aggregierten Variablen bestanden in der Regel aus der Kombination der bestkorrelierten Variablen aus den Bereichen Klima und Boden. Die Nutzung von nicht-linearen Modellen führte oft zu Verbesserungen in der Aussagekraft der Modelle. Insgesamt wurden sehr hohe Genauigkeiten, als $\mathrm{R}^{2}$ korr angegeben, erzielt. Die Mehrheit der beobachteten Effekte in der standortbasierten Modellierung lassen einen überdurchschnittlichen Einfluss der Wasserverfügbarkeit für das Pappel und Weidenwachstum erkennen. Darüber hinaus wurde in der Modellierung erkannt, dass die Bodeneigenschaften für das Wachstum tendenziell von größerer Bedeutung sind als die klimatischen Kenngrößen.

Die Auswertung der Ertragsteigerung und ihrer Faktoren in Folgerotationen brachte interessante Ergebnisse hervor. Die Ertragssteigerung war klon- und bodenartspezifisch. Nur für die Max-Klone wurden signifikante Ertragssteigerungsfaktoren ermittelt. Bei den Balsampappelklonen war die Ertragssteigerung ab der dritten Rotation marginal. Das verdeutlicht, wie dünn die Klonwahl für die kurze Umtriebszeit unter Nutzung des Stockausschlags ist. Die Ertragssteigerung von wuchsstarken Weidenklonen wie Zieverich und Tora über viele Rotationen ist vielversprechend. Leider lagen für die Entwicklung von Ertragssteigerungsfaktoren für Weidenklone nicht genügend Informationen vor.

Die wesentliche Schwäche der vorliegenden Arbeit war die kurze Beobachtungszeit in Form einer einzigen dreijährigen Rotation. Dementsprechend sind die Aussagen der Ertragsmodellierung bezogen auf die lange Nutzungsdauer einer KUP stark begrenzt. Durch die Herleitung von Ertragsteigerungssfaktoren für die Max-Klone konnte eine Prognose für die Ertragsentwicklung gegeben werden. Aufgrund der größeren Unsicherheiten, welche mit der Entwicklung der Ertragssteigerungsfaktoren behaftet waren, sollten diese vorsichtig angewandt werden. Weitere Grenzen der Versuchsreihe sind die festgelegte Bestandesdichte und Umtriebszeit.

Die Stärken der vorliegenden Arbeit liegen in der präzisen Identifizierung und vor allem Quantifizierung leistungsbeinflussender Standorteigenschaften sowie in der Ermittlung von genauen standort- und klonspezifischen Ertragsmodellen für die erste Rotation von KUP im überregionalen Gültigkeitsbereich. Das weitere Vorgehen sollte wie folgt ablaufen:

- Die Versuchsflächen im Verbundvorhaben sollten langfristig beobachtet werden um das Verhalten der verschiedenen Klone nach einem oder mehreren Rückschnitten hinsichtlich ihrer Überlebensrate und Dimensionsentwicklung zu untersuchen.

- Weitere vielversprechende Weidenzüchtungen zum Beispiel aus England und Schweden sollten unter hiesigen Standortbedingungen auf ihre Ertragsleistungen geprüft werden. Des Weiteren ist die landeseigene Weidenzüchtung voranzutreiben.

- Die Kosten und der Nutzen der Bewässerung von KUP-Flächen sollte erforscht werden. Ein Schwerpunkt der Forschung sollte auf die Auswirkung der Bewässerung in unterschiedlichen Abschnitten der Vegetationsperiode zielen.

- Die Transpirationsregulierung im Sinne einer erhöhten Wassernutzungseffizienz sowie die Hitzetoleranz der Gewächse, insbesondere der Weidenklone, sollte untersucht werden.

- Es ist anzunehmen, dass eine Verbesserung der Bodenphysik eine Ertragssteigerung zur Folge hat. Daher sollte der Einfluss verschiedener Maßnahmen zur bodenphysikalischen Standortmelioration auf das Wachstum, zum Beispiel in Form einer Tiefenlockerung im Etablierungsjahr oder in Form einer Zugabe von strukturgebenden organischen Substanzen wie Kompost oder Rottemist, geprüft werden.

- Es empfiehlt sich vor der Anlage einer KUP eine umfassende bodenkundliche Standortcharakterisierung durchzuführen.

- Es wäre sinnvoll, die empirischen Beobachtungen im Bereich der leistungsbeeinflussenden Faktoren durch Versuche unter kontrollierten oder semikontrollierten Bedingungen oder mit Hilfe von mechanistischen Waldwachstumsmodellen zu validieren. 
- Es sollte geprüft werden, ob eine Verschiebung der relevanten Faktoren für das Wachstum sowohl bei den einzelnen leistungsbeeinflussenden Faktoren als auch bei den standortbasierten Modellen in Folgerotationen stattfindet. Durch weitere Beobachtung der Versuchsflächen ist eine Erweiterung der zeitlichen Validität zu erzielen.

- Für die ermittelten Modelle wäre eine Validierung mittels externer Daten zu empfehlen.

- Anhand der Analyse zur Ertragssteigerung wurde deutlich, dass die Züchtung von Pappelklonen für die Bewirtschaftung mit Stockausschlag vorangetrieben werden muss. 


\section{Zusammenfassung}

Der Anbau von schnell wachsenden Gehölzen auf Ackerflächen gilt mit Maximalerträgen des durchschnittlichen Gesamtzuwachses an Trockenbiomasse (dGZ) von bis $\mathrm{zu} 16,0 \mathrm{t}_{\text {(atro) }}$ ha $^{-1} \mathrm{a}^{-1}$ (BIERTÜMPFEL et al. 2012) und der unter den Bioenergielinien besten Energiebilanz (WBA 2007) als einer der Hoffnungsträger unter den nachwachsenden Rohstoffen. Trotz des hohen Potentials dieses Landnutzungssystems ist seine Flächenausdehnung in Deutschland mit derzeit ca. 4.000 ha eher marginal (STROHM et al. 2012). Einer der Hauptgründe für diese Situation ist die mangelnde Planungssicherheit für den Landwirt. Weil die das Wachstum beeinflussenden Standortfaktoren für Pappel und Weide nicht hinreichend identifiziert und quantifiziert sind, können bundesweite sichere Ertragserwartungen in Abhängigkeit von bodenkundlichen und klimatischen Standortbedingungen auf derzeitiger Informationsgrundlage nicht gegeben werden. Das Hauptziel dieser Arbeit ist daher die Entwicklung überregional gültiger standortbasierter Ertragsmodelle zur Abschätzung von Ertragserwartungen für Pappel und Weide auf Ackerflächen im Kurzumtrieb.

Um dieses Ziel zu erreichen, wurden unter Einbeziehung möglichst aller Regionen Deutschlands auf Standorten, die für einen Anbau schnellwachsender Baumarten in Frage kommen, nach einheitlichem Versuchsdesign 38 Versuchsflächen angelegt. Der Versuch war vollrandomisiert in vierfacher Wiederholung. Ausgesuchte Klone für den Versuch waren die Pappelklone Max 1 ( $P$. nigra $\mathrm{x}$ P. maximowiczii), Hybride 275 (H 275, P. maximowiczii x $P$. trichocarpa) und AF 2 ( $P$. nigra $\mathrm{x}$ $P$. deltoides) sowie die Weidenklone Inger $(S$. triandra $\mathrm{x} S$. viminalis) und Tordis $(S$. viminalis $\mathrm{x}$ S. schwerinii) x $S$. viminalis). Die Pflanzung erfolgte mit einem Pflanzabstand von 1,8*0,5 $\mathrm{m}$ im einreihigen Verband. Dies entspricht einer Bestandesdichte von $11.111 \mathrm{Stk}^{\mathrm{ha}}{ }^{-1}$. Die ausgewählte Umtriebszeit betrug drei Jahre. Für die Erfassung der Massenleistung wurden Zuwachsparameter wie die Höhe, der Durchmesser in 0,1 $\left(\mathrm{d}_{0,1}\right)$ sowie in 1,3 m Stammhöhe $\left(\mathrm{d}_{1,3}\right)$ und der Biomassezuwachs im jährlichen Turnus erhoben. Die Aufnahmen fanden auf insgesamt 24 Pflanzplätzen je Parzelle statt. Des Weiteren erfolgte eine Standortcharakterisierung mit Erfassung klimatischer sowie bodenkundlicher Kennwerte.

Aus den aufgenommenen Standortparametern wurden für die Modellierung Variablen definiert. Im Bereich des Klimas setzten sich die Variablen von Temperatur (T), Niederschlag (N) und Trockenheitsindex (TI) aus Summen oder Mittelwerten ausgewählter Zeitfenster des Jahres und der Vegetationsperiode zusammen. Aus dem Bereich des Bodens wurden als Variablen Kennwerte der Reichsbodenschätzung wie die Zustandsstufe (ZS), die Bodenzahl (BZ) und die Ackerzahl (AZ) verwendet. Die gewählten Kennwerte der Textur waren die prozentualen Gehalte an Sand $\left(\mathrm{S}_{[\%]}\right)$, Schluff $\left(\mathrm{U}_{[\%]}\right)$ und Ton $\left(\mathrm{T}_{[\%]}\right)$. Variablen der Porenraumkenngrößen waren die nutzbare Feldkapazität (nFK), die Luftkapazität (LK) und die Trockenrohdichte (TRD). Sowohl Textur- als auch Porenraumvariablen bezogen sich auf eine Bodentiefe von 0 bis $60 \mathrm{~cm}$. Als abhängige Variable wurde der dGZ in $t_{(\text {atro })}$ je Hektar und Jahr am Ende der ersten dreijährigen Rotation verwendet. Von den 38 Standorten konnten schließlich nur 20 für die Modellierung verwendet werden. Fünf Versuchsflächen wurden aufgrund massiver Störungen oder unvorhergesehener Managementprobleme im Anwuchsjahr bereits vor Ablauf der Beobachtungszeit aufgegeben. Gründe für das Ausscheiden der weiteren Versuchsflächen waren eine stark abweichende Vornutzung als Bergbaufolgelandschaft $(n=3)$, nicht terrestrische organische Bodenarten $(\mathrm{n}=2)$, stark saure $\mathrm{pH}-$ Werte $(\mathrm{n}=1)$ sowie massiver Unkrautdruck $(\mathrm{n}=3)$. Darüber hinaus wurden nach einem flächigen Ausfall drei Versuchsflächen komplett nachgebessert. Eine Versuchsfläche wurde im Jahr 2009 angelegt. Da diese Versuchsflächen die dritte Wuchsperiode nicht erreichten, wurden sie nicht in der Modellierung verwendet.

Zur Präzisierung der Ergebnisse kamen nach Hauptbodenart getrennte Standortcluster sowie die Aggregierung von Variablen zum Einsatz. Die Standortcluster waren zunächst alle untersuchten Standorte 
$(\mathrm{n}=20)$, die Hauptbodenart der Sande $(\mathrm{n}=8)$, der Schluffe $(\mathrm{n}=5)$ sowie der Lehme $(\mathrm{n}=7)$. Die Variablenaggregierung erfolgte über Multiplikation und Division. Für die Identifizierung leistungsbeeinflussender Standorteigenschaften sowie zur Entwicklung standortbasierter Ertragsmodelle wurden die statistischen Methoden der Korrelationsanalyse sowie die univariate und multiple lineare sowie nicht-lineare Regressionsanalyse verwendet. Aufgrund von Ausfällen durch Mortalität konnten nicht für jeden Klon Korrelationen bzw. Regressionen gerechnet werden. Im Standortcluster aller untersuchten Standorte sowie der Schluffe wurden Max 1, AF 2, Inger und Tordis berücksichtigt. Im Standortcluster der Sande konnten alle Prüfglieder, im Standortcluster der Lehme hingegen nur Max 1, AF 2 und Inger berücksichtigt werden.

Im Folgenden werden die Hauptergebnisse dieser Arbeit aufgelistet:

- Die Weidenklone zeigten mit $86 \%$ eine im Mittel ca. $10 \%$ höhere signifikante Anwuchsleistung als die Klone der Pappel. Max 1 hatte unter den Pappeln mit $84 \%$ die beste, H 275 mit $60 \%$ die schlechteste Anwuchsrate.

- Die Anzahl an Höhentrieben war bei den Weiden mit durchschnittlich 1,9 signifikant höher als die der Pappel. Unter den Pappeln hatte H 275 eine signifikant geringere Anzahl an Höhentrieben im Vergleich zu den verbleibenden Klonen.

- Über alle Versuchsflächen variierten die mittleren Bestandeshöhen zwischen 0,4 m und 3,3 m in der ersten, zwischen 0,7 und 5,9 $\mathrm{m}$ in der zweiten sowie zwischen 1,0 und 7,6 $\mathrm{m}$ in der dritten Wuchsperiode (WP). Durchschnittlich waren Tordis und AF 2 mit jeweils 5,2 und 5,1 m Höhe die stärksten Prüfglieder.

- Über alle Versuchsflächen wurde eine Variation im mittleren $d_{1,3}$ zwischen 0,2 und $1,7 \mathrm{~cm}$ in der ersten, zwischen 0,4 und 3,5 cm in der zweiten sowie zwischen 0,4 und 5,0 cm in der dritten WP festgestellt. In der dritten WP waren alle Pappelklone den Weidenklonen signifikant überlegen. Das mittlere jährliche $\mathrm{d}_{1,3}$-Wachstum schwankte bei den Weiden zwischen 0,7 bis $0,9 \mathrm{~cm}$. Bei den Pappeln lag es bei 1,0 cm. Die $\mathrm{d}_{0,1}$ waren durchschnittlich 20 bis $40 \%$ stärker als die $\mathrm{d}_{1,3}$.

- Der dGZ hatte eine gattungsspezifische Entwicklung für jede WP. In der ersten und zweiten WP dominierten die Weidenklone signifikant mit einem dGZ von bis zu 6,9 $\mathrm{t}_{\text {(atro) }}$ ha $^{-1} \mathrm{a}^{-1}$ in der ersten und bis zu 10,1 $\mathrm{t}_{\text {(atro) }} \mathrm{ha}^{-1} \mathrm{a}^{-1}$ in der zweiten WP. In der dritten WP wurden dGZ von 0,1 bis hin zu 11,0 $\mathrm{t}_{\text {(atro) }} \mathrm{ha}^{-1} \mathrm{a}^{-1}$ ermittelt, dort war keine Gattung der anderen überlegen. Über alle Standorte hinweg waren AF 2 und Tordis die stärksten Klone.

- $\mathrm{Zu}$ den leistungsstärksten Standorten gehörten die Lössböden. Weitere Böden, die Spitzenleistungen aufwiesen, waren anlehmige und lehmige Sande. Ebenfalls ertragreich waren die organischen Standorte, auf denen dGZ von bis zu 10,0 $\mathrm{t}_{\text {(atro) }} \mathrm{ha}^{-1} \mathrm{a}^{-1}$ erreicht wurden. Schwere, tonige Böden zeigten mittlere dGZ zwischen 1,0 und 5,0 $\mathrm{t}_{\text {(atro) }}$ ha $^{-1} \mathrm{a}^{-1}$. Sandige Böden mit schlechter Wasserverfügbarkeit wiesen die niedrigsten Wachstumsraten auf.

- Im Bereich der Biomassefunktionen wurden zwischen der Wahl von $\mathrm{d}_{0,1}$ oder $\mathrm{d}_{1,3}$ als unabhängige Variable keine signifikanten Unterschiede festgestellt. Die Funktion des Pappelklons AF 2 hatte einen statistisch belegten, deutlich flacheren Verlauf als die Funktionen der anderen beiden Pappelklone.

- Bei den Pappeln hatte die durchschnittliche Höhe einen marginalen Einfluss auf den Funktionsverlauf, bei den Weiden war der Einfluss nicht vorhanden. Es wurden allgemeingültige Funktionen für die Pflanzdichte von 11.111 Stk. ha ${ }^{-1}$ entwickelt.

- Bei den Sand- und Lehmböden wurden negative Korrelationen zwischen dGZ und Temperaturen in der Vegetationsperiode, insbesondere in den Monaten Juli bis September, festgestellt. Die stärksten Korrelationen wurden bei den Sandböden für beide Weidenklone gleichermaßen 
festgestellt $(r=-0,55)$. Positive Korrelationen mit dem dGZ wurden bei den Schluffbodenarten festgestellt. Dort wurden $r$-Werte von 0,27 bis 0,84 registriert.

- Niederschlagsvariablen hatten bei allen Klonen und Standortclustern stets eine positive Auswirkung auf das Wachstum von Pappel und Weide. Bei Berücksichtigung aller Versuchsflächen waren die Niederschläge im Zeitfenster von Mai bis Juli am wichtigsten. Die r-Werte der verschiedenen Klone schwankten zwischen 0,28 und 0,57. Die r-Werte betrugen bei den Sandböden zwischen 0,64 und 0,87. Bei den Schluffen und Lehmen schwankten die r-Werte zwischen jeweils 0,39 und 0,75 sowie 0,36 und 0,60 . Am bedeutsamsten für das Pappel- und Weidenwachstum in Sandböden war die Niederschlagssumme im Zeitfenster Juni bis Juli. In Schluff- und Lehmböden war das Zeitfenster von Mai bis Juni.

- Die Nutzung von Trockenheitsindex- statt Niederschlagsvariablen brachte nur eine geringe Verbesserung in den Korrelationen, insgesamt konnten annähernd dieselben Beobachtungen hinsichtlich bedeutender Zeitfenster und Stärke der Korrelationen wie beim Niederschlag getroffen werden. Weder beim Niederschlag noch beim Trockenheitsindex konnten Unterschiede zwischen Klonen oder Gattungen festgestellt werden.

- Für alle Standorte erreichten die Kennwerte der Bodenschätzung eine mäßige Korrelation mit dem Wachstum. Die r-Werte schwankten zwischen 0,28 und 0,53. Überraschenderweise zeigte ZS, mit Ausnahme des Sandclusters, die höchste Bedeutung für das Wachstum. Bei den Sanden zeigte AZ die besseren Werte. Der Effekt der Kennwerte der Bodenschätzung war bei den Schluffböden am stärksten. Dort wurden r-Werte von bis zu 0,97 erreicht.

- Anhand der Betrachtung aller Standorte sowie der einzelnen Standortcluster wurde sichtbar, dass $\mathrm{U}_{[\%]}$ die wichtigste Texturvariable für das Pappel- und Weidenwachstum ist. Die r-Werte variierten bei Berücksichtigung aller Standorte zwischen 0,33 und 0,46, bei den Sanden zwischen 0,75 und 0,83 sowie bei den Lehmen zwischen 0,79 und 0,89. Bei den Sanden wurden zusätzlich inverse Korrelationen zwischen $\mathrm{S}_{[\%]}$ mit dem dGZ festgestellt, und zwar fast genauso stark wie mit $\mathrm{U}_{[\%]}$.

- Für alle Standorte gleichzeitig betrachtet, hatte die nFK über alle geprüften Größen die höchste Bedeutung für das Wachstum. Es wurden je nach Klon r-Werte zwischen 0,56 und 0,61 ermittelt. Unerwartet wurden bei den Sandböden eher mäßige Beziehungen mit dem Porenraumvariablen, insbesondere zwischen der nFK mit dem dGZ, festgestellt. Bei den Schluffböden hatte die LK und die TRD eine entscheidende Rolle in der Ertragsbildung. Die r-Werte der Korrelation lagen absolut betrachtet zwischen 0,69 und 0,90. Bei den Lehmböden hatten sowohl nFK als LK sehr starke Beziehungen zum dGZ. Dort wurden r-Werte bis zu 0,93 registriert.

- Insgesamt wurden im Bereich des Bodens keine Unterschiede zwischen den Gattungen und Klonen festgestellt.

- Sowohl die Flächenvornutzung als auch der Bodentyp zeigten einen statistisch gesicherten Einfluss auf dem dGZ. Ein höherer Grundwasserspiegel hatte einen signifikanten positiven Einfluss auf den dGZ.

- Aggregierte Variablen warfen höhere r-Werte auf als nicht aggregierte. Über alle Standorte waren für alle Klone die besten Variablen $\left(\mathrm{nFK} * \mathrm{TI}_{5,7}\right)$. Im Standortcluster der Sande war die beste aggregierte Variable für die Pappel $\left(\mathrm{S}_{[\%]} / \mathrm{TI}_{6,7}\right)$ bzw. für die Weide $\left(\mathrm{S}_{[\%]} * \mathrm{~T}_{7,8}\right)$. Bei den Schluffen waren die bestkorrelierten aggregierten Variablen für einen Klon stets unterschiedlich, zum Beispiel für Max $1\left(\mathrm{BZ} * \mathrm{TI}_{4,5}\right)$. Bei den Lehmen waren die aggregierten Variablen $\left(\mathrm{nFK} * \mathrm{TI}_{5,6}\right)$ sowie $\left(\mathrm{nFK} * \mathrm{~N}_{5,6}\right)$ mit dem dGZ am besten korreliert.

- Die standortbasierten Ertragsmodelle waren allesamt univariat und bestanden oft aus aggregierten Variablen. Weder multiple lineare noch nicht-lineare Regressionen brachten signifikante Modelle hervor. 
- Bei Berücksichtigung aller Standorte waren für Max 1, Inger und Tordis ( $\mathrm{nFK} * \mathrm{TI}_{5,7}$ ), bzw. für AF $2\left(n F K * \mathrm{~N}_{5,7}\right)$ Modellprädiktoren. Die Modelle wurden jeweils mit dem inversen oder sigmoidalen Ansatz gerechnet und hatten $\mathrm{R}^{2}$ korr zwischen 0,45 und 0,64 zur Folge bei RMSE von durchschnittlich 2,0 $\mathrm{t}_{(\text {atro })} \mathrm{ha}^{-1} \mathrm{a}^{-1}$.

- Die Aufteilung in Standortcluster verbesserte die Genauigkeit der Modelle merklich. Im Standortcluster der Sande hatten die Modelle $\mathrm{R}^{2}$ korr von 0,77 bis 0,97 und RMSE von 0,95 bis zu 1,36 $\mathrm{t}_{\text {(atro) }} \mathrm{ha}^{-1} \mathrm{a}^{-1}$. Sie setzten sich für Max 1 und AF 2 aus $\left(\mathrm{U}_{[\%]} * \mathrm{~N}_{6,7}\right)$, für $\mathrm{H} 275$ $\left(\mathrm{S}_{[\%]} / \mathrm{TI}_{6,7}\right)$ sowie für Inger und Tordis aus $\left(\mathrm{S}_{[\%]} * \mathrm{~T}_{7,8}\right)$ zusammen.

- Im Standortcluster der Schluffe konnten signifikante Modelle einzig für die Klone Max 1 und Tordis ermittelt werden. Die Modelle hatten als Prädiktor für Max $1\left(\mathrm{BZ} * \mathrm{TI}_{4,5}\right)$ und für Tordis alleine BZ. Es wurden jeweils $\mathrm{R}^{2}$ korr von 0,84 bzw. 0,95 und RMSE zwischen 0,22 und $0,62 \mathrm{t}_{\text {(atro) }} \mathrm{ha}^{-1} \mathrm{a}^{-1}$ ermittelt. In den Lehmböden setzten sich die Modelle für Max 1 und Inger aus der Variable $\mathrm{nFK}$, für AF 2 aus $\left(\mathrm{nFK} * \mathrm{~N}_{5,6}\right)$ zusammen. Die $\mathrm{R}^{2}$ korr variierten zwischen 0,86 und 0,98 bei RMSE zwischen 0,56 und $1,21 \mathrm{t}_{\text {(atro) }} \mathrm{ha}^{-1} \mathrm{a}^{-1}$.

- Für fast alle Modelle war der Einfluss des bodenbezogenen Anteils der aggregierten Variablen stärker ausgeprägt als jene des klimabezogenen Anteils.

- Eine signifikante Ertragssteigerung in Folgerotationen war nur bei den Max-Klonen gegeben. Bei den Balsampappeln wurden ab der dritten Rotation kaum Ertragssteigerungen festgestellt.

- Für insgesamt sechs Rotationen (dreijährig) unter Nutzung standortbasierter Modelle und bodenartspezifischer Ertragssteigerungsfaktoren sind für die Herleitung von Erträgen für Max 1 klare Abstufungen im dGZ zwischen den Hauptbodenarten zu erkennen. Bei Lehmböden wurde ein dGZ von über 12,0 $\mathrm{t}_{\text {(atro) }} \mathrm{ha}^{-1} \mathrm{a}^{-1}$ kaum erreicht. Bei Sandböden werden diese nur im stärksten Drittel des modellierten Bereiches erreicht. Mehr als die Hälfte des modellierten Bereiches der Schluffböden würden einen dGZ von 12,0 $t_{(\text {atro) }}$ ha $^{-1} \mathrm{a}^{-1}$ übersteigen. Bei den wuchsstärksten Schluffböden würden die größten dGZ laut Berechnungen bei $16,5 \mathrm{t}_{\text {(atro) }} \mathrm{ha}^{-1} \mathrm{a}^{-1}$ liegen.

Der größte Wert dieser Arbeit liegt in der präzisen Identifizierung und vor allem Quantifizierung der leistungsbeeinflussenden Standorteigenschaften und der Entwicklung genauer bodenartspezifischer Ertragsmodelle für Pappel und Weide auf Ackerflächen im Kurzumtrieb. Die Mehrzahl der Erfahrungen, die im Bereich des Bodens gemacht wurden, bestätigte sich. Bei den klimatischen Variablen wurde das Prozessverständnis über den gegenwärtigen Stand des Wissens deutlich erweitert. Es empfiehlt sich, die Modelle auf längere Umtriebszeiten und variierende Bestandesdichten auszudehnen und innerhalb eines längeren Beobachtungszeitraumes zu verifizieren. 


\section{Site-based yield modeling of short rotation coppice with poplar and willow clones}

\section{Summary}

The cultivation of fast growing tree species on arable land is deemed to be one of the most promising alternatives amongst the approaches to the cultivation of renewable resources currently available. The reasons for this are the high maximum yields associated with the average total biomass increment (durchschnittlicher Gesamtzuwachs, dGZ) of up to $16.0 \mathrm{odt} \mathrm{ha}^{-1} \mathrm{a}^{-1}$ (BIERTÜMPFEL et al. 2012) and the fact that this approach exhibits the best energy balance amongst the various bioenergy lines (WBA 2007). In spite of the great potential offered by this land use approach, its application in Germany remains marginal, currently occupying an approximate area of just 4000 ha (STROHM et al. 2012). One of the principle reasons for this situation is the insufficient planning security offered to landowners. As the site factors influencing the growth of poplar and willow have not yet been sufficiently identified and quantified, it is not possible to provide reliable national yield expectations as a function of the prevailing soil and climate conditions at a particular site on the basis of the data currently available. The main objective of this study, therefore, was to develop a supraregional sitebased yield model for the assessment of yield expectations for poplar and willow grown on arable land over short rotations.

In order to achieve this goal, a total of 38 research sites were established across as many regions of Germany as possible, incorporating all sites suitable for the cultivation of fast growing tree species, using a standardised experimental design. The experiment was full randomized with four repetitions. The clones selected for the experiment were the poplar clones Max 1 ( . nigra x P. maximowiczii), Hybride 275 (H 275, P. maximowiczii x P. trichocarpa) and AF 2 (P. nigra $\times$ P. deltoides), and the willow clones Inger (S. triandra $\mathrm{x} S$. viminalis) and Tordis $((S$. viminalis $\mathrm{x} S$. schwerinii) $\mathrm{x}$ S. viminalis). Planting occurred in single rows at a spacing of $1.8 \mathrm{~m} \times 0.5 \mathrm{~m}$. This corresponds to a stocking density of 11111 plants $\mathrm{ha}^{-1}$. The chosen rotation length was three years. The growth parameters recorded to determine the mass growth were the height, the diameter at $0.1 \mathrm{~m}$ in height $\left(\mathrm{d}_{0.1}\right)$ and at $1.3 \mathrm{~m}$ in height $\left(\mathrm{d}_{1.3}\right)$, and the biomass increment. These growth parameters were recorded annually. The recordings took place for a total of 24 planting sites per parcel. Each site was also characterised, on the basis of climate and soil data.

From the site parameters recorded, variables were defined for modelling purposes. The climate variables comprised temperature $(\mathrm{T})$, precipitation $(\mathrm{N})$ and aridity index $(\mathrm{TI})$ derived from total or average values from selected periods during the year and during the vegetation period. Parameters of the German soil appraisal such as the condition grade (Zustandsstufe, ZS), the soil quality index value (Bodenzahl, BZ) and the arable land quality index value (Ackerzahl, AZ) were used as soil variables. The selected texture parameters were the proportions of sand $\left(\mathrm{S}_{[\%]}\right)$, silt $\left(\mathrm{U}_{[\%]}\right)$ and clay $\left(\mathrm{T}_{[\%]}\right)$. Variables of the pore space parameters were the available field moisture capacity (nFK), the air capacity (LK) and the dry bulk density (TRD). Both the texture and the pore space variables referred to a soil depth of 0 to $60 \mathrm{~cm}$. The dGZ, measured in odt ha ${ }^{-1} \mathrm{a}^{-1}$, at the end of the first three-year rotation was chosen as the dependent variable. Only 20 of the 38 sites could ultimately be used for modelling purposes. Five experimental sites were abandoned during the observation period due to either massive disturbances or unforeseen management problems in the year of establishment. Reasons for the exclusion of further experimental sites were severe deviations due to prior use of the land for mining purposes $(n=3)$, non-terrestrial organic soil types $(n=2)$, very acidic $\mathrm{pH}$ values $(\mathrm{n}=1)$ and massive weed competition $(n=3)$. Following a complete crop failure, three other experimental sites were wholly re-established. One additional experimental site was established in 2009. These 
experimental sites were not used for modelling purposes as they failed to complete the third year of cultivation in time. To increase the precision of the results, site clusters were derived, differentiated by main soil type, and the variables were aggregated. The site clusters initially comprised all available experimental sites $(n=20)$, the predominantly sandy sites $(n=8)$, the silty sites $(n=5)$ and the loamy sites $(n=7)$. The aggregation of the variables took place by means of multiplication and division.

The statistical methods employed to identify the performance-influencing site characteristics and for the development of site-based yield models were correlation analysis, univariate and multiple linear regression, and non-linear regression analysis. Correlations and/or regressions were not possible for every clone due to losses as a result of mortality. For the site clusters all sites and silty sites, the clones considered were Max 1, AF 2, Inger and Tordis. All clones could be considered for the sandy sites, whereas for the loamy sites only Max 1, AF 2 and Inger could be considered.

The main results of the study are outlined in the following:

- At $86 \%$, the willow clones exhibited a significantly higher initial growth than the poplar clones of an average of approximately $10 \%$. Max 1 was the best of the poplars with $84 \%$, whereas $\mathrm{H} 275$ had the poorest initial growth rate of $60 \%$.

- The number of vertical shoots was significantly higher for the willows than for the poplars with an average of 1.9. Amongst the poplars, H 275 had a significantly lower number of vertical shoots than the other clones.

- The average stand heights varied between 0.4 and $3.3 \mathrm{~m}$ across all experimental sites in the first growth period (WP), between 0.7 and $5.9 \mathrm{~m}$ in the second and between 1.0 and $7.6 \mathrm{~m}$ in the third. On average, Tordis and AF 2 were the strongest species with heights of $5.2 \mathrm{~m}$ and $5.1 \mathrm{~m}$, respectively.

- Across all experimental sites a variation in the mean $d_{1.3}$ of between 0.2 and $1.7 \mathrm{~cm}$ was observed in the first WP, between 0.4 and $3.5 \mathrm{~cm}$ in the second and between 0.4 and $5.0 \mathrm{~cm}$ in the third. By the third WP, all poplar clones were significantly superior to the willows. The mean annual $\mathrm{d}_{1.3}$ growth varied between 0.7 and $0.9 \mathrm{~cm}$ in the willows. In the case of the poplars the figure was $1.0 \mathrm{~cm}$. The $\mathrm{d}_{0.1}$ values were an average of 20 to $40 \%$ higher than the $\mathrm{d}_{1.3}$.

- The dGZ exhibited a genus-specific development for each WP. In the first and second WP the willow clones dominated significantly, with a dGZ of up to 6.9 odt ha $\mathrm{a}^{-1} \mathrm{a}^{-1}$ in the first and up to $10.1 \mathrm{odt} \mathrm{ha}^{-1} \mathrm{a}^{-1}$ in the second. In the third WP dGZ values of 0.1 to $11.0 \mathrm{odt} \mathrm{ha}^{-1} \mathrm{a}^{-1}$ were recorded, with no clear advantage to either genus. AF 2 and Tordis were the strongest clones across all sites.

- The loess soils were amongst the most productive sites. Other soils revealing high growth performances were slightly loamy and loamy sands. The organic sites achieving a dGZ of up to $10.0 \mathrm{odt} \mathrm{ha}^{-1} \mathrm{a}^{-1}$ also proved productive. Heavy, clayey soils exhibited mean dGZ values of between 1.0 and 5.0 odt ha $\mathrm{a}^{-1}$. Sandy soils with poor water availability produced the lowest growth rates.

- With respect to the biomass functions, no significant differences were observed between the selection of either $\mathrm{d}_{0.1}$ or $\mathrm{d}_{1.3}$ as independent variables. The poplar clone AF 2 had a statistically verified flatter curve than that exhibited by the functions for the other poplar clones.

- The average height had a marginal influence on the biomass function curves for the poplars. No such influence was apparent for the willows. Generally applicable biomass functions were developed for stands with a stocking density of 11111 plants ha $^{-1}$.

- Negative correlations between dGZ and temperatures in the vegetation period, especially in the months July to September, were observed on the sandy and loamy soils. The strongest correlations on the sandy soils were identified for the two willow clones (in both cases 
$\mathrm{r}=-0.55)$. Positive correlations with the $\mathrm{dGZ}$ were revealed for the silty soil types, where $\mathrm{r}$ values of 0.27 to 0.84 were registered.

- Precipitation variables always had a positive effect on the growth of poplar and willow across all clones and site clusters. Taking into consideration all experimental sites, precipitation in the period from May to July was most important. The r-values for the different clones varied between 0.28 and 0.57 . The r-values for the sandy soils were between 0.64 and 0.87 . In the case of the silts and the loams the r-values ranged between 0.39 and 0.75 and 0.36 and 0.60 , respectively. Of greatest significance for the growth of poplars and willows on sandy soils was the total precipitation in the period June to July. In the case of silty and loamy soils it was the period from May to June.

- The application of aridity index variables rather than precipitation variables resulted in only a slight improvement in the correlations. Overall the observations with regard to the important timeframe and the strength of the correlations were largely the same as those revealed for precipitation. No differences between clones or genera could be identified using either precipitation or the aridity index.

- The parameters of the German soil appraisal revealed a moderate correlation with growth for all sites. The r-values ranged from 0.28 to 0.53 . Surprisingly, ZS revealed, with the exception of the sandy soil cluster, the greatest relevance for growth. In the case of the sandy soils the AZ produced better values. The effect of the parameters of the German soil appraisal was highest for the silty soils, where r-values of up to 0.97 were revealed.

- From the appraisal of all sites and of the individual site clusters it became apparent that $\mathrm{U}_{[\%]}$ represents the most important texture variable for poplar and willow growth. The r-values for all sites varied between 0.33 and 0.46 , between 0.75 and 0.83 for the sandy soils and between 0.79 and 0.89 for the loamy soils. Additionally, in the case of the sandy soils, inverse correlations between the percent sand content and the $\mathrm{dGZ}$ were identified. These were almost equally as strong as for $\mathrm{U}_{[\%]}$.

- Considered for all sites simultaneously, the nFK had the greatest significance for growth across all of the parameters examined. Depending on the clone, $r$-values of between 0.56 and 0.61 were calculated. Unexpectedly, only moderate relationships with the pore space variables were identified for the sandy soils, especially between the $\mathrm{nFK}$ and the dGZ. In the case of the silty soils the LK and TRD played decisive roles in the yield development. The absolute $\mathrm{r}$-values revealed by the correlation lay between 0.69 and 0.90 . On the loamy soils $\mathrm{nFK}$ and LK were both strongly related to dGZ, with r-values of up to 0.93 registered.

- Overall there were no differences identified between the genera and clones with respect to soil.

- Both the previous land use and the soil type exerted a statistically significant influence on the dGZ. A higher groundwater level revealed a significant positive influence on the dGZ.

- Aggregated variables produced higher r-values than non-aggregated. The best variables across all sites and clones were $\left(\mathrm{nFK} * \mathrm{TI}_{5.7}\right)$. In the case of the sandy soils cluster, the best aggregated variable for poplar was $\left(\mathrm{S}_{[\%]} / \mathrm{TI}_{6.7}\right)$ and $\left(\mathrm{S}_{[\%]} * \mathrm{~T}_{7.8}\right)$ for willow. In the case of the silty soils, the best correlated aggregated variables for a particular clone varied; for example, $\left(\mathrm{TI}_{4.5} * \mathrm{BZ}\right)$ for Max 1. The aggregated variables $\left(\mathrm{nFK} * \mathrm{TI}_{5.6}\right)$ and $\left(\mathrm{nFK} * \mathrm{~N}_{5.6}\right)$ revealed the best correlations in the loamy soils.

- The site-based yield models were all univariate and often comprised aggregated variables. Neither multiple linear nor non-linear regressions produced significant models.

- Under consideration of all sites, the model predictors for Max 1, Inger and Tordis were $\left(\mathrm{nFK} * \mathrm{TI}_{5.7}\right)$ and $\left(\mathrm{nFK} * \mathrm{~N}_{5.7}\right)$ for AF 2. Each model was calculated with the inverse or 
sigmoid approach and revealed an $\mathrm{R}^{2}$ korr between 0.45 and 0.64 with a RMSE of an average of 2.0 odt ha ${ }^{-1} \mathrm{a}^{-1}$

- The division into site clusters improved the accuracy of the models considerably. In the sandy site cluster, the models exhibited an $\mathrm{R}^{2}$ korr of 0.77 to 0.97 and an RMSE of 0.95 to 1.36 odt ha ${ }^{-1} \mathrm{a}^{-1}$. These comprised $\left(\mathrm{U}_{[\%]} * \mathrm{~N}_{6.7}\right)$ for Max 1 and $\mathrm{AF} 2,\left(\mathrm{~S}_{[\%]} / \mathrm{TI}_{6.7}\right)$ for $\mathrm{H} 275$ and $\left(\mathrm{S}_{[\%]} * \mathrm{~T}_{7.8}\right)$ for Inger and Tordis.

- For the silty site cluster, significant models could only be determined for the clones Max 1 and Tordis. The model predictor for Max 1 was $\left(\mathrm{BZ}^{*} \mathrm{TI}_{4.5}\right)$ and for Tordis solely (BZ). The calculated $\mathrm{R}^{2}$ korr values were 0.84 and 0.95 with a corresponding RMSE of 0.22 and 0.62 odt ha ${ }^{-1} \mathrm{a}^{-1}$, respectively. For the loamy soils the models for Max 1 and Inger comprised the variable $\mathrm{nFK}$, for $\mathrm{AF} 2$ the variables $\left(\mathrm{nFK} * \mathrm{~N}_{5.6}\right)$. $\mathrm{R}^{2}$ korr varied between 0.86 and 0.98 with RMSE between 0.56 and 1.21 odt ha $^{-1} \mathrm{a}^{-1}$.

- The influence of the soil-related proportion of the aggregated variables was more pronounced for almost all models than the climate-related proportion.

- A significant increase in yield in further rotations was evident only for the Max clones. In the case of the balsam poplars, yield increases after the third rotation were found to be negligible.

- Over a total of six rotations (3 years each), using site-based models and soil type specific yield increase factors for the derivation of yields for the clone Max 1, there were clear graduations apparent in the dGZ between the main soil types. On loamy soils a dGZ of over 12.0 odt ha $^{-1} \mathrm{a}^{-1}$ was rarely achieved. On sandy soils these yields were achieved only in the strongest third of the area modelled. More than half of the modelled area for silty sites surpassed a dGZ of $12.0 \mathrm{odt} \mathrm{ha}^{-1} \mathrm{a}^{-1}$. According to the calculations, the most productive silty sites would attain highest dGZ values of 16.5 odt ha $^{-1} \mathrm{a}^{-1}$.

The greatest merit of this study lies in the precise identification and especially the quantification of the performance-influencing site characteristics and the development of accurate, soil type specific yield models for poplar and willow cultivated on arable land over short rotations. The majority of the experiences made with respect to soils to date could be verified. In the case of the climate variables, the understanding of the prevailing process has been advanced considerably compared to the previous level of knowledge. It is recommended, however, that the models be expanded to apply to longer rotation lengths and variable stocking densities, and that these be verified over a longer period of observation. 


\section{Modelamiento del rendimiento de plantaciones de rotación corta con clones de álamo y sauce en función de variables del sitio}

\section{Resúmen}

El cultivo de especies arbóreas de crecimiento rápido en suelos agrícolas representa uno de los recursos renovables más prometedores del futuro. Entre las razones, destacan los altos rendimientos que alcanzan 16,0 toneladas secas por hectáreas anualmente $\left(\mathrm{t}_{\text {(seca) }} \mathrm{ha}^{-1} \mathrm{a}^{-1}\right)$ (BIERTÜMPFEL et al. 2012) y un adecuado balance de energético entre las diversas líneas de bioenergía (WBA 2007). A pesar del alto potencial de este sistema de uso de suelo, su extension total en Alemania alcanza sólo a 4.000 ha.

Una de las principales razones para esta superficie marginal, es la falta de seguridad en la planificación del cultivo para el agricultor. Los factores de sitio que condicionan el crecimiento de álamos y sauces no han sido identificados ni cuantificados de forma suficiente, por lo cual no es posible con la base de información actual entregar pronósticos de rendimiento en función de parámetros climáticos y edafológicos. Consecuentemente el objetivo principal de este trabajo es desarrollar modelos de rendimiento de álamos y sauces cultivados en sitios agrícolas en corta rotación en función de parámetros de sitio en el nivel supraregional.

Se establecieron un total 38 sitios experimentales, procurando incluir todas las regiones edafoclimáticas de Alemania, que son consideradas aptas para el cultivo de estas especies de crecimiento rápido. El diseño experimental fue de bloques completos al azar con con cuatro repeticiones. Los clones seleccionados fueron los álamos Max 1 (P. nigra x P. maximowiczii), Hybride 275 (H 275, P. maximowiczii x P. trichocarpa) y AF 2 (P. nigra x P. deltoides) y para los clones de sauce se incluyó a Inger ( $S$. triandra x $S$. viminalis) y Tordis ( $S$. viminalis x $S$. schwerinii) x S. viminalis). La plantación fue realizada en fila simple con una distancia de $1,8 \mathrm{~m}$ entre filas $* 0,5 \mathrm{~m}$ en la fila. Esto es equivalente a una densidad de establecimiento de 11.111 individuos por hectárea. La duración de rotación elegida asciende a tres años. Para la evaluación del rendimiento se obtuvieron anualmente parámetros: la altura total $(\mathrm{m})$, el diámetro medido en $0,1\left(\mathrm{~d}_{0,1}\right)$ y en $1,3 \mathrm{~m}$ de altura $\left(\mathrm{d}_{1,3}\right)$ asi como el incremento en biomasa. Las mediciones tuvieron lugar en un total de 24 individuos por parcela. Además se realizó una caracterización de sitio con registro de variables y parámetros climáticos y edafológicos.

Se registraron diversas variables y parámetros de sitio, los cuales fueron base para el posterior análisis de modelamiento del incremento de la biomasa. Para el clima se registraron: la temperatura (T), las precipitaciones $(\mathrm{N})$ y el índice de aridez (TI), formadas como sumatorias o promedios de distintos intervalos de tiempo del año o del periodo vegetacional. Para el suelo se utilizaron como variables parámetros de la evaluación oficial alemana de suelos como la "condición del suelo" (Zustandsstufe, ZS), el "número de suelo" (Bodenzahl, BZ) y el "número de campo" (Ackerzahl, AZ). Los parámetros del área de la textura del suelo elegidos como variables fueron los contenidos porcentuales de arena $\left(\mathrm{S}_{[\%]}\right)$, limo $\left(\mathrm{U}_{[\%]}\right)$, y arcilla $\left(\mathrm{T}_{[\%]}\right)$. Las variables del área del cuerpo poroso del suelo fueron la capacidad de campo (nFK), la capacidad de aire (LK) y la densidad aparente del suelo (TRD). Las variables de textura y del cuerpo poroso incluyeron una profundidad del suelo entre 0 y $60 \mathrm{~cm}$. Como variable dependiente fue elegida el rendimiento promedio anual (dGZ) en $\mathrm{t}_{\text {(seca) }} \mathrm{ha}^{-1} \mathrm{a}^{-1}$, incluyendo una rotación de tres años. De los 38 sitios experimentales inicalmente planificados sólo pudieron ser incluidos para el análisis de modelamiento 20 sitios. Cinco sitios fueron cancelados durante el periodo de observación a causa de masivas dificultades y problemas de manejo en el año de plantación. Las razones para la no consideración de los restantes sitios son diversas y tienen relación con problemas de alta acidez de suelo $(n=1)$, uso anterior del suelo $(n=3)$, tipos de suelos $(n=2)$ y presencia de 
vegetación competidora $(\mathrm{n}=3)$. Todos aspectos que alterarían la evaluación objetiva de los factores de análisis considerados para este trabajo (genotipo x sitio). Por lo demás hubo que establecer nuevamente tres sitios experimentales que fallaron completamente en el año de plantación, además un sitio experimental fue establecido en el año 2009. Ya que estos sitios no alcanzaron el tercer periodo de crecimiento, no pudieron ser tomados en cuenta en el modelaje.

Con el fin de obtener resultados más precisos se realizaron agrupaciones de sitios en cluster en función del grupo textural asi como de la combinación de variables. La agrupación de sitios fue primero todos los sitios examinados $(n=20)$, luego en función del grupo textural de arenas $(n=8)$, del grupo textural de limos $(n=5)$, asi como de los suelos francos $(n=7)$, la combinación de variables se realizó mediante multiplicación y división.

Para la identificación de los parámetros de sitios que condicionan el rendimiento, asi como para el desarrollo de los modelos de rendimiento en base a parámetros de sitios, se utilizaron los métodos estadísticos del análisis de correlación y de regresión univariante, múltiple linear y no-linear. Producto de las pérdidas por mortalidad, no pudieron calcularse correlaciones y regresiones para cada clon. En el cluster de todos los sitios experimentales, asi como de los suelos limosos pudieron ser considerados los clones Max 1, AF 2, Inger y Tordis. En el cluster de los suelos arenosos pudieron ser considerados todos los clones, por otra parte en el cluster de los suelos francos solo Max 1, AF 2 e Inger.

Entre los principales resultados del presente trabajo se destacan:

- Los clones de sauce alcanzaron en promedio un $86 \%$ de sobrevivencia significativamente mayor a los álamos en $10 \%$. El clon Max 1 alcanzó entre los clones de álamo la mayor sobrevivencia con un promedio $84 \%$, H 275 tuvo con $60 \%$ la más baja.

- El número de vástagos de los clones de sauce fue con en promedio 1,9 significativamente mayor que para los álamos. Entre los clones de álamo H 275 tuvo una cantidad significativamente menor de vástagos que el resto de clones.

- Considerando todos los sitios experimentales, las alturas totales promedio variaron entre $0,4 \mathrm{y}$ 3,3 $\mathrm{m}$ en el primer periodo de crecimiento (WP), en el segundo entre 0,7 y 5,9 $\mathrm{m}$ y en el tercero entre 1,0 y 7,6 m. Los clones Tordis y AF 2 fueron con alturas promedio de 5,2 y 5,1, respectivamente,los clones más desarrollados.

- Bajo consideración de todos los sitios experimentales, se registró una variación del $\mathrm{d}_{1,3}$ promedio entre 0,2 y $1,7 \mathrm{~cm}$ en el primer WP, entre 0,4 y $3,5 \mathrm{~cm}$ en el segundo y entre 0,4 y $5,0 \mathrm{~cm}$ en el tercero. Al finalizar el tercer WP los clones de álamo mostraron un significativo mayor diámetro en comparación con los clones de sauce. El crecimiento anual del $\mathrm{d}_{1,3}$ varió en los clones de sauce entre 0,7 y $0,9 \mathrm{~cm}$. Para los álamos el promedio alcanzó al $1,0 \mathrm{~cm}$ por año. Los valores de $\mathrm{d}_{0,1}$ fueron en promedio entre 20 y $40 \%$ mayores que los $\mathrm{d}_{1,3}$.

- El desarrollo del dGZ en cada WP fue dependiente del género. En el primer y segundo WP dominaron los clones de sauce en términos de rendimiento con dGZ de hasta $6,9 \mathrm{t}_{\text {(seca) }} \mathrm{ha}^{-1} \mathrm{a}^{-1}$ en el primer WP y hasta $10,1 \mathrm{t}_{\text {(seca) }} \mathrm{ha}^{-1} \mathrm{a}^{-1}$ en el segundo WP. En el tercer WP se obtuvieron dGZ más variados, entre 0,1 y $11,0 \mathrm{t}_{\text {(seca) }} \mathrm{ha}^{-1} \mathrm{a}^{-1}$, sin embargo no hubo un género dominante.

- Los sitios de mayor rendimiento fueron aquellos de sustrato limoso. Otros suelos con alto potencial fueron arenas francas, asi como suelos orgánicos en los que se obtuvieron dGZ de hasta $10,0 \mathrm{t}_{\text {(seca) }} \mathrm{ha}^{-1} \mathrm{a}^{-1}$. Suelos arcillosos mostraron dGZ entre 1,0 y $5,0 \mathrm{t}_{\text {(seca) }} \mathrm{ha}^{-1} \mathrm{a}^{-1}$. Suelos arenosos con baja disponibilidad de agua tuvieron los rendimientos más bajos.

- En las funciones de biomasa no se obtuvieron diferencias significativas en la elección de $\mathrm{d}_{0,1} \mathrm{o}$ $\mathrm{d}_{1,3}$ como variable independiente. La función de producción del clon AF 2 tuvo una pendiente significativamente un curso más baja que las restantes de los clones de álamo. 
- La altura promedio no mostró influencia significativa como variable independiente en las funciones de álamo ni de sauce. Finalmente se sólo se ajustaron funciones generales para la densidad de plantación de 11.111 individuos $\mathrm{ha}^{-1}$.

- En los suelos arenosos y francos se registraron correlaciones negativas entre dGZ y las temperaturas del periodo vegetacional, especialmente en los meses de Julio a Septiembre. Las mayores correlaciones se obtuvieron para los clones de sauce (para ambos clones $r=-0,55$ ). En los suelos limosos ocurrieron correlaciones positivas, variando los valores del coeficiente de correlación entre 0,27 y 0,84 .

- Las variables de las precipitaciones tuvieron para todos los clones y grupos de sitios un efecto positivo para el crecimiento de ambas especies de clones. Considerando todos los sitios, las precipitaciones entre mayo y julio fueron las más relevantes. La correlación entre esta variable y los clones variaron entre 0,28 y 0,57. Para los sitios, especialmente para arenas, la correlación vario entre 0,64 y 0,87 . Los sitios con texturas intermedias, limosa y franca, la correlación fluctuó entre 0,39 y 0,75 , asi como entre 0,36 y 0,60, respectivamente. El intervalo de tiempo más relevante para el crecimiento de álamos y sauces en los suelos arenosos fue entre junio y julio. En cambio, para los suelos limosos y francos, el intervalo más importante fue entre mayo y junio.

- La utilización del índice de aridez, en vez de las variables de precipitaciones, generó una leve mejora en la correlación. En general, se observaron resultados similares correspondientes a los intervalos de tiempo relevantes y al nivel de correlación. $\mathrm{Ni}$ en las variables de las precipitaciones o del índice de aridez se observaron diferencias entre clones o géneros.

- Considerando todos los sitios experimentales, se obtuvo una correlación mediana entre párametros de la evaluación oficial alemana de suelos y el crecimiento. La correlación varió entre 0,28 y 0,53 . Inesperadamente tuvo ZS, con excepción del grupo de las arenas, el mayor efecto sobre el crecimiento. En las arenas mostró AZ las mejores correlaciones. El efecto de los párametros de la evaluación oficial alemana de suelos fue el mayor en los suelos limosos, alcanzando registros de 0,97 .

- Contemplando todos los sitios experimentales asi como los grupos individuales fue evidente que $\mathrm{U}_{[\%]}$ es la variable de textura más importante para el crecimiento de álamos y sauces. La correlación varió entre 0,33 y 0,46 considerando todos los sitios experimentales, entre 0,33 y 0,46 en las arenas, entre 0,75 y 0,83 en los suelos francos. Además se registraron correlaciones negativas en las arenas entre $\mathrm{S}_{[\%]}$ y el dGZ. Estas tuvieron un valor comparable con las correlaciones entre $\mathrm{U}_{[\%]}$ y el dGZ.

- Considerando todos los sitios experimentales tuvo nFK la mayor influencia en el crecimiento. Dependiendo del clon, se registraron correlaciones entre 0,56 y 0,61. Inesperadamente se observaron en los suelos arenosos sólo relaciones medias entre variables del cuerpo poroso y el dGZ, especialmente con el nFK. En los suelos limosos tuvieron las variables LK y TRD un efecto decisivo en el rendimiento. Los valores absolutos de correlación oscilaron entre 0,69 y 0,90. En los suelos francos tuvieron ambas variables $\mathrm{nFK}$ y LK una relación muy fuerte con el dGZ, registrándose valores cercanos a 0,93.

- En los análisis de correlación y regresión no se observaron diferencias entre los clones o géneros en las variables del área del suelo.

- El uso anterior del terreno asi como el tipo de suelo mostraron un efecto significativo con el dGZ. Un nivel freático más alto tuvo un efecto positivo con el incremento de biomasa.

- Las variables compuestas mostraron mayores correlaciones que las no compuestas. Considerando todos los sitios experimentales y todos los clones, la variable ( $\left.\mathrm{nFK} * \mathrm{TI}_{5,7}\right)$ mostró el mejor desempeño. En el grupos de las arenas fue $\left(\mathrm{S}_{[\%]} / \mathrm{TI}_{6,7}\right)$ la mejor variable para los clones de álamo y $\left(\mathrm{S}_{[\%]} * \mathrm{~T}_{7,8}\right)$ para los clones de sauce. En los suelos limosos las variables 
agregadas mostraron la mejor correlación según el clon, para Max 1 por ejemplo fue (BZ * $\left.\mathrm{TI}_{4,5}\right)$. En los francos fueron las variables $\left(\mathrm{nFK} * \mathrm{TI}_{5,6}\right)$ y $\left(\mathrm{nFK} * \mathrm{~N}_{5,6}\right)$, las que mostraron la mayor correlación con el dGZ.

- Los modelos de rendimiento, en función a parámetros de sitio, fueron todos univariantes y tenían como componente principal una variable compuesta. Ni modelos lineares múltiples ni regresiones no-lineares dieron como resultado modelos significativos.

- Bajo consideración de todos los sitios experimentales fueron para Max 1, Inger y Tordis $\left(\mathrm{nFK} * \mathrm{TI}_{5,7}\right)$, para AF $2\left(\mathrm{nFK} * \mathrm{~N}_{5,7}\right)$ los predictores del modelo. Los modelos fueron calculados con el enfoque inverso o sigmoidal y los coeficientes de determinación variaron entre 0,45 y 0,64 con RMSE promedio de 2,0 $\mathrm{t}_{\text {(seca) }} \mathrm{ha}^{-1} \mathrm{a}^{-1}$.

- La agrupación de los sitios experimentales mejoró claramente la exactidud de los modelos. En las arenas mostraron los modelos coeficientes de determinación entre 0,77 y 0,97 y RMSE entre 0,95 y $1,36 \mathrm{t}_{\text {(seco) }} \mathrm{ha}^{-1} \mathrm{a}^{-1}$. Los modelos incluyeron las variables de $\left(\mathrm{U}_{[\%]} * \mathrm{~N}_{6,7}\right)$ para Max 1 y AF 2, asi como por $\left(\mathrm{S}_{[\%]} / \mathrm{TI}_{6,7}\right)$ para $\mathrm{H} 275$ y por $\left(\mathrm{S}_{[\%]} * \mathrm{~T}_{7,8}\right)$ para Inger y Tordis.

- En los sitios con suelos limosos se obtuvieron modelos significativos sólo para los clones Max 1 y Tordis. Los modelos tuvieron para Max $1\left(\mathrm{BZ} * \mathrm{TI}_{4,5}\right)$ como predictor, para Tordis sólo (BZ). Se registraron coeficientes de determinación entre 0,84 y 0,95 y RMSE entre 0,22 y $0,62 \mathrm{t}_{\text {(seca) }} \mathrm{ha}^{-1} \mathrm{a}^{-1}$. En los suelos francos los modelos consideraron la variable nFK para Max 1 e Inger, asi como $\left(\mathrm{nFK} * \mathrm{~N}_{5,6}\right)$ para AF 2.

- El efecto del componente edafológico tuvo para casi la totalidad de los modelos mayor importancia que el componente climático.

- Un aumento significativo del rendimiento en rotaciones posteriores fue sólo registrado para los clones Max. Los álamos balsámicos a partir de la tercera rotación practicamente no mostraron un aumento del rendimiento.

- Usando los modelos de rendimiento a base de parámetros de sitio, y los factores de aumento de rendimiento específicos para distintas texturas de suelo, se registraron diferencias del dGZ entre los principales grupos texturales para en total seis rotaciones (de tres años). En los suelos francos practicamente no se observaron dGZ superiores a 12,0 $\mathrm{t}_{\text {(seca) }} \mathrm{ha}^{-1} \mathrm{a}^{-1}$. En los suelos arenosos sólo pudo ser alcanzado este nivel de rendimiento en el tercio más fuerte del intervalo modelado. Mas de la mitad del intervalo modelado en los suelos limosos superaría un límite de 12,0 $\mathrm{t}_{\text {(seca) }} \mathrm{ha}^{-1} \mathrm{a}^{-1}$ del dGZ. En los suelos limosos más fértiles se obtendrían dGZ máximos de $16,5 \mathrm{t}_{\text {(seca) }} \mathrm{ha}^{-1} \mathrm{a}^{-1}$.

El mayor valor de este trabajo, yace en la precisa identificación y cuantificación de los parámetros de sitio, que condicionan el rendimiento de cultivos clonales asociados a álamos y sauces; así como en la identificación de los factores que se relacionan con el aumento de rendimiento, como son las distintas texturas de suelo de uso agrícola en plantaciones de rotaciones cortas. La mayoría de las experiencias del pasado en el ámbito del suelo, fueron corroboradas por este estudio. En las variables climáticas se expandió enormemente el nivel de comprensión de los procesos más allá del estado actual de conocimiento, de todas maneras se recomienda expandir los modelos a rotaciones más largas y diferentes densidades de plantaciones, y verificar su comportamiento con un periodo de observación más largo. 


\section{Literatur}

AG BODEN (AD-HOC ARBEITSGRUPPE BODEN) (2005): Bodenkundliche Kartieranleitung. 5. Auflage. Bundesanstalt für Geowissenschaften und Rohstoffe in Zusammenarbeit mit den Staatlichen Geologischen Diensten. Schweizerbart'sche Verlagsbuchhandlung, Hannover.

AGENTUR FÜR ERNEUERBARE ENERGIEN (2012): Jährliche Ausgaben Deutschlands für den Import fossiler Energieträger von 2000 bis 2011. Stand 08/2012. Berlin (URL: http://www.unendlich-vielenergie.de/uploads/media/Energieausgaben_Deutschland_aug12_linie.pdf, Zugriff am 09.07.2013).

AgENTUR FÜR ERNEUERbARE ENERGIEN (2013): FAKTEN. Die wichtigsten Daten zu den Erneuerbaren Energien. Schnell und kompakt. Stand 05/2013. Berlin (URL: http://www.unendlichviel-energie.de/uploads/tx_nawikeebasket/AEE_TalkingCards_2013_Jun13.pdf, Zugriff am 09. Juli 2013).

AHMAN I.; LARSSON, S. (1994): Genetic improvement of willow (Salix) as a source of bioenergy. Proceeding. In: Norwegian Journal of Agricultural Sciences (Hrsg.): The International Energy Agency (IEA), The Bioenergy Agreement. Task VIII. Konferenz vom 4. bis 9. September 1994, Biri. Supplement $\mathrm{N}^{\circ} 18$, As, S. 47-56.

ALI, W. (2009): Modelling of biomass production potential of poplar in short rotation on agricultural lands of Saxony, Germany. Dissertation. Fachrichtung Forstwissenschaften, Technische Universität Dresden, Dresden.

Alloway, B.J. (1995): Vorgänge in Böden und das Verhalten von Schwermetallen. In: Alloway, B.J. (Hrsg.): Schwermetalle in Böden. Springer, Berlin, Heidelberg.

ALRIKSSON, B. (1997): Influence of site factors on Salix growth with emphasis on nitrogen response under different soil conditions. Dissertation. Acta Universitatis agriculturae Sueciae, Silvestria 46. Swedish University of Agricultural Sciences, Uppsala.

AMichev, B.Y.; Johnston, M.; VAn ReES, K.C.J. (2010): Hybrid poplar growth in bioenergy production systems: Biomass prediction with a simple process-based model (3PG). Biomass and Bioenergy 34: 687-702.

AMicheV, B. Y.; HANGS, R. D.; VAN ReES, K. C. J. (2011): A novel approach to simulate growth of multi-stem willow in bioenergy production systems with a simple process-based model (3PG). Biomass and Bioenergy 35: 473-488.

Amthauer Gallardo, D.; Seymour, D. (2011): Rindenanteile und Schegehalte von Pappel- und Weidenhybriden. Vortrag. In: NORDWESTDEUTSCHE FORSTLICHE VERSUCHSANSTALT UND KomPETENZZENTRUM HeSSENROHSTOFFE (HeRo) E.V. Symposium „Züchtung und Ertragsleistung schnellwachsender Baumarten im Kurzumtrieb - Erkenntnisse aus drei Jahren FastWOOD, ProLoc und Weidenzüchtung“ am 21. bis 22. September 2011, Hannoversch Münden (URL: http://www.herohessen.de/downloads/vortraege_veranstaltungen/KUP_2012/10_AmthauerD_RindenanteileAschegeha lte.pdf, Zugriff am 13.08.2012).

ANONYMUS (1973): Pappeleingangsbuch Weserkamp. Interne Dokumentation. Forschungsinstitut für schnellwachsende Baumarten. Hannoversch Münden.

ARgus, G. W. (1986): The genus Salix (Salicaceae) in the southeastern United States. Systematic botany monographs Band 9. American society of plant taxonomist, Ann Arbor.

ARMSTRONG, A.; Johns, C.; TUBBY, I. (1999): Efects of spacing and cutting cycle on the yield of poplar grown as an energy crop. Biomass and Bioenergy 17: 305-314. 
Aylott, M.J.; Casella, E.; Tubby, I.; Street, N. R.; Smith, P.; Taylor, G. (2008): Yield and spatial supply of bioenergy poplar and willow short-rotation coppice in the UK. New Phytologist 178: 358-370.

BÄrwolfF, M.; Vetter, A.; Böhm, C; Hoffmann, J.; SchmidT, C. (2011): Projekt AgroforstEnergie. Was bringen Streifen-KUP? Energiepflanzen 2/2011: 9-11.

BAUER, FR.W. (1938): Furnierpappeln im deutschen Wald : ein Beitrag zur Sicherstellung der deutschen Holzversorgung. Parey, Berlin.

Bemmann, A.; Gerold, D.; Mantau, U. (2010): Perspektiven von Kurzumtriebsplantagen für den Holzmarkt. In: BemmAnN, A.; KNUST, C. (Hrsg.): AGROWOOD Kurzumtriebsplantagen in Deutschland und europäische Perspektiven. Weißensee, Berlin, S. 243-245.

BEMmanN, A.; BÖHNISCH, B. 2011. Abschlussbericht des Verbundprojektes "GREENERGY" Anbau schnellwachsender Baumarten auf Grünlandstandorten zur Erhöhung des Rohstoffpotenzials für die energetische Holzverwendung - Phase I: Machbarkeitsstudie. Institut für Internationale Forstund Holzwirtschaft, Technische Universität Dresden, Professur für Landwirtschaftliche Betriebslehre, Universität Halle, Institut für Technikfolgenabschätzung und Systemanalyse, Karlsruher Institut für Technologie (URL http://boku.forst.tu-dresden.de/pdf/Abschlussbericht_Schua.pdf, Zugriff am 12. Juni 2013).

Bergante, S.; Facciotto, G.; MinotTa, G. (2010): Identification of the main site factors and management intensity affecting the establishment of Short-Rotation-Coppices (SRC) in Northern Italy through stepwise regression analysis. Central European Journal of Biology 5: 522-530.

BFH (BUNDESFORSCHUNGSANSTALT FÜR FORST- UND HOLZWIRTSCHAFT) (2001): Die Robinie und ihr Holz. Hamburg (URL: http://www.bfafh.de/bibl/pdf/robinie.pdf, Zugriff am 24. Juni 2013).

BfN (BUNDESAMT FÜR NATURSCHUTZ) (2012): Energieholzanbau auf landwirtschaftlichen Flächen. Auswirkungen von Kurzumtriebsplantagen auf Naturhaushalt, Landschaftsbild und biologische Vielfalt. Anbauanforderungen und Empfehlungen des Bundesamts für Naturschutz. Bonn (URL: http://www.bfn.de/fileadmin/MDB/documents/themen/erneuerbareenergien/bfn_energieholzanbau_lan dwirtschaftliche_flaechen.pdf, Zugriff am 04. April 2013).

BIERTÜMPFEl, A.; RUdel, A.; Werner, A.; VetTER, A. (2009): 15 Jahre Energieholzversuche in Thüringen. Thüringer Landesanstalt für Landwirtschaft (TLL), Jena (URL http://www.tll.de/ainfo/pdf /ehol1009.pdf, Zugriff am 12. Oktober 2010)

BIERTÜMPFEL, A.; GrAF, T.; VeTTER, A. (2012): Feldversuchsbericht 2010 und 2011 Ölfrüchte und Nachwachsende Rohstoffe. Thüringer Landesanatlt für Landwirtschaft (TLL), Jena (URL http://www.tll.de/ainfo/pdf/fvb_0312.pdf, Zugriff am 03. Januar 2012)

Blanco, P. (1993): Salix. In: Castroviejo, S.; Aedo, C.; Cirujano, S.; Laínz, M.; Montserrat, P.; Morales, R.; Muñoz Garmendia, F.; Navarro, C.; Paiva, J.; Soriano, C. (Hrsg.): Flora Ibérica. Plantas vasculares de la Península Ibérica e Islas Baleares, Vol. 3: Plumbaginaceae (partim) Capparaceae. Consejo de Investigaciones Cientifícas, Madrid, S. 477-517 (URL: http://www.floraiberica.es/ floraiberica/texto/pdfs/03_070_02_salix.pdf, Zugriff am 12. Oktober 2012).

BLE (BUndesanstalt FÜR LANDWIRTSCHAFT UND ERNÄHRUNG) (2012): Die Pappel. Klone, Klonmischungen und Familieneltern. Bonn (URL http://www.ble.de/SharedDocs/Downloads/ 02_Kontrolle/07_SaatUndPflanzgut/Pappelklone_mischungen.pdf?_blob=publicationFile, Zugriff am 11. Dezember 2012) . 
Blume, K. (1951): Neue Ertragstafeln für die Pappel. In: Hesmer, H. (Hrsg.): Das Pappelbuch. Verlag des deutschen Pappelvereins, Bonn, S. 192-199.

BMELV (BUNDESMINISTERIUM FÜR ERNÄHRUNG, LANDWIRTSCHAFT UND VERBRAUCHERSCHUTZ) (2012): Statistisches Jahrbuch über Ernährung, Landwirtschaft und Forsten 2012. Landwirtschaftsverlag Münster, Münster.

BMU (BUNDESMINISTERIUM FÜR UMWELT, NATURSCHUTZ UND REAKTORSICHERHEIT); BMELV (BUNDESMINISTERIUM FÜR ERNÄHRUNG, LANDWIRTSCHAFT UND VERBRAUCHERSCHUTZ) (2010): Nationaler Biomasseaktionsplan für Deutschland Beitrag der Biomasse für eine nachhaltige Energieversorgung. Berlin (URL http://www.bmelv.de/SharedDocs/Downloads/Broschueren/ BiomasseaktionsplanNational.pdf?_blob=publicationFile, Zugriff am 15. November 2011)

BMU (BUNDESMINISTERIUM FÜR UMWELT, NATURSCHUTZ UND REAKTORSICHERHEIT) (2013): Zeitreihe zur Entwicklung der erneuerbaren Energien in Deutschland. Berlin (URL http://www.erneuerbare-energien.de/fileadmin/Daten_EE/Bilder_Startseite/Bilder_Datenservice/PDFs _XLS/ ee-energiedaten_2012_bf.pdf, Zugriff am 09. August 2013)

BMWi (BUNDESMINISTERIUM FÜR WIRTSCHAFT UND TECHNOLOGIE) (2010): Energiekonzept für eine umweltschonende, zuverlässige und bezahlbare Energieversorgung. Berlin (URL http://www.bundesregierung.de/Content/DE/_Anlagen/2012/02/energiekonzept-final.pdf?_blob =pub licationFile, Zugriff am 23. Februar 2013)

BöDEN, E., KeChEL, H. G. (1984): Rinden-, Blatt- und Triebkrankheiten an Pappeln. Merkblatt des Forschungsinstituts für schnellwachsende Baumarten Nr. 10. Hannoversch Münden.

BOELCKE, B. (2006): Schnellwachsende Baumarten auf landwirtschaftlichen Flächen - Leitfaden zur Erzeugung von Energieholz. Ministerium für Ernährung, Landwirtschaft, Forsten und Fiescherei Mecklenburg-Vorpommern, Schwerin.

Boelcke, B.; KAHLE, P. (2008): Energieholzproduktion mit Weiden und Pappeln - Ertragsbildung und Grundnährstoffbedarf. Pflanzenbauwissenschaften 12: 78-85.

BOHNENS, J.; FRIEDRICH, E. (1990): Züchterische Maßnahmen zur Steigerung und Sicherung der Produktion und anbautechnische Untersuchungen zur Biomasseproduktion in forstlichen Schnellwuchsplantagen: Schlußbericht. Forschungsinstitut für schnellwachsende Baumarten, Hannoversch Münden.

Bonnemann, A. (1980): Die Eigenschaften des Pappelholzes. Schriften des Forschungsinstitutes für schnellwachsende Baumarten Band 1. DRW-Verlag, Leinfelden-Echterdingen.

Bonnor, G.M; De Jong, R.J.; Boudewyn, P.; Flewelling, J.W. (1995): A guide to the STIM growth Model. Information Report BC-X-353. Natural Resources Canada, Canadian Forest Service, Pacific Forestry Centre, Victoria.

Bossel, H. (1994): TREEDYN 3 forest simulations model, mathematical model, program documentation, and simulation results. Berichte des Forschungszentrums Waldökosysteme der Universität Göttingen, Reihe B, Band 35, Göttingen.

BOTKIN, D.B.; JANAK, J.F.; WALLIS, J.R. (1972): Some ecological consequences of a computer model of forest growth. Journal of Ecology 60: 849-872.

BRosIUS, F. (1988): SPSS 8 Professionelle Statistik unter Windows. Bonn (URL http://www.molar.unibe.ch/help/statistics/spss/23_lineare_regression.pdf, Zugriff am 13. August 2011) 
BROWN, L.R. (2012): Full planet, empty plates: The new geopolitics of food scar. Norton \& Company, New York.

BROZIO, S.; PIORR, H.P.; ZEIDLER, M.; TORKLER, F. (2009): Methodik zur Ermittlung standortsspezyfischer Bioenergiepotenziale unter Berücksichtigung von Nachhaltigkeitskriterien am Beispiel des Landes Brandenburg. Proceeding. In: Kage, H.; Kersebaum, K.C.; Müller, T. (Hrsg.): Modellierung des Systems Nutzpflanze-Boden - Herausforderungen des 21. Jahrhunderts. Kurzfassungen der Beiträge zum Workshop vom 26. bis 27. Februar 2009 in Kiel. Berichte der Gesellschaft für Pflanzenbauwissenschaften Band 4, Göttingen, S. 49-55.

BUNGART, R.; HÜTTL, R. (2004): Growth dynamics and biomass accumulation of 8-year-old hybrid poplar clones in a short-rotation plantation on a clayey-sandy mining substrate with respect to plant nutrition and water budget. European Journal of Forest Research 123: 105-115.

BuRGER, F. (2010): Bewirtschaftung und Ökobilanzierung von Kurzumtriebsplantagen. Dissertation. Fakultät Wissenschaftszentrum Weihenstephan für Ernährung, Landnutzung und Umwelt, Technische Universität München, München.

BURGER, F. (2011): Energiebilanz klar positiv - Kurzumtriebsplantagen. AFZ der Wald 13/2011: 2021.

BundESWALDGESETZ vom 2. Mai 1975 (BGB1. I S. 1037), das zuletzt durch Artikel 1 des Gesetzes vom 31. Juli 2010 (BGBl. I S. 1050) geändert worden ist.

CANNELL, M.G.R.; SMITH, R.I. (1980): Yields of minirotation closely spaced hardwoods in temperate regions - review and appraisal. Forest Science 26: 415-428.

CANNEL, M.G.R. (1988): The scientific background. In: Hummel, F.C.; PALZ, W.;GRASSI, G.(Hrsg.): Biomass forestry in Europe: a strategy for the future. Elsevier, New York.

CHRISTERSSON, L. (1986): High technology biomass production by Salix clones on a sandy soil in southern Sweden. Tree Physiology 2: 261-272.

CoHEN, J. (1988): Statistical power analysis for the behavioral sciences. 2. Auflage. Lawrence Erlbaum Associates, Hillsdale.

Coyle, D.; Coleman, M.; Durant, J.; Newman, L. (2006): Survival and growth of 31 Populus clones in South Carolina. Biomass and Bioenergy 30: 750-758.

CuRL, E.A.; Truelove, B. (1986): The Rhizosphere. Springer, Berlin, Heidelberg.

DANFORS, B.; LEDIN, S.; RoSENQVIST, H. (1998): Short rotation willow coppice - Growers manual. The Swedish Institute for Agricultural Engineering, Uppsala.

DeBell, D.; HARRINGTON, C. (1993): Deploying genotypes in short-rotation plantations: mixtures and pure cultures of clones and species. The Forestry Chronicle 69: 705-713.

Deckmyn, G.; Laureysens, I.; Garcia, J.; MuYs, B.; Ceulemans, R. (2004): Poplar growth and yield in short rotation coppice: model simulations using the process model SECRETS. Biomass and Bioenergy 26: 221-227.

DE Jong, R.J.; ZuMrawi, A.A.; Boudewyn, P.; FleWELling, J.W. (1995): STIM User's Manual (for Windows) Ver 2.2a. FRDA Report No. 237. (Canada-British Columbia Partnership Agreement on Forest Resource Development, B. C. Ministry of Forests, Quebec.

DE MARTONNE E. (1926): Une nouvelle fonction climatologique: L'indice d'aridité. La Meteorologie 8:449-458. 
DESCH, G. (2011): Empirische Modelle. Vorlesungsskript (URL: http://www.uni-graz.at/ desch/Lehr Ver/empirmodelle.pdf, Zugriff am 23. April 2013).

Dickmann, D.I.; NGuYen, P.V.; Pregitzer, K.S. (1996): Effects of irrigation and coppicing on above-ground growth, physiology, and fine-root dynamics of two field-grown hybrid poplar clones. Forest Ecology and Management 80: 163-174.

DIN (DEUTSCHES InSTITUT FÜR NORMUNG) (1997): DIN EN 350-2, Dauerhaftigkeit von Holz und Holzprodukten - Natürliche Dauerhaftigkeit von Vollholz - Teil 2: Leitfaden für die natürliche Dauerhaftigkeit und Tränkbarkeit von ausgewählten Holzarten von besonderer Bedeutung in Europa.

DIN (DEUTSCHES INSTITUT FÜR NORMUNG) (1997): DIN ISO 10390, Bodenbeschaffenheit Bestimmung des pH-Wertes.

DIN (DEUTSCHES INSTITUT FÜR NORMUNG) (1997): DIN ISO 10693, Bodenbeschaffenheit Bestimmung des Carbonatgehaltes - Volumetrisches Verfahren.

DIN (DEUTSCHES INSTITUT FÜR NORMUNG) (1997): DIN ISO 10694, Bodenbeschaffenheit Bestimmung von organischem Kohlenstoff und Gesamtkohlenstoff nach trockener Verbrennung (Elementaranalyse).

DIN (DEUTSCHES INSTITUT FÜR NORMUNG) (1997): DIN ISO 11261, Bodenbeschaffenheit Bestimmung von Gesamt-Stickstoff - Modifiziertes Kjeldahl-Verfahren.

DIN (DEUTSCHES INSTITUT FÜR NORMUNG) (1998): DIN ISO 11272, Bodenbeschaffenheit Bestimmung der Trockenrohdichte.

DIN (DEUTSCHES INSTITUT FÜR NORMUNG) (1998): DIN ISO 11277, Bodenbeschaffenheit Bestimmung der Partikelgrößenverteilung in Mineralböden - Verfahren mittels Siebung und Sedimentation.

DitTert, K.; WÄtzel, J.; SATTELMAChER, B. (2006): Responses of Alnus glutinosa to anaerobic conditions-mechanisms and rate of oxygen flux into the roots. Plant Biology 8: 212-223.

Dobbertin, M.; Gioggiola, A. (2006): Baumwachstum und erhöhte Temperaturen. In: WoHlgemuth, T. (Hrsg.): Forum für Wissen: Wald und Klimawandel. Eidg. Forschungsanstalt für Wald, Schnee und Landschaft, S. 35-45.

DÖHRER, K. (1991): Praktische Erfahrungen mit der Anlage großer Holzfelder. Die Holzzucht 45: 27 30.

DÖHRER, K. (2010): Erfahrungen mit der Anlage von Pappel-Kurzumtriebsplantagem auf landwirtschaftlichen Flächen. Karl Döhrer, Diemelstadt.

DOWSLEY, B. (1987): Study of the correlation of diurnal growth patterns to climatic variables in a Salix energy plantation. Newfoundland Forest Research Center Information Report N-X-260. Canadian Forestry Service, Pacific Forestry Centre, Victoria.

ECKENWALDER, J.E. (1996): Systematics and evolution of Populus. In: STETTLER, R.F.; BRADSHAW, H.D. Jr.; Heilman, P.E.; HinCKLEY, T.M. (Hrsg.): Biology of Populus and its implications for management and conservation. NRC Research Press, National Research Council of Canada, Ottawa, Ontario, Canada, S. 7-32.

ECKERSTEN, H. (1994): Modelling daily growth and nitrogen turnover for a short-rotation forest over several years. Forest Ecology and Management 69: 57-72. 
ECKersten, H.; Noronha-SANNERVIK, A.; TORSSEll, B.; NyMAn, P. (2006): Modelling radiation use, water and nitrogen in willow forest. Report from the Department of Crop Production Ecology (VPE) Band 2. Swedish University of Agricultural Sciences, Uppsala.

EISENREICH, H. (1956): Schnellwachsende Holzarten. Deutscher Bauernverlag, Berlin.

Engel, J.; KNOCHE, D. (2011): Energie aus dem Stock - Zur Bewirtschaftung der Robinie im Schnellumtrieb. Proceeding. In: MINISTERIUM FÜR INFRASTRUKTUR UND LANDWIRTSCHAFT DES LANDES BRANDENBURG; LANDESBETRIEB FORST BRANDENBURG; LANDESKOMPETENZZENTRUM FORST EBERSWALDE (Hrsg.): Wissenstransfer in die Praxis. Beiträge zum 6. Winterkolloquium am 24. Februar 2011 in Eberswalde. Eberswalder Forstliche Schriftenreihe Band 47, Eberswalde, S. 26-36.

ERNEUERBARE-ENERGIEN-GESETZ vom 25. Oktober 2008 (BGBl. I S. 2074), das durch Artikel 1 des Gesetzes vom 17.August 2012 (BGBl. I S. 1754) geändert worden ist.

EtTALA., M. (1988): Short-Rotation tree plantations at sanitary landfills. Waste Management \& Research 6: 291-302.

Evans, S.; Baldwin, M.; Henshall, P.; Matthews, R.; Morgan, G.; Poole, J.; Taylor, P.; TUBBY, I. (2007): Final Report: Yield models for Energy: Coppice of Poplar and willow. Volume A Empirical Models. Report to DTI (B/W2/00624/00/00 URN). Forest Research, Farnham.

FACHVERBAND BIOGAS E.V. (2012): Maisanbaufläche 2012: geringer Anstieg bestätigt BiogasPrognose. Freising (URL: http://www.biogas.org/edcom/webfvb.nsf/id/DE_PM-20-12/\$file/12-0820_PM_Maisanbaustatistik.pdf, Zugriff am 20.08.2012).

FANG, S.; XU, X.; LU, S.; TANG, L. (1999): Growth dynamics and biomass production in shortrotation poplar plantations: 6-year results for three clones at four spacings. Biomass and Bioenergy 17: 415-425.

FAO (FOOD AND AGRICULTURE ORGANISATION OF THE UNITED NATIONS) (1979): Poplars and willows in wood production and land use. FAO Forestry Series No. 10. International Poplar Commission. Rom.

FAO (FoOd AND AgRiculture ORGANiSATION OF THE United NATIONS) (2013): The state of food and agriculture 2013. Food systems for better nutrition. Rom (URL: http://www.fao.org /docrep/018/i3300e/i3300e.pdf, Zugriff am 30. August 2013).

FERM, A.; HYTÖNEN, J.; VUORI, J. (1989): Effect of spacing and nitrogen fertilization on the establishment and biomass production of short rotation poplar in Finland. Biomass 18: 95-108.

FIEDLER, H.J. (2001): Böden und Bodenfunktionen in Ökosystemen, Landschaften und Ballungsgebieten. Forum EIPOS Band 7, Expert Verlag, Renningen.

Filat, M.; ChIRA, D.; NiCA M.S.; DogarU, M. (2010) First year development of poplar clones in bio-mass short rotation coppiced experimental cultures. Annals of Forest Research 53: 151-160.

FINKE, P.A.; WÖSTEN, J.H.M.; JANSEN, M.J.W. (1996): Effects of uncertainty in major input variables on simulated functional soil behaviour. Hydrological Processes 10: 661-669.

FRIEDRICH, E. (1999): Anbautechnische Untersuchungen in forstlichen Schnellwuchsplantagen und Demonstration des Leistungsvermögens schnellwachsender Baumarten. In: BUNDESMINISTERIUM FÜR ERNÄHRUNG, LANDWIRTSCHAFT UND FORSTEN (Hrsg.): Modellvorhaben „Schnellwachsende Baumarten". Zusammenfassender Abschlussbericht. Schriftenreihe Nachwachsende Rohstoffe Band 13. Landwirtschaftsverlag Münster, Münster, S. 19-150. 
FRÖHLICH, D. (2009): Raumzeitliche Dynamik der Parameter des Energie-, Wasser- und Spurengashaushalts nach Kleinkahlschlag. Dissertation. Fakultät für Forstwissenschaften und Waldökologie, Universität Göttingen, Göttingen.

FRÖHLICH, H.J.; DIETZE, W. (1975): Die Pflanzung von Pappeln. Merkblatt des Forschungsinstituts für schnellwachsende Baumarten Nr. 4. Hannoversch Münden.

FRÖHLICH, H.J.; GROSSCURTH, W. (1973): Züchtung, Anbau und Leistung der Pappeln. Mitteilungen der Hessischen Landesforstverwaltung Band 10, Hessische Forstliche Versuchsantstalt und Forschungsinstitut für Pappelwirtschaft, Hannoversch Münden.

Griese, C.; LeOnhaRdT, A.; LARSEN, B.J. (1993): Ökophysiologische Untersuchungen an verschiedenen Pappelklonen. In: HÜTTERMANN, A. (Hrsg.): Anbau von Pappel bei mittlerer Umtriebszeit - Produktionsbiologie, Nutzungstechnologien und Ökonomie. Schriften aus der Forstlichen Fakultät der Universität Göttingen und der Niedersächsischen Forstlichen Versuchsanstalt Band 110, Sauerländer, Frankfurt am Main, S. 20-37.

GROSSCURTH, W. (1982): Ertragstafeln für Schwarz- und Balsampappeln. Schriften des Forschungsinstitutes für schnellwachsende Baumarten Band 2. DRW-Verlag, LeinfeldenEchterdingen.

Grote, R.; Bellmann, K.; ERHARD, M.; SucKOw, F. (1997): Evaluation of the forest growth model FORSANA. Summary Report Nr. 32, Potsdamer Institut für Klimaforschung, Potsdam.

GRÜNEwald, H.; BRANDT, B.; SCHNEIDER, B. (2007): Agroforestry systems for the production of woody biomass for energy transformation purposes. Ecological Engineering 29: 319-328.

GUIDI, W.; PICCIONI, E.; BONARI, E. (2008): Evapotranspiration and crop coefficient of poplar and willow short-rotation coppice used as vegetation filter. Bioresource Technology 99: 4832-4840.

GURGEL, ANDREAS (2011): schriftliche Mitteilung vom 21. Januar 2011, Landesforschungsanstalt für Landwirtschaft und Fischerei Mecklenburg-Vorpommern, Gülzow.

HALl, D.G.M.; ReEVE, M.J.; ThOMASson, A.I.; Wright, V.F. (1977): Water retention, porosity and density of field soils. Soil Survey Technical Monograph Nr. 9. Rothamsted Experimental Station, Lawes Agricultural Trust, Harpenden.

HALlgReEn, S. (1989): Growth response of Populus hybrids to flooding. Annals Science Forest 46: 361-372.

HANSEN, E. (1991): Poplar woody biomass yields:a look to the future. Biomass and Bioenergy 1: 1-7

HARRACH, T. (2008): Fragen und Anregungen zur Bewertung bester Böden (Höchstleistungsstandorte). Proceeding. In: Vortrags- und Exkursionstagung zur Bodenschätzung, 11.12. September 2008, Weimar. Arbeitsgruppe „Bodenschätzung und Bodenbewertung, Deutsche Bodenkundliche Gesellschaft, Göttingen (URL: http://eprints.dbges.de/48/, Zugriff am 03. April 2012)

HARTGE, K.H.; HORN, R. (1992): Die physikalische Untersuchung von Böden. Schweitzerbart, Stuttgart.

HARTMANN, K.-U. (2010): Entwicklung eines Ertragsschätzers für Kurzumtriebsbestände aus Pappel. Dissertation. Fachrichtung Forstwissenschaften, Technische Universität Dresden, Dresden.

Hauhs, M; Kastner-Maresch, A.; Rost-Siebert, K. (1995): A model relating forest growth to ecosystem-scale budgets of energy and nutrients. Ecological Modelling 83: 29-243. 
HeILMAN, P.E.; STETTLER, R.F. (1985): Genetic variation and productivity of Populus trichocarpa T. and G. and its hybrids. II. Biomass production in a 4-year plantation. Canadian Journal of Forest Research 15: 382-388.

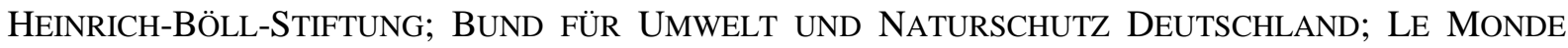
DIPLOMATIQUE (2013): Fleischatlas - Daten und Fakten über Tiere als Nahrungsmittel. Berlin (URL: http://www.boell.de/sites/default/files/fleischatlas2013_v_web.pdf, Zugriff am 30.08.2013).

HERZOG, F. (1997): Konzeptionelle Überlegungen zu Agroforstwirtschaft als Landnutzungsalternative in Europa. Zeitschrift für Kulturtechnik und Landesentwicklung 38: 32-35.

HEYn, N.; WaChENDORF, C. (2011): ProLoC Zwischenbericht, Teilprojekt Bodenkunde. FKZ: 22001908. Fachgebiet Bodenbiologie und Pflanzenernährung, Universität Kassel, Witzenhausen.

HEYN, N.; WACHENDORF, C. (2012): PROLOC Endbericht, Teilprojekt Bodenkunde. FKZ: 22001908. Fachgebiet Bodenbiologie und Pflanzenernährung, Universität Kassel, Witzenhausen.

HILLEL, D. (1998): Environmental Soil Physics. Academic Press, New York.

HofmANN, M. (1995): Schnellwachsende Baumarten für den Kurzumtrieb - Aspekte der Pflanzenzüchtung und Ergebnisse zur Kloneignung auf verschiedenen Standorten. Die Holzzucht 49: $3-8$.

HOFMANN, M. (1998): Bewirtschaftung schnellwachsender Baumarten auf landwirtschaftlichen Flächen im Kurzumtrieb. Merkblatt des Forschungsinstituts für schnellwachsende Baumarten Nr. 11. Hannoversch Münden.

HOFMANN, M. (1999): Bereitstellung von genetisch hochwertigem Vermehrungsgut für Kurzumtriebsbestände. In: BUNDESMINISTERIUM FÜR ERNÄHRUNG, LANDWIRTSCHAFT UND FORSTEN (Hrsg.): Modellvorhaben „Schnellwachsende Baumarten“. Zusammenfassender Abschlussbericht. Schriftenreihe Nachwachsende Rohstoffe Band 13. Landwirtschaftsverlag Münster, Münster, S. 151239.

HofmanN, M. (2005): Pappeln als nachwachsender Rohstoff auf Ackerstandorten - Kulturverfahren, Ökologie und Wachstum unter dem Aspekt der Sortenwahl. Dissertation. Fakultät für Forstwissenschaften und Waldökologie, Universität Göttingen. Schriften des Forschungsinstitutes für schnellwachsende Baumarten Band 8, Hannoversch Münden.

HofmAnN. M; LIPPELT, J. (2008): Anlage einer Modellpflanzung mit Pappel-Sortenschau auf dem landwirtschaflichen Versuchsgut Eichhof zur Sicherung der Sortenbasis und zur Sortenpflege: Abschlußbericht. FKZ: 22002406. Kompetenzzentrum HessenRohstoffe (HeRo) e.V., Witzenhausen.

HoRn, H.; SKIBbe, K.; RÖHLE, H. (2013): Wuchsleistung von KUP aus Pappel in Folgerotationen. AFZ der Wald 7/2013: 53-55.

HoYningen-HuENE, J. V. (1983): Die Interzeption des Niederschlages in landwirtschaftlichen Pflanzenbeständen. DVWK-Schrift Nr. 57. Parey, Hamburg, Berlin.

HÜBENER, E. (1989): Anbau und Bewirtschaftung von Pappeln. In JOACHIM, H.F.; HÜBENER, E.; ERNST, E. (Hrsg.): Anbau und Bewirtschaftung von Pappeln: langjährigen Erfahrungen und neue Ergebnisse. IFE-Berichte aus Forschung und Entwicklung Band 15. Institut für Forstwissenschaften Eberswalde. Eberswalde-Finow, S. 37-48.

HUXLEY, P. A. (1999): Tropical agroforestry. Blackwell, Oxford.

IEA (INTERNATIONALE ENERGIEAGENTUR) BIOENERGy (2002): Sustainable production of woody biomass for energy. A position paper prepared by IEA Bioenergy. Rotorua (URL http://np- 
net.pbworks.com/f/IEA+(2002)+Sustianable +Woody+Biomass+Production.pdf, Zugriff am 19 . Januar 2013).

IPCC (InTergovernmental PANel ON Climate Change) (2007): Climate Change 2007 Synthesis Report. Genova (URL http://www.ipcc.ch/pdf/assessment-report/ar4/syr/ar4_syr.pdf, Zugriff am 28. August 2009).

ISEBRANDS, J.G.; RAUSCHER, H.M.; CROW, T.R.; DICKMANN, D.I. (1990): Whole tree growth process models based on structure-functional relationships. In DIXON, R.; MELDAHL, R.; RUARK, G.; WARREN W. (Hrsg.): Forest growth: process modeling of response to environmental stress. Timber Press, Portland, S. 96-102.

ISEBRANDS, J.G.; BURK, T.E. (1992): Ecophysiological growth process models of short rotation forest crops. In Mitchell, C. P. (Hrsg.): Ecophysiology of short rotation forest crops. Elsevier Applied Sciences, London, New York, S. 96-112.

JANSSON, P.E. (1991): Soil water and heat model. Technical description. Report 165. Department of Soil Sciences, Division of Agricultural Hydrotechnics, Swedish University of Agricultural Sciences, Uppsala.

JANSSON, P.E. (1998): Simulating model for soil water and heat conditions. Description of the SOIL model. Report 91:2. Department of Soil Sciences, Division of Agricultural Hydrotechnics, Swedish University of Agricultural Sciences, Uppsala.

JIEN, S.H.; WANG, C.S. (2013): Effects of biochar on soil properties and erosion potential in a highly weathered soil. Catena 110: 225-233.

JinG, Q.; CONIJN, J.G.; JONGSCHAAP, R.E.E.; BINDRABAN, P.S. (2012): Modeling the productivity of energy crops in different agro-ecological environments. Biomass and Bioenergy 46: 618-633.

JOACHIM, H.F. (1953a): Untersuchungen über die Wurzelausbildung der Pappel und die Standortsansprüche von Pappelsorten. Dissertation. Wissenschaftliche Abhandlungen / Deutsche Akademie der Landwirtschaftswissenschaften zu Berlin Band 7. Deutscher Bauernverlag, Berlin.

JOACHIM, H.F. (1953b): Pappelanbau außerhalb des Waldes. Schriftenreihe für die landwirtschaftlichen Produktionsgenossenschaften Band 33. Deutscher Bauernverlag, Berlin.

JOACHIM, H.F. (1989): Über Sorten- und Standortsfragen sowie die forstlichen Möglichkeiten beim Anbau von Pappeln. In: JOACHIM, H.F.; HÜBENER, E.; ERNST, E. (Hrsg.): Anbau und Bewirtschaftung von Pappeln: langjährigen Erfahrungen und neue Ergebnisse. IFE-Berichte aus Forschung und Entwicklung Band 15. Institut für Forstwissenschaften Eberswalde. Eberswalde-Finow, S. 37-48.

JOHANSSON, T.; KARAČIĆ, A. (2011): Increment and biomass in hybrid poplar and some practical implications. Biomass and Bioenergy 35: 1925-1934.

JONSSON, H. (1994): Sammanfattande utvärdering av svenska försökodlingar med Salix 1986-1991. Ramprogram Energiskog, NUTEK 1994: 24.

Jug, A.; HofMAnN-Schielle, C.; MAKeschin, F.; RehFueSS, K.E. (1999): Short-rotation plantations of balsam poplars, aspen and willows on former arable land in the Federal Republic of Germany. II. Nutritional status and bioelement export by harvest of shoot axes. Forest Ecology and Management 121: 67-83.

JURY, W.A.; GARDNER W.R.; GARDNER W.H. (1991): Soil Physics. 5. Auflage. John Wiley \& Sons, Hoboken. 
KARACIC, A.; VERWIJST, T.; WEIH., M. (2003): Above-ground woody biomass production of shortrotation populus plantations on agricultural land in Sweden. Scandinavian Journal of Forest Research 18:427-437.

KarP, A.; Shield, I.; MacalPine, W.; Hanley, J. (2010): Genetische Verbesserung und Züchtung von Biomasse-Weiden für Großbritannien. Proceeding. In: ALFRED TOEPFER AKADEMIE; 3NKOMPETENZZENTRUM; NIEDERSÄCHSICHES MINISTERIUM FÜR ERNÄHRUNG, LANDWIRTSCHAFT VERBRUACHERSCHUTZ UND LANDESENTWICKLUNG; DEUTSCHE LANDWIRTSCHAFTSGESELLSCHAFT (Hrsg.): Schnellwuchsplantagen - Chancen für Klimaschutz, Naturschutz und Landwirtschaft. Tagung am 19. bis 20. Oktober 2010, Schneverdingen (URL: http://www.3-n.info/pdf_files/Vortraege /101020_04_abstract_karp_kup_zuechtung.pdf, Zugriff am 21. Oktober 2011).

KAUlfub, Peter (2011): schriftliche Mitteilung vom 23. Februar 2011, Leibniz-Institut für Agrartechnik Potsdam-Bornim e.V., Potsdam.

KECHEL, H.G. (1984): Untersuchungen über die Resistenz von Pappeln gegenüber dem Erreger des Pappelkrebses, Xanthomonas populi subsp. populi (Ridé) Ridé und Ridé. Schriften des Forschungsinstitutes für schnellwachsende Baumarten Band 3. Hannoversch Münden.

KNOCHE, D. (2001): Forstliche Rekultivierung. Proceeding. In: LAUSITZER Und MitTELDEUTSCHE BERGBAU-VERWALTUNGSGESELLSCHAFT mbH (Hrsg.): Wissenschaftliche Begleitung der ostdeutschen Braunkohlesanierung. Eigenverlag der Lausitzer und Mitteldeutsche BergbauVerwaltungsgesellschaft, Berlin, Dresden, S. 105-131.

Knust, C. (2009): Kurzumtriebsplantagen - Stand des Wissens. In: REEG, T.; BEMmanN, A.; Konold, W.; Murach, D.; SPIECKER, H. (Hrsg.): Anbau und Nutzung von Bäumen auf landwirtschaftlichen Flächen, Wiley-VCH, Weinheim, S. 3-10.

KoOP, B.; KAHLE, P.; LenNARTZ, B. (2005): Evaluierung des Potentials qualifizierter Abdeckungen am Beispiel einer ehemaligen Betriebsdeponie. Proceeding. In: MelchIOR, S.; Berger, K. (Hrsg): Abfallverwertung bei der Rekultivierung von Deponien, Altlasten und Bergbaufolgelandschaften. Fachtagung Abfallverwertung bei der Rekultivierung von Deponien, Altlasten und Bergbaufolgelandschaften vom 31. März bis 01. April 2005, Hamburg. Hamburger Bodenkundliche Arbeiten, Band 56. S. 277-287.

KopP, R. F.; Abrahamson, L. P.; White, E. H.; Burns, K. F.; NowaK, C. A. (1997): Cutting cycle and spacing effects on biomass production by a willow clone in New York. Biomass and Bioenergy 12: $313-319$.

KRIMMINS, J.P. (1990): Modelling the sustainability of forest production and yield for a changing and uncertain future. The Forestry Chronicle 66: 271-280.

Kroiher, F.; Bielefeld, J.; Bolte, A.; Schulter, M. (2008): Die Phytodiversität in Energieholzbeständen: erste Ergebnisse im Rahmen des Projektes NOVALIS. Archiv für Forstwesen und Landschaftsökologie 42: 158-165.

KRUSKAL, W. H.; WALLIS, W. A. (1952): Use of ranks in one-criterion variance analysis. Journal of the American Statistical Association 47: 583-621.

Kuntze, H.; Roeschmann, G.; SchwerdtFeger, G. (1994): Bodenkunde. 5. Auflage. UTB, Stuttgart.

KUZOVKINA, Y.; QUIGLEY, M. (2005): Willow beyond wetlands: uses of Salix spp. for environmental projects. Water, Air, and Soil Pollution 162: 183-204. 
LABRECQUE, M.; TEODORESCU, T. I. (2005): Field performance and biomass production of 12 willow and poplar clones in short-rotation coppice in southern Quebec (Canada). Biomass and Bioenergy 29: $1-9$.

LAMERSDORF, N.; SChUlte-BISPING, H. (2010): Bodenökologie. In: DeUTSCHE BundesstifTung UMWELT (Hrsg.). Kurzumtriebsplantagen. Handlungsempfehlungen zur naturverträglichen Produktion von Energieholz in der Landwirtschaft. Ergebnisse aus dem Projekt NOVALIS S. 14-25

LANDGRAF, D.; BÖCKER, L. (2010): Kurzumtriebsplantagen auf Sonderstandorten. In: KNUST, C.; BEmmanN, A. (Hrsg.): AGROWOOD - Kurzumtriebsplantagen in Deutschland und europäische Perspektiven. Weißensee Verlag, Berlin. S. 54-64

LANDSBerg J.J.; KAUfMAnN, M.R.; BinldeY, D.; Isebrands, J.; Jarvis, P.G. (1991): Evaluating progress towards closed forest models based on fluxes of carbon, water and nutrients. Tree Physiology 9: $1-15$.

LANDSBERG, J.J.; WARING, R.H. (1997): A generalized model of forest productivity using simplified concepts of radiation-use efficiency, carbon balance, and partitioning. Forest Ecology and Management 95: 209-228.

Larsson, S.; Bullard, M.J.; Christian, D.G.; Knight, J.D.; Lainsbury, M.A.; Parker, S.R. (2001): Commercial varieties from the swedish willow breeding programme. In: AssociATION OF APPLIED BiOLOGISTS (Hrsg.): Biomass and Energy Crops II, Workshop vom 18 .bis 21. December 2001, York, S. 193-198.

LEDIN, S.; WiLlebrand, E. (1995): Handbook on how to grow short rotation forests. Swedish University of Agricultural Sciences, Uppsala.

LeWANDOWski, I.; VetTer, A. (2000) Biomasseentstehung. In KaltschmitT, M.; HaRTMann, H.; HofBAuER, H. (Hrsg.): Energie aus Biomasse: Grundlagen, Techniken und Verfahren. Springer, Berlin. S. 41-74.

LiBBy, W. (1980): What is a safe number of clones per plantation? In: HeYBroeK, H.M.; STEPHAN, B.; VON WEISSENBERG, K. (Hrsg.): Resistance to diseases and pest in forest trees. Proceedings of the $3^{\text {rd }}$ International Workshop on the genetics of host-parasite interactions in forestry. 14-21 September 1980 Wageningen, Niederlande. S. 342-360

LIESEBACH, M.; WÜHLISCH, G.V.; MuHS, H.J. (1999): Eignung der Baumart Aspe und Prüfung von Aspenhybriden für die Biomasseerzeugung in Kurzumtriebsplantagen. In: BUNDESMINISTERIUM FÜR ERNÄHRUNG, LANDWIRTSCHAFT UND FORSTEN (Hrsg.): Modellvorhaben „Schnellwachsende Baumarten“. Zusammenfassender Abschlussbericht. Schriftenreihe Nachwachsende Rohstoffe Band 13. Landwirtschaftsverlag Münster, Münster, S. 240-313

LiNDROTH, A.; BÄTH, A. (1999): Assessment of regional willow coppice yield in Sweden on basis of water availability. Forest Ecology and Management 121: 57-65.

LÖSCH, R. (2001): Wasserhaushalt der Pflanzen. Quelle \& Meyer, Wiebelsheim.

LTZ (LANDWIRTSCHAFTLICHES TECHNOLOGIEZENTRUM AUgustenBERG) (2010): Anlage und Bewirtschaftung von Kurzumtriebsflächen in Baden-Württemberg. Eine praxisorientierte Handreichung. Rheinstetten-Forchheim (URL: http://www.mlr.baden-wuerttemberg.de/mlr/bro/ Kurzumtriebsflaechen.pdf, Zugriff am 17. August 2012).

LÜDEMANN, G.H. (1998): Schnellwachsende Baumarten in Wald und Landschaft Norddeutschlands. Gesellschaft zur Förderung Schnellwachsender Baumarten in Norddeutschland, Eutin. 
LUXMOORE, R.J.; THARP, M.L.; POST, W.M. (2008): Simulated biomass and soil carbon of loblolly pine and cottonwood plantations across a thermal gradient in southeastern United States. Forest Ecology and Management 254: 291-299.

MACPHERSON, G. (1995): Home-grown energy from short-rotation coppice. Farming Press Books, Ipswich.

MAIER, J.; VetTER, R. (2004): Erträge und Zusammensetzung von Kurzumtriebs-Gehölzen (Weide, Pappel, Blauglockenbaum). Proceeding. In: InSTITUT FÜR AGRARTECHNIK BoRNIM E.V. (Hrsg.): Energieholzproduktion in der Landwirtschaft. Seminar am 29. Januar 2004 in Potsdam, S. 87-92.

MAKESCHIN, F. (1999): Short rotation forestry in Europe. Introduction and conclusions. Forest Ecology and Management 121: 1-7.

MAKESCHIN, F.; REHFUESS, K.E. (1994): Standorts- und ernährungskundliche Untersuchungen zur Nutzung landwirtschaftlicher Flächen für die Erzeugung von Biomasse mit schnellwachsenden Baumarten: Schlußbericht zum BMFT-Projekt; Zusammenfassung der Ergebnisse für die Jahre 1988 bis 1993; Teil A. FKZ: BMFT 03C-177-3. Lehrstuhl für Bodenkunde und Standortslehre, Universität München, München.

MANN, H.; WhITNEY, D. (1947): On a test of whether one of two random variables is stochastically larger than the other. Annals of Mathematical Statistics 18: 50-60.

MANTAU, U. (2012): Holzrohstoffbilanz Deutschland - Entwicklungen und Szenarien des Holzaufkommens und der Holzverwendung von 1987 bis 2015. Informationssysteme für Rohstoffe (INFRO), Zentrum für Holzwirtschaft, Universität Hamburg, Hamburg (URL: http://literatur.vti.bund.de/digbib_extern/dn051281.pdf, Zugriff am 30. März 2013).

MARQUARDT, D. (1963): An algorithm for least-squares estimation of nonlinear parameters, SIAM. Journal of Applied Mathematics 11: 431-441.

MATZDORF, B.; ReutTer, M.; HÜBNER, C. (2010): Gutachten-Vorstudie Bewertung der Ökosystemdienstleistungen von HNV-Grünland (High Nature Value Grassland) Abschlussbericht. Institut für Sozioökonomie, Leibniz-Zentrum für Agrarlandschaftsforschung (ZALF) e.V., Müncheberg (URL: http://www.bfn.de/fileadmin/MDB/documents/themen/recht/oekosdienstleist_ hnv.pdf., Zugriff am 27. Mai 2013).

MAYER, K. (2009): Kurzumtriebsversuche der Landeskammer für Land- und Forstwirtschaft Steiermark, dem Versuchsreferat des Landes Steiermark und der E-Steiermark. In: VERSUCHSREFERAT STEIERMARK (Hrsg.): Versuchsbericht 2008. Hatzendorf (URL: http://www.versuchsreferat.com/Versuchsbericht2008/Energieholz.pdf, Zugriff am 12. März 2011).

MC Alpin, R.G.; Brown, C.L.; Herrick, A.M.; RuARK, H.E. (1966): "Silage" sycamore. Forest Farmer 26: 6-7.

MC MURTRIE, R.E.; LANDSBERG, J.J.; LINDER, S. (1989): Research priorities in field experiments on fast-growing tree plantations: Implications of a mathematical production model. In: PEREIRA, J.S.; LANDSBERG, J.J. (Hrsg.): Biomass production by fast-growing trees. NATO ASI Series Volume 166. Kluwer Academic Publishers, Dordrecht, Boston, London, S. 181-207.

Meiresonne, L.; Nadezhdina, N.; Cermak, J.; van Slycken, J.; Ceulemans, R. (1999): Measured sap flow and simulated transpiration from a poplar stand in Flanders (Belgium). Agricultural and Forest Meteorology 96: 165-17. 
Mitchell, C.P.; Stevens E.A.; WATTERS, M.P. (1999): Short- rotation forestry- operations, productivity and costs based on experience gained in the UK. Forest Ecology and Management 121: 123-136

Mitscherlich, G.; Sonntag, G. (1982): Pappelversuche. V. Modell für eine Regenerata- und Neupotz-Pappel-Ertragstafel im Oberrheingebiet. Allgemeine Forst- und Jagdzeitung 153: 213-219.

MoHREN, G.M.J. (1987): Simulation of forest growth, applied to douglas fir stands in the Netherlands. Dissertation. Agricultural University Wageningen, Wageningen.

MuHS, H.J. (1986): Kurzumtriebsplantagen. AFZ der Wald 51/52: 1313-1316.

MuHS, H.J.; WÜHLISCH, G.V.; LIESEBACH, M. (1994): Nutzung landwirtschaftlicher Flächen zur Biomasseerzeugung mit schnellwachsenden Baumarten: Erfahrungen mit der Baumart Aspe. Abschlußbericht. FKZ: BMFT 0319150B8. Institut für Forstgenetik, Bundesforschungsanstalt für Forst- und Holzwirtschaft, Großhansdorf.

Müller, R. (1951): Der Anbau der Pappel. In: Hesmer, H. (Hrsg.): Das Pappelbuch. Verlag des deutschen Pappelvereins, Bonn. S. 111-141.

Murach, D.; Hartmann, H.; Murn, Y.; Schultze, M.; Ali, W.; Röhle, H. (2009): Standortsbasierte Leistungsschätzung in Agrarholzbeständen in Brandenburg und Sachsen. In: REEG, T.; Bemmann, A.; Konold, W.; Murach, D.; SpIECKer, H. (Hrsg.): Anbau und Nutzung von Bäumen auf landwirtschaftlichen Flächen, Wiley-VCH, Weinheim, S. 29-40.

NAGEL, J. (1997): BWIN - Programm zur Bestandesanalyse und Prognose. Handbuch zur Version 3.0. Niedersächsische Forstliche Versuchsanstalt Göttingen, Göttingen.

NAGEL, J. (2001): Skript Waldmesslehre (URL: http://www.lwvlu.com/wamel.pdf, Zugriff am 03. November 2009).

NaIR, S.S.; Kang, S.; Zhang, X.; MigueZ, F.E.; IZaurralde, R.C.; Post, W.M.; DietZe, M.C., LYND, L.R., WULLSCHLEGER, S.D. (2012): Bioenergy crop models: descriptions, data requirements, and future challenges. Global Change Biology 4: 620-633.

NAumanN, G. (1980): Die Pappel in der Landschaft des Niederrheins. Proceeding. In: HöHERE ForstBeHÖRDE RhEINLAND (Hrsg.): Die Pappel am Niederrhein. Tagung am 20. März 1980, Mönchengladbach. Mitteilungen und Berichte aus dem Bereich der höheren Forstbehörde Rheinland, Heft 4. Rheinischer Landwirtschafts-Verlag, S. 21-31.

Neubert. F.; Boll, T.; Zimmermann, K.; Bergfeld, A. (2013): Aktuelle Hemmnisse und Empfehlungen für den weiteren Ausbau von Kurzumtriebsplantagen in Deutschland: Ergebnisse einer Online-Umfrage von Praktikern. Vortrag. In: Internationaler Kongress der Fachagentur Nachwachsende Rohstoffe - Agrarholz 2013, vom 19. bis 20. Februar 2013, Berlin.

NEUMEISTER, CARSTEN (2009): mündliche Mitteilung vom 22. Mai 2009, Ingenieurbüro Biomasseconsulting, Ketzerbachtal.

Newsholme, C. (1992): Willows: The Genus Salix. Timber Press, Oregon.

NIEDER, R.; KÖSTER, W.; DAUK, H.B; BRINKMANN, S. (2003): Nährstoffüberschüsse in Deutschland von 1950 bis 2000: Quellen, Senken und Wirkungen auf die Umwelt. I. N-Überhang der Landwirtschaft. Zeitschrift für Landnutzung und Landentwicklung 44: 172-178.

NOAA (NATIONAL OCEANIC AND ATMOSPHERIC ADMINISTRATION) (2012): Trends in atmospheric carbon dioxide. Boulder (URL: http://www.esrl.noaa.gov/gmd/ccgg/trends/weekly.html, Zugriff am 13. Mai 2013). 
NOLEPPA, S; VON WITZKE, H. (2012): Tonnen für die Tonne: Ernährung - Nahrungsmittelverluste Flächenverbrauch. World Wide Fund For Nature (WWF) - Deutschland, Berlin (URL: http://www. wwf.de/fileadmin/fm-wwf/Publikationen-PDF/studie_tonnen_fuer_die_tonne.pdf, Zugriff am 13. Juni 2013).

O'Loughlin, G.; Huber, W.; CHOCAT, B. (1996): Rainfall-runoff processes and modelling. Journal of Hydraulic Research 34: 733-751.

PaCala, S. W.; Canham, C. D.; Silander, J.A. Jr. (1993): Forest models defined by field measurements: I. The design of a northeastern forest simulator. Canadian Journal of Forest Research 23: $1980-1988$.

Pacala, S.W.; Canham, C.D.; Saponara, J.; Silander, J.A. Jr.; Kobe, R.K.; Ribbens, E. (1996): Forest models defined by field measurements: II. Estimation, error analysis and dynamics. Ecological Monographs 66:1-43.

PAPPELINSTITUT NoRDRHEIN-WeSTFAlen (1948): Pappel-Standorte und Standortsbedingungen. Pappel-Merkblatt des Pappelinstituts Nordrhein-Westfalen Nr. 2. Dalheim-Rödgen.

Perny, B.; STEYRER, G. (2009): Massenvermehrung der Blattwespe Nematus pavidus in einer Waldviertler Weiden-Kurzumtriebsfläche. Forstschutz Aktuell 47: 22-25.

PERSSON, G.; LINDROTH, A. (1994): Simultaneous evaporation from short-rotation forest: variations within and between seasons. Journal of Hydrology 156: 21-45.

Petzold, R.; FEgER, K.-H.; RÖHLE, H. (2010): Standörtliche Voraussetzungen für Kurzumtriebsplantagen. In: BEMMANN, A.; KNUST, C. (Hrsg.): AGROWOOD Kurzumtriebsplantagen in Deutschland und europäische Perspektiven. Weißensee, Berlin, S. 44-53

PETZOLD, R. (2013): Standortsökologische Aspekte und Anbaupotenziale von Kurzumtriebsplantagen in Sachsen. Dissertation. Fachrichtung Forstwissenschaften, Technische Universität Dresden, Dresden.

PHILIPPOT, S. (1996): The suitability of existing models of short rotation forestry for modelling coppice - related growth processes: a literature review. Report Nr. 51. Department of Short Rotation Forestry, Swedish University of Agricultural Sciences, Uppsala.

PINON, J. (1992): Variability in the Genus Populus in Sensivity to Melampsora Rusts. Silvae Genetica 41: 25-34.

PINON, J.; FREY, P. (2005): Interactions between poplar clones and Melampsora populations and their implications for breeding for durable resistance. IN: PEI, M.H.; MCCRACKEN. A.R. (Hrsg.): Rust diseases of willow and poplar. CABI Publishing, Oxfordshire, Cambridge, S. 139-154.

PISTOCCHI, C.; GUIDI, W.; PICCIONI, E.; BONARI, E. (2009): Water requirements of poplar and willow vegetation filters grown in lysimeter under Mediterranean conditions: results of the second rotation. Desalination 246: 37-146.

PASTOR, J.; Gardner, R.H.; DAle, V.H.; POST, W.M. (1987): Successional changes in nitrogen availability as a potential factor contributing to spruce declines in boreal North America. Canadian Journal of Forest Research 17: 1394-1400.

PlagGenbORG, B. (1989): Schnellwachsende Weichlaubhölzer im Kurzumtrieb, bei Verwendung von Kompost aus der anaeroben Vergärung als Düngemittelsubstitut: eine Alternative für die Landwirtschaft. Informationen zu Naturschutz und Landschaftspflege in Nordwestdeutschland Band 5. Biologische Schutzgemeinschaft Hunte-Weser-Ems. BSH-Verlag, Wardenburg.

PRETZSCH, H. (2001): Modellierung des Waldwachstums. Blackwell, Berlin, Wien. 
PRETZSCH, H. (2002): Grundlagen der Waldwachstumsforschung. Blackwell, Berlin, Wien.

Pretzsch, H.; BIBer, P.; Dursky, J. (2002): The single tree-based stand simulator SILVA: construction, application and evaluation. Forest Ecology and Management 162: 3-21.

RÄTZEL, K. (1969): Die Ertragsleistung der Robustapappel im geschlossenen Reinbestand. Allgemeine Forst und Jagzeitung 140: 239-250.

RAu, H. M.; SchulzKe, R.; AlbReChT, J. (1988): Steigerung und Sicherung der Holzproduktion durch Auswahl, Prüfung und züchterische Verbesserung geeigneten Ausgangsmaterials bei schnellwachsenden Baumarten. Schriften des Forschungsinstitutes für schnellwachsende Baumarten Band 5. Hannoversch Münden.

Rauscher H.M.; Isebrands, J.G.; Host, G.E.; Dickson, R.E.; Dickmann, D.I.; Crow, T.R.; MICHAEL, D.A. (1990): Ecophys: an ecosphysiological growth process model for juvenile poplar. Tree Physiology 7: 255-281.

Renger, M.; Bohne, K.; Facklam, M.; Harrach, T.; RieK, W.; SchäFer, W.; Wessolek, G.; ZACHARIAS， S. (2008): Ergebnisse und Vorschläge der DBG-Arbeitsgruppe „Kennwerte des Bodengefüges“ zur Schätzung bodenphysikalischer Kennwerte. Berlin (URL http://www.boden.tuberlin.de/fileadmin/fg77/_pdf/publikationen/bodenphysikalischeKennwerte.pdf, Zugriff am 20 . Dezember 2008).

RIBEIRO C.; BETTERS, D. (1995): Single rotation vs coppice systems for short rotation intensive culture plantations - optimality conditions for volume production. Biomass and Bioenergy 8: 395-400.

RICHARDS, F.J. (1959): A flexible growth function for empirical use. Journal of Experimental Botany 10: 290-300.

Robinson, K.; KARP, A.; TAYLOR, G. (2004): Defining leaf traits linked to yield in short-rotation coppice Salix. Biomass and Bioenergy 26: 417-431.

RÖHLE, H. (1995): Zum Wachstum der Fichte auf Hochleistungsstandorten in Südbayern. Habilitationsschrift. Forstwissenschaftliche Fakultät, Universität München. Mitteilungen aus der Staatsforstverwaltung Bayerns 48. Heft. Bayerisches Staatsministerium für Ernährung, Landwirtschaft und Forsten, München.

RÖHLE, H.; GEROLD, D.; MÜNDER, K.; SCHRÖDER, J. (2004): BWINPro-S @ (Software) Technische Universität Dresden / Dendro-Institut Tharandt e.V.

RÖHle, H.; HaRtMann, K.-U.; STEInKe, C.; WOlF, H. (2005): Wuchsleistung von Pappel und Weide im Kurzumtrieb. AFZ der Wald 60/14: 745-747

Röhle, H.; HartmanN, K.-U.; Gerold, D.; Steinke, C.; SCHRÖDER, J. (2006): Überlegungen zur Aufstellung von Biomassefunktionen für Kurzumtriebsbestände. Allgemeine Forst- und Jagdzeitung 177: $178-187$.

RÖHLe, H.; BöCKeR, L.; Feger, K.-L.; Petzold, R.; Wolf, H.; Ali, W. (2008): Anlage und Ertragsaussichten von Kurzumtriebsplantagen in Ostdeutschland. Schweizerische Zeitschrift für Forstwesen 159: 133-139.

RöHle, H.; AlI, W. (2009): Production Potential of Poplar in Short Rotation Plantations in Saxony. In: Lithuanian University of Agriculture (Hrsg.): Rural Development 2009. Tagung am 15. bis 17. Oktober 2009. Proceedings Volume 4, Book 2, Kaunas, S. 190-195.

RöHLe, H.; Hartmann, K.-U.; SteinKe, C.; Murach, D. (2009): Leistungsvermögen und Leistungserfassung von Kurzumtriebsbeständen. In: REEG, T.; BEMMANN, A.; KONOLD, W.; 
MURACH, D.; SPIECKER, H. (Hrsg.): Anbau und Nutzung von Bäumen auf landwirtschaftlichen Flächen. Wiley-VCH, Weinheim, S. 41-55.

RÖHLE, H. (2012): Nachhaltig wirtschaften in einer begrenzten Welt? Im Angesicht der Krise erst recht!. In: VEREIN ZUM SCHUTZ DER BERGWELT E.V (Hrsg): Jahrbuch des Vereins zum Schutz der Bergwelt, 76./77. Jahrgang, München, S. 65-80.

RÖHRICHT, C.; Kiesewalter, S. (2008): Anbau und Ernte schnellwachsender Baumarten im Kurzumtrieb - Versuchsergebnisse. (URL: http://www.smul.sachsen.de/lfl/publikationen/jsp/inhalt .jsp?seite $=$ detail \&pub_id=3870\&count $=10 \&$ navi $=88 \&$ sort $=E R S C H \_J A H R \& a n z=10 \&$ sqla $=0 k \& s q l b=$ ok\&von=\&suche=T, Zugriff am 13. März 2010).

RÖHRICHT, C. (2009): Anbau und Wirtschaftlichkeit von KUP. Stand und Ergebnisse in Sachsen. Vortrag. In: Sächsisches Landesamt für Umwelt, Landwirtschaft und Geologie (Hrsg.): Workshop Energieholz auf KUP am 03. Dezember 2009, Köllitsch (URL: http://www.landwirtschaft. sachsen.de/landwirtschaft/download/03_Roehricht_2009_12_03.pdf, Zugriff am 10. Oktober 2011)

ROYLE, D.; OSTRY, M. (1995): Disease and pest control in the bioenergy crops poplar and willow. Biomass and Bioenergy 9: 69-79.

Rowell, D.L. (1997): Bodenkunde. Untersuchungsmethoden und ihre Anwendungen. Springer, Berlin, Heidelberg.

RUNNING, S.W.; GOWER, S. T. (1991): FOREST-BGC, a general model of forest ecosystem processes for regional applications. II. Dynamic carbon allocation and nitrogen budgets. Tree Physiology 9: 147160.

RUST, I. (2006): Aktualisierung der Bodenschätzung unter Berücksichtigung klimatischer Bedingungen. Dissertation. Fakultät Agrarwissenschaften, Universität Göttingen, Göttingen.

RutTer, A.J.; Kershaw, K.A.; RoBins, P.C.; MorTOn, A.J. (1971): A predictive model of rainfall interception in forests. I. Derivation of the model from observations in a plantation of corsican pine. Agricultural Meteorology 9: 367-384.

SACHS, L.; HEDDERICH, J. (2009): Angewandte Statistik. Methodensammlung mit R. Springer, Berlin, Heidelberg.

SAMPSON, D.A.; CEUlEMANS, R. (2000): SECRETS: simulated carbon fluxes from a mixed coniferous/deciduous Belgian forest. In: Ceulemans, R.J.M.; Veroustraete, F.; GOND, V.; VAN RENSBERGEN, J.B.H.F. (Hrsg.): Forest ecosystem modelling, upscaling and remote sensing. SPB Academic Publishing, The Hague, S. 95-108.

SANDS, P.J.; BATTAGLIA, M.; MUMMERY, D. (2000): Application of process-based models to forest management: experience with PROMOD, a simple plantation productivity model. Tree Physiology 20: 383-392.

SCHACK, H.; HILDEBRAND, E.E. (1988): Einfluss der mechanischen Beanspruchung eines Sandbodens auf das Wurzelwachstum von Forstpflanzen. Allgemeine Forst- und Jagdzeitung 159: 27-34.

SCHEFFER, F.; SCHACHTSCHABEL, P. (2002): Lehrbuch der Bodenkunde. Spektrum, Heidelberg.

SCHILDBACH, M.; LANDGRAF, D.; BÖCKER, L. (2008): Steckhölzer zur Begründung von Kurzumtriebsplantagen. AFZ der Wald 18/2008: 992-993.

SCHIRMER, R. (1996): Aspekte der Pflanzenzüchtung schnellwachsender Baumarten für Energiewälder. Proceeding. In: BAYERISCHE ANSATALT FÜR WALD- UND FORSTWIRTSCHAFT (Hrsg.): Schnellwachsende Baumarten, ihr Anbau und ihre Verwertung. Tagung am 25. Januar 1996, Neustadt. S. 7-17. 
SCHIRMER, R. (2011): Sortenprüfung von Pappelklonen - Voraussetzung für einen erfolgreichen Energieholzanbau. Proceeding. In: WERNER, D.M.; HAASE, B. (Hrsg.): Holzproduktion auf forstgenetischer Grundlage im Hinblick auf Klimawandel und Rohstoffverknappung. 28. Internationale Tagung der Arbeitsgemeinschaft (ARGE) für Forstgenetik und Forstpflanzenzüchtung vom 4. bis 6. November 2009 in Treis-Karden (Mosel). Mitteilungen aus der Forschungsanstalt für Waldökologie und Forstwirtschaft Rheinland-Pfalz Band 69, S. 123-129.

SCHLEPPHORST, RAINER (2013): mündliche Mitteilung 09. Oktober 2013, Hochschule für Nachhaltige Entwicklung Eberswalde, Eberswalde.

SCHIRMER, RANDOLF (2012): Schriftliche Mitteilung vom 14. Mai 2013, Bayerischer Amt für forstliche Saat- und Pflanzenzucht, Teisendorf.

SChMEeR, M.; Loges, R.; NANNEN, D.; SenbayRam, M.; TAUbe, F. (2009): Lachgasemissionen auf intensiv genutztem Grünland in Abhängigkeit von Bodenverdichtung und Stickstoffdüngung. Mitteilungen der Arbeitsgemeinschaft Grünland \& Futterbau 10: 79-82.

SCHMitZ-LENDERS, B. (1948): Pappel-Ertragstafeln: mit einer Pappel-Massentafel im Anhang. Schaper Verlag, Hannover-Waldhausen.

SCHMITZ-LENDERS, B. (1956): Mein Pappel-Testament: Erfahrungsgewinne und Forschungsergebnisse auf dem Gebiet des Pappel-Waldbbaues und der Pappel-Ertragskunde. Sauerländer, Frankfurt am Main.

SCHOBER, R. (1987): Ertragstafeln wichtiger Baumarten bei verschiedener Durchforstung. Sauerländer, Frankfurt am Main.

Scholz, V.; Beier, W.; Ellerbrock, R.; Hellebrand, J.; HöHn, A.; Kaulfuss, P.; KrÜGer, K.; KÜHLING, M.; PAGEL, R. (1999): Forschungsbericht des ATB. Umwelt- und technologiegerechter Anbau von Energiepflanzen. Institut für Agrartechnik Bornim e. V. (ATB), Landesanstalt für Landwirtschaft Brandenburg, Zentrum für Agrarlandschafts- und Landnutzungsforschung Müncheberg e. V., Potsdam-Bornim.

SCHOLZ, V.; ElLERBROCK, R. (2002): The growth productivity, and environmental impact of the cultivation of energy crops on sandy soils in Germany. Biomass and Bioenergy 23: 81-92.

SchOlZ, V.; Hellebrand, H. J.; HÖHN, A. (2004): Energetische und ökologische Aspekte der Feldholzproduktion. In: ATB POTSDAM BORNIM (Hrsg.): Energieholzproduktion in der Landwirtschaft - Potenzial, Anbau, Technologie, Ökologie und Ökonomie. Seminar am 29. Januar 2004 in Potsdam-Bornim. Bornimer Agrartechnische Berichte Heft 35, S. 15-31.

SCHOlZ, V.; KeRn, J.; KAUlfuss, P. (2010): Environmental effects of energy crop cultivation results of a long-term field trial. Agronomy Research 8: 445-452.

SChUlZ, U.; BRAuner, O.; SACHS, D.; TÜRING, M. (2008): Insekten an Pappeln und Weiden - erste Ergebnisse aus dem Projekt NOVALIS und Auswertung von Wirtspflanzenangaben. In: VERBUNDFORSCHUNGSVORHABEN DENDROM (Hrsg.): Holzerzeugung in der Landwirtschaft. Cottbuser Schriften zur Ökosystemgenese und Landschaftsentwicklung, Band 6. Brandenburgische Technische Universität Cottbus, Cottbus, S. 171-173.

SCHULZKE, RALF (2012): Mündliche Mitteilung vom 06. September 2012, Förderverein für nachwachsende Rohstoffe e.V., Hannoversch Münden.

SeVel, L.; Nord-LARSEN, T.; RAUlund-RASMUSSEN, K. (2012): Biomass production of four willow clones grown as short rotation coppice on two soil types in Denmark. Biomass and Bioenergy 46: 664672. 
SHAPIRO, S.S.; WILK, M.B. (1965): An analysis of variance test for normality (complete samples). Biometrika 52: 591-611.

SIEVÄNEN, R. (1983): Growth model for mini-rotation plantations. Communicationes Instituti Forestalis Fenniae Band 117. Finnish Forest Research Institute. Helsinki.

SiREN, G.; SENNERBY-Forse, L.; Ledin, S. (1987): Energy plantations-short rotation forestry. In: HALl, D.O.; OVEREND, R. (Hrsg.): Biomass: Regenerable energy. John Wiley and Sons, Chichester, S. 143-199.

SCHRÖDER, J.; RÖHLE, H.; MÜNDER, K. (2005): Simulation und Bewertung von Managementoptionen mit dem Waldwachstumssimulator BWINPro-S. Forst und Holz 60: 411-415.

Soriano, C. (1993): Populus. In: Castroviejo, S.; Aedo, C.; Cirujano, S.; Laínz, M.; Montserrat, P.; Morales, R.; Muñoz Garmendia, F.; Navarro, C.; Paiva, J.; Soriano, C. (Hrsg.): Flora Ibérica. Plantas vasculares de la Península Ibérica e Islas Baleares, Band 3: Plumbaginaceae (partim) - Capparaceae. Consejo de Investigaciones Cientifícas, Madrid, S. 471-477 (URL: http://www.floraiberica.es/floraiberica/texto/pdfs/03_070_01_Populus.pdf, Zugriff am 12. Oktober 2012).

SOUCH, C.A.; STEPHENS, W. (1998): Growth, productivity and water use in three hybrid poplar clones. Tree Physiology 18: 829-835.

STEINBECK, K. (1999): Thirty years of short-rotation hardwoods research. Proceeding. In: HAYwOOD, J.D. (Hrsg.): Tenth biennial southern silvicultural research conference vom 16. bis 18. Februar 1999, Shreveport. USDA Forest Service, General Technical Report SRS-30, Asheville, S. 63-66.

Stimm, B.; BlaschKe, H.; RothKegel, W.; RupPeRt, O. (2013): Stabilität hat tiefe Wurzeln. Die ideale Forstpflanze: Aspekte einer funktionalen Beziehung zwischen Wurzel und Spross. LWF aktuell 93: 9-14.

STOLL, B. (2011): Vergleich unterschiedlicher Anbaumethoden von Energiepflanzen. Dissertation. Fakultät für Forstwissenschaften und Waldökologie, Universität Göttingen, Göttingen.

STOUT, A.B.; SCHREINER E.J. (1933): Results of a project in hybridizing poplars. Journal of Heredity. 24: $216-229$.

STRECK, G. (2004): Einführung in die Statistik: für Geoökologen und andere Naturwissenschaftler. Books on Demand Gmbh. Norderstedt.

Strohm, K.; Schweinle, J.; Liesebach, M.; Osterburg, B.; RöDl, A.; Baum, S.; Nieberg, H. (2012): Kurzumtriebsplantagen aus ökologischerund ökonomischer Sicht. Arbeitsberichte aus der VTI-Agrarökonomie No. 06/2012. Braunschweig (URL: http://literatur.vti.bund.de/digbib_extern /bitv/dn050857.pdf, Zugriff am 03. April 2013).

STRONG, T.; HANSEN, E. (1993): Hybrid poplar spacing/productivity relations in short rotation intensive culture plantations. Biomass and Bioenergy 4: 255-261.

Tallis, M.J.; Casella, E.; Henshall, P.A.; AYlott, M.J.; Randle, T.H.; Morison, J.I.L.; TAYLOR, G. (2013): Development and evaluation of ForestGrowth-SRC a process-based model for short rotation coppice yield and spatial supply reveals poplar uses water more efficiently than willow.Global Change Biology Bioenergy 5: 53-66.

TGL 37868 (1987): Fachbereichstandard. Pappel und Baumweide. Wirtschaftssorten. Ministrium für Land-, Forst- und Nahrungsgüterwirtschaft, Hauptabteilung Forstwirtschaft, Berlin.

Tiessen, H.; Cuevas, E.; Chacon, P. (1994): The role of soil organic matter in sustaining soil fertility. Nature 371: 783-785. 
TISDALL, J. M.; OADES, J. (1982): Organic matter and water-stable aggregates in soils. Journal of Soil Science 33: 141-163.

TRINKAUS, P. (1998): Short rotation forestry: Discussion of 10 Austrian principles from the viewpoint of preservation of environment and nature. Biomass and Bioenergy 15: 109-114.

Trnka, M.; Trnka, P.; Fialova, J.; Koutecky, V.; Fajman, M.; Zalud, Z.; HejduK, S. (2008): Biomass prodcution and survival rates of selected poplar clones grwon under a short-rotation system on arable land. Plant and Soil Environment 54: 78-88.

UHLMANN, ASTRID (2013): mündliche Mitteilung vom 22. Februar 2013, Bundesanstalt für Landwirtschaft und Ernährung, Bonn.

Ulber, R.; MufFler, K.; TIPPKÖTTER, N.; HiRTH, T.; SELl, D. (2011): Introduction to renewable resources in the chemical industry. In: Ulber, R.; SELl, D.; HIRTH, T. (Hrsg.): Renewable raw materials - New feedstocks for the chemical industry. Wiley-VCH, Weinheim, S. 1-6.

UNSELD, R. (1999): Kurzumtriebsbewirtschaftung auf landwirtschaftlichen Grenzertragsböden: Biomassenproduktion und bodenökologische Auswirkungen verschiedener Baumarten. Dissertation. Fakultät für Forstwissenschaften, Universität Freiburg. Shaker, Aachen.

Vande Walle, I.; Van Camp, N.; Van de Casteele, L. Verheyen, K., Lemeur, R. (2007): Shortrotation forestry of birch, maple, poplar and willow in Flanders (Belgium) I- Biomass production after 4 years of tree growth. Biomass and Bioenergy 31: 267-275.

VDLUFA (VERBAND DEUTSCHER LANDWIRTSCHAFTLICHER UNTERSUCHUNGS- UND FORSCHUNGSANSTALTEN) (1991): Die Untersuchung von Böden - Band 1. Speyer.

VETTERLEIN, E. (1983): Beziehung zwischen hydraulischer Leitfähigkeit und Bodenwassergehalt in Sand-lehm-, Lehm- und Tonsubstraten. Archiv für Acker- Pflanzenbau und Bodenkunde 27: 417-426.

VERORDNUNG (EG) Nr. 1122/2009 DER KOMMISSION vom 30. November 2009 mit Durchführungsbestimmungen zur Verordnung(EG) Nr. 73/2009 des Rates hinsichtlich der Einhaltung anderweitiger Verpflichtungen, der Modulation und des integrierten Verwaltungs- und Kontrollsystems im Rahmen der Stützungsregelungen für Inhaber landwirtschaftlicher Betriebe gemäß der genannten Verordnung und mit Durchführungsbestimmungen zur Verordnung(EG) Nr. 1234/2007 hinsichtlich der Einhaltung anderweitiger Verpflichtungen im Rahmen der Stützungsregelung für den Weinsektor.

VERORDNUNG (EG) Nr. 73/2009 DES RATES vom 19. Januar 2009 mit gemeinsamen Regeln für Direktzahlungen im Rahmen der gemeinsamen Agrarpolitik und mit bestimmten Stützungsregelungen für Inhaber landwirtschaftlicher Betriebe und zur Änderung der Verordnungen (EG) Nr. 1290/2005, (EG) Nr. 247/2006, (EG) Nr. 378/2007 sowie zur Aufhebung der Verordnung (EG) Nr. 1782/2003

WANG, Y.P.; JARVIS, P.G. (1990): Description and validation of an array model - MAESTRO. Agricultural and Forest Meteorology 51: 257-280.

WBA (WISSENSCHAFTLICHER BEIRAT FÜR Agrarpolitik BEIM BUNDESMINISTERIUM FÜR ERNÄHRUNG LANDWIRTSCHAFT UND VERBRAUCHERSCHUTZ) (2007): Nutzung von Biomasse zur Energiegewinnung - Empfehlungen an die Politik. Berlin (URL: http://www.bmelv.de/SharedDocs/ Downloads/Ministerium/Beiraete/Agrarpolitik/GutachtenWBA.pdf?_blob=publicationFile, Zugriff am 16. März 2009).

WeILGUNI, V. (2006): Regionalisierung des Niederschlags. In: Blöschl, G.; Godina, R.; Merz, R. (Hrsg.): Methoden der hydrologischen Regionalisierung. ÖWAV - Seminar im Lebensministerium am 
18. bis 19. Mai 2006, Wien. Wiener Mitteilungen, Wasser-Abwasser-Gewässer, Band 197. Technische Universität Wien, Wien, S. 71-92.

WeInsteIn, D.A.; Beloin, R.M.; YANAI, R.D. (1991): Modeling changes in red spruce carbon balance and allocation in response to interacting ozone and nutrient stresses. Tree Physiology 9:127146.

WeISGERBER, H. (1980): Heutige Kenntnisse über die Formenvielfalt und Standortsansprüche von Pappeln sowie über deren Anbaumöglichkeiten in der Ebene. Proceeding. In: HÖHERE FORSTBEHÖRDE RHEINLAND (Hrsg.): Die Pappel am Niederrhein. Tagung am 20. März 1980, Mönchengladbach. Mitteilungen und Berichte aus dem Bereich der höheren Forstbehörde Rheinland, Heft 4. Rheinischer Landwirtschafts-Verlag, S. 7-20.

WENSEL, L.C.; BIGING, G.S. (1987): The CACTOS system for individual-tree growth simulation in the mixed conifer forests of California. In: EK, A.R.; SHIFLEY, S.R.; BURK, T.E. (Hrsg.): Forest Growth Modeling and Prediction. IUFRO Konferenz am 23. bis 27. August, Minneapolis, S. 175-183.

Werner, A.; VetTer, A.; REINHOLD, G. (2006): Leitlinie zur effizienten und umweltvertäglichen Erzeugung von Energieholz. Thüringer Landesanstalt für Landwirtschaft (TLL), Jena (URL: http://www.dendrom.de/daten/downloads/vetter_leitlinie\%20energieholz\%202006.pdf, Zugriff am 10. November 2012).

WIDIN, K.; SCHIPPER, A. (1981): Effect of Melampsora medusae leaf rust infection on yield of hybrid poplars in the north central United States. European Journal of Forest Pathology 11: 438-448.

WiLleBRAND, E.; LEDIN, S.; VERWIJST, T. (1993): Willow coppice systems in short rotation forestry: Effects of plant spacing, rotation length and clonal composition on biomass production. Biomass and Bioenergy 4: 323-331.

WitTich, R. (1951): Die Standorte der Pappel. In: Hesmer, H. (Hrsg.): Das Pappelbuch. Verlag des deutschen Pappelvereins, Bonn, S. 98-110.

WÖSTEN, J.H.M.; PACHEPSKY, Y.A.; RAWLS, W.J. (2001): Pedotransfer functions: bridging the gap between available basich soil data and missing hydraulic characteristics. Journal of Hydrology 251: 123-150.

ZALESNY, R.S.; WieSE, A.H.; BAUER, E.O.; RIEMENSChNEIDER, D.E. (2009): Ex situ growth and biomass of Populus bioenergy crops irrigated and fertilized with landfill leachate. Biomass and Bioenergy 33: 62-69.

Zasada, J.C.; Douglas, D.A.; Buechler, W. (2008): Salix L. willow. In: BonNer, F.T.; KARRFALT, R.P. (Hrsg.): Woody plant seed manual. United States Department of Agriculture, Forest Service. Agriculture Handbook 727. Hamden, S. 1000-1009 (URL: http://w3.cenn.org/wssl/programs /Woody_Plant_Seed_Manuall.pdf, Zugriff am 01. April 2011).

ZeCh, W.; Hintermaier-ERHARD, G. (2002): Böden der Erde. Spektrum, Heidelberg.

ZSUFFA, L.; GAMBLES, R.L. (1992): Improvement of energy-dedicated biomass production systems. Biomass and Bioenergy 2: 11-15. 


\section{Anhang}

Anhang 1: Übersicht der Porengrößen, Porendurchmesser, Wasserspannung, Porenraumbereiche sowie ihre Vorkommen in Böden (aus SCHEFFER und SCHACHTSCHABEL 2002, ergänzt durch FIEDLER 2001)

Anhang 2: Wassergehalt, Roh- und Darrdichten der untersuchten Pappel- und Weidenklone (ungleiche Buchstaben zeigen signifikante Unterschiede, in Klammer Standardabweichung - aus AMTHAUER GALLARDO und SEYMOUR 2011) .................178

Anhang 3: Klimastationen zur Ergänzung der Datenlage für die Ermittlung der langjährigen Klimawerte

Anhang 4: Box-Whiskers Plots der mittleren Trockenheitsindices 2008, 2009, 2010 sowie des Zeitfensters zwischen 1990 und 2010 über alle Standorte für die Bezugszeitfenster a) Jahr und b) VP

Anhang 5: Mittlere Monats-, Vegetationsperiode- und Jahres-Trockenheitsindexmittelwerte im Untersuchungszeitraum 2008-2010 von acht ausgewählten Standorten. Relative Vergleiche zum langjährigen Mittel wurden mittels Farbgebung gekennzeichnet.

Anhang 6: Anwuchsrate [\%] der Pappelklone Max 1, H 275, AF 2 sowie der Weidenklone Inger und Tordis im Anlagejahr 2008.

Anhang 7: Überlebensrate [\%] der Pappelklone Max 1, H 275, AF 2 sowie der Weidenklone Inger und Tordis in der zweiten Wuchsperiode (2009).

Anhang 8: Höhenentwicklung [m] der Pappelklone Max 1, H 275 und AF 2 sowie der Weidenklone Inger und Tordis für jede Wuchsperiode und Standortcluster.

Anhang 9: $\mathrm{d}_{0,1}$-Entwicklung [cm] der Pappelklone Max 1, H 275 und AF 2 sowie der Weidenklone Inger und Tordis für jede Wuchsperiode und Standortcluster.

Anhang 10: $d_{1,3}$-Entwicklung der Pappelklone Max 1, H 275 und AF 2 sowie der Weidenklone Inger und Tordis für jede Wuchsperiode und Standortcluster.

Anhang 11: Höhe- $\mathrm{d}_{1,3}$-Verhältnis der untersuchten Gattungen Pappel und Weide in Abhängigkeit der Bestandesmittelhöhe. Dargestellt sind Parzellenmittelwerte

Anhang 12: Höhe- $\mathrm{d}_{0,1}-$ Verhältnis der untersuchten Gattungen Pappel und Weide in Abhängigkeit der Bestandesmittelhöhe. Dargestellt sind Parzellenmittelwerte.

Anhang 13: $\mathrm{d}_{1,3}-\mathrm{d}_{0,1}-$ Verhältnis der untersuchten Gattungen Pappel und Weide in Abhängigkeit der Bestandesmittelhöhe. Dargestellt sind Parzellenmittelwerte

Anhang 14: dGZ-Mittelwerte $\left[\mathrm{t}_{(\text {atro) }} \mathrm{ha}^{-1} \mathrm{a}^{-1}\right]$ der dritten Wuchsperiode der Pappelklone Max 1, H 275 und AF 2 sowie der Weidenklone Inger und Tordis im Bodenartcluster S (ungleiche Buchstaben zeigen signifikante Unterschiede innerhalb der Prüfglieder eines Standorts)

Anhang 15: dGZ-Mittelwerte $\left[\mathrm{t}_{\text {(atro) }} \mathrm{ha}^{-1} \mathrm{a}^{-1}\right]$ der dritten Wuchsperiode der Pappelklone Max 1, H 275 und AF 2 sowie der Weidenklone Inger und Tordis im Bodenartcluster U (ungleiche Buchstaben zeigen signifikante Unterschiede innerhalb der Prüfglieder eines Standorts)

Anhang 16: dGZ-Mittelwerte $\left[\mathrm{t}_{\text {(atro) }} \mathrm{ha}^{-1} \mathrm{a}^{-1}\right]$ der dritten Wuchsperiode der Pappelklone Max 1, H 275 und AF 2 sowie der Weidenklone Inger und Tordis im Bodenartcluster L (ungleiche Buchstaben zeigen signifikante Unterschiede innerhalb der Prüfglieder eines Standorts)

Anhang 17: Statistische Kennwerte der Biomassefunktionen für Max 1 mit der allometrischen Anpassung und der $d_{1,3}$ als unabhängige Variable

Anhang 18: Statistische Kennwerte der Biomassefunktionen für H 275 mit der allometrischen Anpassung und der $d_{1,3}$ als unabhängige Variable

Anhang 19: Statistische Kennwerte der Biomassefunktionen für AF 2 mit der allometrischen Anpassung und der $d_{1,3}$ als unabhängige Variable

Anhang 20: Statistische Kennwerte der Biomassefunktionen für Inger mit der allometrischen Anpassung und der $d_{1,3}$ als unabhängige Variable

Anhang 21: Statistische Kennwerte der Biomassefunktionen für Tordis mit der allometrischen Anpassung und der $d_{1,3}$ als unabhängige Variable

Anhang 22: Statistische Kennwerte der Biomassefunktionen für Max 1 mit der allometrischen Anpassung und der $d_{0,1}$ als unabhängige Variable

Anhang 23: Statistische Kennwerte der Biomassefunktionen für H 275 mit der allometrischen Anpassung und der $d_{0,1}$ als unabhängige Variable

Anhang 24: Statistische Kennwerte der Biomassefunktionen für AF 2 mit der allometrischen Anpassung und der $d_{0,1}$ als unabhängige Variable 
Anhang 25: Statistische Kennwerte der Biomassefunktionen für Inger mit der allometrischen Anpassung und der $d_{0,1}$ als unabhängige Variable

Anhang 26: Statistische Kennwerte der Biomassefunktionen für Tordis mit der allometrischen Anpassung und der $\mathrm{d}_{0,1}$ als unabhängige Variable

Anhang 27: Allgemeingültige Biomassefunktionen für die Bestandesdichte 11.111 Stk. ha ${ }^{-1}$ der Pappelklone Max 1, H 275 , AF 2 sowie der Weidenklone Inger und Tordis mit dem d0,1 als unabhängige Variable

Anhang 28: Statistische Kennwerte der Biomassefunktionen der Pappelklone Max 1, H 275, AF 2 sowie der Weidenklone Inger und Tordis mit dem $\mathrm{d} 0,1$ als unabhängige Variable.

Anhang 29: Korrelationsanalyse zwischen Porenraumvariablen hergeleitet beim Feuchtigskeitsäquivalenten sowie nach dem Standard beim pF 1,8 und dem dGZ für die untersuchten Klone in den Standortclustern ALL, S, U und L..........................197

Anhang 30: Analyse zur Variablen-Interaktion im Standortcluster ALL, $r>0,80$ sind unterstrichen angegeben 198

Anhang 31: Analyse zur Variablen-Interaktion im Standortcluster S, r > 0,80 sind unterstrichen angegeben 198

Anhang 32: Analyse zur Variablen-Interaktion im Standortcluster $U, r>0,80$ sind unterstrichen angegeben 199

Anhang 33: Analyse zur Variablen-Interaktion im Standortcluster L, $r>0,80$ sind unterstrichen angegeben

Anhang 34: dGZ des Klons Max 1 bei Standorten unterschiedlicher Vornutzung in der dritten Wuchsperiode (deskriptiv berücksichtigte Flächen)

Anhang 35: dGZ des Klons Max 1 bei Standorten unterschiedlichen Bodentyps in der dritten Wuchsperiode (deskriptiv berücksichtigte Flächen)

Anhang 36: Modelle in denen die multiple nicht-lineare Regressionen höhere $\mathrm{R}^{2}$ korr als die univariate Modelle erzielten....200

Anhang 37:Amplitude der zur Modellierung verwendeten aggregierte Variablen .201

Anhang 38:Amplitude der zur Modellierung verwendeten nicht-aggregierte Variablen. 201 
Anhang 1: Übersicht der Porengrößen, Porendurchmesser, Wasserspannung, Porenraumbereiche sowie ihre Vorkommen in Böden (aus SCHEFFER und SCHACHTSCHABEL 2002, ergänzt durch FIEDLER 2001).

\begin{tabular}{llllll}
\hline Porengröße & $\begin{array}{l}\text { Porendurchmesser } \\
{[\mu \mathrm{m}]}\end{array}$ & $\begin{array}{l}\text { Wasserspan- } \\
\text { nung }[\mathrm{hPa}]\end{array}$ & $\mathrm{pF}$ & $\begin{array}{l}\text { Porenraum- } \\
\text { bereich }\end{array}$ & Vorkommen \\
\hline Makroporen & $>2000$ & -4 & $<0,6$ & $\mathrm{LK}$ & Spalten, Risse \\
Weite Grobporen & $>50$ & $-4--60$ & $0,6-1,8$ & $\mathrm{LK}$ & $\begin{array}{l}\text { Sandböden } \\
\text { Schluff- und } \\
\text { Enge Grobporen }\end{array}$ \\
Mittelporen & $50-10$ & $-60--300$ & $1,8-2,5$ & $\mathrm{nFK}^{\mathrm{a}}$ & $\begin{array}{l}\text { Lehmböden } \\
\text { Feinporen }\end{array}$ \\
\hline
\end{tabular}

* Je nach Definition befindet sich die Grenze zwischen LK und nFK in diesem Bereich

Anhang 2: Wassergehalt, Roh- und Darrdichten der untersuchten Pappel- und Weidenklone (ungleiche Buchstaben zeigen signifikante Unterschiede, in Klammer Standardabweichung - aus AMTHAUER GALLARDO und SEYMOUR 2011).

\begin{tabular}{llccc}
\hline Gattung & Klon & $\begin{array}{c}\text { Wassergehalt } \\
{[\%]}\end{array}$ & $\begin{array}{c}\text { Rohdichte } \\
{\left[\mathrm{kg} \mathrm{m}^{-3}\right]}\end{array}$ & $\begin{array}{c}\text { Darrdichte } \\
{\left[\mathrm{kg} \mathrm{m}^{-3}\right]}\end{array}$ \\
\hline \multirow{2}{*}{ Pappel } & Max 1 & $54,6 \mathrm{c}$ & $340(40)$ & $450(50)$ \\
& H 275 & $50,8 \mathrm{a}$ & $385(25)$ & $510(30)$ \\
& AF 2 & $56,7 \mathrm{~d}$ & $305(20)$ & $370(10)$ \\
\multirow{2}{*}{ Weide } & Inger & $51,4 \mathrm{ab}$ & $390(20)$ & $500(30)$ \\
& Tordis & $52,5 \mathrm{~b}$ & $450(40)$ & $510(10)$ \\
\hline
\end{tabular}


Anhang 3: Klimastationen zur Ergänzung der Datenlage für die Ermittlung der langjährigen Klimawerte.

\begin{tabular}{|c|c|c|}
\hline Sto.-Nr. & Standortname & Ergänzende Klimastation \\
\hline 1 & Emmendingen & Emmendingen Mün. \\
\hline 2 & Lilienthal & Freiburg \\
\hline 3 & Aulendorf & Pfullendorf \\
\hline 4 & Forchheim & Karlsruhe \\
\hline 5 & Ladenburg & Heidelberg \\
\hline 6 & Kupferzell & Öhringen \\
\hline 7 & Kaisheim & Donauwörth-Osterweiler \\
\hline 8 & E.Wald Welzow & Cottbus \\
\hline 9 & Löwenberg & Zehdenik \\
\hline 11 & Cahnsdorf & Lübben Blumenfelde \\
\hline 12 & Kummerow & Angermünde \\
\hline 13 & Grünewalde & Klettwitz \\
\hline 14 & Welzow Süd & Cottbus \\
\hline 15 & Potsdam-Bornim & Potsdam \\
\hline 16 & Lehmkaute & Giessen \\
\hline 17 & Unterrieden & Göttingen \\
\hline 18 & Gülzow & Teterow \\
\hline 19 & Werlte & Bremen \\
\hline 20 & Borlinghausen & Kassel \\
\hline 22 & Haus Düsse & Lippstadt-Börkenförde \\
\hline 24 & Königshov. Höhe & Heinsberg-Schleiden \\
\hline 25 & Klein Altendorf & Neuenahr, Bad-Ahrweiler \\
\hline 27 & Bärenrode & Harzgerode \\
\hline 28 & Bernburg & Bernburg/Saale \\
\hline 29 & Hayn & Harzgerode \\
\hline 30 & Iden & Seehausen \\
\hline 31 & Schlag Adler & Kubschütz \\
\hline 32 & Thammenhain I & Klitzschen \\
\hline 33 & Thammenhain II & Klitzschen \\
\hline 35 & Threnthorst & Lübeck-Blankensee \\
\hline 36 & Oberdorla & Ebeleben \\
\hline 37 & Ü. d. Erdengraben & Jena (Sternwarte) \\
\hline 38 & Heiliges Marpingen & Tholey \\
\hline
\end{tabular}


Anhang 4: Box-Whiskers Plots der mittleren Trockenheitsindices 2008, 2009, 2010 sowie des Zeitfensters zwischen 1990 und 2010 über alle Standorte für die Bezugszeitfenster a) Jahr und b) VP.

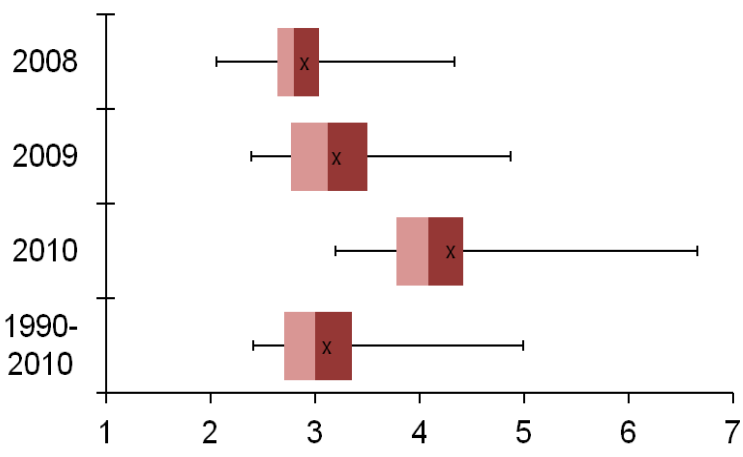

a)

Jahres-Trockenheitsindexmittelwert

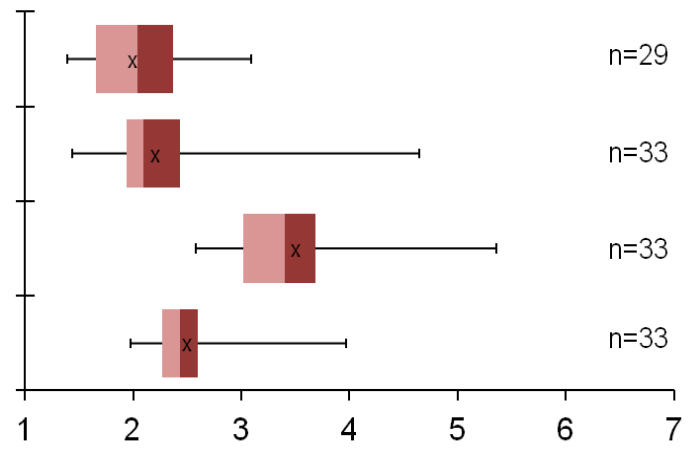

b)

VP-Trockenheitsindexmittelwert

Anhang 5: Mittlere Monats-, Vegetationsperiode- und Jahres-Trockenheitsindexmittelwerte im Untersuchungszeitraum 2008-2010 von acht ausgewählten Standorten. Relative Vergleiche zum langjährigen Mittel wurden mittels Farbgebung gekennzeichnet.

\begin{tabular}{|c|c|c|c|c|c|c|c|c|c|}
\hline \multirow[b]{2}{*}{ Zeitraum } & \multicolumn{8}{|c|}{ Trockenheitsindex } & \multirow[b]{2}{*}{$\begin{array}{l}\text { Abweichung vom } \\
\text { langjährigen } \\
\text { Mittelwert }\end{array}$} \\
\hline & $\begin{array}{l}\text { Sto. Nr. } 1 \\
\text { "Emmen- } \\
\text { dingen" }\end{array}$ & $\begin{array}{l}\text { Sto. Nr. } 15 \\
\text { "Potsdam } \\
\text { Bornim" }\end{array}$ & $\begin{array}{c}\text { Sto. Nr. } 17 \\
\text { "Unterrie- } \\
\text { den" }\end{array}$ & $\begin{array}{l}\text { Sto. Nr. } 19 \\
\text { "Werlte" }\end{array}$ & $\begin{array}{l}\text { Sto. Nr. } 25 \\
\text { "Klein- } \\
\text { Altendorf" }\end{array}$ & $\begin{array}{l}\text { Sto. Nr. } 29 \\
\text { "Hayn" }\end{array}$ & $\begin{array}{l}\text { Sto. Nr. } 31 \\
\text { "Schlag } \\
\text { Adler" }\end{array}$ & $\begin{array}{l}\text { Sto. Nr. } 37 \\
\text { "ü. d. Erd- } \\
\text { engraben" }\end{array}$ & \\
\hline Januar & 2,9 & 5,2 & 3,6 & 6,0 & 2,9 & 4,2 & 5,6 & 2,5 & $+[>40 \%]$ \\
\hline Februar & 3,5 & 2,6 & 3,6 & 4,2 & 2,9 & 3,7 & 2,8 & 2,8 & $+[30-40 \%]$ \\
\hline März & 4,1 & 2,9 & 3,5 & 4,9 & 4,8 & 3,6 & 3,9 & 2,9 & $+[20-30 \%]$ \\
\hline April & 2,8 & 1,2 & 1,7 & 1,2 & 1,9 & 2,5 & 1,6 & 3,1 & $+[10-20 \%]$ \\
\hline Mai & 3,2 & 2,5 & 2,5 & 1,9 & 2,6 & 3,6 & 3,5 & 2,6 & $+[0-10 \%]$ \\
\hline Juni & 1,8 & 1,4 & 2,3 & 1,5 & 2,5 & 2,0 & 2,2 & 1,3 & - $[0-10 \%]$ \\
\hline Juli & 3,4 & 1,6 & 2,8 & 3,1 & 2,1 & 2,6 & 3,1 & 3,0 & $-[10-20 \%]$ \\
\hline August & 4,4 & 2,0 & 2,5 & 3,2 & 2,8 & 2,3 & 4,5 & 2,7 & - [20 - $30 \%]$ \\
\hline September & 2,8 & 3,0 & 2,4 & 2,3 & 2,1 & 3,0 & 3,1 & 2,2 & - $[30-40 \%]$ \\
\hline Oktober & 3,7 & 3,2 & 2,3 & 3,6 & 1,7 & 2,9 & 3,3 & 2,3 & - $[>40 \%]$ \\
\hline November & 3,1 & 3,9 & 4,2 & 4,2 & 2,5 & 5,2 & 3,9 & 4,2 & \\
\hline Dezember & 6,9 & 6,8 & 4,8 & 4,3 & 4,5 & 8,7 & 7,2 & 7,4 & \\
\hline Jahr & 3,6 & 3,0 & 3,0 & 3,4 & 2,8 & 3,7 & 3,7 & 3,1 & \\
\hline VP & 3,1 & 2,1 & 2,5 & 2,4 & 2,4 & 2,7 & 3,3 & 2,4 & \\
\hline
\end{tabular}


Anhang 6: Anwuchsrate [\%] der Pappelklone Max 1, H 275, AF 2 sowie der Weidenklone Inger und Tordis im Anlagejahr 2008.

\begin{tabular}{|c|c|c|c|c|c|c|}
\hline \multirow{2}{*}{$\begin{array}{l}\text { Sto. } \\
\text { Nr. }\end{array}$} & \multirow{2}{*}{ Standortname } & \multicolumn{5}{|c|}{ Anwuchsrate [\%] } \\
\hline & & $\operatorname{Max} 1$ & Н 275 & AF 2 & Inger & Tordis \\
\hline 1 & Emmendingen & 98 & 79 & 96 & 100 & 97 \\
\hline 2 & Lilienthal & 85 & 74 & 95 & 99 & 98 \\
\hline 3 & Aulendorf & 94 & 87 & 98 & 98 & 97 \\
\hline 4 & Forchheim & 90 & 67 & 83 & 90 & 83 \\
\hline 5 & Ladenburg & 75 & 26 & 67 & 88 & 47 \\
\hline 6 & Kupferzell & 71 & 14 & 84 & 70 & 35 \\
\hline 7 & Kaisheim & 94 & 83 & 96 & 99 & 98 \\
\hline 8 & E.Wald Welzow & 92 & 60 & 78 & 77 & 67 \\
\hline 9 & Löwenberg & 84 & 89 & 87 & 94 & 86 \\
\hline $11^{*}$ & Cahnsdorf & 40 & 6 & 12 & 21 & 37 \\
\hline 12 & Kummerow & 92 & 83 & 82 & 81 & 90 \\
\hline 13 & Grünewalde & 100 & 94 & 98 & 99 & 100 \\
\hline 14 & Welzow Süd & 89 & 61 & 76 & 85 & 75 \\
\hline 15 & Potsdam Bornim & 67 & 61 & 94 & 89 & 90 \\
\hline 16 & Lehmkaute & 74 & 18 & 74 & 92 & 87 \\
\hline 17 & Unterrieden & 93 & 89 & 97 & 100 & 100 \\
\hline 18 & Gülzow & 94 & 84 & 100 & 100 & 100 \\
\hline 19 & Werlte & 77 & 6 & 82 & 93 & 88 \\
\hline 20 & Borlinghausen & 93 & 59 & 90 & 92 & 99 \\
\hline 22 & Haus Düsse & 97 & 88 & 97 & 95 & 95 \\
\hline 24 & Königshov. Höhe & 92 & 77 & 90 & 96 & 96 \\
\hline 25 & Klein Altendorf & 99 & 96 & 92 & 99 & 100 \\
\hline 27 & Bärenrode & 86 & 61 & 47 & 78 & 63 \\
\hline 28 & Bernburg & 92 & 67 & 96 & 99 & 91 \\
\hline 29 & Hayn & 96 & 89 & 90 & 96 & 94 \\
\hline 30 & Iden & 91 & 74 & 83 & 84 & 72 \\
\hline 31 & Schlag Adler & 98 & 95 & 98 & 99 & 100 \\
\hline $32^{*}$ & Thammenhain I & 27 & 2 & 17 & 74 & 35 \\
\hline $33^{*}$ & Thammenhain II & 36 & 5 & 47 & 56 & 55 \\
\hline 35 & Threnthorst & 98 & 76 & 98 & 100 & 98 \\
\hline 36 & Oberdorla & 72 & 21 & 76 & 95 & 96 \\
\hline 37 & Ü. d. Erdengraben & 97 & 45 & 92 & 100 & 99 \\
\hline 38 & Heiliges Marpingen & 93 & 98 & 85 & 91 & 92 \\
\hline
\end{tabular}

*Bezeichnet die in 2009 komplett nachgebesserten Standorte 
Anhang 7: Überlebensrate [\%] der Pappelklone Max 1, H 275, AF 2 sowie der Weidenklone Inger und Tordis in der zweiten Wuchsperiode (2009).

\begin{tabular}{|c|c|c|c|c|c|c|}
\hline \multirow{2}{*}{$\begin{array}{l}\text { Sto. } \\
\text { Nr. }\end{array}$} & \multirow{2}{*}{ Standortname } & \multicolumn{5}{|c|}{ Überlebensrate [\%] } \\
\hline & & Max 1 & Н 275 & AF 2 & Inger & Tordis \\
\hline 1 & Emmendingen & 100 & 86 & 98 & 100 & 99 \\
\hline 2 & Lilienthal & 90 & 81 & 100 & 92 & 99 \\
\hline 3 & Aulendorf & 89 & 89 & 89 & 93 & 91 \\
\hline 4 & Forchheim & 96 & 100 & 97 & 95 & 97 \\
\hline 5 & Ladenburg & 99 & 98 & 99 & 100 & 98 \\
\hline 6 & Kupferzell & 99 & 99 & 99 & 99 & 97 \\
\hline 7 & Kaisheim & 93 & 99 & 94 & 99 & 98 \\
\hline 8 & E.Wald Welzow & 95 & 98 & 95 & 95 & 98 \\
\hline 9 & Löwenberg & 87 & 77 & 86 & 95 & 89 \\
\hline 11 & Cahnsdorf & 67 & 60 & 71 & 96 & 98 \\
\hline 12 & Kummerow & 100 & 98 & 99 & 100 & 99 \\
\hline 13 & Grünewalde & 98 & 94 & 96 & 98 & 99 \\
\hline 14 & Welzow Süd & 92 & 89 & 82 & 89 & 86 \\
\hline 15 & Potsdam Bornim & 98 & 95 & 96 & 97 & 93 \\
\hline 16 & Lehmkaute & 93 & 92 & 97 & 91 & 93 \\
\hline 17 & Unterrieden & 94 & 100 & 97 & 100 & 100 \\
\hline 18 & Gülzow & 98 & 98 & 84 & 96 & 97 \\
\hline 19 & Werlte & 94 & 76 & 96 & 94 & 97 \\
\hline 20 & Borlinghausen & 97 & 99 & 95 & 96 & 95 \\
\hline 22 & Haus Düsse & 99 & 95 & 97 & 98 & 95 \\
\hline 24 & Königshov. Höhe & 96 & 91 & 95 & 100 & 99 \\
\hline 25 & Klein Altendorf & 99 & 95 & 92 & 98 & 100 \\
\hline 27 & Bärenrode & 97 & 95 & 68 & 97 & 93 \\
\hline 28 & Bernburg & 95 & 99 & 96 & 98 & 93 \\
\hline 29 & Hayn & 98 & 95 & 91 & 97 & 99 \\
\hline 30 & Iden & 93 & 96 & 99 & 99 & 97 \\
\hline 31 & Schlag Adler & 98 & 96 & 98 & 99 & 100 \\
\hline 32 & Thammenhain I & 96 & 91 & 94 & 96 & 97 \\
\hline 33 & Thammenhain II & 95 & 89 & 93 & 97 & 97 \\
\hline 35 & Threnthorst & 97 & 57 & 98 & 100 & 98 \\
\hline 36 & Oberdorla & 97 & 91 & 99 & 95 & 96 \\
\hline 37 & Ü. d. Erdengraben & 96 & 71 & 94 & 98 & 99 \\
\hline
\end{tabular}


Anhang 8: Höhenentwicklung [m] der Pappelklone Max 1, H 275 und AF 2 sowie der Weidenklone Inger und Tordis für jede Wuchsperiode und Standortcluster.

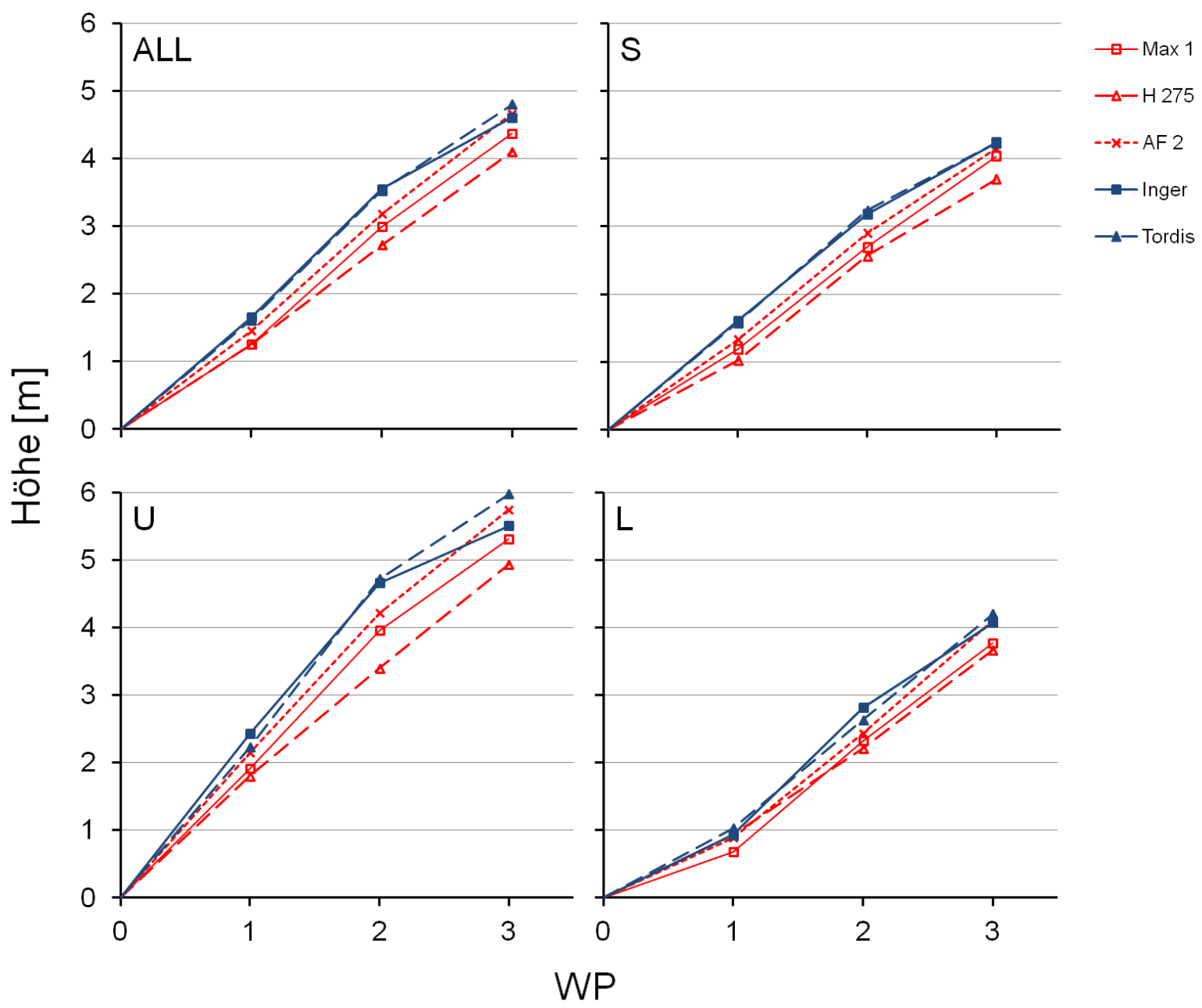


Anhang 9: $\mathrm{d}_{0,1}$-Entwicklung [cm] der Pappelklone Max 1, H 275 und AF 2 sowie der Weidenklone Inger und Tordis für jede Wuchsperiode und Standortcluster.

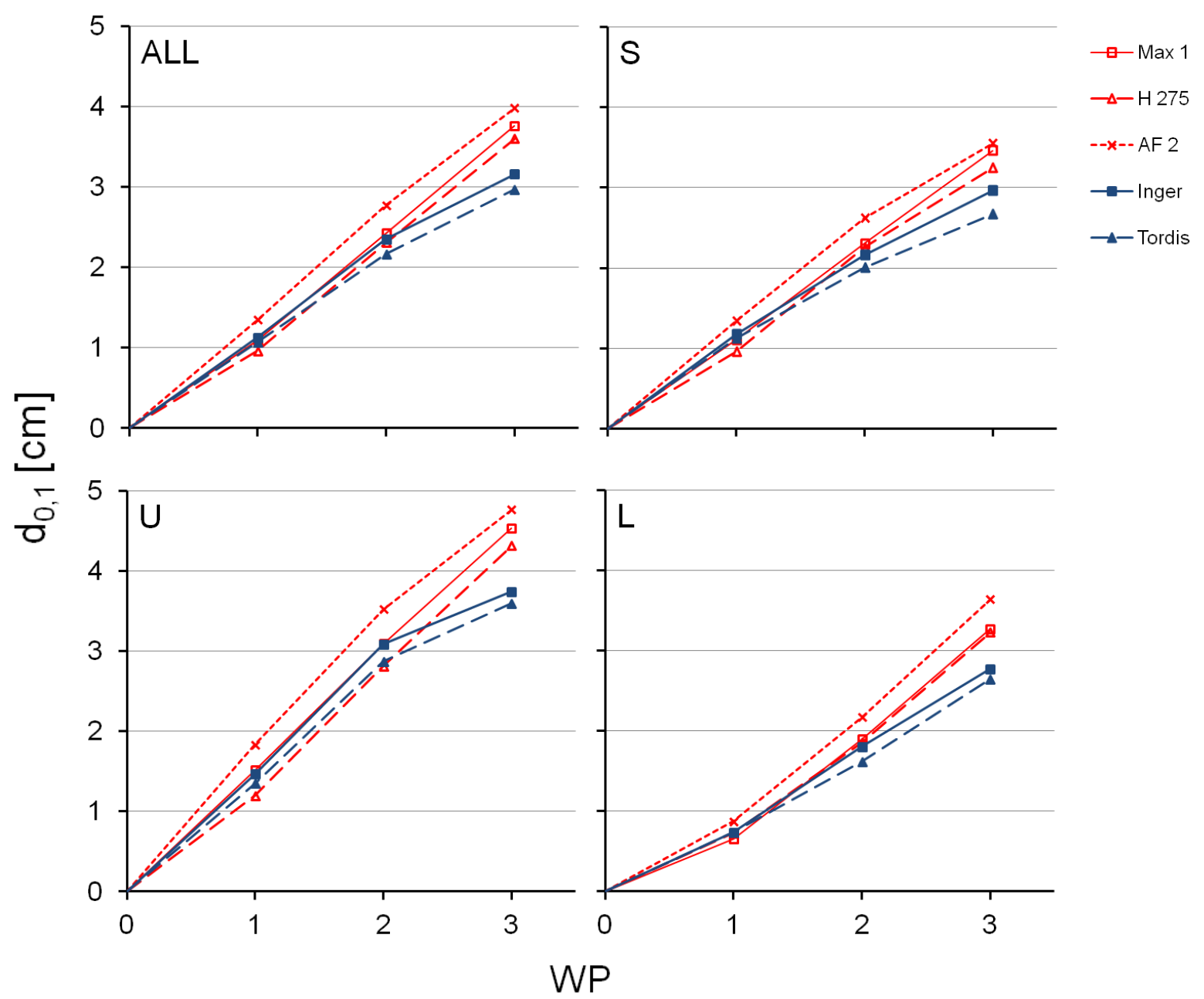


Anhang 10: $\mathrm{d}_{1,3}$-Entwicklung der Pappelklone Max 1, H 275 und AF 2 sowie der Weidenklone Inger und Tordis für jede Wuchsperiode und Standortcluster.

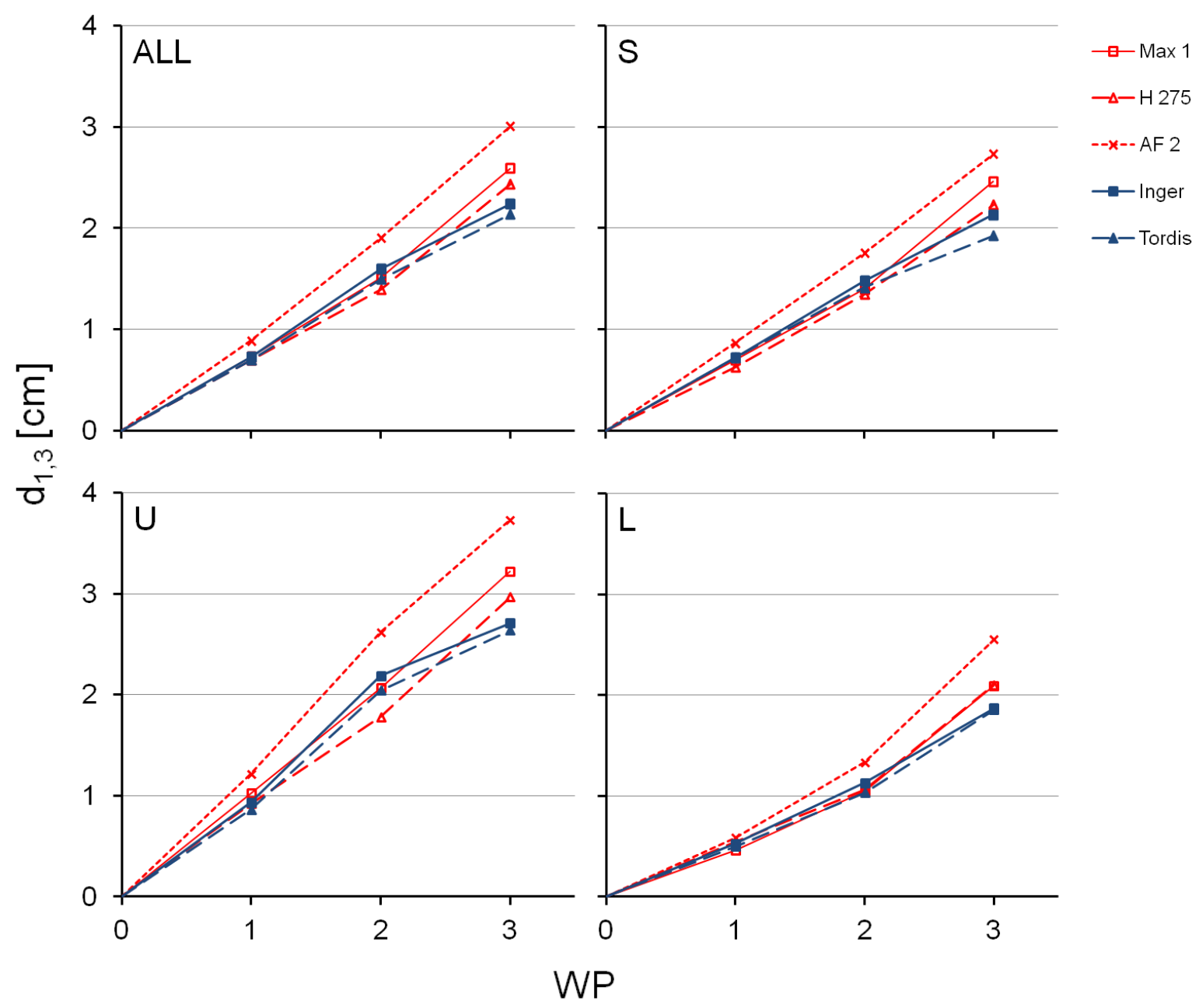


Anhang 11: Höhe- $\mathrm{d}_{1,3}$-Verhältnis der untersuchten Gattungen Pappel und Weide in Abhängigkeit der Bestandesmittelhöhe. Dargestellt sind Parzellenmittelwerte.

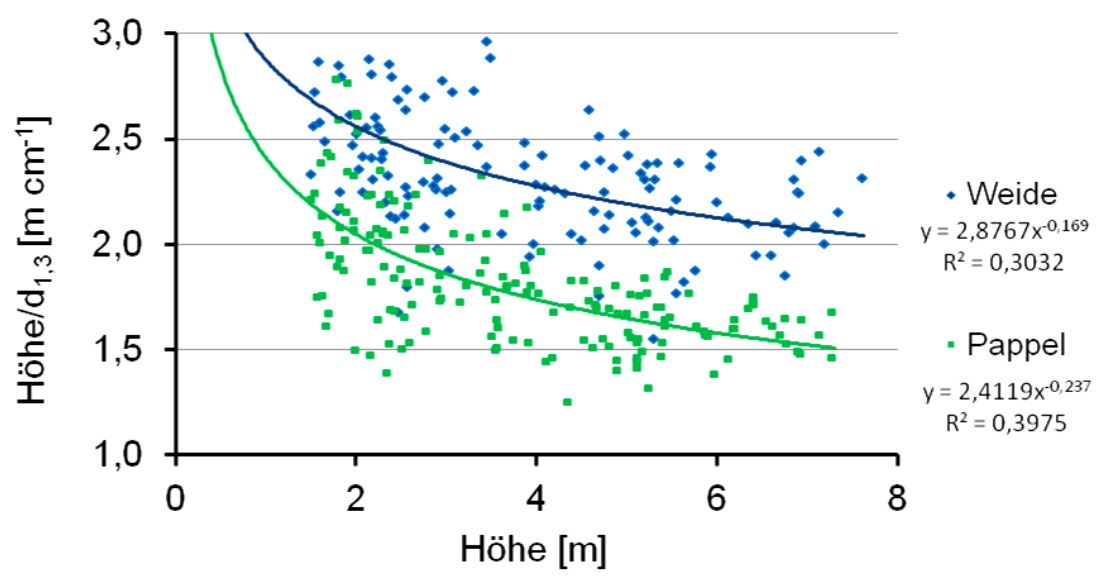

Anhang 12: Höhe- $\mathrm{d}_{0,1}$-Verhältnis der untersuchten Gattungen Pappel und Weide in Abhängigkeit der Bestandesmittelhöhe. Dargestellt sind Parzellenmittelwerte.

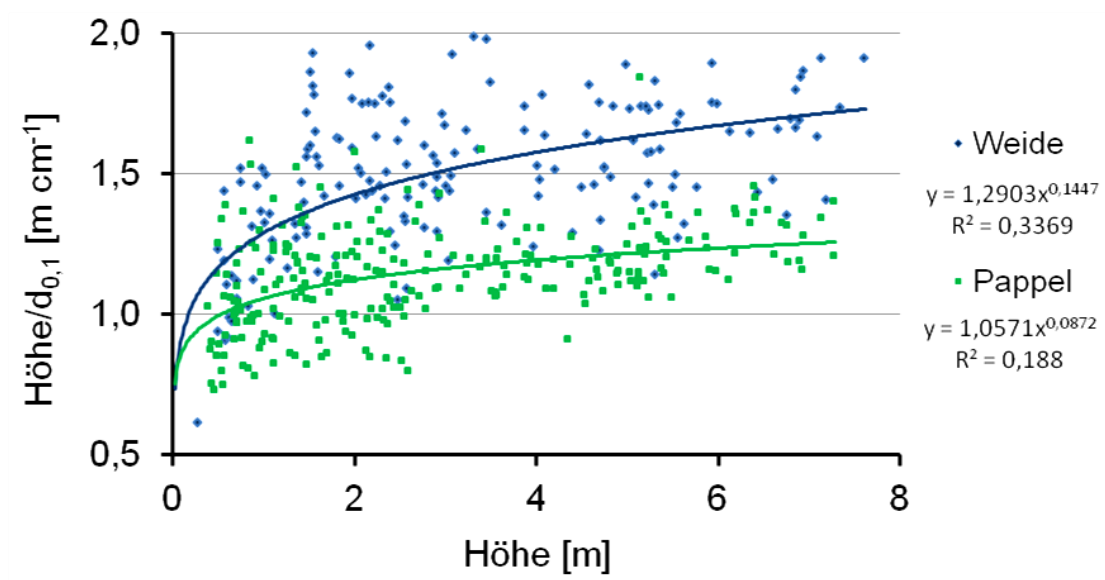

Anhang 13: $\mathrm{d}_{1,3}-\mathrm{d}_{0,1}$-Verhältnis der untersuchten Gattungen Pappel und Weide in Abhängigkeit der Bestandesmittelhöhe. Dargestellt sind Parzellenmittelwerte.

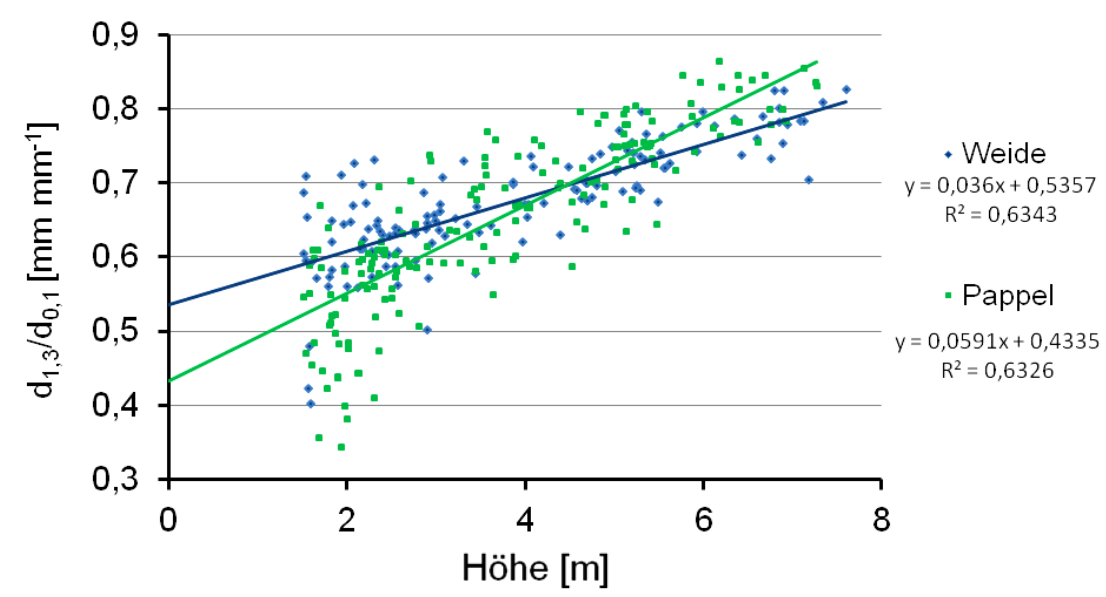


Anhang 14: dGZ-Mittelwerte $\left[\mathrm{t}_{\text {(atro) }} \mathrm{ha}^{-1} \mathrm{a}^{-1}\right]$ der dritten Wuchsperiode der Pappelklone Max 1, H 275 und AF 2 sowie der Weidenklone Inger und Tordis im Bodenartcluster S (ungleiche Buchstaben zeigen signifikante Unterschiede innerhalb der Prüfglieder eines Standorts).

\begin{tabular}{ccccccccccccc}
\hline & & \multicolumn{10}{c}{$\mathrm{dGZ}\left[\mathrm{t}_{\text {(atro }} \mathrm{ha}^{-1} \mathrm{a}^{-1}\right]$} \\
\cline { 3 - 12 } Gattung & Klon & & Sto. Nr. & Sto. Nr. & Sto. Nr. & Sto. Nr. & Sto. Nr. & Sto. Nr. & Sto. Nr. & Sto. Nr. & Sto. Nr. & Sto. Nr. \\
14 & 30 & 18 & 12 & 4 & 13 & 15 & 31 & 19 & 17 \\
\hline \multirow{2}{*}{ Pappel } & Max 1 & $0,17 \mathrm{~b}$ & $0,37 \mathrm{c}$ & $1,39 \mathrm{a}$ & $1,96 \mathrm{a}$ & $5,46 \mathrm{a}$ & $4,63 \mathrm{ab}$ & $4,50 \mathrm{~b}$ & $7,36 \mathrm{c}$ & $6,43 \mathrm{~b}$ & $9,91 \mathrm{a}$ \\
& H 275 & $0,25 \mathrm{a}$ & $0,50 \mathrm{bc}$ & $0,55 \mathrm{c}$ & $1,97 \mathrm{a}$ & $4,41 \mathrm{bc}$ & $3,87 \mathrm{~b}$ & $2,99 \mathrm{~b}$ & $8,89 \mathrm{ab}$ & n.v. & $10,21 \mathrm{a}$ \\
& AF 2 & $0,20 \mathrm{~b}$ & $1,46 \mathrm{a}$ & $0,77 \mathrm{~b}$ & $0,85 \mathrm{~b}$ & $4,52 \mathrm{~b}$ & $3,58 \mathrm{~b}$ & $5,92 \mathrm{a}$ & $6,61 \mathrm{c}$ & $7,26 \mathrm{~b}$ & $8,76 \mathrm{a}$ \\
\multirow{2}{*}{ Weide } & Inger & $0,18 \mathrm{~b}$ & $0,84 \mathrm{ab}$ & $1,39 \mathrm{a}$ & $1,89 \mathrm{a}$ & $2,76 \mathrm{c}$ & $3,43 \mathrm{c}$ & $3,75 \mathrm{~b}$ & $8,08 \mathrm{bc}$ & $9,23 \mathrm{a}$ & $9,13 \mathrm{a}$ \\
& Tordis & $0,08 \mathrm{~b}$ & $0,41 \mathrm{c}$ & $0,75 \mathrm{bc}$ & $1,72 \mathrm{a}$ & $2,16 \mathrm{~d}$ & $4,75 \mathrm{a}$ & $3,52 \mathrm{~b}$ & $9,11 \mathrm{a}$ & $9,14 \mathrm{a}$ & $9,97 \mathrm{a}$ \\
\hline
\end{tabular}

Anhang 15: dGZ-Mittelwerte $\left[\mathrm{t}_{(\text {atro) }} \mathrm{ha}^{-1} \mathrm{a}^{-1}\right]$ der dritten Wuchsperiode der Pappelklone Max 1, H 275 und AF 2 sowie der Weidenklone Inger und Tordis im Bodenartcluster U (ungleiche Buchstaben zeigen signifikante Unterschiede innerhalb der Prüfglieder eines Standorts).

\begin{tabular}{|c|c|c|c|c|c|c|c|c|c|}
\hline \multirow{2}{*}{ Gattung } & \multirow{2}{*}{ Klon } & \multicolumn{8}{|c|}{$\mathrm{dGZ}\left[\mathrm{t}_{(\text {atro) }} \mathrm{ha}^{-1} \mathrm{a}^{-1}\right]$} \\
\hline & & $\begin{array}{c}\text { Sto. Nr. } \\
7\end{array}$ & $\begin{array}{c}\text { Sto. Nr. } \\
2\end{array}$ & $\begin{array}{c}\text { Sto. Nr. } \\
22\end{array}$ & $\begin{array}{c}\text { Sto. Nr. } \\
36\end{array}$ & $\begin{array}{c}\text { Sto. Nr. } \\
1\end{array}$ & $\begin{array}{c}\text { Sto. Nr. } \\
37\end{array}$ & $\begin{array}{c}\text { Sto. Nr. } \\
24\end{array}$ & $\begin{array}{c}\text { Sto. Nr. } \\
28\end{array}$ \\
\hline \multirow{3}{*}{ Pappel } & $\operatorname{Max} 1$ & $0,72 \mathrm{c}$ & $6,07 \mathrm{a}$ & $5,00 \mathrm{~b}$ & $4,92 \mathrm{~b}$ & $7,59 \mathrm{a}$ & $7,39 \mathrm{a}$ & $7,06 \mathrm{~b}$ & $10,14 a b$ \\
\hline & Н 275 & $1,62 \mathrm{~b}$ & $2,71 \mathrm{~b}$ & $5,28 \mathrm{~b}$ & n.v. & $5,25 \mathrm{~b}$ & n.v. & $3,70 \mathrm{c}$ & $8,04 \mathrm{c}$ \\
\hline & AF 2 & $2,65 \mathrm{a}$ & $6,44 \mathrm{a}$ & $5,75 \mathrm{~b}$ & $7,12 \mathrm{a}$ & $6,30 \mathrm{~b}$ & $5,82 \mathrm{~b}$ & $7,02 \mathrm{~b}$ & $11,05 \mathrm{a}$ \\
\hline \multirow{2}{*}{ Weide } & Inger & $2,38 \mathrm{a}$ & $1,60 \mathrm{c}$ & $3,25 \mathrm{c}$ & $7,04 \mathrm{a}$ & $7,08 \mathrm{ab}$ & $6,71 \mathrm{~b}$ & $9,49 \mathrm{a}$ & $10,60 \mathrm{ab}$ \\
\hline & Tordis & $2,25 \mathrm{a}$ & $1,45 \mathrm{c}$ & $7,92 \mathrm{a}$ & $6,73 \mathrm{a}$ & $7,76 \mathrm{a}$ & $7,33 a b$ & $9,43 a b$ & $10,00 \mathrm{~b}$ \\
\hline
\end{tabular}

* Die H 275 wurde aufgrund einer kleinerer Wiederholungsanzahl als die restlichen Prüfglieder nicht in der Varianzanlyse mit Mittelwertvergleich berücksichtigt

Anhang 16: dGZ-Mittelwerte $\left[\mathrm{t}_{(\mathrm{atro})} \mathrm{ha}^{-1} \mathrm{a}^{-1}\right]$ der dritten Wuchsperiode der Pappelklone Max 1, H 275 und AF 2 sowie der Weidenklone Inger und Tordis im Bodenartcluster L (ungleiche Buchstaben zeigen signifikante Unterschiede innerhalb der Prüfglieder eines Standorts).

\begin{tabular}{ccccccccccc}
\hline & & \multicolumn{10}{c}{$\mathrm{dGZ}\left[\mathrm{t}_{\text {(atro }} \mathrm{ha}^{-1} \mathrm{a}^{-1}\right]$} \\
\cline { 3 - 11 } Gattung & Klon & Sto. Nr. & Sto. Nr. & Sto. Nr. & Sto. Nr. & Sto. Nr. & Sto. Nr. & Sto. Nr. & Sto. Nr. & Sto. Nr. \\
& & 8 & $35^{*}$ & 27 & 5 & 20 & 6 & 16 & 29 & 25 \\
\hline \multirow{2}{*}{ Pappel } & Max 1 & $0,45 \mathrm{a}$ & $1,55 \mathrm{a}$ & $1,02 \mathrm{~b}$ & $2,49 \mathrm{~b}$ & $2,06 \mathrm{ab}$ & $3,77 \mathrm{a}$ & $3,25 \mathrm{~b}$ & $8,41 \mathrm{a}$ & $9,61 \mathrm{a}$ \\
& H 275 & $0,46 \mathrm{a}$ & $0,26 *$ & $0,94 \mathrm{~b}$ & n.v. & $1,50 \mathrm{~b}$ & n.v. & n.v. & $7,49 \mathrm{a}$ & $9,07 \mathrm{a}$ \\
& AF 2 & $0,48 \mathrm{a}$ & $0,85 \mathrm{c}$ & n.v. & $6,69 \mathrm{a}$ & $1,48 \mathrm{~b}$ & $3,84 \mathrm{a}$ & $4,83 \mathrm{a}$ & $6,90 \mathrm{a}$ & $9,25 \mathrm{a}$ \\
\multirow{2}{*}{ Weide } & Inger & $0,29 \mathrm{a}$ & $1,85 \mathrm{a}$ & $1,46 \mathrm{a}$ & $2,37 \mathrm{~b}$ & $1,82 \mathrm{ab}$ & $2,76 \mathrm{~b}$ & $4,72 \mathrm{a}$ & $8,27 \mathrm{a}$ & $6,95 \mathrm{c}$ \\
& Tordis & $0,14 \mathrm{a}$ & $0,94 \mathrm{~b}$ & $1,12 \mathrm{~b}$ & n.v. & $1,96 \mathrm{a}$ & n.v. & $4,70 \mathrm{ab}$ & $8,08 \mathrm{a}$ & $8,01 \mathrm{~b}$ \\
\hline
\end{tabular}

* Auf dem Sto. Nr. 35 „Threnthorst“ wurde der Klon H 275 nicht in den Mittelwertvergleich miteinbezogen aufgrund einer deutlich schwächeren Überlebensrate.

** Die H 275 wurde aufgrund einer kleinerer Wiederholungsanzahl als die restlichen Prüfglieder nicht in der Varianzanlyse mit Mittelwertvergleich berücksichtigt. 
Anhang 17: Statistische Kennwerte der Biomassefunktionen für Max 1 mit der allometrischen Anpassung und der d1,3 als unabhängige Variable.

\begin{tabular}{cccccccc}
\hline Sto. Nr. & $\begin{array}{c}\text { Höhe } \\
{[\mathrm{m}]}\end{array}$ & $\mathrm{n}$ & $\mathrm{R}^{2}$ & $\mathrm{R}^{2}$ korr & $\mathrm{p}$ & $\mathrm{a}_{0}$ & $\mathrm{a}_{1}$ \\
\hline 3 & 6,92 & 56 & 0,98 & 0,98 & 0,000 & 0,089 & 2,202 \\
4 & 5,04 & 20 & 0,98 & 0,98 & 0,000 & 0,137 & 1,905 \\
5 & 2,30 & 9 & 0,85 & 0,81 & 0,000 & 0,182 & 1,739 \\
6 & 4,88 & 56 & 0,96 & 0,96 & 0,000 & 0,112 & 2,181 \\
9 & 5,48 & 66 & 0,94 & 0,94 & 0,000 & 0,115 & 2,143 \\
10 & 5,71 & 30 & 0,98 & 0,98 & 0,000 & 0,105 & 2,253 \\
13 & 5,00 & 88 & 0,96 & 0,96 & 0,000 & 0,115 & 2,109 \\
15 & 4,37 & 74 & 0,97 & 0,97 & 0,000 & 0,099 & 2,364 \\
17 & 7,26 & 77 & 0,99 & 0,99 & 0,000 & 0,074 & 2,417 \\
18 & 2,34 & 47 & 0,87 & 0,86 & 0,000 & 0,173 & 1,662 \\
20 & 3,45 & 54 & 0,92 & 0,91 & 0,000 & 0,143 & 1,991 \\
22 & 5,14 & 55 & 0,99 & 0,99 & 0,000 & 0,110 & 2,141 \\
25 & 6,93 & 67 & 0,95 & 0,94 & 0,000 & 0,099 & 2,232 \\
31 & 6,40 & 72 & 0,95 & 0,95 & 0,000 & 0,108 & 2,180 \\
35 & 2,92 & 71 & 0,83 & 0,82 & 0,000 & 0,205 & 1,497 \\
36 & 5,43 & 21 & 0,99 & 0,99 & 0,000 & 0,116 & 2,183 \\
37 & 5,87 & 76 & 0,95 & 0,95 & 0,000 & 0,101 & 2,228 \\
\hline & & & & & & &
\end{tabular}


Anhang 18: Statistische Kennwerte der Biomassefunktionen für H 275 mit der allometrischen Anpassung und der $d_{1,3}$ als unabhängige Variable.

\begin{tabular}{cccccccc}
\hline Sto. Nr. & $\begin{array}{c}\text { Höhe } \\
{[\mathrm{m}]}\end{array}$ & $\mathrm{n}$ & $\mathrm{R}^{2}$ & $\mathrm{R}^{2}$ korr & $\mathrm{p}$ & $\mathrm{a}_{0}$ & $\mathrm{a}_{1}$ \\
\hline 1 & 6,40 & 79 & 0,84 & 0,83 & 0,000 & 0,148 & 1,948 \\
2 & 3,55 & 73 & 0,95 & 0,94 & 0,000 & 0,099 & 2,192 \\
3 & 5,90 & 67 & 0,97 & 0,97 & 0,000 & 0,083 & 2,291 \\
6 & 2,66 & 14 & 0,98 & 0,98 & 0,000 & 0,158 & 1,916 \\
9 & 4,52 & 53 & 0,93 & 0,93 & 0,000 & 0,149 & 1,982 \\
10 & 4,43 & 19 & 0,98 & 0,97 & 0,000 & 0,131 & 2,147 \\
13 & 4,66 & 89 & 0,94 & 0,94 & 0,000 & 0,135 & 2,028 \\
14 & 1,46 & 6 & 0,88 & 0,81 & 0,005 & 0,200 & 1,832 \\
15 & 4,02 & 86 & 0,92 & 0,92 & 0,000 & 0,111 & 2,215 \\
16 & 2,61 & 24 & 0,84 & 0,83 & 0,000 & 0,140 & 1,963 \\
17 & 6,75 & 82 & 0,99 & 0,99 & 0,000 & 0,093 & 2,289 \\
20 & 3,37 & 54 & 0,81 & 0,80 & 0,000 & 0,140 & 2,015 \\
22 & 5,34 & 73 & 0,97 & 0,97 & 0,000 & 0,123 & 2,069 \\
25 & 6,76 & 73 & 0,92 & 0,92 & 0,000 & 0,079 & 2,385 \\
31 & 6,54 & 71 & 0,97 & 0,97 & 0,000 & 0,109 & 2,141 \\
32 & 3,16 & 80 & 0,87 & 0,87 & 0,000 & 0,159 & 1,788 \\
\hline
\end{tabular}


Anhang 19: Statistische Kennwerte der Biomassefunktionen für AF 2 mit der allometrischen Anpassung und der $d_{1,3}$ als unabhängige Variable.

\begin{tabular}{cccccccc}
\hline Sto. Nr. & Höhe [m] & $\mathrm{n}$ & $\mathrm{R}^{2}$ & $\mathrm{R}_{\text {korr }}^{2}$ & $\mathrm{p}$ & $\mathrm{a}_{0}$ & $\mathrm{a}_{1}$ \\
\hline 1 & 6,39 & 64 & 0,96 & 0,95 & 0,000 & 0,088 & 2,152 \\
3 & 6,89 & 36 & 0,96 & 0,95 & 0,000 & 0,044 & 2,551 \\
4 & 4,89 & 24 & 0,85 & 0,83 & 0,000 & 0,126 & 1,741 \\
5 & 3,54 & 32 & 0,80 & 0,79 & 0,000 & 0,144 & 1,607 \\
6 & 4,73 & 34 & 0,96 & 0,96 & 0,000 & 0,089 & 2,121 \\
9 & 5,11 & 54 & 0,98 & 0,98 & 0,000 & 0,068 & 2,250 \\
10 & 5,56 & 22 & 0,92 & 0,92 & 0,000 & 0,079 & 2,142 \\
13 & 4,81 & 82 & 0,97 & 0,97 & 0,000 & 0,072 & 2,292 \\
15 & 5,37 & 89 & 0,96 & 0,96 & 0,000 & 0,079 & 2,328 \\
16 & 4,51 & 23 & 0,87 & 0,85 & 0,000 & 0,074 & 2,224 \\
17 & 7,13 & 83 & 0,96 & 0,96 & 0,000 & 0,085 & 2,190 \\
18 & 2,36 & 45 & 0,90 & 0,89 & 0,000 & 0,099 & 1,754 \\
20 & 3,67 & 62 & 0,94 & 0,94 & 0,000 & 0,120 & 1,849 \\
22 & 5,77 & 67 & 0,95 & 0,94 & 0,000 & 0,055 & 2,500 \\
25 & 7,27 & 66 & 0,97 & 0,96 & 0,000 & 0,053 & 2,501 \\
31 & 6,18 & 62 & 0,96 & 0,96 & 0,000 & 0,059 & 2,434 \\
32 & 3,55 & 75 & 0,93 & 0,92 & 0,000 & 0,127 & 1,657 \\
35 & 2,17 & 70 & 0,81 & 0,80 & 0,000 & 0,133 & 1,645 \\
36 & 5,97 & 17 & 0,95 & 0,94 & 0,000 & 0,062 & 2,337 \\
37 & 5,11 & 66 & 0,97 & 0,97 & 0,000 & 0,085 & 2,178 \\
\hline & & & & & & &
\end{tabular}

Anhang 20: Statistische Kennwerte der Biomassefunktionen für Inger mit der allometrischen Anpassung und der $\mathrm{d}_{1,3}$ als unabhängige Variable.

\begin{tabular}{cccccccc}
\hline Sto. Nr. & Höhe [m] & $\mathrm{n}$ & $\mathrm{R}^{2}$ & $\mathrm{R}^{2}$ korr & $\mathrm{p}$ & $\mathrm{a}_{0}$ & $\mathrm{a}_{1}$ \\
\hline 2 & 3,55 & 12 & 0,93 & 0,91 & 0,000 & 0,105 & 2,205 \\
11 & 2,76 & 11 & 0,84 & 0,80 & 0,000 & 0,144 & 1,792 \\
13 & 4,49 & 30 & 0,91 & 0,90 & 0,000 & 0,111 & 2,230 \\
15 & 5,21 & 64 & 0,96 & 0,96 & 0,000 & 0,087 & 2,361 \\
16 & 2,76 & 6 & 0,98 & 0,97 & 0,000 & 0,118 & 2,032 \\
18 & 2,47 & 30 & 0,84 & 0,83 & 0,000 & 0,138 & 1,999 \\
20 & 4,02 & 46 & 0,89 & 0,88 & 0,000 & 0,128 & 2,008 \\
22 & 4,75 & 39 & 0,89 & 0,88 & 0,000 & 0,104 & 2,308 \\
25 & 6,60 & 52 & 0,93 & 0,92 & 0,000 & 0,076 & 2,487 \\
32 & 4,09 & 73 & 0,89 & 0,89 & 0,000 & 0,104 & 2,106 \\
35 & 2,57 & 46 & 0,87 & 0,86 & 0,000 & 0,126 & 1,974 \\
37 & 5,76 & 13 & 0,99 & 0,99 & 0,000 & 0,117 & 2,047 \\
\hline
\end{tabular}


Anhang 21: Statistische Kennwerte der Biomassefunktionen für Tordis mit der allometrischen Anpassung und der $\mathrm{d}_{1,3}$ als unabhängige Variable.

\begin{tabular}{cccccccc}
\hline Sto. Nr. & Höhe $[\mathrm{m}]$ & $\mathrm{n}$ & $\mathrm{R}^{2}$ & $\mathrm{R}^{2}$ korr & $\mathrm{p}$ & $\mathrm{a}_{0}$ & $\mathrm{a}_{1}$ \\
\hline 3 & 6,89 & 37 & 0,97 & 0,96 & 0,000 & 0,102 & 2,374 \\
9 & 4,39 & 12 & 0,92 & 0,90 & 0,000 & 0,138 & 1,898 \\
10 & 6,20 & 14 & 0,92 & 0,90 & 0,000 & 0,134 & 2,187 \\
11 & 3,05 & 15 & 0,95 & 0,94 & 0,000 & 0,108 & 2,181 \\
15 & 5,20 & 51 & 0,92 & 0,92 & 0,000 & 0,109 & 2,416 \\
17 & 7,60 & 25 & 0,99 & 0,99 & 0,000 & 0,089 & 2,493 \\
20 & 4,58 & 26 & 0,87 & 0,86 & 0,000 & 0,109 & 2,427 \\
31 & 6,80 & 9 & 0,99 & 0,99 & 0,000 & 0,081 & 2,525 \\
32 & 4,06 & 70 & 0,93 & 0,93 & 0,000 & 0,113 & 2,206 \\
35 & 3,62 & 41 & 0,80 & 0,79 & 0,000 & 0,112 & 2,035 \\
37 & 6,35 & 38 & 0,94 & 0,94 & 0,000 & 0,103 & 2,371 \\
\hline
\end{tabular}


Anhang 22: Statistische Kennwerte der Biomassefunktionen für Max 1 mit der allometrischen Anpassung und der $\mathrm{d}_{0,1}$ als unabhängige Variable.

\begin{tabular}{|c|c|c|c|c|c|c|c|}
\hline Sto. Nr. & Höhe [m] & $\mathrm{n}$ & $\mathrm{R}^{2}$ & $\mathrm{R}_{\text {korr }}$ & $\mathrm{p}$ & $\mathrm{a}_{0}$ & $a_{1}$ \\
\hline 3 & 6,92 & 58 & 0,96 & 0,96 & 0,000 & 0,025 & 2,622 \\
\hline 4 & 5,04 & 22 & 0,95 & 0,95 & 0,000 & 0,027 & 2,570 \\
\hline 5 & 6,93 & 27 & 0,83 & 0,81 & 0,000 & 0,034 & 2,227 \\
\hline 6 & 4,88 & 57 & 0,95 & 0,94 & 0,000 & 0,031 & 2,467 \\
\hline 8 & 1,47 & 57 & 0,84 & 0,83 & 0,000 & 0,016 & 2,758 \\
\hline 9 & 5,48 & 68 & 0,92 & 0,91 & 0,000 & 0,024 & 2,520 \\
\hline 10 & 5,71 & 30 & 0,97 & 0,97 & 0,000 & 0,027 & 2,666 \\
\hline 11 & 1,83 & 38 & 0,91 & 0,90 & 0,000 & 0,024 & 2,660 \\
\hline 12 & 2,51 & 10 & 0,94 & 0,92 & 0,000 & 0,037 & 2,223 \\
\hline 13 & 5,00 & 89 & 0,93 & 0,93 & 0,000 & 0,032 & 2,573 \\
\hline 14 & 1,10 & 47 & 0,96 & 0,95 & 0,000 & 0,022 & 2,570 \\
\hline 15 & 4,37 & 94 & 0,95 & 0,95 & 0,000 & 0,019 & 2,893 \\
\hline 17 & 7,26 & 78 & 0,96 & 0,96 & 0,000 & 0,053 & 2,368 \\
\hline 18 & 2,34 & 74 & 0,95 & 0,95 & 0,000 & 0,015 & 2,905 \\
\hline 20 & 3,45 & 61 & 0,94 & 0,94 & 0,000 & 0,021 & 2,968 \\
\hline 22 & 5,14 & 57 & 0,98 & 0,97 & 0,000 & 0,021 & 2,923 \\
\hline 23 & 1,20 & 41 & 0,93 & 0,93 & 0,000 & 0,020 & 2,875 \\
\hline 25 & 6,93 & 67 & 0,93 & 0,93 & 0,000 & 0,077 & 2,069 \\
\hline 31 & 6,40 & 72 & 0,94 & 0,94 & 0,000 & 0,069 & 2,205 \\
\hline 32 & 3,09 & 85 & 0,95 & 0,95 & 0,000 & 0,039 & 2,238 \\
\hline 33 & 1,91 & 45 & 0,82 & 0,81 & 0,000 & 0,035 & 2,214 \\
\hline 35 & 2,92 & 87 & 0,96 & 0,95 & 0,000 & 0,021 & 2,902 \\
\hline 36 & 5,43 & 21 & 0,98 & 0,98 & 0,000 & 0,035 & 2,495 \\
\hline 37 & 5,87 & 76 & 0,96 & 0,96 & 0,000 & 0,054 & 2,293 \\
\hline
\end{tabular}


Anhang 23: Statistische Kennwerte der Biomassefunktionen für H 275 mit der allometrischen Anpassung und der $\mathrm{d}_{0,1}$ als unabhängige Variable.

\begin{tabular}{|c|c|c|c|c|c|c|c|}
\hline Sto. Nr. & Höhe [m] & $\mathrm{n}$ & $\mathrm{R}^{2}$ & $\mathrm{R}_{\text {korr }}$ & $\mathrm{p}$ & $\mathrm{a}_{0}$ & $a_{1}$ \\
\hline 1 & 6,40 & 80 & 0,86 & 0,86 & 0,000 & 0,070 & 2,117 \\
\hline 2 & 3,55 & 81 & 0,93 & 0,93 & 0,000 & 0,033 & 2,512 \\
\hline 3 & 3,87 & 67 & 0,93 & 0,93 & 0,000 & 0,035 & 2,395 \\
\hline 6 & 2,66 & 41 & 0,88 & 0,87 & 0,000 & 0,015 & 2,944 \\
\hline 8 & 1,61 & 69 & 0,89 & 0,88 & 0,000 & 0,020 & 2,611 \\
\hline 9 & 4,52 & 59 & 0,91 & 0,91 & 0,000 & 0,027 & 2,413 \\
\hline 10 & 4,43 & 20 & 0,96 & 0,96 & 0,000 & 0,022 & 2,722 \\
\hline 11 & 2,36 & 43 & 0,89 & 0,88 & 0,000 & 0,020 & 2,731 \\
\hline 12 & 2,59 & 20 & 0,81 & 0,78 & 0,000 & 0,017 & 2,731 \\
\hline 13 & 4,66 & 89 & 0,94 & 0,94 & 0,000 & 0,038 & 2,379 \\
\hline 14 & 1,46 & 81 & 0,97 & 0,97 & 0,000 & 0,024 & 2,667 \\
\hline 15 & 4,02 & 93 & 0,96 & 0,96 & 0,000 & 0,031 & 2,471 \\
\hline 16 & 2,61 & 62 & 0,90 & 0,90 & 0,000 & 0,026 & 2,614 \\
\hline 17 & 6,75 & 84 & 0,98 & 0,98 & 0,000 & 0,032 & 2,629 \\
\hline 18 & 1,63 & 74 & 0,91 & 0,91 & 0,000 & 0,024 & 2,500 \\
\hline 20 & 3,37 & 55 & 0,85 & 0,84 & 0,000 & 0,028 & 2,636 \\
\hline 22 & 5,34 & 74 & 0,93 & 0,93 & 0,000 & 0,035 & 2,530 \\
\hline 23 & 1,55 & 59 & 0,88 & 0,87 & 0,000 & 0,025 & 2,475 \\
\hline 25 & 6,76 & 73 & 0,88 & 0,88 & 0,000 & 0,038 & 2,447 \\
\hline 31 & 6,54 & 76 & 0,99 & 0,99 & 0,000 & 0,045 & 2,460 \\
\hline 32 & 3,16 & 82 & 0,95 & 0,95 & 0,000 & 0,041 & 2,281 \\
\hline 33 & 1,54 & 34 & 0,89 & 0,89 & 0,000 & 0,025 & 2,652 \\
\hline 35 & 2,71 & 36 & 0,95 & 0,94 & 0,000 & 0,020 & 2,729 \\
\hline
\end{tabular}


Anhang 24: Statistische Kennwerte der Biomassefunktionen für AF 2 mit der allometrischen Anpassung und der $\mathrm{d}_{0,1}$ als unabhängige Variable.

\begin{tabular}{|c|c|c|c|c|c|c|c|}
\hline Sto. Nr. & Höhe [m] & $\mathrm{n}$ & $\mathrm{R}^{2}$ & $\mathrm{R}_{\text {korr }}^{2}$ & $\mathrm{p}$ & $\mathrm{a}_{0}$ & $a_{1}$ \\
\hline 1 & 6,39 & 65 & 0,94 & 0,94 & 0,000 & 0,048 & 2,362 \\
\hline 3 & 6,89 & 36 & 0,90 & 0,89 & 0,000 & 0,023 & 2,585 \\
\hline 4 & 4,89 & 24 & 0,82 & 0,80 & 0,000 & 0,020 & 2,714 \\
\hline 5 & 3,54 & 35 & 0,91 & 0,90 & 0,000 & 0,030 & 2,297 \\
\hline 6 & 4,73 & 34 & 0,93 & 0,93 & 0,000 & 0,038 & 2,202 \\
\hline 8 & 1,69 & 66 & 0,83 & 0,83 & 0,000 & 0,016 & 2,784 \\
\hline 9 & 5,11 & 54 & 0,94 & 0,94 & 0,000 & 0,026 & 2,452 \\
\hline 10 & 5,56 & 25 & 0,93 & 0,93 & 0,000 & 0,017 & 2,739 \\
\hline 11 & 2,32 & 17 & 0,94 & 0,94 & 0,000 & 0,021 & 2,605 \\
\hline 13 & 4,81 & 83 & 0,94 & 0,94 & 0,000 & 0,029 & 2,542 \\
\hline 14 & 1,41 & 58 & 0,94 & 0,93 & 0,000 & 0,021 & 2,702 \\
\hline 15 & 5,37 & 94 & 0,96 & 0,96 & 0,000 & 0,031 & 2,574 \\
\hline 16 & 4,51 & 24 & 0,84 & 0,83 & 0,000 & 0,026 & 2,378 \\
\hline 17 & 7,13 & 83 & 0,92 & 0,92 & 0,000 & 0,051 & 2,286 \\
\hline 18 & 2,36 & 79 & 0,93 & 0,93 & 0,000 & 0,018 & 2,681 \\
\hline 20 & 3,67 & 65 & 0,95 & 0,95 & 0,000 & 0,027 & 2,756 \\
\hline 22 & 5,77 & 69 & 0,94 & 0,94 & 0,000 & 0,020 & 2,898 \\
\hline 23 & 1,27 & 73 & 0,93 & 0,93 & 0,000 & 0,020 & 2,471 \\
\hline 25 & 7,27 & 66 & 0,93 & 0,92 & 0,000 & 0,054 & 2,228 \\
\hline 31 & 6,18 & 62 & 0,94 & 0,94 & 0,000 & 0,034 & 2,566 \\
\hline 32 & 3,55 & 79 & 0,94 & 0,94 & 0,000 & 0,039 & 2,211 \\
\hline 33 & 2,23 & 74 & 0,86 & 0,86 & 0,000 & 0,028 & 2,240 \\
\hline 35 & 2,17 & 83 & 0,95 & 0,95 & 0,000 & 0,017 & 2,926 \\
\hline 36 & 5,97 & 17 & 0,96 & 0,95 & 0,000 & 0,043 & 2,304 \\
\hline 37 & 5,11 & 67 & 0,92 & 0,91 & 0,000 & 0,032 & 2,488 \\
\hline
\end{tabular}


Anhang 25: Statistische Kennwerte der Biomassefunktionen für Inger mit der allometrischen Anpassung und der $\mathrm{d}_{0,1}$ als unabhängige Variable.

\begin{tabular}{cccccccc}
\hline Sto. Nr. & Höhe $[\mathrm{m}]$ & $\mathrm{n}$ & $\mathrm{R}^{2}$ & $\mathrm{R}^{2}$ korr & $\mathrm{p}$ & $\mathrm{a}_{0}$ & $\mathrm{a}_{1}$ \\
\hline 2 & 3,87 & 22 & 0,86 & 0,85 & 0,000 & 0,032 & 2,550 \\
5 & 2,91 & 6 & 0,95 & 0,91 & 0,000 & 0,026 & 2,957 \\
11 & 2,76 & 17 & 0,96 & 0,95 & 0,000 & 0,027 & 2,904 \\
13 & 4,49 & 30 & 0,82 & 0,81 & 0,000 & 0,057 & 2,153 \\
14 & 1,60 & 35 & 0,91 & 0,91 & 0,000 & 0,029 & 2,426 \\
15 & 5,21 & 65 & 0,94 & 0,93 & 0,000 & 0,040 & 2,429 \\
17 & 7,33 & 30 & 0,85 & 0,84 & 0,000 & 0,117 & 1,921 \\
18 & 2,47 & 39 & 0,94 & 0,94 & 0,000 & 0,018 & 3,087 \\
20 & 4,02 & 50 & 0,85 & 0,85 & 0,000 & 0,045 & 2,183 \\
22 & 4,75 & 39 & 0,88 & 0,87 & 0,000 & 0,018 & 3,058 \\
25 & 6,60 & 52 & 0,91 & 0,91 & 0,000 & 0,031 & 2,631 \\
32 & 4,09 & 80 & 0,90 & 0,89 & 0,000 & 0,047 & 2,194 \\
35 & 2,57 & 48 & 0,97 & 0,97 & 0,000 & 0,030 & 2,556 \\
37 & 5,76 & 14 & 0,96 & 0,95 & 0,000 & 0,049 & 2,300 \\
\hline
\end{tabular}

Anhang 26: Statistische Kennwerte der Biomassefunktionen für Tordis mit der allometrischen Anpassung und der $\mathrm{d}_{0,1}$ als unabhängige Variable.

\begin{tabular}{|c|c|c|c|c|c|c|c|}
\hline Sto. Nr. & Höhe [m] & $\mathrm{n}$ & $\mathrm{R}^{2}$ & $\mathrm{R}_{\text {korr }}^{2}$ & $\mathrm{p}$ & $a_{0}$ & $a_{1}$ \\
\hline 1 & 7,12 & 16 & 0,91 & 0,90 & 0,000 & 0,104 & 2,011 \\
\hline 3 & 6,89 & 37 & 0,97 & 0,96 & 0,000 & 0,045 & 2,495 \\
\hline 9 & 4,39 & 12 & 0,84 & 0,80 & 0,000 & 0,033 & 2,490 \\
\hline 10 & 6,20 & 17 & 0,96 & 0,96 & 0,000 & 0,026 & 2,849 \\
\hline 11 & 3,05 & 23 & 0,89 & 0,88 & 0,000 & 0,040 & 2,299 \\
\hline 13 & 4,81 & 20 & 0,95 & 0,94 & 0,000 & 0,043 & 2,443 \\
\hline 14 & 1,55 & 30 & 0,85 & 0,84 & 0,000 & 0,032 & 2,892 \\
\hline 15 & 5,20 & 53 & 0,97 & 0,97 & 0,000 & 0,036 & 2,773 \\
\hline 17 & 7,60 & 25 & 0,98 & 0,98 & 0,000 & 0,026 & 3,051 \\
\hline 18 & 1,83 & 63 & 0,90 & 0,90 & 0,000 & 0,019 & 3,175 \\
\hline 20 & 4,58 & 30 & 0,93 & 0,93 & 0,000 & 0,027 & 2,985 \\
\hline 31 & 6,91 & 11 & 0,96 & 0,95 & 0,000 & 0,070 & 2,324 \\
\hline 32 & 4,06 & 76 & 0,94 & 0,94 & 0,000 & 0,045 & 2,448 \\
\hline 33 & 2,07 & 64 & 0,85 & 0,84 & 0,000 & 0,023 & 3,095 \\
\hline 35 & 3,62 & 46 & 0,95 & 0,95 & 0,000 & 0,031 & 2,767 \\
\hline 37 & 6,35 & 38 & 0,94 & 0,94 & 0,000 & 0,056 & 2,407 \\
\hline
\end{tabular}


Anhang 27: Allgemeingültige Biomassefunktionen für die Bestandesdichte 11.111 Stk ha ${ }^{-1}$ der Pappelklone Max 1, H 275 , AF 2 sowie der Weidenklone Inger und Tordis mit dem $\mathrm{d}_{0,1}$ als unabhängige Variable.

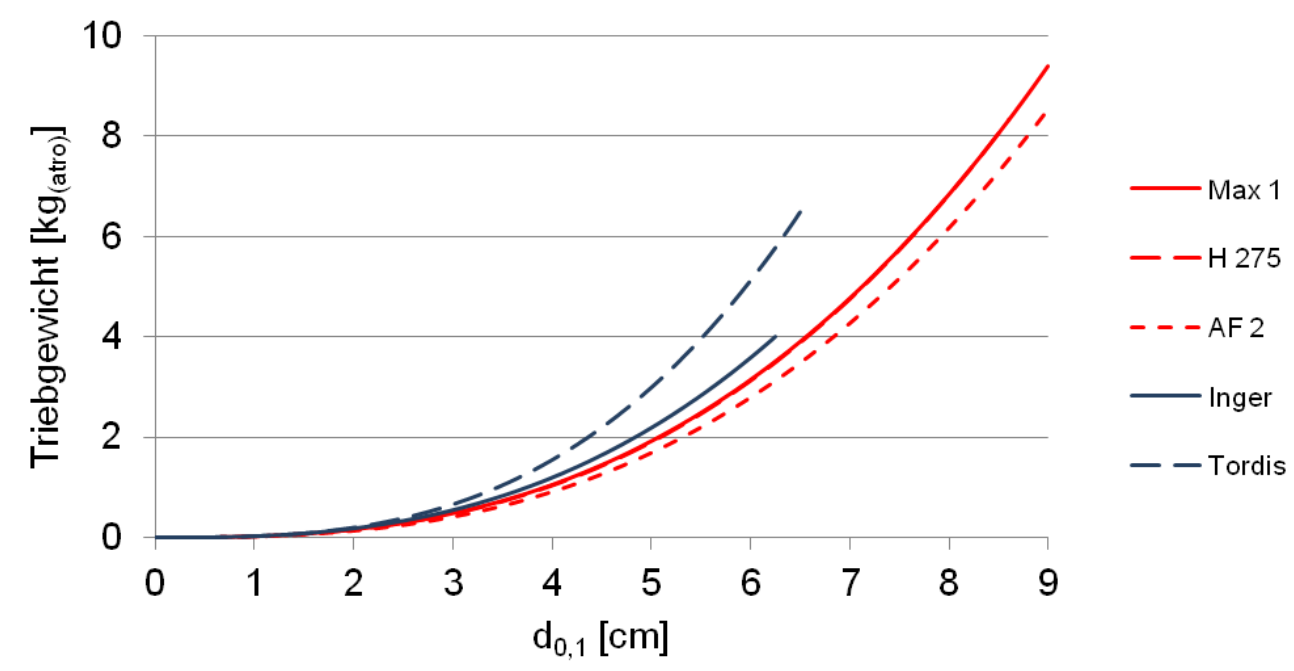

Anhang 28: Statistische Kennwerte der Biomassefunktionen der Pappelklone Max 1, H 275, AF 2 sowie der Weidenklone Inger und Tordis mit dem $\mathrm{d}_{0,1}$ als unabhängige Variable.

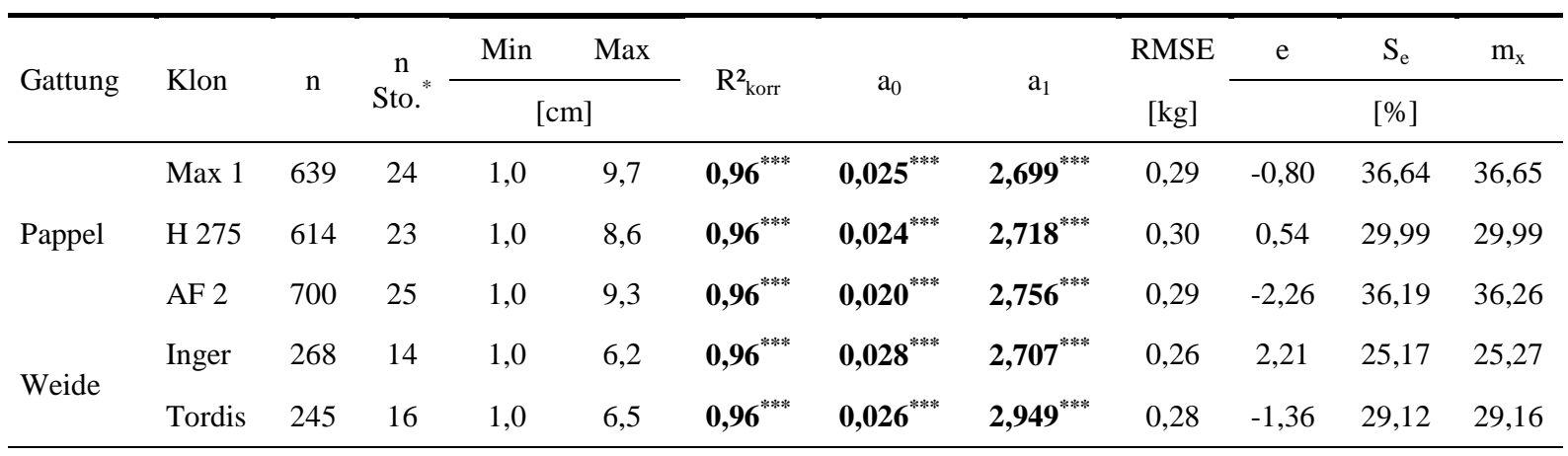


Anhang 29: Korrelationsanalyse zwischen Porenraumvariablen hergeleitet beim Feuchtigskeitsäquivalenten sowie nach dem Standard beim $\mathrm{pF}$ 1,8 und dem dGZ für die untersuchten Klone in den Standortclustern ALL, S, U und L.

\begin{tabular}{|c|c|c|c|c|c|c|c|c|}
\hline \multirow{2}{*}{$\begin{array}{l}\text { Standort- } \\
\text { cluster }\end{array}$} & \multirow{2}{*}{ Gattung } & \multirow{2}{*}{ Klon } & \multirow{2}{*}{$\mathrm{n}$} & \multicolumn{5}{|c|}{$\mathrm{R}$} \\
\hline & & & & nFK FÄ & LK FÄ & nFK & LK & $\mathrm{nFK}+\mathrm{LK}$ \\
\hline \multirow{4}{*}{ ALL } & \multirow{2}{*}{ Pappel } & Max 1 & 20 & $0,55^{*}$ & $-0,08$ & $0,52^{*}$ & $-0,12$ & 0,15 \\
\hline & & $\mathrm{AF} 2$ & 20 & $0,61^{* *}$ & $-0,22$ & $0,61^{* *}$ & $-0,22$ & 0,10 \\
\hline & \multirow{2}{*}{ Weide } & Inger & 20 & $0,61^{* *}$ & $-0,05$ & $0,56^{*}$ & $-0,08$ & 0,21 \\
\hline & & Tordis & 18 & $0,66^{* *}$ & $-0,27$ & $0,61^{* *}$ & $-0,31$ & 0,01 \\
\hline \multirow{5}{*}{$S$} & \multirow{3}{*}{ Pappel } & Max 1 & 8 & 0,26 & $-0,69$ & 0,23 & $-0,66^{(*)}$ & $-0,61$ \\
\hline & & Н 275 & 7 & 0,12 & $-0,60$ & 0,08 & $-0,56$ & $-0,59$ \\
\hline & & AF 2 & 8 & 0,31 & $-0,69$ & 0,31 & $-0,69^{(*)}$ & $-0,60$ \\
\hline & \multirow{2}{*}{ Weide } & Inger & 8 & 0,44 & $-0,53$ & 0,36 & $-0,49$ & $-0,36$ \\
\hline & & Tordis & 8 & 0,49 & $-0,55$ & 0,41 & $-0,52$ & $-0,36$ \\
\hline \multirow{4}{*}{$\mathrm{U}$} & \multirow{2}{*}{ Pappel } & Max 1 & 5 & 0,14 & 0,66 & 0,25 & $0,81^{(*)}$ & 0,59 \\
\hline & & $\mathrm{AF} 2$ & 5 & 0,59 & 0,84 & 0,59 & $0,84^{(*)}$ & 0,77 \\
\hline & \multirow{2}{*}{ Weide } & Inger & 5 & 0,07 & 0,59 & 0,21 & 0,69 & 0,50 \\
\hline & & Tordis & 5 & 0,61 & 0,88 & 0,67 & $0,92^{*}$ & 0,86 \\
\hline \multirow{3}{*}{$\mathrm{L}$} & \multirow{2}{*}{ Pappel } & Max 1 & 7 & $0,85^{*}$ & 0,75 & $0,86^{*}$ & 0,60 & $0,78^{*}$ \\
\hline & & $\mathrm{AF} 2$ & 7 & $0,84^{*}$ & 0,49 & $0,84^{*}$ & 0,49 & 0,71 \\
\hline & Weide & Inger & 7 & $0,92^{* *}$ & $0,86^{*}$ & $0,93^{* *}$ & $\mathbf{0 , 8 0 ^ { * }}$ & $0,92^{* *}$ \\
\hline
\end{tabular}


Anhang 30: Analyse zur Variablen-Interaktion im Standortcluster ALL, $r>0,80$ sind unterstrichen angegeben.

\begin{tabular}{lccccccc}
\hline $\mathrm{r}$ & $\mathrm{U}_{[\%]}$ & $\mathrm{nFK}$ & $\mathrm{BZ}$ & $\mathrm{N}_{5,7}$ & $\mathrm{~N}_{6,7}$ & $\mathrm{TI}_{5,7}$ & $\mathrm{TI}_{6,7}$ \\
\hline $\mathrm{U}_{[\%]}$ & 1 & $\mathbf{0 , 6 1 *}^{* *}$ & $\underline{\mathbf{0 , 8 0}^{* *}}$ & 0,18 & 0,21 & 0,20 & 0,23 \\
$\mathrm{nFK}$ & & 1 & $\mathbf{0 , 6 6 * *}^{* *}$ & $-0,04$ & 0,14 & $-0,02$ & 0,18 \\
$\mathrm{BZ}$ & & & 1 & 0,18 & 0,12 & 0,15 & 0,08 \\
$\mathrm{~N}_{5,7}$ & & & & 1 & $\underline{\mathbf{0 , 8 6}}$ & $\underline{\mathbf{0 , 9 4}^{* *}}$ & $\mathbf{0 , 7 7 ^ { * * * }}$ \\
$\mathrm{N}_{6,7}$ & & & & & 1 & $\mathbf{0 , 7 9}^{* *}$ & $\underline{\mathbf{0 , 9 7}}$ \\
$\mathrm{TI}_{5,7}$ & & & & & & 1 & $\mathbf{0 , 7 8 * *}$ \\
$\mathrm{TI}_{6,7}$ & & & & & & & 1 \\
\hline
\end{tabular}

Anhang 31: Analyse zur Variablen-Interaktion im Standortcluster $S, r>0,80$ sind unterstrichen angegeben.

\begin{tabular}{lcccccccc}
\hline $\mathrm{r}$ & $\mathrm{S}_{[\%]}$ & $\mathrm{U}_{[\%]}$ & $\mathrm{LK}$ & $\mathrm{BZ}$ & $\mathrm{T}_{7,8}$ & $\mathrm{~T}_{7,9}$ & $\mathrm{~N}_{6,7}$ & $\mathrm{TI}_{6,7}$ \\
\hline $\mathrm{S}_{[\%]}$ & 1 & $\underline{\mathbf{- 0 , 9 7 ^ { * * }}}$ & $\mathbf{0 , 7 3 ^ { * }}$ & $-0,49$ & 0,08 & 0,10 & $\mathbf{- 0 , 7 1 *}^{*}$ & $\mathbf{- 0 , 7 2 ^ { * }}$ \\
$\mathrm{U}_{[\%]}$ & & 1 & $-0,68$ & 0,43 & $-0,05$ & $-0,06$ & 0,60 & 0,61 \\
$\mathrm{LK}$ & & & 1 & $\mathbf{- 0 , 7 3 ^ { * }}$ & $-0,07$ & $-0,07$ & $-0,59$ & $-0,55$ \\
$\mathrm{BZ}$ & & & & 1 & $-0,42$ & $-0,43$ & 0,19 & 0,25 \\
$\mathrm{~T}_{7,8}$ & & & & & 1 & $\underline{\mathbf{0 , 9 9 *}}$ & $-0,14$ & $-0,27$ \\
$\mathrm{~T}_{7,9}$ & & & & & & 1 & $-0,13$ & $-0,27$ \\
$\mathrm{~N}_{6,7}$ & & & & & & & 1 & $\mathbf{0 , 9 9 *}$ \\
$\mathrm{TI}_{6,7}$ & & & & & & & & 1 \\
\hline
\end{tabular}


Anhang 32: Analyse zur Variablen-Interaktion im Standortcluster U, r $>0,80$ sind unterstrichen angegeben.

\begin{tabular}{lccccccccc}
\hline $\mathrm{r}$ & $\mathrm{LK}$ & $\mathrm{TRD}$ & $\mathrm{BZ}$ & $\mathrm{T}_{7,8}$ & $\mathrm{~T}_{7,9}$ & $\mathrm{~N}_{4,5}$ & $\mathrm{~N}_{5,6}$ & $\mathrm{TI}_{4,5}$ & $\mathrm{TI}_{5,6}$ \\
\hline $\mathrm{LK}$ & 1 & $\underline{\mathbf{- 0 , 9 9 * *}}$ & $\underline{\mathbf{0 , 9 4}}$ & 0,44 & 0,53 & 0,42 & 0,70 & 0,45 & $\underline{0,82}$ \\
$\mathrm{TRD}$ & & 1 & $\underline{\underline{\mathbf{0 , 9 2}}}$ & $-0,41$ & $-0,51$ & $-0,41$ & $-0,72$ & $-0,43$ & $\underline{-0,83}$ \\
$\mathrm{BZ}$ & & & 1 & 0,56 & 0,67 & 0,51 & 0,60 & 0,54 & 0,72 \\
$\mathrm{~T}_{7,8}$ & & & & 1 & $\underline{\mathbf{0 , 9 8}}$ & $\underline{\mathbf{0 , 9 6}}$ & 0,64 & $\underline{\mathbf{0 , 9 8}}$ & 0,65 \\
$\mathrm{~T}_{7,9}$ & & & & & 1 & $\underline{\mathbf{0 , 9 6}}$ & 0,68 & $\underline{\mathbf{0 , 9 7}}$ & 0,70 \\
$\mathrm{~N}_{4,5}$ & & & & & & 1 & 0,78 & $\underline{\mathbf{0 , 9 9 *}}$ & 0,74 \\
$\mathrm{~N}_{5,6}$ & & & & & & & 1 & 0,76 & $\underline{\mathbf{0 , 9 8}}$ \\
$\mathrm{TI}_{4,5}$ & & & & & & & & 1 & 0,74 \\
$\mathrm{TI}_{5,6}$ & & & & & & & & & 1 \\
\hline
\end{tabular}

Anhang 33: Analyse zur Variablen-Interaktion im Standortcluster L, r > 0,80 sind unterstrichen angegeben.

\begin{tabular}{lccccccc}
\hline $\mathrm{R}$ & $\mathrm{U}_{[\%]}$ & $\mathrm{nFK}$ & $\mathrm{LK}$ & $\mathrm{T}_{7,9}$ & $\mathrm{~T}_{\mathrm{VP}}$ & $\mathrm{N}_{5,6}$ & $\mathrm{TI}_{5,6}$ \\
\hline $\mathrm{U}_{[\%]}$ & 1 & $\underline{\mathbf{0 , 8 4}}$ & 0,65 & $-0,48$ & $-0,45$ & 0,68 & $\underline{\mathbf{0 , 8 3}^{*}}$ \\
$\mathrm{nFK}$ & & 1 & $\mathbf{0 , 7 8}^{*}$ & $-0,65$ & $-0,65$ & 0,28 & 0,57 \\
$\mathrm{LK}$ & & & 1 & $\underline{\underline{\mathbf{0 , 9 0 * *}}}$ & $\underline{\underline{\mathbf{0 , 8 4}}}$ & 0,17 & 0,65 \\
$\mathrm{~T}_{7,9}$ & & & & 1 & $\underline{\mathbf{0 , 9 8}}$ & 0,15 & $-0,46$ \\
$\mathrm{~T}_{\mathrm{VP}}$ & & & & & 1 & 0,24 & $-0,40$ \\
$\mathrm{~N}_{5,6}$ & & & & & & 1 & 0,75 \\
$\mathrm{TI}_{5,6}$ & & & & & & & 1 \\
\hline
\end{tabular}


Anhang 34: dGZ des Klons Max 1 bei Standorten unterschiedlicher Vornutzung in der dritten Wuchsperiode (deskriptiv berücksichtigte Flächen).

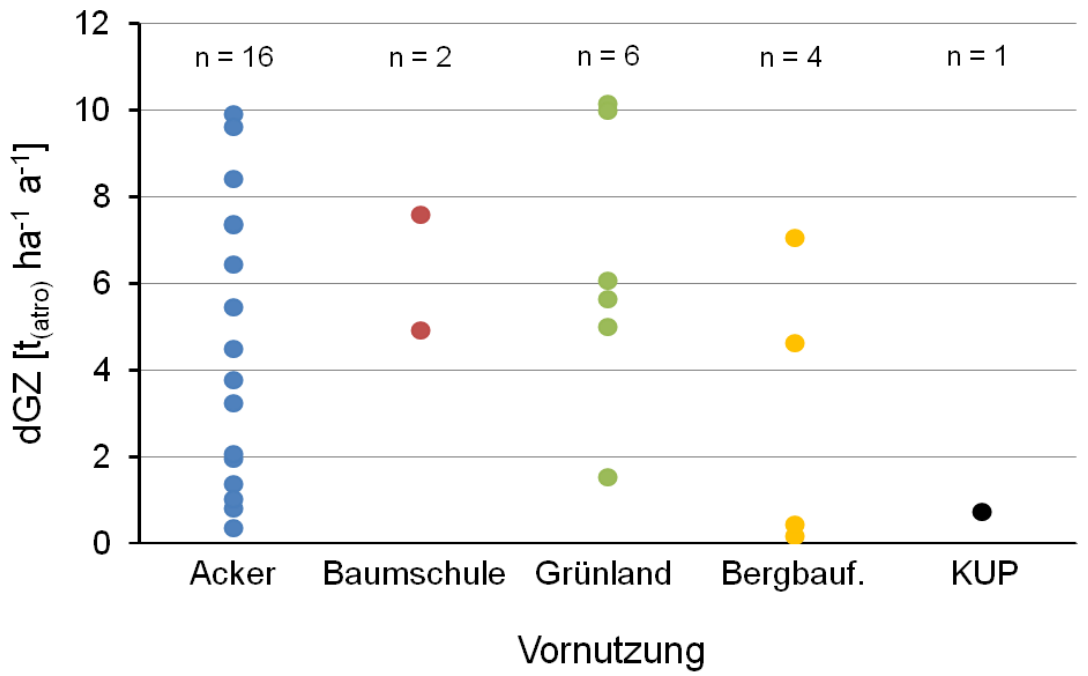

Anhang 35: dGZ des Klons Max 1 bei Standorten unterschiedlichen Bodentyps in der dritten Wuchsperiode (deskriptiv berücksichtigte Flächen).

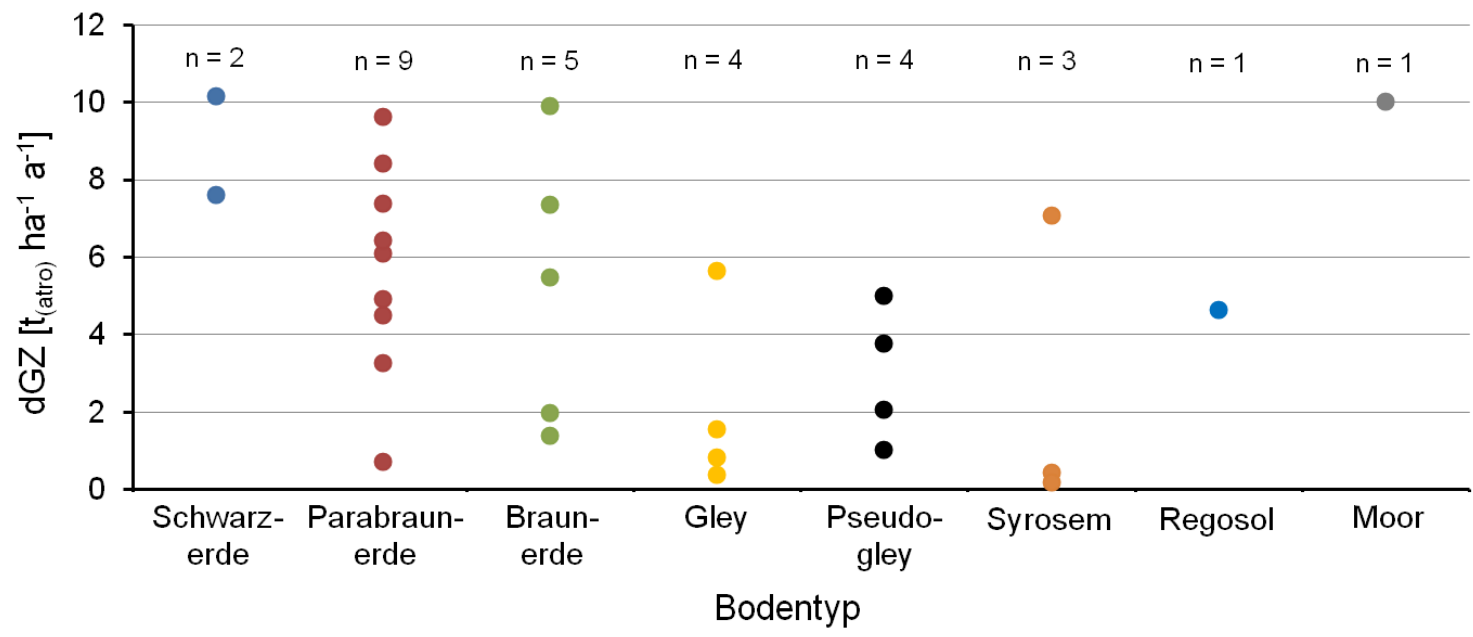

Anhang 36: Modelle in denen die multiple nicht-lineare Regressionen höhere $\mathrm{R}^{2}{ }_{\text {korr }}$ als die univariate Modelle erzielten.

\begin{tabular}{|c|c|c|c|c|}
\hline Standortcluster & Klon & Modellanpassung & Modellausdruck & $\mathrm{R}_{\text {korr }}^{2}$ \\
\hline \multirow{4}{*}{ ALL } & \multirow{2}{*}{ Max 1} & Univariate Reg. & $\mathrm{dGZ}=\mathrm{a} 0+\left(\mathrm{a} 1 /\left(\mathrm{nFK}^{*} \mathrm{TI}_{5,7}\right)\right)$ & 0,47 \\
\hline & & Mnlr & $\mathrm{dGZ}=\mathrm{a} 0+(\mathrm{a} 1 / \mathrm{nFK})+\left(\mathrm{a} 2 / \mathrm{TI}_{5,7}\right)$ & 0,48 \\
\hline & \multirow{2}{*}{ Tordis } & Univariate Reg. & $\mathrm{dGZ}=\mathrm{a} 0+\left(\mathrm{a} 1 /\left(\mathrm{nFK}^{*} \mathrm{TI}_{5,7}\right)\right)$ & 0,64 \\
\hline & & Mnlr & $\mathrm{dGZ}=\mathrm{a} 0+(\mathrm{a} 1 / \mathrm{nFK})+\left(\mathrm{a} 2 / \mathrm{TI}_{5,7}\right)$ & 0,65 \\
\hline \multirow{4}{*}{$\mathrm{S}$} & \multirow{2}{*}{ Inger } & Univariate Reg. & $\mathrm{dGZ}=\mathrm{a} 0+\mathrm{a} 1 *\left(\mathrm{~S}_{[\%]} * \mathrm{~T}_{7,8}\right)$ & 0,85 \\
\hline & & Mnlr & $\mathrm{dGZ}=\mathrm{a} 0+\left(\mathrm{a} 1 * \mathrm{~S}_{[\%]}\right)+\left(\mathrm{a} 2 / \mathrm{T}_{7,8}\right)$ & 0,89 \\
\hline & \multirow{2}{*}{ Tordis } & Univariate Reg. & $\mathrm{dGZ}=\mathrm{a} 0+\mathrm{a} 1 *\left(\mathrm{~S}_{[\%]} * \mathrm{~T}_{7,8}\right)$ & 0,81 \\
\hline & & Mnlr & $\mathrm{dGZ}=\mathrm{a} 0+\left(\mathrm{a} 1 * \mathrm{~S}_{[\%]}\right)+\left(\mathrm{a} 2 / \mathrm{T}_{7,8}\right)$ & 0,82 \\
\hline \multirow{2}{*}{$\mathrm{L}$} & \multirow{2}{*}{ Inger } & Univariate Reg. & $\mathrm{dGZ}=\mathrm{e}^{\wedge}(\mathrm{a} 0+\mathrm{a} 1 * \mathrm{nFK})$ & 0,98 \\
\hline & & Mnlr & $\mathrm{dGZ}=\mathrm{a} 0 *\left((\mathrm{a} 1 * \mathrm{nFK})+\left(\mathrm{a} 2^{\wedge}\left(\mathrm{N}_{5,6} * \mathrm{LK}\right)\right)\right)$ & 0,99 \\
\hline
\end{tabular}

Die mnlr (Multiple nicht lineare Regression) Modelle hatten keine komplett signifikante Parametersets 
Anhang 37:Amplitude der zur Modellierung verwendeten aggregierte Variablen.

\begin{tabular}{lccc}
\hline $\begin{array}{l}\text { Standort- } \\
\text { cluster }\end{array}$ & Modell & Min & Max \\
\hline \multirow{2}{*}{$\mathrm{ALL}$} & $\left(\mathrm{nFK} * \mathrm{TI}_{5,7}\right)$ & 43,4 & 112,1 \\
& $\left(\mathrm{nFK} * \mathrm{~N}_{5,7}\right)$ & 1093,3 & 3049,1 \\
\hline & $\left(\mathrm{U}_{[\%]} * \mathrm{~N}_{6,7}\right)$ & 351,1 & 4083,3 \\
$\mathrm{~S}$ & $\left(\mathrm{~S}_{[\%]} / \mathrm{TI}_{6,7}\right)$ & 12,2 & 27,9 \\
& $\left(\mathrm{~S}_{[\%]} * \mathrm{~T}_{7,8}\right)$ & 2494,5 & 3503,2 \\
\hline $\mathrm{U}$ & $(\mathrm{BZ})$ & 50 & 100 \\
\hline \multirow{2}{*}{$\mathrm{L}$} & $\left(\mathrm{BZ} * \mathrm{TI}_{4,5}\right)$ & 181,2 & 565,9 \\
\hline & $(\mathrm{nFK})$ & 7,3 & 11,1 \\
\hline
\end{tabular}

Anhang 38:Amplitude der zur Modellierung verwendeten nicht-aggregierte Variablen.

\begin{tabular}{lccc}
\hline $\begin{array}{l}\text { Standort- } \\
\text { cluster }\end{array}$ & Variable & Min & Max \\
\hline \multirow{2}{*}{ ALL } & $\mathrm{nFK}[\mathrm{cm}]$ & 6,3 & 14,3 \\
& $\mathrm{~N}_{5,7}[\mathrm{~mm}]$ & 144,0 & 232,3 \\
& $\mathrm{TI}_{5,7}$ & 5,7 & 9,0 \\
\hline \multirow{2}{*}{$\mathrm{S}$} & $\mathrm{U}[\%]$ & 3,5 & 27,0 \\
& $\mathrm{~N}_{6,7}[\mathrm{~mm}]$ & 86,9 & 151,3 \\
& $\mathrm{~S}[\%]$ & 65,0 & 91,4 \\
& $\mathrm{TI}_{6,7}$ & 3,1 & 5,4 \\
$\mathrm{U}$ & $\mathrm{BZ}^{*}$ & 50 & 100 \\
& $\mathrm{TI}_{4,5}$ & 3,6 & 6,2 \\
\hline & $\mathrm{nFK}[\mathrm{cm}]$ & 7,3 & 11,1 \\
& $\mathrm{~N}_{5,6}[\mathrm{~mm}]$ & 99,1 & 128,8 \\
\hline
\end{tabular}

